RECEIVED BY THC AUG IST

Department of Energy

Contract No. EG.77·C.01-4042

\title{
National Solar Energy Education Directory
}

\section{First Edition}

In cooperation with the of fice of U.S. Congressman George E. Brown, Jr., John Kimball, Staff Assistarit, and the Congressional Solar Coalition
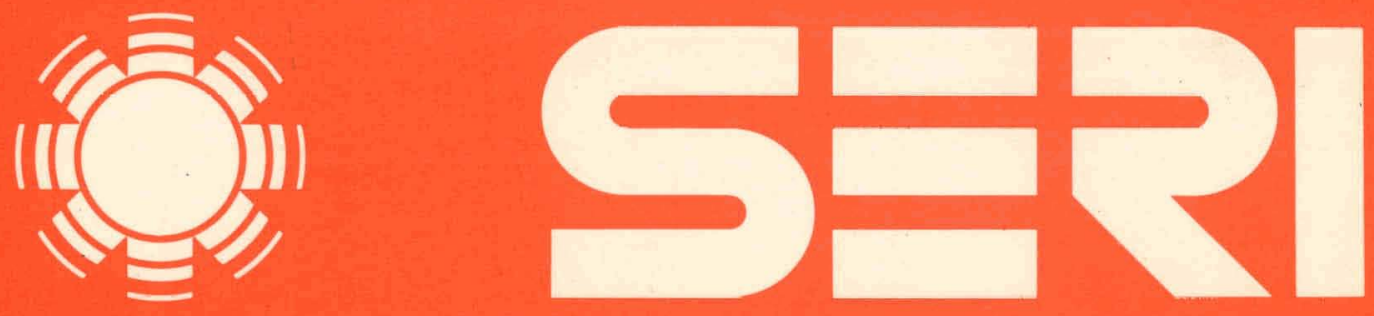

Prepared by

George Corcoleotes

Katherine Kramer

Kevin O'Connor

Jo Ann Silversmith

Solar Energy Research Institute

1536 Cole Boulevard

Golden, Colorado 80401

A Division of Midwest Research institute 


\section{DISCLAIMER}

This report was prepared as an account of work sponsored by an agency of the United States Government. Neither the United States Government nor any agency Thereof, nor any of their employees, makes any warranty, express or implied, or assumes any legal liability or responsibility for the accuracy, completeness, or usefulness of any information, apparatus, product, or process disclosed, or represents that its use would not infringe privately owned rights. Reference herein to any specific commercial product, process, or service by trade name, trademark, manufacturer, or otherwise does not necessarily constitute or imply its endorsement, recommendation, or favoring by the United States Government or any agency thereof. The views and opinions of authors expressed herein do not necessarily state or reflect those of the United States Government or any agency thereof. 


\section{DISCLAIMER}

Portions of this document may be illegible in electronic image products. Images are produced from the best available original document. 
Printed in the United States of America Available from:

National Technical Information Service

U. S. Department of Commerce

5285 Port Royal Road

Springfield, VA 22161

Price:

Microfiche - $\$ 3.00$

Printed Copy - $\$ 11.00$

Also available from:

Superintendent of Government Documents

U. S. Government Printing office

Washington, DC 20402

Stock Number: 061-000-00210-3

\begin{abstract}
NOTICE
This report was prepared as an account of work sponsored by the United States Government. Neither the United States nor the United States Department of Energy, nor any of their employees, nor any of their contractors, subcontractors, or their employees, makes any warranty, express or implied, or assumes any legal liability or responsibility for the accuracy, completeness or usefulness of any information, apparatus, product or process disclosed, or represents that its use would not infringe privately owned rights.
\end{abstract}




\section{MASTER}

$S E R I / S P-42-141$

UC Category: UC-13

\section{First Edition
Prepared by \\ Academic Programs Branch \\ Kevin $\mathrm{O}^{\prime}$ Connor \\ George Corcoleotes \\ Data Base Systems \\ Jo Ann Silversmith Katherine A. Kramer}

JANUARY $19 \% 9$
This report was prepared as an account of work sponsored by the United States Government. Neither the United States nor the United States Department of Energy, not any of their emplayees, nos any of their contractors, subcontractors, of their employees, makes any warranty, express of implied, or assumes any legal or usefor responsibulity, for the accuracy, completeness or use ful iess of any informetion, apparatus, product or process disclosed, or represents that its use would not infringe privately owned rights.

\section{SOLAR ENERGY RESEARCH INSTITUTE \\ 1536 Cole Boulevard \\ Golden, Colorado 80401}

A Division of Midwest Research Institute

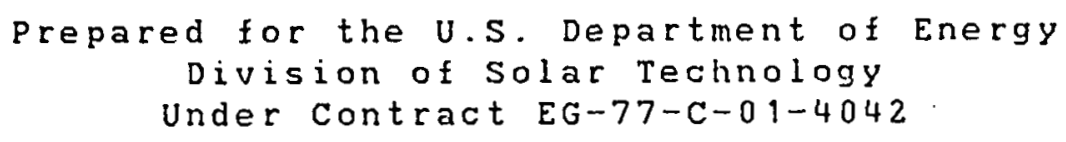

Approved for:

Solar Energy Research Institute

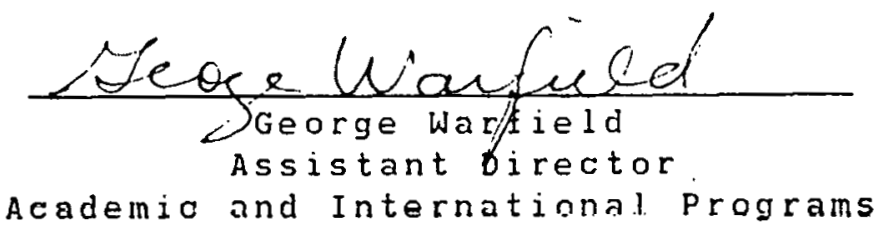




\section{ACKNOWLEDGEMENTS}

The preparation of this report was accomplished by a combined effort of many people. In the Academic programs Branch, kevin $0^{\prime}$ Connor had overall project management responsibility and served as the contact person with John.kimball who coordinated the survey eftort from U.S. Congressman Brown's office. George Corcoleotes, Academic Programs, had overall responsibility for data collection. He was responsible for receiving, reviewing and editing the survey returns.

Jo Ann Silversmith, Katherine Kramer, SPIRES consultants, and the staff of the Database systems Branch designed and built the computerized database, supervised the data input, and provided technical assistance. 
The production of the "1978-79 National Solar Energy Education Directory" (NSEED) represents the culmination of a project initiated in June of 1978. At the Solar Energy Research Institute (SERI), a need for an Educational Data Base (EDB) has been established. The created EDB becomes part of the Congressionally-mandated Solar Energy Information Data Bank (SEIDB).

SERI, having learned from John Kimball of the office of U.S. Congressman George E. Brown, Jr.. that they were planning a National survey of post-secondary educational institutions, agreed to become the survey information processing agent for the sponsoring organizations - Congressman. Brown's office and the Congressional solar Coalition. Approximately 8,000 surveys were mailed to some 3,200 educational institutions in July of 1978. This Directory, along with information from other noted sources, represents information placed in the data base as of December, 1978.

We hope you find the Directory useful. We wili appreciate your brief evaluation of the Directory by returning the User Evaluation sheet on page xuiii of the Directory. 


\section{TABLE OF CONTENTS}

\section{Description}

Page

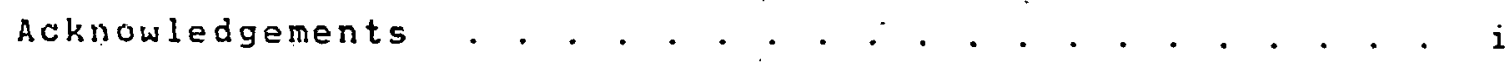

Foreword . . . . . . . . . . . . . . . . . . . . . . . . . . . . .

Table of contents. . . . . . . . . . . . . . . . . . . . . . . i i

Use and Interpretation of the Directory . . . . . . . . . vi

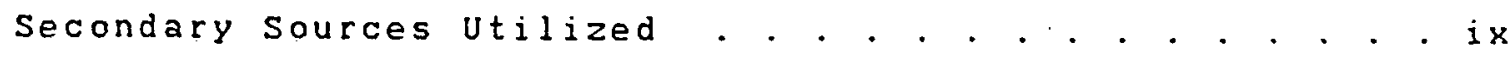

Cover Letter -
"First National Solar Energy Education Survey" . . . . . xi

National Solar Energy Education Survey Form . . . . . . kii

Selected Sources of Information . . . . . . . . . . . . . xvi

User Evaluation sheet . . . . . . . . . . . . . . . . . . . . xuiji

Alabama. . . . . . . . . . . . . . . . . . . . . . . . . . . 1

Alaska . . . . . . . . . . . . . . . . . . . . . . . . . . . . .

Arizona... . . . . . . . . . . . . . . . . . . . . . . . . . . . . 5

Arkancas . . . . . . . . . . . . . . . . . . . . . . . . . . 11

California . . . . . . . . . . . . . . . . . . . . . . . . . . . . . . . 14

Colorado . . . . . . . . . . . . . . . . . . . . . . . 45

Connecticut . . . . . . . . . . . . . . . . . . . . . . . . 58

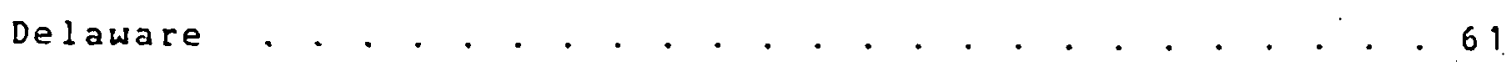

District of Columbia

Florida. . . . . . . . . . . . . . . . . . . . . . . . . . . . 64

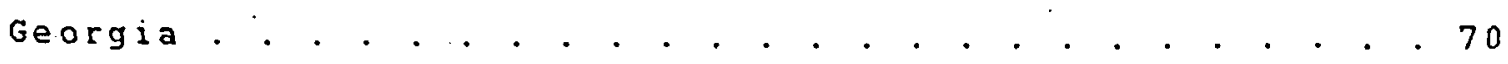


Guam . . . . . . . . . . . . . . . . . 73

Hawaii . . . . . . . . . . . . . . . . . 74

Idaho . . . . . . . . . . . . . . . . . . . . . . . 75

Illinois . . . . . . . . . . . . . . . . . . . . . . . . 78

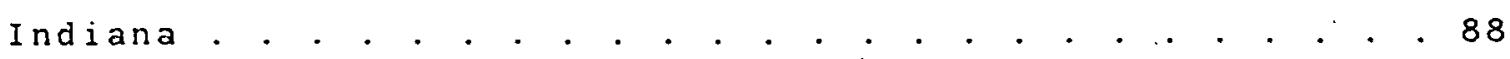

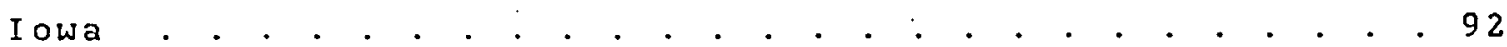

Kansas . . . . . . . . . . . . . . . . . . . . . . . . . . . . 98

kentucky . . . . . . . . . . . . . . . . . . . . . . . . . . . 104

Louisiana.. . . . . . . . . . . . . . . . . . . . 106

Maine. . . . . . . . . . . . . . . . . . . . . . . 108

Maryland . . . . . . . . . . . . . . . . . . . . . . 110

Massachusetts. . . . . . . . . . . . . . . . . . . 113

Michigan . . . . . . . . . . . . . . . . . . . 123

Minnesota. . . . . . . . . . . . . . . . . . . . . 135

Mississippi . . . . . . . . . . . . . . . . . . . . . . . 139

Missour1 . . . . . . . . . . . . . . . . . . . . . . . . . . . 141

Montana. . . . . . . . . . . . . . . . . . . . . . . 146

Nebraska . . . . . . . . . . . . . . . . . . . . . 148

Nêuäda

New Hampshire. . . . . . . . . . . . . . . . . . 153

New jersey . . . . . . . . . . . . . . . . . . . . . . . . . . . 155

New Mexico . . . . . . . . . . . . . . . . . . . . . . . . . . 162

Hew Yort: . . . . . . . . . . . . . . . . . . . . 165

North Carolina

North Dakota . . . . . . . . . . . . . . . . . . . . 199 
ohio . . . . . . . . . . . . . . . . . . . . . 192

Oklahoma . . . . . . . . . . . . . . . . . . . . . . . . . . 199

oregon . . . . . . . . . . . . . . . . . . . . . . . . . . 202

Pennsyluania . . . . . . . . . . . . . . . . . . 2006

Puerto Rico... . . . . . . . . . . . . . . . . . . . . . 215

Rhode Island..................... . . . . . . 216

South Carolina . . . . . . . . . . . . . . . 217

South Dakota . . . . . . . . . . . . . . . . . . . . . . 221

Tennessee... . . . . . . . . . . . . . . . . 222

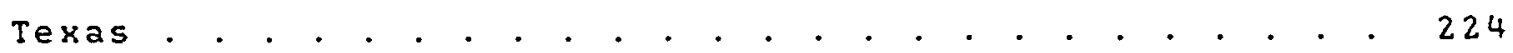

Utah . . . . . . . . . . . . . . . . . . . . . . 242

vermont. . . . . . . . . . . . . . . . . . . . 245

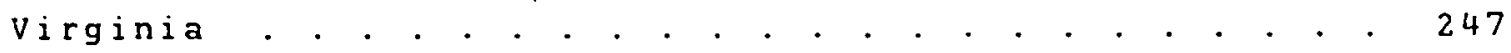

Washington . . . . . . . . . . . . . . . . . . . . . . 252

West virginia. . . . . . . . . . . . . . . . . . . . . 255

Wisconsin. . . . . . . . . . . . . . . . . . . . . . . . 257

wyoming . . . . . . . . . . . . . . . . . . . . . . . 265

Index to Institutions................. . . . . 269

Index to Programs and Curricula . . . . . . . . . . . . 277 


\section{USE AND INTERPRETATION OF THIS DIRECTORY}

IMPORTANT NOTICE: Several important points need to be understood by the user of this Directory. The information contained in the Directory is derived from responses to a national survey of all post-secondary educational institutions. Its completeness is based on those responses and the secondary sources utilized as noted on page ix. The accuracy and reliability of the information is based on those responses and, in some cases, our best judgement on data interpretation. This is a first effort. While we feel the information should be useful to many, we know the next $D i-$ rectory will be more complete and accurate. Should individuals desire additional information relative to activities of particular institutions, they should call or write the institutions:

DIRECTORY ORGANIZATION: The organization of the directory lists institutions alphabetically by institution type within state. A complete alphabetical index of institutions is found in the back of the Directory along with a cross reference to program and curriculum. titles. Within each institution, programs and curricula offered, if any, are listed following the institution name, ID number (found in parentheses to the right of the institution name), address and phone number. All solar-related courses are then 1 is ted alphabetically by course title. If a course is offered within a program or curriculum, the program or curriculum name with which it is associated is printed.

Institution names and addresses for mailing labels and for creating the initial computerized data base were obtained from the National Center for Education statistics. Approximately 8,000 surveys were mailed to some 3,200 educational institutions. Of these, some 2,100 instutitions responded. Over 600 institutions indicated offering at least one solar-related course. Over 125 educational institutions indicated offering a program or curriculum. By coinbining the survey responses with secondary sources of information (courses, programs or curricula marked by an "*") the Directory contains entries for nearly 700 institutions.

EDITING NOTES: In the compilation of the directory of post-secondary educational institutions offering solar-related courses, programs and curricula, every reasonable effort 
was made to obtain accurate and reliable information. Both phone calls and follow-up mail requests were used to gather additional information where necessary. Where information was difficult to interpret, and contact was not made with the respondent, a best judgement of the data submitted was entered into the file. Every survey instrument was read, coded and edited. In order to conform with character limitations in certain fields, some responses were abbreviated.

Specific data element responses were examined for consistency with other data elements, e.g. Did the total contact hour field represent the sum of the subfields of contact hours? In some cases, interpretations were made relative to the programicurriculum classification (page $\times 11$ defines program and curriculum). The most common judgements which were made concerned the question of solar-related courses. With a slight stretch of interpretation, an institution could develop a long list of "solar-related" courses. Hence, some courses submitted which appeared only remotely related to solar were not included in the directory. In future updates, a solar-related course is defined as one in which at least one-third of the contact hours are spent teaching direct solar-related topics.

Probably the most difficult question to interpret on the survey was question 11 on the first page of the survey (see page xiii). The question reads: "Students completing the Program/Curriculum would generally be classified as...". While the attempt of this question was to elicit responses relative to the expertise obtained by the students as a result of completing the program or curriculum, the responses very often reflected the kinds of students involved in the program or curriculum. Therefore, in the Directory we have named the responses to the question with the heading "students Taking or Completing offering".

Institutions which only had planned courses, programs, or curricula were not included in the Directory. Relative to course topic information, only those topics taught extensively were listed in the course details.

DIRECTORY UPDATE: As the Directory is used, data errors and ommissions will be noted. If errors in the reported records are found, the pages may be copied and returned to SERI with corrections noted. If additional 
programs, courses, and/or curricula should be included, the survey form on pages xiii - xv may be copied and completed. Return changes, additions, or deletions to:

Solar Energy Education Data Base

Attn: George Corcoleotes

Solar Energy Research Institute

1536 Cole Boulevard

Golden, Colorado. 80401

For questions concerning updates call SERI at (303) 231-1831. 
In addition to the responses received from the initial survey, several sources of information were utilized to make the data base more complete. Each entry derived from secondary sources has been "starred" $(*)$ to call attention to the user that the information provided for that institution has been derived from a source other than the primary survey.

Institutions and organizations which appear in the Directory as having information supplied from secondary sources are encouraged to submit complete information on their educational offerings by completing a copy of the survey Response Form found on pages xili - $\mathrm{kV}$.

Following is a list of secondary sources utilized in the compilation of the Directory:

1. Alternative Sources of Enerqy, Nos. 21 \& 31, Rt. 2, Milaca, MN 56353 .

2. California Educational opportunities for Solar Energy and Energy Conservation at Institutions of Hiqher Education. U.S. Congressman George E. Brown, Jr., 2342 House office Building, Washington, D.C. 20515 .

3. Colleqes and Universities with Solar-Related Courses, Mid-American Solar Energy Complex, 1256 Trapp Road, Eagan, MN 55121.

4. HUD Solar Status-A Special Report, September 1978, U.S. Department of Housing and Urban Development, washington, D.C. 20410.

5. Illinois Solar Energy Education Program Directory, 1977-79, University of Illinois, Urbana, Illinois 61801 .

6. National Solar Heating and Cooling Information Center, P.o. Bux 1607, Rockville, MD 20850.

7. Report from California, California Community Colleges, November 2, 1978, Barbara S. Pratt, California Community Colleges, 1238 "S" Street, Sacramento, CA 95814 . 
8. Solar Action Office, One Ashburton Place, Boston, MA 02108 .

9. Solar Energy Source Book, Christopher w. Martz, Solar Energy Institute of America, $11106 \mathrm{th}$ street, NW, Washington, D.C. 20001 . 
GEORGE E. BROWN, JR.

2342 HOUSE OFFICE BUILDINO

WASHINGTON, D.C. 20515

(202) 225-6161

COMMITTEES

AGRICULTURE

SCIENCE AND TECHNOLOGY CHAIRMAN, SUBCOMMITTEE ON ENVIRONMENT AND THE ATMOSPHERE

TECHNOLOGY ASSESSMENT BOARD
Congress of the anited tates

\section{蒠ouse of Representatives}

\author{
rasbington, 㚼.C. 20515
}

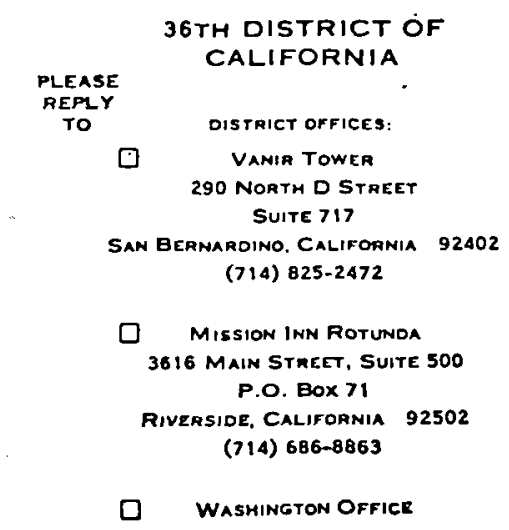

FIRST NATIONAL SOLAR ENERGY EDUCATION SURVEY

July, 1978

T0: Presidents of Educational Institutions, Institute Heads, Deans and Department Directors offering Educational Programs in Solar Energy

FROM: U.S. Rep. George E. Brown, Jr.

U.S. Rep. James M. Jeffords

U.S. Rep. James J. Blanchard
U.S. Senator Charles H. Percy

U.S. Senator Thom as J. Mintyre

The Congressional Solar Coalition is interested in your participation in a cooperative effort to create a data base of all solar energy-related programs and courses currently being offered by our nation's post-secondary educational institutions.

Two organizations have agreed to work with the collected information. The Solar Energy Research Institute (SERI) in Golden, Colorado will have responsibility for the creation of a computerized data base which will become part of the legislatively mandated Solar Energy Information Data Bank (SEID B). SER Is Information Systems Branch will receive the survey returns, edit and process the data into a computerized data base, and create the appropriate retrieval, reporting, updating, and analysis mechanisms. The compilation of collected material will be made available in a published Solar Energy Educational Directory. As an interim measure, before the computerized data base at SE RI is fully operational, the $\mathrm{National} \mathrm{Solar} \mathrm{Heating} \mathrm{and} \mathrm{Cooling} \mathrm{Information} \mathrm{Center} \mathrm{(NSHCIC)} \mathrm{will}$ have the capability of providing a listing of solar educational offerings on a state-bystate basis free of charge to anyone by calling their toll-free number: (800) 523-2929.

The suggested deadline for returning the survey is Septe m ber 15, 1978. For the purpose of this survey, wind, biom ass conversion, and ocean thermal energy conversion should also be considered as solar technologies. New courses, curricula, and updates will be accepted any time following the September 15 th date. However, this is not preferred. Even if you do not offer solar courses, please complete and return the first page of the form.

We appreciate your participation and, for your help, the Solar Energy Research Institute will be sending you a listing of the programs in your state. We hope you will be able to use the compiled information for your own course and/or curriculum development.

We would also like to use this opportunity to make sure that you are aware of the Higher Education Act Amendments of 1976. Of particular im portance is an amendment introduced by Sen. Thomas J. McIntyre, (N.H.), that allows Federal Funds to be used for solar education program $s$ in post-secondary vocational education schools. You might want to investigate your own possible uses of federal funds for the development of new or additional solar energy curricula. 


\section{Solar Energy Educational Survey Instructions}

If your institution is not offering any solar related programs, please complete only the first page of the survey, so indicating. If solar offerings are made by your institution, we would be happy to receive any additional information which you feel would be helpful.

T5. : $:$ page survey form is designed to elicit information on solar-related programs, cois : . .nd/or curricula currently being offered or planned to be offered by educational insticutions. Your assistance in making the form entries as complete as possible will be greatly appreciated. There are two sections to be completed: (1) Educational Institutions and (2) Course Information (2 pages). Some detailed instructions may help in answering certain questions. Note that for the purpose of this survey wind, biomass coriversion, and ocean thermal energy conversion should also be considered as solar technologies.

\section{Educational Institutions}

Q. 4. If your institution offers or plans to offer a solar curriculum or program, answer "yes" to question 4 and complete questions 5 through 12 for each curriculum or program offered or planned. Duplicate the form if necessary.

Q. 5-12. In questions 5 through 12 , information relative to solar curricula and programs is requested, as distinguished from single course of ferings. A curriculum is a set of integrated courses leading toward a degree or certificate. A program is loosely defined as a formal academic experience usually combining course-work and research in applied solar technology, but not necessarily leading to a degree in solar expertise. Specialized summer programs and research institutes are examples. The answer to Q. 8, Head of Prog./Curr.,'should be the main contact person for someone desiring more information about the program or curriculum. Question 12 estimates the proportion of students successfully placed in solar-related jobs.

Q. 13-14. Please answer question 14, regardless of the answer given in question 13. If your institution is presently offering solar courses ("Yes" to question 13), it may also be developing additional courses.

\section{Course Information}

Hlease complete the Course Information Section (2 pages) for each separate, solar-related course, either presently offered or planned to be offered. Duplicate the course information section if necessary to cover all solar courstes uffercd. Any questions you may liave cuncerning the survey form may be answered by George Corcoleotes at SERI (303) 231-1831 or Kevin O'Connor 2311825.

SERI, Arademice Programs

Attn: George Corcoleotes

1536 Cole Boulevard

Golden, Colorado 80401

Thank you very much for your participation. 


\section{Educational Data Base - Educational Institutions}

1. Institution Name:

2. Address:

3. Institution Type:
$\square 4$ Yr. Coll./Univ./Grad. School
[ Vocational/Technical School
$\square$ Community/Junior College
$\square$ Other (describe)

4. Does your institution offer/plan a Solar Curriculum or Program? ${ }^{\circ}$ Yes $\square \quad$ No $\square$ If "No", go to Question 13.

Please complete Questions 5-12 for each solar energy Curriculum or Program offered or planned. It more than one Program/Curriculum is offered/planned please make copies of this scction and attach.

5. Is the offering a Program? or Curriculum?

Currently Offered?

Currently Offered?

or Planned?

6. Program/Curriculum Name

7. College/Dept offering Program/Curriculum

8. Head of Prog./Curr.: Phone:

9. What Degree/Title/Certificate, if any, does graduating student reccivc?
$\square$ Ph.D.
$\square \mathrm{MA}$
$\square \mathrm{MS}$
$\square \mathrm{BA} \quad \square \mathrm{BS}$
$\square$ Associate Degree
Sone
$\square$ Other (specify)

10. Name of Degree/Title/Certificate:

11. Students completing Program/Curriculum would generally be classified as (check as many as apply):
$\square$ Architect
$\square$ Educator
$\square$ Researcher
$\square$ Solar Engineer
$\square$ Mech./Elec. Contraclor
$\square$ General Contractor - Specializing in solar design/installation
$\square$ Do-it-yourselfer/Homeowner
$\square$ Other (specify)

$\square$ Solar System Installer:
$\square$ Residential
$\square$ Industrial/Commercial

$\square$ Solar Technician - one trained in instrumentation, controls, design, maintenance, etc

12. Estimate what percentage of graduates of the above Prog./Curr. enters the job market in the field for which they are specifically trained: $\%$

13. Is your institution presently offering solar courses?

$\begin{array}{ll}\text { Yes } & \text { No } \square \\ \text { Yes } & \text { No } \square\end{array}$

14. Are any (additional) solar courses being developed?
$\square$ Electrical $\square$ Plumbing $\square$ Sheet Metal
$\square$ Other (specify)
$\square$ Electrical $\square$ Plumbing $\square$ Sheet Metal
$\square$ Other (specify)
$\square$ Electrical $\square$ Plumbing $\square$ Sheet Metal
$\square$ Other (specify) Person specializing in solar from one of the following trades/skilis:

For all courses, existing or planned, please complete the 2-page Course Information Section. Make extra copies of the section if needed. If no solar-related courses are offered or planned, complete this page only and return.

Thank you very much. 


\section{Educational Data Base - Course Information}

1. Name of Institution:

2. Course Title

3. Course Number: 4. Is Course Currently Offered? $\square \quad$ Or Planned?

5. Course Instructor/Contact: Phone

6. College/Dept. Offering Course:

7. Is Course also taught in conjunction with other Colleges/Depts.? Yes $\square$ No $\square$

8. Most of the students in course are from what Colleges/Depts.? (Please List)

a.

d.

9. Number of Times Course Taught to Date b. e.
C.

f. Don't Kriow

11. Number of Students Completing Course:

\begin{tabular}{lrr}
1973 or carlier & $1974-1975$ & 1976 \\
\cline { 2 - 3 } & 1978 (est.) & 1979 (est.)
\end{tabular}

12. Is Course Offered for Academic Credit? Yes $\square \quad$ No $\square$ 13. \# Credit Hours

14. Typical Academic Level of Student Taking Course: All Levels $\square$ High School Grad. $\square \quad$ Fresh/Soph $\square \quad$ Jr/Sr $\square \quad$ College Grad. $\square \quad$ Postdoctoral $\square$

15. Duration of Course: (\# of Weeks

Hrs./Week ) OR ( \# of Days Hrs./Day

16. Contact Hours: Total Classroom/Lecture On-the-job-training Other (Specify) Laboratory

17. To what extent are the following topics covered in your course? Check only those that apply. Please specify topics not listed, but included in the course.
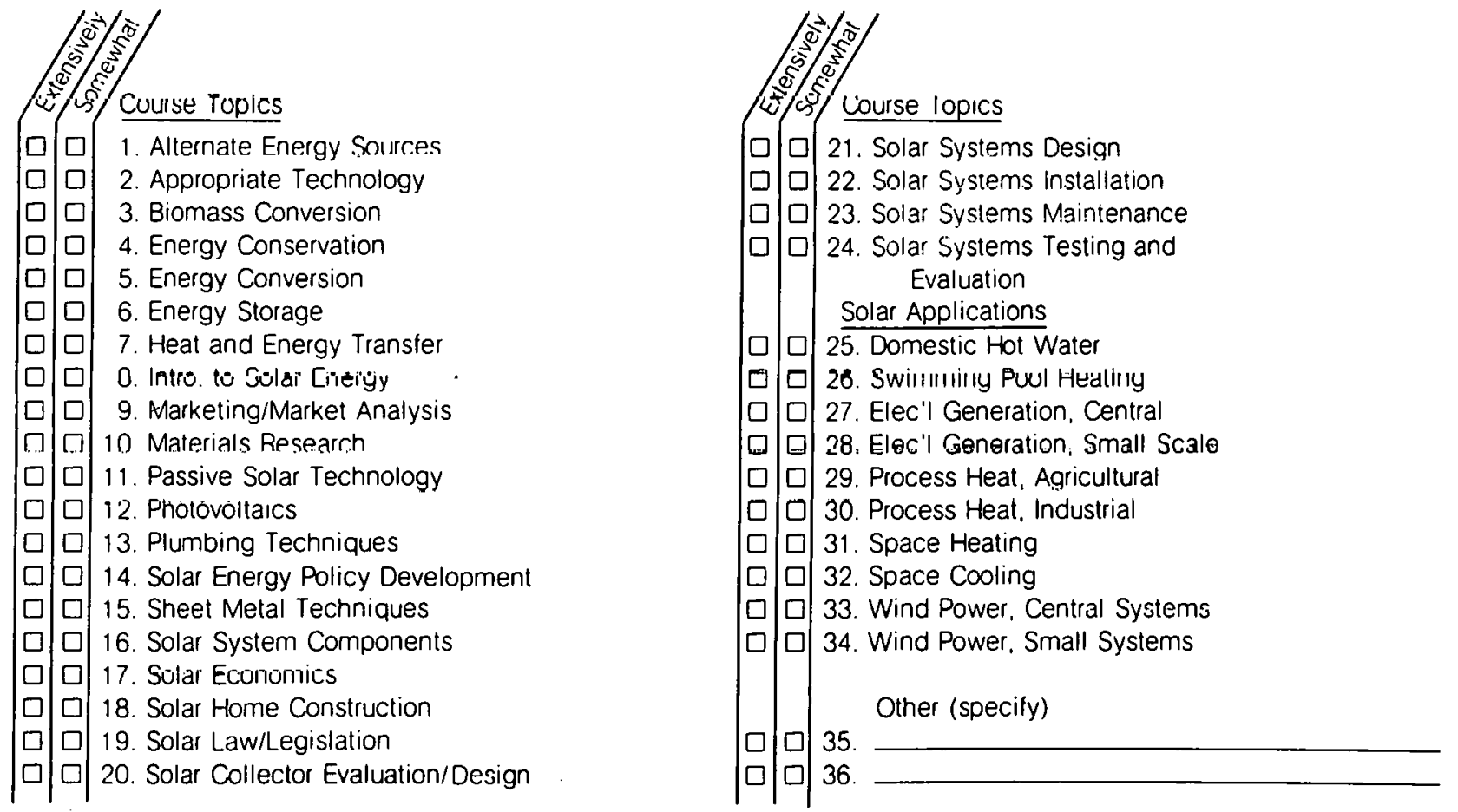

Please complete back of form for this course. Thank you. 


\section{Educational Data Base - Course Information (Continued)}

Materials Used or Proposed for Use in this Course

18. Is a text used in this course? Yes $\square \quad$ No $\square$

19. Name of text

20. Author

The following are used in this course:

21. Slicies: Yes $\square$ No $\square \quad$ 22. Films: Yes $\square$ No $\square \quad$ 23. Demonstrations: Yes $\square$ No $\square$

24. List sources of above materials or describe demonstrations.

25. Course Prerequisites (May be copied from catalog, etc.):

26. Course Description (May be copied from catalog, etc.): 
An abundance of reference materials and other sources of information exists in the field of solar energy. The sources of information cited here will only be highlights of some of the information which can be obtained.

a. Solar Energy Research Institute.

For information about SERI publications contact the SERI Public Information Office, 1536 Cole Boulevard, Golden, Colorado $80401,(303)$ 231-1000. Asts to be placed on the mailing list for the SERI Publications and Presentations Bulletin, a quarterly. publication. The first issue, Volume I-1, was published in November, 1978.

b. US Department of Energy.

1) "Guide to Solar Energy Prograns" (Latest Edition) DOE/ET-0036/1, US Department of Energy Assistant Secretary of Energy Technology Division of Solar Technology Washington, DC 20545

Also available from:

US Government Printing office

Washington, DC 20402 Price: $\$ 2.40$

$S / N 061-000-00042-9$

2) "Solar Energy Task Force Report on Technical Training" (Appropriate for Educational Institutions involved in or anticipating involvement in solar course, program, or curriculum development.) Draft report distributed at the $\mathrm{Na-}$ tional Energy Education, Business and Labor Conference in washington, DC, January 15-17, 1979 .

Available from:

Education Programs Division

US Department of Energy

Forrestal Building, 1000 Independence Ave., SW washington, DC 20585 (202) 376-9211

Also available froin:

SERI, Public Information office

1536 Cole Boulevard 


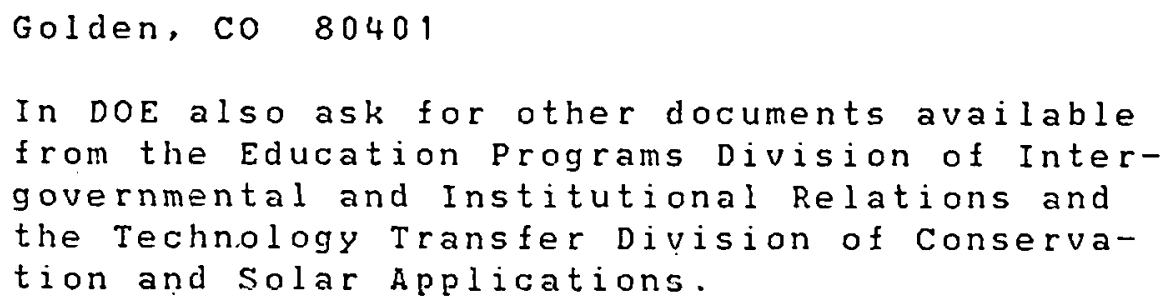

3) Education materials also available from:

US Department of Energy

Technical. Information Center

PO BOX 62

oak Ridge, TN 37830

c. National Solar Heating and Cooling Information Center.

Bibliographies and reference $l$ ists are available along with films and many other resources. Call toll free ( 800$)$ 523-2929. In Pennsyluania call $(800) 462-4933$. Or write:

National Solar Heating and Cooling Information Center PO BOx 1607

Rockville, MD 20850

d. US Office of Education.

For Possible funding sources obtain:

"A 3elecled تulde to f'ederal Energy and Education Assistance"

Energy and Education Action Center US Office of Education, Room 514 Reporters Building, 3007 th street, SW

Washington, DC 20202 (202) 472-7777 
Please take a few minutes to complete the following form. Your comments will be valuable to us.

1. I found the $1978-79$ NSEED:

\section{Extremely useful}

Not very useful, but worth having
Useful

Not worth printing

Comments :

2. I found the information to be:

Sufficiently detailed Not detailed sufficiently

Comments :

3. I would like to see the following cross references in future directories:

a.

b.

4. I found the printing quality to be:

Sat is factory Unsatisfactory

5. I recommend the continuation of an annual publication of the Solar Energy Education Directory:

Yes No

6. I recommend the following additions, deletions, changes to be made in future directories:

7. I would like to see the following special reports from the data base on solar energy education:

Please return form to:

Academic Programs Branch

Attn: Kevin O'Connor

Solar Energy Research Institute

1536 Cole Boulevard

Golden, Colorado 80401
(Optional) Respondent's Name and Address

Name: Phone

Address: 


\section{Colleges/Universities}

ALABAMA A 2 I UNIVERSITY

$(1002)$

Patton Hall

NORMAL, Alabama 35762

(205) 859-7011

\section{SOLAR RELATED COURSES}

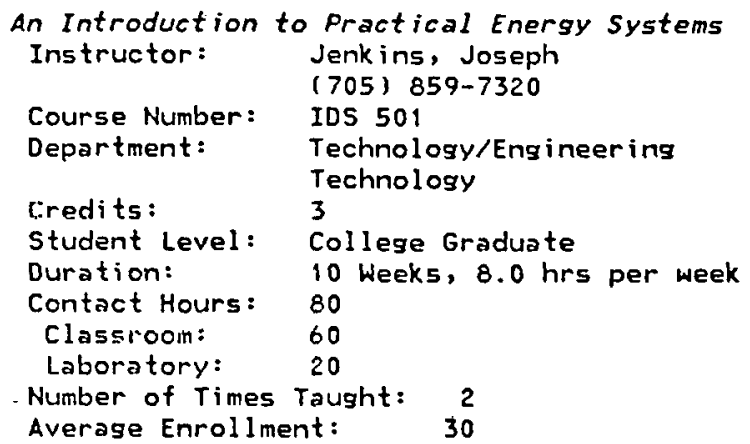

\section{Heat Transfer}

Instructor: Jenkins, Joseph

(205) 859-7320

Course Number: MET 408

Department: Technology/Engineering

Credits: 3

Student Level: Junior or Senior

Duration: 18 Weeks, $3.0 \mathrm{hrs}$ per week

Contact Hours: 54

Classroom: $\quad 54$

Topics Covered Extensively: Heat and

Energy Transfer

Number of Times Taught: 4

Average Enrollment: $\quad 10$

\section{Thermodynamics}

Instructor:

Course Number:

Department:

Jenk ins, Joseph

(205) 859-7320

MET 306

Technology/Eng ineering

Technology

Credits: 3

Student Level: Junior or Senior

Duration: 18 Heeks, 3.0 hrs per week

Contact Hours: $\quad$ b4

Classroom: $\quad 54$

Number of Times Taught: 15

Average Enrollment: $\quad 15$

***********.

\section{ALABAMA IH BIRMIKGHAH, U \\ BIRMINGHAM, Alabama 35294 \\ (205) 934-4011}

SOLAR RELATED COURSES

Ener. Crisis, Homecuners Alternatives

Instructor: Garrison, Aubrey

(205) 87i-7336

Department: Special Studies

Student Level: All levels

Duration: $\quad 6$ Weeks, $2.0 \mathrm{hrs}$ per week

Contact Hours: 12
Topics Covered Extensively: Appropriate Technology; Energy Conservation; Intro. to Solar Energy; Materials Research; Passive Solar Technology; Solar Home Construction

Number of Times Taught: 2

Average Enrollment: $\quad 15$

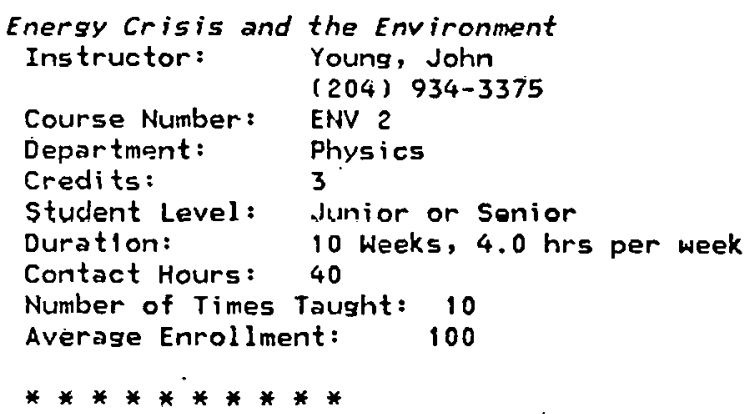

ALABAMA IN HUHTSVILLE, U

$(1055)$

HUNTSVILLE, Alabama 35807

(205) 895-6120

\section{SOLAR RELATED COURSES}

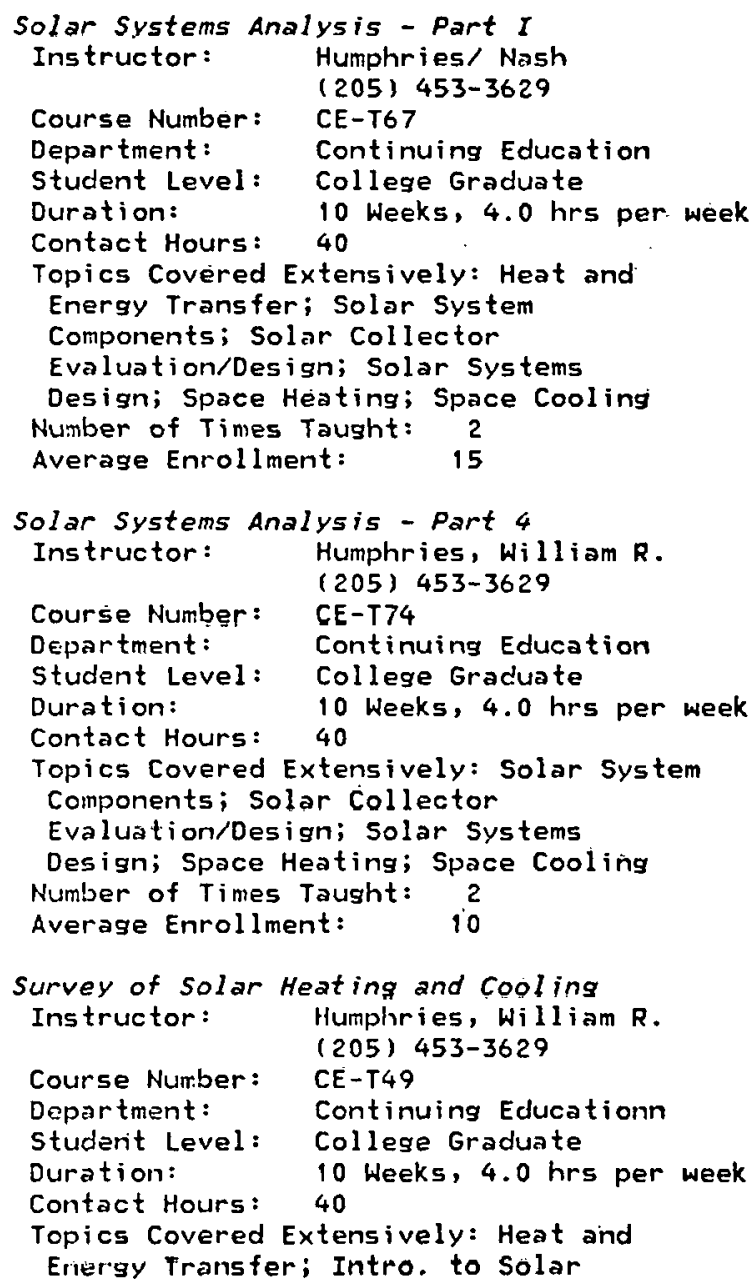


Energy; Solar Collector

Evaluation/Design

Number of Times Taught: 4

Average Enrollment: $\quad 14$

AUBURN U AT MONTGOMERY

$(8310)$

MONTGOMERY, Al abama 36117

(205) 279-9110

\section{SOLAR RELATEO COURSES}

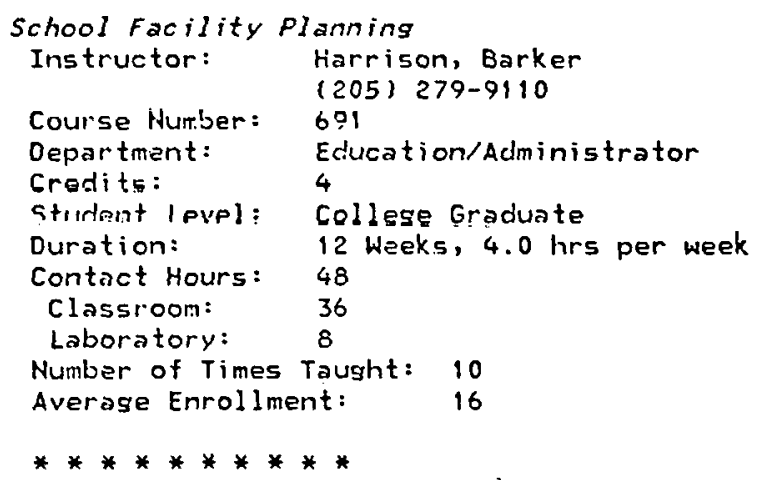

Course Number: 4608490

Department: Architecture \& Fine

Arts

Credits: 3

Student Level: Junior or Senior

Duration: $\quad 10$ Weeks, $3.0 \mathrm{hrs}$ per week

Contact Hours: 30

Number of Times Taught: 2

Average Enrollment: $\quad 15$

\section{MOITEVALLO, UNIVERSITY OF}

$(1004)$

MONTEVALLO, Alabama 35115

(205) 665-2521

solAR RELATED COURSES

Energy and Civilization

Instructor: Kwon, T.H.

$\begin{array}{ll} & (2005) \text { ob } \\ \text { Course Number: } & 120\end{array}$

Department: Physics

Credits: 4

Student Level: Freshman or Sophomore

Duration: 14 Weeks, 3.5 hrs per week

Contact Hours: 50

Classroom: $\quad 36$

Laboratory: 14

Number of Times Taught: 2

Average Enrollment: $\quad 22$ 


\section{colleges/Universities}

ALASKA METH UNIVERSITY

AHICHORAGE, Alaska 99504

( 907 ) 276-8181

\section{SOLAR RELATED COURSES}

\section{Alaska's Energy Resources}

Instructor: Rutledge, Eugene

Course Number: PHYS 492

Department: Arts and Sciences

Credits: 3

Student Level: Jumior or Senior

Duration: 14 Weeks, 2.5 hrs per week

Contact hours: 35

Classrocm: $\quad 35$

Topics Covered Extensively: Alternate

Energy Sources; Appropriate Technology;

Energy Conservation; Energy Conversion;

Energy Storage; Heat and Enersy

Transfer; Intro. to Solar Energy;

Passive Solar Technology; Solar

Economics; Solar Law/Legislation;

Elec'l Generation, Central; Space

Heating; Wind Power, Central Systems;

Wind Power, Small Systems

Number of Times Taught: 1

Average Enrollment: $\quad 10$

\section{Community/Junior Colleges}

ALASKA AHCHORAGE CC, $U$ OF

$(1064)$

ANCHORAGE, Alaska 99504

(907) 279-6602

SOLAR PELATED COURSES

Topics on Solar and Hind Power

Instructor: Wise, James L.

(007) 279-1523

Course Number: ElJVS 194

Department: Natural

Sciences/Mathematics

Credits: 1

Student Level: All levels

Duration:

5 Wecks, 3.0 hrs per week

Contact Hours: 15

Classroom: $\quad 15$

Topics Covered Extensively: Intro. to

Solar Energy; Fassive Solar Technology;

Solar Energy Policy Develcpment; Solar

System Componants; Solar Economics;

Solar Law/Legislation; Wind Power,

Central Systems; Wind Power, Small

Sy'sten's

Number of Times Taught: 1

Average Enrollment: 25

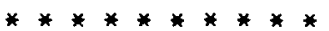

U OF ALASKA KENAI CC

SOLDOTHNA, Alaska 99669

(907) 262-5801

SOLAR RELATED COURSES

Alternative sources I

Instructor: Steffy, D.

(907) 262-5801

Course Number: ET 211

Department: Applied Science

Credits: 3

Student Level: All levels

Duration: $\quad 15$ Weeks, $4.0 \mathrm{hrs}$ per week

Contact Hours: 60

Classroom: $\quad 30$

Laboratory: $\quad 30$

Topics Covered Extensively: Alternate

Energy Sources; Appropriate Technology;

Biomass Conversion; Energy Conversion;

Energy Storage; Heat and Energy

Transfer; Plumbing Techniques; Solar

Econonics; Solar Hoine Construction;

Solar Systems Design; Solar Systems

Installation; Solar Systems

Maintenance; Solar Systems Testing and

Evaluation; Process Heat, Agricultural;

Space Heating; Wind Power, Central

Systems; Wind Power, Small Systems

**********

$U$ OF ALASKA NCRTHLEST CC

NOME, Alaska 99762

(707) 443-2201

SOLAR RELATEO COURSES

Environment Soc iety

Instructor: McGuire, Nancy

Course Number: BIOL103

Department: . Arts and Science

Credits: 3

Student Level: All levels

Duration: 14 Weeks, 3.0 hrs per week

Contact Hours: 42

***********

$U$ OF ALASKA TANAHA VLY CC

(29093)

FAIRBANKS, Alaska 99701

(907) 479-7035

\section{SOLAR RELATED COURSES}

Const. and Analysis Ener. Eff. Homes

Instructor: Raggasch, Robert

(907) 479-7880

Course Number: P.D. 193

Department: Community Interest

Programs

Student Level: All levels

Duration: $\quad 10$ Weeks, $3.0 \mathrm{hrs}$ per week

Contact Hours: 30

Classroom: $\quad 30$

Topics Covered Extensively: Al ternate

Energy Sources 
THIS PAGE

\section{WAS INTENTIONALLY LEFT BLANK}




\section{colleges/Universities}

ARIZOHA STATE UHIVERSITY

(1081)

TEMPE, Arizona 8528

(602) 965-9011

PROGRAMS AND CURRICULA

Energy Conversion and Power Systems

Degree: Pho, MS, 8S, Mechanical

Engineering

Contact: Metzser, D. E. (602) 965-3291

Students Taking or Completing offering:

Trade Specialty

\section{SOLAR RELATED TOURSES}

Direct Energy Conversion 407

Course Number: MEE 487

Department: Mechanical Engineering

Program or

Curriculum: Energy Conversion and

Credits: 3

Stucient Level: Junior or Senior

Duration: 16 Weeks, 3.0 hrs per week

Contact Hours: 48

Classroom: $\quad 48$

Topics Covered Extensively: Energy

Conversion

Averase Enrollment: $\quad 25$

Direct Energy Conversion 583

Course Number: MEE 583

Department: Mechanical Engineering

Program or

Curriculum: Energy Conversion and

Credits: 3 power systems

Student Level: College Graduate

Durstion: 16 Wepks, 3.0 hrs per week

Contact Hours: 48

Classroom: 48

Topics Covered Extensively: Energy

Conversion

Number of Times Taught: 2

Average Enrollment: 5

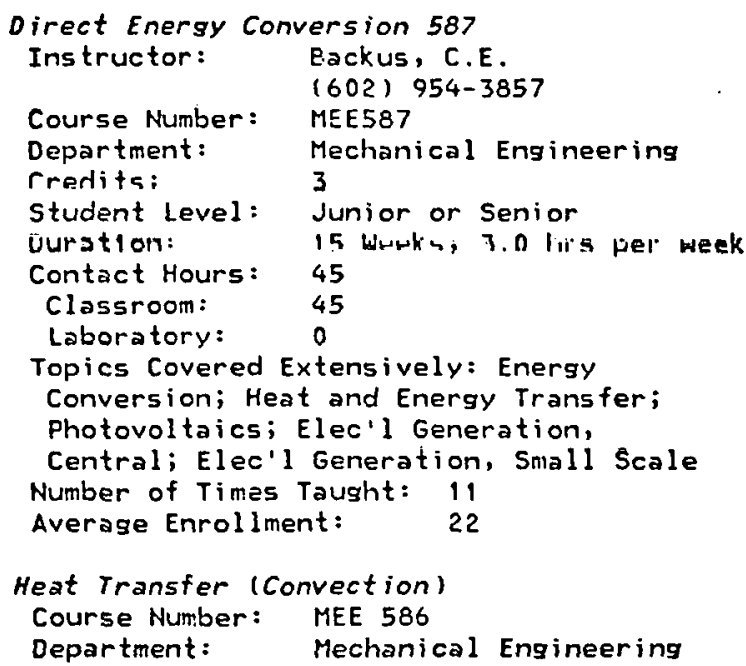

Program or

Curriculum: Energy Conversion and

Credits:

Student Level: College Graduate

Duration:

16 Weeks, $3.0 \mathrm{hrs}$ per week

Contact Hours: 48

Classroom: $\quad 48$

Topics Covered Extensively: Heat and

Energy Transfer

Number of Times Taught: i

Average Enrollment: $\quad 10$

P-S: Energy Technology

Course Number: MEE 498

Department: Mechanical Engineering

Program or

Curriculum: Energy Conversion and

Crodlts! 3 Powser Systems

studont Loval: Jumior or Senior

Duration: 16 Weeks, $3.0 \mathrm{hrs}$ per week

Contact Hours: 48

Clrisgl.0om: $\quad 48$

Number of Times Taught: 1

Average Enrollment: $\quad 15$

$P-S$ : Solar Energy Fundamentals

Course Number: MEE 498

Department: Mechanical Engineering

Program or

Curriculum: Energy Conversion and

Credits : Power Systens

Stucient Level: Junior or Senior

Duration: $\quad 16$ Weeks, $3.0 \mathrm{hrs}$ per week

Contact Hours: 48

Classroom: $\quad 48$

Humber of $\mathrm{Times}$ Tought:

Average Enrollment: $\quad 15$

S: Photovoltaics

Course Number:

artment:

Program or

Curriculum: Energy Conversion and

Credits Power Systems

3

Student Level: Collese Graduate

Duration: $\quad 16$ Weeks, $3.0 \mathrm{hrs}$ per week

Contact Hours: 48

Classroom: $\quad 48$

Tojics Covered Exterrsquely.

Photovoltaics

Number of Times Taught: 1

Average Enrollment:

Solar Energy

Instructor:

Hood, Byard D.

(602) $965-7298$

Course Number: MEE458

Department: Mechanical Engineering

Credits: 3

Student Level: Juriur ur Striol

Duration:

Contact Hours:

Classioom:

15 Weeks, 3.0 hrs per week

45

Topics Covered Extensively: Heat and

Energy Transfer; Intro. to Solar

Energy; Solar Collector 
Evaluation/Design; Solar Systems Design Number of Times Taught: 1

Average Enrollment: $\quad 15$

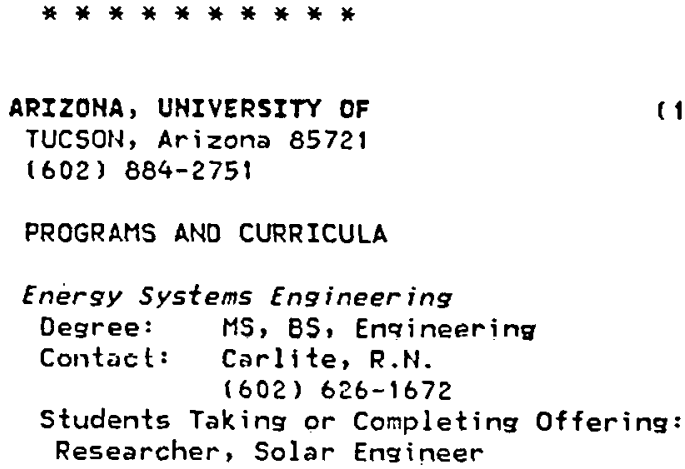

(1083)

Laboratory: 51

Topics Covered Extensively: Alternate Energy Sources; Biomass Conversion; Intro. to Solar Energy; Solar System Components

***********

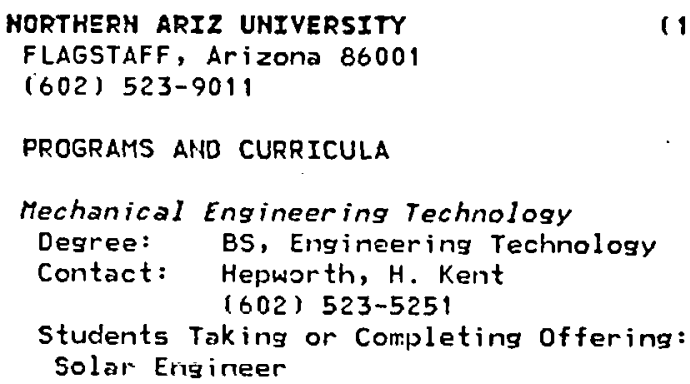




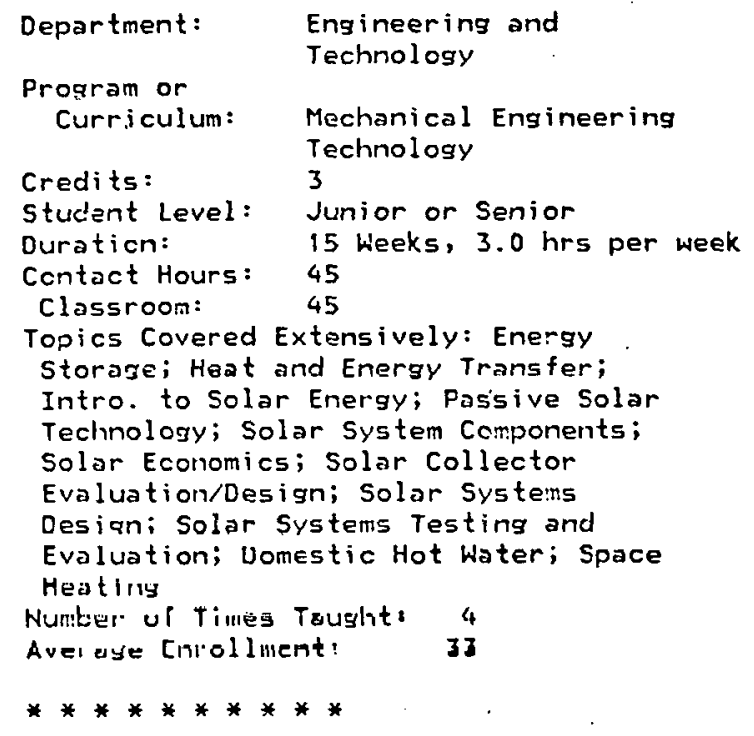

\section{Community/Junior colleges}

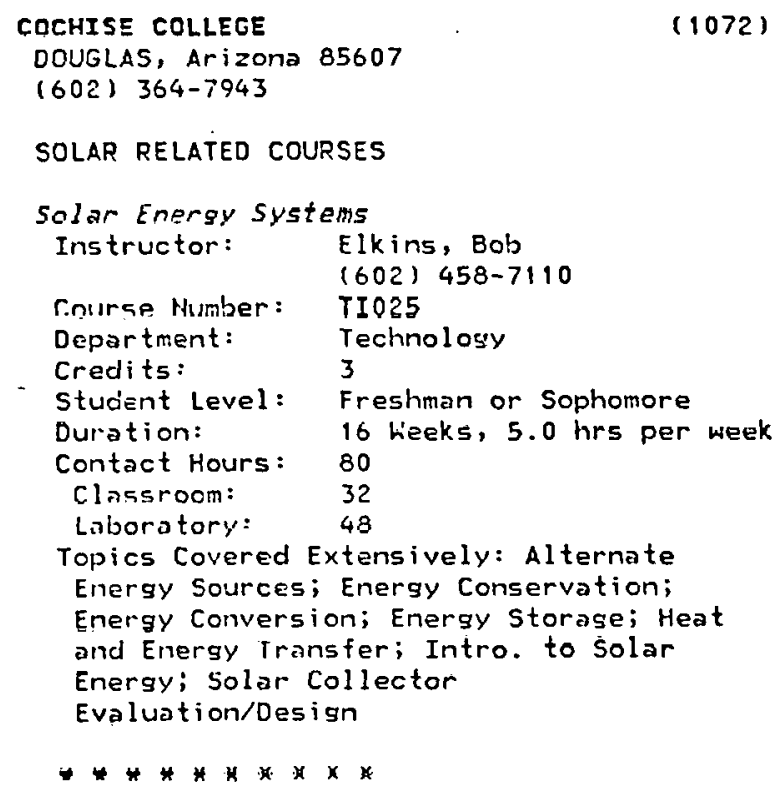

Student Level: All levels

Duration:

Contact Hours:

Classroom:

Laboratory:

1 Weeks, $8.0 \mathrm{hrs}$ per week

8

1

Topics Covered Extensively: Alternate

Energy Sources; Energy Conservation;

Energy Conversion; Energy Storage;

Energy Storage; Intro. to Solar Energy;

Solar System Components; Solar Home

Construction; Solar Systems

Installaticn; Donestic Hot Water

Number of Times Taught: 1

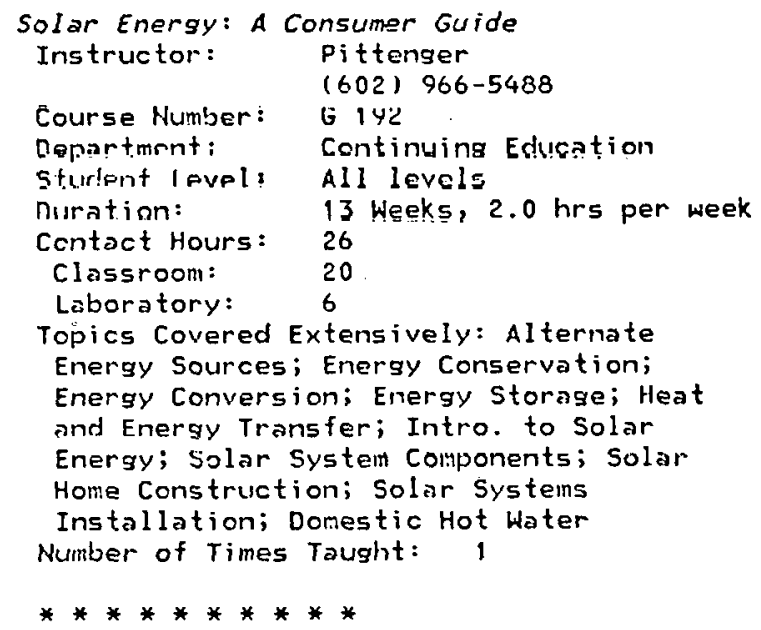


Solar Collector Evaluation/Design;

Solar Systams Design; Solar Systems

Testing and Evaluation; Space Heating;

Space Cooling; Wind Fower, Central

Systems; Wind Power, Simall Systems

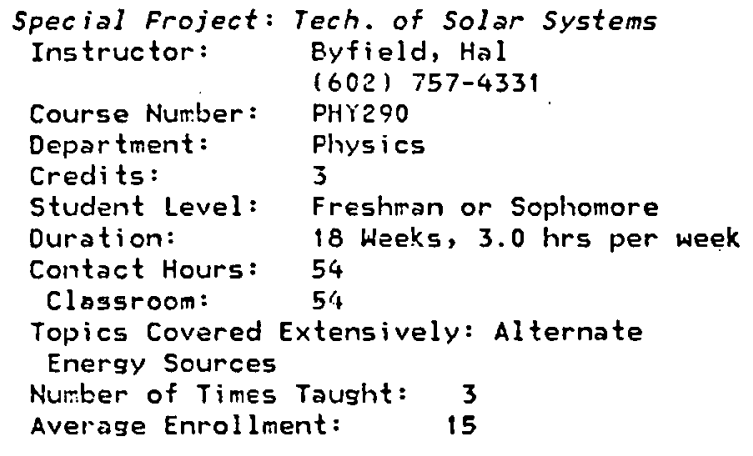

\section{MORTHLAND PIOHEER COLLEGE \\ HOLBROOK, Arizona 66025 \\ (602) 524-6111}

\section{SOLAR RELATED COURSES}

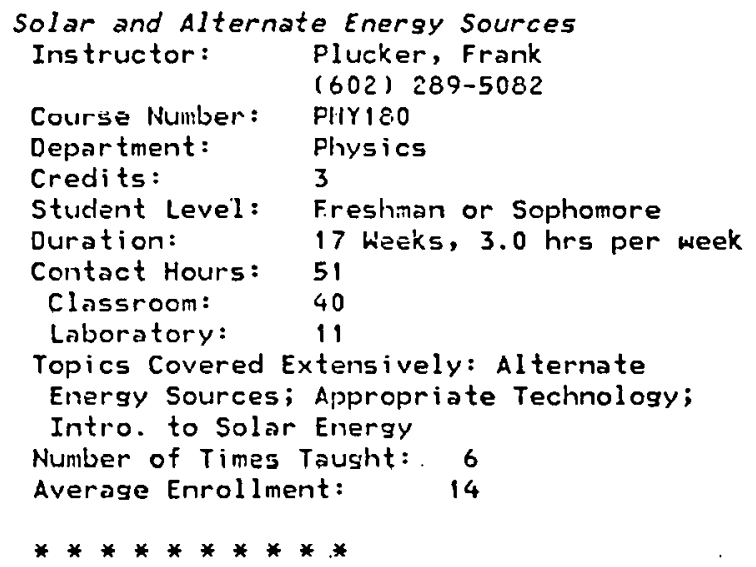

\section{RIO SALADO COMHUHITY COLLEGE}

$(90010)$

10451 Palmera Dr.

Sun City, Arizona 85373

(602) 974-9939

\section{SOLAR RELATED COURSES}

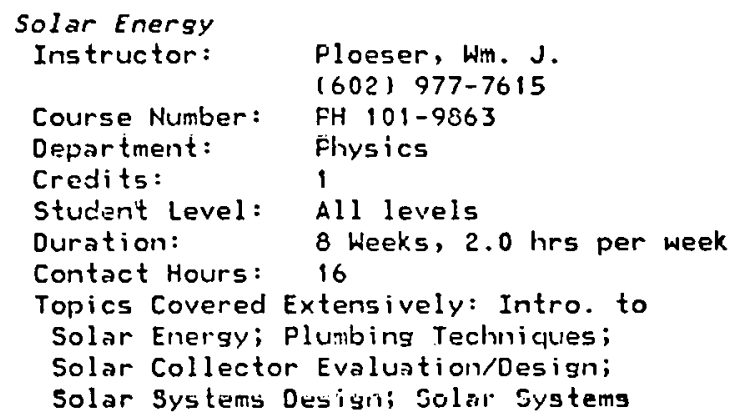

Installation; Domestic Hot Water Number of Times Taught: 2 Average Enrollment: $\quad 25$

***********

YAVAPAI COLLECE

$(1079)$

FRESCOTT, Arizona 86301
$(602) 445-7300$

PROGRAMS AND CURRICULA

Solar Energy Technology

Contact: Minkler, L./ Beverly, G./ Strom, L. (602) 445-7300

students Taking or Completing Offering: Do-it-yourself Homeowiter, Installer-Residential (Solar System)

SOLAR RELATED COURSES

Here Comes the Sun

$\begin{array}{ll}\text { Instructor: } & \text { Minkler, Lyle } \\ \text { Course Number: } & \text { PAS } 1002 \text { 445-5264 }\end{array}$

Department: Science

Program or

Curriculum: Solar Energy Technology

Credits:

Duration:

Contact Hours:

Classroom: 1

5 Weeks, $3.0 \mathrm{hrs}$ per week 15

15

Topics Covered Extensively: Heat and

Energy Transfer; Intro. to Solar

Energy; Solar Econoinics; Solar

Collector Evaluation/Design

Number of Times Tousht: 1

Average Enrollment: $\quad 28$

Methane, Hind-Elec., Hood-Alt. Ener.

Instructor: Reverly, Gary

(602) 445-7300

Course Number: PHS109

Department: Science

Propram or

Curriculum: Solar Energy Technology

Credits:

Uuration:

Contact Hours: 15

Topics Covered Extensively: Alternate Energy Sources; Appropriate Technology; Biomass Conversion; Elec'l Generation, Simall Scale

Number of Times Taught: 4

Average Enrollment: $\quad 14$

Solar Cookers

Instructor:

Minkler, Lyle

(602) 445-5264

Course Number: PHS109

Department: Science

Program or

Curriculum: Solar Energy Tẹchnology

Credits:

Duration: 1

5 Weeks, 3.0 hrs per week Contact Hours: 15

Topics Covered Extensively: Solar

Collector Evaluation/Design 
Solar Greenhouse

Ins tructor:

Beverly, Gary

(602) 445-7300

Course Number: PHS107

Department: Science

Program or

Curriculum:

Credits:

Duration:

Contact Hours:

Solar Energy Technology

1

5 Heeks, $3.0 \mathrm{hrs}$ per week 15

Topics Covered Extensively: Appropriate

Technology; Energy Storage; Heat and

Enersy Transfer; Passive Solar

Technology; Solar System Components;

Solar Systems Design; Space Heating

Number of Times Taught: 5

Average Enrollment: $\quad 16$

Solar Heating, Air and Hater Systems

Instructor:

Minkler, Lyle

(605) 445-5264

Coures Hlumbor: THE101.

Department: Science

Frosram or Curriculum: Solar Energy Techology

Credits:

Duration:

Contact Hours: 1

5 Weeks, $3.0 \mathrm{hrs} \cdot$ per week 15

Topics Covered Extensively: Appropriate Technolcgy; Energy Storage; Solar

System Components; Solar Systems Design

Number of Times Taught: i

Average Enrollment: 15

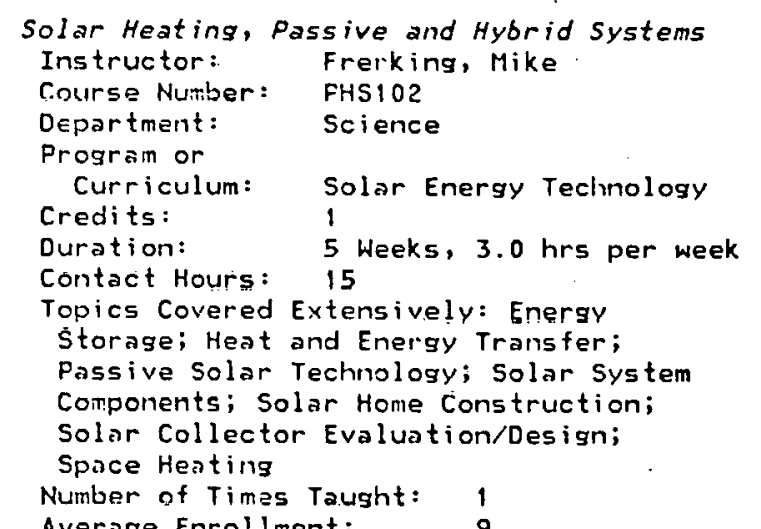

Average Enroliment: 9

Solar Heating, Retrof it systems

Instructor: Minkler, Lyle

Course Number: $(602) 445-7300$

Department: Science

Pregram or

Curriculum: Solar Energy Technology

Credits:

Duration: 5 Weeks, 3.0 hrs per week

Contact Hours: 15

Tcpics Covered Extensively: Appropriate

Technology; Energy Storage; Heat and

Energy. Transfer; Solar System

Compcnents; Solar Economics; Solar

Law/Legislation; Solar Collector

Evaluation/Desisn; Solar Systems

Design; Space Heating
Solar Hot Hater

Instructor:

Course Number:

Department:

Program or

Curriculum:

Credits:

Duration:

Contact Hours:

Eeverly, Gary

$16021445-7300$

FHS 103

Science

Solar Energy Technology

1

5 Weeks, 3.0 hrs per week

Tcpics Covered Extensively: Appropriate

Tectrulogy; Energy Storage; Heat and

Energy Transfer; Plumbing Techniques:

Solar System Conponents; Solar

Economics; Solar Collector

Evaluation/Design; Solar Systems

Design; Domestio Hot Water

Number of Times Taught: 1

Average Enrollment:

4

Solar Laboratory 121

Instructor: Minkler, Lyle

(CO2) 445-7300

Course Number: PHS121

Department: Science

Program or

Curriculum: Solar Energy Technology

Credits:

Duration:

Contact Hours:

Classrooin:

Laboratory: 1

6 Weeks, $4.5 \mathrm{hrs}$ per week

27

6

21

Topics Covered Extensivelyi Solar

Systems Installation; Solar Systems

Mintenance; Solar Systems Testing and

Evaluation; Domestic Hot Water; Space

Heating; Space Cooling

Number of Times Taught:

Average Enrollment:

\section{1}

Solar Laboratory 122

Instructor: Minkler, Lyle

I6n?! 445-7300

Course Number: FHS 122

Department: Science

Program or

Curriculum: Solar Energy Technology

Credits:

Duration:

Contact Hours:

Classroom:

Laborntory:

1

6 Weeks, 4.5 hrs per week

27

6

Topics Covered Extensively: Solar

Systems Installation; Solar Systems

Maintenance: Solar Syctems TeEting and

Evaluation; Domestic Hot Water; Space

Hestiris; Space Cooling

Number of Times Taught:

Average Enrollment:

1
25

Solar Laboratory 123

Instructor:

Minkler, Lyle

Course Number:

(602) 445-7300

Department:

PHS 123

Program or

Curriculum:

Credits:

Duration:
Solar Energy Technology

1

6 Heeks, 4.5 hrs per week 


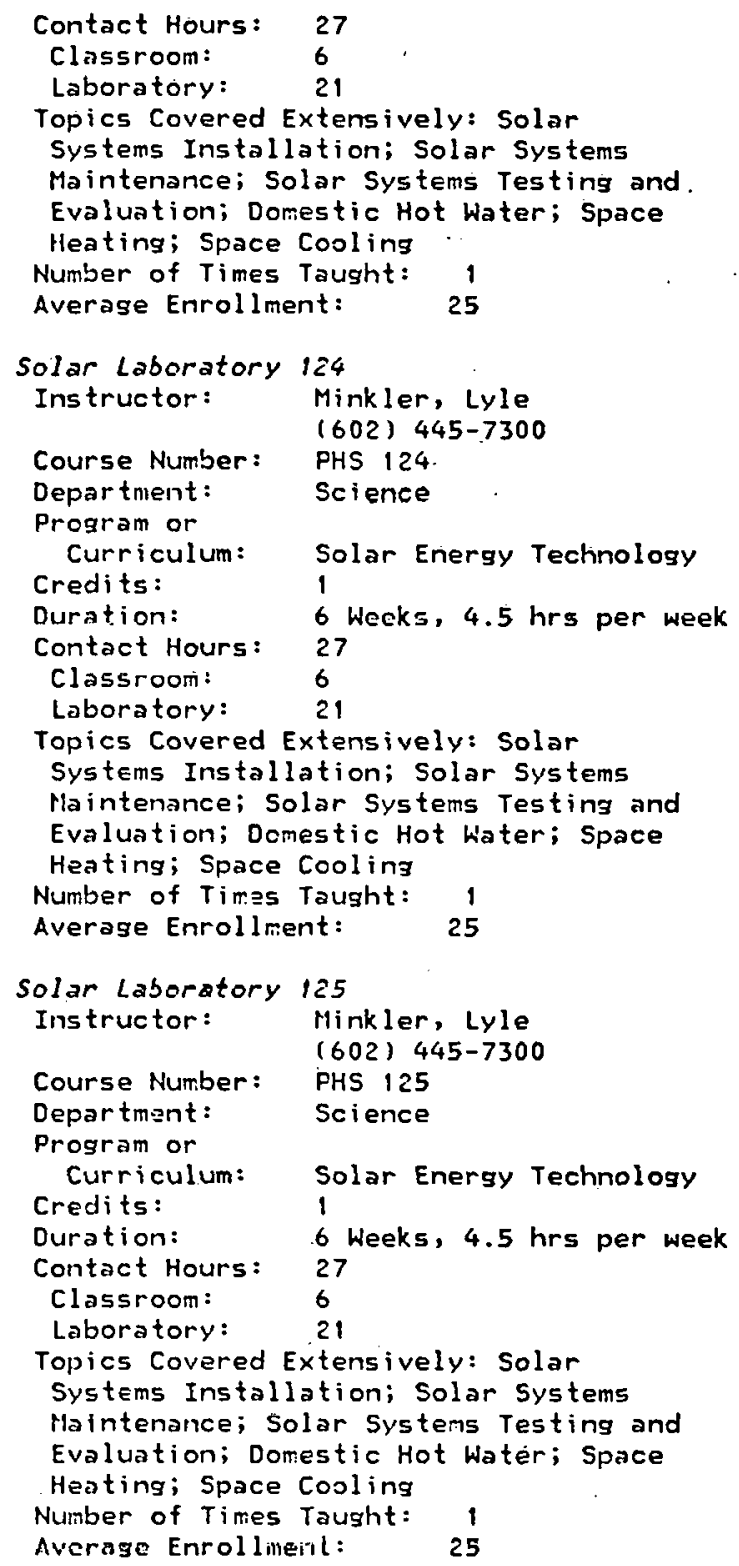




\section{Colleges/Universities}

ARKAHISAS MAIN CAMPUS, U OF

FAYETTEVILLE, Arkansas 72701

(501) 575-2000

\section{SOLAR RELATED COURSES}

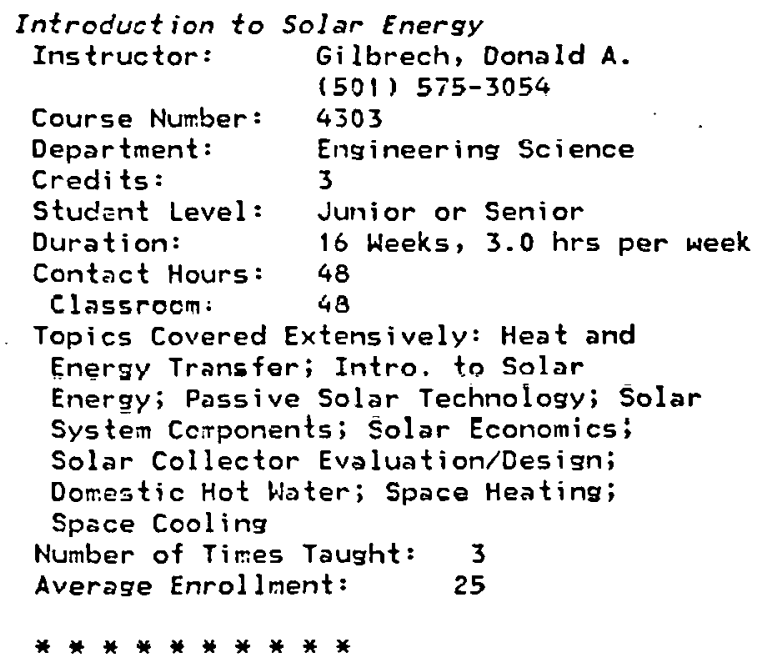

PROGRAIS ANO CURRICULA

Solar Energy horkshop

Deqree: Certificate of Completion

Contact: Feck, Vincent J./ Jordan, Ken (501) 329-2931

Students Taking or Completing Offering: Contractor, Plumber, Sheet Metal Worker

\section{SOLAR RELATED COURSES}

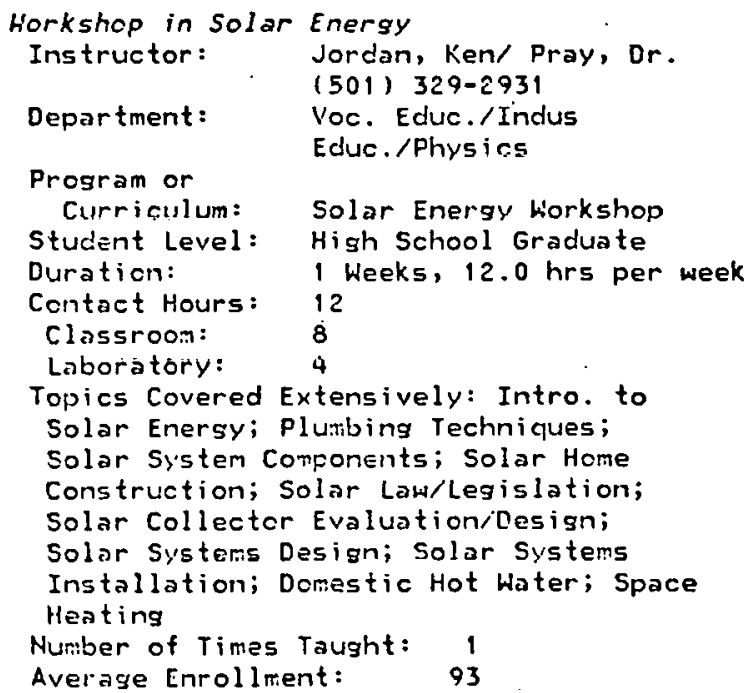

\section{Community/Junior Colleges}

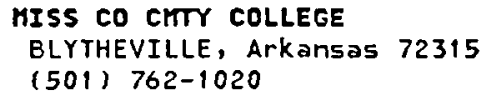




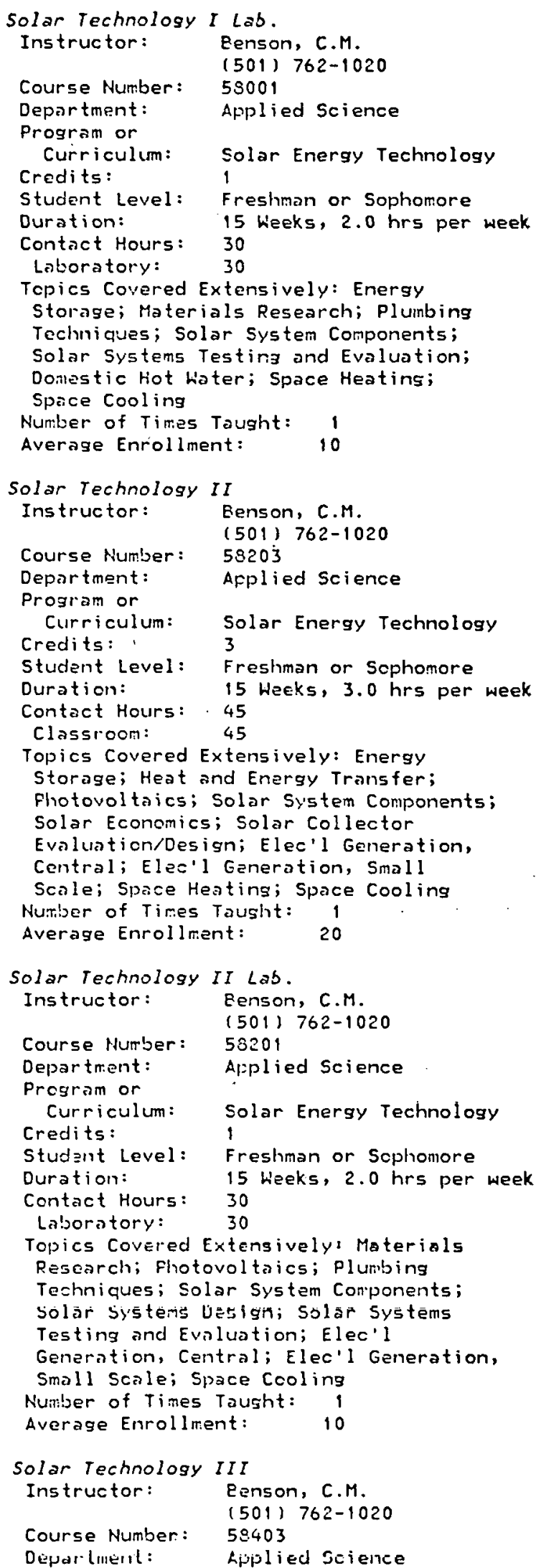

Program or

Curriculum:

Credits:

Student Level: Freshman or Sophomore

Duration: 15 Weeks, 3.0 hrs per week

Contact Hours: 45

Classroom: 45

Tepics Covered Extensively: Energy

Storage; Photovoltaics; Plumbing

Techniques; Solar Energy Policy

Development; Solar Economics; Solar

Systems Design; Domestic Hot hater;

Elec'l Generation, Central; Elec'l

Generation, Small Scale; Process Heat,

Agricultural; Process Heat, Industrial;

Space Heating; Space Cooling

Number of Times Tausht: 1

Average Enrollment:

20

Solar Technology III Lab.

Instructor:

Course Number:

Benson, C.M.

(501) 762-1020

Department:

58409

Prosram or

Curriculum: Solar Energy Technology

Credits:

Student Level: Freshman or Sophomore

Duratic

Contact Hours: 30

Laboratory: $\quad 30$

Topics Covered Extensively: Energy

Storase; Materials Research;

Photovoltaics; Plumbing Techniques;

Solar System Components; Solar Systems

Design; Solar Systems Installation;

Solar Systems Maintenance; Solar

Systems Testing and Evaluation;

Donestic Hot Woter; Elec'l Generation,

Central; Elec'l Generation, Small

Scale; Process Heat, Asricultural;

Process Heat, Industrial; Space

Heating; Space Cooling

Number of Timas Taught:

Average Enrollment:

Solar Topics

Instructor:

Benson, C.M.

Course Number: $\quad \mathbf{5 8 7 0 0}$

Department: Applied Science

Program or

Curriculum: Solar Energy Technology

Credits:

Student Level: Freshman or Sophomore

Duration: 15 Weeks, $2.0 \mathrm{hrs}$ per week

Contact Hours: 30

Classroom: $\quad 15$

Laboratory: 15

Topics Covered Extensively: Energy

Storase; Fhotovoltaics; Plumbing

Techniques; Solar Energy Folicy

Develepment; Solar System Cormonents;

Solar Economics; Solar Home

Construction; Solar Law/Legislation;

Solar Collector Evaluation/Design;

Solar Systams Design; Solar Systens

Installation; Solar Systems

Maintenance; Solar Systems Testing and

Evaluetion

Number of Tinies Taught: 1

Average Enrollment:
1
5 
THIS PAGE

\section{WAS INTENTIONALLY LEFT BLANK}




\section{Colleges/Universities}

CAL INST OF TECHHOLOGY

PASADENA, California 91125

(213) 795-6811

PROGRAMS AND CURRICULA

* Research in. Heat ing and Photovoltaics Contact: Cannon, R.H.

SOLAR RELATED COURSES

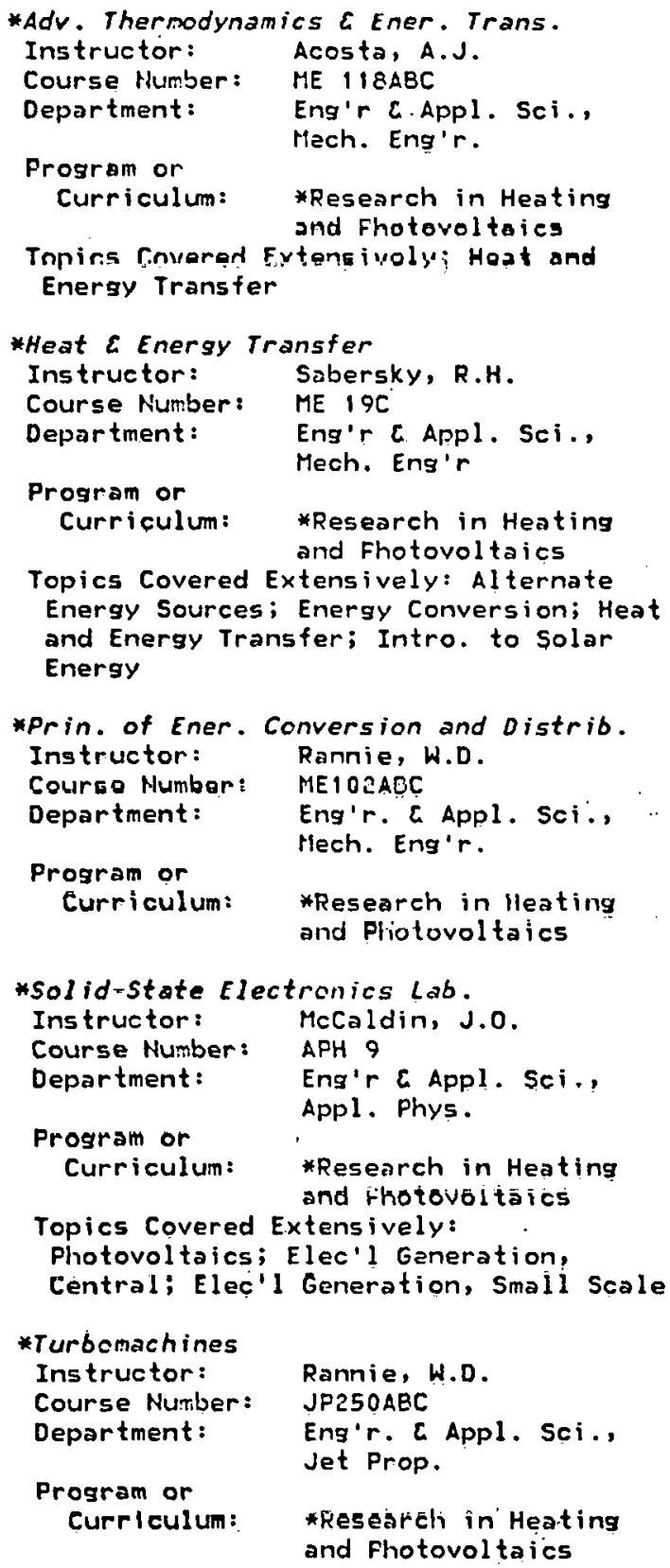

$(1131)$

CAL POLY ST UHI. - SAH LUIS OB

SAN LUIS OBISPO, California 93407

$1805) \quad 546-0111$

\section{PROGRAMS AHD CURRICULA}

Sol. Envirn. Systs./Envir. Engnr.

Degree: BS, Environmental Engineering

Contact: Holtz, Walter E. (E.05) 546-2539

Students Taking or Conpleting offering:

Solar Engineer, Solor Technician, Other

\section{SOLAR RELATED COURSES}

Intro to Environmental Design Sci

Instructor: Pohl, Jens 6 .

(805) $546-284$

Course Number: EOES 221

Department: Architecture

Credits: 3

Dul allurl. iù weeks, 3.U hrs per week

Contret Hours: 30

Classroom: $\quad 20$

Laboratory: $\quad 10$

Topics Covered Extensively: Energy

Conservation; Heat and Energy Transfer;

Intro. to Solar Energy; Solar Home

Construction; Domestic Hot Water; Space

Heating

Mumber of Times Taught: 3

Average Enrollment: $\quad 120$

Solar Energy

Instructor: clark, W.E.

(805) 546-1248

Course Number: EIIVE 221

Department: Environmental

Probrsm or

Curriculum: Sol. Envirn. Systs./

Credits: Envir. Engnr.

Duration:

Contrict Hours: 30

Classroom: $\quad 30$

Topics Covered Extensively: Intro. to

Solar Energy; Solar. System Components;

Space Heating

Number of Times Taught: 18

Average Enrollment: $\quad 35$

Solar Energy Engineering

Instructor: Niles, Philip $W$.

(8).5) 546-2643

Course Number: ENVE 322

Deportment: Environmental

Program or Engineering

Curriculum: Sol. Envirn. Systs./

Credits: 3

Envir. Engnr.

Student Level: Junior or Senior

Duration: 10 Weeks, $5.0 \mathrm{hrs}$ per week

Contact Hours: 50

Classroom: $\quad 20$

Laboratory: $\quad 30$

Topics Covered Extensively: Energy

Storage; Heat and Energy Transfer; 
Solar System Components; Solar

Collector Evaluation/Design; Solar

Systems Design; Domestic Hot Water;

Space Heating

Number of Times Taught: 3

Average Enrollment: $\quad 25$

Solar Energy Systems Analysis

Instructor: Niles, P.W.

(805) 546-2643

Course Number: ErIVE 366

Department: Environmental

Engineering

Program or

Curriculum: Sol. Envirn. Systs./

Credits:

Envir. Engnr.

Student Level: Junior or Senior

Duration:

Contact Hours:

10 Weeks, 5.0 hrs per week

Classroom:

50

50

Topics Covered Extensively: Energy

Storage; Heat and Energy Transfer;

Passive Solar Technology; Solar System

Components; Solar Economics; Domestic

Hot Water; Process Heat, Agricultural;

Process Heat, Industrial; Space

Heating; Space Cooling

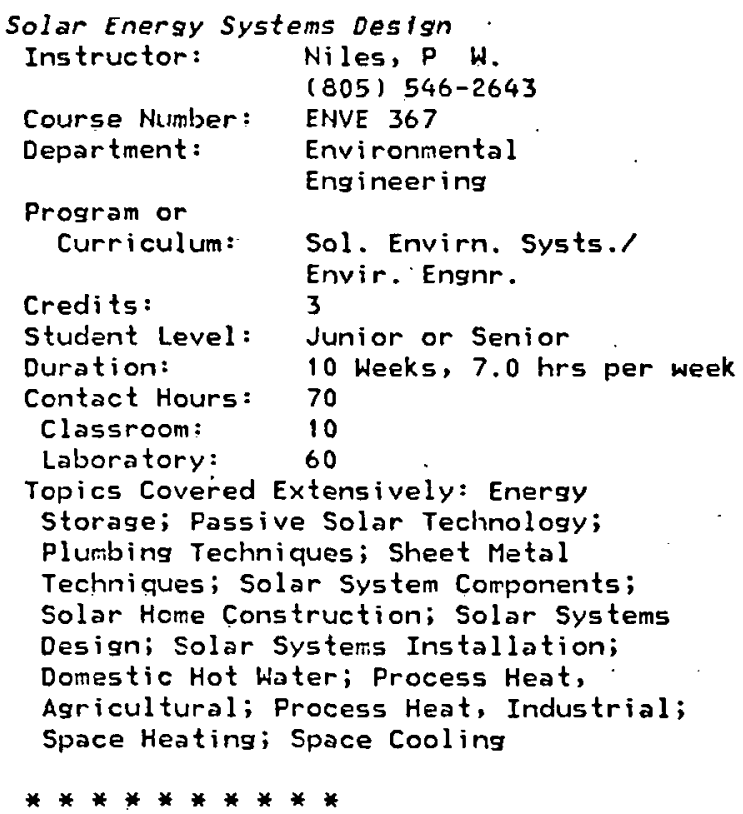

Topics Covered Extensively: Energy Storage; Passive Solar Technology; Plumbing Techniques; Sheet Metal

Techniques; Solar System Components; Solar Home Construction; Solar Systems Design; Solar Systems Installation; Domestic Hot Water; Process Heat, Agricultural; Process Heat, Industrial; Space Heating; Space Cooling

CAL STATE C- DOMINGUEZ HLS

DOMINGUEZ HILLS, California 90747

(213) 532-4300

\section{SOLAR RELATED COURSES}

\footnotetext{
*Energy and Man: Their future Together

Instructor: Gash, Ken/ Rogers,

Richard

Course Hurber: ED $\times 403$

Department: Grad. School of

Eduration

Topics Covered Extensively: Energy
}

Conversion; Solar Economics

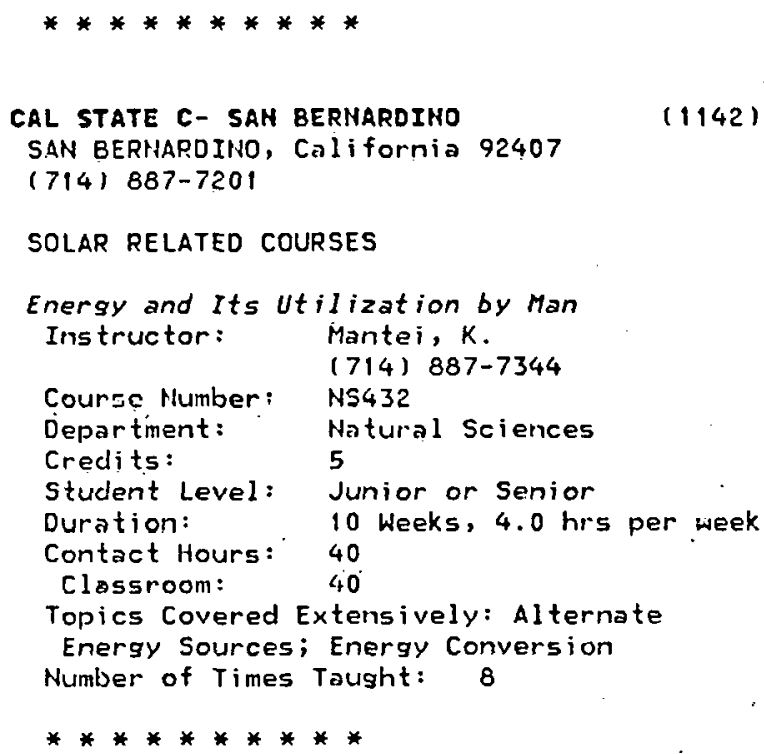

* Solar Technician Train. Classes Program or Curriculum: *Sol. Heat. Tech. Skills Training

Topics Covered Extensively: Appropriate Technology; Energy Conservation; Energy Conversion; Energy Storage; Heat and Energy Transfer; Intro. to Solar Energy; Marketing/Market Arialysis; Passive Solar Technology; Plumbing Techniques; Sheet Matal Techniques; Solar System Components; Solar Economics; Solar Law/Legislation; Solar Collector Evaluation/Design; Solar Systers Design; Solar Systems Installation; Solar Systems Maintenance; Solar Systems Testing and Evaluation; Domestic Hot Water; Space Heating; Space Cooling

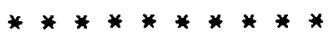




\section{SOLAR RELATEO COURSES}

Solar Enersy Systems

Instructor: $\quad$ Giddle, John R.

$\begin{array}{ll} & (714) \\ \text { Course Number: } & \text { EGR590 } \\ \text { Department: } & \text { Mechan }\end{array}$ 598-0239 Mechanical Engineering

Credits: 4

Student Level: College Graduate

Duration: 12 Heeks, $4.0 \mathrm{hrs}$ per week

Contact Hours: 48

Topics Covered Extensively: Appropriate

Technology; Energy Storage;

Pirotovoltaics; Solar System Components;

Solar Collector Evaluation/Desisn;

Solar Systems Design; Solar Systems

Installation; Sclar Systems

Maintenance; Solar Systems T̈esting and

Evaluation

Nuirber of Times Taught: 1

Averase Enrollment: 32

Solar Thermal Engineering

Eiddle, John R.

(714) 598-0239

Course Number: ME407

Department: Mechanical Engineering

Credits: 4

Student Level: Junior or Senior

Duration: 12 Heeks, 4.0 hrs per week

Contact Hours: 48

Classroom: $\quad 48$

Topics Covered Extensively: Energy

Storage; Heat and Energy Transfer;

Intro. to Solar Energy; Passive Solar

Technology; Solar Econemics; Solar Home

Construction; Solar Collector

Evaluation/Design; Solar Systems

Desism; Doneslic Hut Hater.

Number nf Times Ta!bht: 1

Average Enrollment: $\quad 32$

**********

CAL STATE U- CHICO

(1146)

CHICO, Cali fornia. 95929

(916) 895-5011

\section{SOLAR RELATED COURSES}

$\begin{array}{ll}\text { Alternate Energy } & \text { Systems } \\ \text { Instructor: } & \text { O'Bannon, James E. } \\ & (916) 343-2975 \\ \text { Course Number: } & 109 \\ \text { Department: } & \text { Industry and Technology } \\ \text { Credits: } & 3 \\ \text { Student Level: } & \text { All levels } \\ \text { Duration: } & 3 \text { Heeks, } 17.0 \text { hrs per week } \\ \text { Contact Hours: } & 51 \\ \text { Classrocm: } & 36 \\ \text { Laboratory: } & 15 \\ \text { Topics Covered Extensively: Alternate } \\ \text { Energy Scurces; Intro: to Solar Energy } \\ \text { Nuniser of Times Taught: } 3 \\ \text { Avarage Enrollment: } 40\end{array}$

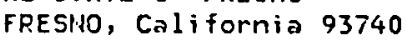

CAL STATE U-FULLERTON

FULLERTON, Cali fornia 92634

$(1137)$

(714) $870-2011$

\section{SOLAR RELATED COURSES}

* Solar Ener. E Eng'r. Appls.

Instructor: Turner, Robert

Course Number: EGPG 472

Department: Mech. Ens'r.

Duiatiull 16 waeks, $3.0 \mathrm{hrs}$ per week Contact Hours: 48

Topics Covered Extensively: Energy Conversion; Enersy Storage; Solar Economics; Solar Collector tvaluation/Uesisn; Solar Systems Design; Solar Systems Testing and Evaluation; Space Heating; Space Cooling

***********

CAL STATE U- HAYHARO

HAYWAREO, California 94542

$(1138)$

(415) $881-3000$

\section{SOLAR RELATED COURSES}

Energy and Environment

Instructur: $\quad$ Guud, R. H.

Course Number: FHY-3650

Department: Fliysics

Credits: 4

Student Level: Junior or Senior

Duration: 10 Weeks, 4.0 hrs per week 
Contact Hours: 40

Number of Times Taught:

Averase Enrollment: $\quad 20$

Environ. Ed. using Sch. and Comm. Fes.

Instructor: Railton, Esther

(415) $881-3027$

Course Number: TED 6415

Department: Teacher Education

Credits:

Student Level: Junior or Senior

Duration: 10 Weeks, 5.0 hrs per week

Contact Hours: $\quad 50$

Classroom: $\quad 30$

Laboratory: 20

Number of Times Taught: 6

Average Enrollment: 25

Environmental LaH

Instructor: $\quad$ Smith, J. Malcolm (4i5) $881-3221$

3460

Course Number:

Credits:

Political Science

4

Student Level: Junior or Senior

Duration: 10 Weeks, $4.0 \mathrm{hrs}$ per week

Contact Hours: 40

Classroom: 40

Topics Covered Extensively: Solar

Law/Legislation

Number of Times Taught: 3

Average Enrollment:

23

Geography of Enersy Resources

Instructor: Fasenhart, Thomas $H$. 1415) $881-3159$

Course Number: 4320

Department: Geography

Credits: 4

Student Level: Junior or Senior

Duration: 10 Weeks, 4.0 hrs per week

Contact Hours: 40

Classroom: $\quad 40$

Tcpics Covered Extensively: Alternate

Energy Sources; Apprcpriate Technology;

Energy Conservation; Energy Conversion;

Energy Storage; Heat and Enersy

Transfer; Intro. to Solar Energy; Solar

Enersy Policy Development; Wind Power,

Central Systems; Wind Power, Small

Systeris

Number of Times Taught: 7

Average Enrollment: $\quad 23$

Public Policy and the Invironment

Instructor: Lewis, Sherman

(415) 881-3221

Course Number: 4171

Department: Political Science

Credits: $\quad 4$

Student Level: Junior or Senior

Duration: 10 Weeks, 4.0 hrs per week

Contact Hours: 40

Classroom: $\quad 40$

Topics Covered Extensively: Al ternate

Energy Sources; Appropriate Techology;

Energy Conservation; Intro. to Solar

Energy; Solar Energy Policy Development

Number of Tines Täuslit: 5
Average Enrollment:

15

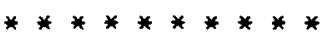

CAL STATE U- LONG BEACH

LONG BEACH, California 90840

$(1139)$

(213) 498-4121

PROGRAMS AND CURRICULA

Ener. Convs., Fower systs. Engineering

Degree: Other, Ener. Convs., Power Systs. Engineering

Contact: Unt, llillard, Jurdanides (213) 498-4407

Students Taking or Completing offering:

Trade Specialty

SOLAR RELATED COURSES

Energy Selection and Conversion

Instructor: Dyer, J.L.

Course Number: ME411

Department: Mechanical Engineering

Program or

Curriculum: Ener. Convs., Power

Credits: Systs. Engineering

Student Level: All levels

$\begin{array}{ll}\text { Duration: } & 15 \\ \text { Contact Hours: } & 45\end{array}$

Topics Covered Extensively: Alternate

Energy Sources; Energy Conversion;

Intro. to Solar Enersy; Elec'1

Generation, Central; Elec'l Generation,

Small Scale; Wind Power, Small Systems

Special Topics in Mech. Ensin.

Instructor: Sungu, Sabri

Course Nuniber: ME405

Department: Mechanical Engineering

Program or

Curriculum: Ener. Convs., Power

Credits: Systs. Engineering

Student Level: Junior or Senior

Duration: 14 Weeks, $3.0 \mathrm{hrs}$ per week

Contaet Houre: 42

Topics Covered Extensively: Appropriate Technology; Energy Conversion; Energy

Storase; Heat and Energy Transfer;

Intro. to Solar Energy; Solar System

Components; Solar Collector

Evaluation/Design; Solar Systems

Design; Domestic Hot Hater; Elec'l

Generation, Central; Elec'l Generation.

Small Scale; Space Heating; Space

Cooling

********** 
CAL STATE U- LOS ANGELES

$(1140)$

LOS ANGELES, California 90032

(213) 224-0111

\section{SOLAR RELATED COURSES}

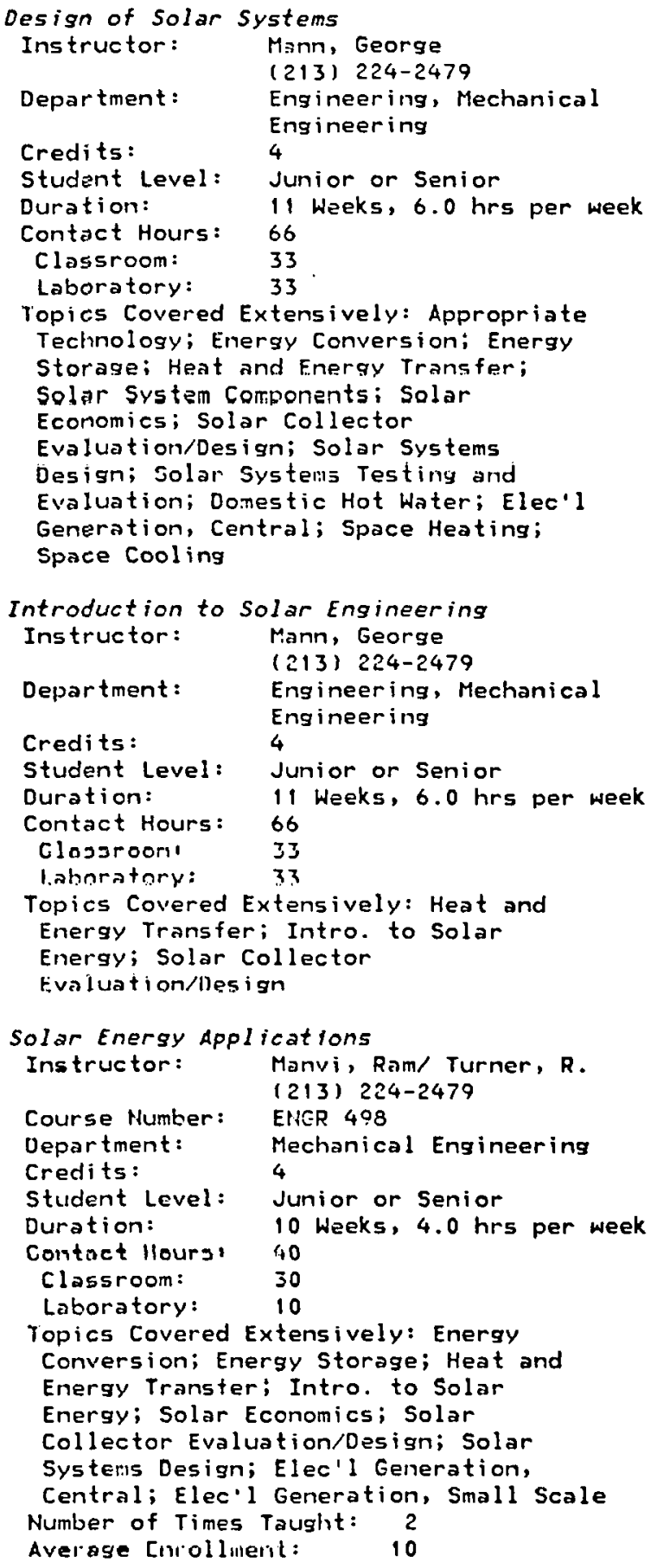

CAL STATE U- MORTHRIDGE

NORTHRIDGE, California 91330

(213) 885-1200

\section{SOLAR RELATED COURSES}

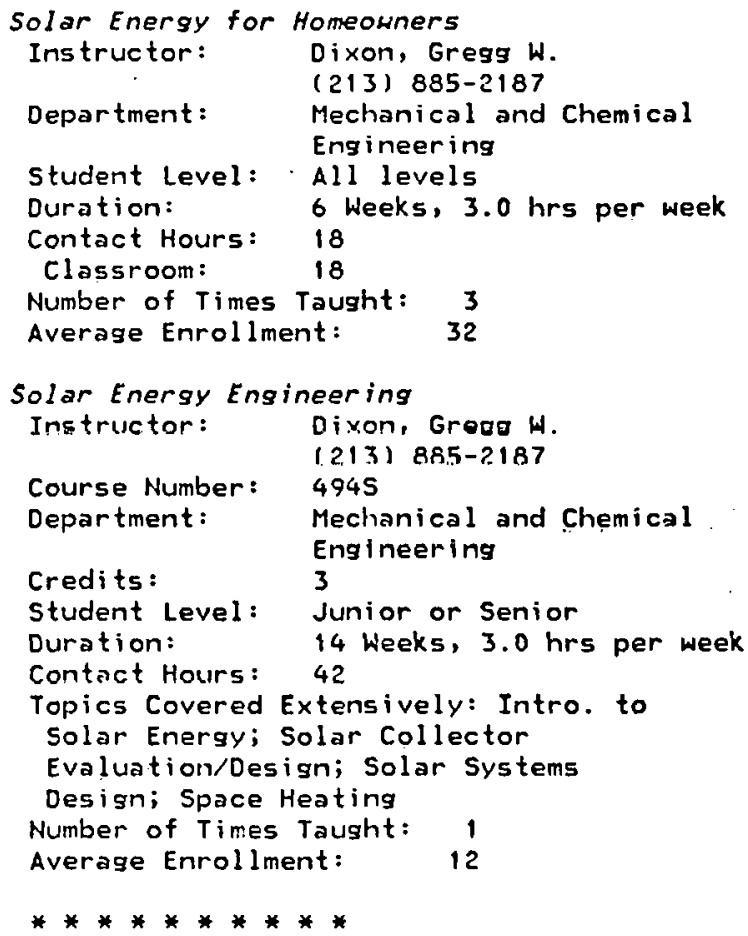


Number of Times Taught: Average Enrollment: 15

Direct Energy Conversion

Instructor: Hu, Chenming

(415) 642-3393

Course Number: EEC 290 G

Department: Elec. Eng. and Computer Sciences

Program or

Curriculum:

Credits:

Solar Engineering

Student Level: College Graduate

Duration: 10 Weeks, 3.0 hrs per week

Contact Hours: 35

Classroom: $\quad$ jo

Topics Covered Extensively: Energy

Conversion; Intro. to Solar Energy;

Photovoltaics; Elec'l Generation, Small scale

Number of Times Taught:

Average Enrollment:

Elementary Solar Electric Systems

Instructor:

Sinith, 0.J.M.

(41.5) 642-7591

Course tumber: EECS 165

Department:

Elec. Eng. C Computer Sci.

Program or

Curriculum: Solar Engineering

Credits: 3

Student Level:

Duration:

Junior or Senior

Contact Hours: 30

Class room:

30

30

Tcpics Covered Extensively: Appropriate

Technology; Energy Conversion; Energy

Storage; Heat and Energy Transfer;

Solar Systems Design; Solar Systems

Installation; Elec'l Generation,

Central; Wind Power, Small Systems

Energy and Power

Instructor:

Lieberman, M.A.

(415) 642-1030

Course Number:

E160

Department:

Engineering

Curriculum:

Credits:

Student Level:

Duration:

Solar Engineering

4

Junior or Senior

Contact Hours: 40

Class room:

40

40

Topics Covered Extensively' Alternate

Energy Sources; Energy Conservation;

Energy Conversion; Heat and Energy

Transfer; Intro. to Solar Energy

Number of Times Taught: 7

Average Enroliment:

100

Energy Conversion Principles

Instructor: Daily, John $W$.

(415) 642-0238

Course Number:

IIE 145

Department:

Program or

Curriculum: Solar Engineering

Credits:
Student Level: Junior or Senior

Duration:

Contact Hours: 40

Topics Covered Extensively: Alternate

Energy Sources; Appropriate Technology;

Energy Conservation; Energy Conversion;

Energy Storage; Photovoltaics

Number of Times Taught: 7

Average Enrollment: 22

Physics of Solar Radiation

Instructor:

Portis, Alan $M$.

(415) 642-3697

Course Number: 1808

Department: Phyics

Program or

Curriculum: Solar Engineering

Credits:

Student Level: Junior or Senior

Duration:

Contact Hours: 40

Classroom: $\quad 40$

Topics Covered Extensively: Energy

Conversion; Heat and Energy Transfer;

Intro. to Solar Energv; Materials

Research; Passive Solar Technclorv;

Photovoltaics; Solar. Collector

Evaluation/Design; Solar Systems

Design; Space Heating

Physics of Solar Radiation

Instructor:

Portis, Alan M.

(415) 642-3697

Course Number: $180 \mathrm{~A}$

Department: . Physics

Program or

Curriculum: Solar Engineerinz

Credits:

4

Student Level:

Duration:

Contact Hours:

Classroom:

Junior or Senior

10 Weeks, 4.0 hiss per week

40

40

Topics Covered Extensiveiy: Energy

Conversion; Heat and Energy Transfer;

Intro. to Solar Energy; Materials

Research; Passive Solar Technology;

Photovoltaics; Solar Collector

Evaluation/Design; Solar Systems Design

Sol. Ener. for BIdss., Homes, Pools

Instructor: Pike, Nanette

(415) 642-4151

Department:

Continuing Education in

Engineering

Student Level:

College Graduate

Duration:

1 Weeks, 16.0 hrs per week

Contact Hours: 16

Topics Covered Extensively: Intro. to

Solar Energy; Passive Solar Technology;

Photovoltaics; Solar Collector

Evaluation/Design; Solar Systems Design

Number of Times Taught: 9

Average Enrollment:

90

Solar Cells-Basic to Advanced Systems

Instructor: Barry, Helen

(415) 642-4151

Department:

Continuing Education in

Student Level:
Engineering

College Graduate 


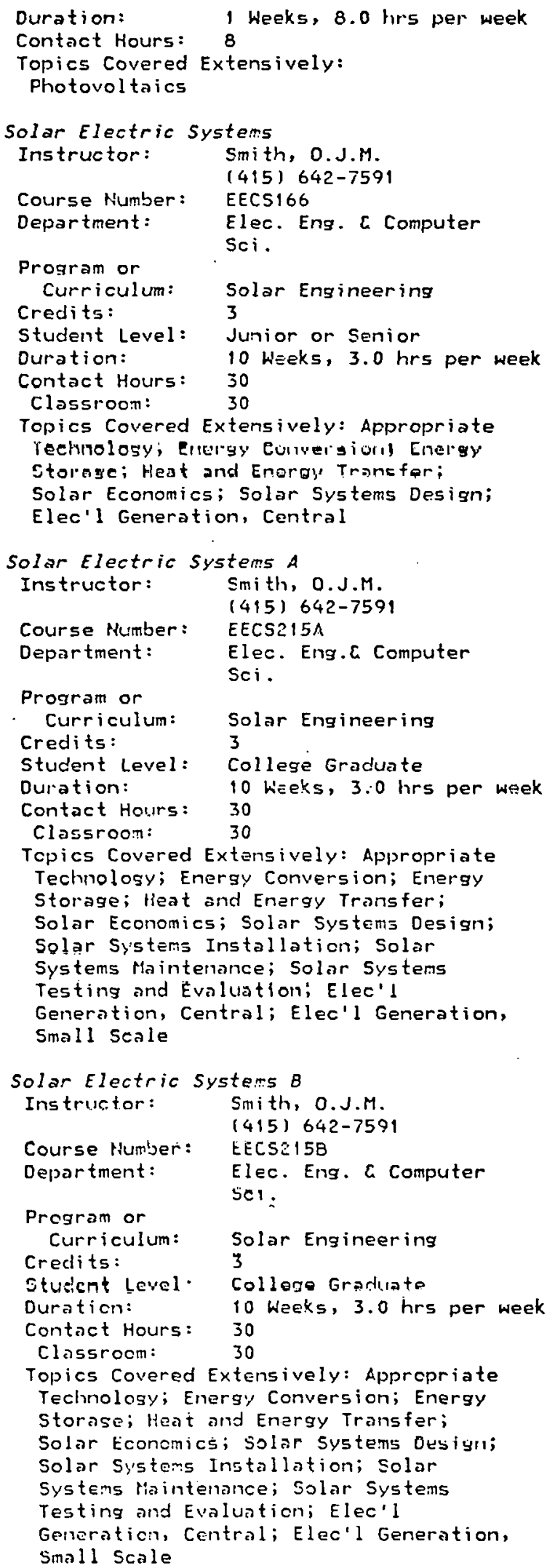

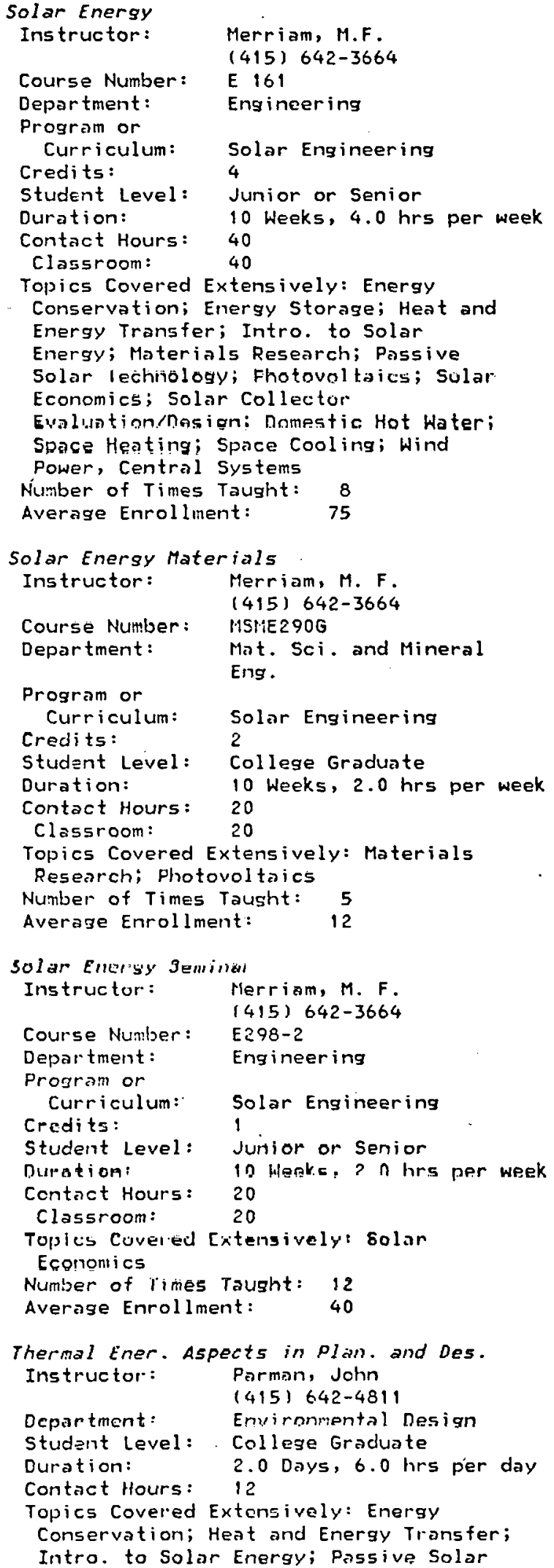


Technology; Solar System Components;

Domestic Hot hater; Space Heating;

Space Cooling; Wind Power, Small

Sy's tems

Number of Times Taught: 1

Average Enrollment: $\quad 14$

Thermal Radiation ME253

Instructor: Daily, John W.

(415) 642-0238

Ccurse Number: ME253

Department: Mechanical Engineering

Program or

Curriculum: Solar Engineering

Credits:

4

Student Level: Collese Graduate

Duraticn: 10 heeks, 4.0 hrs per week

Contact Hours: 40

Topics Covered Extensively: Heat and

Energy Transfer

Number of Times Taught: 7

Average Enrollment: $\quad 28$

Hind Energy

Instructor: Pike, Nanette

(415) 642-4151

Department: Continuing Education in

Engineering
Student Level: $\quad$ College Graduate

Duration: 1 heeks, $8.0 \mathrm{hrs}$ per wepk

Contact Hours: 8

Topics Covered Extensively: Wind Power,

Central Systems; Wind Power, Small

Systems

Number of Times Taught: 2

Averase Enrollment: $\quad 35$

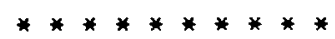

CAL- OAVIS, U OF

DAVIS, California 95616

(1313)

(916) 752-1011

PROGRAMS ARID CURRICULA

Graduate Solar Energy

Degree: MA, MS, Atmospheric Sciences

r.nntart: Conulson, Kinsell

(916) $752-1450$

Students Taking or Completing Offering:

Educator, Researcher

\section{SOLAR RELATED COURSES}

Radiation Instrumentation and Measurement

Instructor: Flecchini, R. G.

(916) 752-7097

Course Number: 128

Department: Atmospheric Science

Prosrim or

Curriculum: Graduate Solar Energy

Credits: 3

Student Level: Junior or Senior

Duration: 10 Weeks, 5.0 hrs per week

Contact Hours: 50

Solar and felated Energy Sources

Instructor: Flocchini, R. G.

(916) $752-7097$
Course Number: 203

Department: Resource Science

Prosram or

Curriculum: Graduate Solar Energy

Credits:

3

Student Level: College Graduate

Duration: 10 Heeks, $3.0 \mathrm{hrs}$ per week

Contact Hours: $\quad 30$

Topics Covered Extensively: Alternate

Energy Sources; Biomass Conversion;

Fhotovoltaics; Wind Fower, Central

Systems; Wind Power, Small Systems

Solar Energy Applications

Instructor:

Flocchini, R. G.

(916) 752-7097

Course Number: 103

Department:

Resource Science

Program or

Curriculum: Graduate Solar Energy

Credits:

Student Level: Junior or Senior

Duration: 10 Weeks, $3.0 \mathrm{hrs}$ per week

Contact Hours: 30

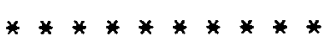

CAL- LOS ANGELES, U OF

LOS ANGELES, California 90024

$(1315)$

(213) 825-4321

PROGRAMS AND CURRICULA

Architecture/Urban Design

Degree: MA, OTHER, Architecture and

Urban Planning

Contact: Moore, Charles

(213) 825-8959

Students Taking or Completing offering:

Architect, Educator, Researcher

Ener. Conserving Des. Elective

Sequence

Degree: MA, OTHER, Architecture and Urban Planning

Contact: Moore, Charles

(213) 825-8950

Students Taking or Completing Offering:

Architect, Researcher

\section{SOLAR RELATED COURSES}

Building Climatology

Instructor: Givoni, Baruch/ Milne,

Murray

Course Number: 442

Department: Architecture/Urban

Program or

Curriculum: Ener. Conserving Des.

Credits: Elective Sequence

Student Level: College Graduate

Duration: 10 Weeks, 4.0 hrs per week

Contact Hours: 40

Classroom: $\quad 40$

Topics Covered Extensively: Heat and Energy Transfer; Solar Systens Design; 
Space Heating; Space Cooling

Number of Times Tausht: 3

Average Enrollment: $\quad 40$

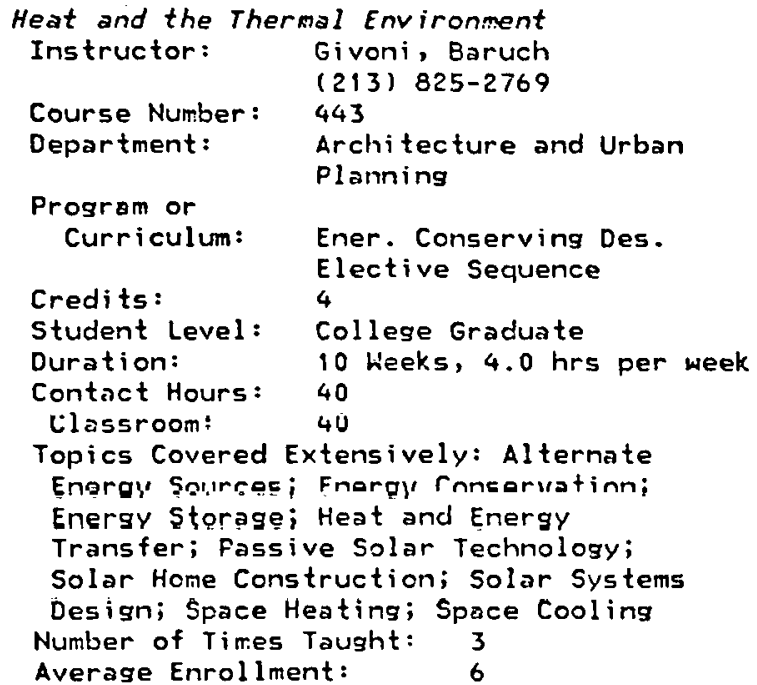

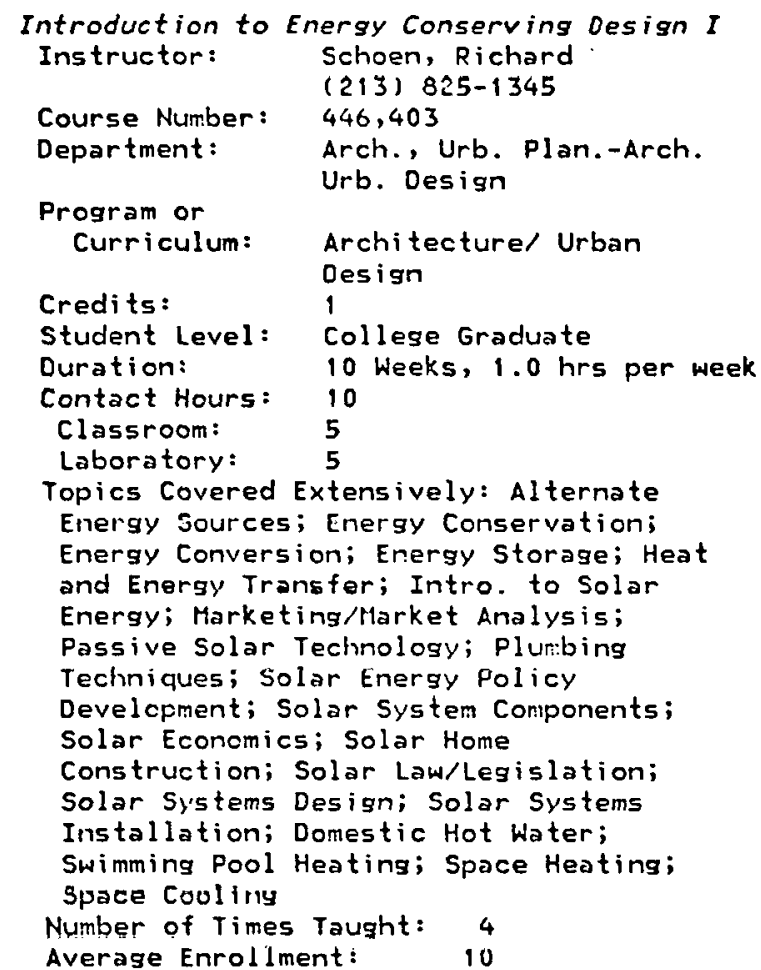

$\begin{array}{cc}\text { Introduction to Energy Conserving Design II } \\ \text { Instructor: } & \begin{array}{l}\text { Schoen, Richard } \\ (213) 825-1345\end{array} \\ \text { Course Number: } & 446,403 \\ \text { Department: } & \begin{array}{l}\text { Arch, Urb. Plan.-Arch. } \\ \text { Urb. Design }\end{array} \\ \text { Program or } & \\ \text { Curriculum: } & \text { Architecture/ Urban } \\ & \text { Design } \\ \text { Credits: } & 1 \\ \text { Student Level: } & \text { College Graduate }\end{array}$

Duration:

Contact Hours: 10

Classroom: $\quad 5$

Laboratory: 5

Topics Covered Extensively: Alternate Energy Sources; Energy Conservation; Energy Conversion; Energy Storage; Heat and Energy Transfer; Intro. to Solar Energy; llarketing/Market Analysis; Passive Solar Techmology; Plumbing Techniques; Solar Energy Policy

Development; Solar System Components;

Solar Economics; Solar Home

Construction; Solar Law/Legislation;

Solar Sy'stems Design; Solar Systems

Installation; Domestic Hot Water;

Swimining Pool Heating; Space Heating;

Space Coolling

Number of Times Taught: 4

Average Fnroliment: in

Introduction to Energy Conserving Design $\bar{I} \bar{I} \bar{I}$

Instructor: Schoen, Richard

Course Nlumber: 446,403

Department: Arch. Urb. Plan.-Arch.

Program or

Curriculum: Architecture/ Urban

Credits:

Student Level: College Graduate

Duration: 10 Weeks, 1.0 hrs per week

Contact Hours: 10

Classroom: $\quad 5$

Laboratory: .5

Topics Covered Extensively: Alternate Energy Sources; Energy Conservation; Energy Conversion; Energy Storage; Heat and Energy Transfer; Intro. to Solar Energy; Marketing/Market Analysis; Passive Solar Technology; Plumbing Techniques; Solar Energy folicy

Development; Solar System Components;

Solar Economics; Solar Home

Construction; Solar Law/Legislation;

Solar Systems Design; Solar Systems

Installation; Domestic Hot Water;

Swimining Pool Heating; Space Heating;

Space Cooling

Number of Times Taught: 4

Average Enrollment: $\quad 10$

New Ener. Tech.-Res. Conv. Constraints

Instructor: Buchberg, $H$.

Course Number: $134 \mathrm{~A}$

Credits: 4

Stuoient Level: Junlor or Senior

Duration: $\quad 10$ Weeks, $4.0 \mathrm{hrs}$ per week

Contact Hours: 40

Classroom: $\quad 40$

Topics Covered Extensively: Alternate Energy Sources; Energy Conservation; Energy Conversion

Number of Times Taught: 5

Average Enrollment: $\quad 20$

Proj. in Arch.: Computer Aided Design

Instructor: Milne, Murray

(213) $825-7370$ 


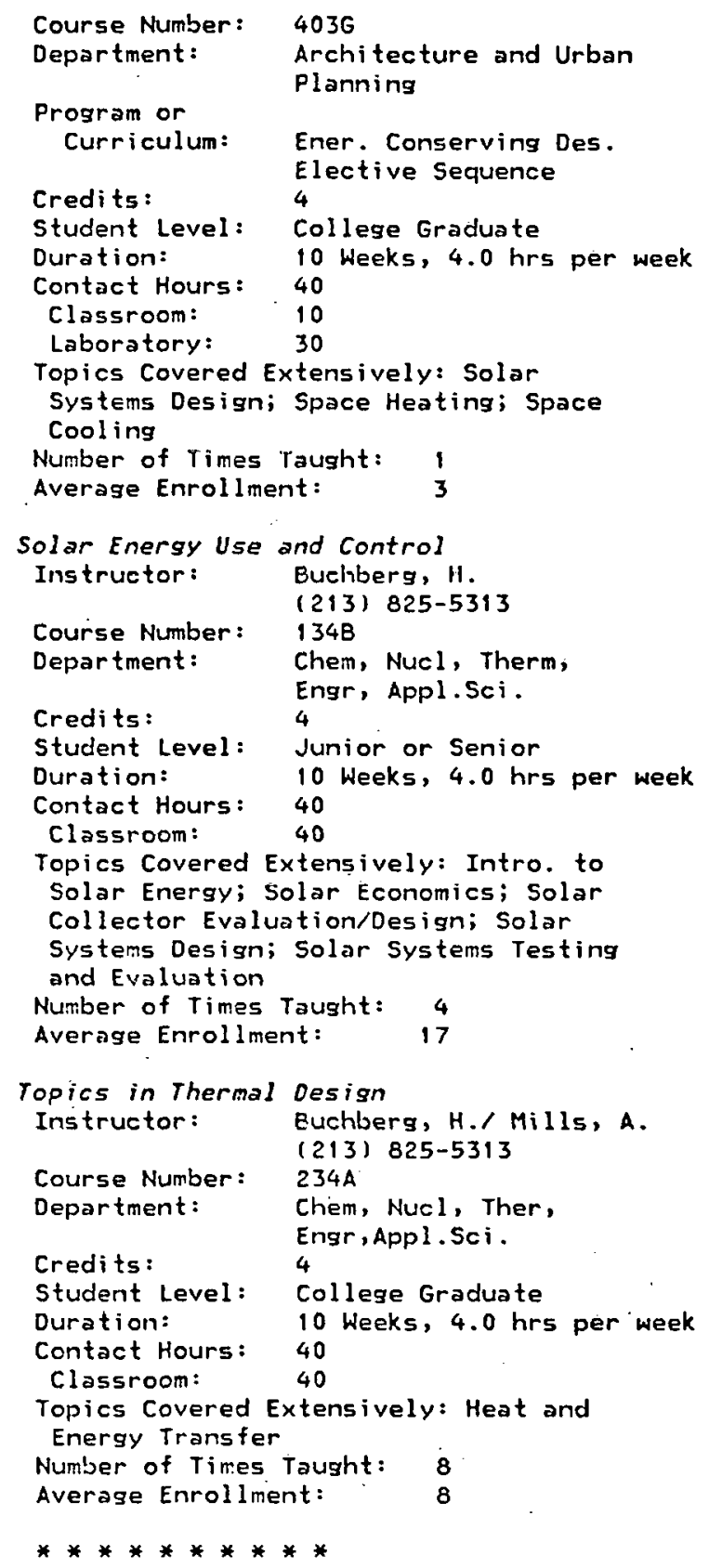

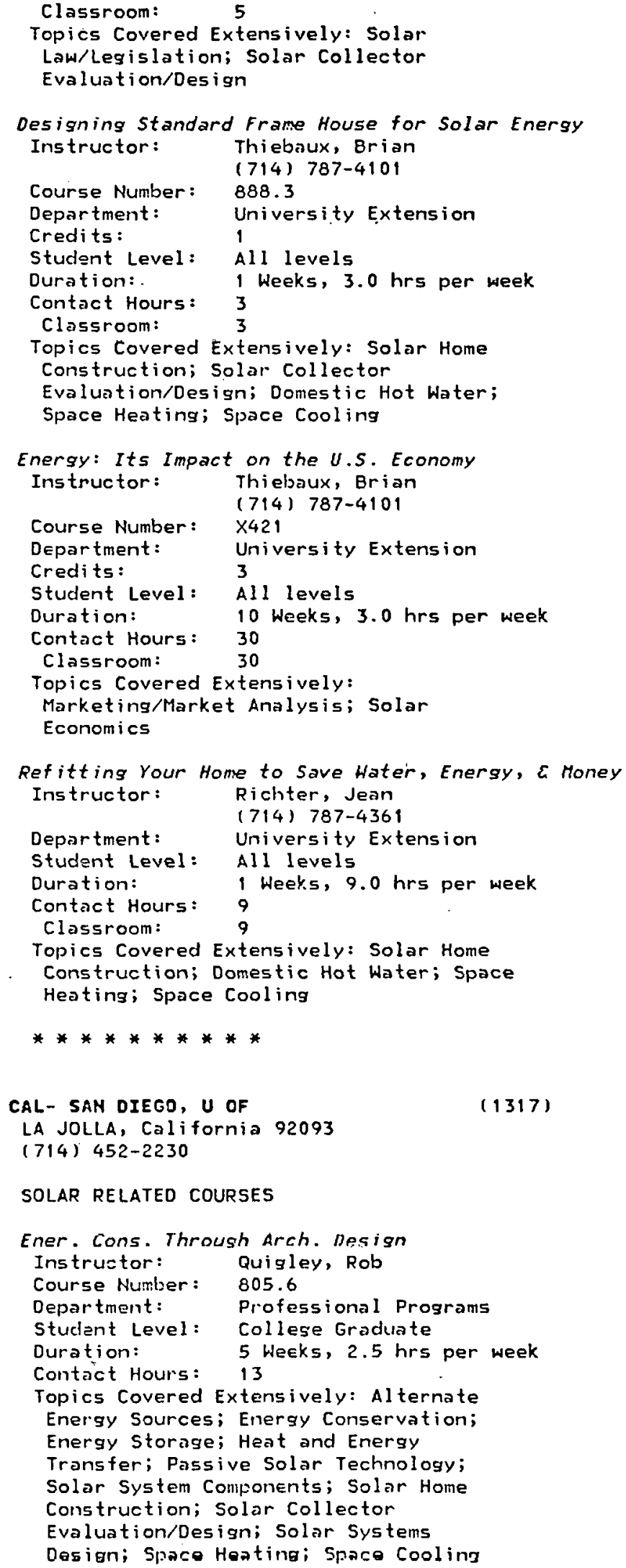




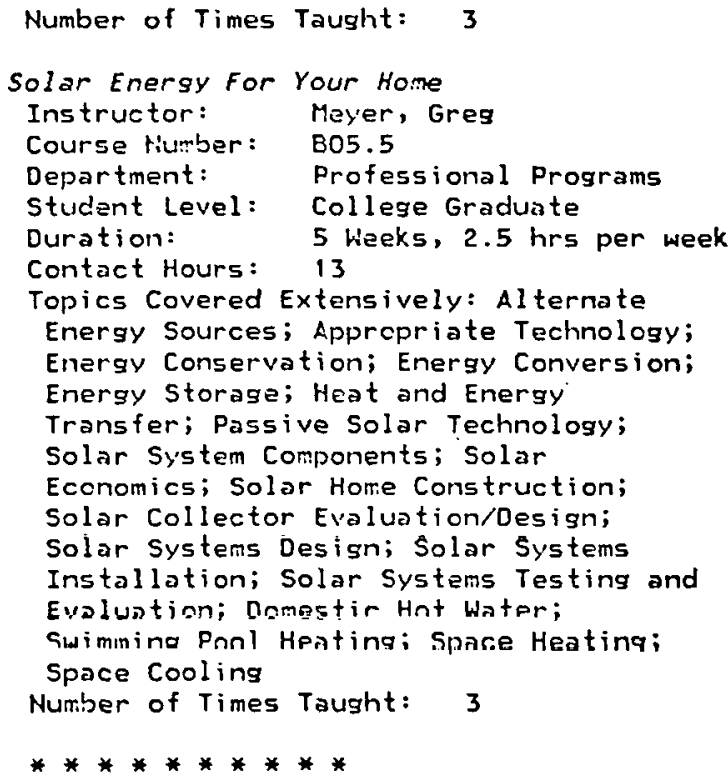

Conservation; Intro. to Solar Enersy; Passive Solar Technology; Solar System Components; Solar Collector

Evaluation/Design; Solar Systems Design

*The Sun

Instructor: Menger, Eva/ Hammond,

George

Course Number: OAKES 38

Department: Oakes College

Topics Covered Extensively: Intro. to Solar Energy

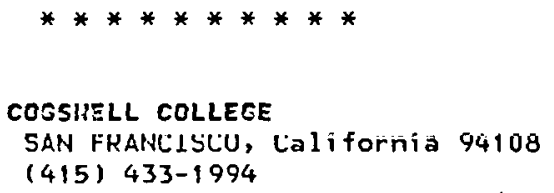

HARVEY HUDD COLLEGE

CLAREMONT, California 91711

(714) 626-8511

\section{SOLAR RELATED COURSES}

freshwan projects

Wolf, Robert

\section{Course Number:}

Deportment:

Credits:

student Level:

Duration:

Cuntact llours:

Classroom: (714) $621-8000$

\section{FY 4}

Freshman Division

1

Freshman or Sophomore

7 Weeks, 3.0 hrs per week 21

21

Topics Covered Extensively: Alternate

Energy Sources; Appropriate Technology;

Energy Conservation; Energy Storage;

Heat and Energy Transfer; Intro. to

Solar Energy; Passive Solar Technology; 
Solar Economics; Solar Collector Evaluation/Desisn; Solar Systems Design; Solar Sy'stems Installation; Solar Systems Maintenance; Solar Systems Testing and Evaluation; Domestic Hot Water; Swinming Pool Heating; Space Heating Nuriber of Times Tausht: 2 Averase Enrollment: $\quad 30$

**********

HUMEOLOT STATE U

ARCATA, Califernia 95521

(1149)

$(707) 826-3011$

\section{SOLAR RELATED COURSES}

\section{Solar Energy: Thermal Processes \\ Instructor: Borgers, Tom R. (707) 826-3255 \\ Course Number: EliGR. 184 \\ Department: Engr./Chem. \\ Credits: 3 \\ Student Level: Junior or Senior \\ Duration: 10 Heeks, $3.0 \mathrm{hrs}$ per week \\ Contact Hours: 30 \\ Classroom: 30 \\ Topics Covered Extensively: Heat and \\ Eneisy Transfer; Intro. to Solar Energy \\ Number of Times Taught: 3 \\ Average Enrollment: 7}

\section{LA VERIIE COLLEGE}

$(1216)$

LA VERHE, California 91750

(714) 593-351

\section{SOLAR RELATED COUPSES}

Energy Colloquium

Instructor: Green, Richard $H$.

Credits: (714) 593-3511

Student Level: All levels

Duration: 14 heaks, $3.0 \mathrm{hrs}$ per week

Tepics Coveled Exlemively: Alternate

Energy Sources; Appropriate Technology;

Biomass Conversion: Passive Solar

Technolozy; Fhotovoltaics; Solar Energy

Policy Developnent; Solar Economics;

Solar Law/Legislation; Wind Power,

Small Systems

\section{Energy Options}

Instrurtor:

Arnold, Geo.

(714) 593-7792

Course Number: FHYSICS

Dèpartnient: Pliysics

Credits:

Student Level: Junior or Senior

Duration: $\quad 14$ Weeks, 4.0 hrs per week

Contact Hours: 56

Tcpics Covered Extensively: Alternate

Energy Scurces; Intro. to Solar Energy

Nurber of Times Taught: 1

Average Enrollment:
***********

LOHA LINDA UMIVERSITY

LOMA LINDA, California 92354

$(1218)$

(714) 796-7311

SOLAR RELATED COURSES

Practical Solar Energy

Instructor:

halls, Art

(714) 785-2218

Course Number: Itlos 283

Department: Industrial Studies

Credits:

4

Duration:

Contact Hours: 60

Classroom: $\quad 30$

Topics Covered Extensively: Heat and Energy Transfer; Intro. to Solar

Energy; Solar System Components; Solar

Collector Evaluation/Design

Nuniber of Times Taught: 2

Average Enrollment:

9

**********

LOYOLA MARYMOURT U

LOS AHGELES, California 90045

(213) 642-2700

\section{SOLAR RELATED COURSES}

Thermal Aspects of Cesign

Instructor: Callihan, J.P.

(213) 642-2827

Course Number: ME 571

Department: Machancial Engineering

Credits: 4

Student Level: Junior or Senior

Duration: 15 Weeks, $4.0 \mathrm{hrs}$ per week

Contact Hours: 60

Clissroom: 60

Topics Covered Extensively: Energy

Conversion; Heat and Energy Transfer;

Intro. to Solar Energy; Solar Collector

Evaluation/Design; Solar Systems

Design; Space tieating

Number of Tin:es Taught: 2

Average Enrollnient:

10

***********

HEH COLLEGE OF CALIFORMIA

$(10831)$

SAN FPAIICISCO, California 94110

(415) 626-1694

FROGRAMS AND CURRICULA

Econ.,Ener. Formation of Horld

Conscious

Degree: MA, BA, Humanities-Energy, Economics, Derisn

Contact: Beru, Jelalce!din (415) 6:6-1694

Stuclents Taking or Completing Offering:

Educator, Researcher, Trade Specialty 


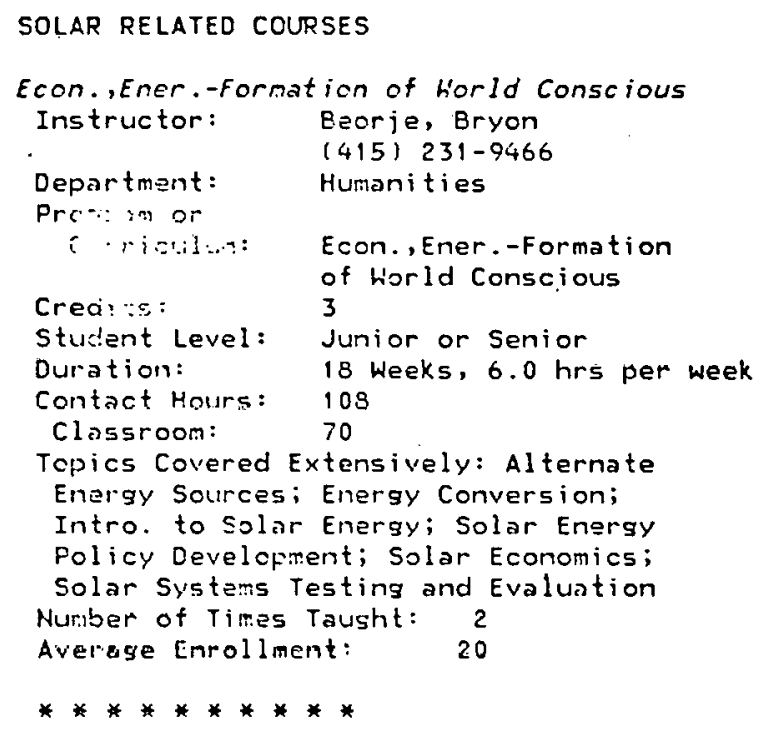

NORTHROP UNIVERSITY

(1248)

INIJLEHOOD, California 90306

(213) $776-3410$

PROERAMS AND CURRICULA

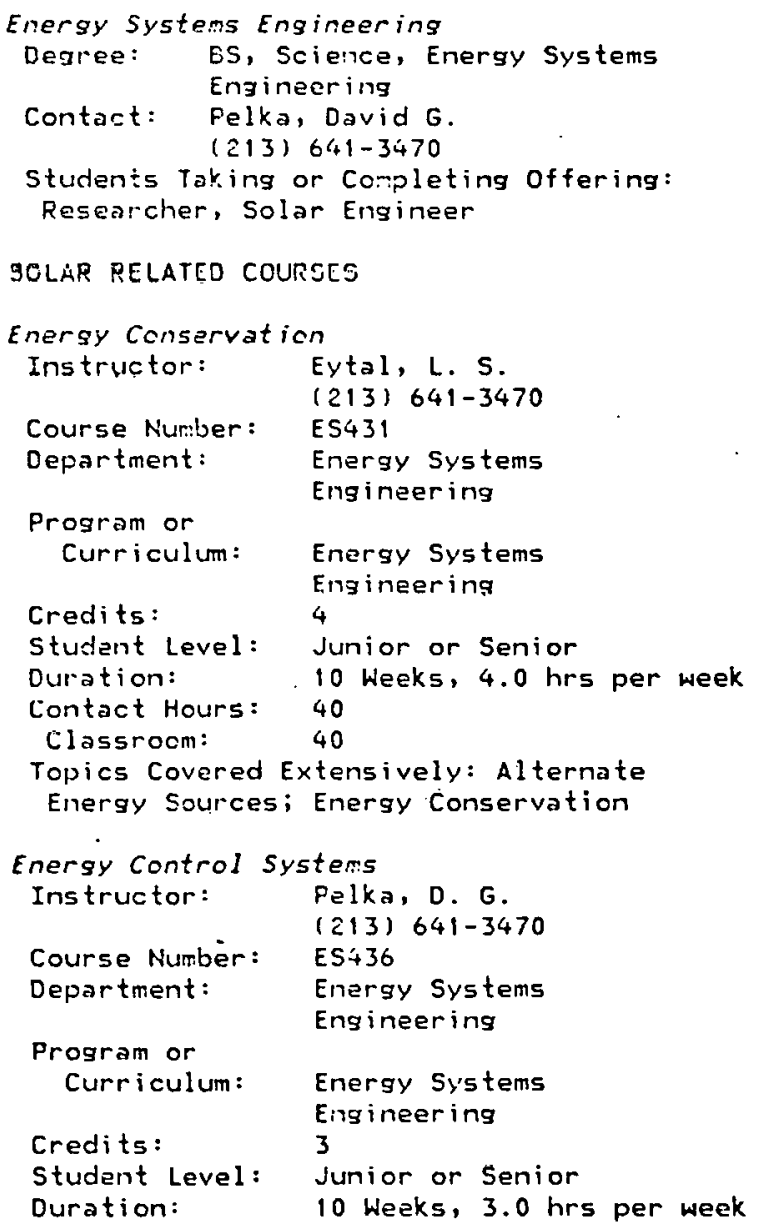

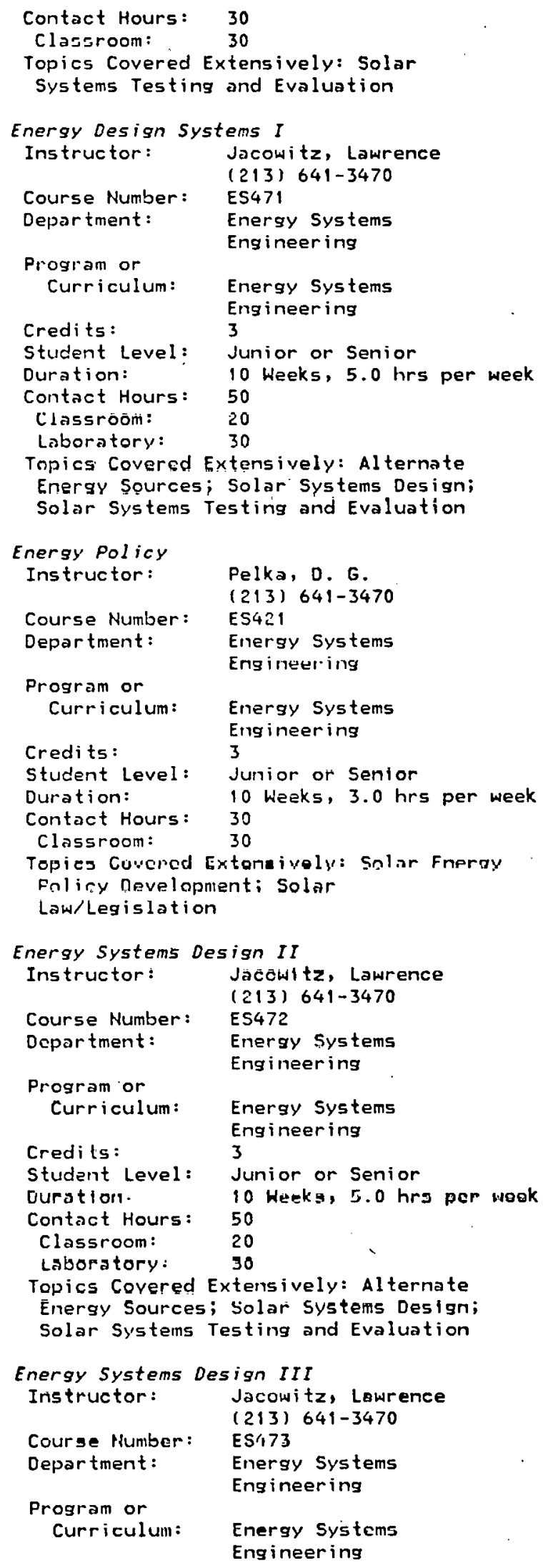




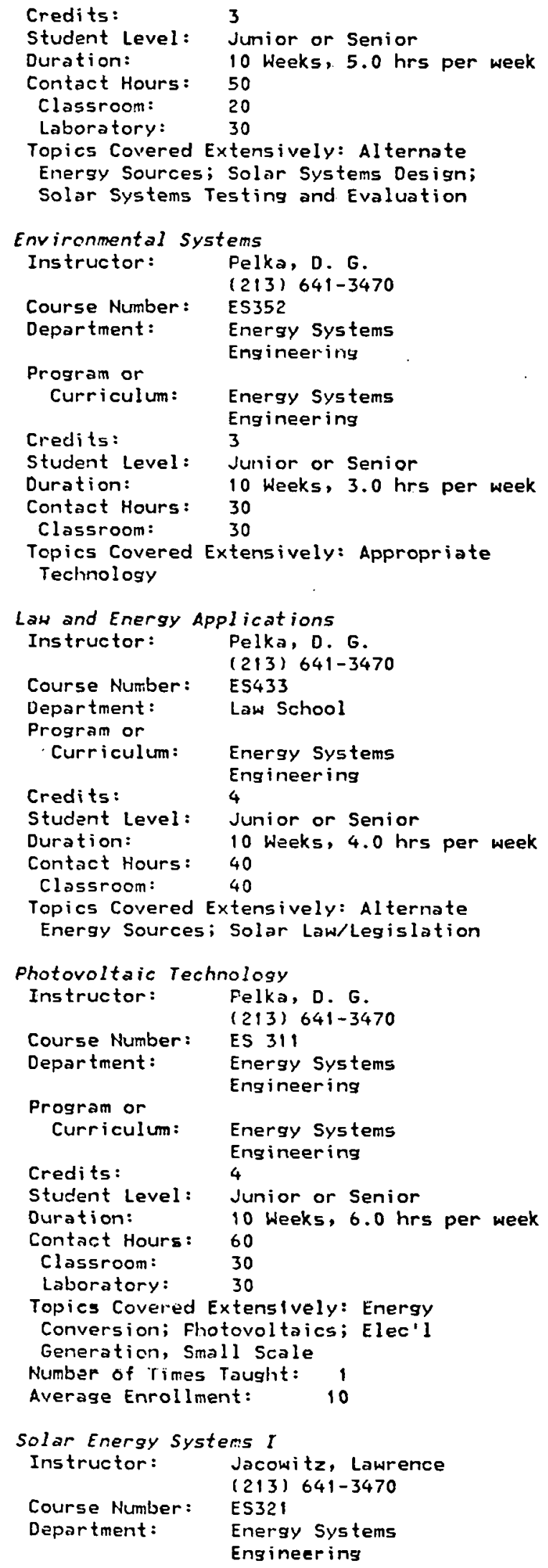

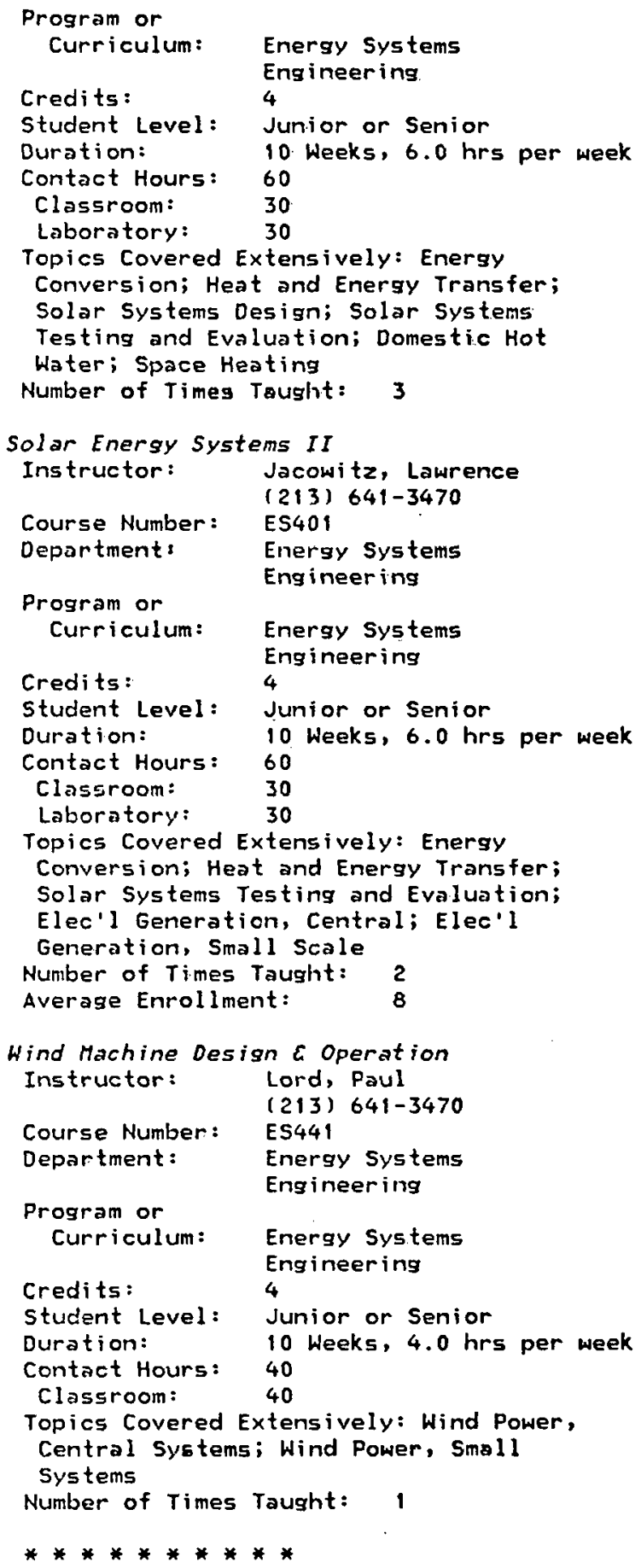


REDLAKOS, UIIIVERSITY OF

(714) 793-2121

\section{SOLAR RELATED COURSES}

*Energy Alt, - Priorities, Policies Instructor: Krantz, Reinhold J.

Course Number: 25

Department: Arts \& Sci. - Eng'r

Topics Covered Extensively: Alternate Energy Sources; Solar Economics

***********

SAH DIEGO STRTE $U$

SAN DIEGO, California 92182

( 7111 ) $286-5000$

\section{SOLAR RELATED COURSES}

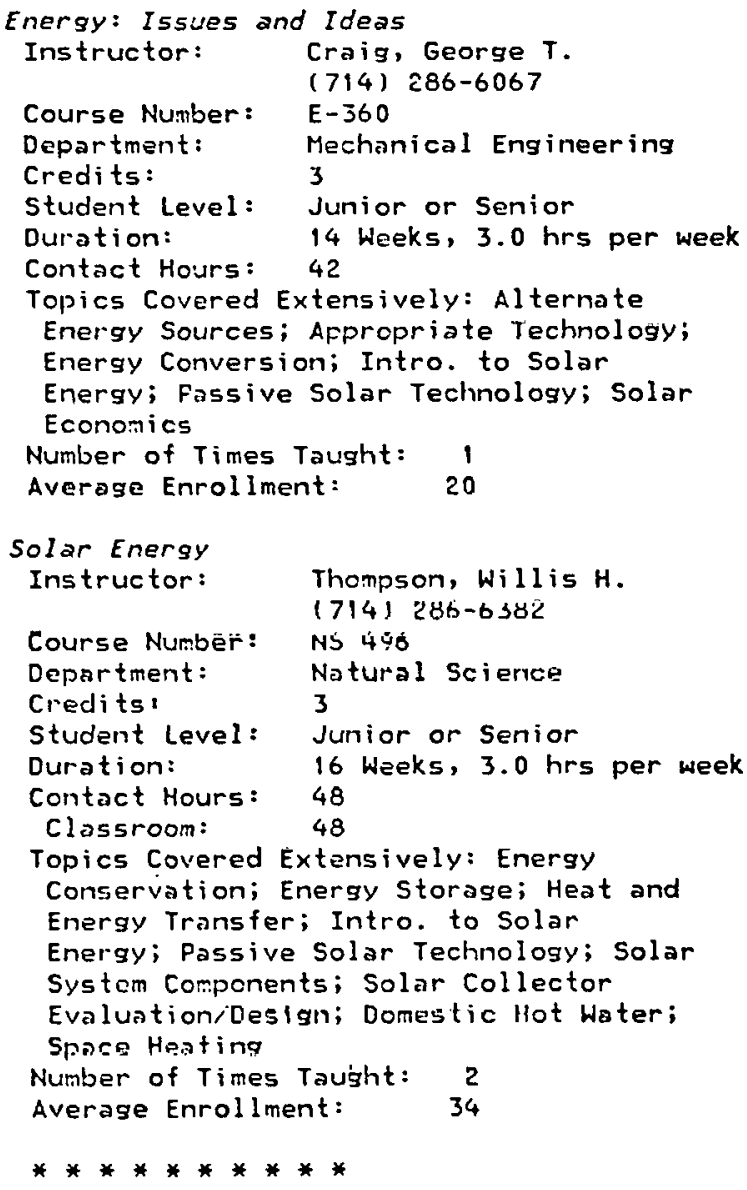

SAN FRANCISCO STATE U

SAN FRANCISCO, California 94132

(415) 469-2141

\section{SOLAR RELATED COURSES}

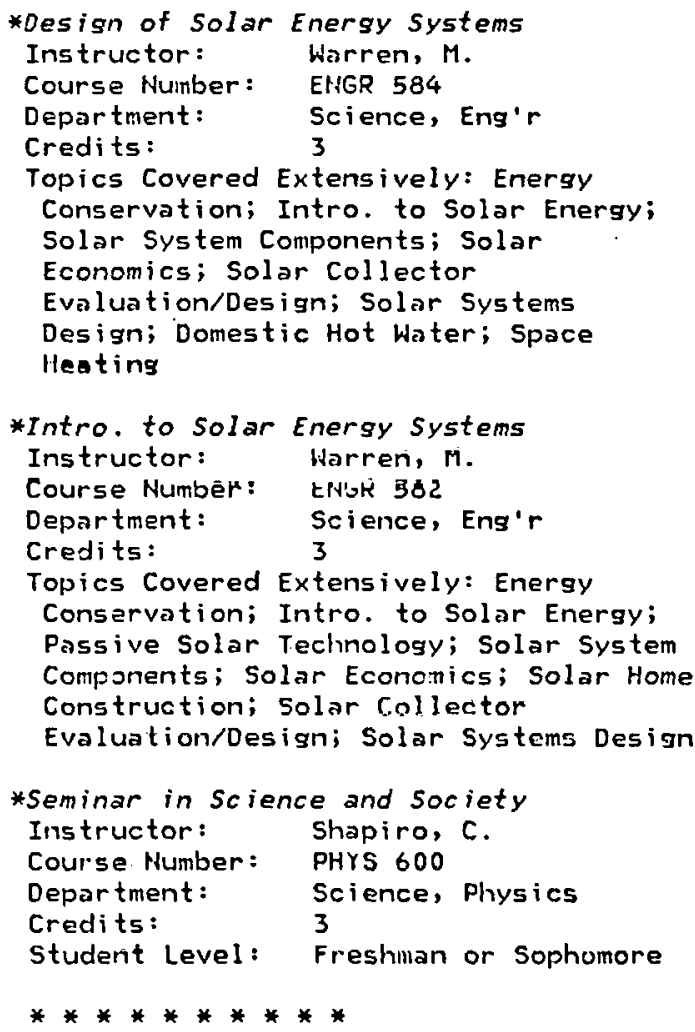

SAN FRANCISCO, U OF

SAH FRAPICI8CO, Galifornio 94117

$\cdot(1325)$

$(115) \quad 666=n 6 \cap n \cap$

PROGRAMS AND LURRICULA

Environmental Planning $E$ Management Degree. MS, Environm. Plan. and Management

Contact: Fetulla, Joseph M. (415) 666-6254

SOLAR RELATED COURSES

$\begin{array}{ll}\begin{array}{l}\text { Ecosicence } \\ \text { Instructor: }\end{array} & \begin{array}{l}\text { Gruhn, Thomas } \\ (415) 666-0208\end{array} \\ \begin{array}{l}\text { Course Number: } \\ \text { Department: }\end{array} & \begin{array}{l}\text { IDS } 250 \\ \text { Continuing Education } \\ \text { Program or }\end{array} \\ \text { Curriculum: } & \text { Environmental Planning } \\ & \mathcal{L} \text { Management } \\ \text { Credits: } & 4 \\ \text { Student Level: } & \text { Collese Graduate } \\ \text { Duration: } & 14 \text { Weeks, } 4.0 \text { hrs per week } \\ \text { Contact Hours: } & 56\end{array}$




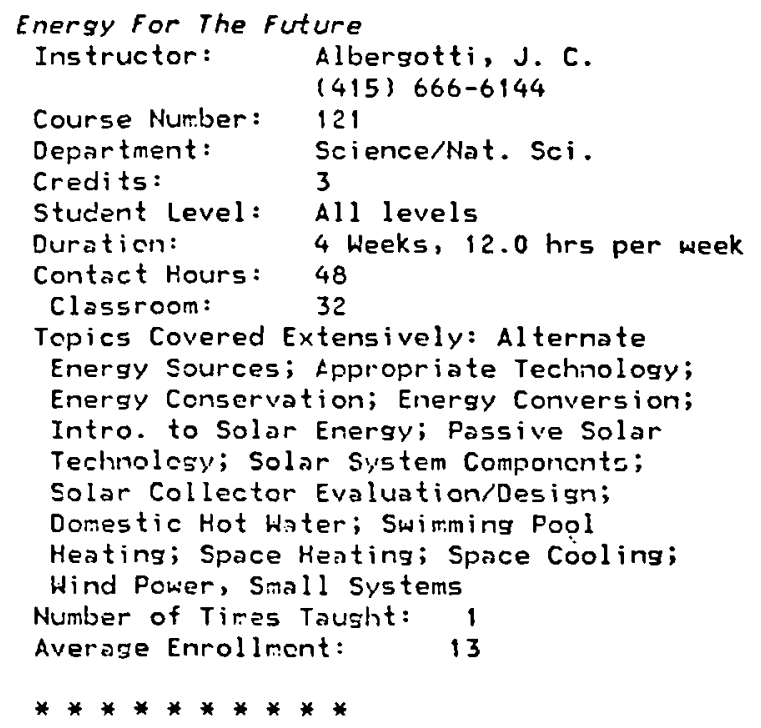

SAN JOSE, California 95192 (408) 277-2000

FROGRAMS ARID CURRICULA

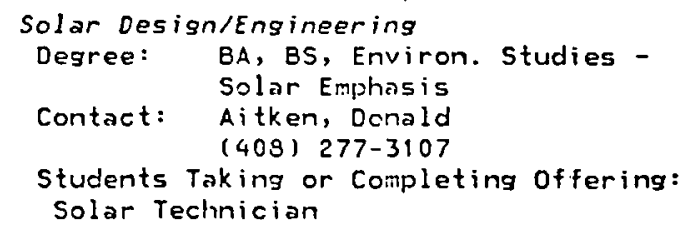

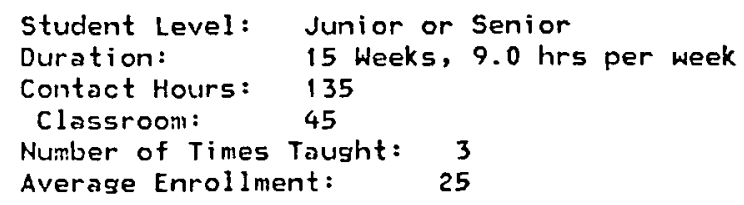




\section{SOLAR RELATED COURSES}

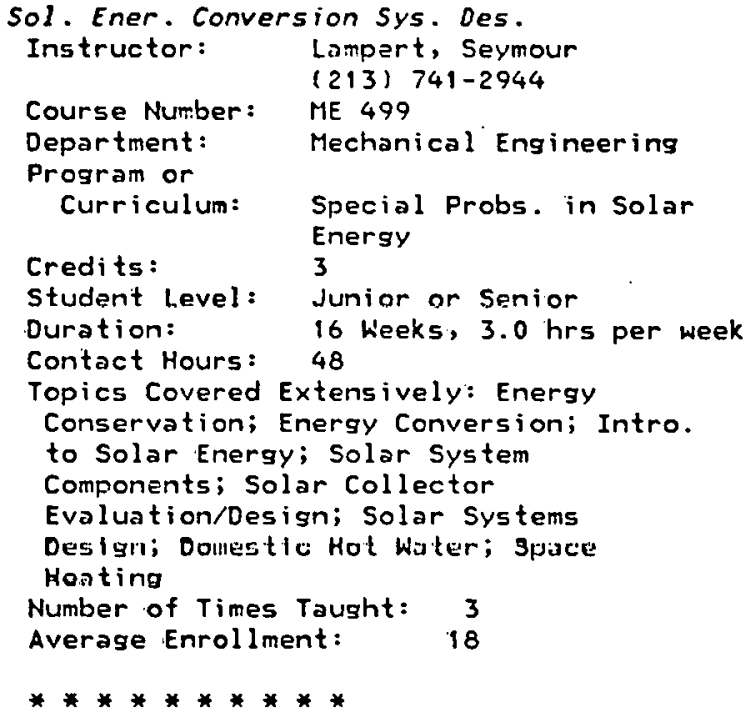

\section{Community/Junior Colleges}

AMERICAN RIVER COLLEGE

SACRAMENTO, California 95841

(916) 484-8011

SOLAR RELATED COURSES

*Alternative Energy Courses
(9552)
ANTELOPE VALLEY COLLEGE

$(1113)$

LANCASTER, California 93534

(805) 947-0160

\section{SOLAR RELATED COURSES}

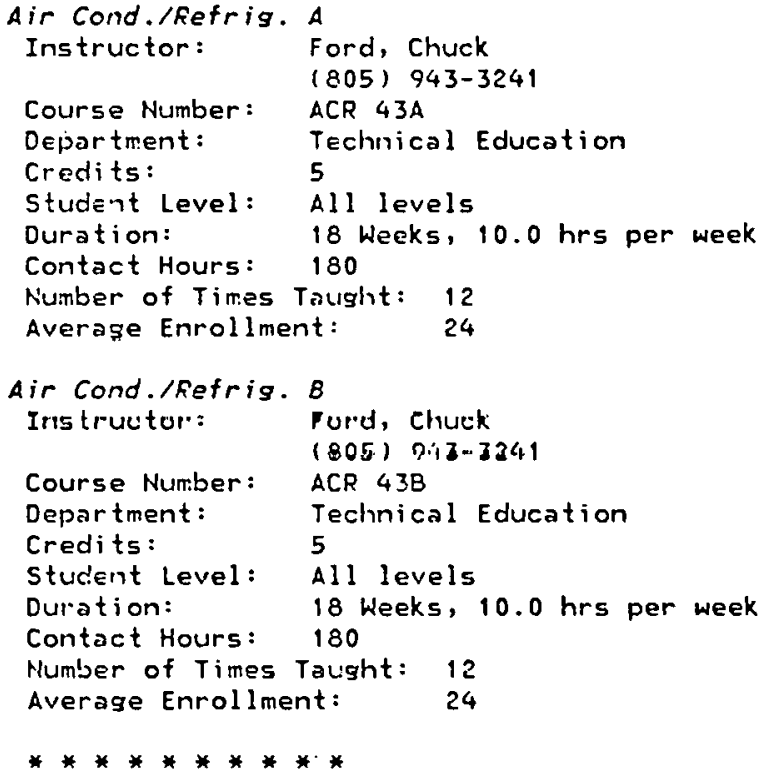




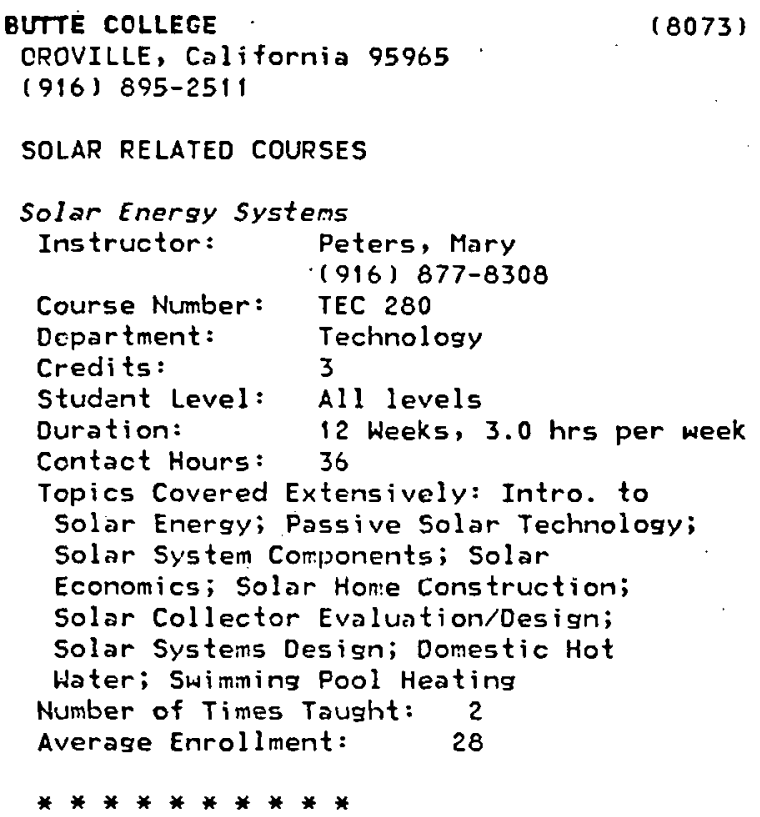

(8073)

$(1124)$

\section{SOLAR RELATED COURSES}

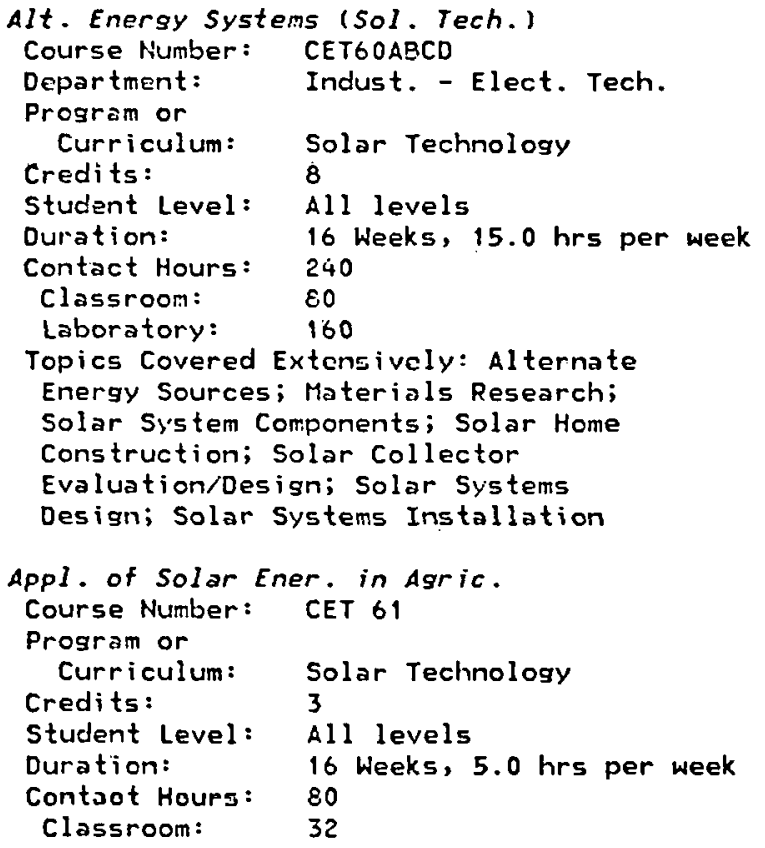




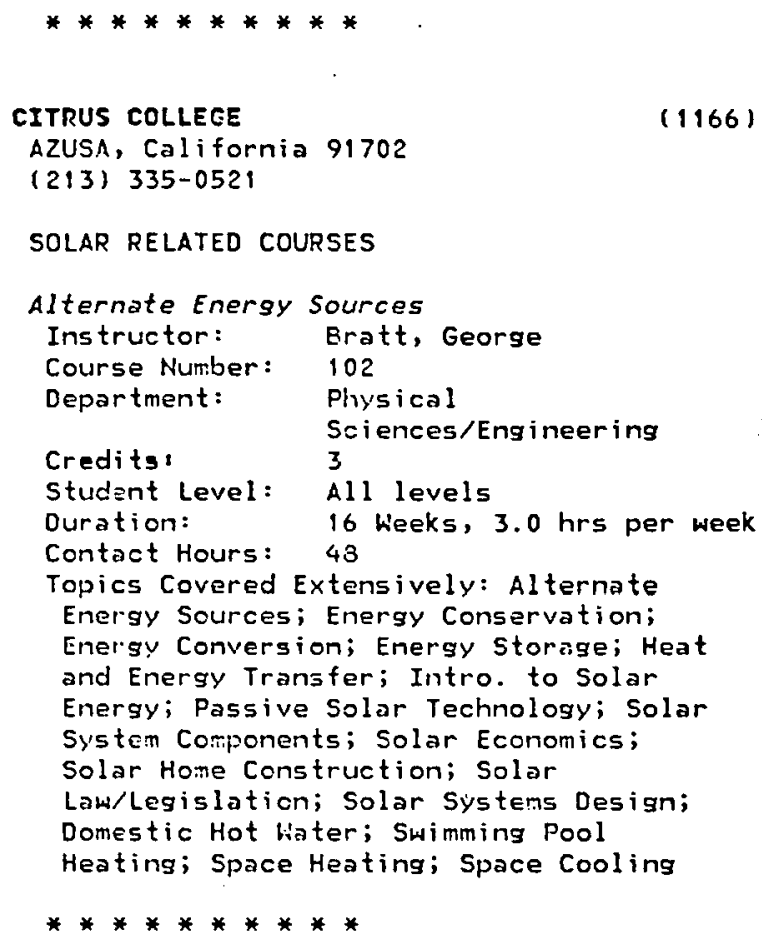

SOLAR RELATED COURSES

* Course on Alternate Energy

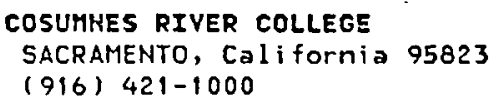

\section{SOLAR RELATED COURSES}

EO 47, Alternate Energy Systems

Instructor: House, Harold

Course Number: 3108-01

Department: Environmental Design

Program or

Curriculum: Envir. Des. -Intro.

Credits: 2

Duration: $\quad 8$ Weeks, $3.0 \mathrm{hrs}$ per week

Contact Hours: 24

Classroom: 24

Topics Covered Extensively: Alternate Energy Sources; Appropriate Technology;

Intro. to Solar Energy; Photovoltaics

Number of Times Taught: 3

Average Enrollment: $\quad 15$

Intro. to Solar Energy Systems

Instructor: House, Harold

Course Number: ED 31

Department: Environmental Design

Program or

Curriculum: Envir. Des. -Intro.

Credits: ,

Duration: 4 Weeks, $16.0 \mathrm{hrs}$ per week

Contact Hours: 64

Classroom: $\quad 48$

Laboratory: 16

Topics Covered Extensively: Energy Storage; Heat and Energy Transfer; Intro. to Solar Energy; Fassive Solar Technology; Solar System Components; Solar Home Construction; Solar Collector Evaluation/Design; Solar Systems Desian; Space Heating; Space Cooling

Residential Energy Conservation

Instructor: House, Harold

Course Number: 3105-01

Department: Environmental Design

Prograni or

Curriculum: Envir. Des. Intro.

Credits:

Duration:

Contact Hours:

Classroom: Sol. Ener. Systems

2

3 Weeks, 8.0 hrs per week

24 24

Topics Covered Extensively: Appropriate

Technology; Energy Conservation; Energy Storase; Heat and Energy Transfer;

Intro. to Solar Energy;

Marketing/Market Analysis; Passive 
Solar Technology; Solar Home

Construction; Solar Systems Design;

Domestic Hot Water: Space Heating;

Space Cooling

Number of Times Taught: 3

Averase Enrollment: $\quad 15$

\section{CUESTA COLLEGE}

SAN LUIS OBISPO, California 93406

$(1192)$

(805) 544-2943

SOLAR RELATEO COURSES

App. of Solar Energy Systems

Instructor: Lagomarsino, Peter (805) 543-2943

Cour"se Hurtuer:

CT6O

Department:

Construction Technology

Credits: 4

Student Level: Freshman or Sophomore

Duration: 18 Weeks, 6.U hrs per week

Contact Hours: 108

Classroom: $\quad 54$

Laboratory: $\quad 54$

Tcpics Covered Extensively: Energy

Storase; Heat and Energy Transfer;

Intro. to Solar Energy; Solar System

Components; Domestic Hot Water; Space

Heating; Space Cooling

Number of Times Taught: 2

Average Enrollment:

18

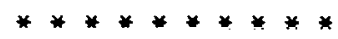

DE AHEA COLLEGE

CUPERTINO, California 95014

(4480)

(408) $996-4567$

SOLAR RELATED COURSES

Design of Sol. Ener. Sys.-Heat. and Cool. A

Instructor: k'edel, $R$.

Course Number: $379 \mathrm{~A}$

Department: Engineering

Credits: 2

Student Level: All levels

Duration: $\quad 12$ heeks, $2.0 \mathrm{hrs}$ per week

Contact Hours: 24

Classroom: 24

Number of Times Taught: 4

Average Enrollment: $\quad 20$

Design of Sol. Ener. Sys.-Heat. and Cool. B

Instructor:

Wedel1, R.

(408) 493-4411

Course Number: 3798

Department : Engineering

Credits:

Student Level: All levels

Duration: 12 Weeks, $2.0 \mathrm{hrs}$ per week

Contact Hours: 24

Classroom: 24

Topics Covered Extensively: Solar

Economics; Solar Collector

Evaluation/Design; Solar Systems Design
Number of Times Taught: 4

Average Enrollment: $\quad 20$

Utilization of Solar Energy

Course Number: 369

Department: Engineering

Credits: 3

Student Level: All levels

Duration: 12 Weeks, 3.0 hrs per week

Contact Hours: 36

Classroom: 18

Laboratory: $\quad 18$

***********

DESERT, COLLEGE OF THE

PALM DESERT, California 92260

$(1182)$

(714) 346-8041

SOLAR RELATEO COURSES

Introduction to Solar Energy

Instructor: Marzicola, John

(714) 346-8041

Course Number: ARCH 13

Department: Engineering and

Credits: 3

Technology

Student Level: All levels

Duration: $\quad 18$ Weeks, 3.0 hrs per week

Contact Hours: 54

Classroom: $\quad 54$

Topics Covered Extensively: Alternate

Energy Sources; Appropriate Technology;

Energy Conservation; Intro. to Solar

Energy; Solar Home Construction; Solar

Law/Legislation; Domestic Hot Water;

Swimming Pool Heating; Space Heating;

Space Cooling

Number of Times Taught: 3

Average Enrollment: 42

$* * * * * * * * * *$

diálo VALLEy College

(1191)

PLEASANT HILL, California 94523

(415) $685-1230$

SOLAR RELATEO COURSES

Energy and Buildings

Course Number: 150

nepartment: arrh /Fnginopring

Credits: 3

Gunstion: 16 Wceks, 6.0 hrs per week

Contact Hours: 96

Classroom: $\quad 48$

Laboratory: 48

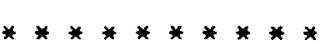


EVEPGREEN VALLEY COLLEGE

SAN JOSE, California 95121

(408) 274-7900

PROGPAMS AND CUPRICULA

* Solar Technician

Students Taking or Completing offering:

Solar Technician

SOLAR RELATED COLRSES

* Solar and Energy Seminar

* Solar house

* Tho Courses on Solar

Program or

Curriculum: *Solar Technician

$* * * * * * * * * *$

\section{FEATHER RIVER COLLEGE}

(8597)

GUINCY, California 9597

(916) 283-0202

\section{SOLAR RELATED COURSES}

Solar Utilization and Energy--Wise Construction Instructor: Mortin, Bill (916) 283-1197

Course Number: PHYS. SCI.75

Department: Physical Science

Credits: 2

Student Level: All levels

Duration: 18 Weeks, 2.0 hrs per week

Contact Hours: 36

Classrocm: $\quad 36$

Topics Covered Extensively: Appropriate

Technology; Energy Conservation; Energy Conversion; Energy Storage; Heat and

Energy Transfer; Intro. to Solar

Enersy; Passive Solar Technology; Solar

Home Construction; Domestic Hot Water;

Space Heating; Space Cooling

Nurber of Times Taught: 1

Average Enrollment: $\quad 15$

**********

FOOTHILL COLLEGE

LOS ALTOS HILLS, California 94022

(1199)

(415) $948-8590$

\section{SOLAR RELATED COURSES}

Solar Energy

Instructor:

Course Humber:

Blanchard/ Heinemann/

Schiavo

Department:

Engineering $\&$

Technology

Credits:

Student Level:

Duration:

4

Contact Hours: 40

Topics Covered Extensively: Intro, to

Solar Energy
Number of Times Taught: 4 Average Enrollment: 30

$* * * * * * * * * *$

FRESHO CITY COLLEGE

FRESHO, California 93704

$(1307)$

(209) 442-4600

SOLAR RELATED COURSES

Solar Energy

Instructor: Mortensen, David

(209) 442-8215

Department: Earth/Physical Science

Credits: 2

Student Level: All levels

Duration: 6 Weeks, $6.0 \mathrm{hrs}$ per week

Contact Hours : 36

Classroom: $\quad 36$

Topics Covered Extensively: Solar

Economics; Solar Home Construction;

Solar Collector Evaluation/Design;

Solar Systems Design; Domestic Hot

Hater

Number of Times Taught: 2

Average Enrollment: $\quad 40$

\section{solar systems}

Instructor:

Wash, Dennis C.

(209) 442-4600

Course Number: AC-55

Department: Technical-Industrial

Credits: 3

Student Level: All levels

Duration: 18 Weeks, $4.0 \mathrm{hrs}$ per week

Contact Hours: 72

Classroom: $\quad 36$

Laboratory: $\quad 36$

Topics Covered Extensively: Alternate

Energy Sources; Energy Conservation;

Energy Storage; Heat and Energy

Transfer; Intro. to Solar Energy;

Materials Research; Passive Solar

Technology; Photovoltaics; Plumbing

Techniques; Solar System Components;

Solar Home Construction; Solar

Collector Evaluation/Design; Solar

Systems Design; Solar Systems

Installation; Solar Systems

Maintenance; Solar Systeris Testing and

Evaluation; Domestic Hot Water;

Swimming Pool Heating; Space Heating;

Space Cooling

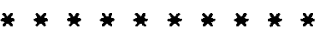

FULLERTOH COLLEGE

FULLERTON, California 92634

(1201)

(714) 879-8000

\section{SOLAR RELATED COURSES}

* Solar Heat ing

Topics Covered Extensively: Space Heating 
**********

\section{GAVILAN COLLEGE}

(1202)

GILROY, California 95020

(408) $847-1400$

\section{SOLAR RELATED COURSES}

Sol. Ener. H/hater Sys. Install.

Instructor: Hansen, John

$$
\text { (403) } 847-1400
$$

Course Number: $66 \mathrm{~A}$

Department: Occupational Education Credits: 3

Student Level: All levels

Duration: $\quad 12$ Weeks, $5.0 \mathrm{hrs}$ per week

Contact Hours: 60

CIassrocm: $\quad 24$

I sluniatnry: 36

Tnnirs Rnvered Extrensivelv: Eneray

Storage; Heat and Energy Transfer;

Intro. to Solar Energy; Plumbing

Techniques; Solar System Components;

Solar Home Construction; Solar Systems

Design; Solar Systems Installation;

Solar Systems Maintenance; Domestic Hot

Water; Swimming Fool Heating

Number of Tin:es Taught: 2

Average Enrollment: $\quad 25$

***********

\section{GLENDALE CMTY COLLEGE}

GLENDALE, California 91208

(1203)

(213) 240-1000

SOLAR RELATED COURSES

*Energy Alternatives

************

LOHG BEACH CITY COLLEGE

$(1219)$

LONG BEACH, California 90808

(213) 420-4111

PROGRAMS AND CURRICULA

* Air Cond. E Refrig.

SOLAR RELATED COURSES

* Solar sestent

Department: Air Cond. $t$ Refrig.

Program or

Curriculum: *Air Cond. C Refrig.
LOS ANGELES FIERCE COLLEGE

hOODLATD HILLS, California 91371

$(1226)$

(213) 347-0551

SOLAR RELATED COURSES

*Energy \& Power

Instructor:

Course Number:

Department:

Duraticn:

PHY SC 13

Topics Covered Extensively: Alternate Energy Sources; Energy Conservation;

Intro. to Solar Energy

* Man $\varepsilon$ His Environ: Phys. Processes

Instructor: . Meyer, W. Craig

(213) 347-0551

Course Number: EN ST 1

nepal tment: 1 ife : Farth ssience

Topics Covered Extensiyely: Alternate

Energy Sources; Solar Systems Design

$* * * * * * * * * *$

LOS AMGELES TRADE TECH COLLEGE

$(1227)$

LOS AlIGELES. California 90015

(213) 746-0800

SOLAR RELATED COURSES

Energy Manasement in Bulidings

Instructor: Adams, $\mathrm{N}$.

Course llumber: EM 189

Department: Electrical-Mechanical Creditg: 3

Student Level: High School Graduate

Duration: 20 Weeks, 3.0 hrs per week

Contact Hours: 60

Topics Covercd Extensively: Energy

Conservation; Heat and Energy Transter

Solar Energy

Instructor:

Aclams, $N$.

(213) 746-0800

Course liumber:

ENIT 183

Department:

Electrical-Mechanical

Credits: Dept.

Credits:

Student Level: High School Graduate

Dui áli uii

co Hecks, 3:0 lips per" weck

Contact Hours: 60

Topics Covered Extensively: Energy

Conversion; Emergy Storsge; Heat and

Energy Transfer; Intro, to Solar

Energy; Plumbing Techniques; Solar

System Components; Solar Economics;

Solar Home Construction; Solar

Collector Evaluation/Design; Solar

Systens Design; Solar Systems

Instollation; Domestic Hot Water; Space

lleating

Nurber of Times Taught: 1

Average Eirollment: $\quad 30$

$* * * * * * * * *$ 
HARIN, COLLEGE OF

$(1178)$

KENTFIELD, Cali fornia 94904

(415) 457-8811

SOLAR RELATED COURSES

Energy Efficient Design

Instructor: Sartor, Dale

Department: Aclult Education

Student Level: All levels

Duration: 10 Weeks, $3.0 \mathrm{hrs}$ per week

Contact Hours: 28

Classroom: $\quad 20$

Laboratory: 8

Topics Covered Extensively: Alternate

Energy Sources; Appropriate Technology;

Energy Conservation; Intro. to Solar

Energy; Passive Solar Technology; Solar

System Components; Solar Eccnomics;

Solar Home Construction; Solar

Collector Evaluaticn/Design; Solar

Systers Design; Solar Systems

Installation; Solar Systems

Maintenance; Solar Systeris Testing and

Evaluation; Domestic Hot Water;

Swiming Pool Heating; Process Heat,

Industrial; Space Heating; Wind Fower,

Small Systems

Number of Times Taught: 9

Average Enrollment: $\quad 50$

$* * * * * * * * * *$

MERCED COLLECE

MERCED, California 95340

$(1237)$

(209) 723-4321

SOLAR RELATED COURSES

Fesidential Application-Solar Energy

Instructor: Cox, James $W$.

Course Number: $\quad$ IT-40

Department: Industrial Technology

Credits: 3

Student Level: High School Graduate

Duration: 18 Weeks, $3.0 \mathrm{hrs}$ per week

Contact Hours: 54

Classroon: $\quad 54$

Topics Covered Extensively: Alternate Energy Sources; Appropriate Technology;

Enersy Conservation; Energy Conversion;

Energy Storage; Heat and Energy

Transfer; Intro. to Solar Energy;

Passive Solar Technology;

Pinotovoltaics; Solar Energy Policy

Development; Solar System Components;

Solar Economics; Solar Home

Construction; Solar Collector

Evaluationidesign; Solar Systems

Design; Solar Systens Testing and

Evaluation; Domestic Hot Hater;

Swimming.Fool Heating; Space Heating;

space Cooling
MODESTO JUHIOR COLLEGE

(1240)

MODESTO, California 95350

(209) 526-2000

PROGRAMS AHD CURRICULA

External - Project Sunrise

Contact: Wilson, E. William

(209) 526-2000

\section{SOLAR RELATED COURSES}

Solar Energy Applications

Instructor: Wilson, E. William

(209) 526-2000

Course Number: PS 368

Departinent: Dept. Engineering, Physical Science \& Mathematics

Program or

Curriculum: External - Project

Credits: 3 Sunrise

Student Level: All levels

Duration: $\quad 16$ Weeks, $3.0 \mathrm{hrs}$ per week

Contact Hours: 48

Classroom: $\quad 42$

Laboratory: 6

Topics Covered Extensively: Alternate

Energy Sources; Energy Conservation;

Energy Storase; Passive Solar

Technology; Solar System Components;

Solar Economics; Solar Home

Construction; Domestic Hot Water; Space

Heating; Space Cooling; Wind Power,

Small Systems

Nimber of Times Taught: 4

Average Enrollment: $\quad 35$

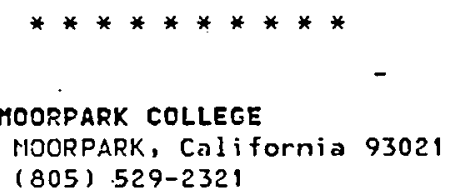

$(7115)$

(805) .529-2321

\section{SOLAR RELATED COURSES}

Solar Heat ing Construction Inst iturte

Instructor: Ainge, Ken

(805) 529-2321

Course Number: ET $89 B$

Department: Technology

Credits:

Student Level: All levels

Duration: 9 Weeks, 3.0 hrs per week

Contact Hours: 27

Classroom: 27

Topics Covered Extensively: Appropriate

Technolozy; Domestic Hot Water

Number of Times Taught: 1

Average Enrollinent:

15 
MOUHT SAH AKTONIO COLLEGE

(1245)

WALNUT, California 91789

(714) 598-2811

PROGRAMS AFTD CURRICULA

Air Cond., Heat., and Vent.

Degree: $A D$, Air Cond., Heat. and Refrig.

Contact: Dillon, Clifford (714) 594-5611

Students Taking or Completing Offering:

Electrician, Solar Technician, Installer-Residential (Solar System), Installer-Commercial (Solar System), Plumber

\section{SOLAR RELATED COUURSS}

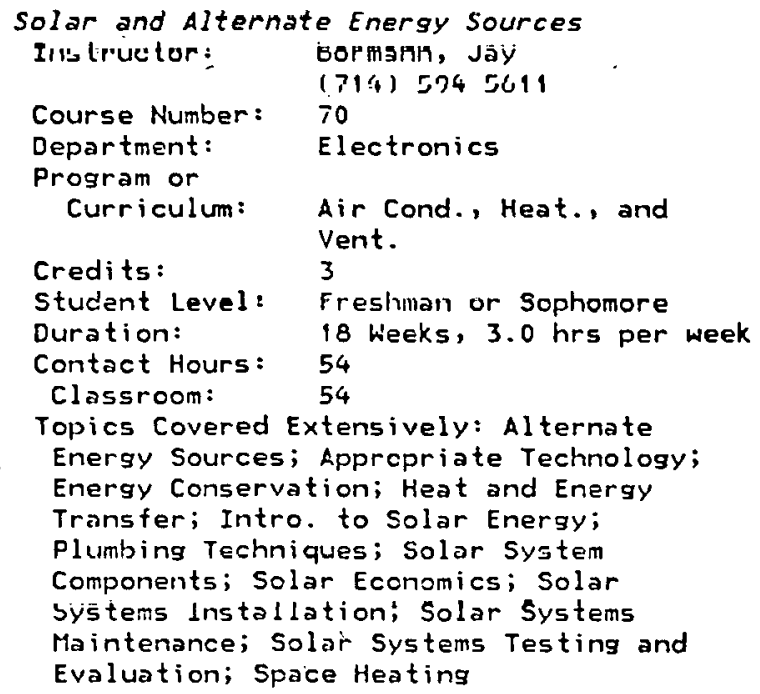

Solar Erergy Systems Installation

Instructor: Eormann, Jay (794) 594-5611

Course Number: $71 / 71 \mathrm{~L}$

Department: Electronics

Program or

Curriculum: Air Cond., Heat., and.

Credits: Vent.

Student Level: Freshman or Sophomore

Duration: 18 Heeks, $6.0 \mathrm{hrs}$ per week

Contact Hours: 108

Classroom: $\quad 54$

Laboratory: $\quad 54$

Topics Covered Extensively: Solar

Syetems Desigm; Solar Syslmis

Installation; Solar Systems

Maintenance; Solar Systems Testing and

Evaluation
IT SAH JACINTO COLLEGE
SAN JACINTO, Cal i fornia 92383
$(714) 654-7321$

$(1246)$

SOLAR RELATED COURSES

Solar Collector Design

Instructor: Caldivell, $B$.

(714) 654-7321

Course Number: EHGR 6

Department: Vocational Education

Credits: 3

Student Level: Freshman or Sophomore

Duration: 18 Weeks, 3.0 hrs per week

Contact Hours: $\quad 54$

Classroom: $\quad 45$

Latoratory: 9

Tepies Covered Extonsively: Solor System

Components; Solar Collector

Evaluation/Design

Number of Times Taught: 1

Average throllment: 34

Solar Energy Applications

Instructor: Caldivell, Benton

Course Number: (714) 654-7321

ERISR 4

Department: Vocational Education

Credits: 3

Student Level: College Graduate

Duration: 18 Weeks, $3.0 \mathrm{hrs}$ per week

Contact Hours: 54

Classroom: $\quad 49$

Laboratory: 5

Topics Covered Extensively: Intro, to

Solar Energy

Number of Times Taught: 2

Average Enrolliment: $\quad 33$

HAPA COLLEGE

NAPA, California 94558

(1247)

(707) 255-2100

SOLAR RELATED COURSES

Solar Energy Horkshop

Instructor: Dean, Anson $R$.

(316) $750-4606$

Department: Continuing Education

Student Level: All levels

Duration: $\quad 6$ Weeks, 3.0 hrs per week

Contact Hours: 18

Tnpirs Covered Extensively: Enerzy

Conservation; Intro. to Solar Energy;

Solsh bijetem Components; Solar llorie

Construction; Solar Systems Design;

Solar Systeris Installation; Solar

Systems Maintenance; Donestic Hot

Hater; Swimming Pool Heating

Niumber of Times Taught: 2

Average Enrollment: $\quad 50$

*********** 
OHLCKE COLLEGE

(4481)

FREMONT, California 94537

(415) 657-2100

SOLAR RELATED COURSES

* Tho Courses on Solar

**********

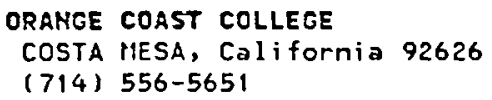

SOLAR RELATED COURSES

Solar

Instructor: Abernathy, Bill J.

(714) $556-5812$

Course Number: 115

Department: Technology

Credits: 3

Student Level: Junior or Senior

Duration: $\quad 18$ heeks, 3.0 hrs per week

Contact Hours: 54

Topics Covered Extensively: Energy

Storage; Intro. to Solar Energy; Solar

System Components; Solar Economics;

Space Heating

Number of Times Taught: 2

Average Enrollment: 30

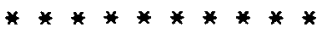

PASADEHA CITY COLLEGE

(1261)

PASADENA, California 91106

(213) 578-7123

\section{SOLAR RELATED COURSES}

\begin{tabular}{|c|c|}
\hline $\begin{array}{l}\text { Energy Sources, } \\
\text { Instruetor: } \\
\text { Course Number: } \\
\text { Department: } \\
\text { Duration: } \\
\text { Contact Hours: } \\
\text { Topics Covered } \\
\text { Energy Sources } \\
\text { Intro. to Sola } \\
\text { Small Systems }\end{array}$ & $\begin{array}{l}\text { Resources E Uses } \\
\text { Ball, D.A. } \\
\text { EN100 } \\
\text { Eng'r \& Tech } \\
18 \text { Weeks, } 3.0 \text { hrs per week } \\
54 \\
\text { Extensively: Alternate } \\
\text {; Appropriate Technolosy; } \\
\text { r Energy; Wind Power, }\end{array}$ \\
\hline $\begin{array}{l}\text { Solar Energy to } \\
\text { Instructor: } \\
\text { Department: } \\
\text { Duration: } \\
\text { Contact Hours: } \\
\text { Topics Covered } \\
\text { Storage; Intro } \\
\text { Passive Sular } \\
\text { Construction; } \\
\text { Evaluation/Des } \\
\text { Design; Domest } \\
\text { Pool Heating; } \\
\text { Scale; Space H }\end{array}$ & $\begin{array}{l}\text { the Consumer } \\
\text { Yanow, Gilbert } \\
(213) 578-7301 \\
\text { Eng'r. C Tech. } \\
9 \text { Weeks, } 3.0 \text { hrs per week } \\
27 \\
\text { Extensively: Energy } \\
\text { to Solar Energy; } \\
\text { Technoluyy; Sular Hume } \\
\text { Solar Collector } \\
\text { igm; Solar Systems } \\
\text { tic Hot Hater; Swimming } \\
\text { Elec'l Generation, Small }\end{array}$ \\
\hline
\end{tabular}

***********
REOHOCOS, COLLEGE OF THE

EUREKA, California 95501

( 707 ) 443-8411

SOLAR RELATED COURSES

Solar Heating A

Instructor:

Mills, David

(707) 443-841

Course Number: ENSC 20A

Department: Env. Sci.

Credits:

Student Level: All levels

Duratic

12 Weeks, 1.0 hrs per week

Contact Hours: 12

Classroom: $\quad 12$

Topics Covered Extensively: Alternate

Energy Sources; Energy Conservation;

Energy Conversion; Energy Storage; Heat

and Energy Transfer; Intro. to Solar

Energy; Fhotovoltaics; Passive Solar

Technology; Solar System Components;

Solar Economics; Solar Home

Construction; Solar Collector

Evaluation/Design; Solar Systems

Design; Solar Systems Installation;

Solar Systems Testing and Evaluation;

Domestic Hot Water; Swimming Pool

Heating; Space Heating

Number of Times Taught: 1

Average Enrollment: $\quad 50$

Solar Heating $B$

Instructor:

Mills, David

(707) 443-8411

Course Number: ENSC $20 \mathrm{~B}$

Department: Env. Sci.

Credits:

1

Student Level: All levels

Duration: 12 Weeks, $1.0 \mathrm{hrs}$ per week

Contact Hours: 12

Classroom: 12

Topics Covered Extensively: Alternate

Energy Sources; Energy Conservation;

Energy Conversion; Energy Storage; Heat

and Energy Transfer; Intro. to Solar

Enerzy; Passive Solar Technology;

Photovoltaics; Solar System Components;

Solar Economics; Solar Hon:e

Construction; Solar Collector

Evaluaticn/Design; Solar Systems

Design; Solar Systems Installation;

Solar Systems Testing and Evaluation;

Doniestic Hot Water; Swimming Pool

Heating; Space Heating

Nunber of Times Taught: 1

Average Enrollment: 50

Solar Heating $C$

Instructor:

Mills, David

(707) 443-8411

Cour'se Hunber: EN3C 200

Department: Env. Sci.

Credits:

Student Level: All levels

Duration:

12 Weeks, 1.0 hrs per week

Contact Hours: 12

Classroom: $\quad 12$

Topics Covered Extensively: Alternate Energy Sources; Energy Conservation; 
Energy Conversion; Energy Storage; Heat and Energy Transfer; Intro. to Solar Energy; Passive Solar Technology; Photovoltaics; Solar System Components; Solar Eccnomics; Solar Home Construction; Solar Collector Evaluation/Desisn; Solar Systems Design; Solar Sy'stems Installation; Solar Systens Testing and Evaluation; Domestic Hot Water; Swimming Pool Heating; Space Heating Number of Times Tausht: Average Enrollment: 50

\section{RIVERSIDE CITY COLLEGE \\ RIVERSIDE, California 92506 \\ (714) 684-3240}

$(1270)$

Sular relateu courses

Solar Cnesy Applicalions

Instructor: Budd, Frank $W$. (714) 684-3240 AC 52A

Course Number: Air Conditioning Departient: Credits: 5

Studant Level: All levels

Duration: $\quad 18$ Weeks, $7.0 \mathrm{hrs}$ per week

Contact Hours: 126

Classroom: $\quad 72$

Laboratory: $\quad 54$

Topics Covered Extensively: Appropriate

Technolozy; Energy Conservation; Energy Storage; lleat and Energy Transfer;

Intro. to Solar Energy; Solar System Components; Solar Collector

Evaluation/Dasign; Solar Systems

Desisn; Solar Systens Installation;

Solar Systens Mointenance; Solar

Systems Testing and Evaluation;

Domestic Hot Water; Swimming Pool

Heating; Space Heating; Space Cooling

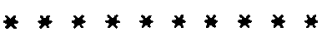

SACPAIEENTO CITY COLLEGE

SACRAMEHTO, California 95822

$(1233)$

(916) 449-7531

\section{SOLAR RELATEO COURSES}

Basic Solar Heating and Cooling systems Instructor: Stockwell, Richard/ Gott, Don (916) 449-7278

Course Nurber: MET 141

Department: Occupational Technology/Mechanical,

Credits: Electrical Technology

Student Level: Freshman or Sophomore

Duration: 18 heeks, 3.0 hrs per week

Contact Hours: 54

Classroom: $\quad 54$

Topics Covered Extensively: Energy

Storage; Intro. to Solar Energy;
Materials Research; Photovoltaics; Plumbing Techniques; Solar System Components; Solar Economics; Solar Law/Legislation; Solar Collector Evaluation/Design; Solar Systems Design; Solar Systen:s Maintenance; Domestic Hot Water; Process Heat, Industrial; Space Heating; Space Cooling

**********

SAN BERHARDIHO VLY COLLEGE

SAN BERNAFDINO, California 92403

(714) 885-0231

(1272)

SOLAR RELATED COUREES

* Two Solar Courses

$* * * * * * * * * *$

SAN DIEGO CC- CITY COLLEGE

SAN DIEGO, California 92101

(8895)

(714) 238-1181

PROGRAMS AND CURRICULA

* Solar Ener. Main. and Tech

Degree: $A D$, Advanced Degree

SOLAR RELATED COURSES

* Tho Courses on Solar Energy Main.,Tech. Program or

Curriculum: *Solar Ener. Main, and Tech.

Topics Covered Extensively: Solar System Conponents; Solar Economics; Solar Collector Evaluation/Design; Solar Systems Installation: Solar Systems Maintenanse; Snlar Systoms Testing and Evaluation

***********

SAN DIEGO CC- EVENIHG COLLEGE

( 7478$)$

SAN DIEGO, California 92101

(714) 238-1181

PROGRAMS AND CURRICULA

Air Cond., Heat., Refrig., and Sol.

Tech.

Degree: AU, UIHER, Air Cond., Heat.,

Refrig., \& Sol. Tech.

Contact: Belker, Loren (714) 238-1181

Students Taking or Completins offering: Solar Technician, Tracle Specialty

SOLAR RELATED COURSES

Adv. Sol. Ser. Main. and Tech.

Instructor: $\quad F a r i s$, Theodore

Course Number: 226 


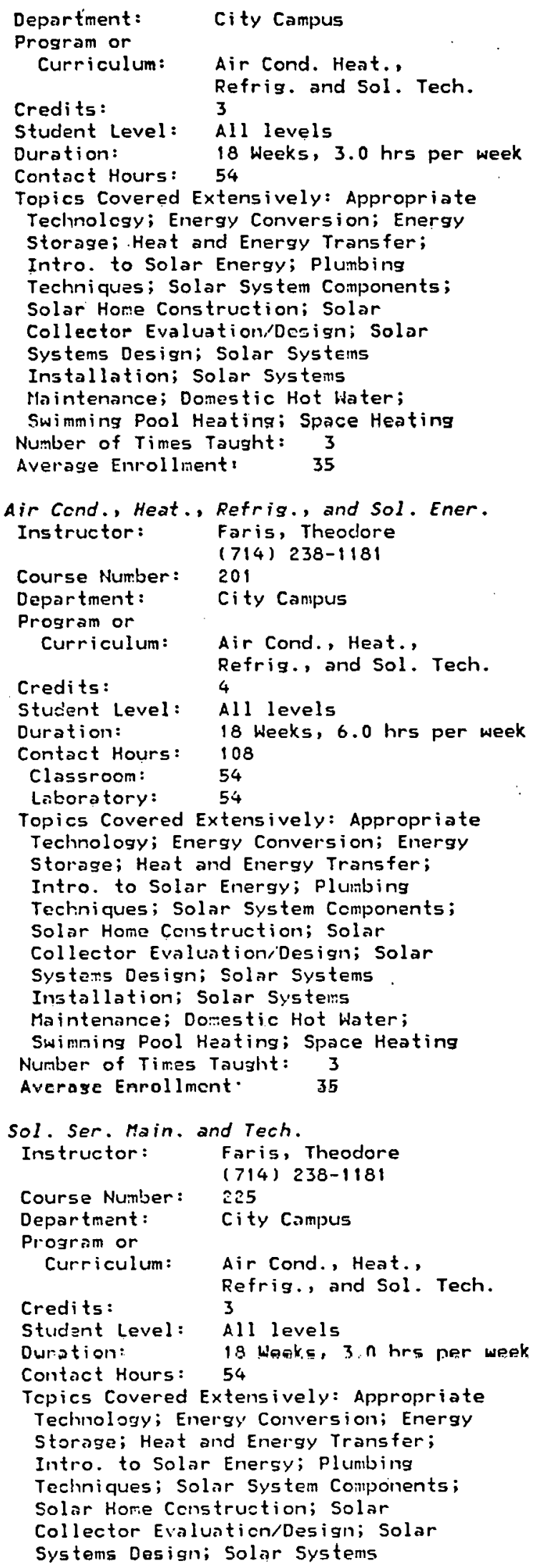

Installation; Solar Systems

Maintenance; Domestic Hot Water;

Swiming Pool Heating; Space Heating Number of Times Taught: 3 Averase Enrollment: $\quad 35$

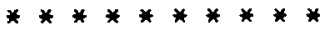

SAN DIECO CC- HESA COLLEGE

SAN DIEGO, California 92111

(1275)

(714) 279-2300

\section{SOLAR RELATED COURSES}

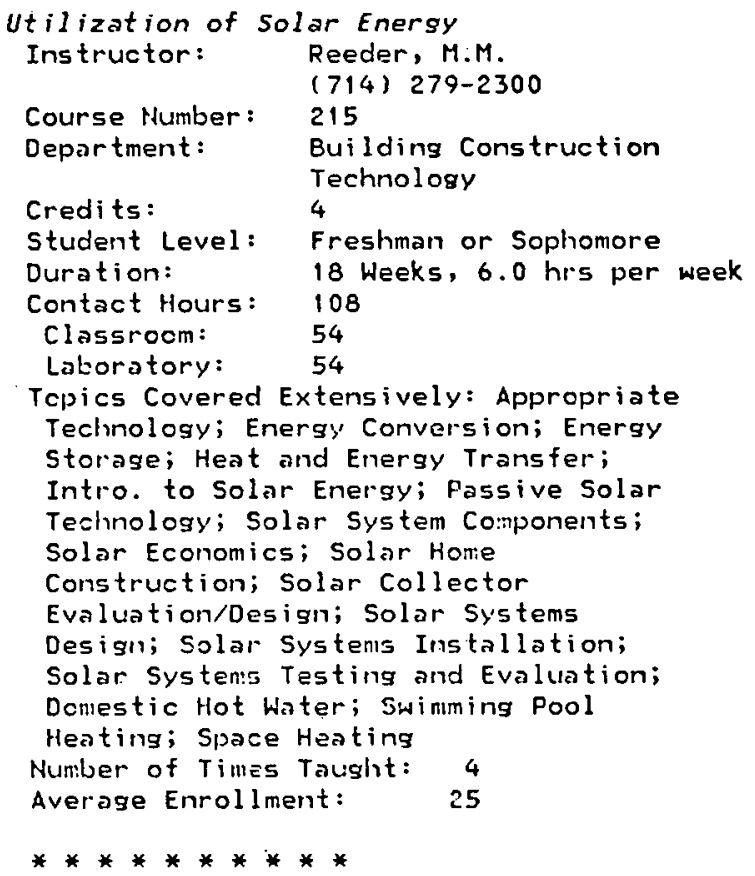

SAN JOAQUIH DELTA COLLEGE

$(1280)$

STOCKTON, California 95207

(209) 478-2011

\section{SOLAR RELATED COURSES}

* Energy Conservation and Alternatives

Instructor: Oliver, James E.

Course Number: NR750

Department: Fhysics

Duration: $\quad 10$ Haeks, 3.0 hrs per week

Contact Hours: 30

Tepics Covered Extensively: Alternate

Energy' Sources; Energy Conservation;

Intro. to Solar Energy 
SAH JOSE CITY COLLECE

(408) 298-2181

\section{PROGRAMS ARTO CURRICULA}

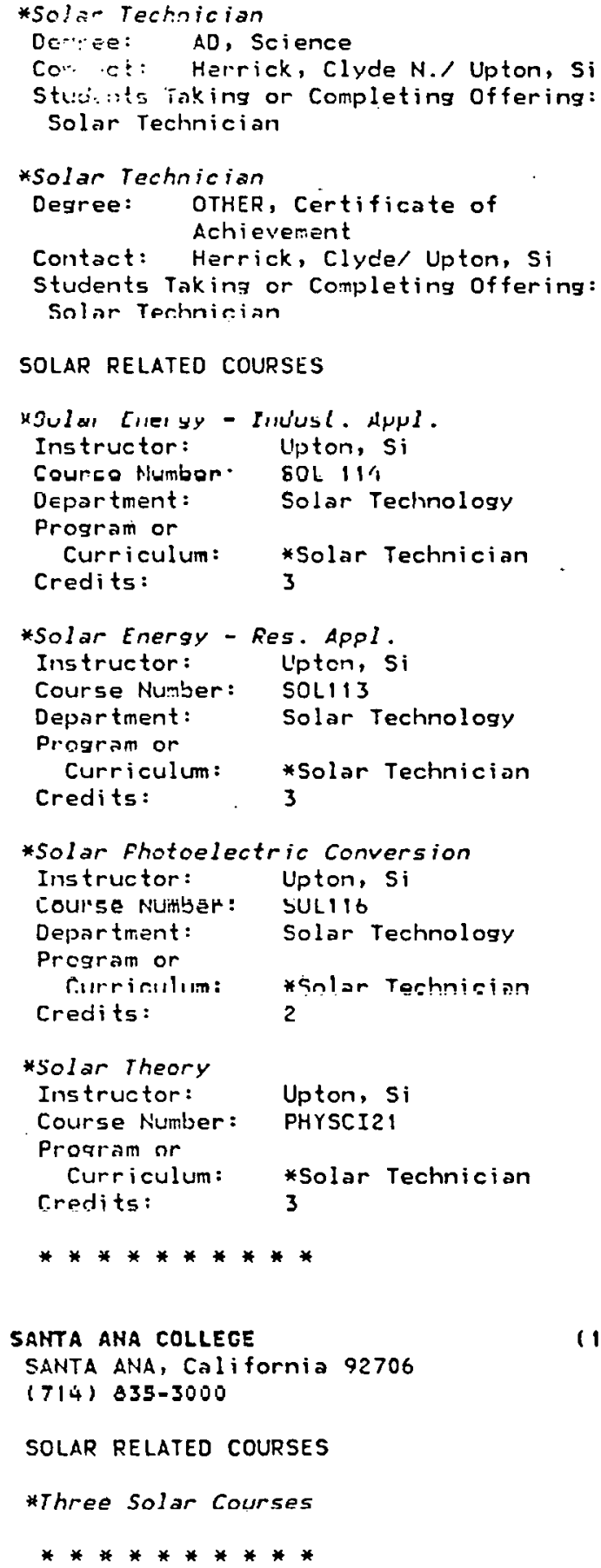

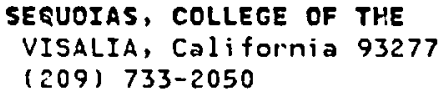

SOLAR RELATED COURSES

*Frontiers of Sci. - Enrer. for Consuners Instructor: Crist, Friend, Dawson Course Number: SCI 10 Department: Natural Science Topics Covered Extensively: Alternate Energy Sources; Solar Economics 
SOUTHHESTERH COLLEGE

(1294)

CHULA VISTA, California 92010

(714) 420-1080

SOLAR RELATED COUPSES

* One Solar Course

$(1338)$

\section{HEST VALLEY COLLEGE \\ SARATOGA, California 95070 \\ (408) $867-2200$}

SOLAR RELATEO COURSES

Introduction of Solar Energy

Instructor: Feamster, John

(408) 925-3095

Department: Engineering

Credits: 3

Student Level: All levels

Duration: 18 Weeks, $3.0 \mathrm{hrs}$ per week

Contact Hours: 54

Classroom: $\quad 54$

Topics Covered Extensively: Appropriate

Technolcgy; Energy Conservation; Energy

Storage; Heat and Energy Transfer;

Intro. to Solar Energy; Passive Solar

Tecinnolczy; Solar System Components;

Solar Economics; Solar Howe

Construction; Solar Collector

Evaluation/Design; Solar Systems

Desisn; Dcmestic Hot Water; Swimming

Pool Heating; Space Heating; Space

Cooling

Number of Times Taught: 8

Average Enrollment: $\quad 40$

**********

\section{other Educational Institutions}

RHTIOCH COLLEGE/WEST

(90520)

1161 Mission St.

San Francisco, California 94103

PROGRAMS AND CURRICULA

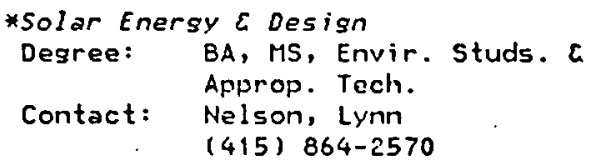

SOLAR RELATED COURSES

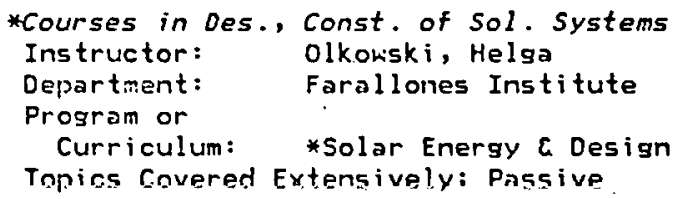

Solar Technology; Solar System Components; Solar Home Construction; Solar Collector Evaluation/Design; Solar Systems Design; Space Heating; Space Cooling

CENTER FOR EMPLOYMEMT TRAIHIHG

$(90350)$

425 So. Market St.

San Jose, California 95113

SOLAR RELATED COURSES

* Building Maintenance

Instructor: Rodriguez, Rudolph

Duration: 6 Weeks

Topics Covered Extensively: Solar System

Components; Solor Collector

Evaluation/Design; Solar Systems

Design; Solar Systems Installation

$* * * * * * * * * *$

OFFICE OF APPROPRIATE TECHHOLOGY

(90530)

PO BoX 1677

Sacramento, California 95808

PROGRAMS AND CURRICULA

* Training Program for Installers (916) 445-1803

Students Taking or Completing offering:

Instoller-Residential (Solar System),

Installer-Commercial (Solar System)

**********

SOLAR TECHWICIAH TRAINING PROGRAN OFFICE OF APFROPRIATE TECHNOLOGY

(90340)

1322 "O" Street

Sacramento, California 95814

PROGRAMS AND CURRICULA

* Solar Technician Training Program

Contact: Trujlllo, JuAm

(916) 322-7190

students Taking or Completing Offering:

Solar Technician

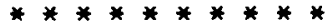

SOLARCOH

$(90490)$

PO Box 14875

San Francisco, California 94114

SOLAR RELATED COURSES

*Installers Horkshop

(415) 648-2159
Department: $\quad \begin{aligned} & \text { Karellen Educational } \\ & \text { Services }\end{aligned}$
Topics Covered Extensively: Solar
Systems Installation


THIS PAGE

\section{WAS INTENTIONALLY LEFT BLANK}




\author{
colleses/Universities \\ ADAMS STATE COLLEGE \\ ALAMOSA, Colorado 81102 \\ (1345) \\ (303) 589-7346

\section{SOLAR RELATED COURSES}

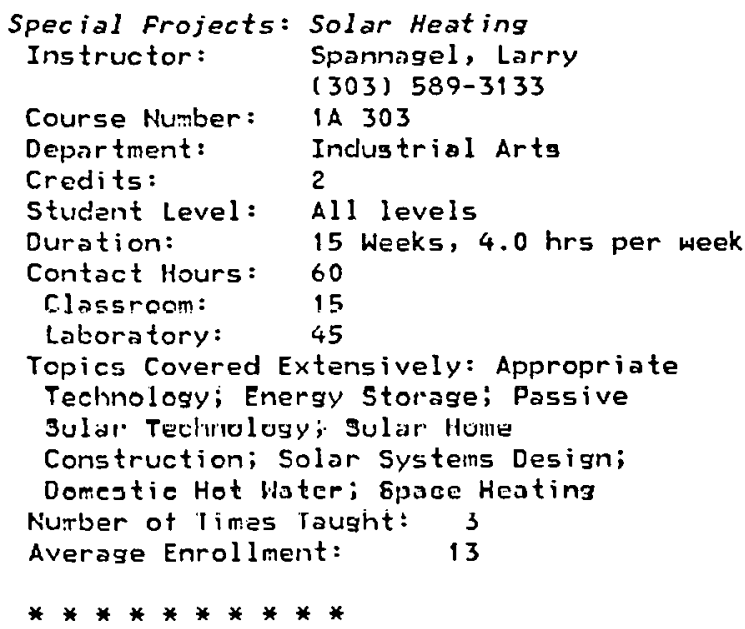

COLO TECHMICAL COLLEGE

COLORADO SFRINGS, Colorado 80907

$(10148)$

(303) 598-0200

\section{PROGRAMS AHD CURRICULA}

Solar Engineering Technology

Desree: BS, AD, Applied Science

Contact: Christensen, Edward

(303) 598-0200

Students Taking or Completing offering:

Solar Engineer, Solar Technician

\section{SOLAR RELATED COURSES}

Associate Seminar

Instructor:

Christensen, Edward

(303) 598-0200

Course Number: SOL 250

Department: Solar Engineering

Technology

Curriculum: Solar Engineering

Technology

Credits: 1

Student level: Freshman or Sophomore

Duration: 11 Heeks, $1.0 \mathrm{hrs}$ per week

Contast Hours: 11

Topics Covered Extensively: Alternate

Enersy Sources

Directed Practice

Instructor: Christensen, Edward

(303) 598-0200

Course Number: SOL 299

Department: Solar Engineering

Program or

Curriculum: Solar Engineering

Technology
Credits: 3

Student Level: Freshman or Sophomore

Duration: $\quad 11$ Weeks, $6.0 \mathrm{hrs}$ per week

Classroom: 66

Number of Times Taught: 14

Average Enrollment: $\quad 6$

\section{Introduction to Energy}

Instructor: 5abo, Julius $J$.

Course Number: SOL 100

Department: Solar Engineering

Technology

Program or

Curriculum: Solar Engineering

Technology

Credits: 3

Student level: All levels

Duration: $\quad 11$ Weeks, $3.0 \mathrm{hrs}$ per week

Contact Hours: 33

Classroom: 33

Topics Covered Extensivelys Alterrate

Energy Sources; Appropriate Technology

Nunber of Times Taught: 10

Average throllment: $\quad$ is

Solar Design I

Instructor:

Christensen, Edward

Course Number: SOL 220

(303) 598-0200

Departnent: Solar Engineering

Program or

Technology

Curriculum:

Credits:

Soler Engineering

Technology

Student Level:

Duration:

Contact Hours: 66

Clissröom:

Laboratory: 44

Topics Covered Extensively: Heat and

Energy Transfar; Solar Sustem

Coriponents; Solar Collector

Evaluation/Design; Solar Systems

Design; Space Heating

Number of Times Taught: 9

Average Enrollment: $\quad 8$

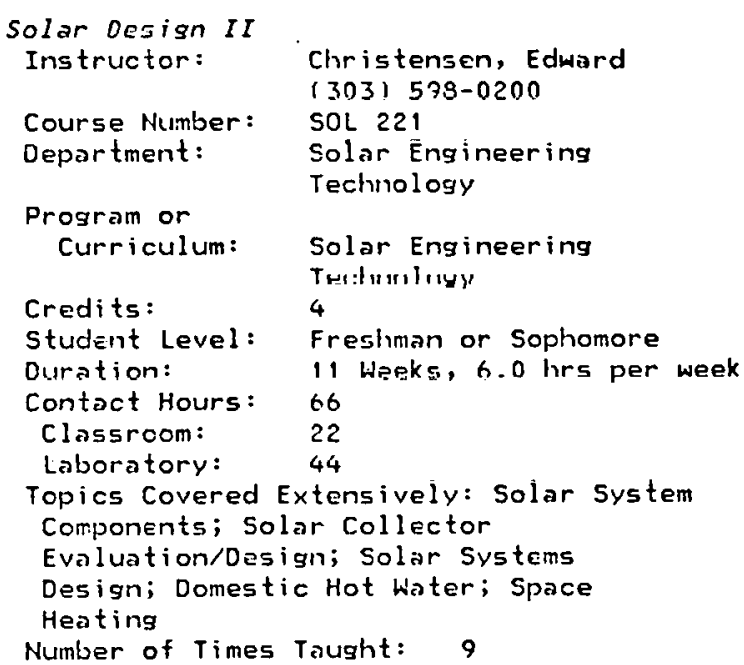

Solar Design II

Instructor:

1303) 538-0200

Course Number: SOL 221

Technology

Program or

Credits:

Trellumiry

Student Level:

Duration:

Classroom:

Components; Solar Collector

Evaluation/Design; Solar Svstems

Heating

Number of Times Taught: 9 
Average Enrollment:

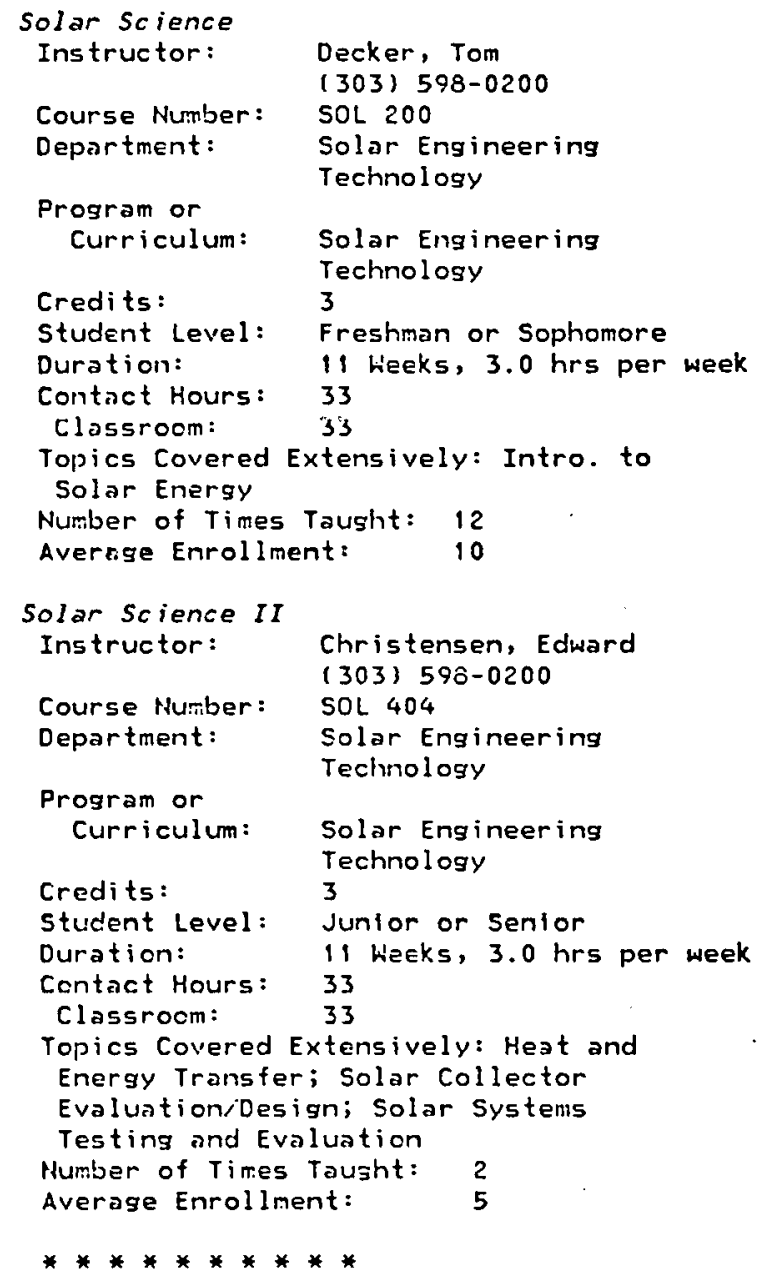

COLO- COLO SFRINES, U OF COLORADO SF?IHISS, COlORAdO 80907

(4509)

(303) 598-3737

PROBRAIIS AISD CURRICULA

Distributed Studies in Energy Science

Degree: BA, Distributed Studies

Contact: Blade, Richard A. (303) 598-3737

Students Taking or Corpleting offering: Researcher, Solar Engineer, Other, Solar Technician

Solar Energy and Energy Sciences

Degree: BS, Resource Systems Engineering

Contact: Wiener, R.

Students Taking or Completing Offering: Solar Engineer
SOLAR RELATED COURSES

Introduction to Energy Sciences 150

Instructor: Grogger, Scott P.I

Blade, R.

(303) 598-3737

Course Number: 150

Department: Phisics and Energy

Sciences

Program or

Curriculum: Distributed studies in

Energy Science

Credits: 3

Student Level: All levels

Duration: 2 Weeks, 40.0 hrs per week

Contact Hours: 80

Classroom: $\quad 64$

Laboratory: 16

Topics Covered Extensively: Al ternate

Energy Sources; Appropriate Technology;

Énergy Conservation; Energy Conversion;

Heat and Energy Transfer; Intro. to

Solar Energy; Space Heating

Nuriber of Times Taught: 2

Average Enrollment:

30

Introduction to Energy Sciences 151

Instructor: Scott, M./ Grogger, P./

Blade, $R$.

(303) 598-3737

Course Number: 151

Department: Physics and Energy

Sciences

Program or

Curriculum: Distributed Studies in

Credits: Energy Science

Student Level: All levels

Duration: 2 Weeks, $40.0 \mathrm{hrs}$ per week

Contact Hours: 80

Classroom: $\quad 64$

Laboratory: 16

Topics Covered Extensively: Alternate

Energy Sources; Appropriate Technolcgy;

Energy Cinservation; Energy Conversion;

Heat and Energy Transfer; Intro. to

Solar Energy; Space Heating

Number of Times Taught: 2

Aveiage [ingollment: $\quad 30$

Methods of Energy Sciences 350

Instructor: Grogser, P./ Blade, R.

(303) 598-3737

Course Humber: 350

Department: Fhysics and Enersy

Sciences

Program or

Curriculum: Distributed Studies in

Credits: 3

Student Level: Junior or Senior

Duration: 16 Weeks, 6.0 hrs per week

Contact Hours: 96

Classroom: 96

Topics Covered Extensively: Alternate

Energy Scurces; Apprcpriate Technology;

Energy Conservation; Enerzy Conversion;

Energy Storage; Heat and Energy

Transfer 
Number of Times Taught: 1

Average Enrollment: 15

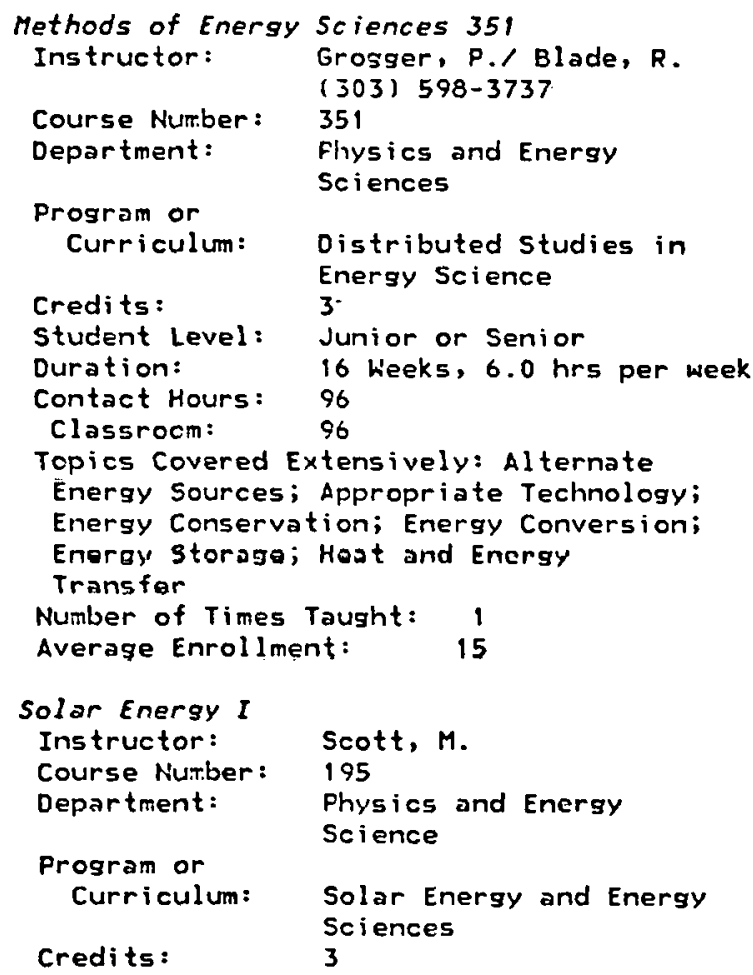

Solar Energy I, 160

Instructor: Scott, Michael

(303) 598-3737

Course Number: 160

Department: Physics and Energy

Science

Program or

Curriculum: Distributed Studies in

Credits:

Energy Science

3

Student Level: All levels

Duration: 15 Weeks, 4.0 hrs per week

Contact Hours: 60

Classrocm: $\quad 40$

Laboratory: 20

Topics Covered Extensively: Energy

Conservation; Energy Conversion; Energy

Storage; Heat and Energy Transfer;

Intro. to Solar Energy; Passive Solar

Technology; Solar Home Construction;

Solar Collector Evaluaticn/Design;

Solar Systems Design; Solar Systems

Installation; Solar Systems Testing and

Evaluation; Domestic Hot Water; Space

Heating

Number of Times Taught: 7

Average Enrollment: $\quad 30$

Solar Energy II

Instructur:

Course Number: 207

scott, $M$.

Department :

Physics C Energy Sci.

Curriculum:
Solar Energy and Energy Sciences

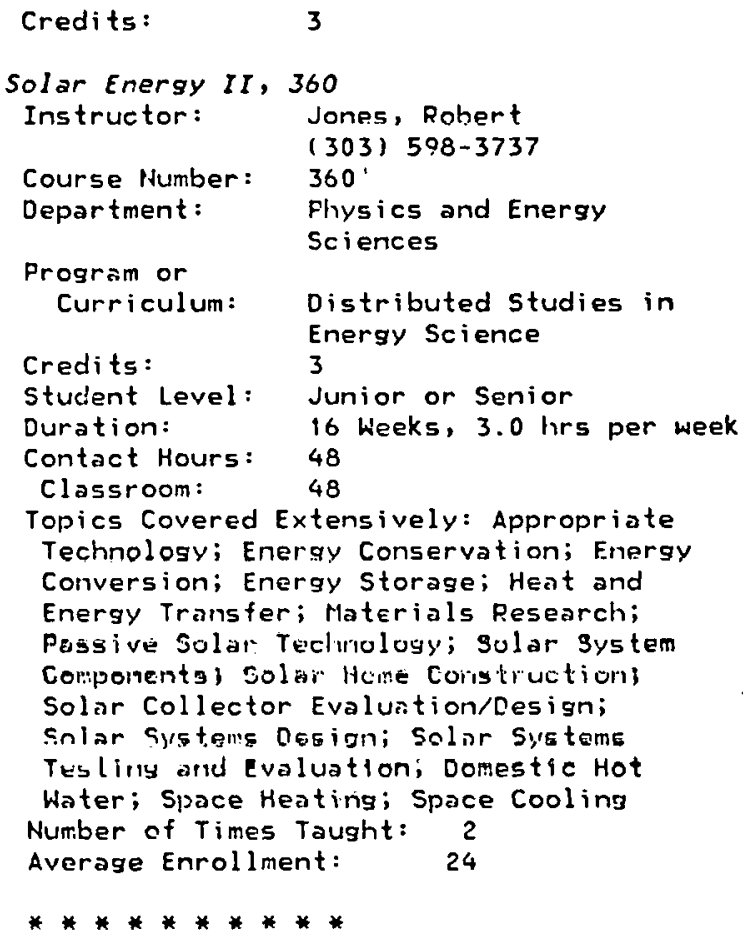




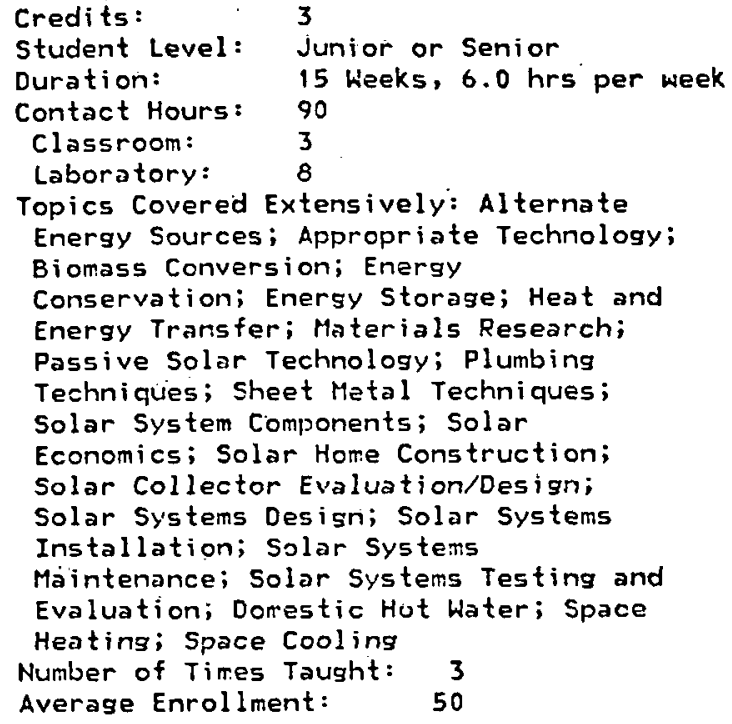

Topics Covered Extensively: Alternate Energy Sources; Appropriate Technology; Biomass Conversion; Energy

Conservation; Passive Solor Technology; Solar Energy Policy Development; Solar Economics; Solar Home Construction; Solar Law/Legislation

Number of Times Taught: 2

Average Enroliment: $\quad 40$

Computer-Aided Thermal Design

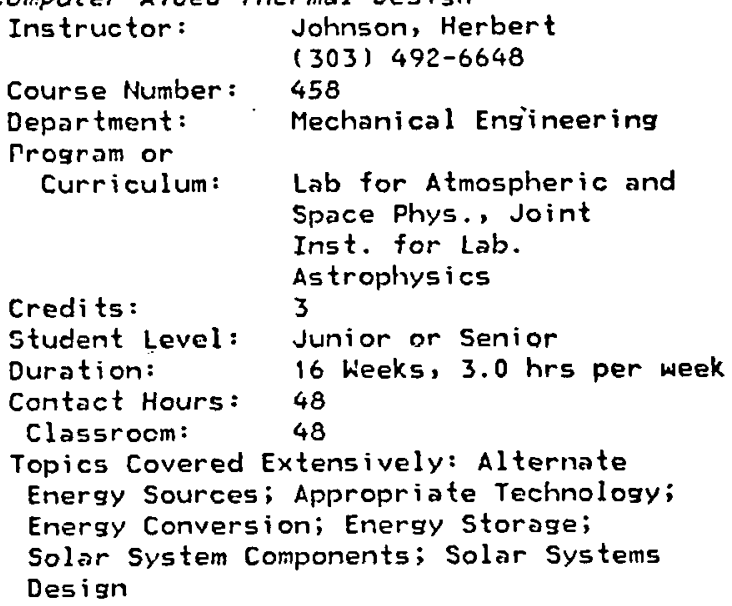

Energy in a Technical Society

Instructor: Bartlett, David

(303) 492-6960 207

Department: Arts and Sci., Phys, /As trophysics

Program or Curriculum: Joint Inst. for Lab. Astrophysics, Lab for Atmospheric and Space Phys.

Credits: 3

Student Level: All levels

Duration: $\quad 16$

Classroom: 48

Topics Covered Extensively: Alternate Energy Sources; Appropriate Technology; Eneray ronservation; Energy Conversion; Heat and Energy Transfer; Intro. to Solar Energy; Solar Collector

Evaluation/Design; Solar Systems Design; Domestic Hot Water; Space Heating

Number of Timas Taught: 7

Average Enrollment: $\quad 50$

Energy Conversion

Instructor: Johnson, Herbert

(303) 492-6648

Course Number: 455

Department: Engineering, Mechanical

Program or Curriculum: Lab for Atmospheric and Engineering Space Phys., Joint Inst. for Lab.

Credits: Rstrophysics 


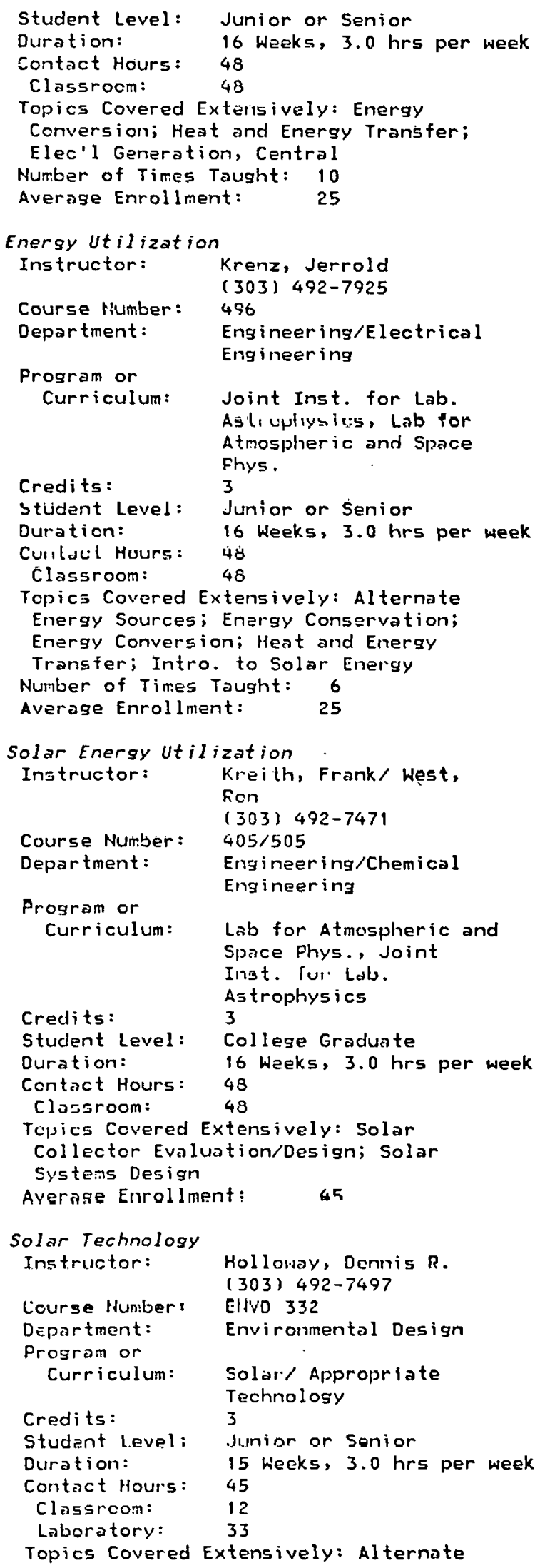

Energy Sources; Appropriate Technology; Energy Conservation; Energy Storage; Heat and Energy Transfer; Intro. to Solar Energy; Materials Research; Passive Solar Technology; Solar Home Construction; Solar Systems Design; Solar Systems Installation; Domestic Hot Water; Space Heating Number of Times Taught: 2 Average Enrollment: $\quad 130$

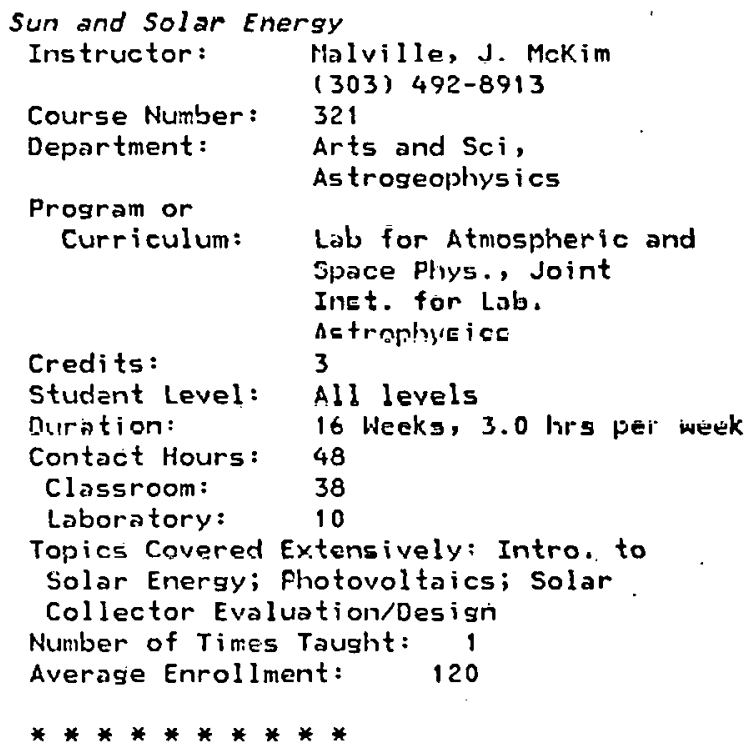

Principles of Solar Energy Systems

Instructor: Mathews, Frank 5.

Course Number: PH419

Department: Fhysics

Credits: 3

Student Level: Junior or Senior

Duration: 16 Weeks, $3.0 \mathrm{hrs}$ per week

Contact Hours: 48

Classroom: $\quad 48$

Tepies Cureied Exletislvely: Energy Conversion; Heat and Energy Transfer; Fhotovoltaics; Solar System Components; Solar Collector Evaluation/Design; Solar Systen:s Designi Solar Syeteme Testing and Evaluation; Elec'l

Generation, Central

Number of Times Taught: 3

Average Enrollment: $\quad 20$

Renewable Energy Sources

Ingtructor: Miller, 3 am

(303) 279-0300

Course Hurber: RE479

Department: Basic Engineering

Credits: 3

Student Level: Junior or Senior
(1348) 


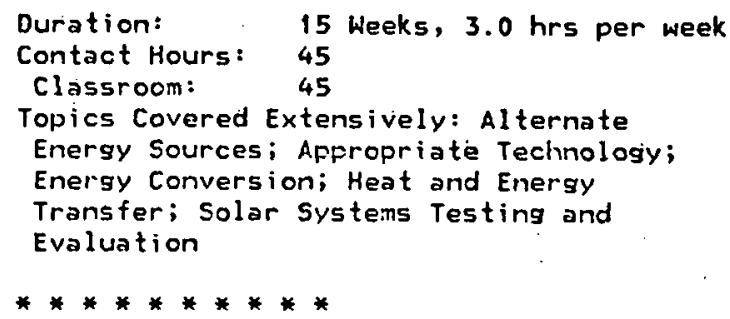

Topics Covered Extensively: Alternate Energy Sources; Afpropriate Technology; Energy Conversion; Heat and Energy Transfer; Solar Systems Testing and Evaluation

\section{PROGRAIS AND CURRICULA}

Solar Energy Applications

Dagree: PhD, MS, Engineering

Students Taking or Completing offering:

Educator, Researcher, Solar Engineer

SOLAR RELATED COURSES

Design of Solar Energy Systems

Course Number: CE/ME 675

Department: Engineering

Program or

Curriculum: Solar Energy

Credits: Applications

Credits: 3

Duration:

College Graduate

15 Weeks, 3.0 hrs per week

Contact Hours: 45

Classrcom: 45

Topics Covered Extensively: Intro. to

Solar Energy; Solar System Components;

Solar Economics; Solar Collector

Evaluation/Design; Solar Systems

Design; Solar Systeris Installation;

Solar Systetis Maintenance; Solar

Systems Testing and Evaluation;

Domestic Hot Water; Elec'l Generation,

Central; Space Heating; Space Cooling

Number of Times Tausht: 3

Average Enrollment: $\quad 30$

Principles of Solar Energy Applications

Couise Hurluei: CEAlle 676

Department: Engineering

Program or

Curriculum: Solar Energy

Credits: Applications

Student Level: Collese Graduate

Duration: 15

Contact Hours: 45

Classroom: 45

Topics Covered Extensively: Intro. to

Solar Energy; Photovoltaics; Solar System Components; Solar Collector

Evaluation/Design; Solar Systens

Design; Solar Systens Installation;

Solar Systems Maintenance; Solar

Systems Testirng and Evaluation;

Domestic Hot Water; Elec'l Generation,

Central; Space Heating; Space Cooling

Number of Times Taught: 3

Average Enrollment:

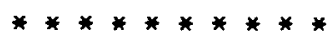

DENVER, UNIVERSITY OF

$(1371)$

DENVER, Coloracio 80210

(303) 753-1964

PROGRAMS AHID CURRICULA

Solar Energy

Contact: Stonely, Paul J. (303) 753-2194

Students Taking or Completing Offering:

Do-it-yourself Honeowner

\section{SOLAR RELATÉD COURSES}

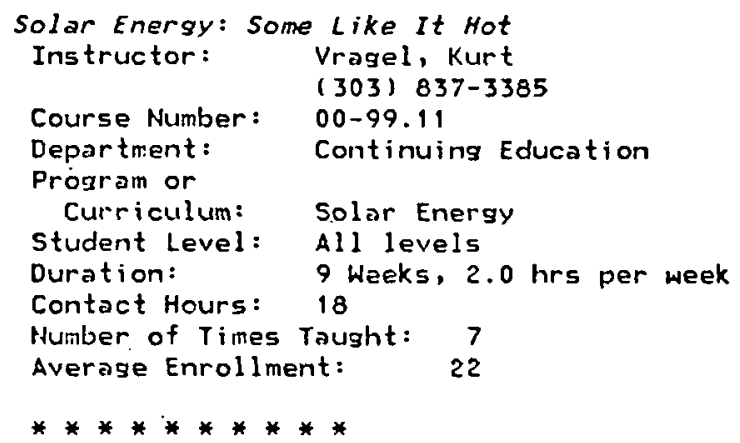

FORT LEIISS COLLEGE

DURANGO, Colorado 81301

(1353)

(303) 247-7661

\section{SOLAR RELATED COURSES}

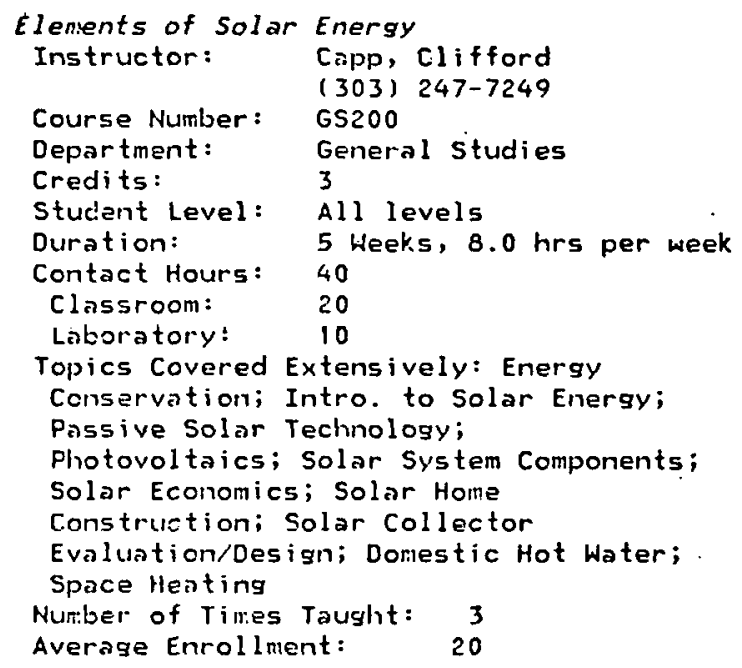


HESA COLLEGE

GRANO JURETION, Colorado 81501

(303) 248-1020

PROGRAMS ARID CURRICULA

Solar fower

Contact: Ramsey, Woodrow

(303) $248-1565$

Students Taking or Completing offering:

Do-it-yourself Homeowner

\section{SOLAR RELATED COURSES}

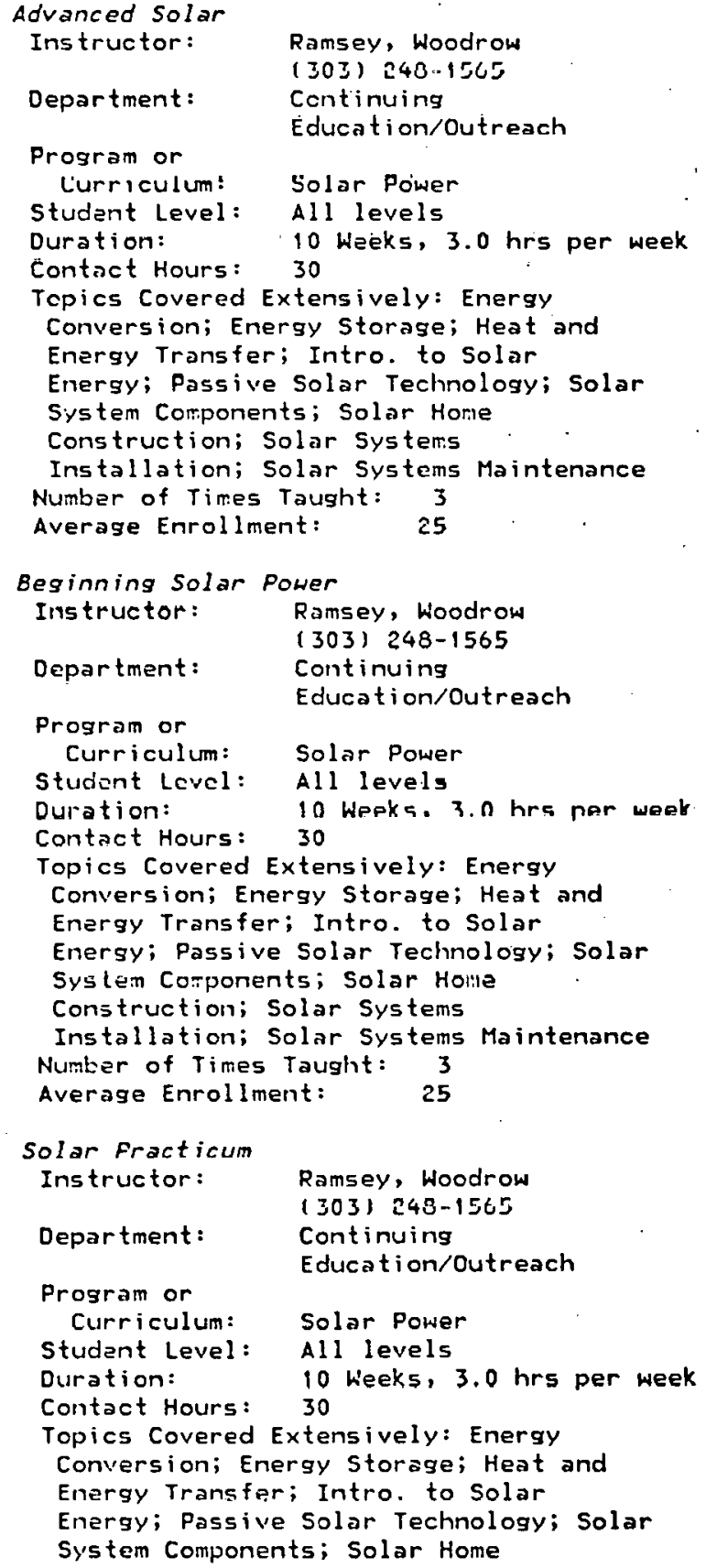

Department:$$
\text { Continuing }
$$$$
\text { Education/Outreach }
$$

Program or

Lurriculum: Solar Poiwer

Student Level: All levels

Duration: $\quad 10$ Weeks, $3.0 \mathrm{hrs}$ per week

Contact Hours: 30

Topics Covered Extensively: Energy

Conversion; Energy Storage; Heat and

Energy Transfer; Intro. to Solar

Enersy; Passive Solar Technology; Solar

System Components; Solar Horie

Construction; Solar Systerrs

Installation; Solar Systems Maintenance

Number of Times Taught: 3

Average Enrollment:

25

Beginning Solar Power

Instructor: Ramsey, Woodrow

Department: Continuing

Program or

Curriculum: Solar Power

Student Level: All levels

Duration: 10 Wpeks. 3.0 hrs ner week

Contact Hours: 30

Topics Covered Extensively: Energy

Conversion; Energy Storage; Heat and

Energy Transfer; Intro. to Solar

Enersy; Passive Solar Technology; Solar

System Components; Solar Home

Construction; Solar Systems

Installation; Solar Systems Maintenance

Number of Times Taught: 3

Average Enrollment: $\quad 25$

Solar fracticum

Instructor: Ramsey, Woodrow

(303) 248-15t5

Department: Continuing

Prosram or

Curriculum: Solar Power

Student Level: All levels

Duration: 10 heeks, $3.0 \mathrm{hrs}$ per week

Contact Hours: 30

Topics Covered Extensively: Energy

Conversion; Energy Storage; Heat and

Energy Transfer; Intro. to Solar

Energy; Passive Solar Technology; Solar

System Components; Solar Home

Construction; solar systems

Installation; Solar Systems Maintenance

Number of Times Taught: 3

Average Enrollment:

25

$* * * * * * * * * *$

RETROPOLITAN ST COLLEGE

$(1360)$

DENVER, Colorado 80204

(303) 629-2400

SOLAR RELATED COURSES

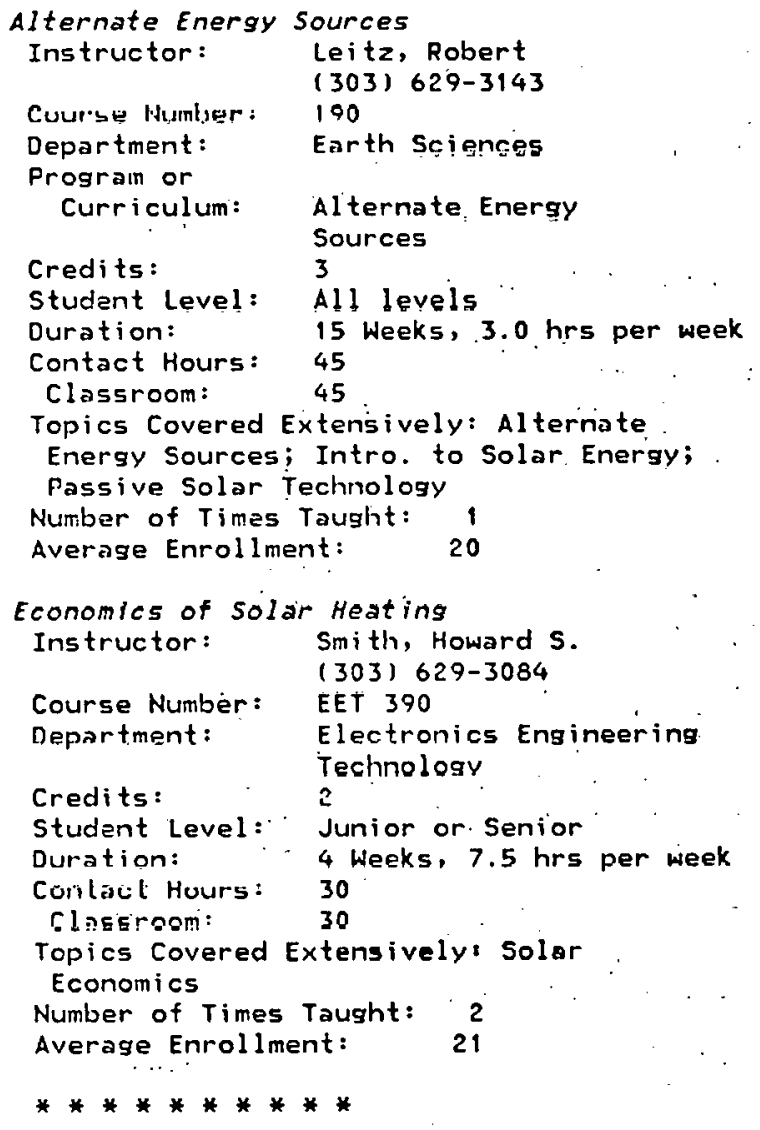

MORTHERH COLORADO; U OF

(1349)

GREELEY, Colorado 80639

(303) 351-1890

SOLAR RELATED COURSES

Applied Solar Energy

Instructor: Fadner, Willard

(303) 351-2962

Course Number: 467

Department: Physics

Credits: 3

Student Level: Junior or. Senior

Duration: $\quad 10$ Weeks, $3.0 \mathrm{hrs}$ per week

Contact Hours: 30

Classroom: $\quad 30$

Topics Covered Extensively: Appropriate

Technology; Energy Conversion; Energy 
Storage; Heat and Energy Transfer;

Intro. to Solar Energy; Solar

Economics; Solar Collector

Evaluaticn/Design; Solar Systems

Design; Elec'l Generation, Central;

Space Heatins

Number of Times Taught: 1

Average Enrollment: $\quad 5$

***********

US AIR FORCE ACADEMY

US AIR FORCE ACAD, COLO, Colorado 80840

(303) $472-1818$

SOLAR RELATED COURSES

Solar Energy Applications

Instructor: Ecien, Anthony

(303) 472-4036

Course Number: CE495

Department: Civil Engr., Engr. Mechanics and Materials

Credit5: 3

Student Level: Junior or Senior

Duration: 16 Weeks, 2.5 hrs per week

Contact Hours: 42

Classroom: . 42

Topics Covered Extensively: Appropriate

Technolosy; Energy Conservation; Energy

Storage; Heat and Energy Transfer;

Intro. to Solar Energy; Solar System

Ccmponents; Solar Economics; Solar

Collector Evaluation/Dasign; Solar

Systens Design; Space Heating

Number of Times Tausht: 5

Average Enrollment:

16

**********

\section{HESTERH ST COLLEGE COLO}

GUNIIISON, Colorado 81230

(1372)

(303) 943-0120

\section{SOLAR RELLATED COURSES}

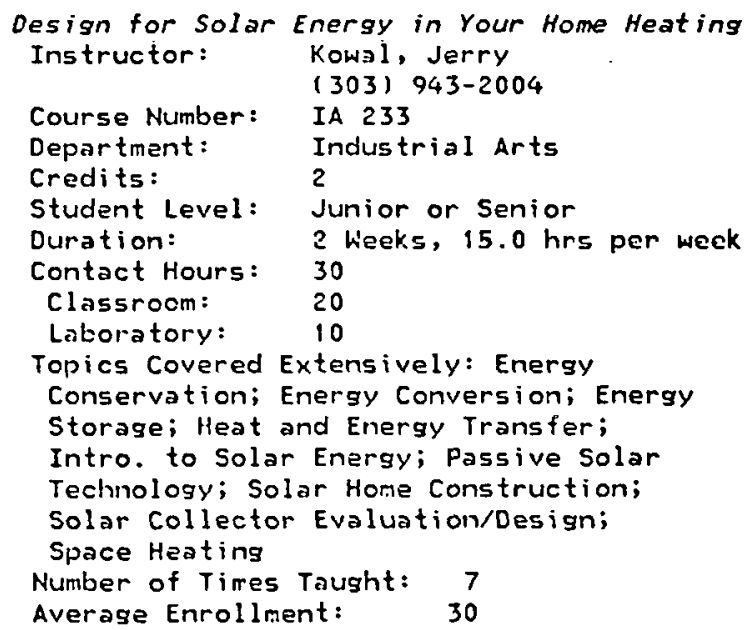

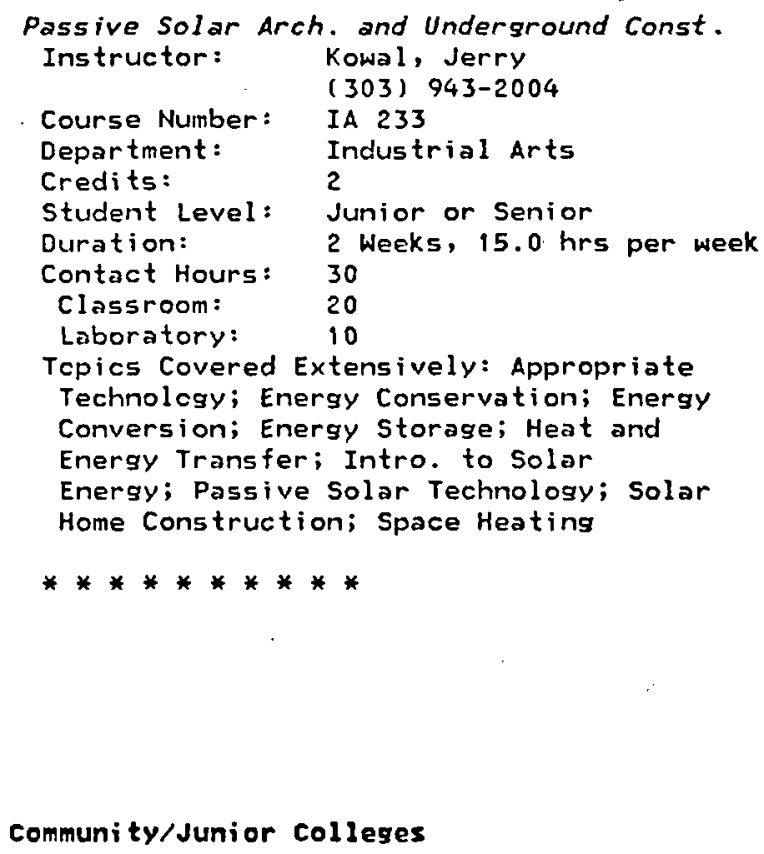

\section{Community/Junior colleges}

COLO ITH COLLEGE HEST CAM

GLERWOOD SPRINGS, Colorado 81601

(4506)

(303) $945-7481$

SOLAR RELATED COURSES

Energy: Sources and Uses

Instructor: Trapani, I. L.

Course Number: GSC 156

Credits: 3

Student Level: Freshman or Sophomore

Duration: 10 Weeks, 3.0 hrs per week

Contact Hours: 30

Topics Covered Extensively: Alternate

Energy Sources; Energy Conservation;

Intro. to Solar Energy; Wind Power,

Central Systems; Wind Power, Small

Systems

Sol. Ener. Bldg.-Design and Const.

Instructor: Shore, Ron

Course Number: BLO 205

Credits: 3

Student Level: All levels

Duration: $\quad 10$ Weeks, $3.5 \mathrm{hrs}$ per week

Contact Hours: 35

Topics Covered Extensively: Energy

Conservation; Heat and Energy Transfer;

Intro. to Solar Energy; Materials

Research; Passive Solar Technolagy;

Solar System Components; Solar Home

Construction; Solar Collector

Evaluation/Design; Solar Systems

Design; Solar Systems Installation;

Space Heating; Space Cooling

Solar and Hind Energy Symposium

Instructor: Dutmers, Gary

Course Number: GSC 27051

Credits:

student Level: All levele 


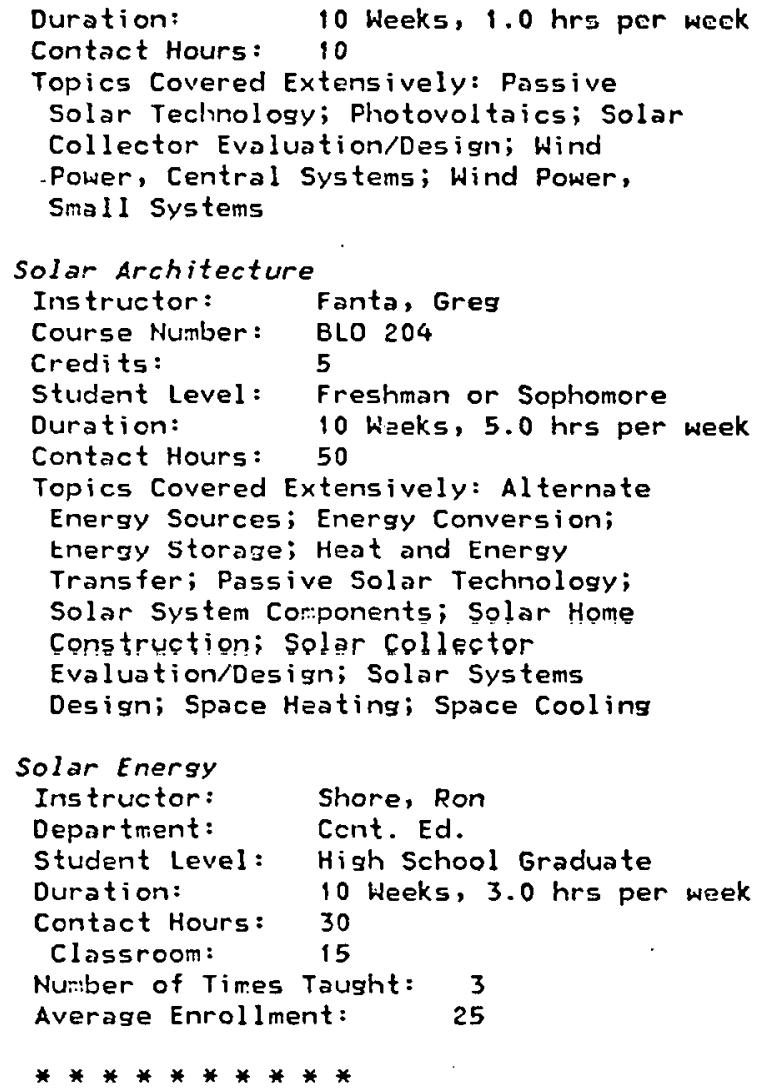

DENYER RED FOCKS CAM, CC OF

GOLDEN, Colorado 80401

(303) 988-6160

\section{PROGRAMS AFID CURRICULA}

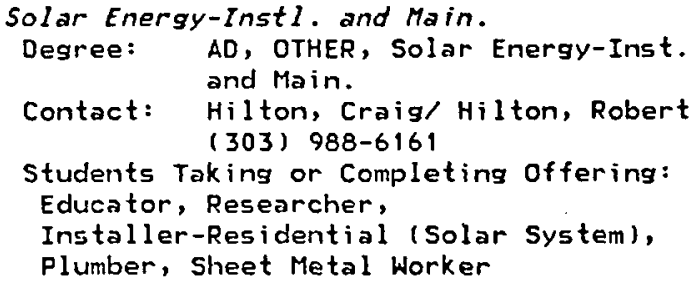


Topics Covered Extensively: Sheet Metal Techniques; Solar System Conponents Number-of Times Tausht: 3 Average Enrollment: $\quad 25$

Basic Solar Controls

Instructor: Hitz, Frank

(303) 9s8-6161

Course Number: S011235

Department: Industrial Occupations

Program or

Curriculum: Solar Energy-Instal.

Credits: 3

Student Level: All levels

Duration: 15 Weeks, $4.0 \mathrm{hrs}$ per week

Contact Hours: 60

Classroom: $\quad 15$

Laboratory: 45

Topics Covered Extensively? Solar system

Combonents; Solar Systems Testing and

Evaluation

Number of Times Taught: 2

Averase Enroliment: $\quad 30$

Basic Solar Systems

\begin{tabular}{|c|c|}
\hline 1structor: & $\begin{array}{l}\text { Hilton, Craig } \\
(303) 988-6161\end{array}$ \\
\hline :ourse liumber: & someco \\
\hline Department: & Industrial Occupations \\
\hline $\begin{array}{l}\text { Program or } \\
\text { Curriculum: }\end{array}$ & $\begin{array}{l}\text { Solar Energy-Inst. and } \\
\text { Main. }\end{array}$ \\
\hline redits: & \\
\hline tudent Level: & All levels \\
\hline uration: & 15 Weeks, 4.0 hrs per week \\
\hline $\begin{array}{l}\text { Contact Hours: } \\
\text { Classroom: }\end{array}$ & $\begin{array}{l}60 \\
15\end{array}$ \\
\hline Laboratory: & 45 \\
\hline $\begin{array}{l}\text { Topics Covered Ex } \\
\text { Energy Scurces; } \\
\text { Intro. to Solar } \\
\text { Techniques; Sol } \\
\text { Solar Systems Ir } \\
\text { Hot Woter; Swimr } \\
\text { Heating }\end{array}$ & $\begin{array}{l}\text { Extensively: Alternate } \\
\text { Energy Conservation; } \\
\text { Energy; Plumbing } \\
\text { lar Hcre Construction; } \\
\text { Installation; Dcmestic } \\
\text { nming Pool Heating; Space }\end{array}$ \\
\hline $\begin{array}{l}\text { lumber of Times } \\
\text { iverage Enrollmer }\end{array}$ & $\begin{array}{lr}\text { Taught: } & 6 \\
\text { ent: } & 20\end{array}$ \\
\hline
\end{tabular}

Blueprint feading for Lonstr. Trades

Instructor: Feister, Clarence

(303) $988-6161$

Course Number: BTR 125

Department: Industriol Div.

Program or

Curriculum: Solar Energy-Instal.

Credits: and Main.

Student Level: All levels

Duration: 4

Contact Hours: 68

Classroom: $\quad 45$

Laboratory: 23

Number of Times Taught: 20

Average Enrollment: $\quad 20$

Bricklaying for Construction Irades

Instructor: Gale, Bud

(303) 938-6161

Course Number: ERI120

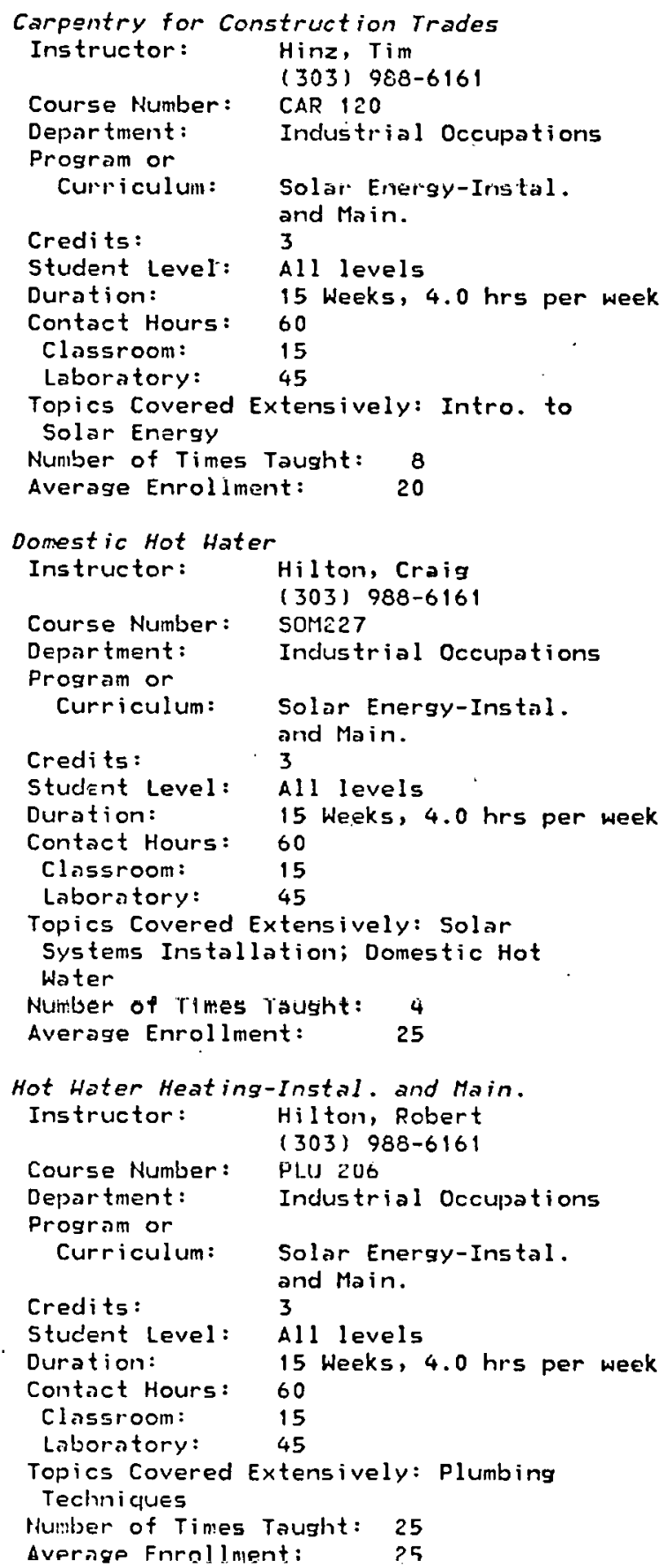




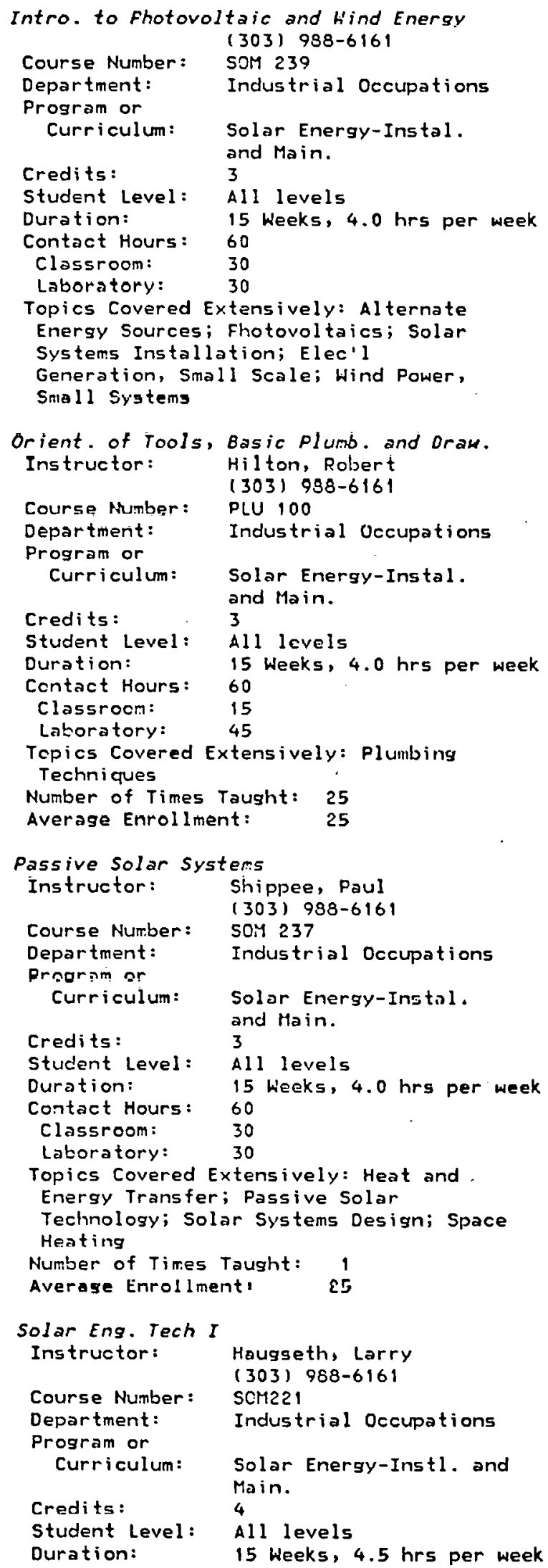

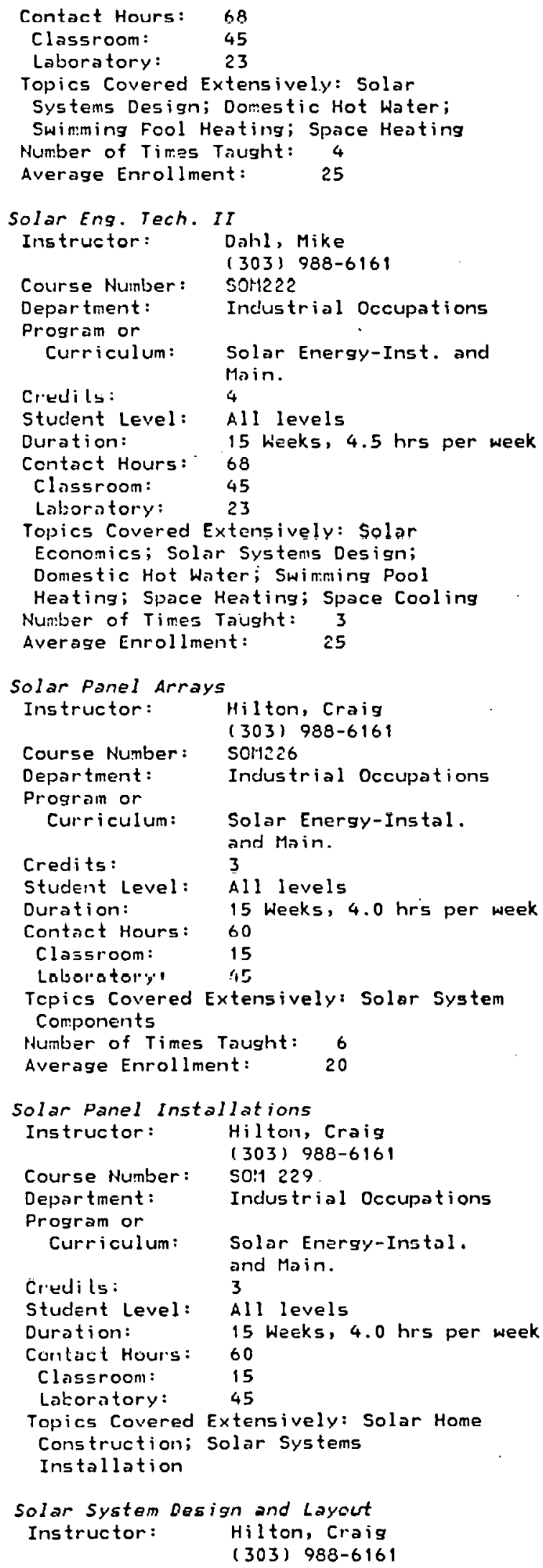




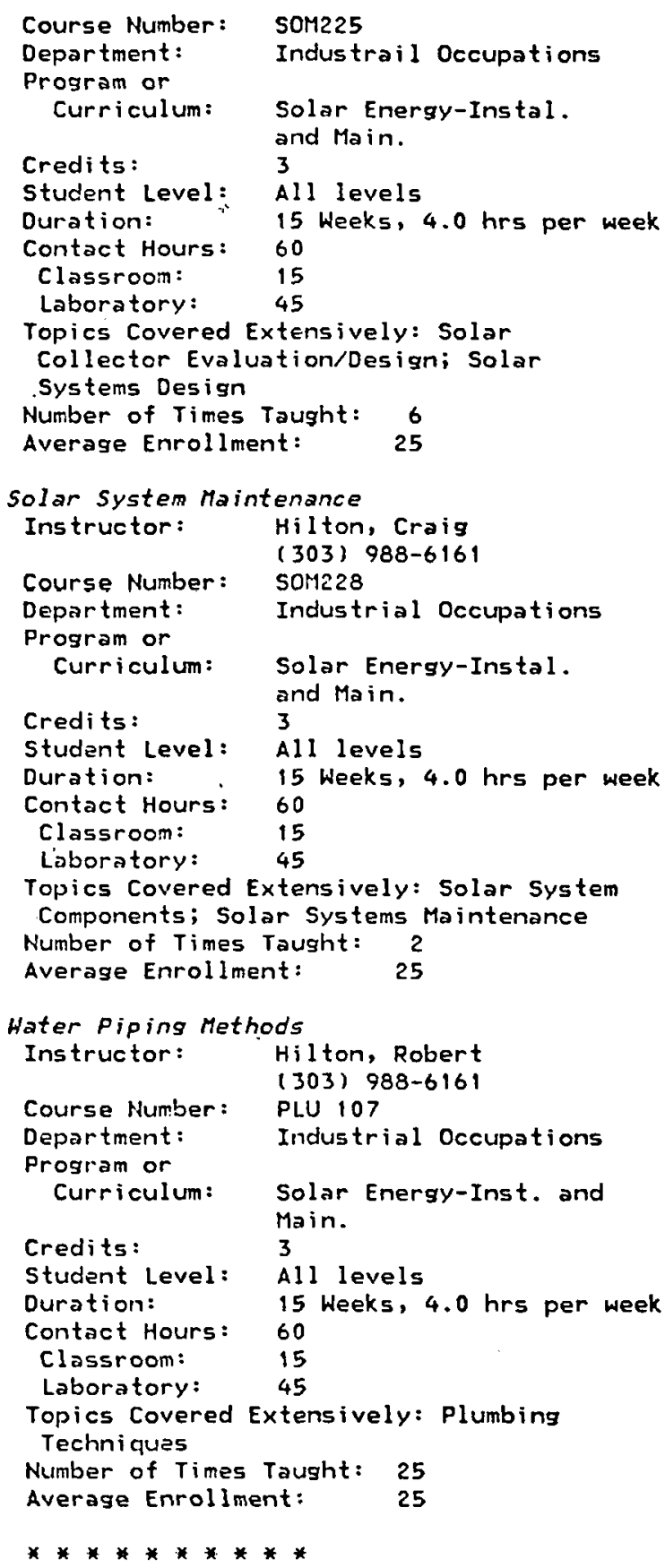

$(1362)$

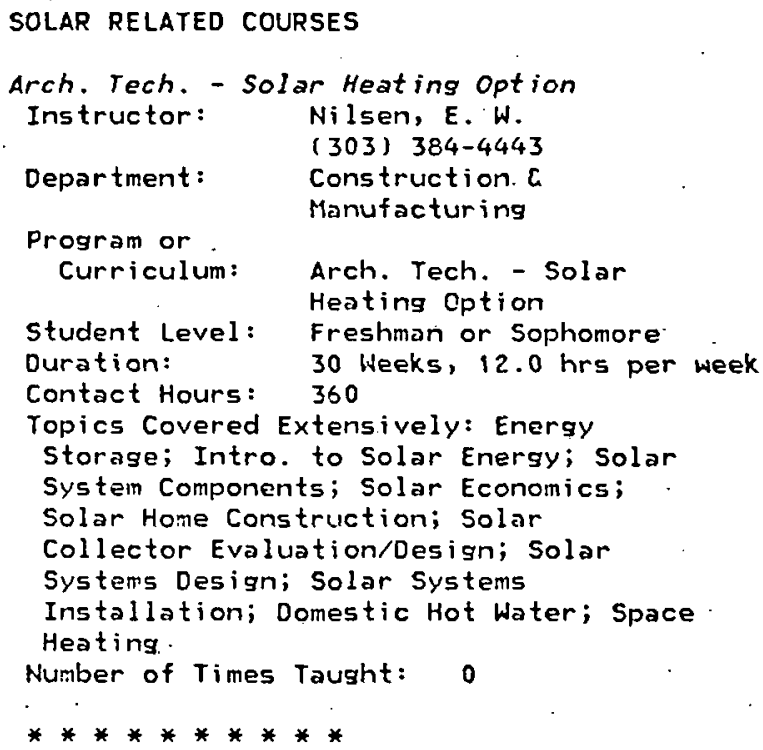

\section{Other Educational Institutions}


COLLEGE OF SOLAR ENERGY

$(90540)$

Box 397

Nederland, Colorado 80466

SOLAR RELATEO COURSES

* Solar Felated Courses

**********

COLORADO OFFICE OF EHERGY

CONEERVATION

1410 Grant St., B-104

(90440)

Denver, Colorado 80203

SOLAR RELATED COURSES

* Solar Energy tiorkshops

**********

DOMESTIC TECHNOLOGY INSTITUTE

(90550)

Box 2043

Evergreen, Colorado 80439

SOLAR RELATED COUPSES

* Solar Energy Horkshops

Instructor: Lillywhite, Malcom

**********

THE ALTERNATE ENERGY IHSTITUTE

$(90310)$

BOX 3100

Estes Park, Colorado 80517

SOLAR RELATED COURSES

* Solar saturday

Topics Covered Extensively: Alternate

Energy Sources

********** 


\section{Colleges/Universities}

CENTRAL CONN ST COLLEGE

(1378)

NEW BPITAIN, Connecticut

(203) 827-7000

\section{SOLAR RELATED COURSES}

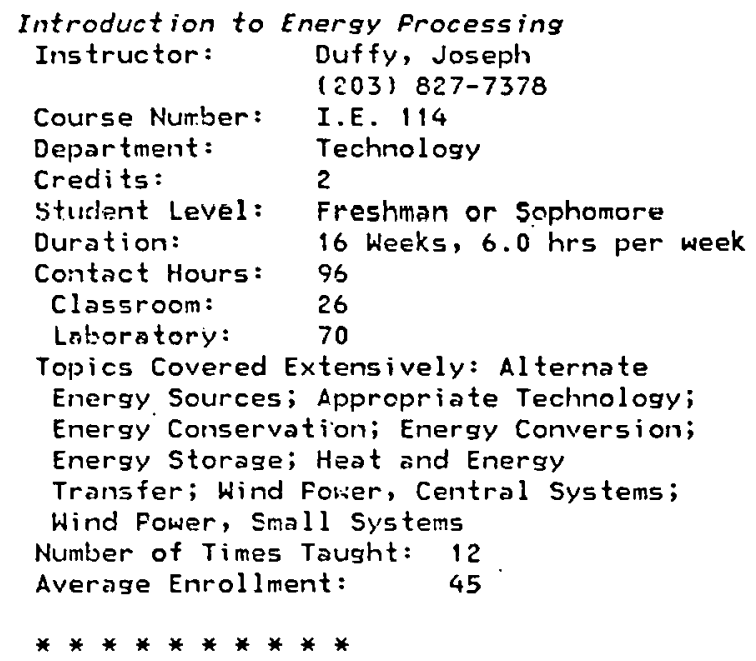

Average Enrollment:

$$
\text { (203) 786-2332 }
$$

Machanical Engineering 3

Junior or Senior

14 Weeks, 3.0 hrs per week

42
GRADUATE CMT. OF RP I $\varepsilon$ CNT.

ENVIROM:AENT \& MAN

$(90260)$

275 Windsor st.

Hartford, Comnecticut 06120

SOLAR RELATED COURSES

* Some Solar Energy Studies

**********

\section{HARTFORD GRADUATE CEITER}

(2804)

HARTFORD, Connecticut

(203) 549-3600

PROGRATS AHID CURRICULA

*Euvirnn. Sci. and Tech.

Contact: Florek, Donald B.

Students Taking or Completing offering:

Architect, Solar Ensineer, Other

SOLAR RELATED COURSES

*Adr. Solar Energy Systems Design Instructor: Florek, Doriald B.

Department: Special Programs

Prozram or

Curriculum! *Environ. Sci. and Tech.

Topics Covered Extensively: Solar System Conponents; Solar Collector

Evaluation/Design; Solar Systems Design

* Solar Energy

Instructor: Florek, Donald B.

Department: Special Prograins

Prosram or

Curriculum: *Environ. Sci. and Tech.

Topics Covered Extensively: Intro. to Solar Energy

* Solar Energy for Bldss.

Instructor: Florek, Donald B.

Department: Special Programs

Prosram or

Curriculum: EEnviron. Sci. and Tech.

Topics Covered Extensively: Passive Solar Technology; Solar Home. Construction; Solar Collector Evaluation/Design; Solar Systems Design

**********

NEH HAVEN, UNIVERSITY OF

(1397) WEST H.AVEN, Connecticut (203) $9311-6321$

\section{SOLAR RELATED COURSES}

* Solar Heating and Cooling

Department: Special studies

Duration: 12 Weeks, 3.0 hrs per week

Contact Hours: 36

Topics Covered Extensively: Space

Heating; Space Cooling 


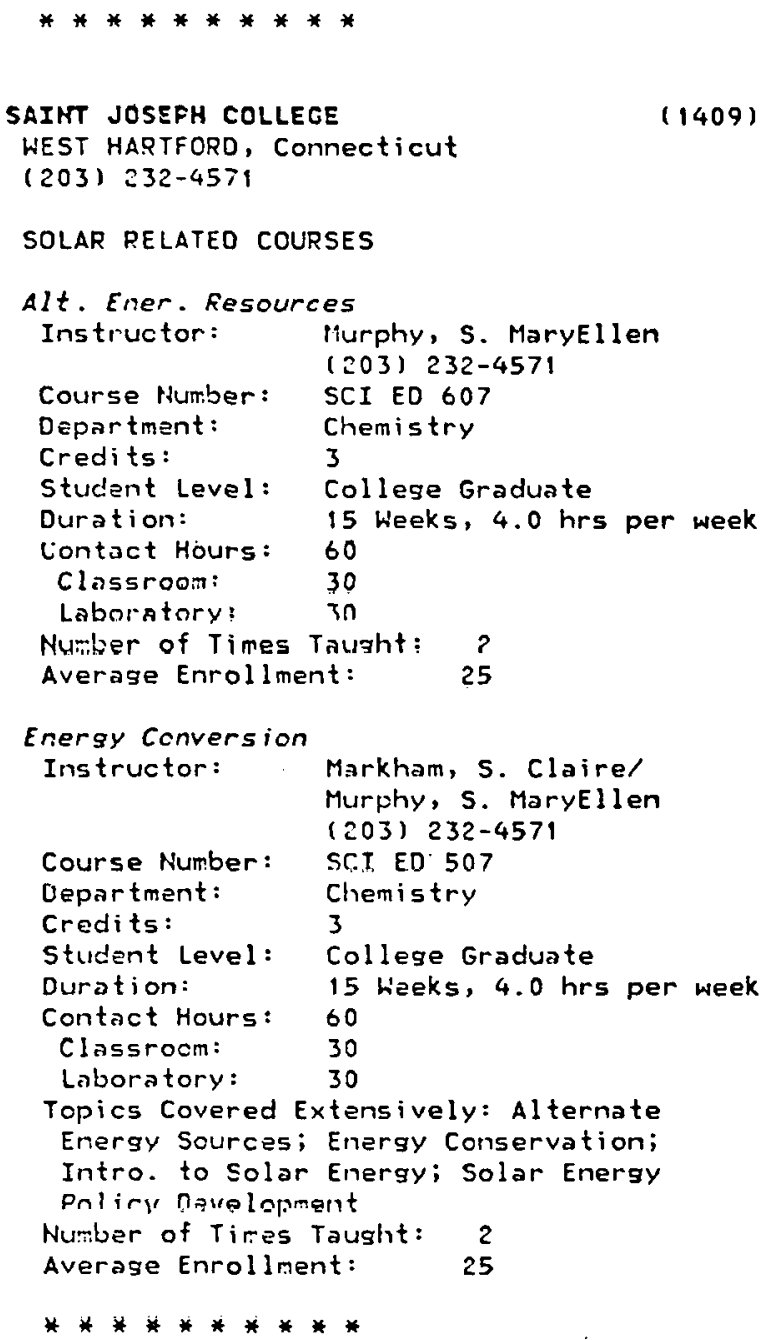

(1409)

Course Number:

Department:

Credits:
Student Level:
Duration:

Duration:

Contact Hours:

Classroom:

413

College of Science in Society 3

All levels

13 Weeks, $3.0 \mathrm{hrs}$ per week 39

33

Topics Covered Extensively: Alternate

Energy Sources; Heat and Energy

Transfer; Intro. to Solar Energy

Number of Times Taught: 3

Average Enrollment:

11

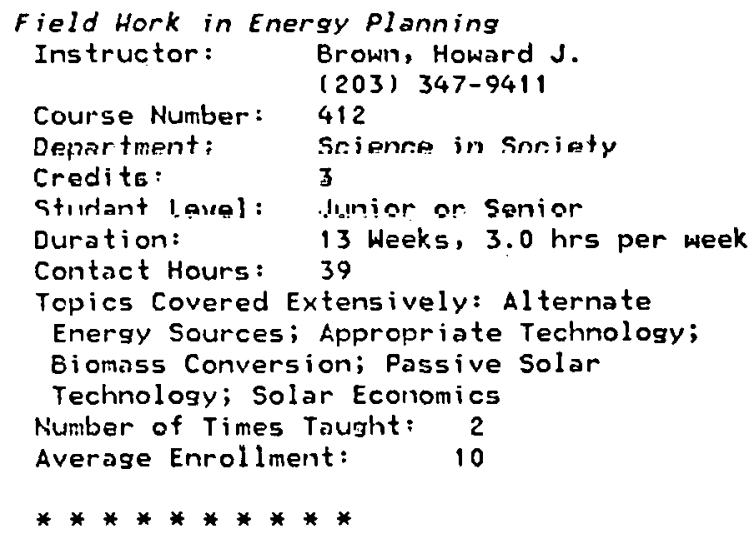

YALE UNIVERSITY

NEW HAVEN, Connecticut

(203) 436-4771

SOLAR RELATED COURSES

Energy Conservation Seminar

Instructor: Watson, Donald (203) 453-6388

Course Number: $C-24$ (B)

Department: Architecture 


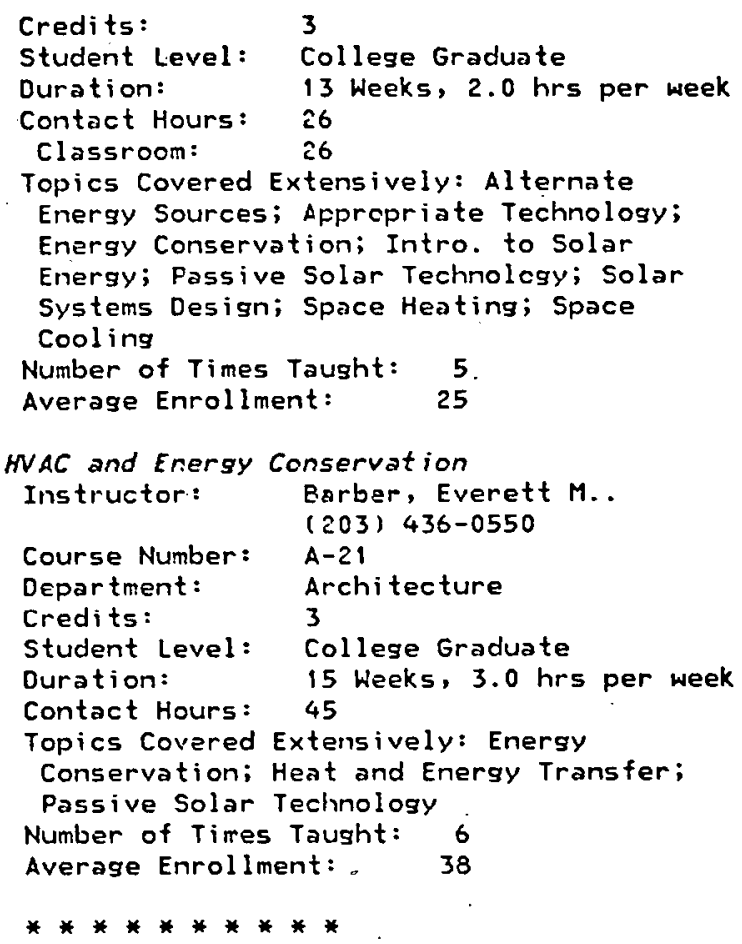

\section{other Educational Institutions}

ENERGY ED. SERVICES OF COHNECTICUT (90280) PO Box 224

Hartford, Connecticut 06103

\section{SOLAR RELATED COURSES}

* Solar Ener. for Homes $\varepsilon$ Bldgs

Topics Covered Extensively: Solar Collector Evaluation/Design; Solar Systems Desisn; Donestic Hot Water; Space Heating

THAMES VLY STATE TECH C NCRWICH, Connecticut

(203) $886-0177$

SOLAR RELATEO COURJES

Solar Energy/Energy Conservation and Usage Department: Evening Uivision Student Level: Hish School Graduate Duration: II Weeks, $3.0 \mathrm{hrs}$ per week Contact Hours: 33

Classroom: $\quad 33$

Number of Times Taught: 1

Average Enrollment: $\quad 30$ 


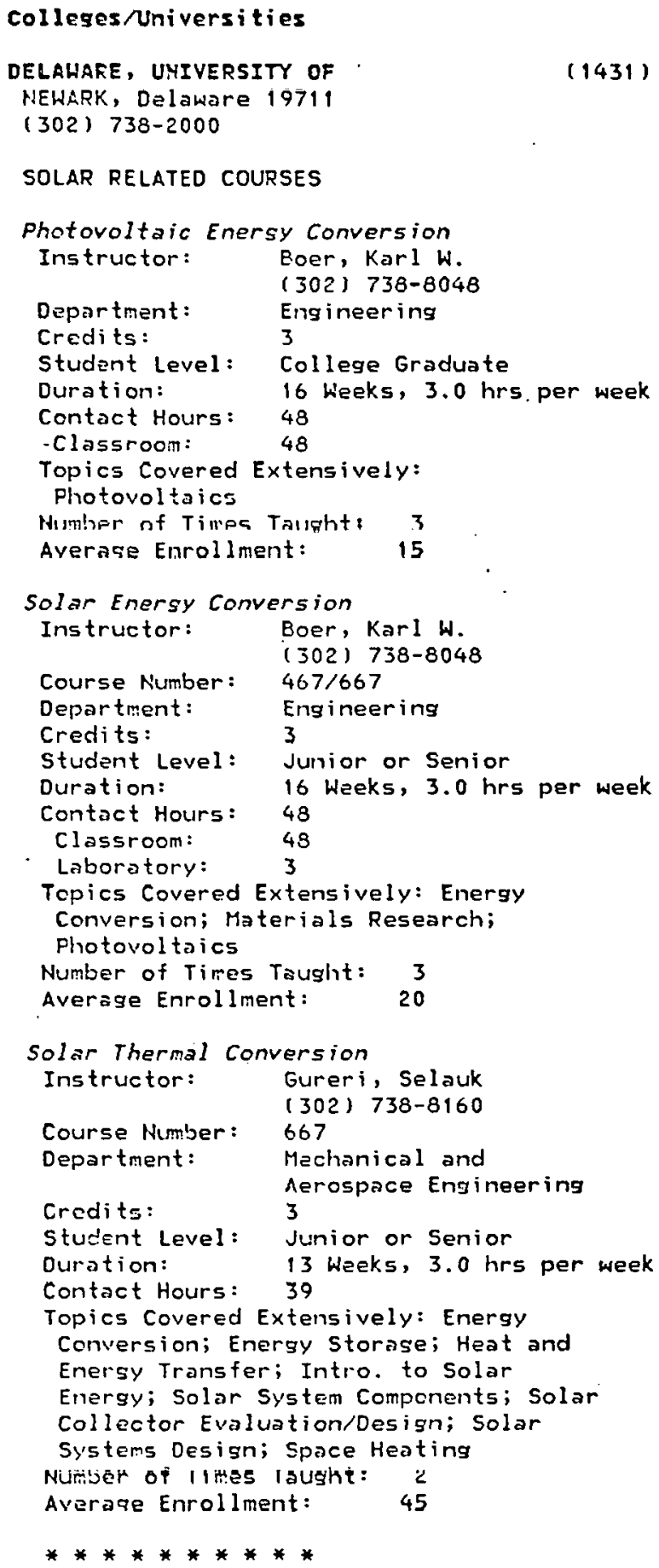

Yocational/Technical colleges
HEHCASTLE COUNTY VOCATIOHAL TECHHICAL SCHOOL

1417 Hewport Rd.

$(90370)$

Wi Imington, Delaware 19804

PROGRAMS AND CURRICULA

* Solar Heating of Buildings

SOLAR RELATED COURSES

* Solar Heating of Buildings

Prosram or

Curriculum: *Solar Heating of Buildinss

Contact Hours: 60

Topics Covered Extensively: Energy

Conversion; Energy Storage; Intro. to Solar Energy; Solar System Components;

Solar Economics; Solar Collector EYaluation/Design; solar Systems Design; Solar Systems Installation; Solar Systems Maintenance; Solar Systems Testing and Evaluation

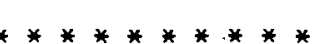




\section{Colleges/Universities}

CATHOLIC $U$ OF AKERICA

WASHINGTON, District of Colombia 20064

(1437)

(202) 635-5000

\section{SOLAR RELATED COURSES}

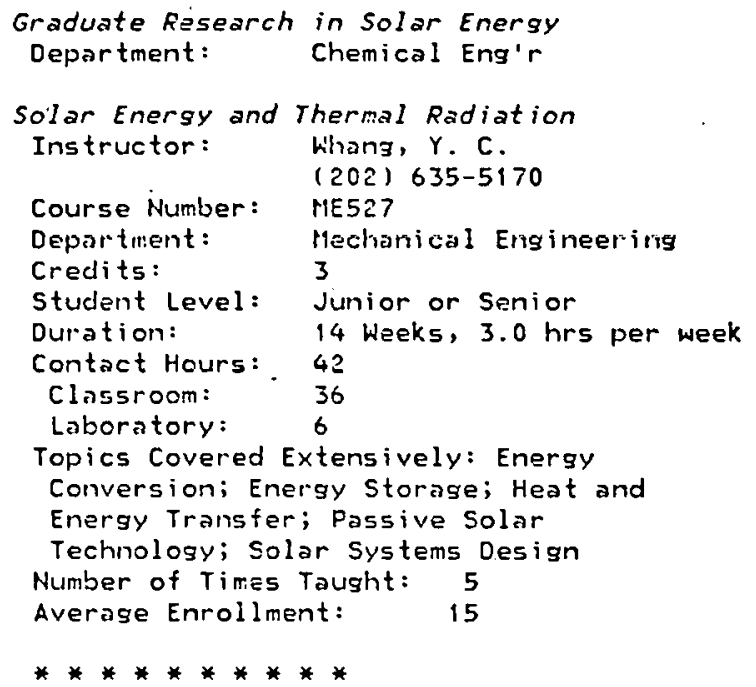

PROGRAMS AIID CUPRICULA

* Enersy Resources E Environ.

Cegree: Fho, MS, (202) 676-6749

\section{SOLAR RELATED COURSES}

* Solar Heat. E Cool.-Res.,Comm.Appls. Department: Continuing Education Topics Covered Extensively: Space Heating; Space Cooling

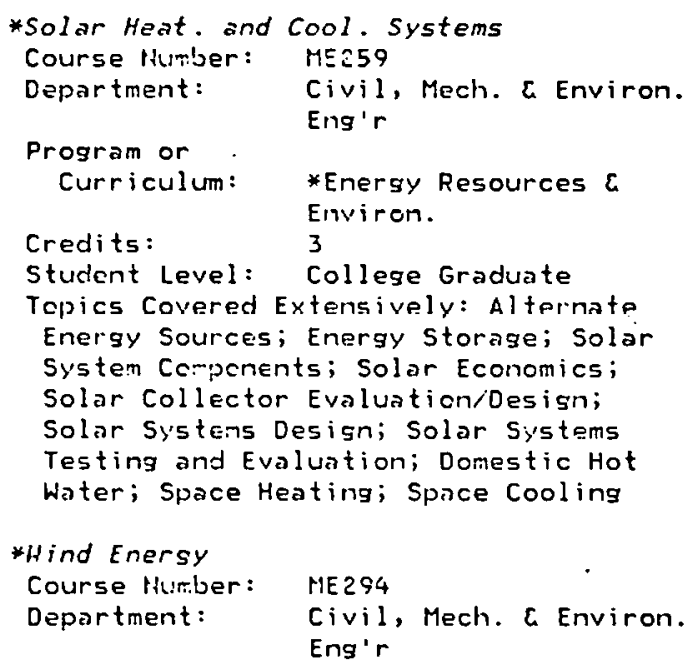

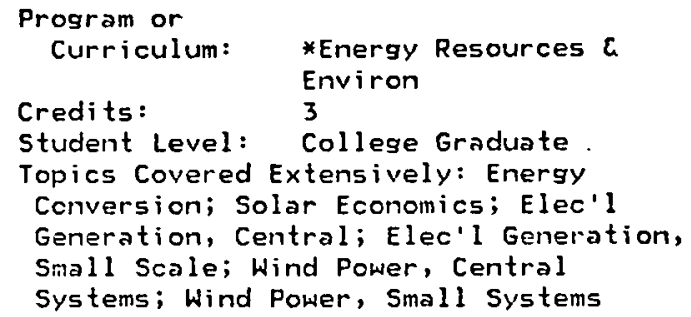

Other Educational Institutions

NATIOHAL TRAINIHG FUHD

1900 "L" Street Hil, Suite 405 ,

Wrshington, District of Colombia 20036

PROGRAIIS ARID CURRICULA

* Sheet Metal - Mpprentice, Journeyman

Contact: Harrington, Mr. (202) 833-9543 
THIS PAGE

\section{WAS INTENTIONALLY LEFT BLANK}




\author{
Colleges Universities \\ BARRY COLLECE \\ MIAMI, Florida 33161 \\ $(9466)$ \\ (305) 758-3392 \\ SOLAR RELATED COURSES \\ Energy Economics \\ Instructor: Wryman, Mark \\ (305) 754-7233 \\ Course Number: ECO 302 \\ Department: Continuing Education \\ Credits: 3 \\ Student Level: Junior or Senior \\ Duration: $\quad 10$ heeks, $3.0 \mathrm{hrs}$ per week \\ Contact Hours: 30 \\ Topics Covered Extensively: Alternate \\ Energy Sources; Appropriate Technology; \\ Energy Conservation; Intro. to Snlar \\ Fnergy: Marketing/Market Analysisi \\ Passive Solar Technology; Solar Energy \\ Policy Development; Solar Economics; \\ Solar Home Constructicn; Solar \\ Law/Legislation; Dcmestic Hot Water; \\ Swimming Pool Heating; Elec'l \\ Generation, Central; Elec'l Generation, \\ Small Scale; Space Cooling; Wind Power, \\ Central Systems; Wind Power, Small \\ Systems \\ Number of Times Taught: 1 \\ Average Enrollment: \\ 12 \\ **********
}

FLORIDA INST TECHMOLOGY

$(1469)$

MELBOURNE, Florida 32901

(305) 723-3701

SOLAR RELATED COURSES

Design of Solar Conversion Systems

Instructor: Alkasab, K. A.

(305) 723-3701

Course Number: ME 5037

Department: Mechanical Engineering

Credits: 3

Student Level: College Graduate

Duration: 10 Heeks, $3.0 \mathrm{hrs}$ per week

Contact Hours: 30

Classroom: $\quad 30$

Topics Covered Extensively: Alternate

Energy Sources; Energy Conversion; Heat and Energy Transfer; Solar System

Components; Solar Systems Design;

Elec'l Generation, Central; Space

Heating; Wind Pcwer, Central Systems;

Wind Power, Small Systems

Number of Times Taught: 2

Average Enrollment: $\quad 10$

Prin. of Solar Energy Conversion

Iristruetur:

Course Number:

Department:

Credits:

Student Level:

Duration:

Alkosab, $K$ : $A$.

(305) 723-3701

ME 5035

Mechanical Engineering

3

College Graduate

10 Weeks, $3.0 \mathrm{hrs}$ per week
Contact Hours: 30

Classroom: $\quad 30$

Topics Covered Extensively: Alternate

Energy Sources; Energy Conversion; Heat and Energy Transfer; Intro. to Solar Energy; Solar System Components; Solar Collector Evaluation/Design; Solar Syster:5 Design; Elec'l Generation, Central; Space Cooling Nuriber of Times Taught: Average Enrollment:

Solar Energy Conversion Systems Instructor: Alkasab, K. A.

(305) 723-3701

Course Number: ME 5036

Department: Mechanical Engineering

Credits: 3

Student Level: College Graduate

Duration: 10 Weeks, $3.0 \mathrm{hrs}$ per week

Contact Hours: 30

Classroom: $\quad 30$

Topics Covered Extensively: Alternate Energy Sources; Energy Conversion; Heat and Energy Transfer; Solar System

Components; Solar Collector

Evaluation/Design; Solar Systems

Design; Elec'l Generation, Central;

Space Cooling

Nunber of Times Taught: 2

Average Enrollment: i2

***********

FLCRIDA INTERMATIONAL U

$(9635)$

MIAMI, Florida 33199

(305) 552-2731

PROGRAMS ANO CURRICULA

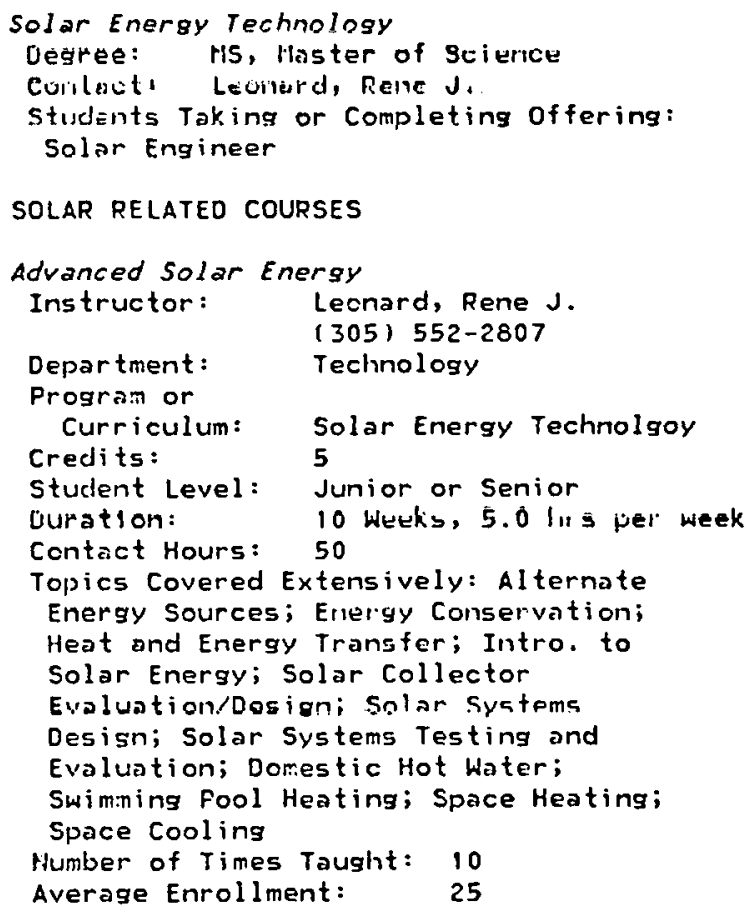




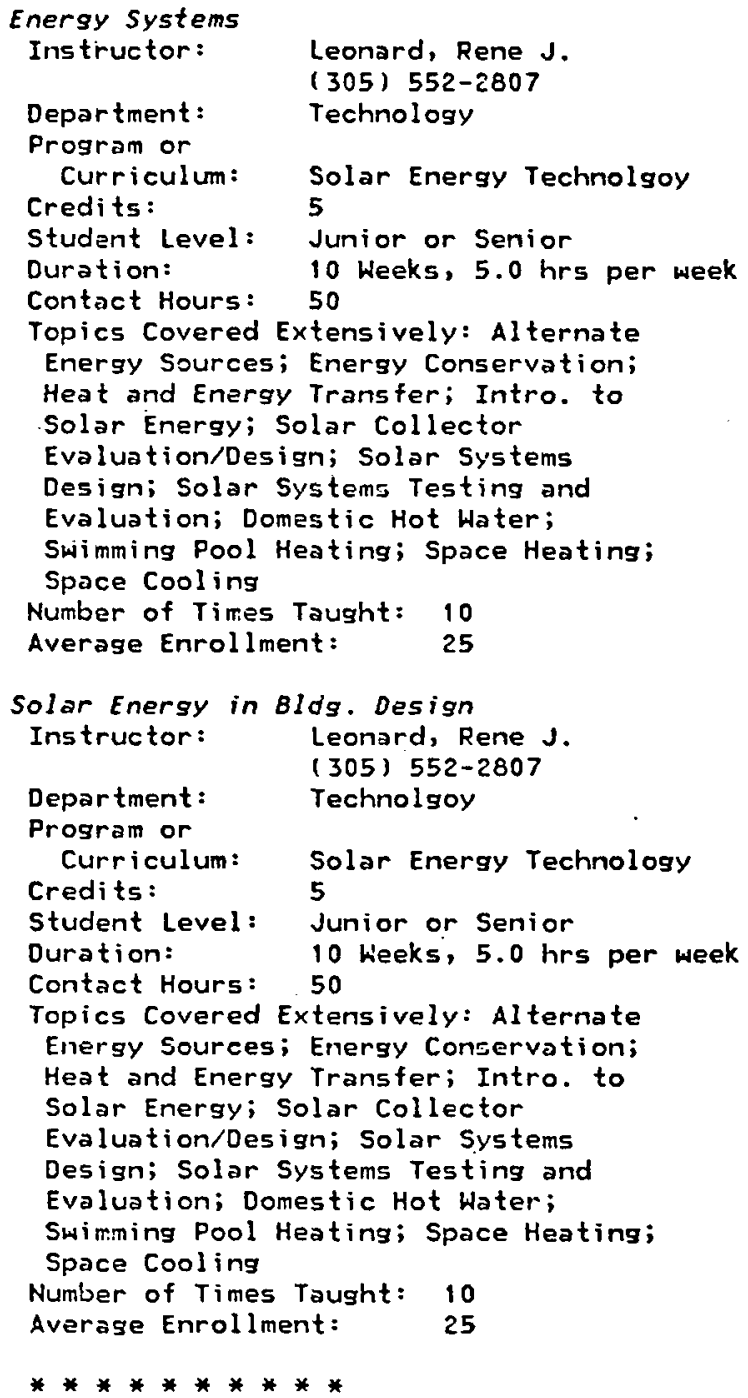

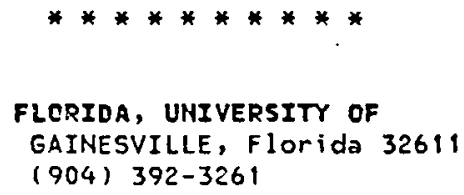




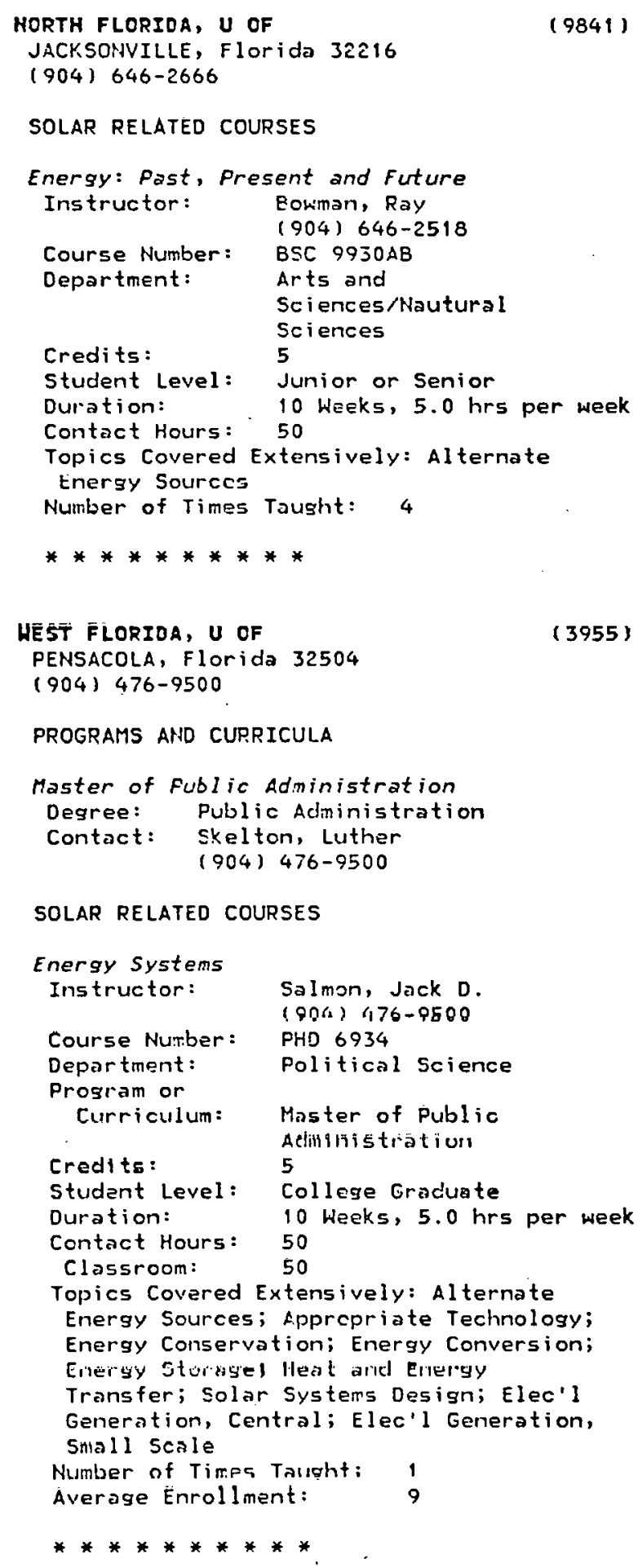

(9841)

(3955)

SULAR RELATEU CUURSES

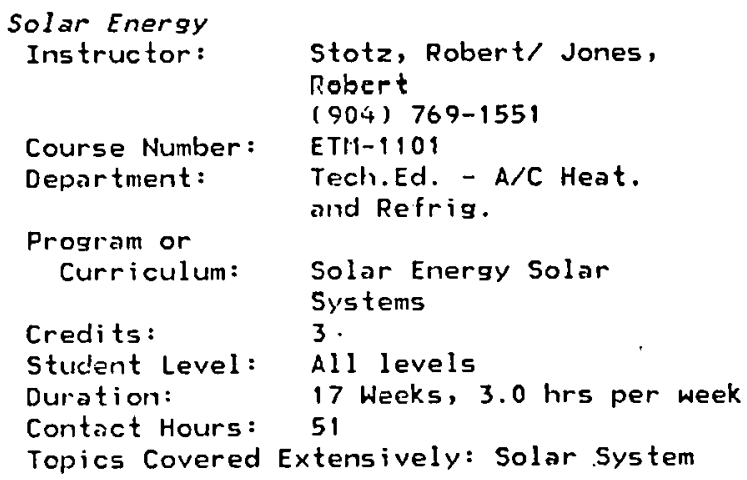




\author{
Components; Solar Home Construction; \\ Solar Collector Evaluation/Design \\ Number of Times Taught: 1 \\ Average Enrollment: 29 \\ solar systems \\ Instructor: Stotz, Robert/ Jones, \\ Robert \\ (904) 769-1551 \\ Course Number: ETM 2102 \\ Department: Tech. Ed. - A/C Heat. \\ and Refrig. \\ Program or \\ Curriculum: Solar Energy Solar \\ Credits: \\ Systems \\ Student Level: All levels \\ Duration: \\ 17 Heeks, 3.0 hrs per week \\ Contact Hours : \\ 51 \\ Topics Covered Extensively: Heat and \\ Enersy Transfer; Fassive Solar \\ Technolosy; Solar System Components; \\ Solar Economics; Solar Home \\ Construction; Solar Collector \\ Evaluation/Design; Solar Systems \\ Desisn; Domestic Hot Water; Space \\ Heating; Space Cooling \\ Number of Tinies Taught: 1 \\ Average Enroliment: 24 \\ **********
}

MIAMI- DADE CITY COLLEGE

$(1506)$

MIAMI, Florida 33176 .

(305) 596-1211

PROGRAMS AND CURRICULA

Air Conditioning Engineering

Technolosy

Degree: AD, Science

Contact: Succop, William (305) 635-4564

students Taking or Conpleting offering: Architect, Installer-Residential (Solar System), Installer-Commercial (Solar

Systeml, Solar Technician

\section{SOLAR RELATED COUREES}

Solar Energy fundamentals

Instructor: Cleland, George

(305) 685-4206

Course Number: ETM 2706

Department: Air Conditioning

Engineering Technology

Program or

Curriculum: Air Conditioning

Credits: Engineering Technology

Student Level: High School Graduate

Duration: 15 Weeks, 3.0 hrs per week

Contact Hours: 45

Topics Covered Extensively: Energy

Conservation; Intro. to Solar Energy;

Passive Solar Technology; Pluribing

Technicues; Solar System Components;

Solar Eccnomics; Solar Home

Construction; Solar Systems Design;
Solar Systems Installation; Domestic

Hot Water; Swimming Pool Heating

Solar Enersy Systems, Commerical

Instructor: Cleland, George

(305) 685-4206

Course Number: ETM $2758 \mathrm{C}$

Department: Air Conditioning

Engineering Technology

Program or

Curriculum: 'Air Conditioning

Credits : Engineering Teclinology

Student Level: High School Graduate

Duration:

Conlact Hours:

Classroom:

Laboratory:

15 Weeks, 4.0 hrs per week

60

30

30

Topics Covered Extensively: Energy

Conservation; Intro, to Solar Energy;

Passive Solar Technology; Plumbing

Techniques; Solar System Components;

Solar Economics; Solar Home

Construction; Solar Systems Design;

Solar Systems Installation; Domestic

Hot Water; Swimming Pool Heating

Solar Energy Systems, fesidential

Instructor: Cleland, George

(305) 685-4206

Course Number: ETM $2756 C$

Department: Air Conditioning

Program or

Curriculum: Air Conditioning

Credits: Engineering Technology

Student Level: High School Gracluate

Duration: 15 Weeks, 4.0 hrs per week.

Contact Hours: 60

Classroom: $\quad 30$

Laboratory: $\quad 30$

Topics Covered Extensively: Energy

Conservation; Intro. to Solar Energy;

Passive Solar Teclmology; Plumbing

Techniques; Solar System Components;

Solar Economics; Solar Home

Construction; Solar Systems Design;

Solar Systems Installation; Domestic

Hot Woter; Silimming Pool Honting

\section{PENSACOLA JUNIOR COLLEgE \\ PENSACOLA, Florido 32504 \\ (904) $476-5410$}

\section{PROGRAMS ANO CURRICULA}

Solar Enersy Technology

Degree: $A D$, Energy Tech. -

Certificate of Completion.

Contact: Lowery, Stanley

(904) $476-5410$ 
SOLAR RELATED COURSES

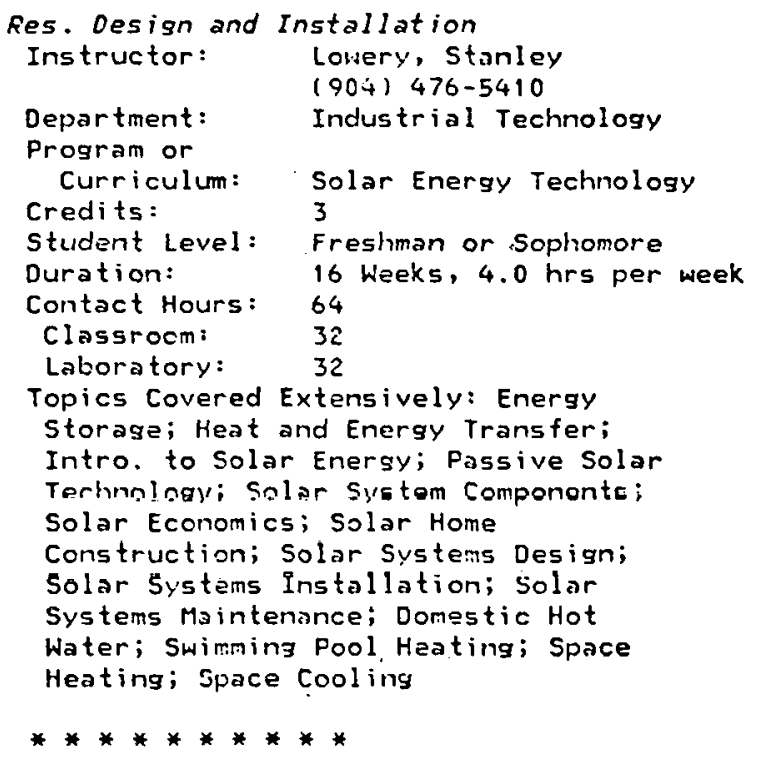

VALENCIA CMTY COLLEGE

(6750)

ORLANDO, Florida 32802

(305) 299-5000

SOLAR RELATED COURSES

Energy

Instructor: McCord, Willian

(305) 299-5000

Course Number: P5-290

Department: Science

Credits: 3

Student Level: Freshman or Sophomore

Duration: 14 Weeks, $3.0 \mathrm{hrs}$ per week

Contact Hours: 42

Classroom: $\quad 30$

Topics Covered Extensively: Alternate

Encrg', Sourees; Energy Congervotions

Energy Storage; Intro. to Solar Energy;

Passive Solar Technology; Domestic Hot

Water

Number of Times Taught: 1

Average Enrollment: 12

Solar Energy for the Home-owner

Instructor: McCord, William M.

(305) 299-5000

Departiment: Open Campus/Continuing

Open

Student Level: All levels

Duration: 1 Weeks, $6.0 \mathrm{hrs}$ per week

Contact Hours: 6

Classroom: 2

Topics Covered Extensively: Intro. to Solar Energy; Donestic Hot Water

***********

\section{Vocational/Technical colleges}

PINELLAS VO-TECH INSTITUTE

(90320)

6100154 th Ave. North

Clearwater, Florida 33516

SOLAR RELATEO COURSES

*Household Ener. Cons./Sol. Energy

Topics Covered Extensively: Energy

Conservation; Domestic Hot Water

* Solar Energy Heating and Cooling

Durationt Weeks

Topics Covered Extensively: Solar System Components; Solar Collector

Evaluation/Design; Solar Systems Design; Domestic Hot Water; Space Heating; Space Cooling

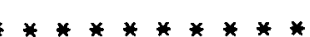


SCUTH FLORIDA TECHNICAL IHSTITUTE

$(9.0020)$

201 W. Sunrise Blvd.

Ft. Lauderdale, Florida 33311

(305) 764-3432

PROGRAMS AHJ CURRICULA

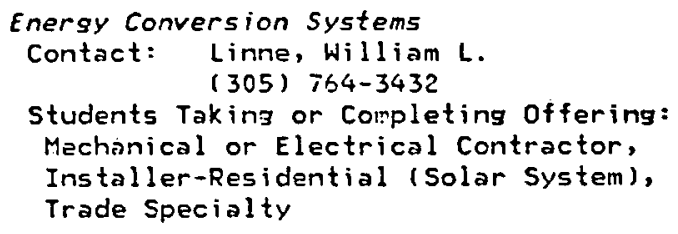

SOLAR RELATED COURSES

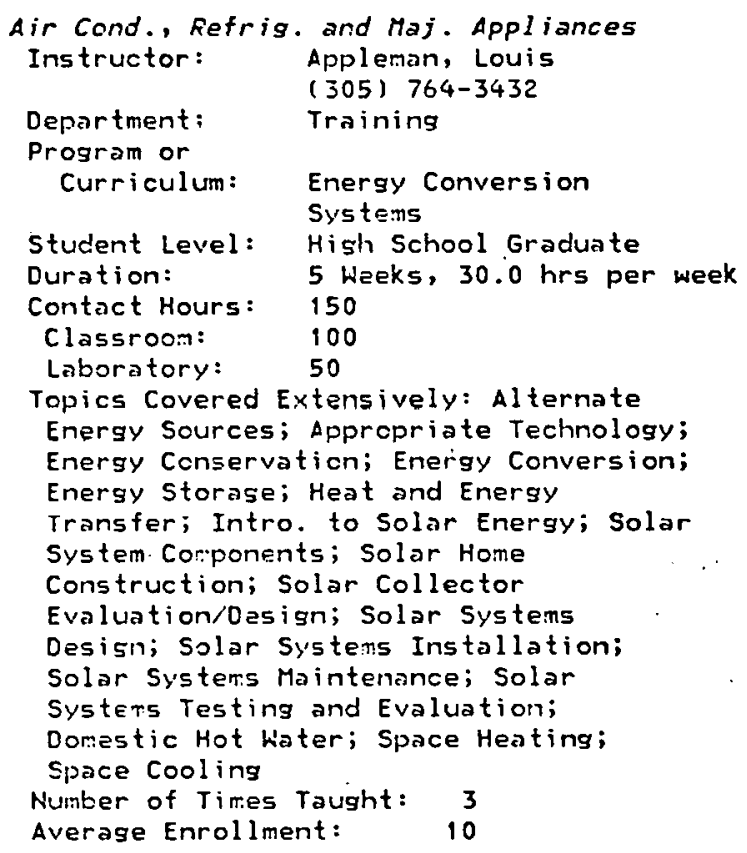

**********

\section{other Educational Institutions}

FLORIDA SOLAR ENERGY CEHTER

300 State Rd. 401

Cape Canaveral, Florida 32920

SOLAR RELATED COURSES

* Short Courses, Horkshops, Seminars 

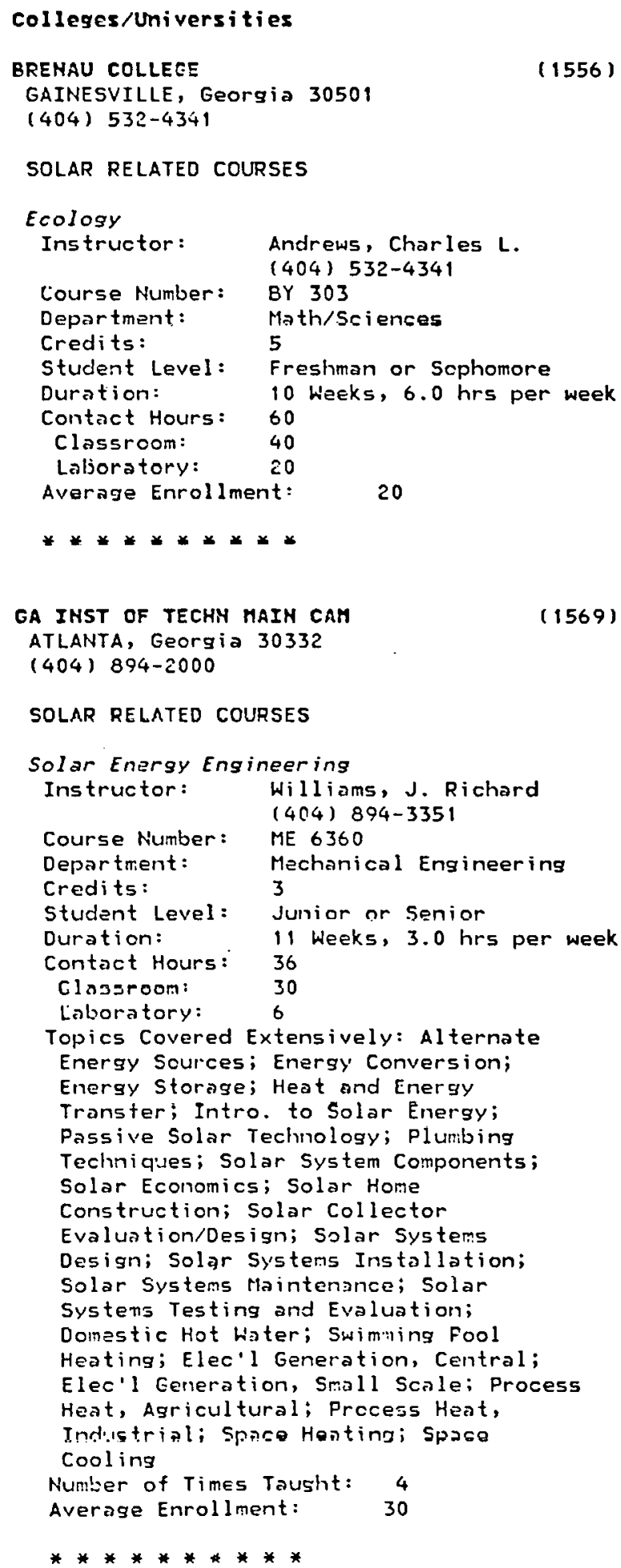

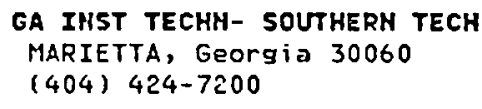




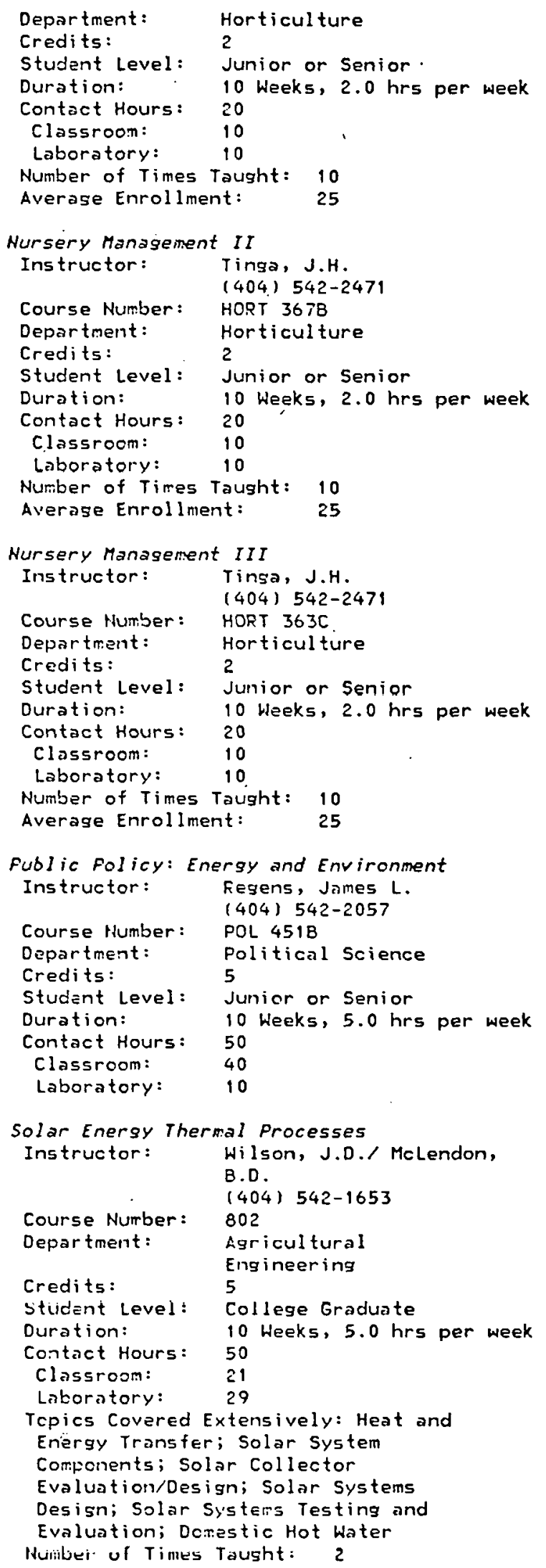

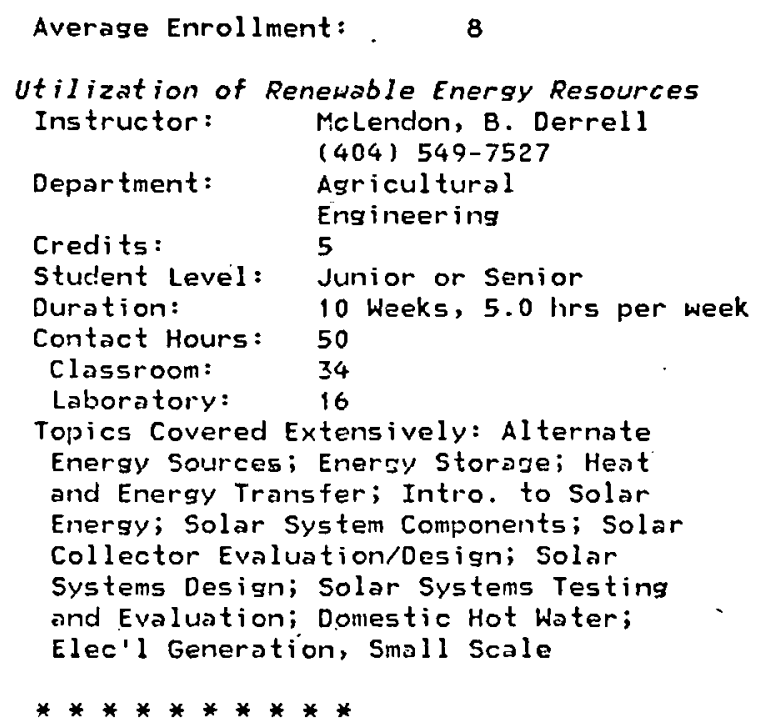

\section{Community/Junior colleges}

BRUHSHICK JUIIOR COLLEGE

ERUNSHICK, Georgia 31520

(912) 264-7211

PROGRAMS AND CURRICULA

*Vocational - Technical

**********

DEKALB COHMUNITY COLLEGE

CLARKSTON, GeOrgia 30021

(404) 292-3994

PROGRAMS AND CURRICULA

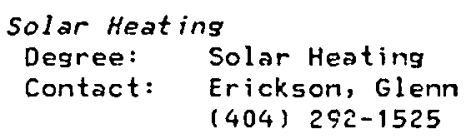

Solar Heat ing

Instructor:

Penland, William D.

(404) 292-1525

Department: Heating/Air

Program or Conditioning

Curriculum: Solar Heating

Student Level: All levels

Duration: 14 Neeks, 24.0 hrs per week

Contact Hours: $\quad 300$

Classroom: $\quad 200$

Laboratory: $\quad 100$
$(1558)$

(1562) 
THIS PAGE

\section{WAS INTENTIONALLY LEFT BLANK}




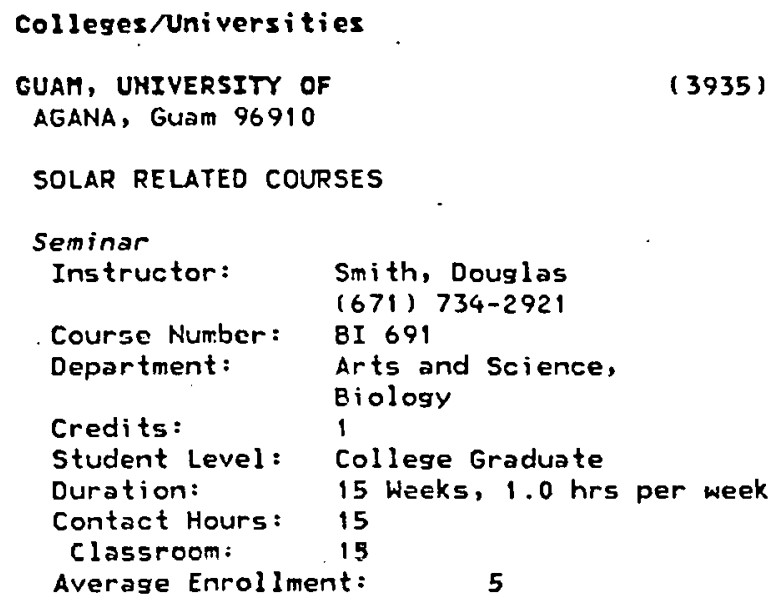




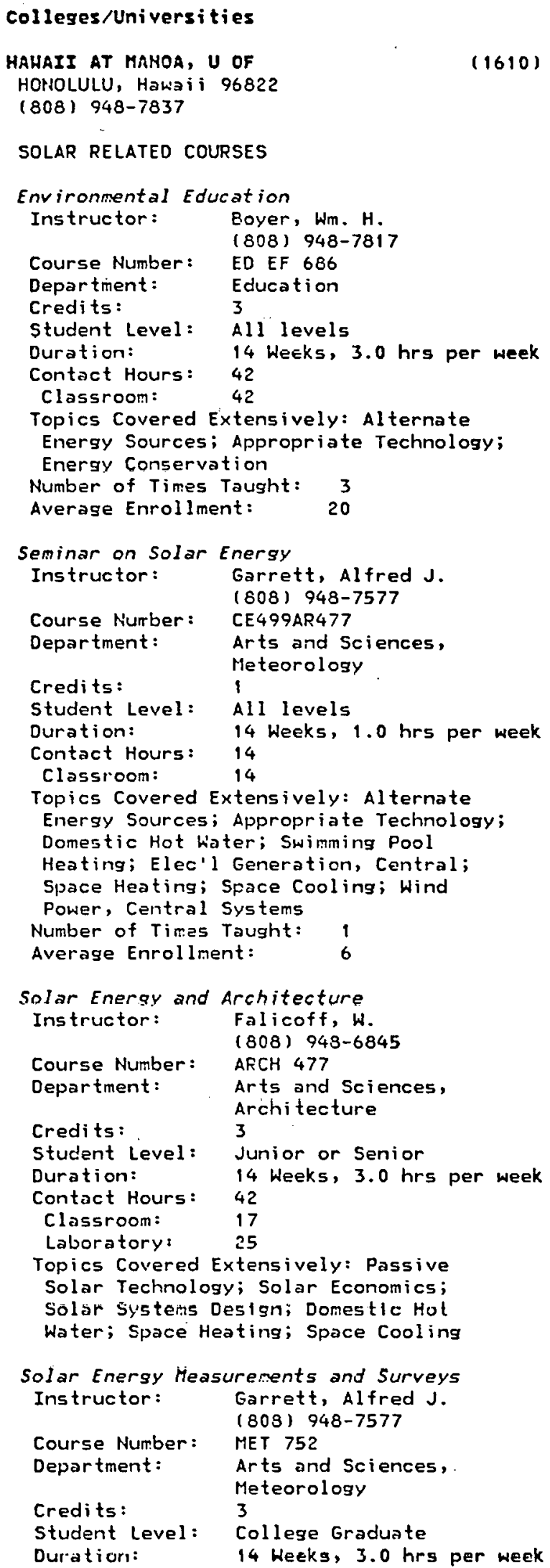

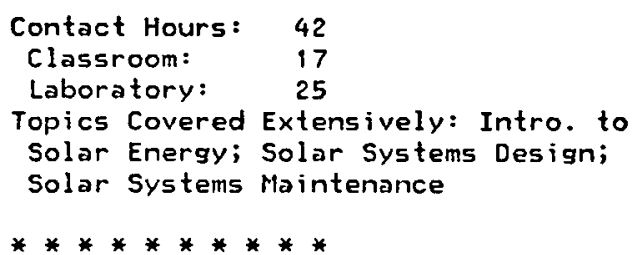

$(1612)$ 


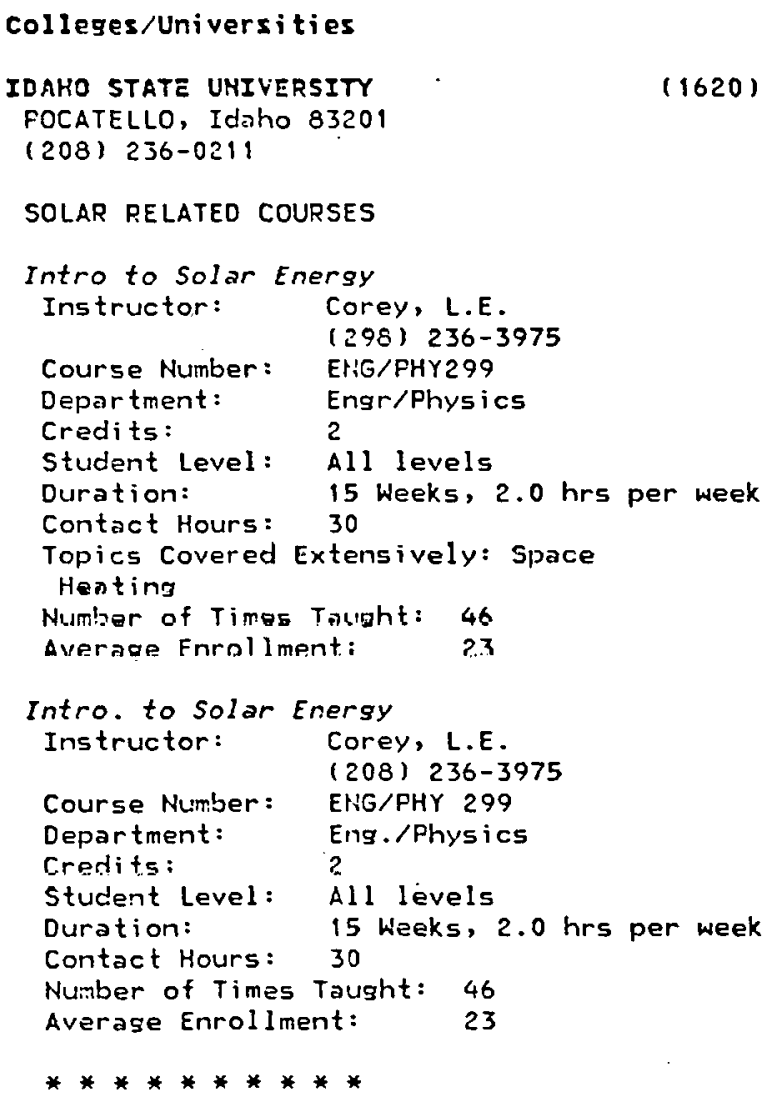

$(1620)$ 


\section{Classroom: 51}

Topics Covered Extensively: Appropriate Technolosy; Energy Conservation; Heat and Energy Transfer; Passive Solar Technolosy; Plumbing Techniques; Space Heating; Space Cooling

Number of Times Tausht:

Average Enrollment:

$$
4
$$

65

\section{Arch. - Solar Energy Design \\ Instructor: Eder, Anton \\ (208) 885-6272 \\ Course Number: 404 \\ Department: Art and Architecture \\ Credits: 3 \\ Student Level: Junior or Senior \\ Duration: $\quad 16$ Weeks, 3.0 hrs per week \\ Contact Hours: 48 \\ Classroom: \\ 48}

Topics Covered Extensively: Energy

Conservation; Enersy Storage; Intro. to

Solar Energy; Passive Solar Technology;

Solar System Components; Solar

Economics; Solar Collector

Evaluation/Design; Solar Systems

Desisn; Domestic Hot Hater; Space

Heating

Number of Times Tausht: 2

Average Enroliment: $\quad 100$

Direct Energy Conversion

Instructor: Hagen, Jack I.

(208) 885-6555

Course Number: EE 420

Department: Electrical Engineering

Program or

Curriculum: Solar Energy Workshops

Credits:

3

Student Level: College Graduate

Duration: 18 heeks, $3.0 \mathrm{hrs}$ per week

Contact Hours: $\quad 54$

Classroom: 54

Topics Covered Extensively: Alternate

Energy Sources; Energy Conservation;

Energy Conversion; Intro. to Solar

Energy; Fhotovoltaics; Solar Collector

Evaluation/Design; Eles'l Generation,

Central; Elec'l Generation, Small Scale

Number of Timas Tausht: 1

Averase Enrollment:

10

Fower Technology

Instructor:

Cassetto, James M.

(208) 885-6492

Course Nuniber: 316

Department:

Program or

Curriculum

Credits:

Industrial Educ.

3

Student Level: Junior or Senior

Duraticn:

Contact Hours:

Classroom:

Laboratory:

Power Technology

3

18 Weeks, 6.0 hrs per week

108

54

54

Topics Covered Extensively: Alternate

Energy Sources; Appropriate Technology;

Energy Conservation; Energy Conversion;

Energy Storage; Heat and Energy

Transfer; Intro. to Solar Energy;

Passive Solar Technolugy;
Photovoltaics; Solar System Components;

Solar Economics; Solar Home

Construction; Solar Collector

Evaluation/Design; Solar Systems

Design; Solar Systems Installation;

Solar Systems Maintenance; Solar

Systers Testing and Evaluation;

Domestic Hot Water; Swiming Pool

Heating; Elec'1 Generation, Small

Scale; Process Heat, Industrial; Space

Heating; Wind Power, Central Systems;

Wind Power, Small Systems

Number of Times Taught: 1

Average Enrollment: $\quad 15$

Solar Energy Systems

Instructor: Warner, R. E.

Course Number: MEE 435

Department: Mechanical Engineering

Program or

Curriculum: Solar Enersy

Credits:

Student Level: Junior or Senior

Duration: 18 Weeks, 3.0 hrs per week

Contact Hours: 54

Classroom: $\quad 52$

Laboratory: 2

Topics Covered Extensively: Appropriate

Technology; Energy Conversion; Energy

Storase; Heat and Energy Transfer;

Intro. to Solar Energy; Solar System

Components; Solar Collector

Evaluation/Design; Solar Systems

Design; Solar Systems Testing and

Evaluation; Domestic Hot Water; Elec'l

Generation, Central; Space Heating

Number of Times Tausht: 2

Averase Enrollment: $\quad 18$

Horkshop Solar Energy Tech

Instructor: Cassetto, James

Course lumber: 403

Department: Education Industrial

Program or

Curriculum: Power Technology

Credits:

Student Level: All levels

Duration:

Contact Hours: 36

Classroom: $\quad 18$

Laboratory: 18

Topics Covered Extensively: Alternate

Energy Sources; Appropriate Technology;

Biomass Conversion; Energy

Conservation; Energy Conversion; Energy

Storage; Heat and Energy Transfer;

Intro. to Solar Energy; Passive Solar

Technolozy; Photovoltaics; Solar System

Cor:ponents; Solar Eccucmics; Solar Home

Construction; Solar Collector

Evaluation/Design; Solar Systems

Desisn; Solar Systems Installation;

Solar Systems Maintenance; Solar

Systems Testing and Evaluation;

Domestic Hot Water; Swimning Pool

Heating; Elec'l Generation, Small

Scale; Process Heat, Industrial; Space lleating

Humber of Times Taught: 3

Average Enrollment: $\quad 35$ 
THIS PAGE

\section{WAS INTENTIONALLY LEFT BLANK}




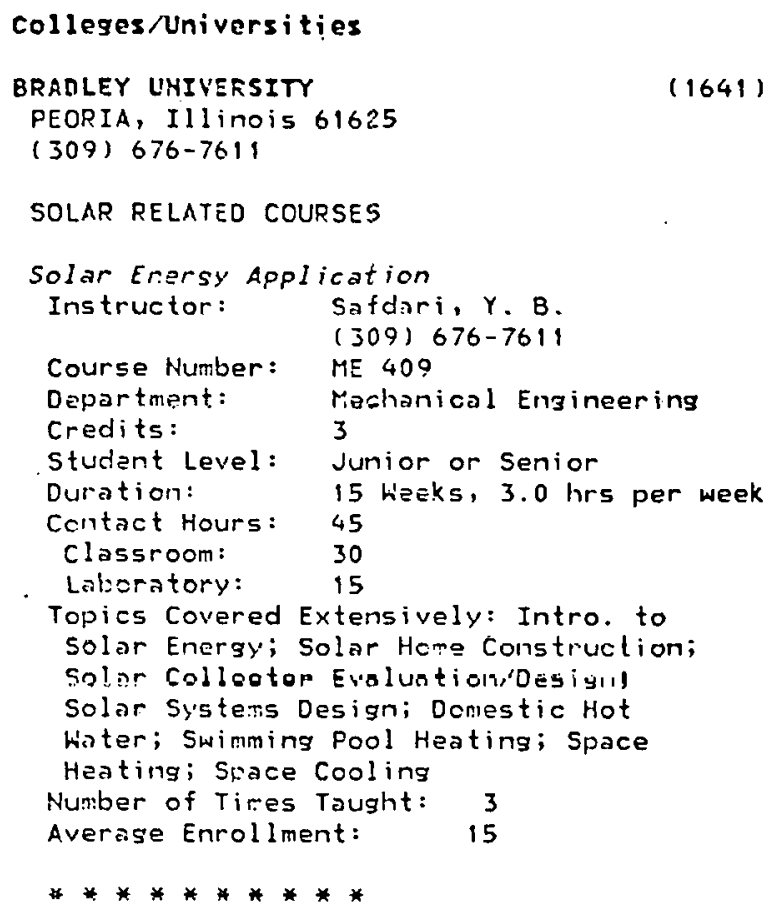

\section{CHICAGO STATE UNIVERSITY}

CHICAGO, Illinois 60628

$\{312\} 995-2000$

SOLAR RELATEO COURSES

Conservation of Energy Resources

Instructor: Cutler, Irving

(312) 909-2 isto

Course Number: 345

Department: Geography

Credlts:

Student Level: Jurior or Senior

Duration: 15 Weeks, 3.0 hrs per week

Contact Hours: 45

Classroom: $\quad 45$

Topics Covered Extensively: Alternate

Energy Sources; Energy Conservation;

Energy Conversion; Energy Storage

Number of Times Taught: 2

Average Enrollment: $\quad 18$

Conservation of Natural Resources

Instructor: Cutler, Irving

(312) 995-2186

Course Number: 256

Deportment: Geograpliy.

Credits: 3

Student Level: Freshman or Sophomore

Duration: 15 Weeks, $3.0 \mathrm{hrs}$ per week

Contact Hours: 45

Classroom: $\quad 45$

Topics Covered Extensively: Alternate

Energy Sources; Energy. Conservation;

Energy Storage

Number of Times Taught: 7

Average Enrollment:
$(1694)$

19

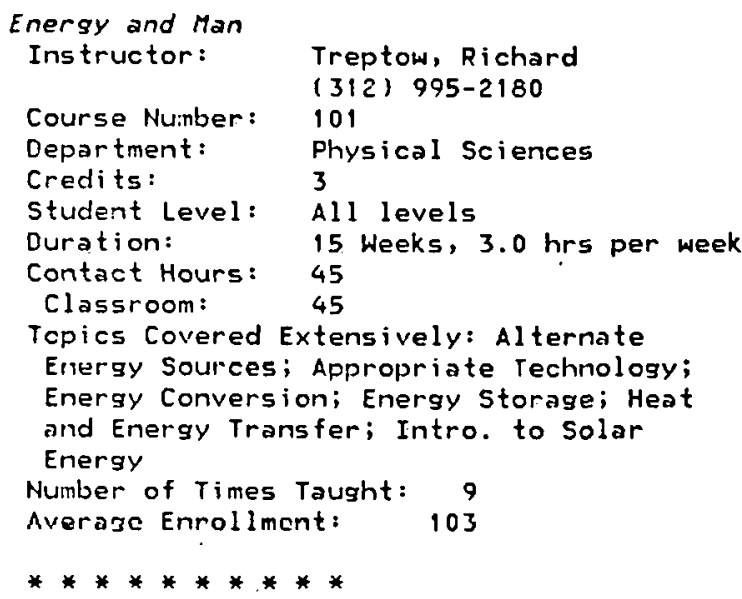

DEPAUL UHIVERSITY

CHICAGO, Illinois 60604

$(1671)$

(312) $321-8000$

\section{SOLAR RELATED COURSES}

Environmental Qual ity

Instructor: Schillinger, E, J.

Course Number: 390

Department: Physics

Credits: 4 
Student Level: College Graduate

Duration:

Contact Hours: 75

Classroom: $\quad 50$

Laboratory: 25

Tcpics Covered Extensively: Alternate

Energy Sources; Energy Conservation;

Energy Conversion; Elec'l Generation, Central

Number of Times Taught: 1

Average Enrollment: $\quad 20$

Probs.(tech. Soc)-frac. Sol. Ener-home

Instructor: R.L.Novak

(312) 321-8192

Course Number: NSH3O3

Departrient: DePaul College

Credits: 4

Student Level: Junior or Senior

Duration: 9 Weeks, 3.0 hrs per week

Contact Hours: 27

Classroom: 27

Topics Covered Extensively: Intro. to

Solar Energy; Passive Solar Technology;

Solar System Components; Solar Home

Construction

Number of Times Taught: 2

Average Enroliment: $\quad 40$

***********

\section{EASTERN ILL UNIVERSITY}

$(1674)$

CHARLESTCH, Illinois 61920

(217) $531-3020$

SOLAR RELATED COURSES

$\begin{array}{ll}\text { Alternate Energy } & \text { Systems } \\ \text { Instructor: } & \text { Kleine, Ric } \\ & (217) 581-2721 \\ \text { Course Number: } & 3933 \\ \text { Department: } & \text { School of Technology } \\ \text { Credits: } & 3 \\ \text { Student Level: } & \text { Junior or Senior } \\ \text { Duration: } & 15 \text { Weeks, } 3.0 \text { hrs per week } \\ \text { Contact Hours: } 45 \\ \text { Classroom: } \quad 30 \\ \text { Laboratory: } \quad 15 \\ \text { Topics Covered Extensively: Alternate } \\ \text { Energy Sources; Energy Conservation; } \\ \text { Energy Conversicn } \\ \text { Number of Times Taught: } 2 \\ \text { Average Enrollment: }\end{array}$

***********

GEORGE HILLIAMS COLLEGE

DOA'NERS GPOVE, Illinois 60515

(1683)

(312) 964-3100

\section{SOLAR RELATED COURSES}

*Energy Technology and the future

Instructor: Clark, Edward T.

Department: IEA

**********
GOVERNORS ST UHIVERSITY

(9145)

PARK FOREST SOUTH, Illinois 60466

(312) 534-5000

\section{SOLAR RELATED COURSES}

Applications of Appropriate Tech.

Instructor: Hagens, Beth

Course Number: 5295

Department: Environmental and

Applied Sciences

Student Level: Junior or Senior

Duration: 15 Heeks, $4.0 \mathrm{hrs}$ per week

Contact Hours: 60

Tepies Covered Extensively: Alternate

Energy Sources; Appropriate Technology;

Energy Conservation; Energy Conversion;

Intro. to Solar Energy;

Marketing/llarket Analysis; Materials

Research; Passive Solar Technology;

Solar Energy Folicy Development; Solar

Economics; Solar Heme Construction;

Solar Law/LEgislation; Solar Collector

Evaluation/Design; Solar Systems

Design; Solar Systens Installation;

Solar Systems Maintenance; Domestic Hot

Water; Space Hesting; Space Cooling;

Wind Power, Small Systems

Number of Times Taught: 2

Average Enrollment: 15

Fundamentals of Appropriate Technology

Instructor: Hagens, Beth

(312) 534-5000

Course Humber: 6255

Department: Environmental $C$ Applied Sciences

Credits:

4

Student Level: Junior or Senior

Duration: 15 heeks, $4.0 \mathrm{hrs}$ per week

Contact Hours: 60

Topics Covered Extensively: Al ternate

Energy Sources; Appropriate Technology;

Energy Conservation; Intro. to Solar

Energy; Passive Solar Technolcgy; Solar

Energy Policy Development; Solar

Econoinics; Solar Harre Construction;

Solar Sy'stens. Design; Elec'l

Generation, Small Scale; Wind Power,

Central Systems; Wind Power, Small

Systems

Number of Times Tausht: 2

Average Enrollment: $\quad 15$

**********

GREENVILLE COLLEGE

GPEEHVILLE, Illinois 62246

(618) 664-1840

SOLAR RELATED COURSES

Solar Energy

Instructor:

Course Number:

Siefken, Hugh

Department:

Credits:

(6.18) 664-1840

FHY 270

Physies

4 
Student Level: Duration: Contact Hours: Classroom: Laboratory:

All levels

6 Weeks, 18.0 hrs per week

108

45

63

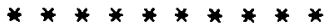

ILL CHICAGO CIRCLE, U OF

(1776)

(312) $996-3000$

SOLAR RELATED COURSES

Building Construction Systems

Instructor: Dudnik, Elliott

(312) 996-3335

Course Number:

ARCH 313

Department:

Architecture

Credits:

6

Student Level: Junior or Seriiör

Duration: 10 Weeks, $15.0 \mathrm{hrs}$ per week

Contact Hours: 150

Topics Covered Extensively: Al ternate

Energy Sources; Appropriate Technology;

Energy Conservation; Energy Storage;

Heat and Energy Transfer

Average Enrollment: $\quad 10$

Solar Energy

Instructor: Simon, H. A.

(312) 996-8530

Course Number: ENPE 391

Department: Energy Engineering

Credits: 4

Student Level: Junior or Senior

Duration: 10 Weeks, 4.0 hrs per week

Contact Hours: 40

Topics Covered Extensively: Heat and

Energy Transfer; Solar Collector

Evaluation/Design

**********

ILL UPBAHA CAHEUS, U OF

URBAHA, Illinois 61801

$(1775)$

(217) 333-1000

\section{SOLAR RELATED COURSES}

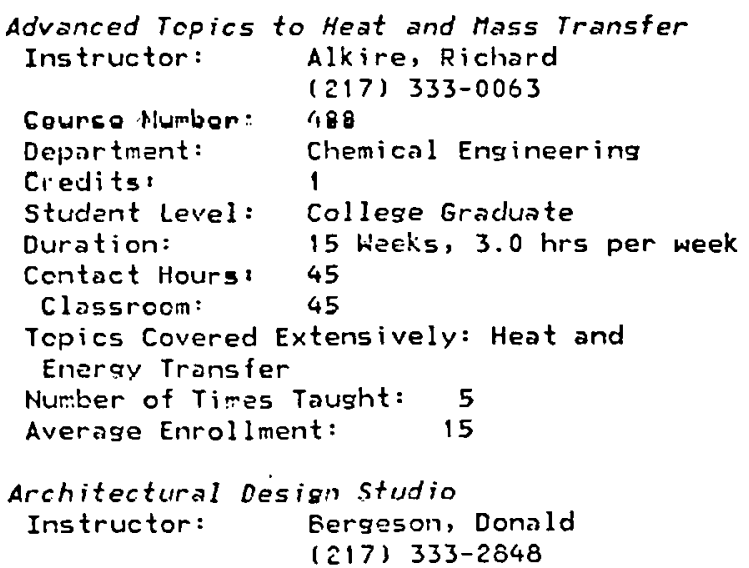

Course Number: 371

Department: Architecture

Credits: 5

Student Level: Junior or Senior

Duration: 15 heeks, 15.0 hrs per week

Contact Hours: 225

Topics Covered Extensively: Energy

Conservation; Intro. to Solar Energy

Electrochemical Engineering

Instructor: Alkire, Richard

Course Number: 388

Department: Chemical Engineering

Credits: 3

Student Level: Junior or Senior

Duration: 15 Weeks, 3.0 hrs per week

Contoet Houre: $\quad 45$

Classroom: 45

Tcpics Covered Extensively: Energy

Conservation; Energy Conversion; Energy

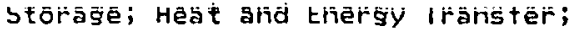

Materials Research

Number of Times Taught:

4

Energy Alternatives and Societal Values

Instructor:

Bond, Charles $E$.

(217) 367-8905

Course Number: AAE 280

Department: Aeroriautical ard

Astronautical

Engineering

Credits: 4

Student Level: All levels

Duration: 15 heeks, $4.0 \mathrm{hrs}$ per week

Contact Hours: 60

Classroom: $\quad 60$

Topics Covered Extensively: Alternate

Energy Sources; Appropriate Technology;

Energy Conservation

Number of Tines Taught: 7

Average Enrollment:

20

Energy Imolications for Euilding Design

Instructor: $\quad S m i t h$, Rolsert

(217) $333-2848$

Course Number: $301 E 5$

Department: Architecture

Creditz:

Student Level: College Graduate

Duration: 15 Heeks, 4.0 hrs per week

Contact Hourg: 60

Clessroom: 60

Topics Covered Extensively: Appropriate

Technology; Energy Conservation

Humber of Tim.se Tought: 1

Average Enrollment:

10

Geology of Energy

Instructor: Langenheim, R. L.

Course Number: 105

(217) 333-1338

Department: Geology

Credits: 2

Student Level: Freshman or Sophomore

Duration: 15 Weeks, 2.0 hrs per week

Contact Hours: 30

Classrocin: $\quad 30$

Number of Times Taught: 4

Averase Enrollment: $\quad 15$ 


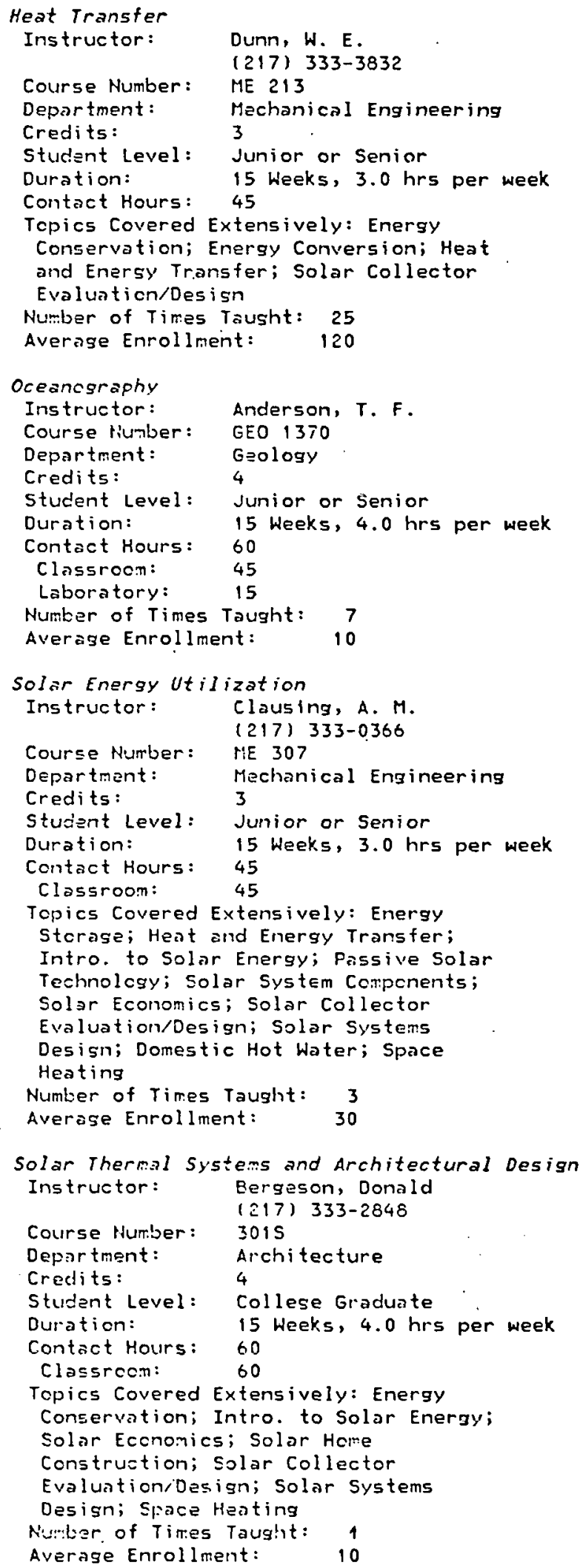

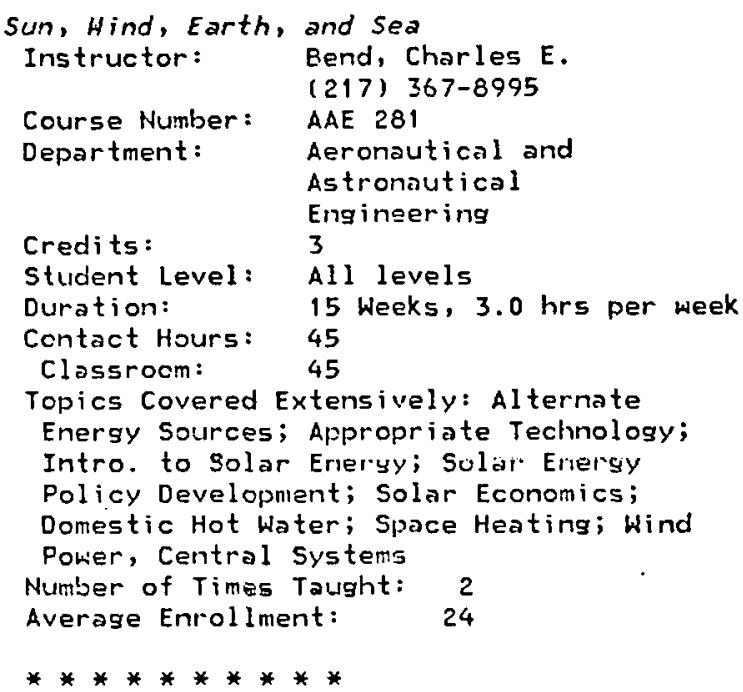


Topics Covered Extensively: Passive Solar Technology; Solar System Components; Solar Home Construction

Solar E Goographical Energy

Instructor: Lavan, Zalman

Course Nuriber: 449

Department: Mechanical and Aero. Engr.

Credits: 3

Student Level: Jumior or Senior

Duration: 15 Heeks, 3.0 hrs per week

Contact Hours: 45

Classroom: $\quad 45$

Topics Covered Extensively: Energy

Storase; Heat and Energy Transfer;

Solar System Corponents; Solar

Economies; Solar Collectorr

Evaluation/Desisn; Solar Systems

Design; Domestic Hot Hater; Space

Heating; Space Coolling

number of Times Taushl.

Average Enrollment:

20

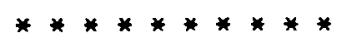

\section{ILLINOIS STATE UHIVERSITY \\ NORMAL, Illinois 61761 \\ (309) 438-2111}

(1692)

PROGRAMS AND CURRICULA

Technolegy for Industry-Energy

Degree: BS, Industrial Technology

Contact: Israel, Everett $\mathrm{N}$. (309) 438-3661

Students Taking or Completing offering:

Contractor, Other

\section{SOLAR RELATED COURSES}

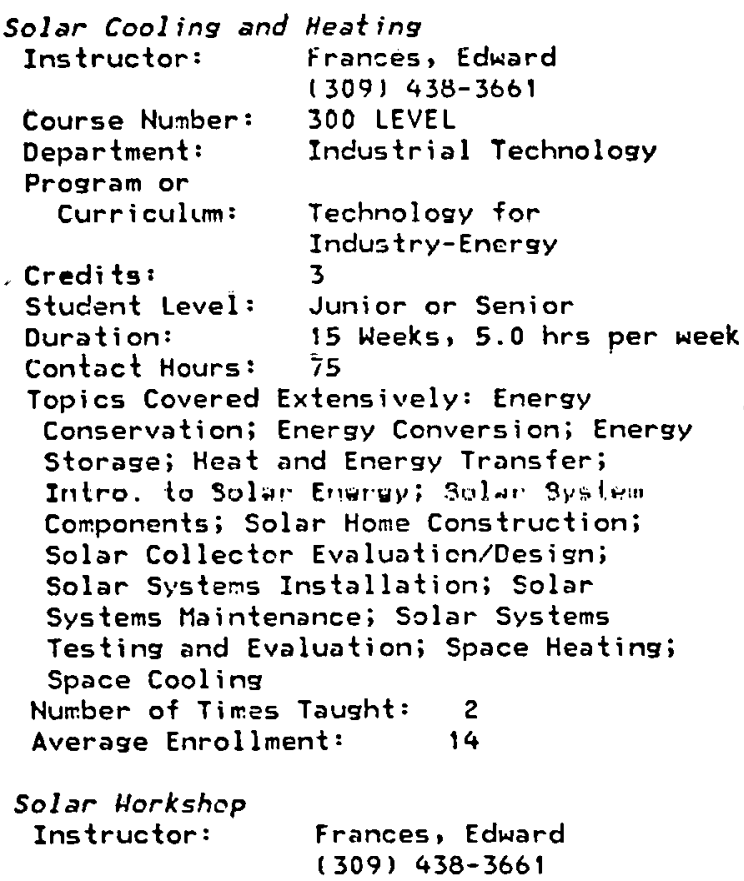

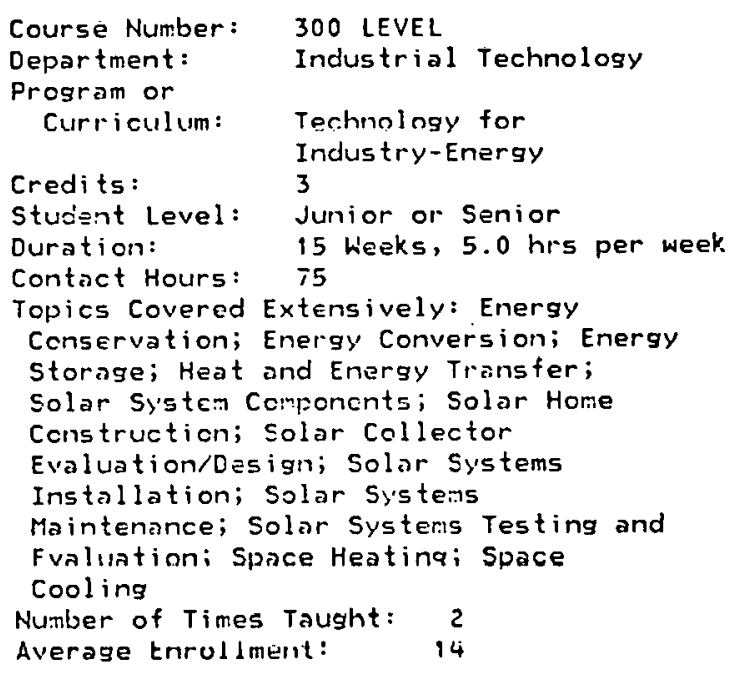


Conversion; Photovoltaics; Elec'l

Generation, Small Scale

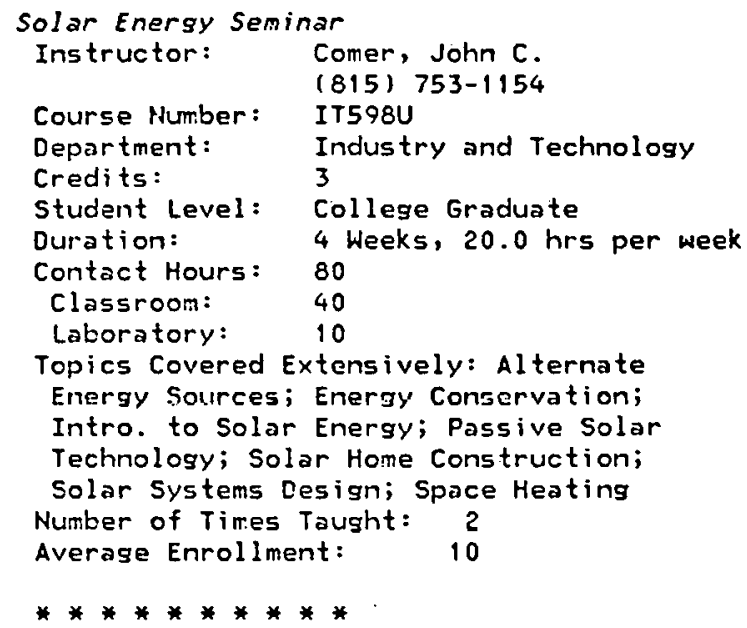

\section{NORTHWESTERN UHIVERSITY \\ EVANSTON, Illinois 60201 \\ (312) 492-3741}

PROGRAMS AND CURRICULA

Solar Energy Principles and

Applications

Degree: NO, Departmental Engineering

Contact: Thodos, George

SOLAR RELATEO COURSES

Solar Energy Principles and Applications

Instructor: Thodos, George

(312) 492-3452

Course Number: 710-C65

Department: Chemical Engineering

Program or

Curriculum: Solar Energy Principles

Credits: 4 and Applications

Student Level: Junior or Senior

Duration: in weoks, $4.0 \mathrm{hrs}$ per week

Contact Hours: 40

Topics Covered Extensively: Intro. to

Solar Energy; Solar System Components Number of Times Taught: 2

Average Enrollment: $\quad 18$

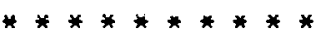

PRINCIPIA COLLEGE

(1744)

ELSAH, Illinois 62028

(618) 374-2131

\section{SOLAR RELATED COURSES}

$\begin{array}{ll}\text { Energy Efficient Living } \\ \text { Instructor: } & \text { Holzberlein, Thomas M. } \\ \text { Course Number: } & 172 \\ \text { Department: } & \text { Fhysics } \\ \text { Credits: } & 5\end{array}$

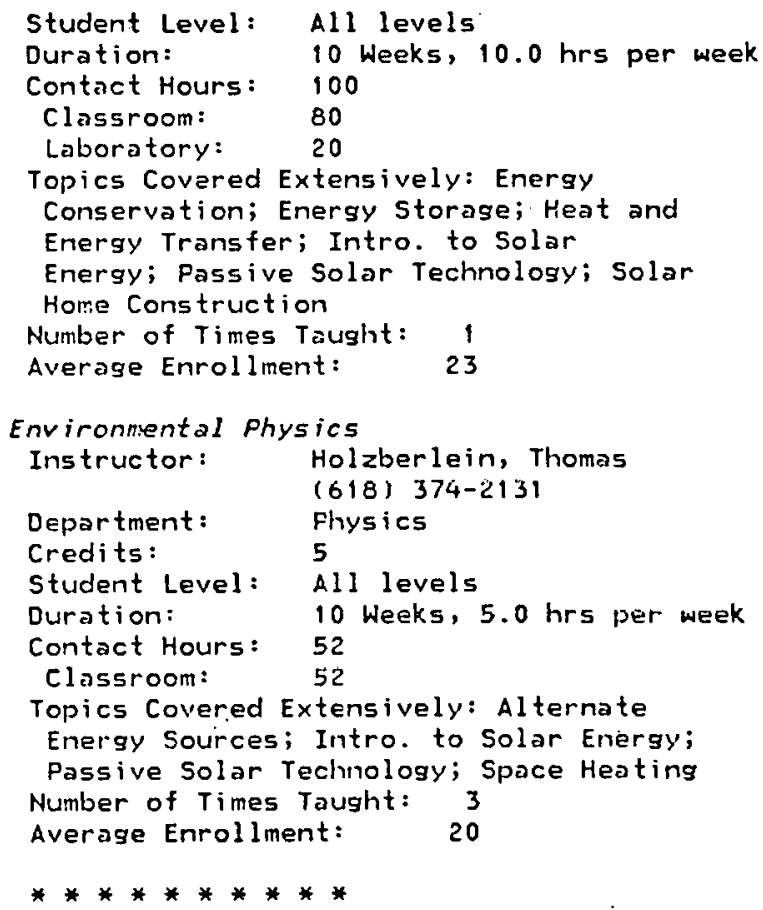


Heating; Space Cooling Number of Times Tausht: Average Enrollment:

SOUTHERM ILLINOIS U CARBOHDL CARBOHOALE, Illinois 62901 (618) 453-2121

\section{SOLAR RELATED COURSES}

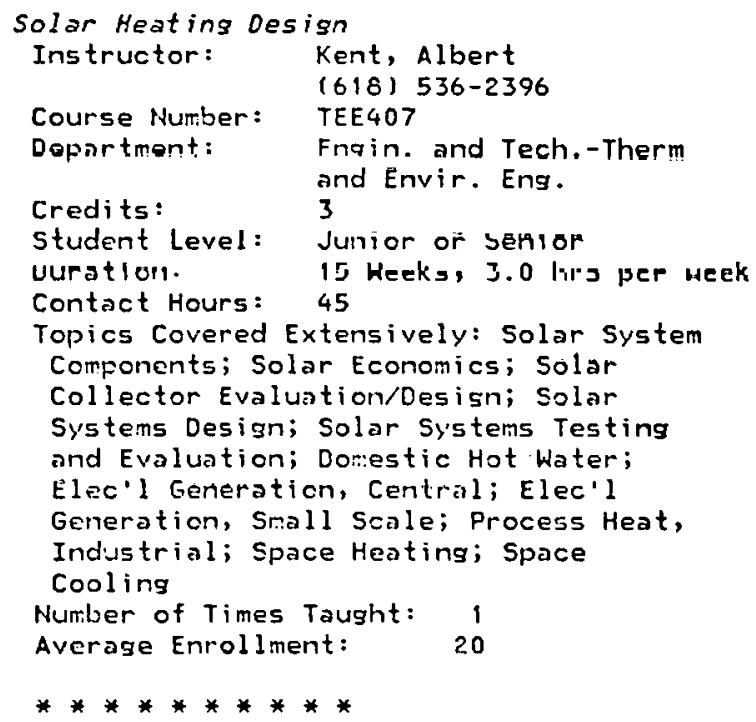

CARL SARDBURG COLLEGE

(7265)

GALESBURG, Illinois 61401

(309) 344-2518

PROGRAMS AND CURRICULA

*adult Cont inuing Edu.

Contact: Rudd, Lanny

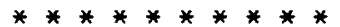

(1652)

CHICAGO, Illinois 60601

(312) 269-8000

SOLAR RELATED COURSES

* Basic Consuner Ed. Courses - Sol. products

×

DUFAGE, COLLEGE OF

GLEN ELLYN, IIlinois 60137

(6656)

(312) $858-2800$

\section{SOLAR RELATED COURSES}

Introduction to Solar Energy

Departinent: Extension Division

Studeint Level: All levels

Duration: $\quad 3$ Heeks, $20.0 \mathrm{hrs}$ per week

Contact Hours: 60

Classrcom: $\quad 39$

Laboratory: 21

Topics Covered Extensively: Intro. to Solar Enersy

Number of Times Taught: 3 Average "Enrollment: $\quad 25$

**********

ILL ESTH CC OLNEY CEN C

OLPEY, Ill inois 62450

(618) $395-4351$

PROGRAMIS AND CURRICULA

Construction Energy Prosram

Uegree: $A D$, Applied science

Contact: Marrs, Steve

(618) 395-1,351

Students Taking or Completing offering: Installer-Residential (Solar System),

Solar Technician

SOLAR RELATED COURSES

Energy Conservation Thecry

Culver, Ray (618) 395-4351

Course Number: SCI 121

Vepartitient: Fhysics

Program or

Curriculum: Construction Energy

Credits: Program

Student Level: Freshman or Sophomore 


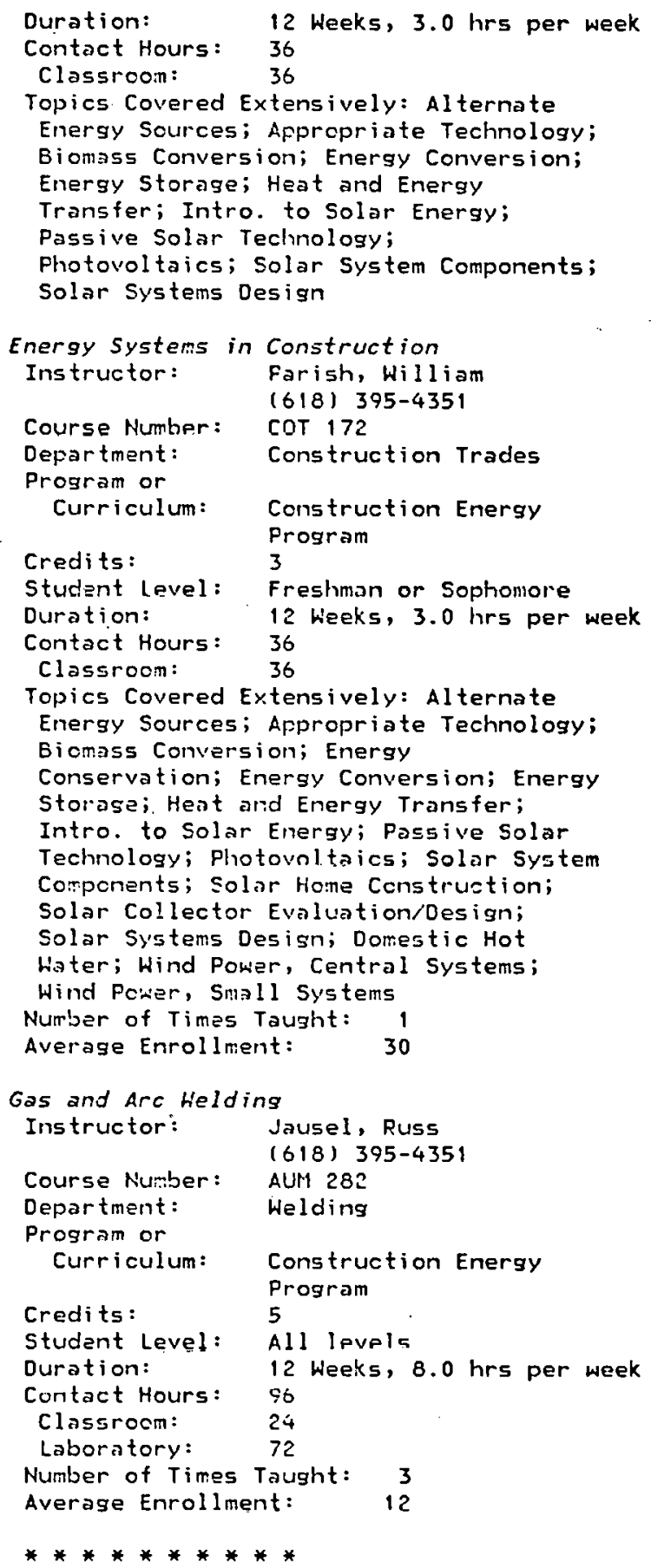

ILL ESTH LINCOLN TRAIL C

(9786)

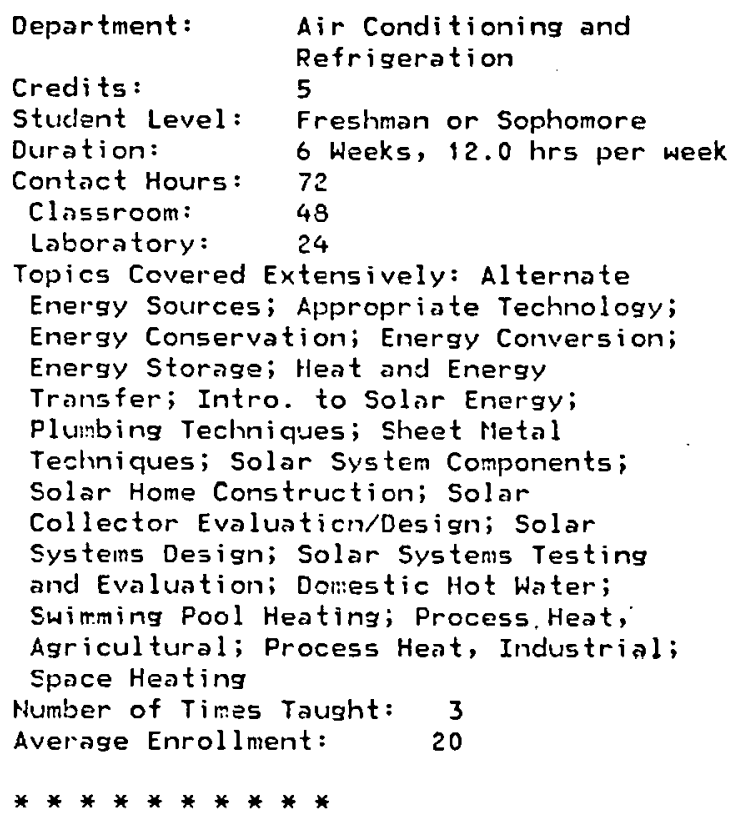

(618) $544-8557$

\section{SOLAR RELATED COURSES}

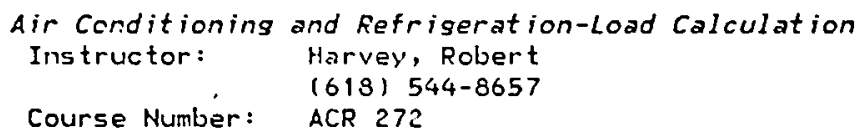




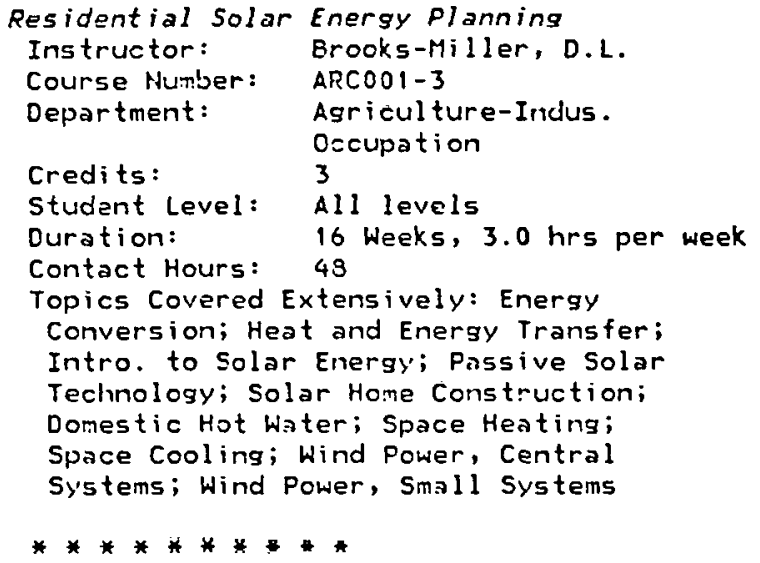

$(8076)$

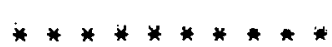

KAHKAKEE CHTY COLLEGE

$(7690)$

KANKAKEE, Illinois 60901

(815) 933-9311

SOLAR RELATED COURSES

Solar Energy Survey

Instructor: Mathers, Kris

(815) 933-0345

Course Number: REFR 1413

Department: Technical Division

Credits: 3

Student Level: All levels

Duration: 16 Weeks, $3.0 \mathrm{hrs}$ per week

Contact Hours: 48

Classroom: $\quad 48$

Tcpics Covered Extensively: Intro. to

Solar Energy; Solar Econcmics; Solar

Systems Design; Solar Systems

Inslallation; Bolar Systama

llaibitenance; Solar Systems Testing and

Craluation

Number of Times Taught: 1

Average Enrollment: 8

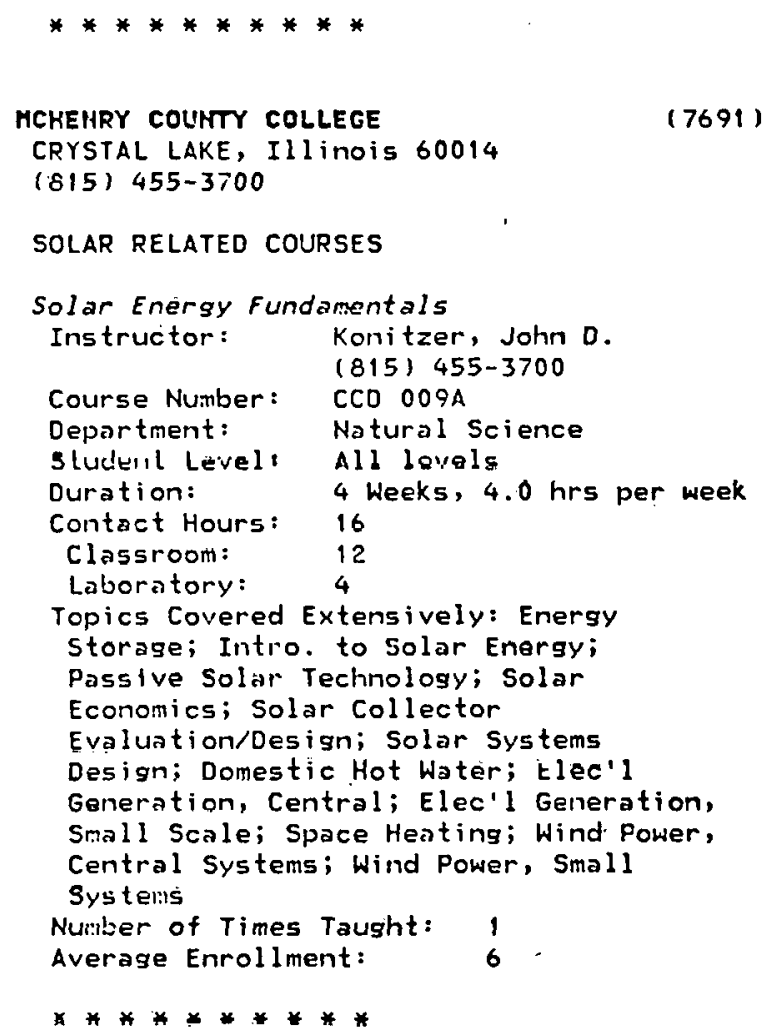


Energy Sources

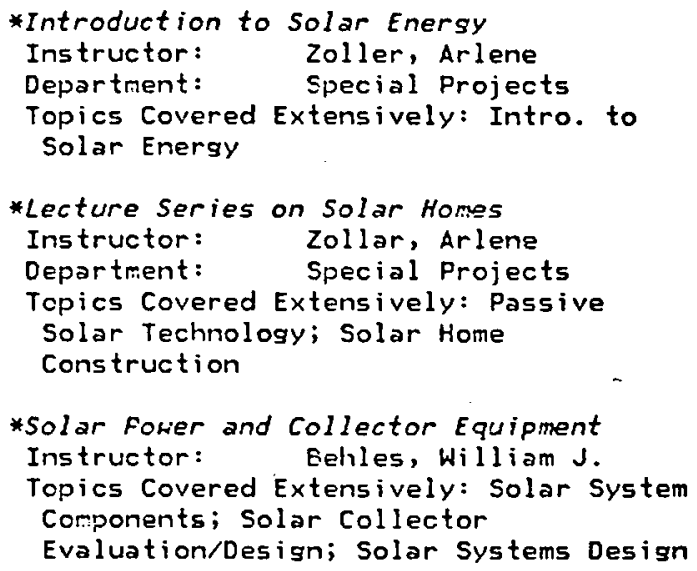

\section{Vocational/Technical Colleges}

THE QUIKCY TECHHICAL SCHOOL

Quincy, Illinois 62301

(90030)

\section{SOLAR RELATED COURSES}

Air Cond., Refrig., Heating Service Instructor: Devlin, David B./ W. G. Dubuque (217) 224-0600

Department: Refrigeration Student Level: High School Graduate Duration: 1 heeks, $30.0 \mathrm{hrs}$ per week Contact Hours: 30

Topics Covered Extensively: Alternate

Energy Sources; Energy Conservation; Energy Storage; Solar System

Components; Solar Systems Design; Space

Heating; Space Cooling Number of Times Taught: 1 Average Enrollment: $\quad 20$

\section{other Educational Institutions}

SOLAR STORE INC

$(90330)$

Box. 841, Dept. bs

Peoria, Illinois 61652

PROGRAMS AND CURRICULA

*Sol. Ener. Ed. for Installers

Contact: Shanks, Diane/ Adsit, $M$.

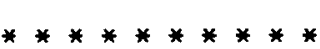




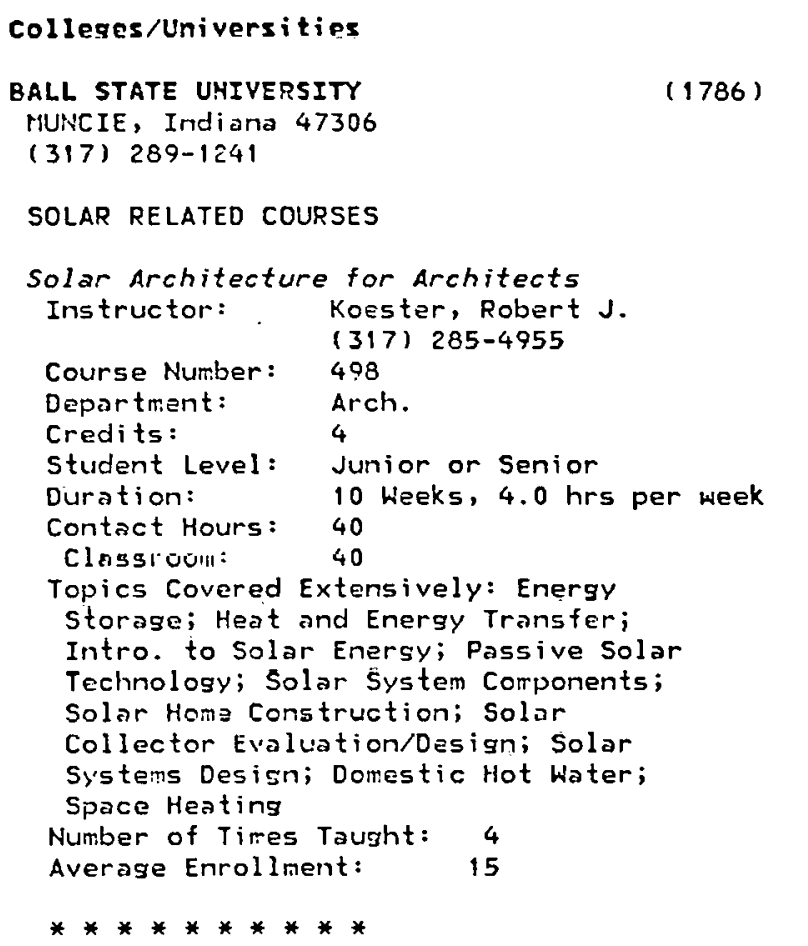

EARLHAM COLLEGE

RICHMOND, Indiana 47374

(317) $962-6551$

\section{SOLAR RELATED COURSES}

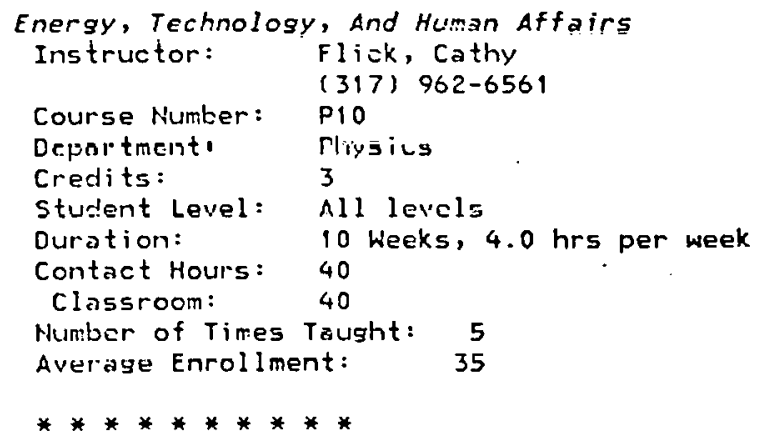

\section{HURTIHGTON COLLEGE}

(1803)

HUNTTHSTON, Indiana 46750

(219) 356-6000

SOLAR RELATED COURSES

Energy Alternatives: Solar Energy

Instructor: Smith, Gerald 0 .

(219) 356-6000

Course Nurber: 207

Deportment: Fhysics

Credits: 4

Stucient Level: All levels

Duration: 4 heeks, $15.0 \mathrm{hrs}$ per weck

Cuntact Hours: 60

Classroom: $\quad 40$
Laboratory: 20

Topics Covered Extensively: Energy

Conversion; Energy Storage; Intro. to

Solar Energy; Passive Solar Technolosy;

Photovoltaics; Solar Home Construction;

Solar Collector Evaluation/Design;

Domestic Hot Water; Elec'l Generation,

Central; Space Heating

$* * * * * * * * * *$

IND NORTHERN GRAD SCH MGMT

$(1806)$

MARION, Indiana 46952

(317) 674-2900

PROGRAMS AND CURRICULA

Masters of Prnfessionol Monsgement

Deqree; MS. NT, Professional

Mannapment

Contact: Morgan, James/ Costa, Dr. Da (317) 674-2900

SOLAR RELATED COURSES

Energy Auditing for Mgrs. and Engrs.

Instructor:

Klima, Karel/ Thumann, Al

Course Number: 561

Department: Ft. Wayne Ext.-Prof.

Mamint. Hosp. Adm.

Curriculum: Masters of Professional

Management

Credits: $\quad 4$

Student Level: College Graduate

nurationi 13 Waeks, 2.0 hre per week

Contact Hours: 26

Classroom: $\quad 26$

Topics Covered Extensively: Energy Lonservation; Solar Systems Testing and Evaluation; Process Heat, Industrial; Space Heating; Space Cooling

Average Enrollment: $\quad 50$

Enersy Conservation for Managers

Instructor: Klima, Karel/Thumann, Al

(404) $074-0100$

Course Number: 560

Department: Ft. Wagine [nt. riul.

Program or

Curiciculum: llasters of Professional

Credits: Manngement

Student Level: College Graduate

Duration: 13 Weeks, $2.0 \mathrm{hrs}$ per week

Contact Hours: 26

Classroom: 26

Topics Covered Extensively: Energy Conservation; Process Hent, Industrial;

Space Heating; Space Cooling

Averise Enrollment:

50 


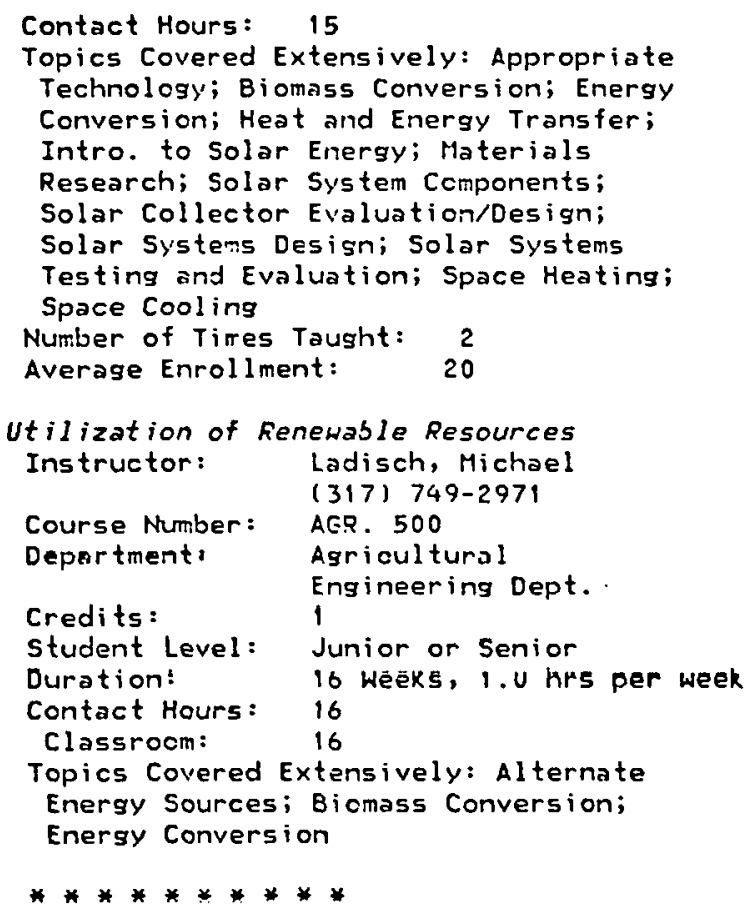

Average Enrollment:

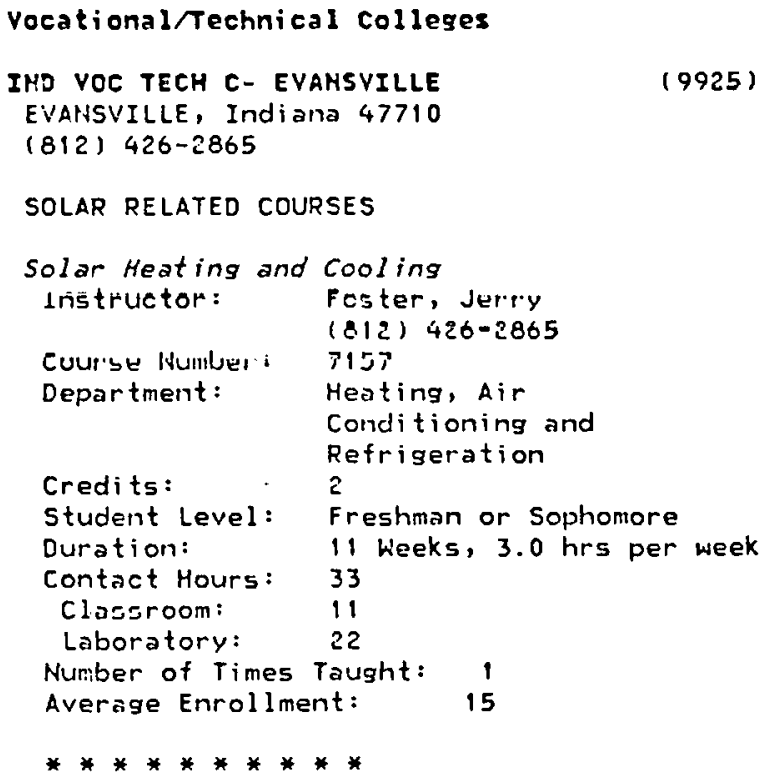

IND VOC TECH - SELLERSBURG

$(10109)$

SELLERSEURG, Indiana 47172

| $8121240-3301$

\section{SOLAR RELATED COURSES}

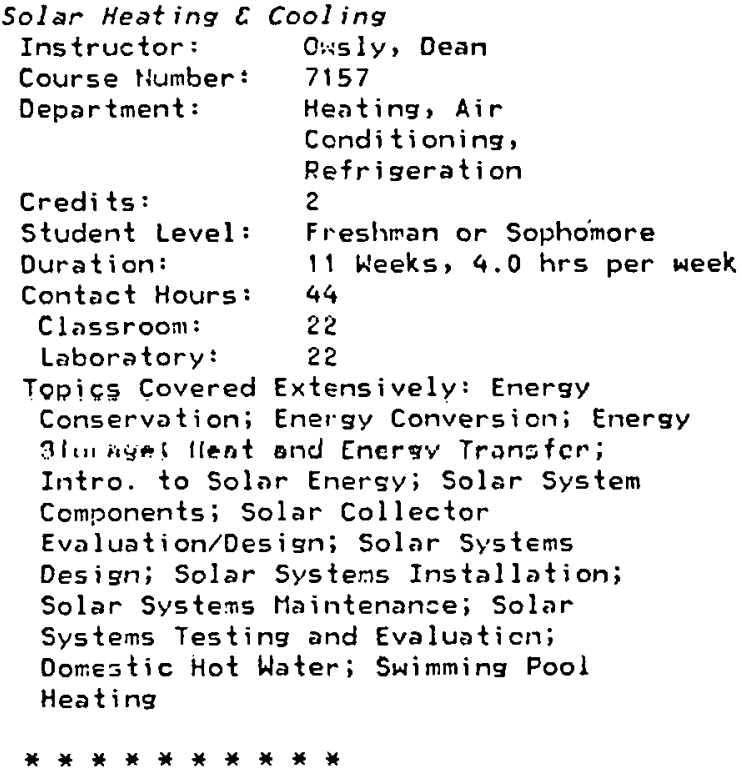


IHDIANA VOCATIOHAL TECHNICAL COLLEGE-

NH

Gary, Indiana 46409

(219) 981,-1111

\section{SOLAR RELATED COURSES}

Solar Energy Seminar

Credits: 1

Student Level: All levels

Duration: i Weeks, $1.5 \mathrm{hrs}$ per week

Contact Hours: 11

Topics Covered Extensively: Solar Energy

Policy Development; Solar System

Components; Solar Law/Legislation;

Solar Collector Evaluation/Design;

Solar Systems Design

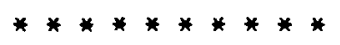

other Educational Institutions

INDIAHAPOLIS CENTER FOR ADVANCED

RESEARCH

1219 Hest Michigan St.

$(90300)$

Indianapolis, Indiana 46202

SOLAR RELATEO COURSES

* Solar Energy Studies

********** 


\section{Colleges/Universities \\ DIVINE HORD COLLEGE \\ EPHORTH, IONA 52045 \\ $(1858)$ \\ (319) 876-3354 \\ SOLAR RELATED COURSES

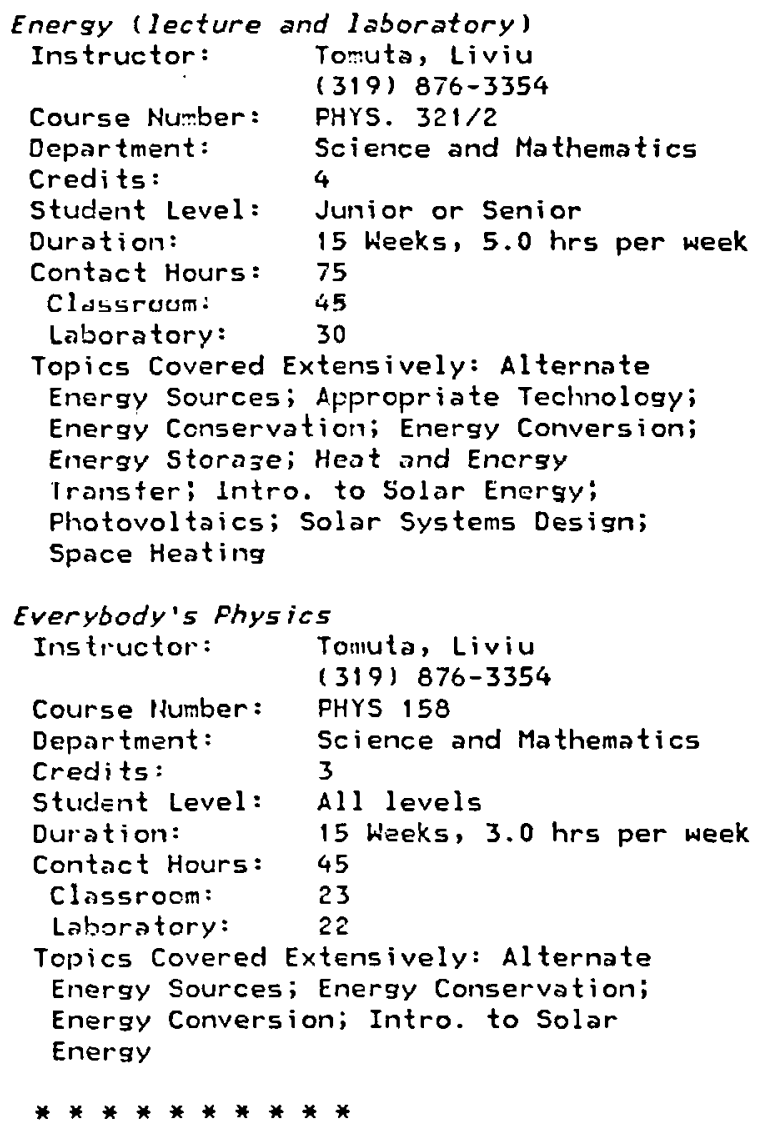

IOHA STATE U SCI C TECHN

$(1869)$

AMES, IOKA 50010

(515) 294-4111

PROGRAMS AYTO CURRICULA

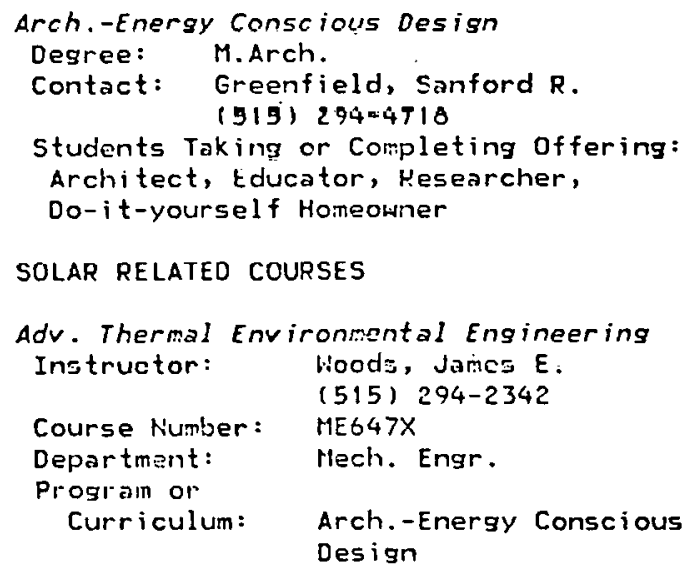

Credits: 4

Student Level: College Graduate

Duration: II Weeks, $8.0 \mathrm{hrs}$ per week

Contact Hours: 88

Classroom: $\quad 22$

Laboratory: 66

Topics Covered Extensively: Alternate Energy Sources; Energy Conservation; Energy Conversion; Energy Storage; Heat and Energy Transfer; Passive Solar Technolosy; Domestic Hot Water; Space Heating; Space Cooling Number of Times Taught:

Average Enrollment:

Design with Solar Energy

Instructor: Kainlauri, Eino 0.

(b)b) 244-4/1

Department: Architecture -

Program or

Curriculum: Arch.-Energy Conscious

Design

Student Level: Collese Graduate

Duration: $\quad 1$

$\begin{array}{cl}\text { Contact Hours: } & 28 \\ \text { Classroom: } & 12\end{array}$

Liboratory: 16

Topics Covered Extensively: Appropriate Technology; Energy Storage; Heat and Energy Transter; Passive Solar Technolosy; Solar Systen Components; Solar Home Construction; Solar Systems Design; Domestic Hot Water; Space

Heating; Space Cooling

Human Thermal Environments

Instructor: Woods, James E.

(515) 294-2342

Course Number: 529

Department: Design/Architecture

Program or

Lurriculum: Arch.-Energy Conscious

Design

Credits: 3

Student Level: Colleze Graduate

Duration: 11 Weeks, 3.0 hrs per week

Contact Hours: 33

Classroom: $\quad 33$

Topics Covered Extensively: Heat and

Energy Transfer; Space Heating; Space

Cooling

Number of Times Taught: 2

Average Enrollment: 8

solar thergy rherhal systeits

Instructor: hoods, James $E$.

Course Number: (515) 294-2343

Department: Mechanical Engineering

Program or

Curriculum: Arch.-Energy Conscious

Credits: 3 Design

Student Level: Junior or Senior

Duration: $\quad 11$ Weeks, $3.0 \mathrm{hrs}$ per week

Contact Hours: 33

Classrooin: $\quad 33$

Topics Covered Extensively: Appropriate

Techrology; Energy Conservation; Energy 
Conversion; Energy Storage; Heat and Energy Transfer; Solar System

Components; Solar Eccnomics; Solar

Collector Evaluaticn/Design; Solar

Systems Desisn; Solar Systems Testing and Evaluation; Domestic Hot Water; Space Heating

Number of Times Taught: 3

Average Enrollment: 24

IOHA, UNIVERSITY OF

(1892)

ICWA CITY, IOWa 52242

(319) 353-2121

\section{SOLAR RELATED COURSES}

Chemistry and the Physics of the Environment Instructor: Frank, L. A.l Frank, C.

W.

Course Number: $11: 25 / 29: 25$

Department: . Liberal

Credits: 4

Student Level: All levels

Duration: $\quad 15$ Weeks, 4.0 hrs per week

Contact Hours: 60

Clessrooin: $\quad 60$

Topics Covered Extensively: Alternate

Energy Scurces; Energy Conversion:

Energy Storage; Intro. to Solar Energy

Number of Times Taught: 6

Averase Enrollment: $\quad 350$

\section{Methods of Direct Energy Conversion}

Instructor: Lonngren, Karl

(319) 353-3696

Course Number: $\quad 545: 176$

Department: Electrical and Computer Engineering

Credits: 3

Student Level: Junior or Senior

Duration: 15 Weeks, 3.0 hrs per week

Contact Hours: 45

Topics Covered Extensively: Alternate Energy Sources; Energy Conversion; Heat and Energy Transfer; Intro. to Solar

Energy; Materials Research;

Photovoltaics

Number of Tin:es Taught: 7

Average Enrollment: $\quad 10$

Solar Energy Applications

Instructor: Spencer, D. L.

Course Humber: 528:148

Deportment: Energy Engineering

Credits: 3

stuciant Level: Junlor or Stmiur.

Duration: 15 Weeks, 3.5 hrs per week

Contact Hours: $\quad 54$

Classrocin: $\quad 44$

Listoretory: $\quad 10$

Topics Covered Extensively: Heat and

Energy Transfer; Intro. to Solar

Energy; Solar Collector

Evaluation/Design

Number of Times Taught: 2
Average Enrollment:

18

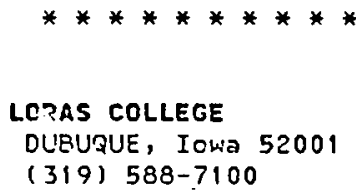

LC?AS COLLEGE

DUBUQUE, IOWa 52001

(319) 588-7100

SOLAR RELATED COURSES

Physics: Energy and the Environment

Instructor: Hutchinson, D. J. (319) 583-7154

Cuurse Number: 9

Department: Phy'sics and Engineering Science

Student Level: All levels

Duration:

15 Weeks, 3.0 hrs per week

Contact Hours:

Classroom: 45

45

Topics Covered Extensively: Alternate Energy Sources; Energy Conservation;

Energy Conversion; Intro. to Solar

Energy; Solar Energy Policy Development

Number of Tines Taught: 4

Average Enrollment:

75

**********

LUTIISR COLLECE

(1874)

DECOPAH, IOWa 52101

(319) 387-2000

SOLAR RELATED COURSES

Solar Energy

Instructor:

Helson, David T.

(319) 387-1226

Department: Physics

Credits:

3

Stucient Level:

Duration:

Contact Hours:

All levels

3 Weeks, 20.0 hrs per week

Classroom:

Laboratory:

60

30

Topics Covered Extensively: Intro. to

Solar Enarsy; Space Heating

Number of Times Taught: 4

Averase Enrollment:

30

MCRTHE?H IOHA, $U$ OF

$(1890)$

CEDAR FALLS, IOWล 50613

(319) 273-2311

SOLAR RELATED COURSES

Alternate Energy Sources

Instructor:

Macouber, Hilliard K.

Department:

(319) 273-2290

Collese of Natural

Credits : Sciences

Stud!ent Level:

Duration:

Contact Hours:
Collese Graduate

5 Waoks, 3.0 hrs per week 15 
Classroom: 12

Topics Covered Extensively: Alternate Energy Sources

Physics $\varepsilon$ the Environment

Instructor: Jensen, Verner

(319) 273-2588

Course Number: $83: 010$

Deportment: Fhsics Dept. of

Credits: Nitural Science

Studant Level: Freshman or Sophomore

Duration: 16 heeks, 3.0 hrs per week

Contact Hours: 48

Number of Times Taught: 10

Average Enrollment: $\quad 80$

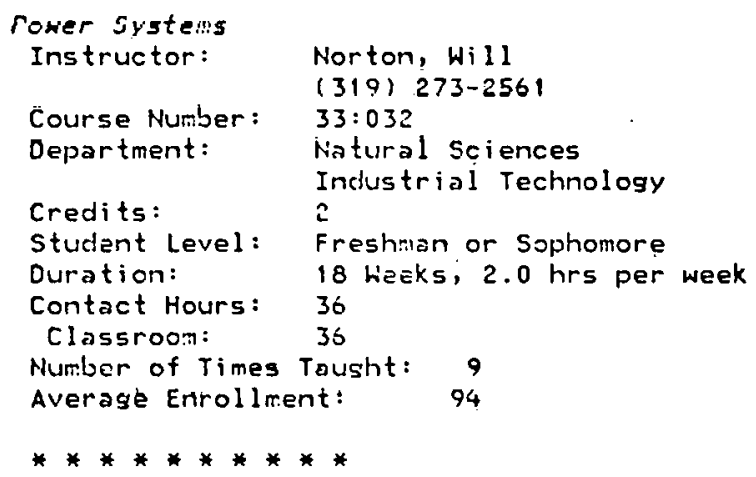

Course Number:

Department:

Credits:

Student Level:

Duration:

Contact Hours:

Classroom:

Number of Times Tausht: 9

Average Enrollment:

Norton, Will

(319) 273-2561

$33: 032$

Niatural Sciences

Industrial Technology

i

Freshman or Sophomore

18 Heeks, 2.0 hrs per week

36

***********

\section{Community/Junior colleges}

DES MOIKES AREA CC

(8735)

AldiCldY, IUWa 50021

(515) 964-6́200

PROGRAMS AND CURRICULA

Solar Energy I and II

Desrcel : Adult [d.

Contact: Rowe, Gordon $N$.

$15151964-6266$

\section{SOIAR RFI ATFN SOIIRGFG}

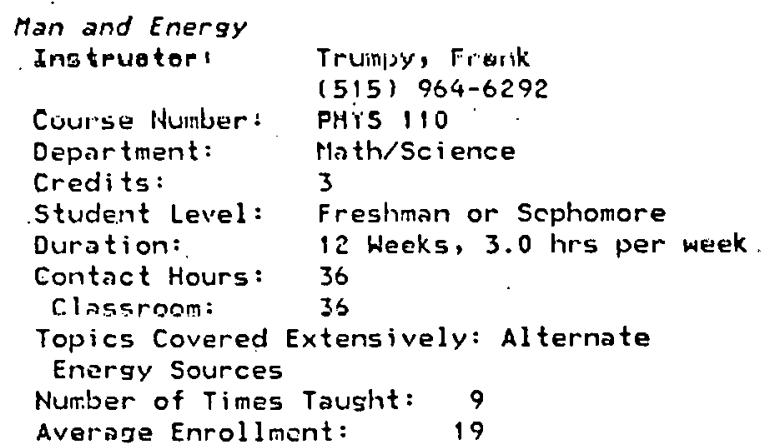

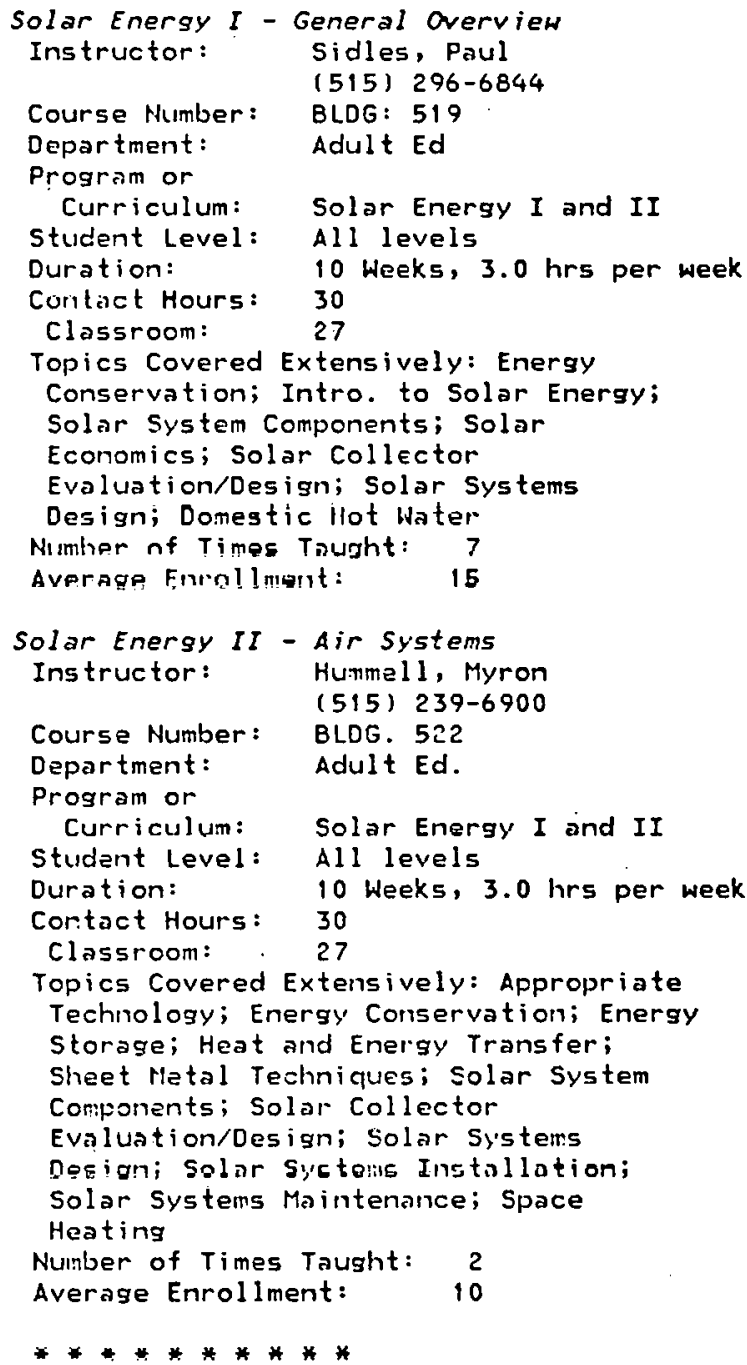


MUSCATINE CHYY COLLECE
MUSCATINE, IOWa 52761

$(1882)$

$(319) \quad 263-8250$

PROGRAMS AND CURRICULA

Solar Carpențy

Degree: $\quad$ SS, Industrial Education

Contact: Melander, Harry (319) 263-8250

Students Taking or Completing Offering:

Installer-Residential (Solar System)

Use of Sol. Ener.-Homeowners, Builders

Degree: Certificate of Completion

Contact: Ohlendorf, Vernon

(319) 263-8250

students Taking or Completing offering:

Do-it-yourself Homeowner

SOLAR RELATED COURSES

Solar Carpentry

Instructor:

Melander, Harry

(319) 263-8250

Department :

Trades

Prosram or

Curriculum: Solar Carpentry

Credits:

61

Student Level: High School Graduate

Duration: $\quad 46$ Weeks, $28.0 \mathrm{hrs}$ per week

Topics Covered Extensively: Energy

Conservation; Energy Conversion;

Passive Solar Technology; Domestic Hot

Water

Number of Times Taught: 1

Averase Enrollment: $\quad 10$

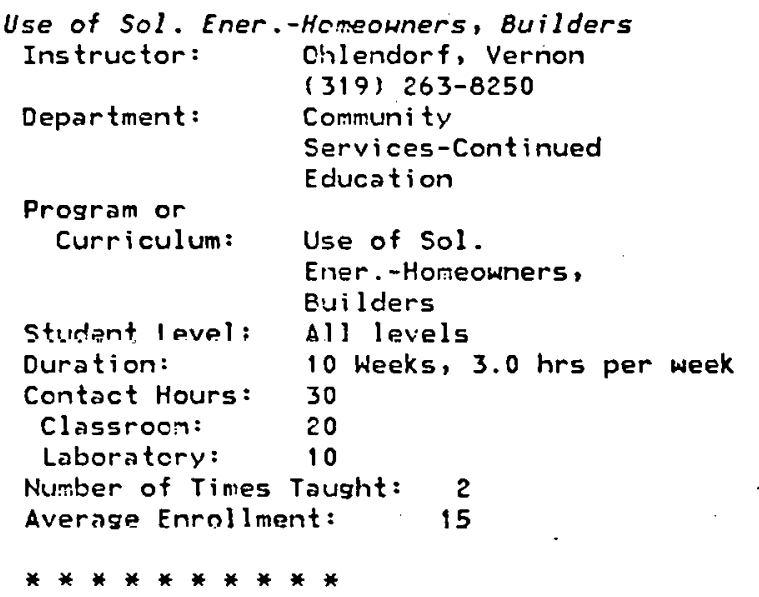

\section{SCOTT CO:MUNITY COLLEGE}

BETTEH:DC?F, IOWA 52722

(4074)

(319) 359-7531

PROGRATS AIID CURRICULA

* Solar Enersetics Technology

Degree: AD, Solar Energetics

Technology

(319) 359-7531

Students Taking or Completing offering:
Architect, Researcher,

Installer-Residential (Solar System), Installer-Commercial (Solar System), Solar Technician

\section{SOLAR RELATED COURSES}

*Courses: Instal.,Repair - Heat.,Ref.,A/C

Program or

Curriculum: *Solar Energetics Technology

Topics Covered Extensively: Energy

Storage; Photovoltaics; Plumbing

Techniques; Sheet Metal Techniques;

Solar System Components; Solar

Collector Evaluation/Design; Solar

Systems Design; Solar Systems

Installation; Solar Systems

Maintenance; Solar Systems Testing and

Evaluation; Domestic Hot Water; Process Heat, Industrial; Space Heating; Space Cool ing.

**********

\section{Vocational/Technical colleges}

WESTERN IOWA TECH

$(7316)$

SIOUX CITY, IOWA 51102

(712) 276-0380

PROGRAMS AND CURRICULA

Solar Systems Technology

Degree: $A D$, Applied Sci. in Sol. Sys.

Tech.

Contact: Chadwick, Richard

(712) 276-0380

Students Taking or Completing offering:

Installer-Residential (Solar system),

Installer-Comercial (Solar System),

Solar Technician, Other

\section{SOLAR RELATED COURSES}

Blueprint Reading

Instructor:

Forsling, $M$. G.

(712) $276-0380$

Course Number: 274-3005

Department: Trades $\&$ Industry

Program or

Curriculum: Solar systems

Credits : 4

Student Level: Freshman or Sophomore

Duration: 12 Weeks, $5.0 \mathrm{hrs}$ per week

Contact Hours: 60

Classroom: $\quad 36$

Laboratory: 24

Topics Covered Extensively: Passive Solar Technology; Plumbing Techniques; Sheet Metal Techniques; Solar System Corponents; Solar Home Construction; solar Collector Evaluation.Design; 
Solar Systems Design; Elec'l

Generation, Small Scale; Space Heating

Average Enrollment:

11

Building Design for Solar Systems

Instructor: Forsling, M. G.

(712) $276-0380$

Course Number: $274-3010$

Department: Trades \& Industry

Program or

Curriculum: Solar Systems

Credits:

Technology

Credits:

Student Level: Freshman or Sophomore

Duration: $\quad 12$ Weeks, $6.0 \mathrm{hrs}$ per week

Contact Hours: 72

Classroom: $\quad 24$

Laboratory: 48

Topics Covered Extensively: Energy

Conservation; Energy Storage; Passive

Solar Technology; Eolar Heme

Construction

Averase Enrollment:

11

Integrated Solar Sci. II

Ins tructor:

Forsling, M. G.

(712) 276-0380

Course Number: 274-3006

Department: Trades $\varepsilon$ Industry

Program or

Curriculum: Solar systems

Credits:

Student Level: Freshman or Sophomore

Duration: 12 Weeks, 5.0 hrs per week

Contact Hours: 72

Classroom: $\quad 48$

Laboratory: 24

Topics Covered Extensively: Energy

Conversion; Elec'l Gereration, Small

Scale; Space Heating; Space Cooling

Average Enrollment:

Introduction to Solar Systems

Instructor: Forsling, M. G.

(712) 276-0380

Course Nuriber: $274-3000$

Department: Trades \& Industry

Program or

Curriculum: Solar Systems

Credits:

Technology

3

Student Level: Freshman or Sophomore

Duration: 12 Weeks, 3.0 hrs per week

Contact Hours: 36

Classroom: $\quad 36$

Average Enrollment:

11

Solar feasability Cost Analysis

Instructor: Forslirg, M. G.

$(712) 276-0380$

Course Number: 274-3012

Department: Trades $\varepsilon$ Industry

Prozram or

Curriculum: Solar Systems

Credits: $\quad 5$

Technology

Student Level: Freshman or Sophomore

Duration: 12 Weeks, $5.0 \mathrm{hrs}$ per week

Contact Hours: 60
Classroom: $\quad 60$

Topics Covered Extensively: Solar

Economics

Averase Enrollment:

11

Solar Systems Application I

Instructor: Forsling, M. G.

Course Number: $\quad 274-3002$

Department: Trades \& Industry

Program or

Curriculum: Solar Systems

Credits: 9

Student Level: Freshman or Sophomore

Duration: 12 Weeks, 13.0 hrs per week

rontact Hours: 155

Classroom: $\quad 60$

Laboratory: 96

Topics Covered Extensively: Energy

3 luldye; Hedt and Energy irshsfer;

Pluntuing Techniques; Solar Collector

Evaluation/Desisn

Average Enrollment:

11

Solar Systems Applications II

Instructor: Forsling, M. G.

(71?) 276-0380

Course Number: 274-3007

Department: Trades \& Industry

Program or

Curriculum: Solar Systems

Credits: 8

Technology

Student Level: Freshman or Sophomore

Durations 12 Weeks, 11.0 hrs per week

Contact Hours: 132

Classroom: $\quad 60$

Laboratory: 72

Topics Covered Extensively: Energy

Conservation; Energy Conversion; Energy

Storage; Plumbing Toohniques; Solar

System Compnnents: Solar rolloptor

Evaluation/Design; Solar Systems

Testing and Evaluation; Space Heating

Averase Enrollment:

11

Solar systems Maintenance

Instructor: Forsling, M. G.

(712) 276-0380

Course Number: 274-3013

Department: Tractes r. Tndustry

Program or

Curriculum: Solar Systems

Credits:

Technolnap

Sludent Level: Freshmon or sophomore

Duration:

Classroom: 24

Laboratory: 48

Topics Covered Extensively: Energy Storage; Solar System Ccmponents; Solar

Systems Maintenance; Solar Systems

Testing and Evaluation; Domestic Hot

Water; Space Heating

Averase Enrollment:

11 


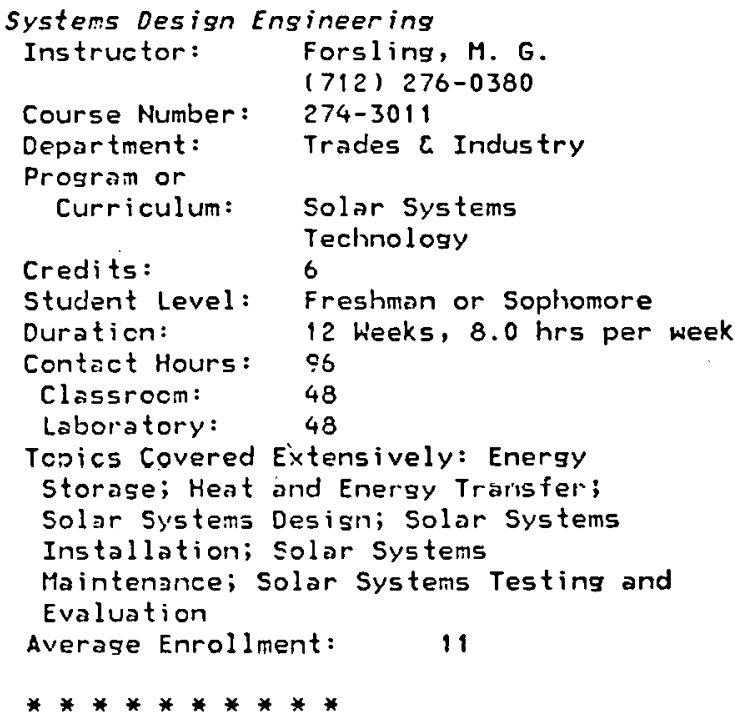




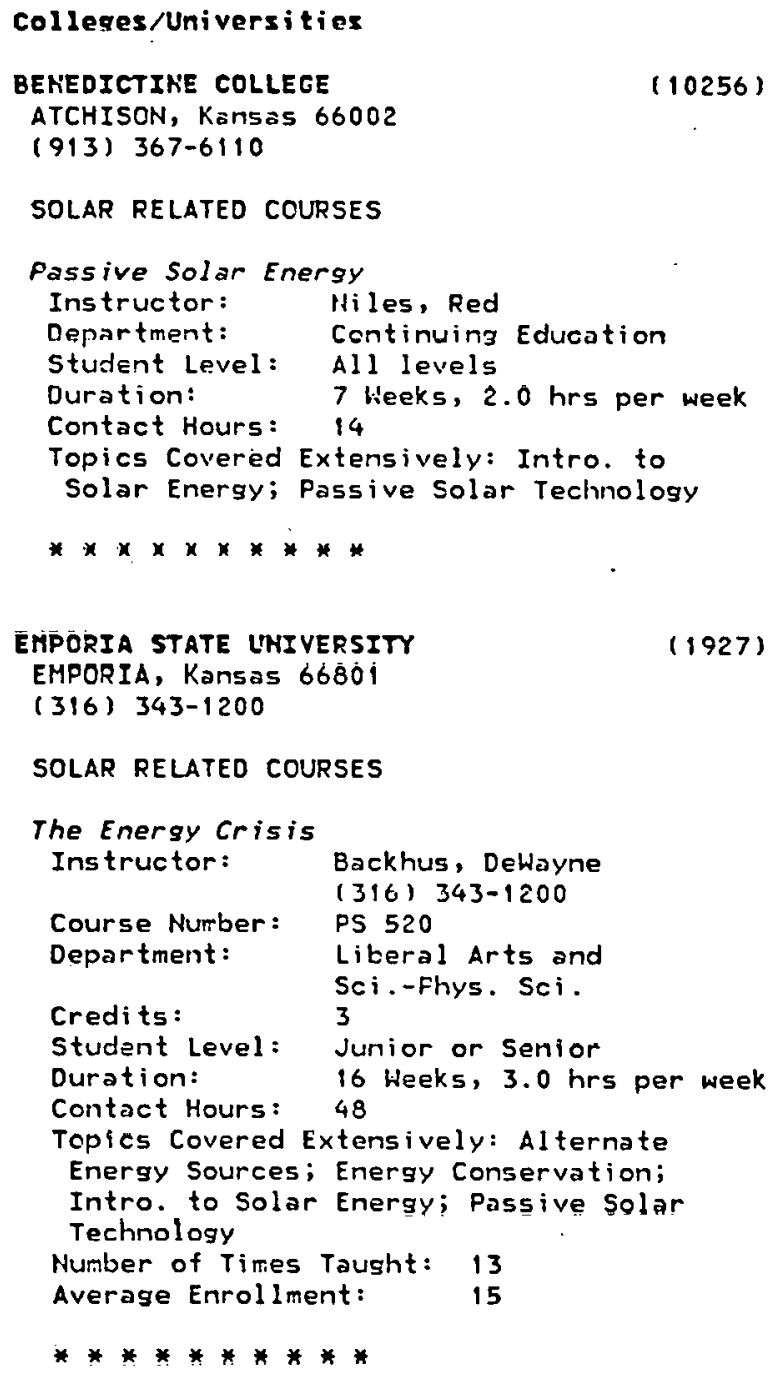

KaNSAS MAIH CAMPUS, $U$ OF

(1948)

LALiREIICE, Kansas 66045

(913) 864-2700

SOLAR RELATED COURSES

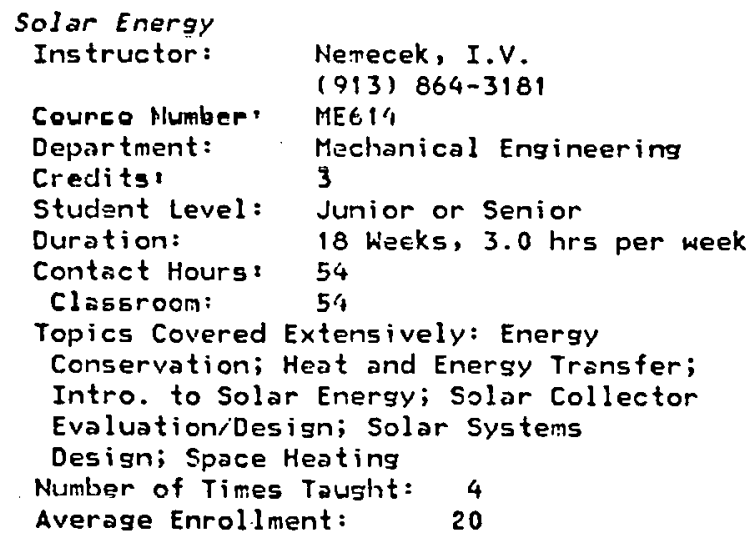

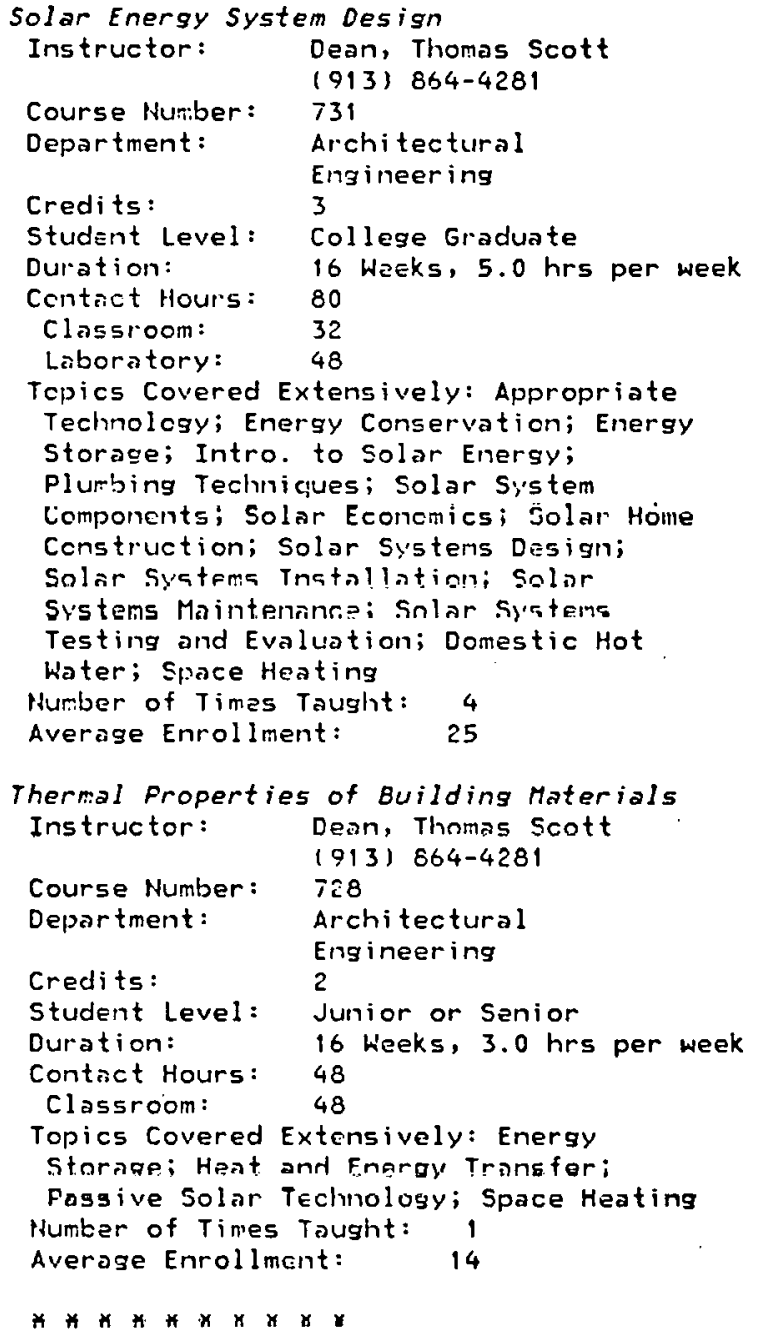




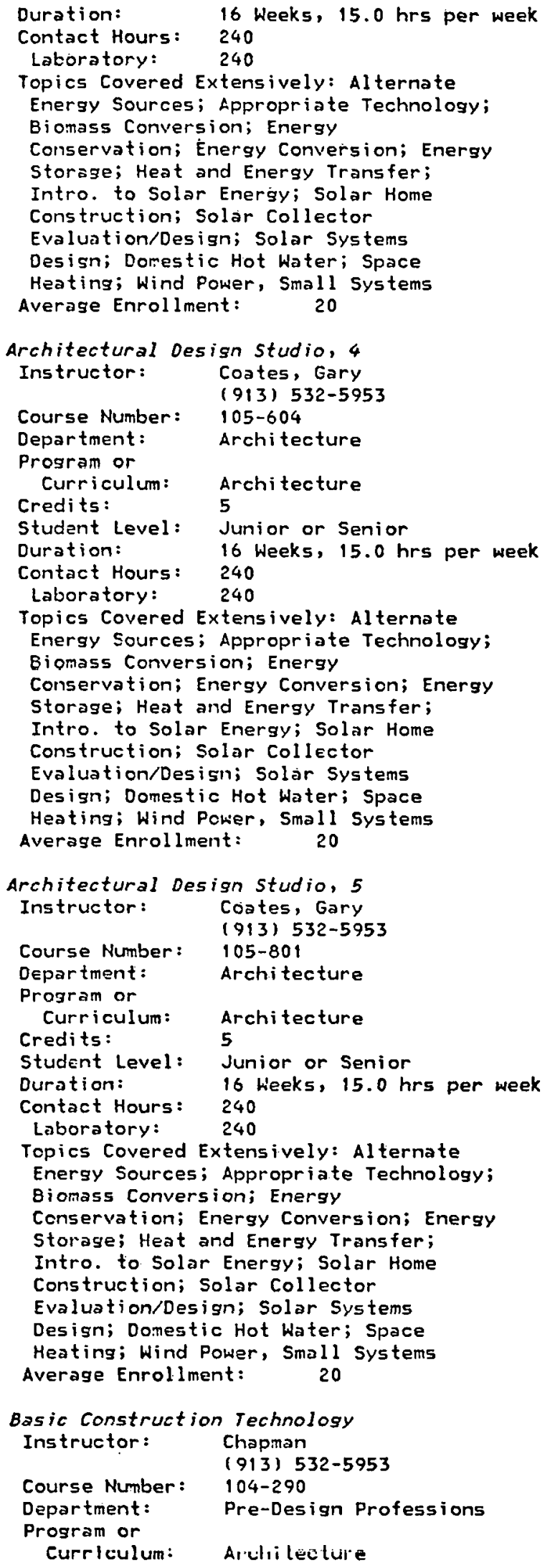

Credits:

fevel: Freshman or Sophomore

Duration:

Contact Hours: 48

Classroom: 8

Number of Times Taught: 5

Average Enrollment: 250

Ener. Use and Control in Agri. Systems

Instructor: Clark, Stanley J.

Course Number: (913) 532-5580

Department: Engineering

Credits: 3

Student Level: Junior or Senior

Duration: 15 Heeks, 5.0 hrs per week

Contact Hours: 75

Classroom: $\quad 30$

Laboratory: 45

Topics Covered Extensively: Alternate

Energy Sources; Biomass Conversion;

Energy Conversion

Number of Tines Taught: 6

Average Enrollment: $\quad 15$

Environmental Design of Farm Buildings

Instructor: Spillman, Charles $K$.

Course Humber: $\quad 505510$

Department: Agricultural

Credits: 3

Student Level: Junior or Senior

Duration: 16 Heeks, 5.0 hrs per week

Contact Hours: 80

Classroom: $\quad 32$

Laboratory: $\quad 48$

Number of Times Taught: 6

Average Enrollinent: $\quad 17$

Environmental Design Studio

Instructor: Miller

(913) 532-5953

Course Number: 104-261

Department: Pre-Design Professions

Program or

Curriculum: Architecture

Credits: 4

Student Level: Freshman or Sophomore

Duration: 16 Weeks, 4.0 hrs per week

Contact Hours: 64

Laboratory: 64

Number of Times Taught: 5

Average Enrollment: $\quad 300$

Environmental Systems in Architecture

Instructor: Jahnke, William R.

(913) 532-5950

Course Number: 105-515.

Department: Architecture

Program or

Curriculum: Archịtecture

Credits: $\quad 3$

Student Level: Junior or Senior

Duration: 16 Weeks, $3.0 \mathrm{hrs}$ per week

Coritact Hours: 48

Topics Covered Extensively: Energy

Conservation; Passive Solar Technology;

Plumbing Techniques; Sheet Metal

Tcohniquos; Solar System romponents; 
Solar Economics; Solar Home Construction; Solar Collector Evaluation/Design; Solar Systems Desisn; Domestic Hot Water; Space Heating; Space Cooling

Number of Times Taught: 6

Averase Enrollment: $\quad 100$

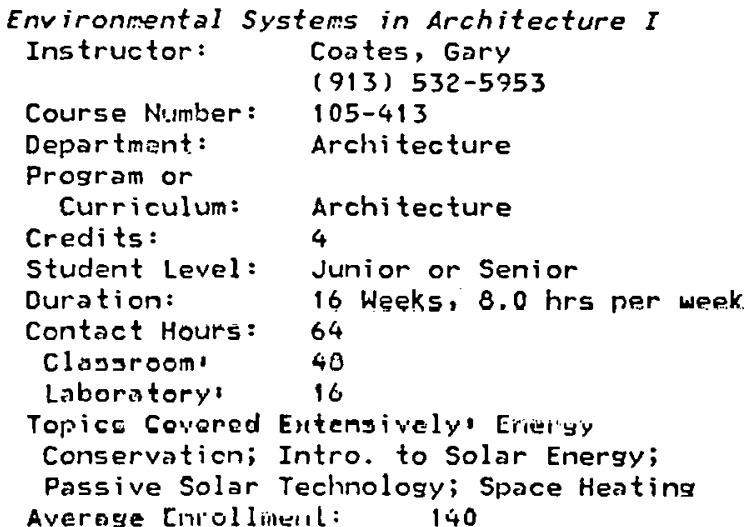

Intro. to Alternate Energy Sources

Instructor: Eckhoff, H. Dean (913) $532-5624$ $500-420$

Course Number: General Engineering Department:

Credits: 3

Student Level :

Duration:

Contact Hours:

Classroom:

All levels

16 Weeks, 3.0 hrs per meek 48

48

Topics Covered Extensively: Alternate Energy Sources; Energy Conservation; Energy Conversion; Heat and Energy Trausfer; Intro. to Solar Enersy; Passive Solar Technolegy; Solar Economics; Solar Home Construction; Solar Collector Evaluation/Design; Solar Systerns Designi Domestic Hot Water; Swiming Pool Heating; Space Heating; Wind Power, Small Systems Number of Times Tausht: 4 Average Enrollment: 10

Solar Energy Conversion Processes Instructor: Eckhoff, N. Dean (913) 532-5624 $500-380$

Course Number: General Engineering Uepartiment: Credits: 3

Student Level: 'All levels

Duration: 16 Weeks, $3.0 \mathrm{hrs}$ per week

Contaot Hours: 40

Classroom: 48

Tcpics Covered Extensively: Al ternate Enersy Sources; Energy Conservation; Energy Conversion; Heat and Energy Transfer; Intro. to Solar Energy; Passive Solar Techolicsy; Solar Ecenomics; Solar Hore Construction; Solar Collector Evaluation/Design; Solar Systens Desisn; Dorsestic Hot Water; Swiming Pool Heating; Space Heating; Wind Power, Small Systems Number of Times Taught: 2
Average Enrollment: $\quad 7$

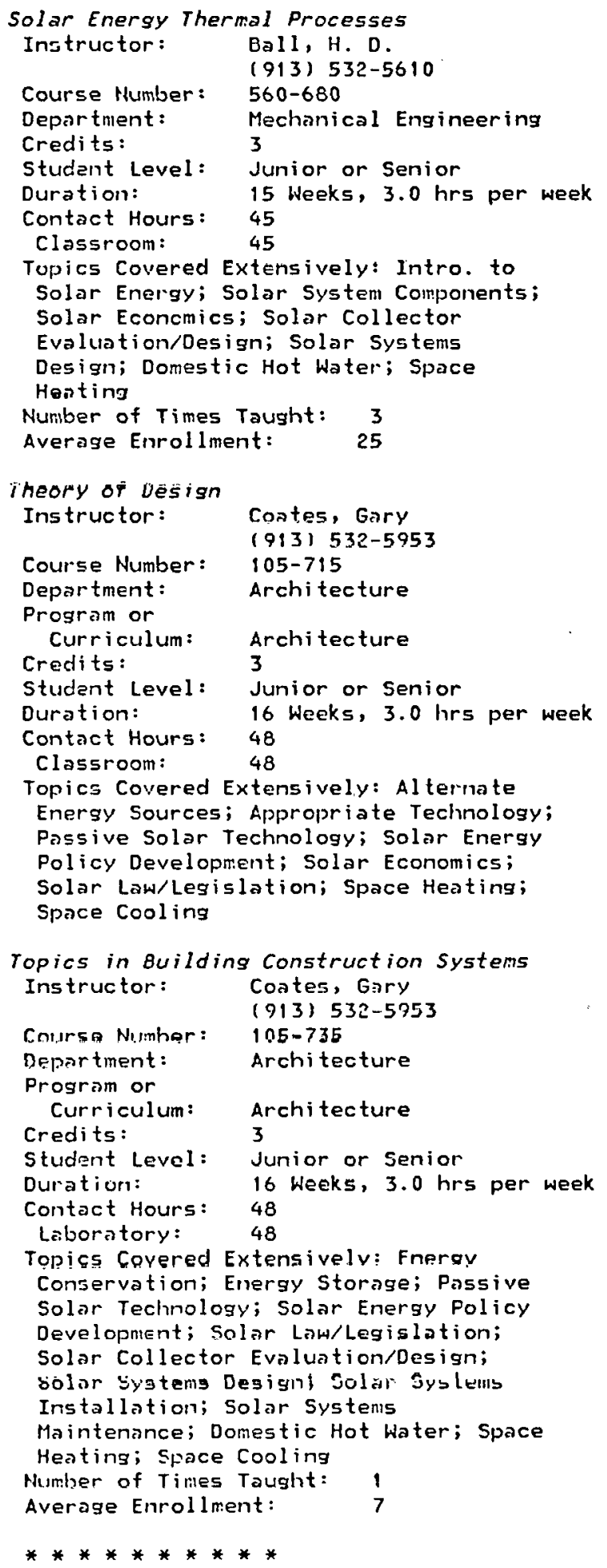


PITTSBURG ST UHIVERSITY

PITTSBURG, Kansas 66762

(316) $231-7000$

\section{SOLAR RELATED COURSES}

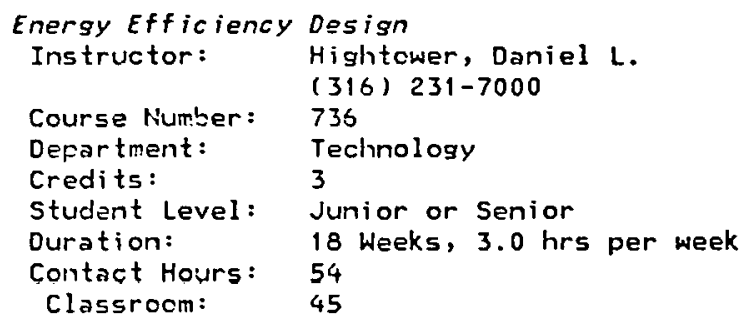

Classrocm:

Topics Covered Extensively: Alternate Energy Sources; Appropriate Technology;

Energy Conservation; Energy Storage; Heat and Energy Transfer; Intro. to

Solar Energy; Passive Solar Technology;

Solar System Components; Solar Home

Construction; Solar Collector

Evaluation/Design; Solar Systems

Design; Space Heating; Space Cooling

Number of Tines Taught: 1

Average Enrolliment:

20

\section{Solar Energy \\ Instructor: \\ Backes, Robert \\ (316) $231-7000$ \\ Course Number: 740 \\ Department: Fhysics \\ Credits: 3 \\ Student Level: Junior or Senior \\ Duration: 8 Weeks, 6.0 hrs per week \\ Contact Hours: 48 \\ Classrocm: $\quad 48$ \\ Tcpics Covered Extensively: Energy \\ Storase; Intro. to Solar Energy; \\ Passive Solar Technology; Solar System \\ Components; Solar Economics; Solar Home \\ Construction; Solar Law/Legislation; \\ Solar Collector Evaluation'Design; \\ Solar Sy'sten's Design; Solar Sy'steins \\ Testing and Evaluation; Domestic Hot Water; Space, Heating \\ **********}

\section{HICHITA STATE UHIVERSITY \\ 1945 Fairmount \\ WICHITA, Kansas 67208 \\ (316) 639-3456}

\section{SOLAR RELATED COURSES}

Energy-Alternatives and Impact

Instructor: Berg, J.R.

(316) 689-3141

Course Number: 690

Department: Geology

Credits: 3

Student Level: Junior or Senior

Duration: 16 Weeks, $3.0 \mathrm{hrs}$ per week

Contact Hours: 48

Classroom: $\quad 48$

Nurber of Times Taught: 4

Averaye Errullimeril: 13

(1950)

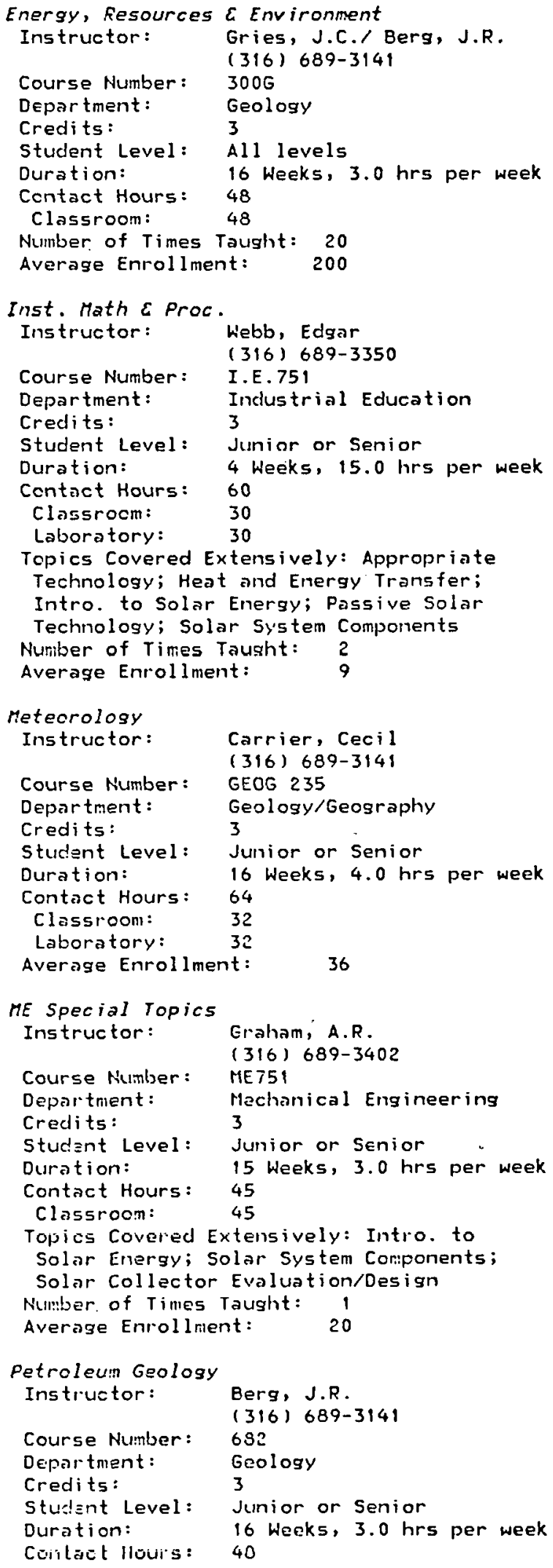




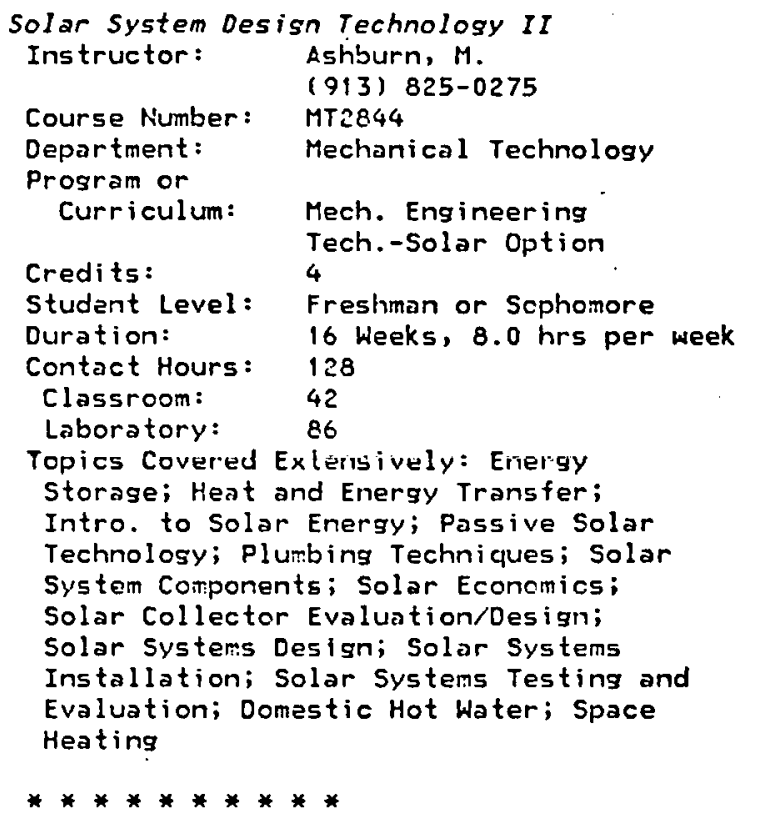

\section{Other Educational Institutions}

1221 Thurston Avenue

Manhattan, Kansas 66502

PROGRAMS AND CURRICULA

Appropriate Technology

Contact: Coates, Gary

( 913$) \quad 532-5866$ 


\section{colleges/Universities}

KEHTUCKY, UNIVERSITY OF

(1989)

LEXINGTON, Kentucky 40506

(606) 258-9000

\section{SOLAR RELATED COURSES}

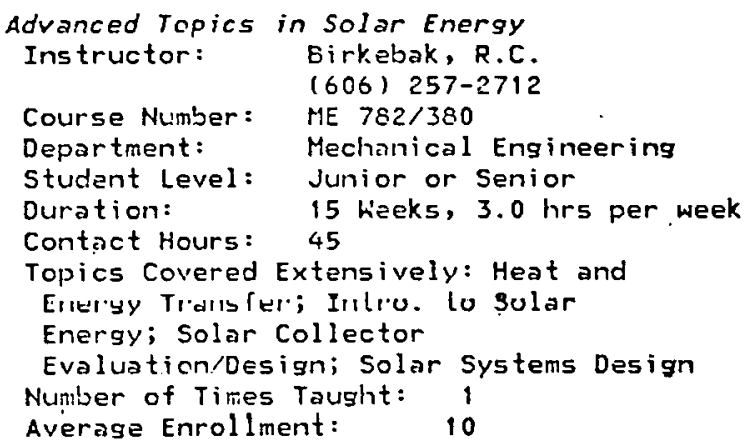

Course Number Mechanical Engineering Department: Junior or Senior Duration: 15 heeks, 3.0 hrs per week Contact Hours: 45

Topics Covered Extensively: Heat and Eritiry Trdusfer; Irrliu. Lo sular Energy; Solar Collector Evaluation/Design; Solar Systems Design Number of Times Taught: 1 Average Enrollment: 10

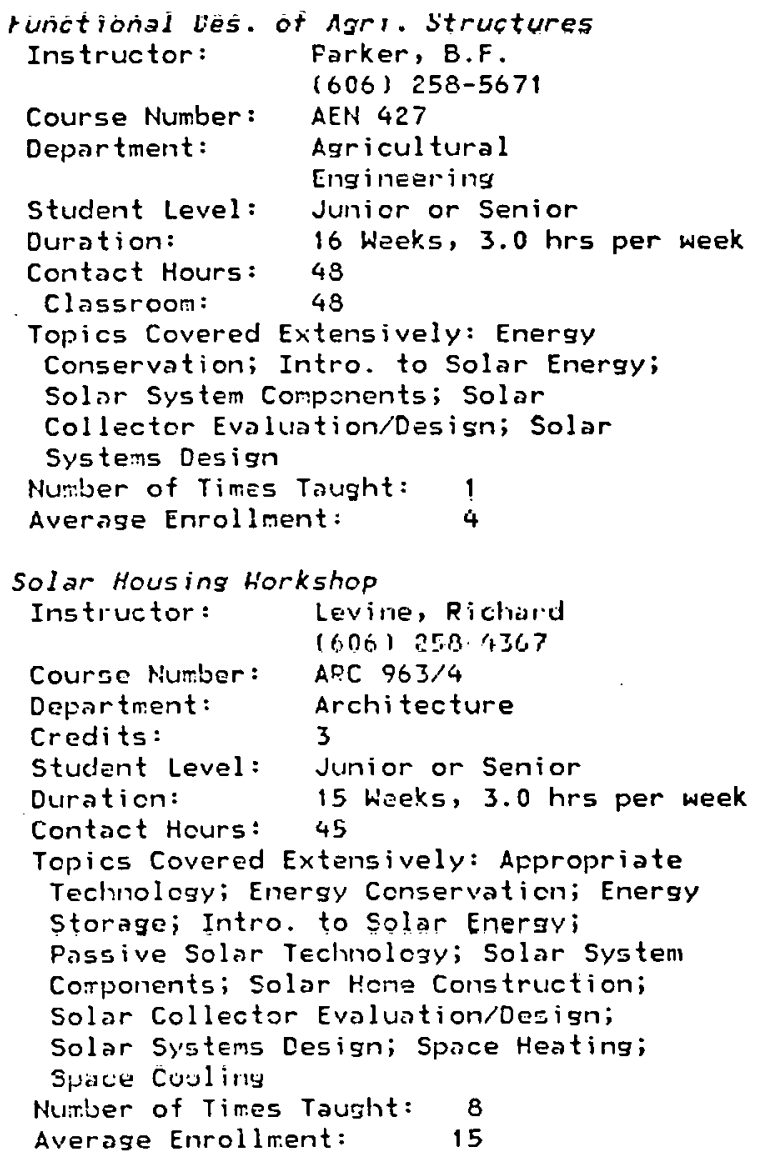

KORTHERH KY UHIVERSITY

HIGHLAND HEIGHTS, Kentucky 41076 (606) 292-5100

SOLAR RELATED COURSES

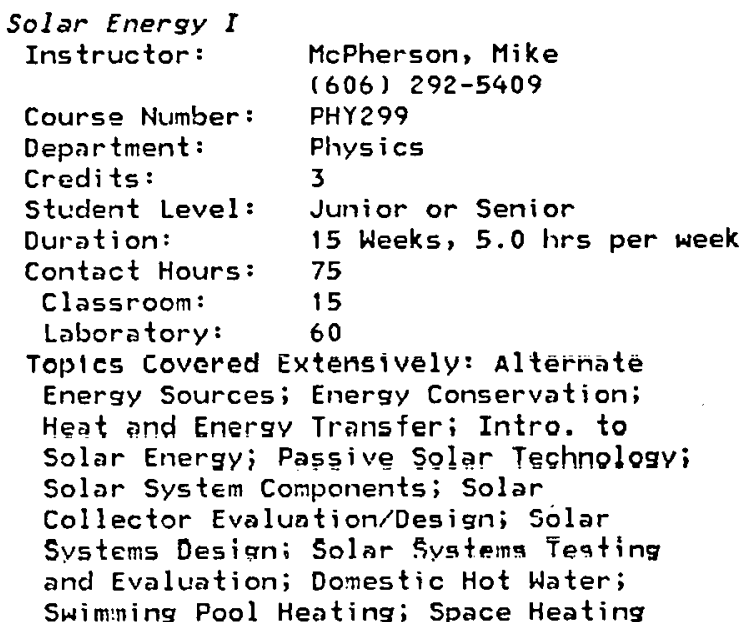

WESTERH KY UHIVERSITY

BOWLING GREEN, Kentucky 42101

(2002) (502) 745-0111

\section{SOLAR RELATED COURSES}

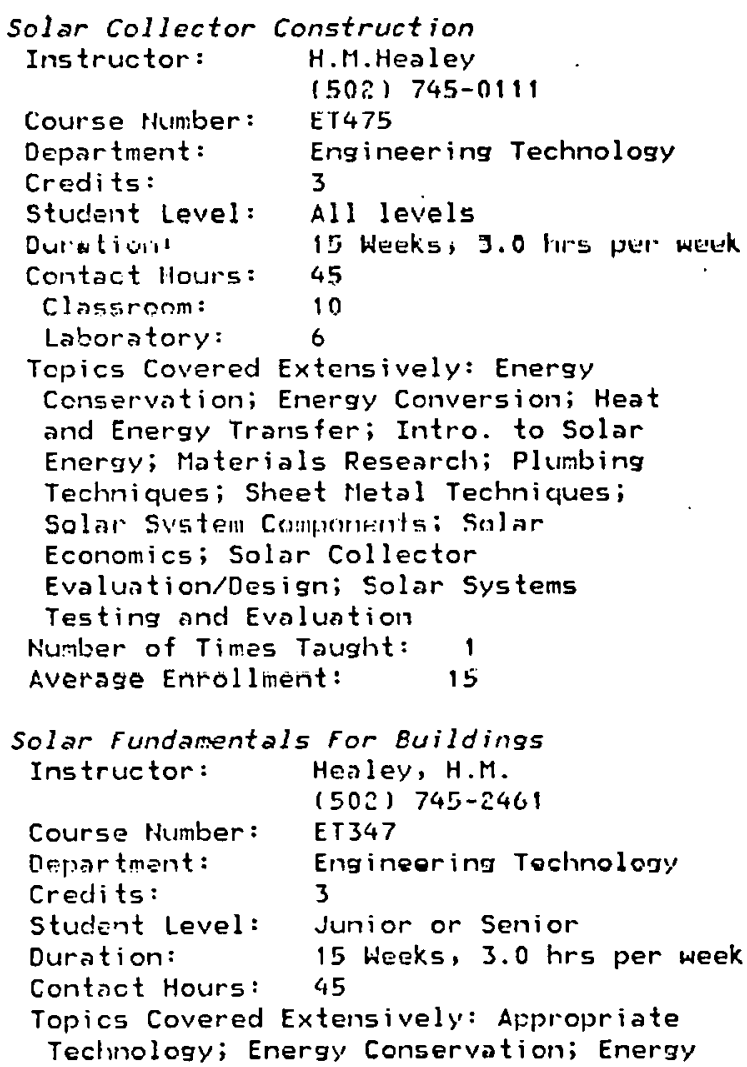


Storage; Heat and Energy Transfer; Intro. to Solar Energy; Passive Solar Technology; Solar System Components; Solar Economics; Solar Home

Construction; Solar Collector Evaluation/Design; Solar Systems

Design; Domestic Hot Water; Space Heating

Number of Times Taught: 0

WKU Solar Heating Systems

Instructor: Healey, H.M.

(502) 745-2461

Department: Engineering Technology

Student level: All levels

Duration: 1

Contact Hours: 32

Classroom: $\quad 28$

Laboratory: 4

Topics Covered Extensively: Energy

Conservation; Energy Storage; Heat and

Energy Transfer; Intro. to Solar

Energy; Solar System Components; Solar

Economics; Solar Collector

Evaluation/Desisn; Solar Systems

Design; Solar Systems Installation;

Solar Systems Maintenance; Solar

Systems Testing and Evaluation;

Domestic Hot Water; Space Heating 


\section{coḷleges/Universities \\ LA STATE U AND REM C \\ BATON ROUGE, Louisiana 70803 \\ $(2010)$ \\ (504) 388-1471 \\ SOLAR RELATED COURSES

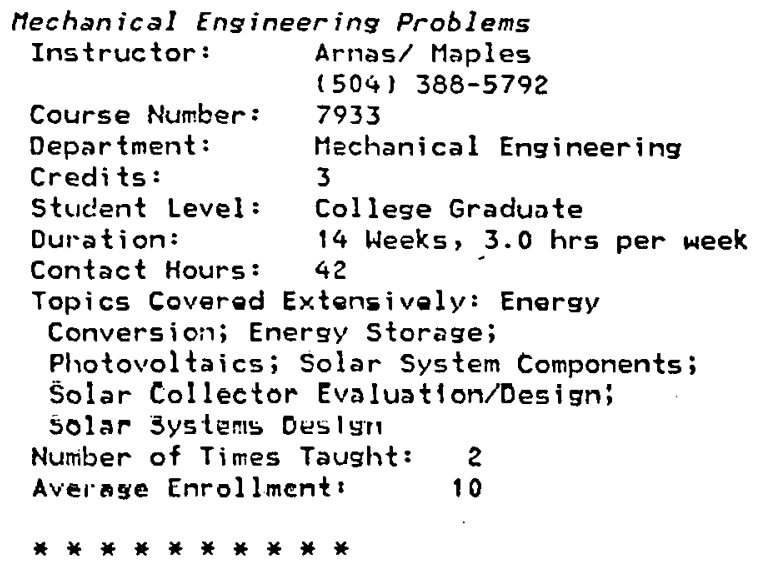

LOUISIAHA TECH UMIVERSITY

(2008)

RUSTON, Louisiana 71272

(318) 257-0211

SOLAR RELATED COUPSES

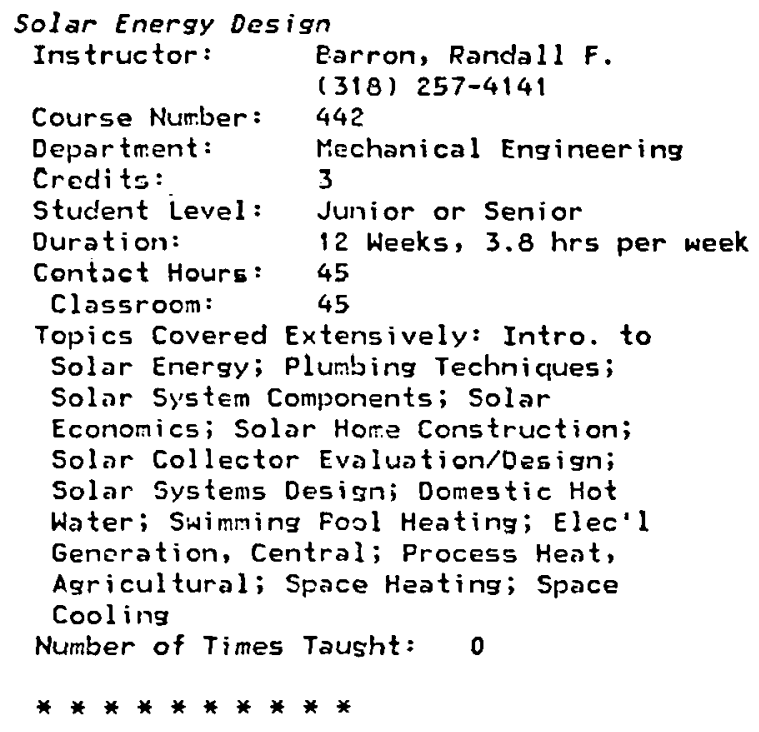

NEW ORLEAHS, UHIVERSITY OF

(2015)

HEW OPLEAHS, LOUisiana 70122

(504) 283-0500

\section{SOLAR RELATED COURSES}

Design of Solar Heat. and Cool. Systems Instructor: Russo, Editin P. (504) 283-0652

Course Nurber: 4770

Department:

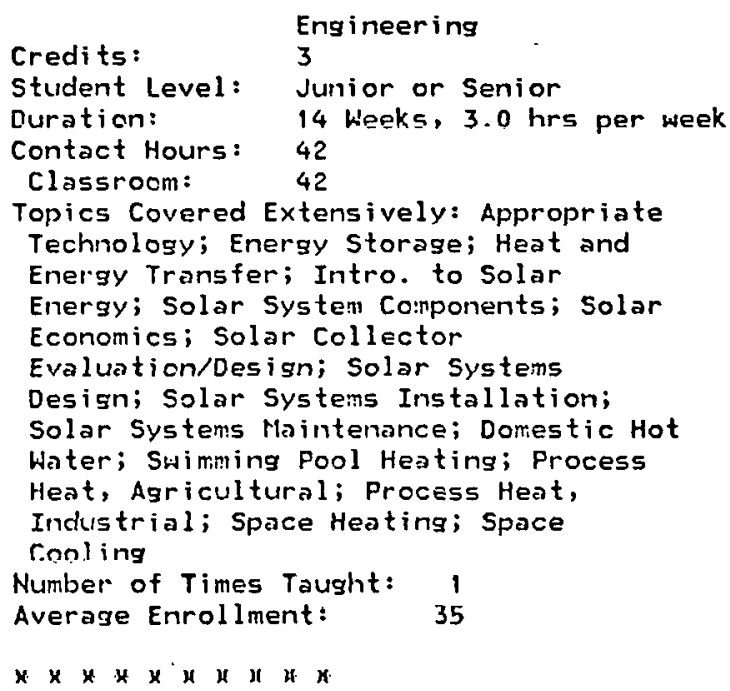

\section{SOLAR RELATED COURSES}

Solar Thermal Processes

Instructor: Hamilton, Dewitt $c$.

(504) 865-6176

Course Number: ME 619

Department: Mech. Enging.

Credits: 3

Student Level: Junior or Senior

Duration: 15 Weeks, 3.0 hrs per week

Contact Hours: $\quad \dot{45}$

L'lassroom: 45

Topics Covered Extensively: Energy

Storage; Heat and Enersy Transfer;

Tntro. to Solar Energy; Solar Collector

Evaluation/Design; Solar Systems

Design; Domestic Hot Water; Space

Heating; Space Cooling

Number of Times Taught: 5

Average Enrollment: $\quad 15$

Cumminity/lumior Colleges

DELGADO COLLEGE

NEW ORLFANS, Louisiana 70119

(4626)

(504) $486-7393$

SOLAR RELATED COURSES

Applied Solar Energy

Instructor: Charbonnet, Lary/ Tou,

(504) 486-7393

Course Number: METI30 


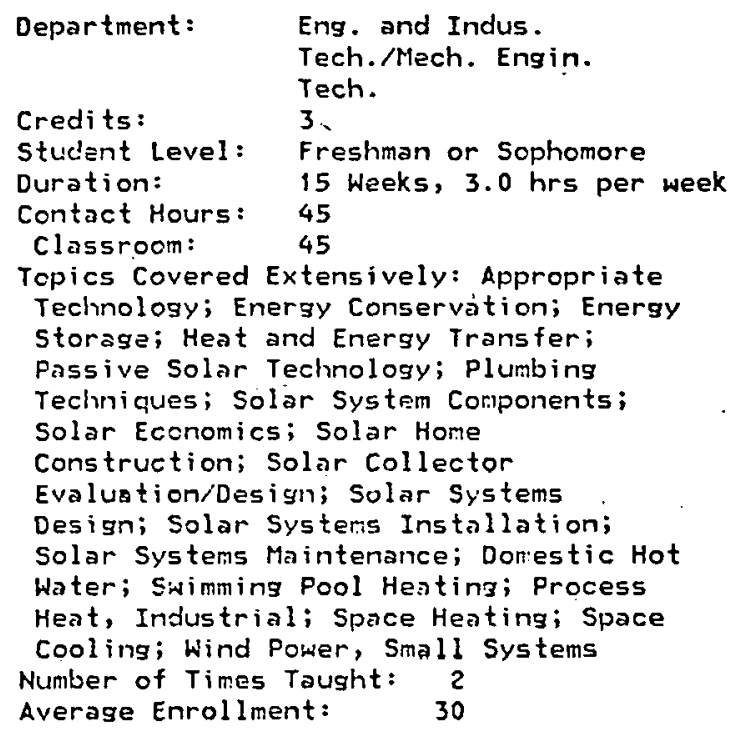




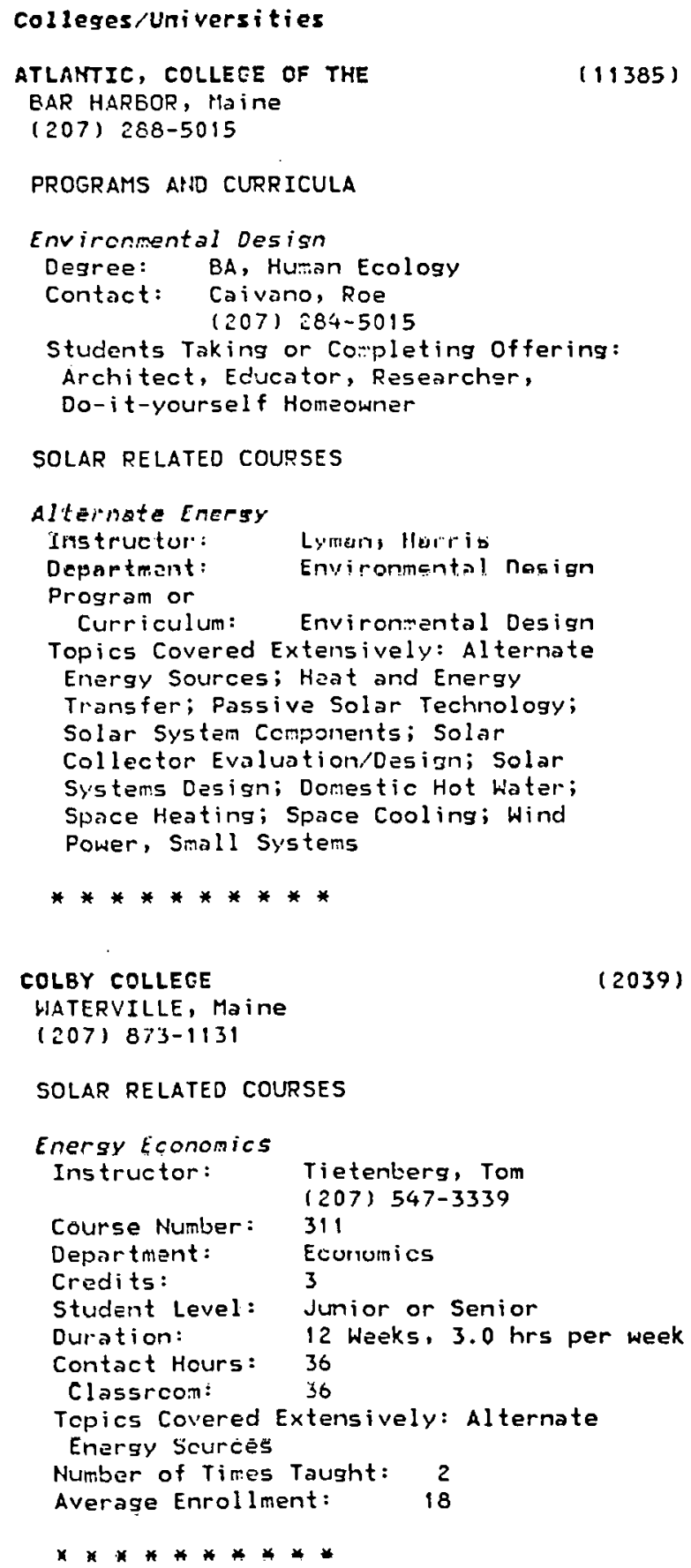

Department: Environmental Studies

Credits: 3

Student Level: Junior or Senior

Duration: 15 Weeks, 3.0 hrs per week

Contact Hours: 45

Classroom: $\quad 45$

Topics Covered Extensively: Alternate

Energy Scurces; Energy Conservation;

Intro. to Solar Energy

Number of Times Taught: 1

Average Enrollment: $\quad 10$

***********

MAINE AT ORONO, U OF

ORGFO, Maine

(207) 581-7011

SOLAR REIATED R.OIRSES

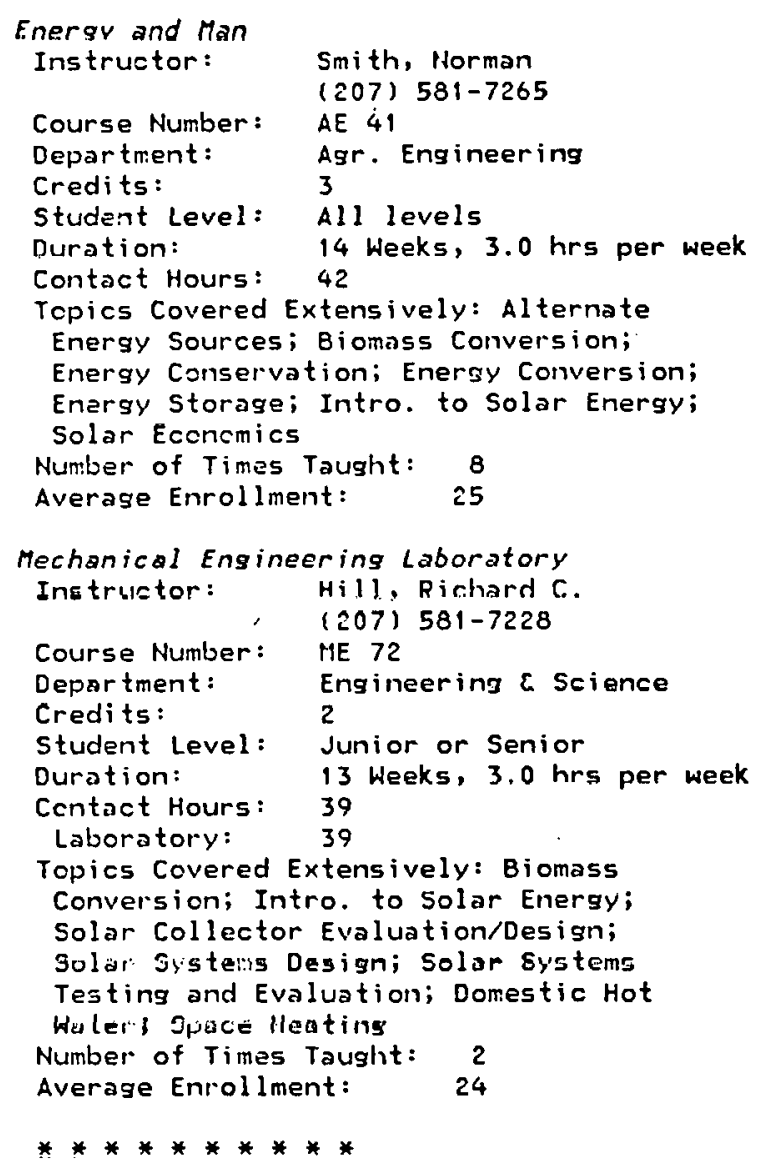

\section{Other Educational Institutions}


CORHERSTCNES, HING SCHOOL OF SHELTER

TECHIIOLCGY

(90090)

54 Cumberland st.

Brunswick, Maine 04011

\section{SOLAR RELATED COURSES}

Advanced New House

Course Number: $B$

Duration: $\quad 3$ Weeks, 35.0 hrs per week Contact Hours: 105

Topics Covered Extensively: Passive

Solar Technology; Solar Home

Construction

Passive Solar Building Desisn.

Course Number:

Duration: 1

Contact Hours: 35

Topics Covered Extensively: Passive

Solar Technology; Solar Home

Construction

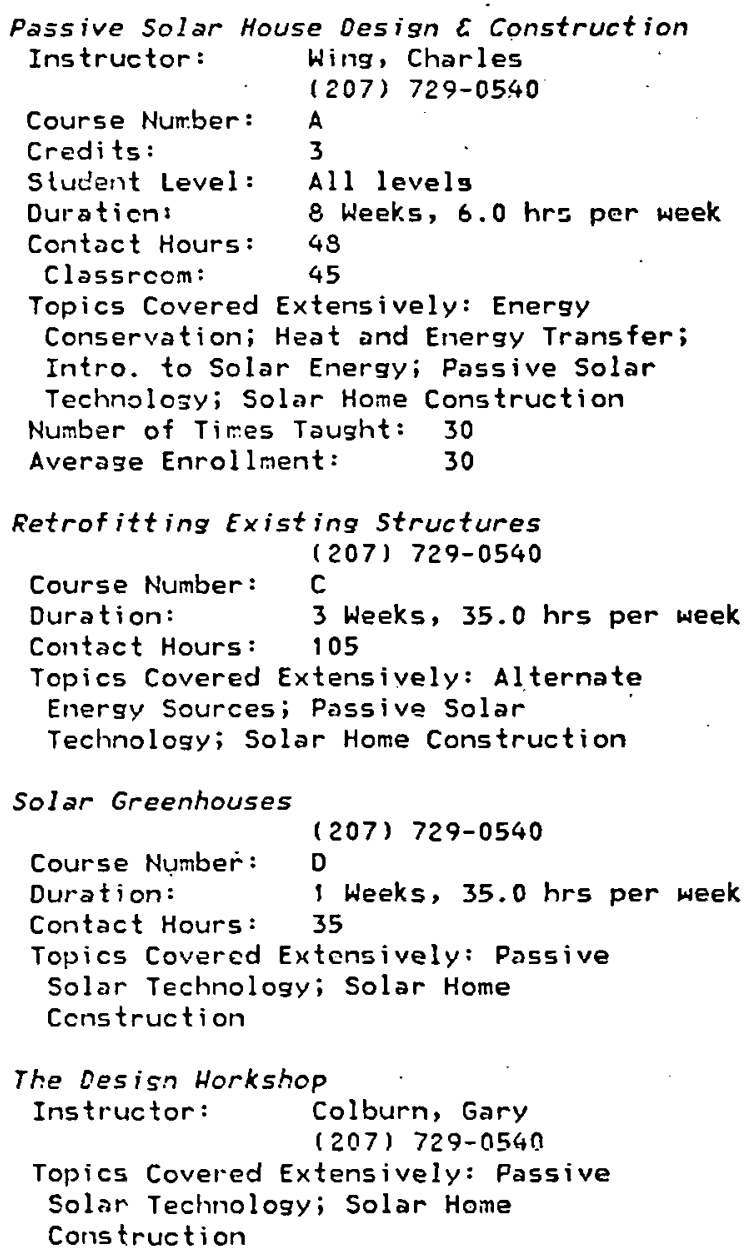

Portland Vocational Center

Portland, Maine 04111

SOLAR RELATED COURSES

* Training in Solar Installation Topics Covered Extensively: Solar Systems Installation

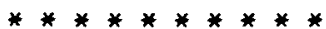

\section{SHELTER IHSTITUTE}

53 Center Street

Bath, Maine 04530

SOLAR RELATED COURSES

* Passive Solar Design

Instructor: Hennin, Patsy (207) $443=9084$

Duration:

15 Weeks

Topics Covered Extensively: Passive Solar Technology; Solar Home

Construction 


\author{
colleges/Universities \\ $(2078)$ \\ LOYOLA COLLEGE \\ BALTIIIORE, Maryland 21210 \\ ( 301 ) $323-1010$

\section{SOLAR RELATED COURSES} \\ Energy and Enyironment \\ Instructor: Haig, Frank $R$. \\ (301) 323-1010 \\ Course Number: \\ FH 150 \\ Department \\ Physics, Eng'r..; \\ Computer Sci. \\ Credits: \\ 4 \\ Student Level: \\ Duration: \\ Junior or Senior \\ Contset Hours : \\ 15 Weeks, 3.0 hrs per week \\ 45 \\ Classronm: \\ 45
}

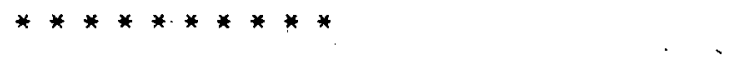

MD CQLLESE PARK CAM, $U$ OF

(2103)

COLLEGE PARK, Maryland 20742

(301) 454-0100

PROGRAMS AFI CURRICULA

$\begin{array}{ll}\text { Mechanical Engr./Solar Energy } \\ \text { Desree: } & \text { BS, Science } \\ \text { Contact: Cunniff, P. F. } & \\ & \text { (301) 454-2410 }\end{array}$

SOLAR RELATED CCURSES

Engineering Applications of Solar Energy

Instructor: Allen, R. W.

(301) 454-4994

Course liurber: Elilie 415

Department: Mechanical Engineering

Program or

Curriculum: Mechanical Engr./ Solar

Student Level: Junior or Senior

Duration: 16 heeks, $3.0 \mathrm{hrs}$ per week

Contact Hours: 43

Classroom: 48

Topics Covered Extensively: Energy

Storase; Heat and Energy Transfer;

Solar Sistem Corponents; Solar

Econerics; Solar Collestor

Evaluaticn/Design; Demestic Hot Water;

Space Heating

Murber of Times Taught: 1

Average Enrollment: $\quad 40$

Envirchmental systems in Architecture

Instructor: Lord, Dayid

(301) 454-3428

Course Number: ASCH 514

Department: Architecture

Program or

Curriculum: Mechanical Engr./ Solar:

Credits:

Energy

Student Level: Junior or Senior

Duration: 14 Weeks, 6.0 hrs per week

Contact Hours: 84
Classroom: $\quad 56$

Topics Covered Extensively: Alternate

Energy Sources; Energy Conservation;

Eneray Storage; Intro. to Solar Energy;

Passive Solar Technology; Solar System

Components; Solar Econcmics; Solar

Sy'stems Design; Domestic Hot Wiater;

Space Heating

Number of Times Taught: 4

Averase Enrollment:

7

Solar Energy Applications in Buildings

Instructor: Allen, R. W.

(301) 454-4994

Course Number: ENES 414

Department: Mechanical Engineering

Urogram or

Curriculum; Mechanical Engr.t Solar

Credits:

Student Level: Junior or Sanior

Duration: 16 Weeks, 3.0 his per week

Contact llours: 48

Classroom: 48

Topics Covered Extensively: Energy

Storage; Passive Solar Technology;

Solar System Components; Solar Home

Construction; Solar Collector

Evaluation/Design; Solar Systems

Desisn; Domestic Hot Water; Space

Heating

Nuriber of Times Taught: 5

Averase Enrollment: 35

**********

TOUSAN STATE IINTYERSTTY

BALTIMICRE, Maryland 21204

(301) 321-2000

SOLAR RELATED COURSES

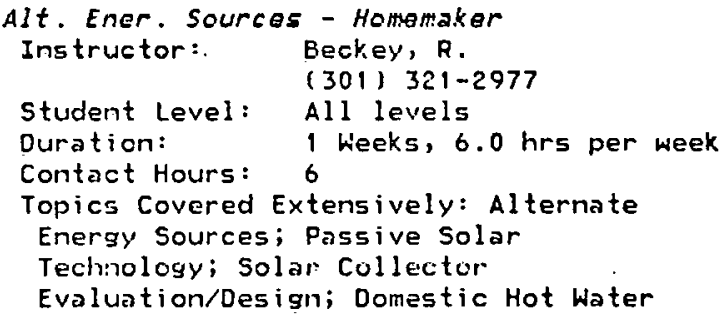

US HAVAL ACADEIT

ANIJAPOLIS, MARYLAND, MarYland 21402

$(2101)$

$13011267-6100$

SOLAR RELATED COURSES

Energy Conversion

Instructor: Hu, C.

(301) 267-3186

Course Number: EM443

Department: Mechanical Engineering

Credits: 3
$(2099)$ 
Student Level: Junior or Senior

Duration: 16 Weeks, 3.0 hrs per week

Contact Hours: 43

Topics Covered Extensively: Alternate

Energy Sources; Energy Conservation;

Energy Conversion; Intro. to Solar

Energy; Solar Economics; Solar

Collector Evaluation/Design; Domestic

Hot hater

Nurber of Times Taught: 5

Averase Enrollment: $\quad \mathbf{5 0}$

Ocean Energy Conversion

Instructor: MicCormick, M.E.

(301.) 267-3873

Course Number: EN474

Department: Maval Systems

Credits: 3

Student Level: Junior or Senior

Duration: 16 Heeks, $3.0 \mathrm{hrs}$ per week

Centact Hours: 48

Classrcom: $\quad 48$

Topics Covered Extensively: Alternate

Energy Sources

Nimber of Times Taught: 1

Average Enrollment: $\quad 30$

**********
Average Enrollment:

12

\section{Community/Junior colleges}

ALLEGANY CHTY COLLEGE

CL:SBERLARID, Maryland 21502

(301) 724-7700

\section{SOLAR RELATED COURSES}

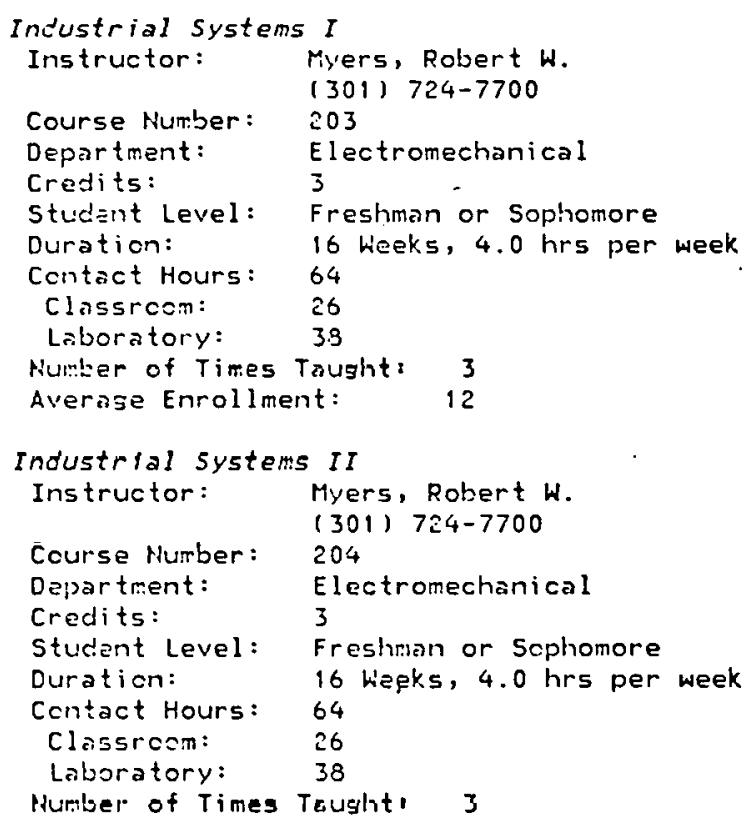

(2057)

HARFORO COAMUIITY COLLEGE

BEL AIR, Maryland 21014

(301) 838-1000

\section{SOLAR RELATED COURSES}

\section{Principles and Applications of Solar Energy Course Humber: 095 \\ Department: Continuing Education \\ Student Level: All levels \\ Duration: $\quad 16$ Weeks, $3.0 \mathrm{hrs}$ per week \\ Contact Hours: 48 \\ Topics Covered Extensively: Intro. to \\ Solar Energy; Passive Solar Technology}

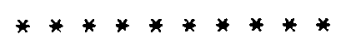

vocational/Technical colleges 
RETS TECH CENTER

$(90050)$

511 Russell Street

Baltimore, Maryland 21230

(301) 727-6863

PROGRAMS AND CURRICULA

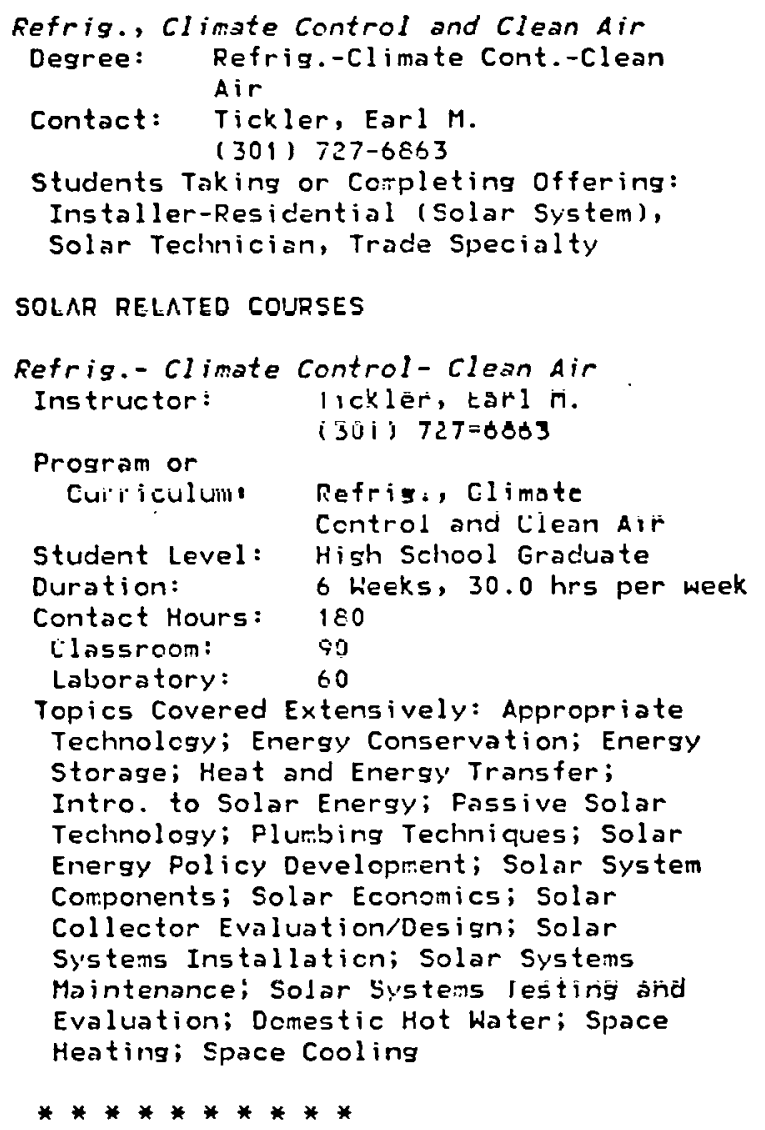




\section{Colleges/Universities}

AHHERST COLLEGE

AMHERST, Massachusetts

(413) 542-2000

SOLAR RELATED COURSES

*Energy

**********

BOSTON COLLECE

CHESTRUT HILL, Massachusetts

(617) 969-0100

\section{SOLAR RELATED COURSES}

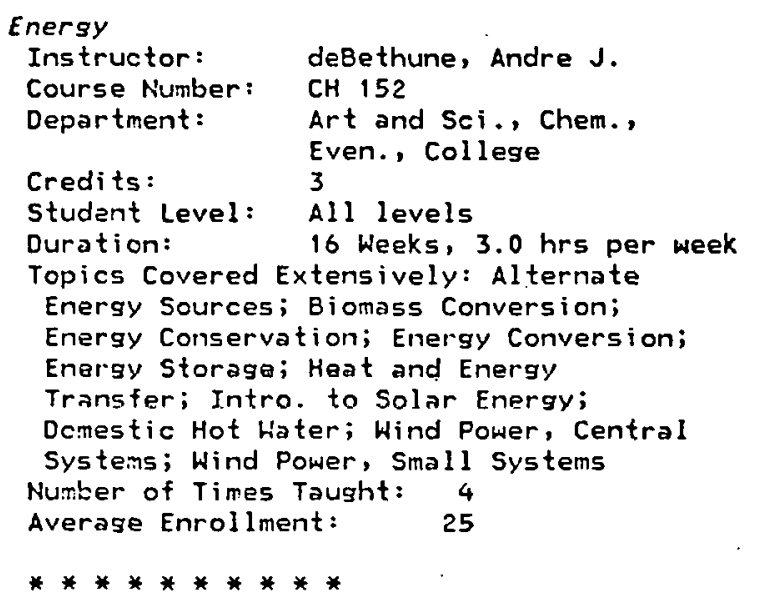

\section{BOSTON UNIVERSITY}

EOSTON, Massachusetts

(617) 353-2000

\section{SOLAR RELATED COURSES}

\section{Man and Energy}

Instructor:

Lichtin, Norman N.

(617) 353-2493

Course Number:

UNI-EY-50

Department:

Credits:

University Professors

4

Student Level: Junior or Senior

Duration: $\quad 13$ Weeks, $3.0 \mathrm{hrs}$ per week

Contact Hours: 39

Classroom: $\quad 39$

Solar Heating

Course Number: MET EM 510

Department: Metropolitan College

Credits: 3

Student Level: Junior or Senior

Duration: .. 15 Heeks, 3.0 hrs per week

Contact Hours: 45

Topics Covered Extensively: Energy

Conservation; Energy Conversion; Energy

Storage; Heat and Energy Transfer;

Solar System Components; Solar

Economics; Solar Collector

Evaluation/Design; Domestic Hot Water;
Space Heating

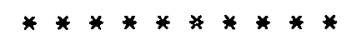

BRIOGELATER STATE COLLEGE

(2183)

BRIDGEWATER, Massachusetts

(617) 697-8321

SOLAR RELATED COURSES

Solar Energy

Instructor: Blackford, Paul A.

Course Number: GE 412

Department: Earth Sripnces and

Geography

Student Level: Junior or Senior

Duration: 14 Weeks, 3.0 hrs per week

Contact Hours: 42

Topics Covered Extensively: Heat and

Energy Transfer; Intro. to Solar

Energy; Passive Solar Technology; Solar

System Components; Solar Economics;

Solar Collector Evaluation/Design;

Solar Systems Design; Domestic Hot

Water; Space Heating

Number of Times Taught:

Average Enrollment:

6
19

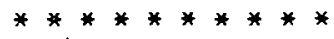

CLARK UMIVERSITY

HORCESTER, Massachusetts

(617) 793-7177

SOLAR RELATED COURSES

Alternative Energy Systems Laboratory

Instructor: Gottlieb, Albert

(617) 793-7439

Course Number: STS 132

Department: Science, Technology and

Student Level: Junior or Senior

Duration: 14 Weeks, $6.0 \mathrm{hrs}$ per week

Contact Hours: 84

Tcpics Covered Extensively: Alternate

Energy Sources; Energy Conversion; Heat and Energy Transfer; Intro. to Solar

Energy; Fhotovoltaics; Solar Collector

Evaluation/Design; Solar Systems

Testing and Evaluation; Domestic Hot

Water; Elec'l Generation, Small Scale;

Space Heating; Wind Power, Small Systems

Number of Times Taught: 2

Average Enroliment: 10

Solar and Wind Energy for Home Use

Instructor: Fussell, John I.

Course Number: $\quad$ (617) 109

Department: Prof. and Cont. Edu.

Credits: 3

Student Level: All levels

Duration: $\quad 14$ Weeks, $3.0 \mathrm{hrs}$ per week

Contact Hours: 42 
Classrcom:

\section{2}

Topics Covered Extensively: Alternate Energy Sources; Appropriate Technology; Energy Conservation; Intro. to Solar Enersy; Fassive Solar Technology; Solar System Components; Solar Economics; Solar Home Construction; Solar Collector Evaluation/Desisn; Solar Systems Design; Domestic Hot Hater; Elec'l Generation, Small Scale; Space Heating; Hind Pcwer, Central Systems; Wind Power, Small Systems

Number of Times Taught: 2

Average Enrollment: $\quad 18$

Solar Energy

Instructor:

Cuì sé Hür,bëi" ,

Davies, John

Department:

5T3 131

Science, Technology, Society

Credits:

Student Level:

All levels

Duration: 14 keeks, $3.0 \mathrm{hrs}$ per week

$\begin{array}{cl}\text { Contact Hours: } & 42 \\ \text { Classroom: } & 42\end{array}$

Topics Covered Extensively: Intro. to

Solar Energy; Space Heating

Number of Times Tausht: 1

Average Enrolliment:

40

***********

\section{HARVARD ULIYERSITY}

(2155)

CAMBRIDGE, Massachuset ts

(617) 495-1000

SOLAR RELATED COURSES

Solar Heat ing: Basic Issues

Instructor: Hapzood, William

Department: Center for Lifelong

Lesinilug

Student Level: All levels

Duration: 6

Contact Hours: 12

Number of Times Taught: 0

$* * * * * * * * *$

LOUELL, UHIVERSITY OF

(2161)

LO'NELL, Massachusotts

(617) 454-7811

\section{PFOGRAME ARD CURRIGULA}

Appl. Fhysics - Solä Cher. Option

Degree: Pho, MS, Fhysics, Solar

Energy Option

Contact: Filiplone, William

$(617) 454-7811$

Students Taking or Completing Offering:

Researcher, Solar Engineer

Solar Energy

Degree: MS, BS, Engineering or

Science

Contact: Filippone, Hilliam

(617) 454-7811
Students Taking or Completing Offering:

Researcher, Solar Engineer

\section{SOLAR RELATED COURSES}

Advanced Solar Energy

Instructor: Filippone, William

(617) 454-7811

Course Number: $24-513$

Department: Nuclear Engineering

Program or

Curriculum: Appl. Phy'sics - Solar

Credits Ener. Option

Student Level: Junior or Senior

Duration: 15 Weeks, 3.0 hrs per week

Contact Hours: 45

Clagsi.00m' 45

Topics Covered Extensively: Energy

Storage; Hent and Energy Transfer;

Intro. to Solar Energy; Fassive Solar

Technology; Photovoltaics; Solar System

Conponents; Solar Economics; Solar

Collector Evaluation/Design; Solar

Systems Design; Domestic Hot hater;

Elec'l Eeneration, Central; Elec'l

Generation, Small Scale; Space Heating;

Space Cooling; Wind Power, Small

Systems

Number of Times Taught: 1

Average Enrolliment: $\quad 10$

Geothermal and Hind Energy Systems

Instructor: Sheff, James $R$.

Course Number: 0

Departiment: Nuclear Engineering

Program or

Curriculum: $\quad 24-530$

Credits: 3

Student Level: Junior or Senior

Duration: 15 Weeks, 3.0 hrs per week

Contaet lloulse 45

Classroom: $\quad 45$

Topics Covered Extensively: Alternato

Energy Sources; Heat and Energy

Tronsfer; Wind Power, Central Systems;

Hind Power, Small Systens

Number of Times Tought: 1

Averase Enrollment: $\quad 15$

Solar Energy

Instructor:

Filippone, William

(617) 454-7811

Course Number: $24-425$

Departmant:

Prosram or

Curriculuma

Credits:

Nucloar Enginsoring

Student Level: Jumior or Senior

Duration:

Contact Hours:

Clissroom:

15 Weeks, 3.0 hrs per week

45

45

Topics Covered Extensively: Heat and

Enersy Transfer; Intro. to Solar

Energy; Passive Solar Technology; Solar

Collector Evaluation/Design; Solar

Systcrs Design; Domestic Hot Linter;

Space Heating

Number of Times Taught: 2 
Average Enrollment:

MASS AMHERST CAMPUS, $U$ OF AMHERST, Massachusetts (413) 545-0111

PROGRAMS AND CURRICULA

Enersy Frosram

Degree: BS, Mechanical

Engineering-Energy Option

Contact: Cremack, Duane (413) 545-2.756

\section{SOLAR RELATED COURSES}

\section{Engineering Wind Fower Systems}

Instructor: Cromach, Duane E.

(413) 545-2756

Course Number: $\quad 153 / 90 \mathrm{C} 9 \mathrm{CH}$

Department: Mechanical Engineering

Program or

Curriculum: Energy Program

Credits:

33

Student Level: Junior or Senior

Duration: 14 Weeks, 3.0 hrs per week

Contact Hours: 42

Classrcom: $\quad 37$

Laboratory: $\quad 5$

Topics Covered Extensively: Appropriate

Technology; Energy Conversion; Solar

Collector Evaluation/Design; Solar

Systens Design; Solar Systems Testing

and Evaluation; Wind Power, Small

Sy'steirs

Number of Tinies Taught: 4

Average Enrollinent: $\quad 20$

Solar and Direct Enersy Conversion

Instructor: MoGowan, J.

Course lumber: $\mathbf{5 7 0}$

Department: Mechanical Engineering

Program or

Curriculum: Energy Program

Credits: 3

Student Level: Junior or Senior

Duration: 14 Weeks, 3.0 hrs per week

Contact Hours: 42

Topics Covered Extensively: Energy

Conversion; Enersy Storage; Heat and

Energy Transfer; Intro. to Solar

Energy; Solar Collector

Evaluation/Design; Solar Systems

Design; Space Heating

Number of Times Taught: 4

Average Enrollment:

20
MASS IHST OF TECHHOLOGY

CAMBRIDGE, Massachusetts

(617) 253-1000

SOLAR RELATED COURSÉS

Desisn with Microclimate

Instructor: Johnson, Tim

(617) 253-5965

Course Number: $4.071 \mathrm{~J}$

Department: Architecture

Program or

Curriculum: Arch. Study

Credits:

12

Studient Level:

All levels

Duration:

13 Weeks, 6.0 hrs per week

Contact Hours: 78

Classroom: $\quad 39$

Laboratory: $\quad 39$

Topics Covered Extensively: Energy

Conservation; Energy Conversion; Energy

Storage; Heat and Energy Transfer;

Intro. to Solar Energy; Materials

Research; Passive Solar Technology

Number of Tin:es Tought: 3

Average Enrollment: $\quad 25$

Energy Economics and Policy

Instructor: Jacoby, H.D.

Ziminerman, M.B.

(617) 253-6607

Course Number: 15:923

Departn:ent: Management - Applied

Prosiam or

Curriculum: Arch. Study

Credits:

9 .

Student Level: College Graduate

Curation:

Contact Hours: 39

Classroom: $\quad 39$

Topics Covered Extensively: Energy

Conservation; Marketing/Market

Analysis; Solar Energy Policy

Development

Nunber of Times Taught: - 5

Average Enrollnent:

25

Energy frod. from Renewable Resources

Instructor: Fay; J.A.

Course Nurber: (617) 253-2236

Department: Mechanical Engimeering

Prosram or

Curriculum: arch. Study

Credits: 12

Studant Level: College Graduate

Duration: 12 Weeks, $3.0 \mathrm{hrs}$ per week

Contact Hours: 36

Classrcom: $\quad 36$

Topics Covered Extensively: Alternate

Energy Sources; Apprcpriate Technology;

Energy Storage; Heat and Energy

Transfer; Passive Solar Ṫechmology;

Fhotovoltaics; Solar Collector

Evaluaticn/Design; Domestic Hot Water;

Space Heating; Space Cooling; Wind

Power, Central Systems; Wirid Power,

Sinall systems

Number of Times Tought: 2

(2178) 


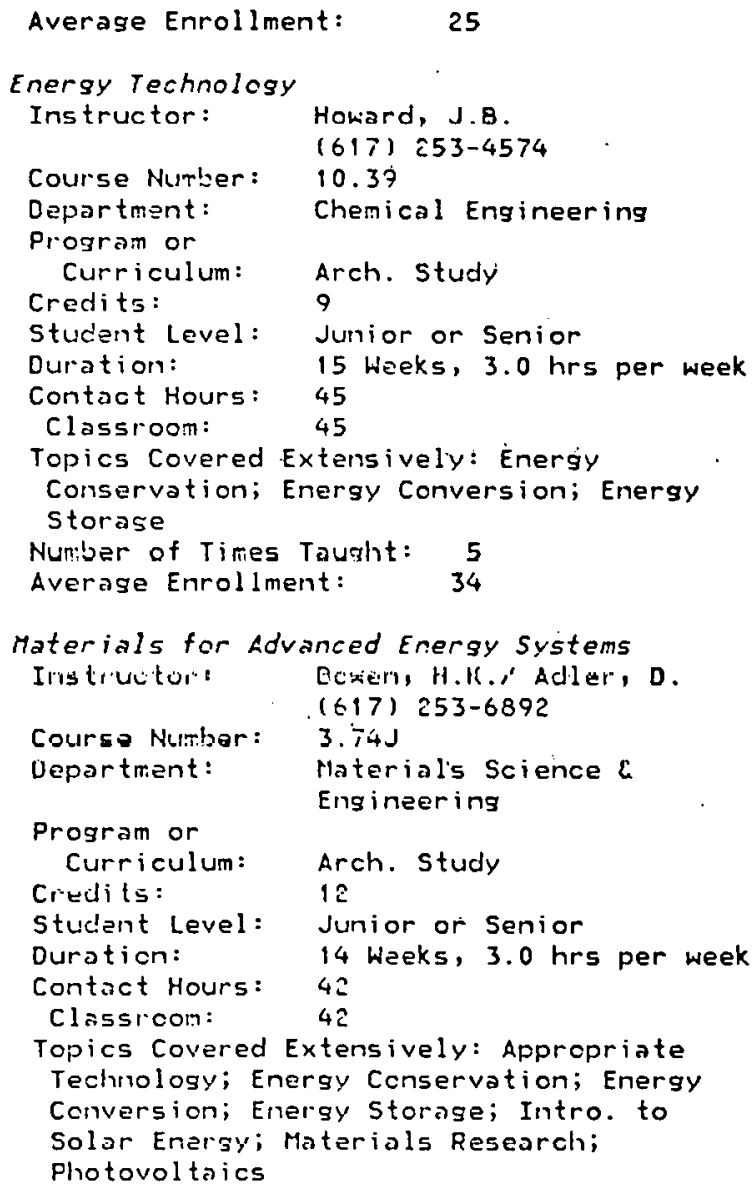

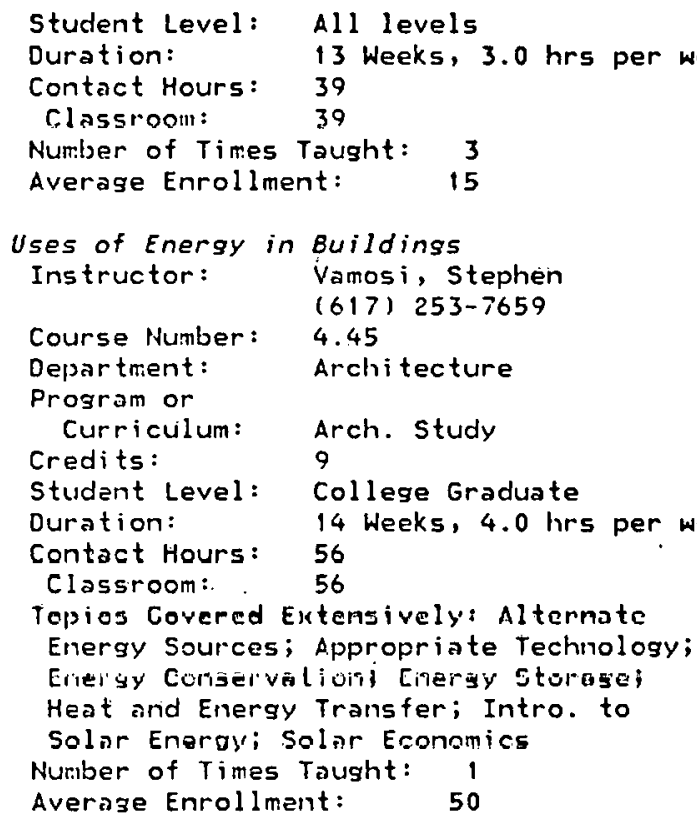


Number of Times Taught: 6 Average Enrollment: $\quad 25$

**********

\section{NORTHEASTERH UHIVERSITY}

(2199)

BOSTON, Massachusetts

(617) 437-2000

\section{SOLAR RELATED COURSES}

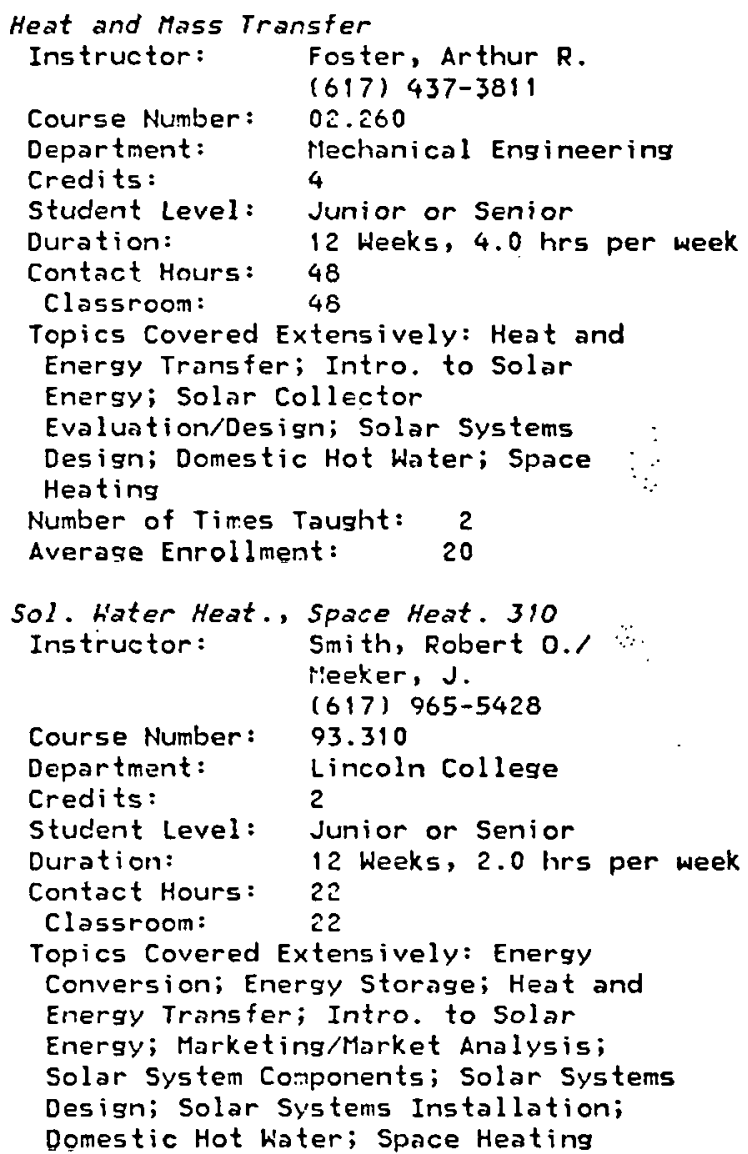

Sol. Hater Heat., Space Heat. 311

Instructor: Smith, Robert 0.1

Meeker, $J$.

(617) 965-5428

Course Number: 93.311

Department: Lincoln College

Credits: 2

Student Level: Junior or Senior

Duration: 12 Weeks, 2.0 hr's pei: wiekek

Contact Hours: 22

Classroom: 22

Topics Covered Extensively: Energy

Conversicn; Energy Storage; Heat and

Energy Transfer; Intro. to Solar

Enersy; Marketing/Market Analysis;

Solar System Compenents; Solar systems

Design; Solar Systems Installation;

Domestic Hot Water; Space Heating

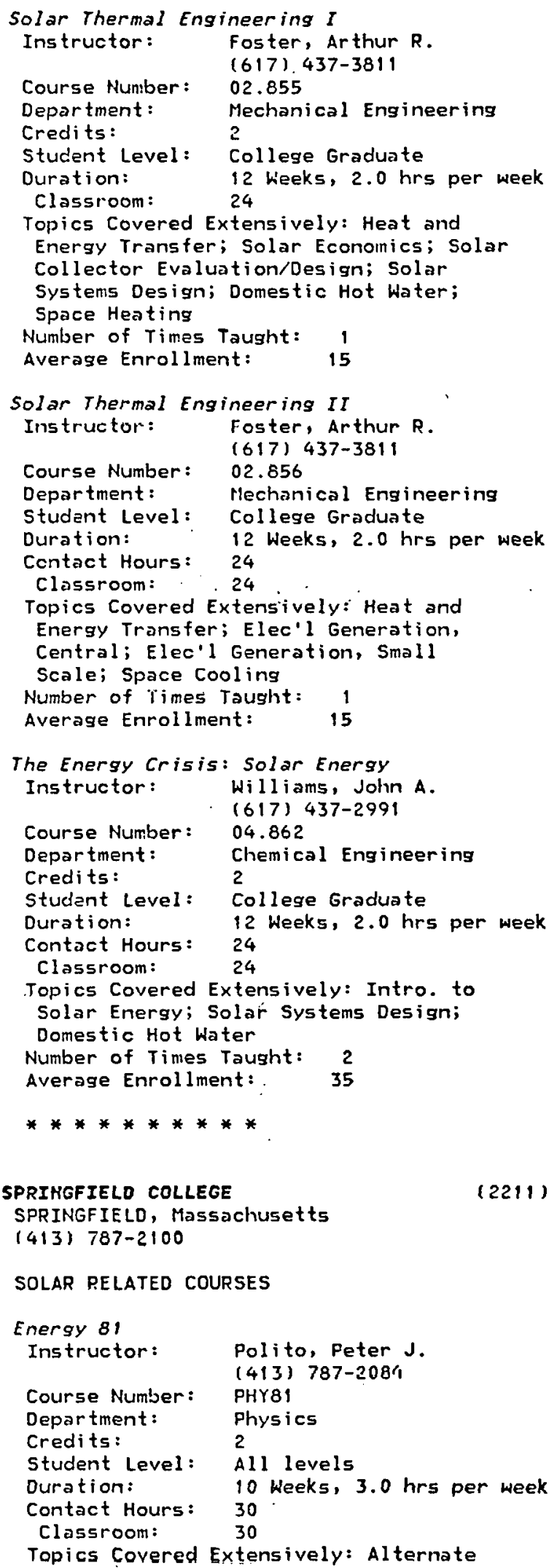


Energy Sources; Appropriate Technology; Energy Conservation; Energy Conversion; Energy Sterase; Heat and Energy Transfer; Intro. to Solar Energy; Passive Solar Technology; Solar System Components; Solar Ecchomics; Solar Home Construction; Domestic Hot Water; Swimming Pcol Heating; Elec'l Generation. Central; Elec'l Generation, Small Scale; Space Heating; Space Cooling; Hind Power, Central Systems; Wind Power, Small Systems

Energy 82

Instructor: Polito, Peter J. (413) 787-2084

Course Number: PHY 82

Uepartment: Hhysics

Credits: 2

Student Level: All levels

Duration: $\quad 10$ heeks, 3.0 hrs per week

Contact Hours: 30

Classroom? 30

Topieg Covered Extensively: Alternate

Energy Sources; Appropriate Technology; Energy Conservation; Energy Conversion;

Energy Storase; Heat and Energy

Transfer; Intro. to Solar Energy;

Passive Solar Technology; Solar System

Components; Solar Econcmics; Solar Home

Construction; Dorrestic Hot Water;

Swimaing Pool Heating; Elec'l

Generaticn, Central; Elec'l Generation,

Small Scale; Space Heating; Space

Cooling; Hind Fower, Central Systems;

Wind Power, Small Systems

Energy 83

Instructor: Politn. Peter .1. (413) 787-2084

Course Number: FHY 83

Department: Fhysics

Credits:

Student Level: All levels

Duration: 10 Weeks, $3.0 \mathrm{hrs}$ per week

Contact Hours: $\quad 30$

Classroom: $\quad 30$

Topics Covered Extensively: Alternate Energy Sources; Appropriate Technology;

Energy Conservation; Energy Conversion;

Energy Storase; Heat and Energy

Transfer; Intro. to Solar Energy;

Fassive Solar Technology; Solar System

Components; Solar Economics; Solar Home

Construction; Domestic Hot Hater;

Swimming Fool Heating; Elec'l

Generation, Central; Elec'l Generation,

Sinall Scale; Space Heatirig; Space

Cooling; hind Poker, Central Systems;

Wind Fower, Small Systems
STHESTH MASS UMIVERSITY
NORTH DARTMOUTH, Massachusetts
(617) 997-9321

$(2210)$

\section{SOLAR RELATED COURSES}
Energy and Energy Alternatives
Instructor: Eento, Robert
(617) 997-9321
Course Number: PH 163
Department: Physics
Credits: 3
Student Level: All levels
Duration: 13 Weeks, $3.0 \mathrm{hrs}$ per week
Contact Hours: 39
Classroom: $\quad 39$
Topics Covered Extensively: Alternate Energy Sources; Appropriate Technology;
Enersy Conservation
Humber of Tin:pa Taught: ?
Averase Enrollment: $\quad 30$
Science, Technology, and Society I
Instructor: Bento, Robert
Course Number: $\quad$ PH 161 997-9321
Departmen
Credits: 3
Student Level: All levels
Duration: $\quad 13$
$\begin{array}{ll}\text { Contact Hours: } & 39 \\ \text { Classroom: } & 26\end{array}$
Laboratóry: 13
Topics Covered Extensively: Al ternate
Energy Sources; Appropriate Technology;
Intro. to Solar Energy
Number of Times Taught: 5
Average Enroliment: $\quad 100$

***********

HEHTHORTH IHST OF TECH

EOSTON, Massacluusetts

(617) 442-9010

\section{SOLAR RELATED COURSES}

* Arch: Solar and Ener. Conservation

Instructor: Balichi, George

Departrient: Architecture

Topics Covered Extensively: Energy Conservation; Intro. to Solar Energy; Passive Solar Technology; Solar Home Construction

$* * * * * * * * * *$

WOPCESTER POLY INSTITUTE

WORLESIER, Massachusetts (617) 753-1411

PROGRAMS ANID CURRICULA

Major Qual ifying Project

Degrea: BS, Science

Contact: Bolz, R.E.

(617) 753-1411

$(29099)$ 


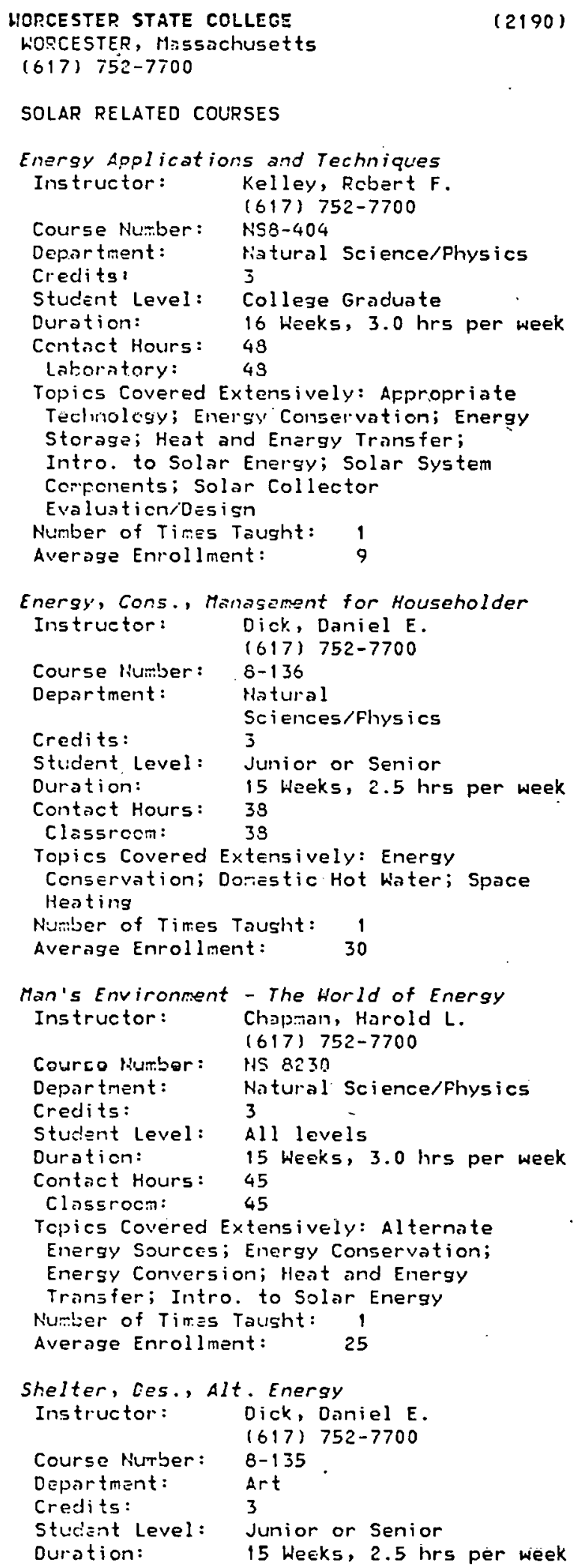

HORCESTEP STATE COLLEGE

K'ORCESTER, Massachusetts

(617) 752-7700

SOLAR RELATED COURSES

Energy Applications and Techniques

Instructor: Kelley, Rcbert F.

(617) 752-7700

Course Number: NS8-404

Department: Niatural Science/Physics

Credits: 3

Student Level: College Graduate

Duration: 16 Heeks, $3.0 \mathrm{hrs}$ per week

Centact Hours: 48

Laborintory: 43

Topics Covered Extensively: Appropriate

Techrolesy; Enersy Conservation; Energy

Storage; Heat and Enargy Transfer;

Intro. to Solar Energy; Solar System

Corponents; Solar Collector

Evaluaticnidesisn

Number of Times Taught: 1

Average Enrollment: 9

Energy, Cons., Manasement for Householder

Instruetor: Dick, Daniel E.

$(617) 752-7700$

Course liumber: 8-136

Department: Natural

Credits:

Sciences/Fhysics

j

Student Level: Junior or Senior

Duration: 15 Heeks, 2.5 hrs per week

Contact Hours: 33

Classrocm: 38

Topics Covered Extensively: Energy

Conservation; Dorestic Hot Water; Space Heating

Number of Times Tausht: 1

Average Enrollment: $\quad 30$

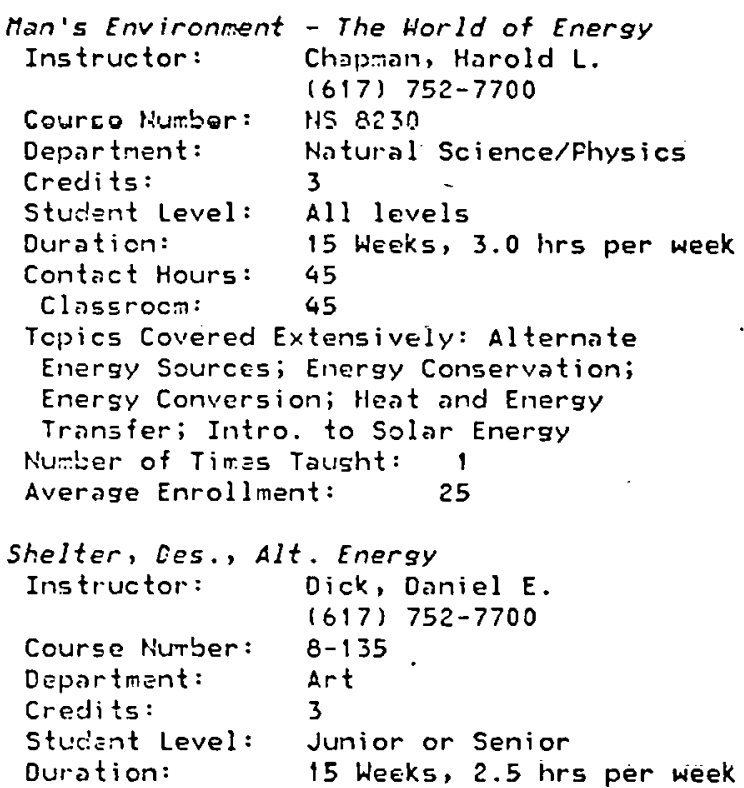

Contact Hours: 38

Classroom: $\quad 38$

Topics Covered Extensively: Al ternate

Energy Sources; Energy Storase; Intro.

to Solar Energy; Passive Solar

Technolcgy; Solar Home Construction;

Solar Systems Design; Space Heating

Number of Times Tausht: 4

Averase Enrollment:

12

\section{Community/Junior collezes \\ BRISTOL COPMLHITY COLLEGE \\ FALL RIVER, Massachusetts \\ (617) 678-2811}

PROGRAMS AND CURRICULA

*Energy Prostam

\section{BUAXER HILL CITY COLLEGE}

CHARLESTO!IN, Massachusetts

(617) $241-8600$

SOLAR RELATED COURSES

Issues in Energy

Instructor:

Chisholm, Francis E.

(617) $241-8600$

Department:

Credits: Soience

Student Level: Freshman or Sophomore

Duration: 15 Weeks, $3.0 \mathrm{hrs}$ per week

Contact Hours: 45

Tcpics Covered Extensively: Alternate

Energy Sources; Energy Conservation;

Intro. to Solar Energy

\section{CAFE COJ CHTY COLLEGE}

(2168)

WEST BARNSTABLE, Massachusetts

(617) 362-2131

PROGRAMS AHID CURRICULA

Energy Systems Technology

Degree: $A D$, Science

Contact: Panitz, Ted

$$
\text { (617) 362-2131 }
$$

Students Taking or Completing Offering:

Solar Technician
(2176)

$(11210)$ 


\section{SOLAR RELATED COURSES}

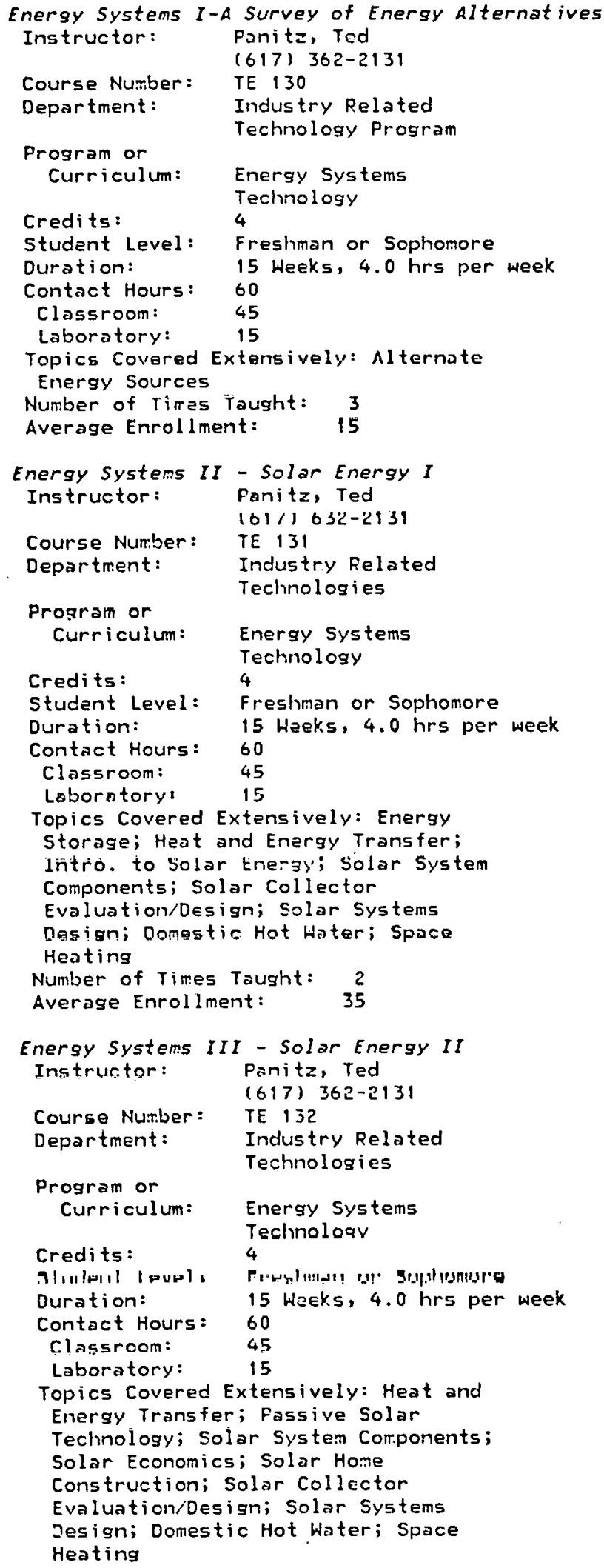

Number of Times Taught: 1 Average Enrollment: 18

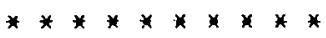


KORTHEAST IHSTITUTE OF INDUSTRIAL TECHNOLOSY

(90060)

41 Phillips st.

Boston, Massachusetts 02114

PROGRAMS AND CURRICULA

Installing Solar Hater Heaters

Degree: Solar hater Systems

Contact: Galvin, G. M.

(617) 523-2813

\section{SOLAR RELATED COURSES}

Installing Solar Hater Heating

Instructor: Smith, Robert 0.1

Lannon, $E$.

(617) 523-2813

Department :

Air Conditioning

Refrigeration Tech.

Program or

Curriculum: Installing Solar Water

Heaters

Student Levẹl: tôllegé bráduatë

Duration: 15 heeks, 2.0 hrs per week

Contact Hours: $\quad 30$

Classroom: $\quad 30$

Number of Times Taught: 4

Averaye Enrollnent: $\quad 30$

THE CAPIBRIDGE SCHOOL - HESTON CHT.

FOR OFEN EDU.

$(90200)$

Weston, Massachusetts

SOLAR RELATED COURSES

*Adapting Heating Systerrs for Solar Use

(617) 965-5428

Topics Covered Extensively: Space

Healing

*adv. Studies in Solar Heating

(617) 965-5428

Topics Covered Extensively: Space Heating

* Basic solar Heating

$161 / 1) 4 b b-b 428$

Topics Covered Extensively: Space

llestins

* Biomass for Energy

$(017) 305.5420$

Topics Covered Extensively: Biomass

Conviersion

* Designing Your Oun Solar System

(617) 965-5428

Topics Covered Extensively: Solar System

Components; Solar Collector

Evaluation/Dasign; Solor Systems

Design; Space Heating

*photovoltaics

(6:7) 965-5428

Topics Covered Extensively:

Photovoltaics
*Power from the Sea

(617) $965-5428$

*Small Hind Mills

(617) 965-5428

Topics Covered Extensively: Wind Power,

Small Systems

* Solar Heat ing Added to Your House

(617) 965-5420

* Solar Heating system design

(617) 965-5428

Topics Covered Extensively: Solar System Components; Solar Collector

Evaluation/Design; Solar Systems Design; Dumestic llot Hater 1 jpace Heating

*Hind Machines

(617) 965-5420

Topics Covered. Extensively: Hind Power, Centrsl Systems; Wind Power, 5imall Systems 


\section{Colleses/Universities}

CERTRAL MICH UNIVERSITY

(2243)

MIOLNT PLEASANT, Michisan 48858

(517) 774-3151

\section{SOLAR RELATED COURSES}

Energy Efficient Design and Cons.

Instructor: Ecker, Louis G./ Nee, Join

(517) 774-3996

Course Number: 697

Departiment: Industrial

Credits: Eriscation/Technology

Stud $\equiv n t$ Level: Junior or Senior

Duration: 6 Heeks, 13.0 hrs per week

Contact Hours: 60

Classroom: $\quad 60$

Laboratory: 20

Topics Covered Extensively: Alternate

Energy Sources; Appropriate Technology;

Energy Conservation; Energy Storage;

Heat and Energy Transfer; Intro. to

Solar Energy; Passive Solar Technology;

Solar Economics; Solar Home

Construction; Solar Collector

Evaluation/Desisn; Solar Sistems

Design; Solar Systers Installation;

Domestic Hot Hater; Swimming Pool

Heating; Space Heating; Space Cooling

Number of Times Taught: 1

Average Enrollment: $\quad 30$

$* * * * * * * * * *$

\section{DETROIT, UMIVERSITY OF}

DETROIT, Michigan 48221

(2323)

(313) $927-1000$

SÓLAR RELATED COURSES

Energy $\varepsilon$ Architecture

Instructor: LaGrassa, Stephen

(313) 927-1532

Course Number: ART 514

Deppartment: Architecture

Credits: 3

Student Level: College Graduate

Duration: $\quad 15$ Weeks, 3.0 hrs per week

Contact Hours: 45

Classroom: $\quad 45$

Number of Times Taught: 1

Average Enrollment: $\quad 15$

***********

EASTEPN MICH UNIVERSITY

$(2259)$

YPSILAPITI, Michigan 48197

(313) 487-1849

SOLAR RELATED COURSES

Solar Energy in Construction

Instructor: Kicklighter, Clois E.

Course Number: 539
Department: Industrial

Technology/Industrial

Education

Credits:

2

Studant Level: College Graduate

Duration:

2 Weeks, 15.0 hrs per week

Contact Hours: 30

Classroom: $\quad 20$

Laboratory: 10

Topics Covered Extensively: Energy

Conservation; Energy Conversion; Energy

Storage; Heat and Energy Transfer;

Intro. to Solar Energy; Materials

Research; Solar System Components;

Solar Home Construction; Solar

Collector Evaluation/Design; Solar

Systens Design; Solar Systems

Installation; Solar Systems Testing and

Evaluation; Domestic Hot Water

Nuliber of Times Taught: 1

Average Enrollment: $\quad 16$

***********

FERRIS STATE COLLEGE

$(2260)$

BIG RAPIDS, Michigan 49307

(616) 796-9971

PROGRAMS AND CURRICULA

Refrig., Heat ing and Air Conditioning

Technology

Uegree:

$A D$, Applied Science in

Refrig., Heating, and Air

Conditioning

Contact: Shane, James B.

(616) 796-9971

Students Taking or Completing offering:

Installer-Comrercial (Solar System), Installer-Residential (Solar System), Solar Technician, Trade Specialty

SOLAR RELATED COURSES

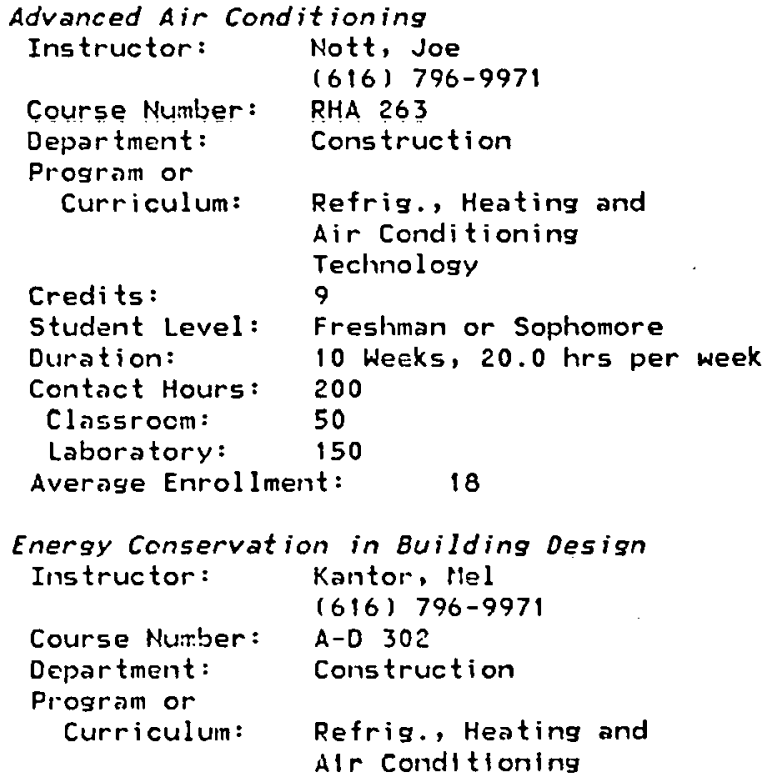




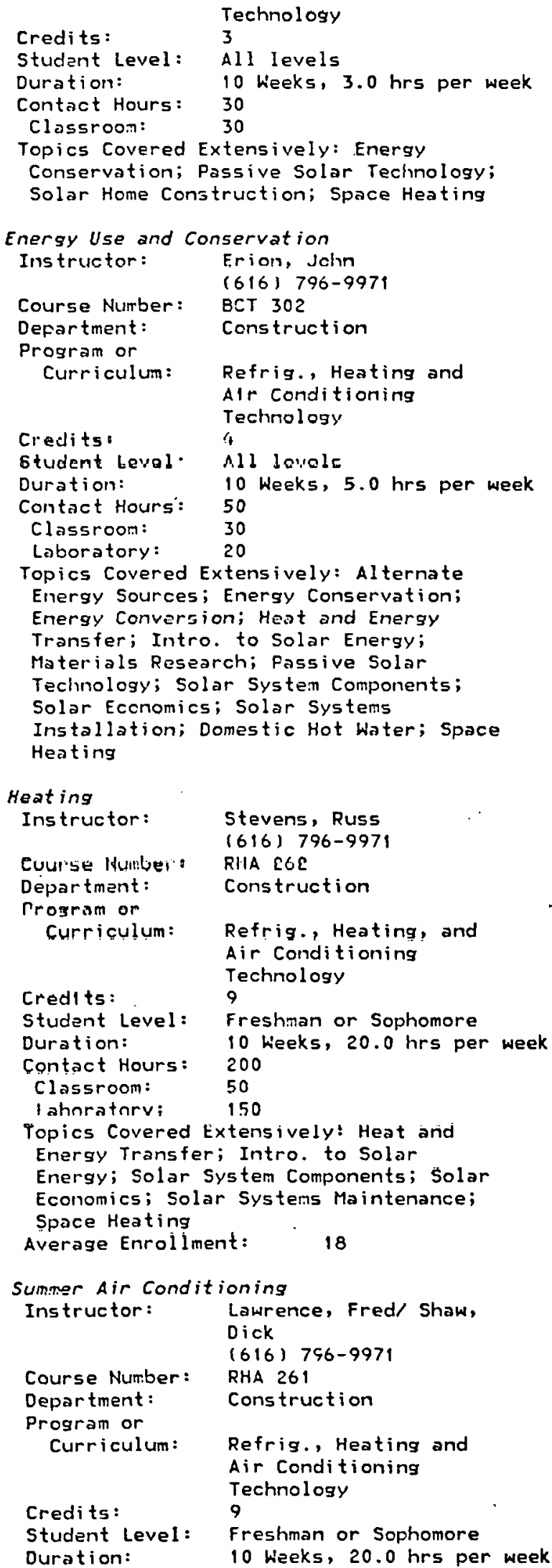

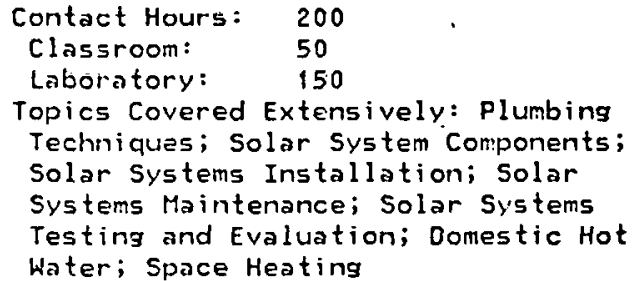

Alt. Enerr. Emph. - Urban, Environ.

Studs.

Degree: BS, Urban, Envi.

Studies-Alt. Ener.

Contact: Bailey, Rod (616) 895-6611

Students Taking or Completing Offering: 
Educator, Do-it-yourself Homeowner, Solar Technician

\section{SOLAR RELATED COURSES}

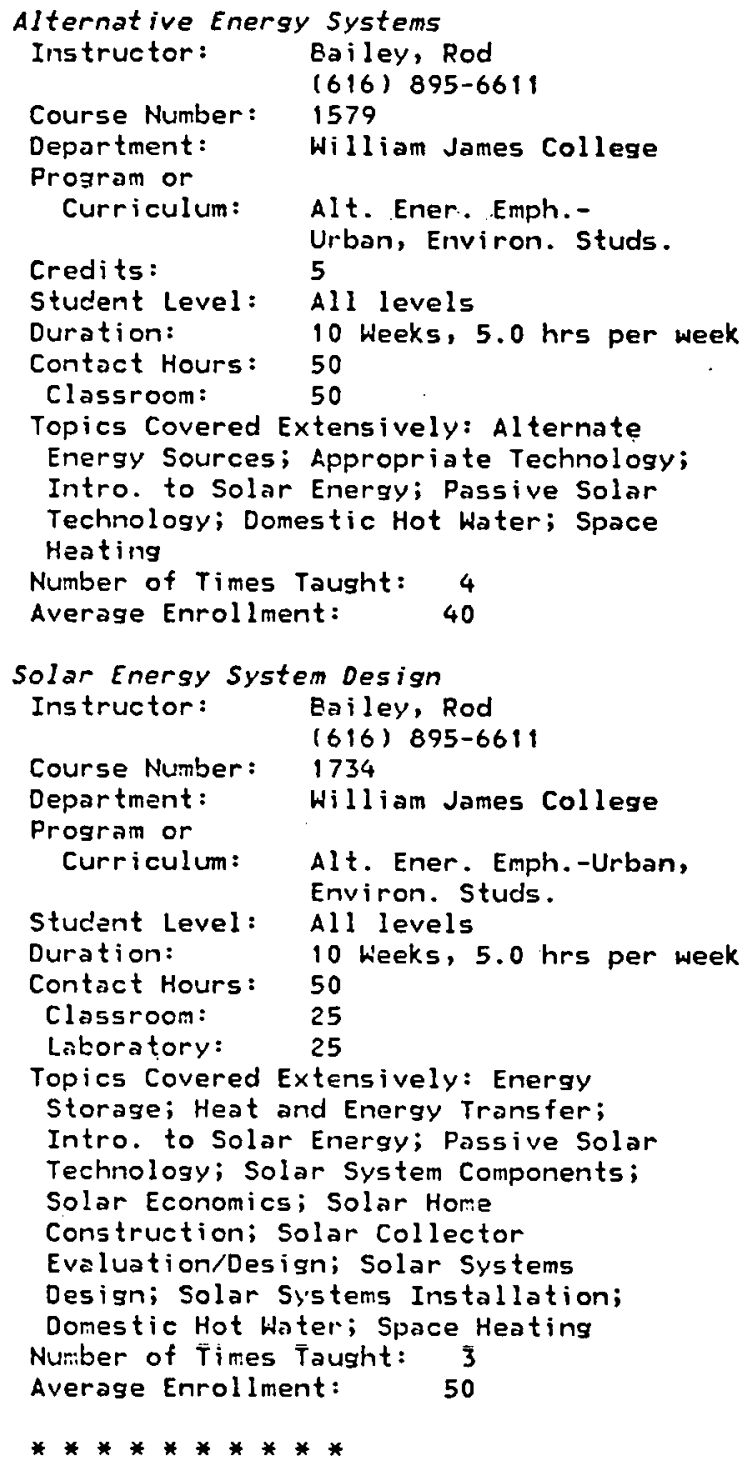

JORDAN COLLEGE

CEDAR SPRINGS, Michigan 49319

(29091)

(616) 696-1180

\section{PROGRAMS ARTD CURRICULA}

Energy and Environmental studies

Degree: BS, Alternate and Envircnitental Studies

Contact: Till, Gordon Vander (616) 696-1180

Students Taking or Completing offering: Educator, Researcher, Solar Technician

\section{SOLAR RELATED COURSES}

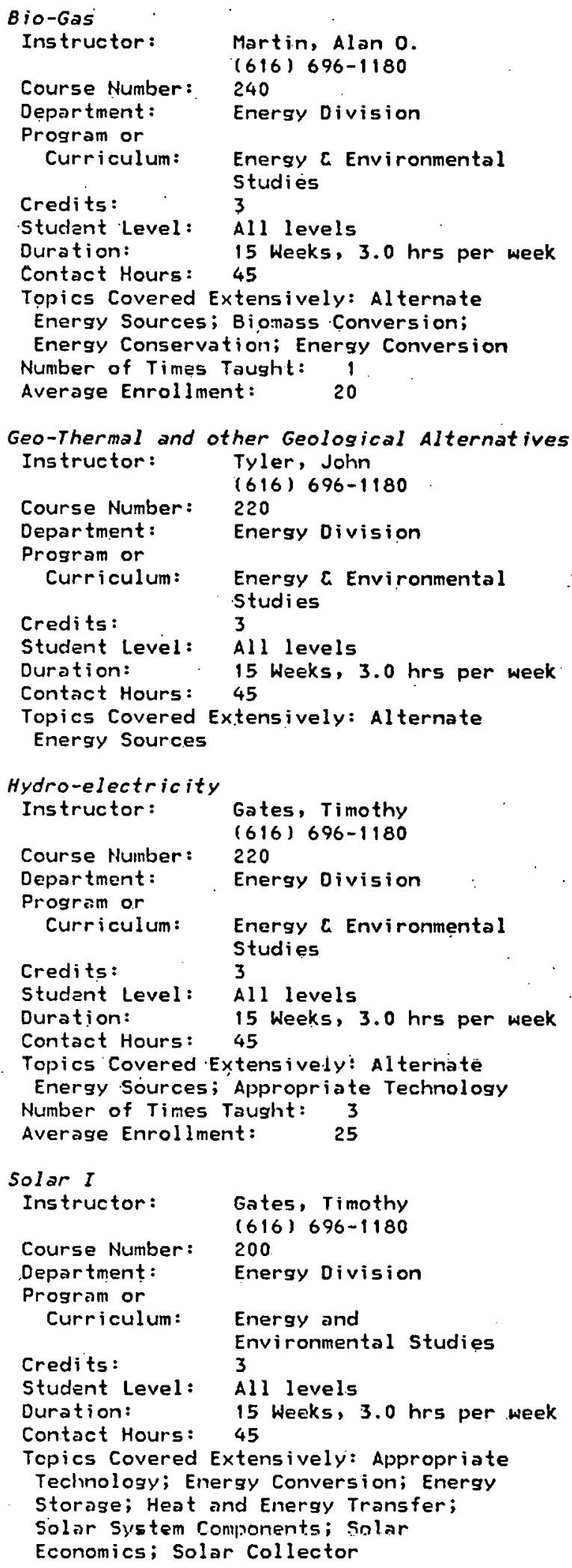


Evaluation/Design; Solar Systems Desisn; Solar Systems Installation; Solar Systens Maintenance; Domestic Hot Water; Space Heating Number of Times Tought: 8 Average Enrollment: 50

Wind Energy Conversion Systems

Instructor: Bregg, Gary

Course Number: 210

Department: (616) 696-1180 210

Program or . Curriculum: Energy Division

Credits: Energy and Environmental Studies

Student Level: All levels

Duration: 15 Weeks, 3,0 hrs per week

Contact Hours: 45

Tuplcs Cuverted Extens lvely: Al ternate

Enargy Souroes; Enepy' Conseriation;

Energy Conversion; Energy Storase;

Materials Research; Hind Power, Small Sygteing

Number of Times Taught: 3

Average Enrollment:

20

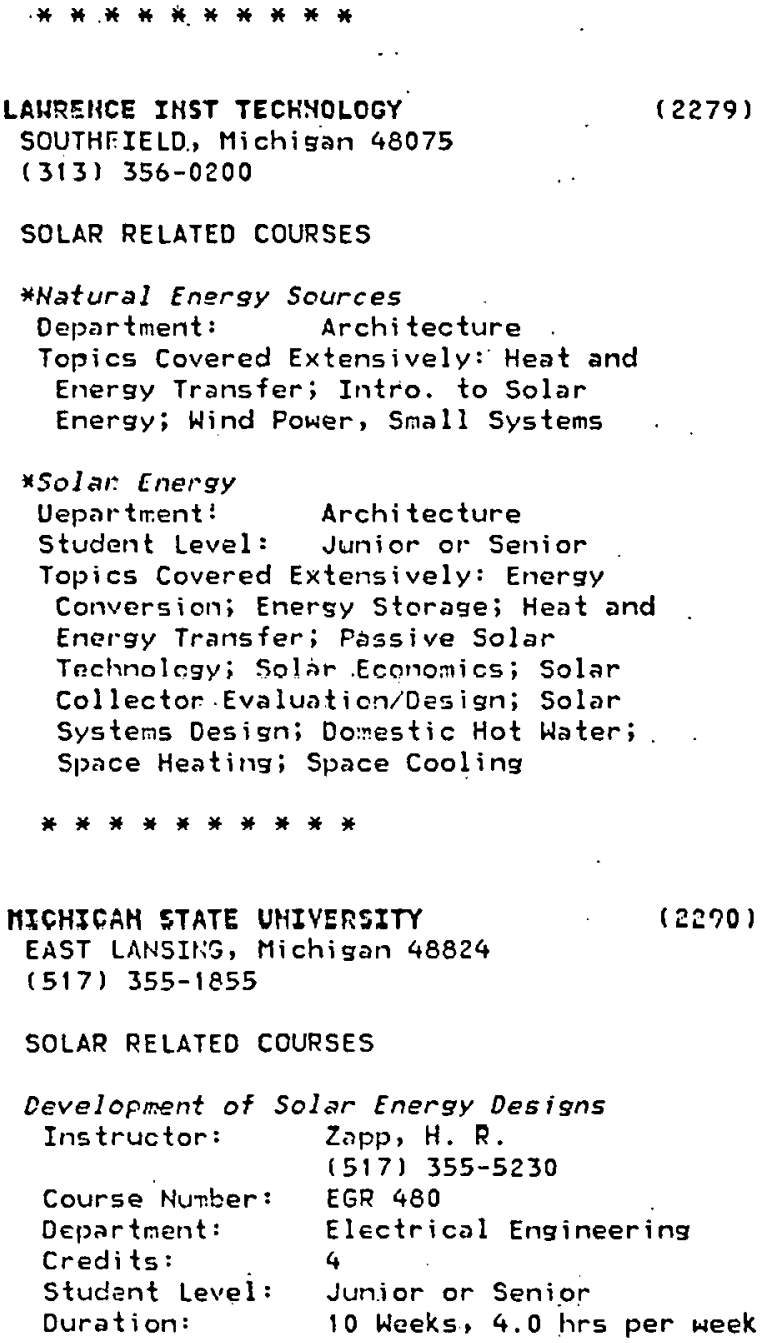

$\begin{array}{cl}\text { Contact Hours: } & 40 \\ \text { Classroom: } & 40\end{array}$

Topics Covered Extensively: Alternate

Energy Sources; Appropriate Technology;

Energy Conservation; Energy Conversion;

Energy Storage; Heat and Energy.

Transfer; Intro. to Solar Energy;

Marketing/ilarket Analysis; Passive

Solar Technology; Photovoltaics; Solar

Energy Policy Development; Solar System Components; Solar Economics; Solar Home

Construction; Solar Collector

Evaluation/Design; Solar Systems

Design; Space Heating; Wind Power,

Central Systems; Wind Power, Small

Systens

Number of Times Taught: 2

Averase Enrollment: $\quad 30$

urrect inergy conversion

Instructor' 1 Kerber, $R$.

Course Number: $\quad$ ME 414 353-9492

Department: Hechanleal Engineering

Credits: 3

Student Level: Junior or Senior

Duration: 10 Weeks, 3.0 hrs per week

Contact llours: 30

Classroom: 30

Topics Covered Extensively: Alternate

Energy Sources; Appropriate Technology;

Energy Conservation; Energy Conversion;

Energy Storase: Heat and Energy

Transfer; Fhotovoltaics; Elec'l

Generation, Small Scale

Number of Times Taught: 5

Average Enrollment:

30

Solar Energy Copversion

Instructor: Dhanak, A. M.

(517) 355-5160

Course Number: ME 490

Departinent: Mechanical Engineering

Credits: 4

Studait Level: Junior or Senior

Duration: $\quad 10$

Contact Hours: 40

Clossroom: 40

Topics Covered Extensively: Energy

Conversion; Energy Storage; Heat and

Energy Transfer; Intro. to Solar

Energy; Domestic Hot Water; Space

Heating; Space Cooling

Number of Times Taught:

Average Enrollment:

1
50

Technology and Utilization of Energy

Instructor:

Dhanak, A. M.

(517) 355-5160

Course Number: HE 300

Department: Mechanical Engineering

Credits: 3

Student Level:. Junior or Senior

Duration: io Weeks, $3.0 \mathrm{hrs}$ per week

Contact Hours: 30

Classroom: $\quad 30$

Topics Covered Extensively: Alternate

Enersy Sources; Energy Conversion

Number of Times Tausht: 7

Averase Enrollment:
60 


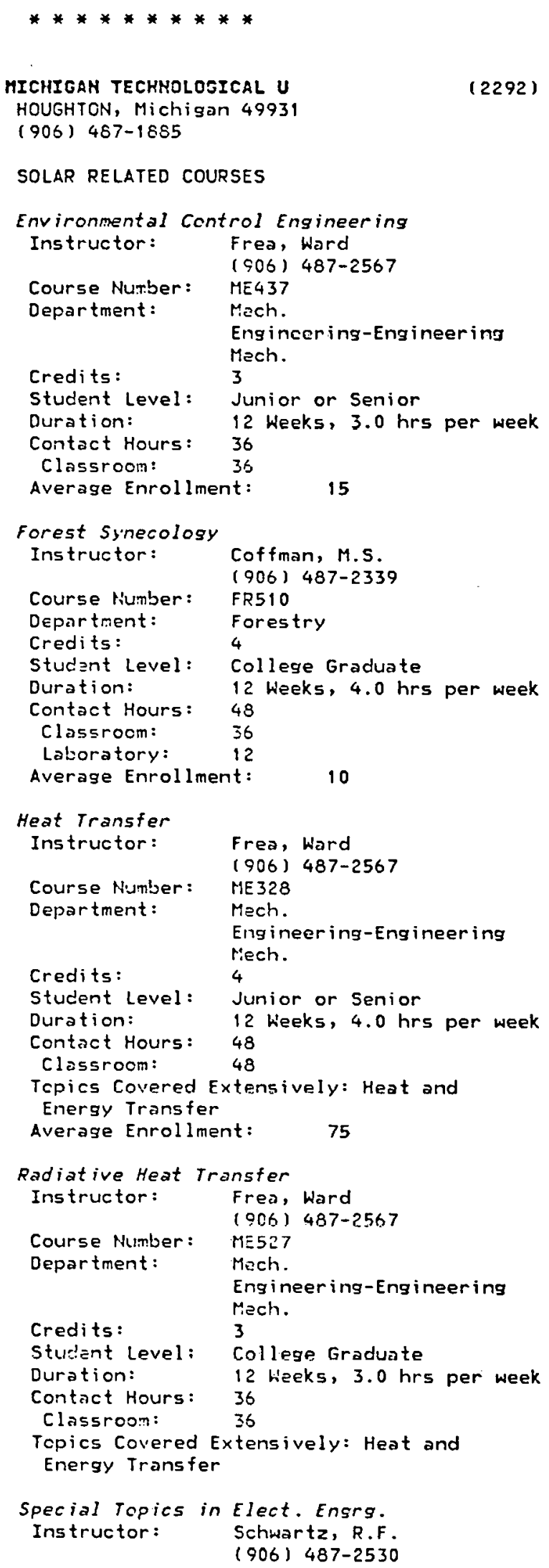

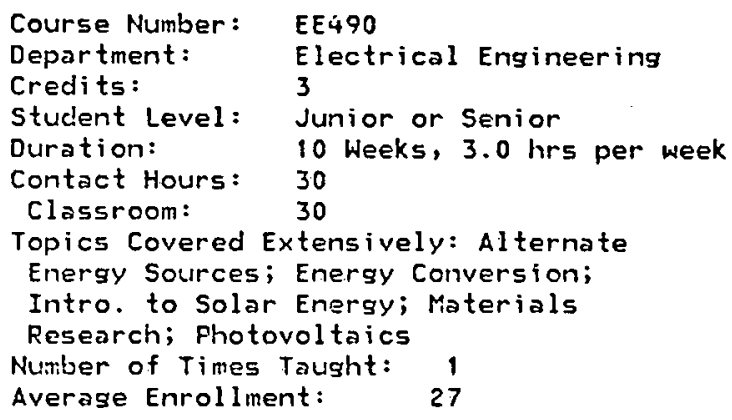




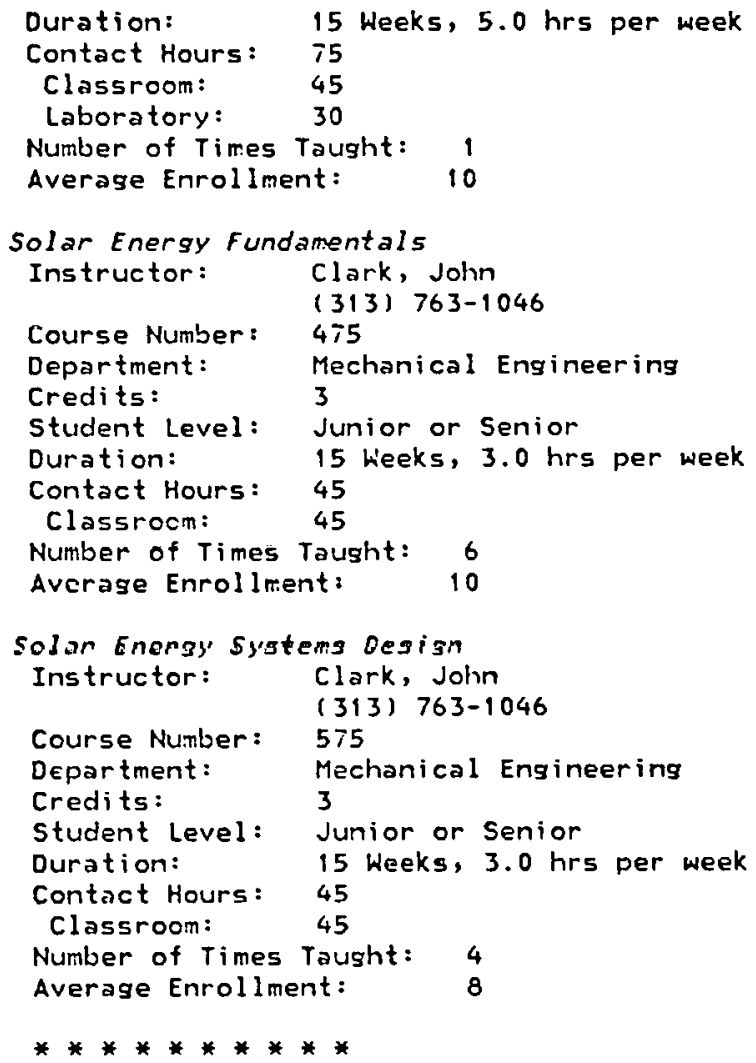

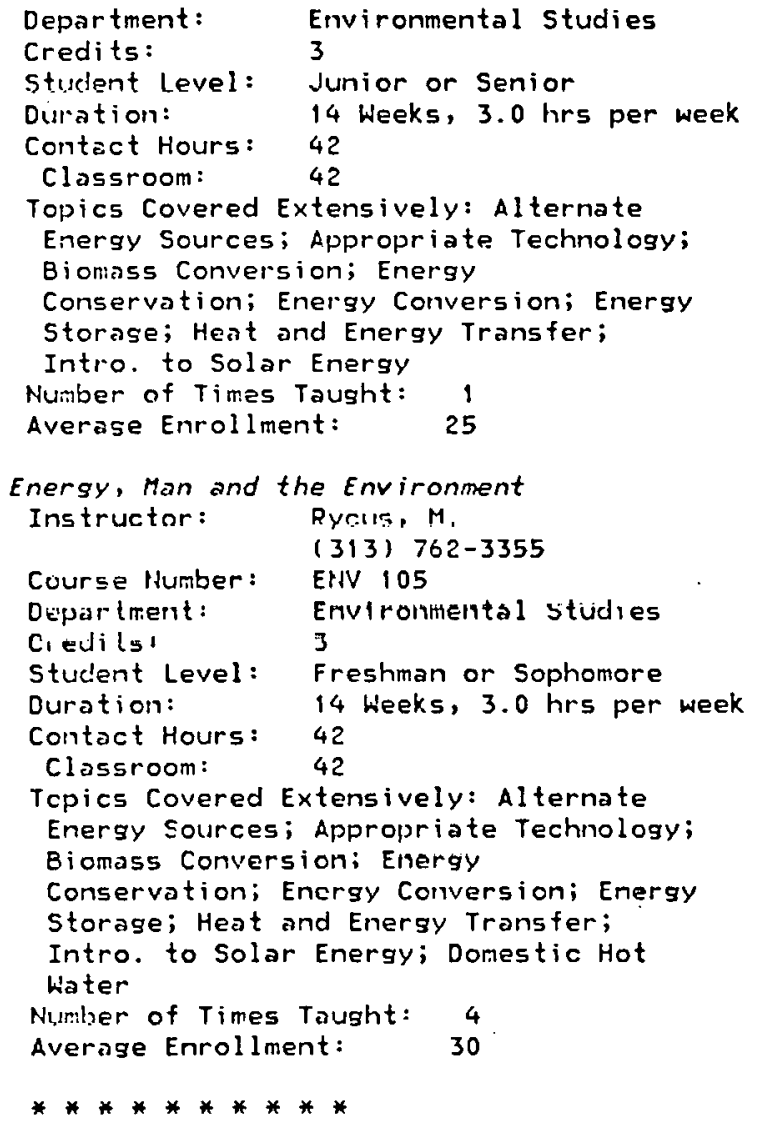

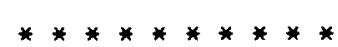


OAKLAND UNIVERSITY

(2307)

ROCHESTER, Michigan 48063

(313) 377-2100

\section{SOLAR RELATED COURSES}

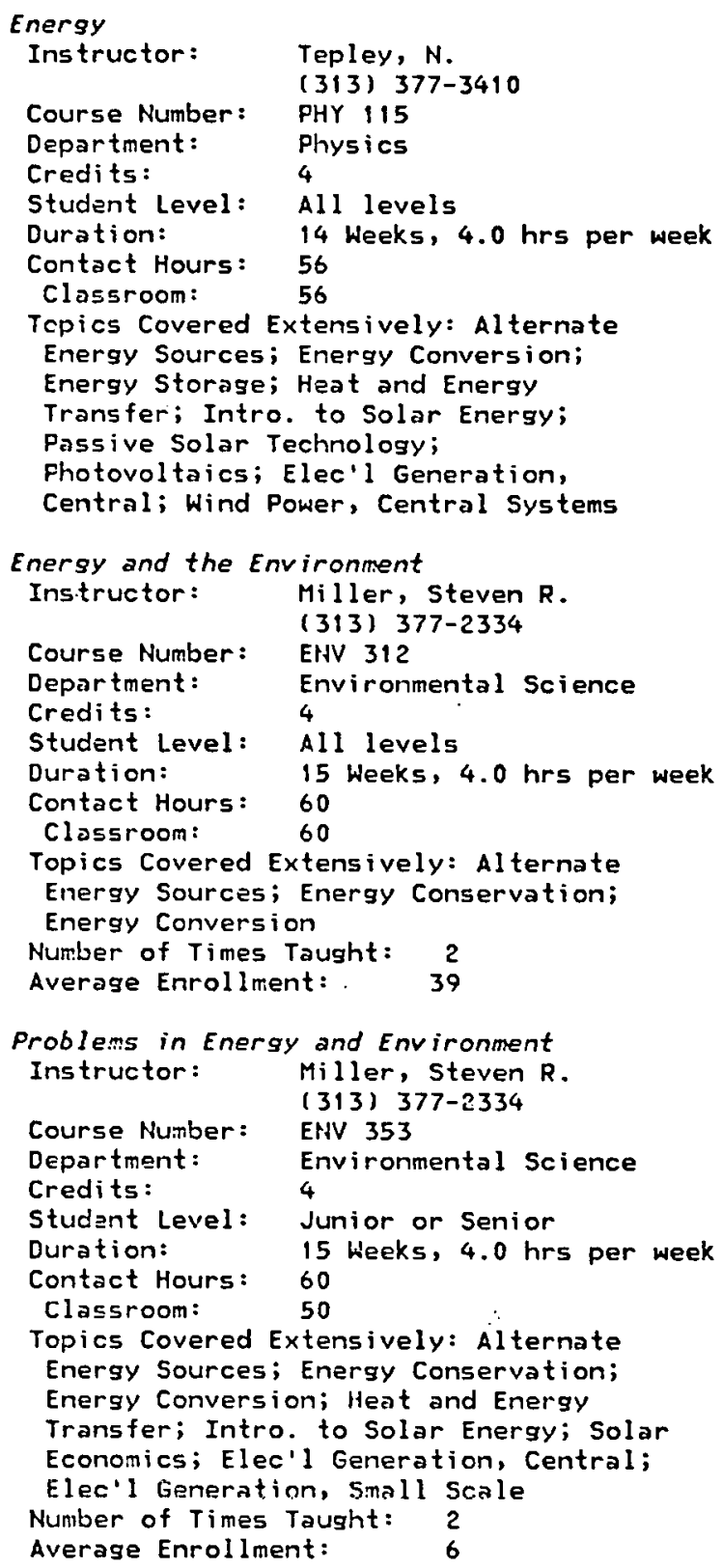

SAGIHAH VLY STATE COLLEGE

(2314)

UNIVERSITY CENTER, Michigan 48710

(517) 793-9800.

\section{SOLAR RELATED COURSES}

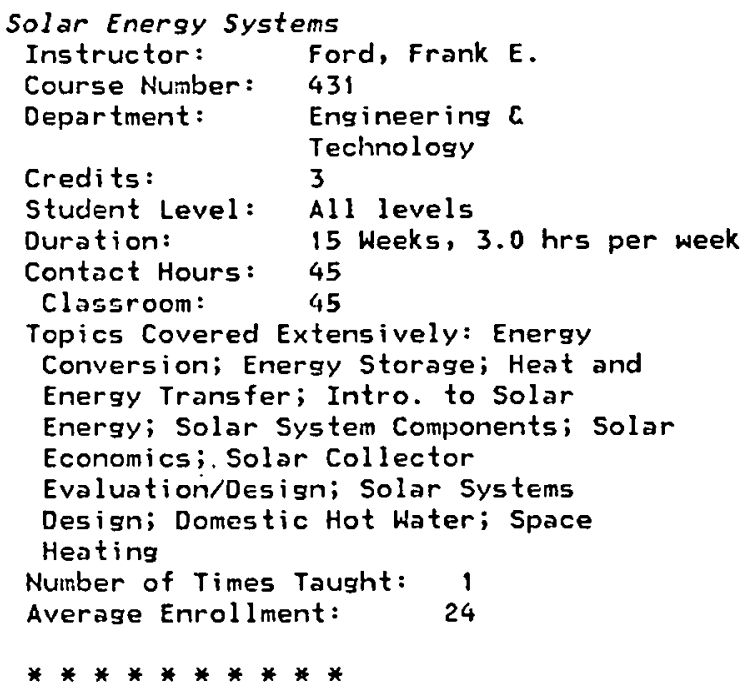

Credits: 4

Student Level: Freshman or Sophomore

Duration: Contact Hours: 44

Classroom: $\quad 11$

Topics Covered Extensively: Alternate

Energy Sources; Energy Conservation

Piumber of Times Taught: 5

Average Enrollment: $\quad 600$ 
Program in Environ. Studies 502

Instructor: Saperstein, A.M.

Course Number: ENV 502

Department: I.E., Physics, Pol.

Sci.

Credits: 4

Student Level: Junior or Senior

Duration: $\quad 30$ Weeks, $4.0 \mathrm{hrs}$ per week

Contact Hours: 120

Number of Times Tausht: 3

Average Errollment: $\quad 10$

Prosram in Environ. Studies 503

Instructor: Saperstein, A.M.

Course Number: ENIV 503

Department: I.E., Physics, Pol.

Sci.

Credits: 4

Student Level: Junior or Senior

Duration: $\quad 3 n$ Woeks. 4 n hrs ner week

renntart Hnura; i?n

Number of Times Tausht: 3

Average Enrollment: $\quad 10$

Program in Environmental studies

Instructor: Saperstein, A.M.

Course Humber: ErV 501

Department: $\quad$ I.E., Physics, Pol.

Credits: 4

Student Level: Junior or Senior

Duration: $\quad 30$ Weeks, $4.0 \mathrm{hrs}$ per week

Contact Hours: 120

Number of Times Tausht: 3

Average Enrollment: $\quad 10$

Residential Solar Energy

Instructor: Bowen, David R.

(313) 577-4631

Course Number: GST 2203

Department: Lifelong Learning,

Univ. Studies/Weekend

Coll. Prog

Credits: 4

Studient Level: Freshman or Sophomore

Duration:

Contact Hours: 34

Classroom: $\quad 34$

Topics Covered Extensively: Alternate

Energy Sources; Energy Storage; Intro.

to Solar Energy; Passive Solar

Technology; Solar Energy Policy

Development; Solar Sy'stem Components;

Solar Economics; Solar Home

Construction; Solar Systems Design;

Solar Systems Installation; Domestic

Hot. Water; Space Heating

Number of Timas Taught: 2

Average Enrollment: $\quad 600$

\section{Senior Seminar}

Instructor:

Course Number:

Department:

Credits:

Stuclent Level:

Duration:

Contact Hours:

Majeske, Penelope K.

( 313 ) 577-4644

4996

Upper Division

4

Junior or Senior

11 Weeks, 4.0 hrs per week 44

Number of Times Taught:

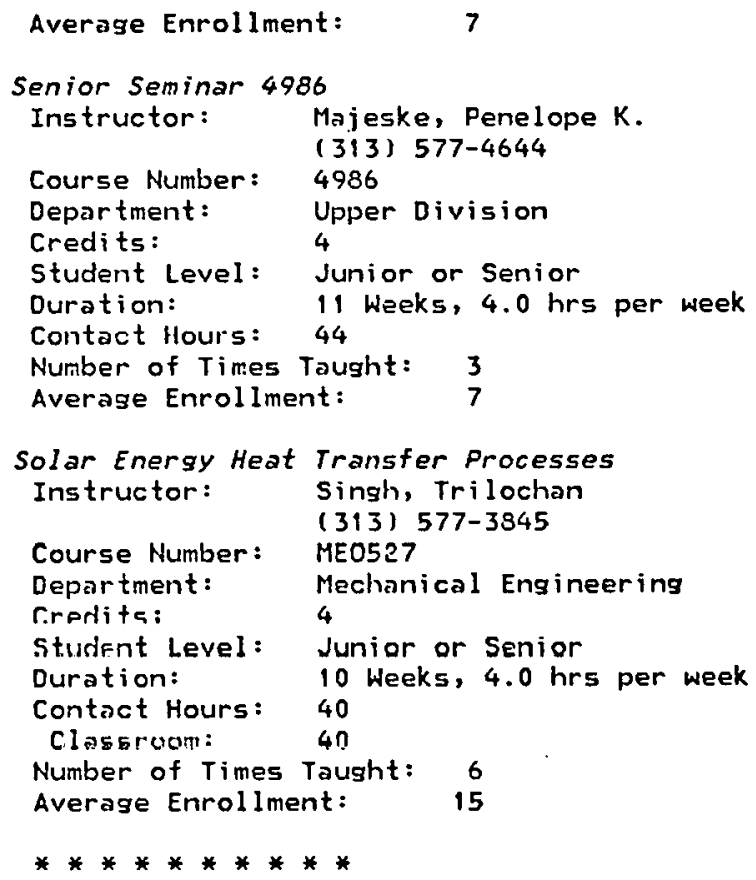

WESTERN MICH UHIVERSITY

(2330)

KalamaZOO, Michigan 49008

(616) 383-1600

SOLAR RELATED COURSES

Solar Energy II

Instructor:

Schubert, R.C.

$(61 \mathrm{~h}) 383-4 n ? 1$

Course Number: 495

Department: Mechanical Engineering

Credits: 3

Student Level: Junior or Senior

Duration: 3 Weeks, $15.0 \mathrm{hrs}$ per week

Contact Hours: $\quad 45$

Classroom: $\quad 45$

Topics Covered Extensively: Alternate

Energy Sources; Appropriate Technology;

Energy Conservation; Energy Conversion;

Energy Storage; Heat and Energy

Transfer; Intro. to Solar Energy;

Passive Solar Technology; Solar System

Compenents; Solar Economics; Solar Home

Construction; Solar Collector

Evaluation/Design; Solar Systems

Design; Solar Systems Testing and

Evaluation; Domestic Hot hater;

Swimming Fool Heating; Space Heating

Number of Times Taught: 1

Average Enrollment:

15

Community/Junior colleges 
CHAS S MOTT CMTY COLLEGE

(2261)

FLINT, Michigan 48503

(313) 762-0200

PROGRAMS APRO CURRICULA

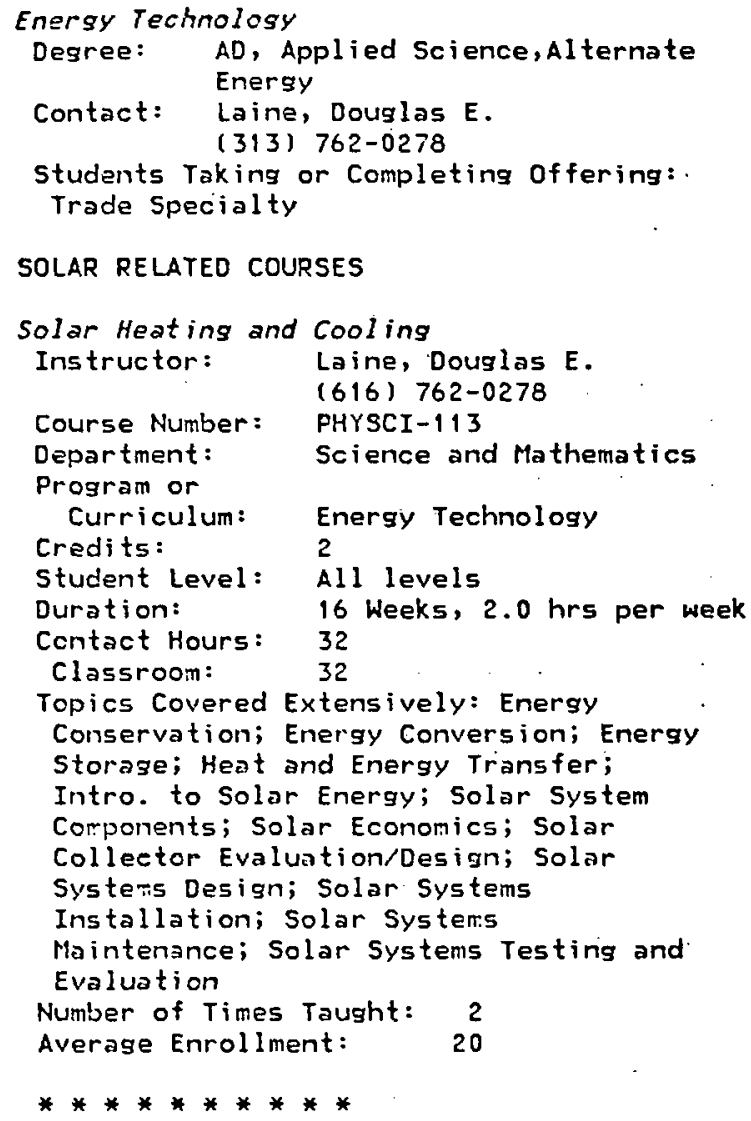

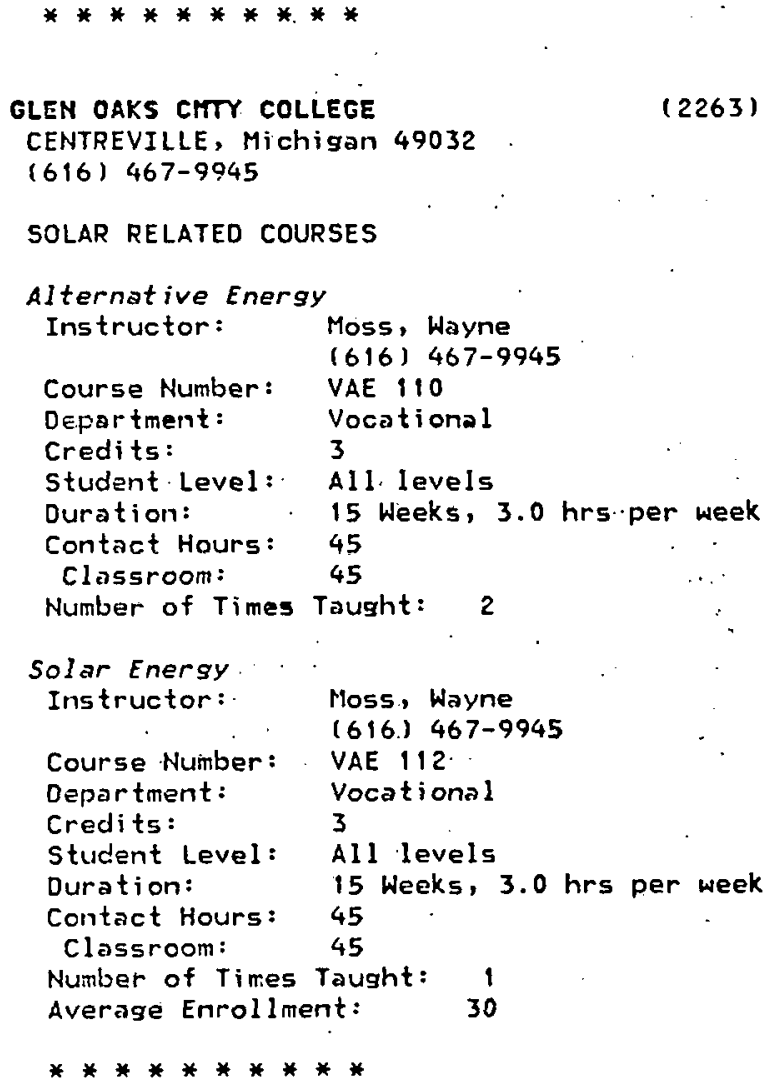

\section{SOLAR RELATED COURSES}

Sol. Sys. - Collector Des. and Cons.

$\begin{array}{ll}\text { Instructor; } & \text { Larson, L. } \\ & (616) 456-4860 \\ \text { Course Humber: } & \text { TE } 245 \\ \text { Department: } & \text { Techinolosy } \\ \text { Program or } & \\ \text { Curriculum: } & \text { Arch. Draft.and Heat., } \\ & \text { Vent., A/ C } \\ \text { Student Level: } & \text { Freshman or Sophomore } \\ \text { Duration: } & 16 \text { Weeks, } 4.0 \text { hrs per week } \\ \text { Contact Hours: } & 64 \\ \text { Classroom: } & 16 \\ \text { Laboratory: } & 32\end{array}$




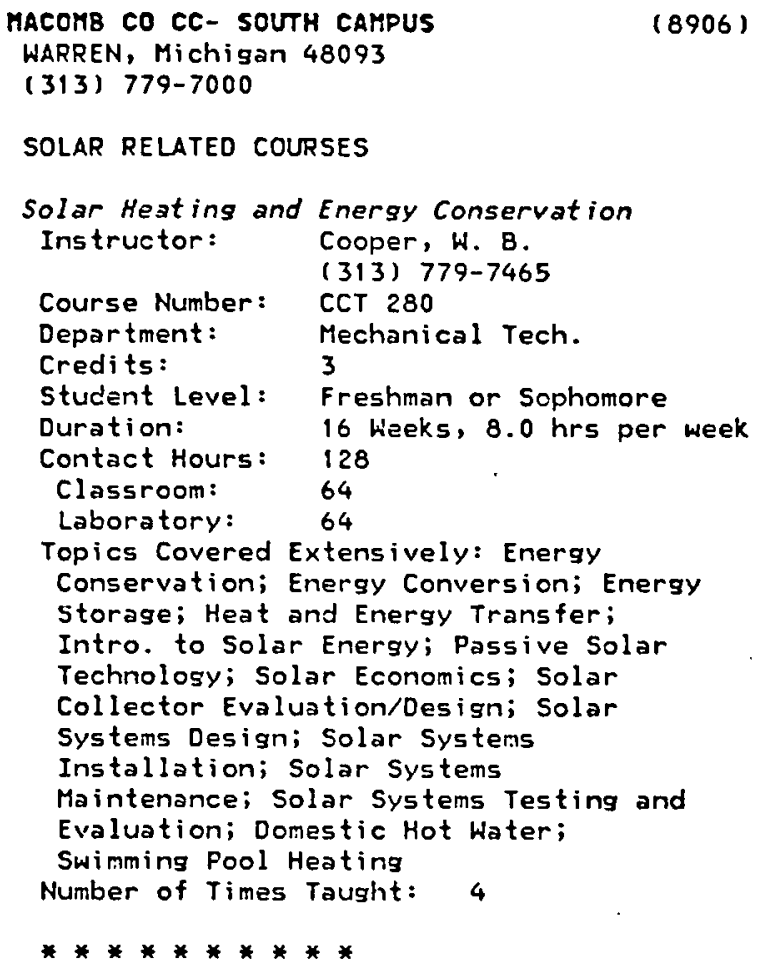

\section{MID MICHIGAN CHTY COLLEGE}

HARRISON, Michigan 48625

$(6768)$

(517) 386-7792

\section{SOLAR RELATED COURSES}

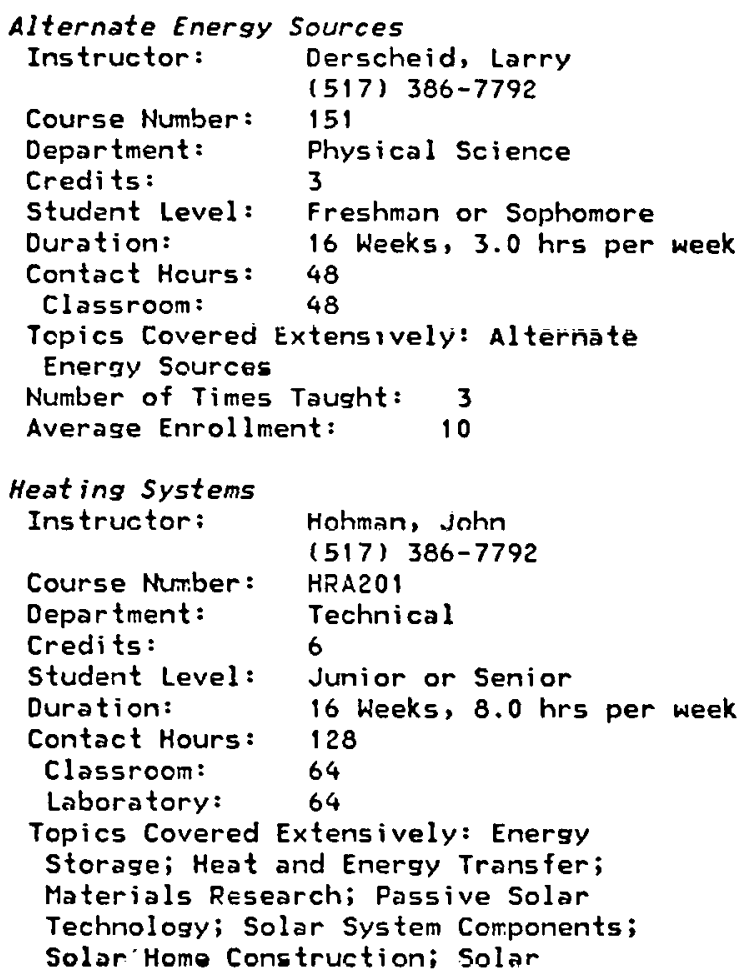

Collector Evaluation/Design; Solar Systems Design; Solar Systems Maintenance; Domestic Hot Water Number of Times Taught: 4 Average Enrollment: $\quad 20$

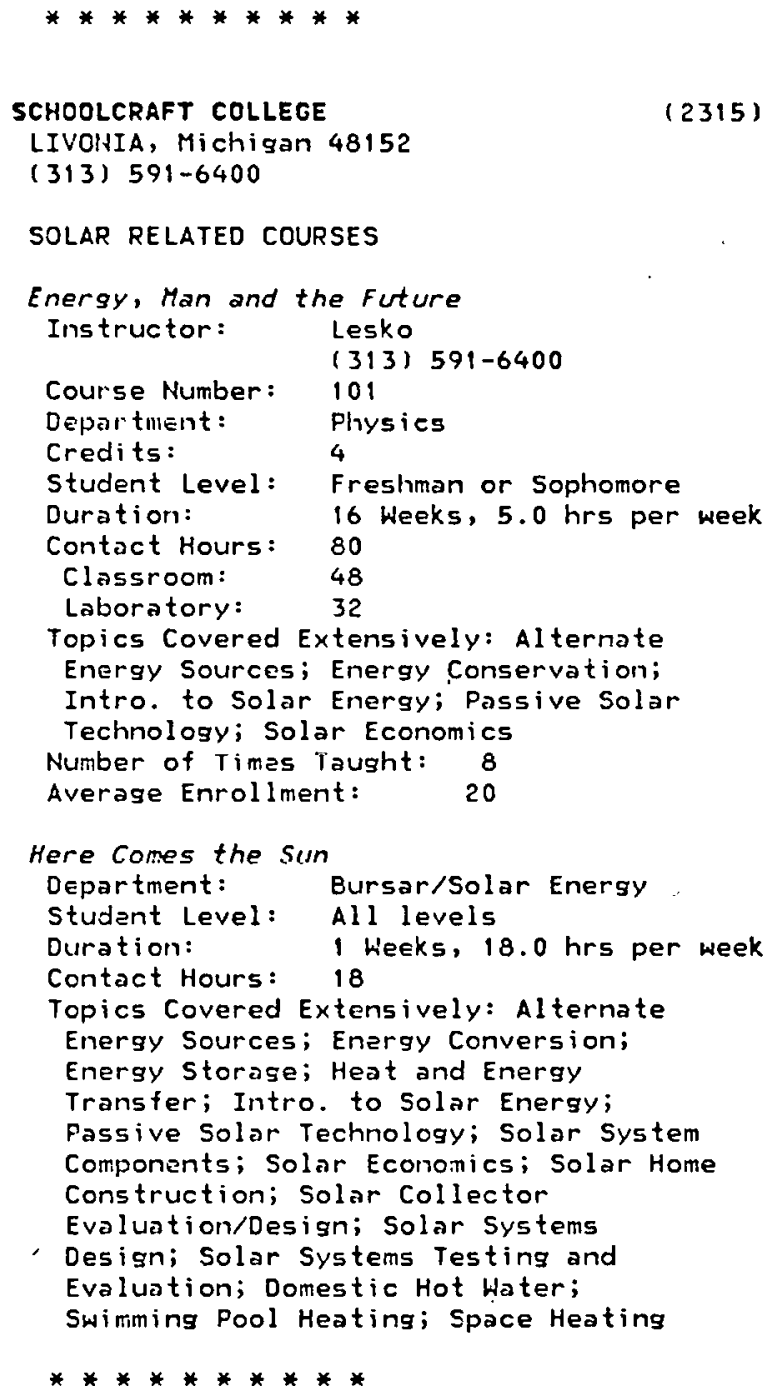

SNT CLAIR CO CMTY COLLEGE

$(2310)$

FORT HUROH, Michigan 48060

(313) 984-3881

SOLAR RELATED COURSES

Alternate Energy, Intro. To Energy

Instructor: Zochowski, Phil

Course Number: (313) 984-3881

Department:

Credits: 100

Student Level:

Duration:

Contact Hours:

Classroom:

Laboratory: 3

All levels

16 Weeks, $4.0 \mathrm{hrs}$ per week 64

32

32 


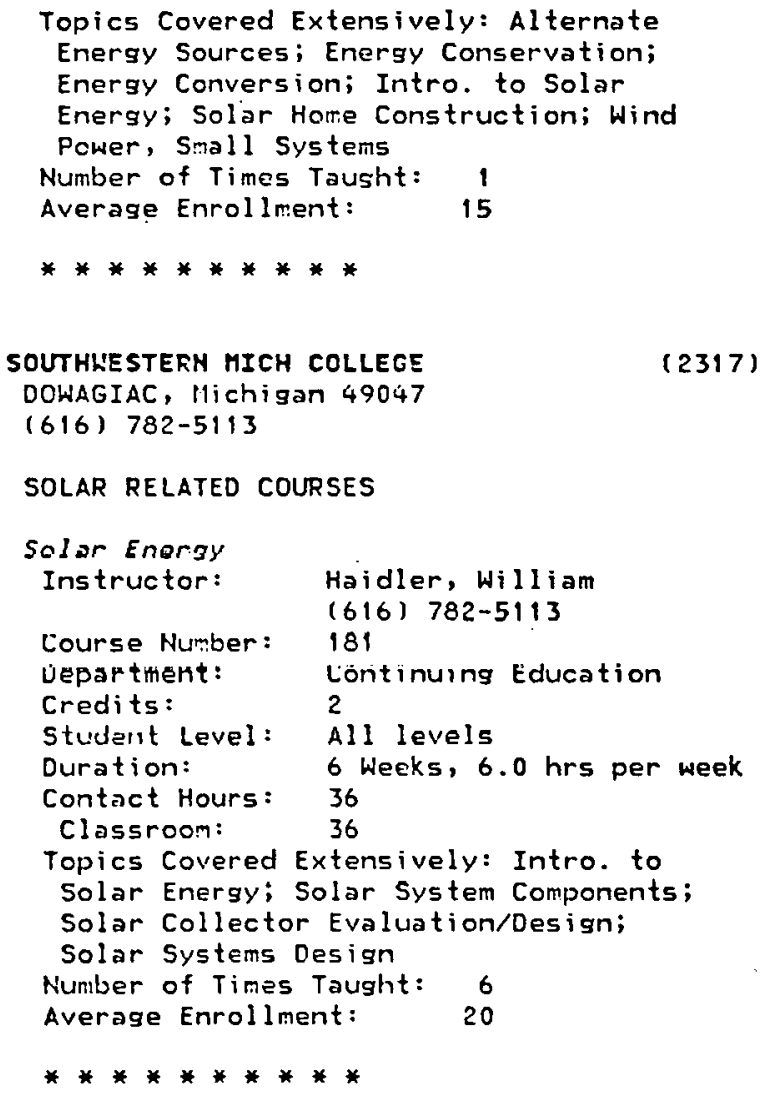

\section{Othor Idueational Institutiona}

\section{SUN STRUCTURES}

201 E. Liberty St.

Ann Arbor, Michigan

SOLAR RELATED COURSES

* 1lt Ener, torkshops 


\author{
Colleges/Universities \\ AUESBURG COLLEGE \\ MINNEAPOLIS, Minnesota 55454 \\ (2334) \\ $(612) 332-5181$ \\ SOLAR RELATED COURSES

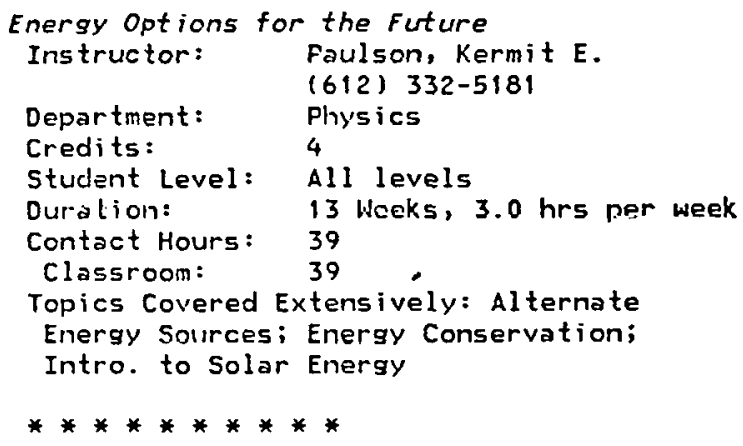

BEMIDJI STATE $U$

(2336)

BEMIDJI, Minnesota 56601

(218) 755-2000

SOLAR RELATED COURSES

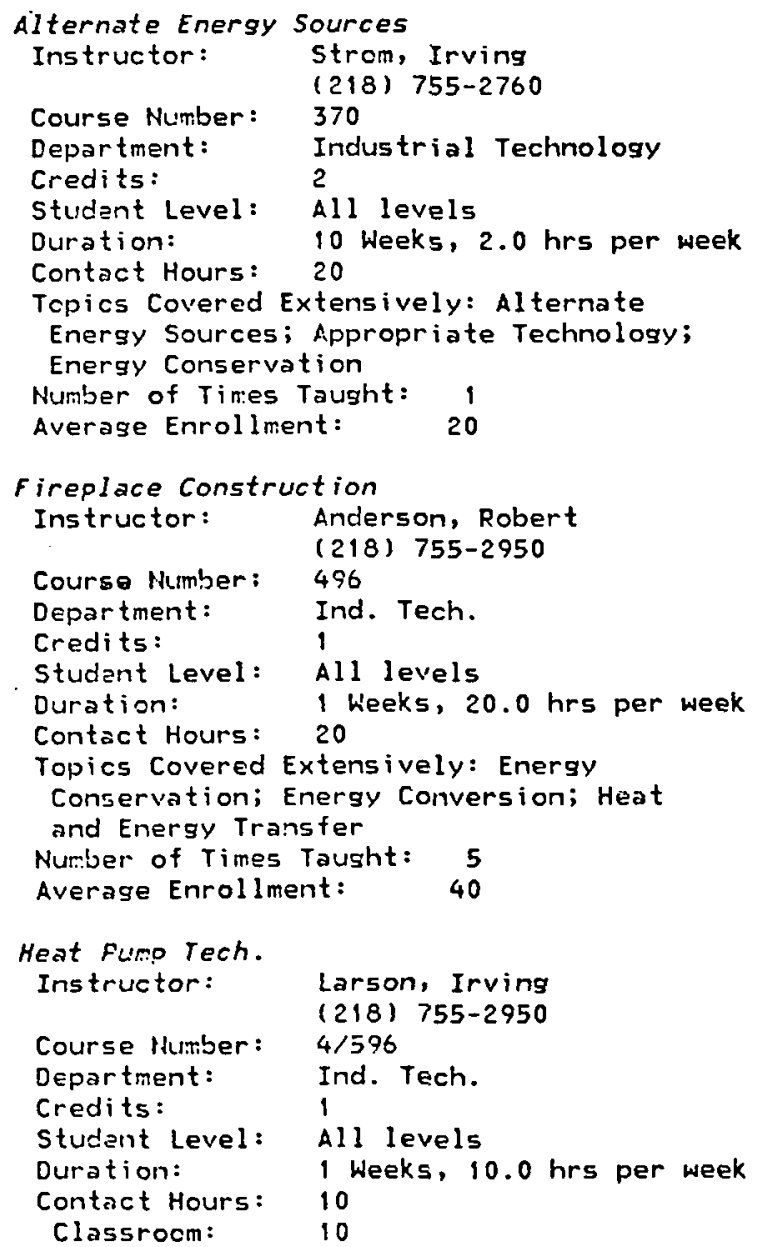

Topics Covered Extensively: Energy Conversion; Energy Storage; Heat and Energy Transfer

Solar Heating (Energy)

Instructor: Larson, Irving

(218) 755-2950

Course Number: 4/596

Department: Ind. Tech.

Credits: 1

Student Level: All levels

Duration: I Weeks, 10.0 hrs per week

Contact Hours: 10

Classroom: $\quad 10$

Tcpics Covered Extensively: Energy Conservation; Energy Conversion; Energy

Storage; Heat and Energy Transfer;

Intro. to Solar Energy; Plumbing

Techniques; Solar System Components;

Solar Home Construction; Solar

Collector Evaluation/Design; Solar

Systems Design; Solar Systems

Installation; Solar Systems

Maintenance; Solar Systems Testing and

Evaluation; Domestic Hot Water; Space

Heating

Number of Times Taught: 3

Average Enrollment: $\quad 25$

Wind Generation (Energy)

Instructor: Larson, Irving

(218) 755-2950

Department: Ind. Tech.

Credits: 1

Student Level: All levels

Duration: 10 Weeks, $1.0 \mathrm{hrs}$ per week

Contact Hours: 10

Classroom: 10

Topics Covered Extensively: Energy

Storage; Wind Power, Central Systems;

Wind Power, Small Systems

Number of Times Taught: 1

Average Enrollment: 20

**********

gUSTAVUS ADOLPHUS COLLEGE

SAINT PETER, Minnesota 56082

(507) $931-4300$

SOLAR RELATED COURSES

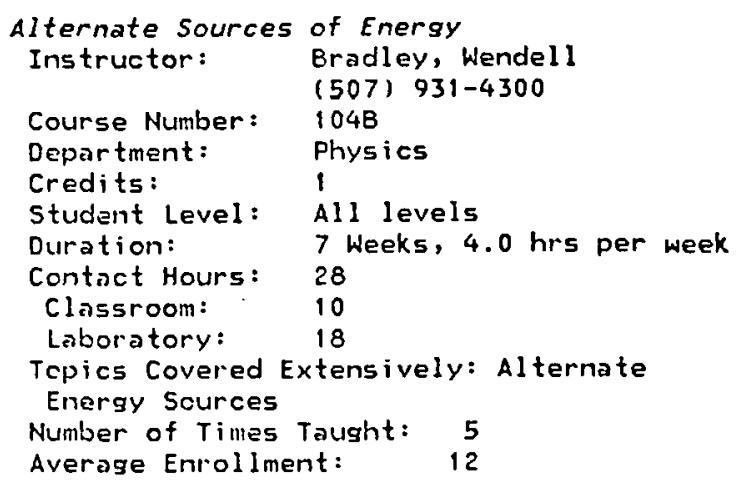




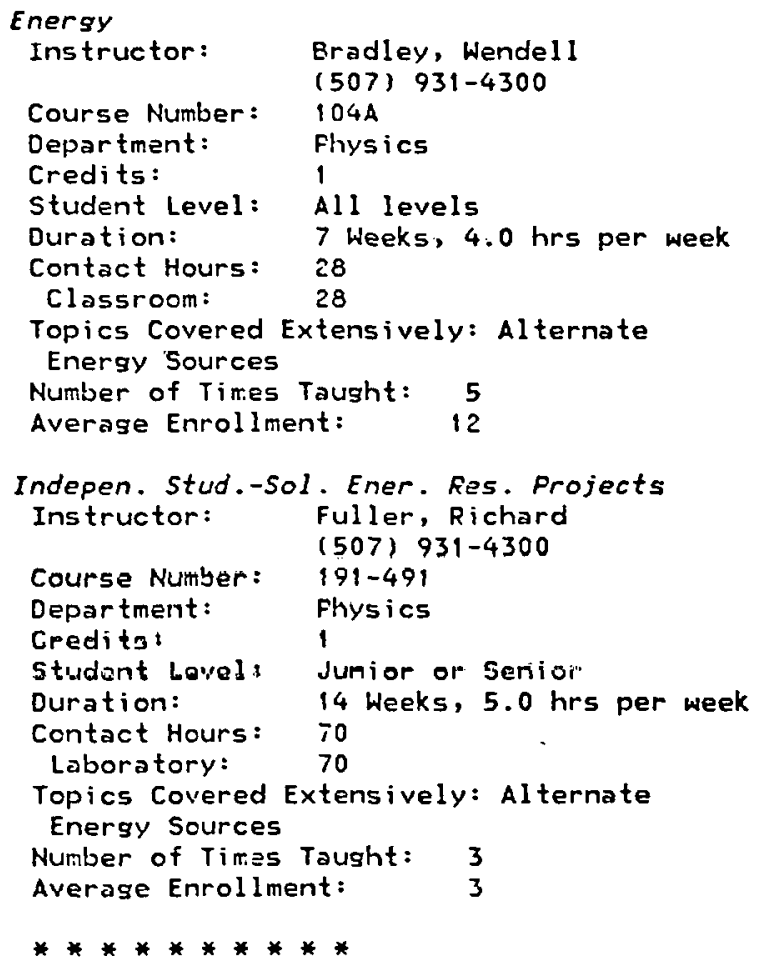

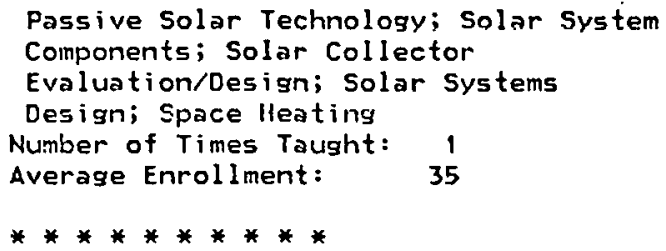

MINM MNPLS SNT PAUL, $U$ OF

(3969)

MINNEAPOLIS, Minniesota 55455

(612) 373-2851

\section{SOLAR RELATED COURSES}

Solar Energy Utilization

Instructor: Lîu, Benjamin Y.II.

Course Number: ME $\mathbf{5 7 1 2}$

Department: Mechanical Engineering

Crédits: 4

Student level: Juhior or Senior

Duration: 10 Weeks, 4.0 hrs per week

Contact Hours: 40

Classroom: $\quad 40$

Topics Covered Extensively: Alternate Energy Sources; Energy Storage; Intro. to Solar Energy; Solar System Components; Solar Economics; Solar Law/Legislation; Solar Cullector Evaluation/Design; Solar Systems Design; Solar Systems Testing and Evaluation; Domestic Hot Water; Process Heat, Industrial; Space Heating; Space Cooling

Number of Times Taught: 4

Averase Enrollment: $\quad 45$

$* * * * * * * * * *$

MINHESOTA DULUTH, $U$ OF

DUL,UTH, Minnesota 55812

(218) $726-80 n n$

SOLAR RELATED COURSES

Energy Resources: Sources, Use and Conservation Instructor: Oakland, Lewis J. (218) 726-7210 PHYS 10CO

Courco Aumber ! Letters $C$ Department: Science-Physics

Credits: 4

Student Level: All levels

Duration: in Wepks. \& $\mathrm{n}$ hrs per meok.

Contact Hours: 40

Cl wsbluem: $\quad 30$

Topics Covered Extensively: Alternate

Energy Sources; Energy Conservation;

Intro. to Solar Energy

Number of Times Taught: 1

Average Enrollment: 35

Environmental studies

Instructor: Sydor, Michael

Course Number: PHY 3050

Department: Letters $C$

Science/Physics 


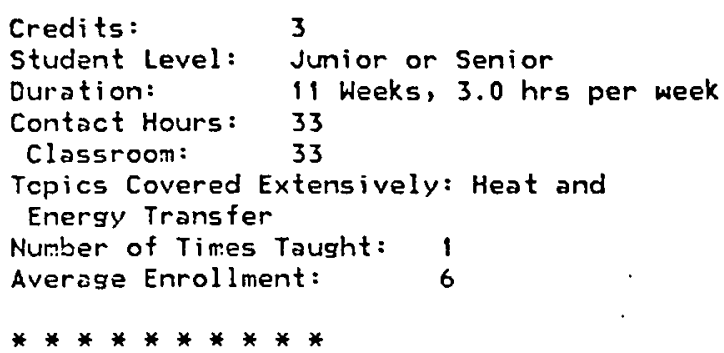

\section{SAINT CLOUD ST UHIVERSITY}

(2377)

SAINT CLOUD, Minnesota 56301

(612) 255-0121

SOLAR RELATED COURSES

Solar Energy

Instructor:

Courfe Nitmher:

Trummel, Donald

(612) 255-2011

Department:

495/595

Credits:

Fhys ics

Student Level:

Duration:

All levels

8 Weeks, $3.0 \mathrm{hrs}$ per week

Contact Hours:

24

19

5

Laboratory:

Number: "of Times Taught:-

Average Enrollment:
SAINT OLAF COLLEGE

(2382)

NORTHFIELD, Minnesota 55057

(507) 663-2222

SOLAR RELATED COURSES

*Physic Dept. Courses

Department: Physics

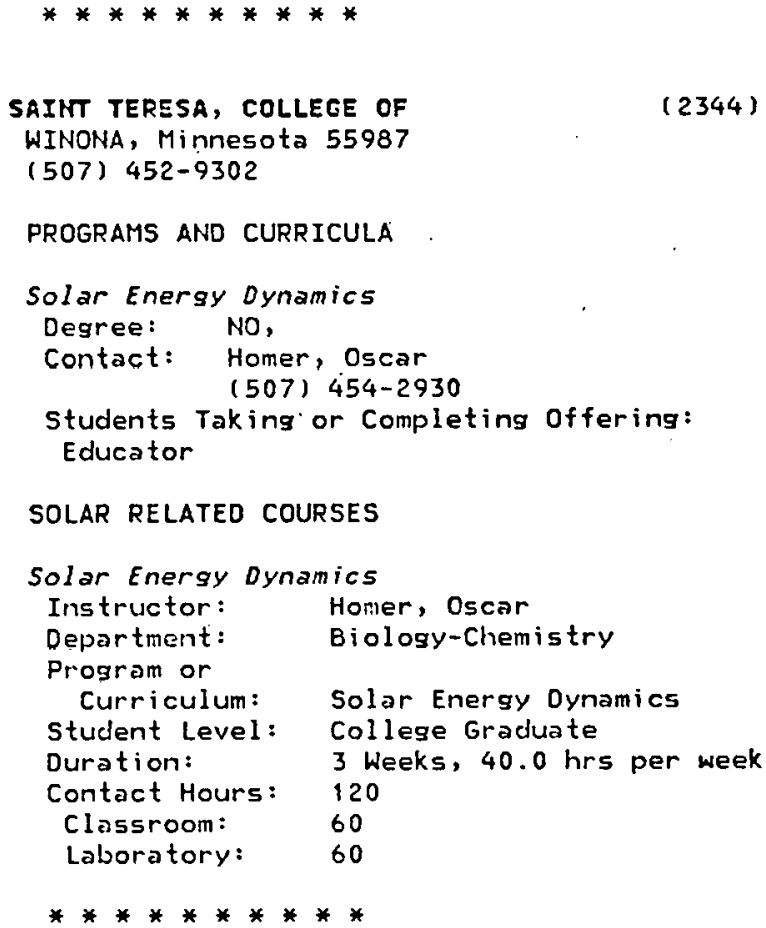

WH MITCHELL COLLEGE LAH

(2391)

SAINT PAUL, Minnesota 55105

(612) 227-9171

\section{SOLAR RELATED COURSES}

Energy Law \& Policy Prince, J. D. (612) 227-9171

Course Number: 393

Department: College of Law Credits: '2

Student Level: College Graduate

Duration: 16 Heeks, 2.0 hrs per week

Contact Hours: 32

Classroom: $\quad 32$

Topics Covered Extensively: Solar Energy

Folicy Uevelopment; Solar

Law/Legislation

Community'Junior Colleges 
LAKENOOD CRTY COLLEGE

(6774)

WHITE BEAR LAKE, Minnesota 55110

(612) 770-1331

PROGRAMS AND CURRICULA

Energy Engineering Technology

Degree: AD, Applied Science-Energy

Contact: Wischriamn, Robert

$1612) 770-1331$

SOLAR RELATED COURSES

Energy Concepts

Instructor:

Course Number:

Department:

(612) 770-1331

Program or

Curriculum:

NS 138

Matiral soience

ciredits

Energy Engineering

Technology

Student Level: Freshman or Sophomore

Duration: 10 Heeks, 4.0 hrs per week

Contact Hours: 40

Classroom: $\quad 40$

Topics Covered Extensively: Alternate

Enersy Sources; Biomass Conversion;

Energy Conservation; Intro. to Solar

Energy; Fassive Solar Technology

Number of Times Taught: 9

Average Enroliment: 30

$* * * * * * * * * *$

ROCHESTER CITY COLLECE

(2373)

ROCHESTER, Minnesota 55901

(507) 285-7210

SOLAR RELATED COURSES

Adv. Sol. Energy for the Homeowner

Department: Civil Engr. Technology

Credits: 3

Student Level: Freshman or Sophomore

Duration: 12 Weeks, $3.0 \mathrm{hrs}$ per week

Contact Hours: 36

Classroom: $\quad 36$

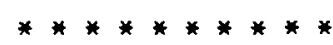




\author{
Colleges/Universities \\ DELTA STATE UHIVERSITY \\ CLEVELAHD, Mississippi 38732 \\ (601) 846-6664 \\ (2403) \\ PROGRAMS AND CURRICULA \\ Energy frogram for High School \\ Teachers \\ Contact: Myers, Richard S. \\ (601) 843-9741 \\ Students Taking or Completing Offering: \\ Educator \\ SOLAR RELATED COURSES
Special Topics in Chemistry-Energy
Instructor: Myers, Richard S.
(601) 843-9741
Course Number: CHE 392
Department: Physical Sciences
Program or
Curriculum: Energy Program for High
Credits: School Teachers
Student Level: College Graduate
Duration: 2 heeks, 28.0 hrs per week
Contact Hours: 56
Classroom: $\quad 56$
Topics Covered Extensively: Alternate
Energy Sources; Intro. to Solar Energy
Number of Times Tausht: 1
24 \\ Average Eniollment:
}

\section{$* * * * * * * * * *$ \\ MISSISSIFPI ST UUIVERSITY \\ MISSISSIPPI STATE, Mississippi 39762 \\ (601) 325-3221 \\ PROGRAMS AYIJ CURRICULA

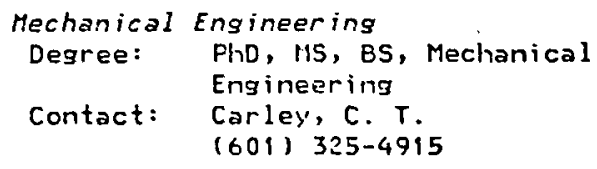

(2423)

\section{SOLAR RELATED COURSES}

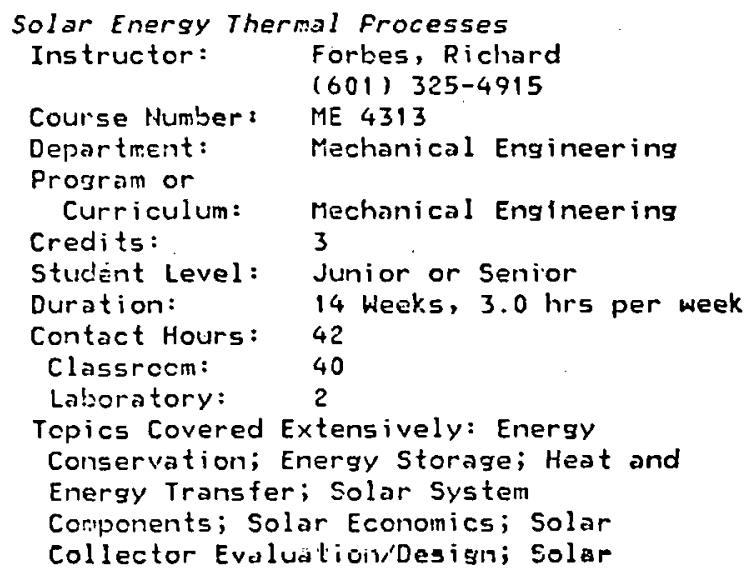

Systems Design; Domestic Hot Water; Space Heating; Space Cooling Number of Tines Taught: 3 Average Enrollment: $\quad 30$
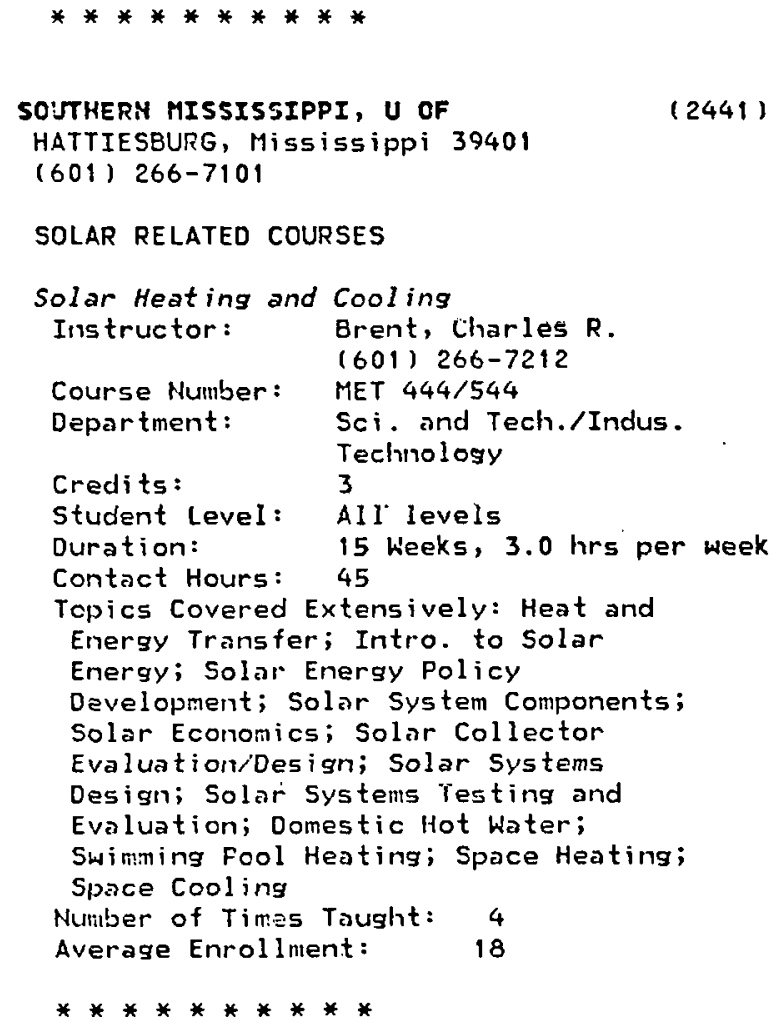

\section{Community/Junior Colleges}

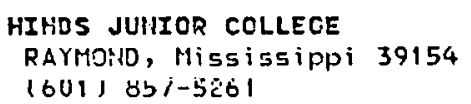


THIS PAGE

\section{WAS INTENTIONALLY LEFT BLANK}




\section{Colleges/Universities}

CEMTRAL METHJDIST COLLEGE

FAYETTE, Mis5ouri 65248

(816) 248-3391

SOLAR PELATED COURSES

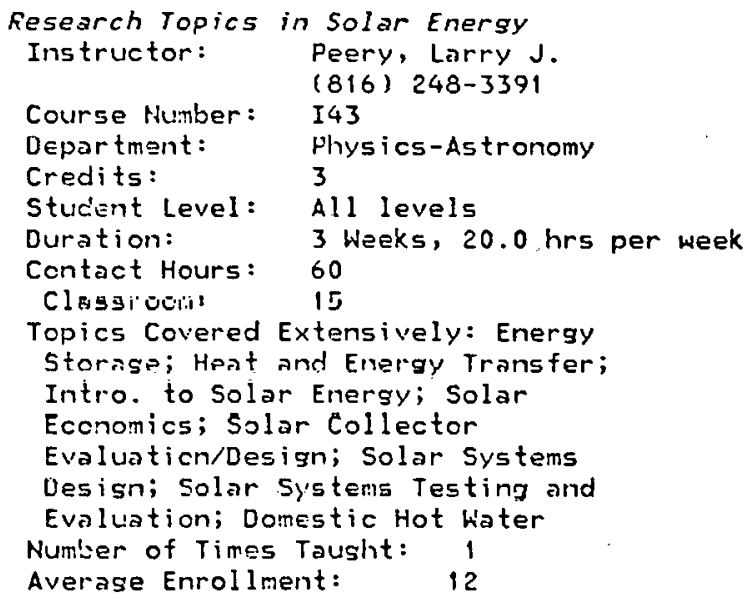

CENTRAL MO ST UNIVERSITY

(2454)

WARRENSSURG, Missouri 64093

(816) 429-4111

\section{SOLAR RELATED COURSES}
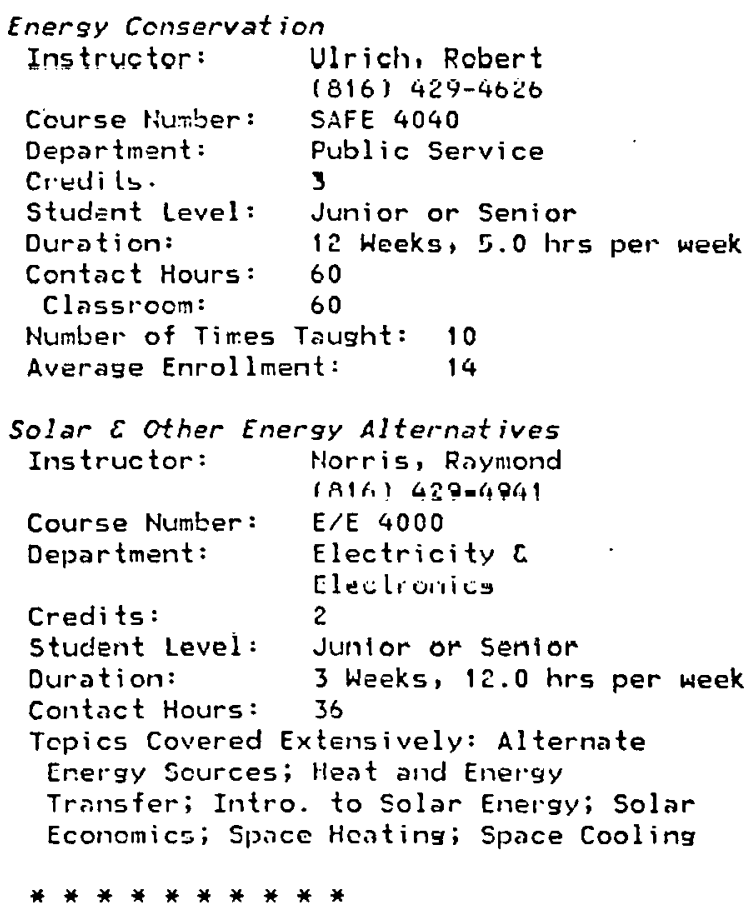

MISSOURI STHN ST COLLEGE

$(2488)$

JOPLIN, Missouri 64801

(417) 624-8100

SOLAR RELATED COURSES

SOLAR RELATEO COURSES

Ener. Systs.., Res-Risks, Benef its Instructor: Meyer, Walter/ Bull, Stanley $R$. (316) $8.98,35.50$

Course Number: NESO

Department: Energy Systenis and

Resources

Lreditas: 2

Student Level: Colleye Graduate

Duration: I Weeks, 40.0 hrs per week

Contact Hours: 40 .

Classroom: $\quad 30$

Laboratory: $\quad 10$

Topics Covered Extensively: Alternate Energy Sources; Energy Conservation; Intro. to Solar Energy

Number of Tiniss Taught: 6

Arerage [arollmenti In

Engin. Eval-Ener. Systs., Fesources

Iistructor: lieyer, wal ter

(314) $882-3550$

Course Nunber: EENAENE315

Department: . Enersy Systems and

Credits:

Student Level: Junlor or Senior

Duration: 16 Weeks, 3.0 hrs per week

Contact Hours: 48 Classroon: 48

Topics Covered Extensively: Alternate Enersy Sources; Energy Conservation; Energy Conversion; Energy Storage; Heat and Energy Transfer; Intro. to Solar Energy 
Number of Times Taught: 3

Average Enrollment: 26

Meet Ener. Crisis-Comp. Risks, Benef it

Instructor: Maver, Halter/ Bull, Stanley R.

(314) $882-3550$

Course Nurijer: HEj01

Department: Enersy Systems and

Resources

Credits: 4

Student level :

Duration: lleze Graduate

Contact Hours: 120

Classroom: $\quad 90$

Laberatory: 30

Topics Covered Extensively: Energy

Conservation; Intro. to Solar Energy

Nuriber of Tim:s Taught: 1

Average Enrollment: 37

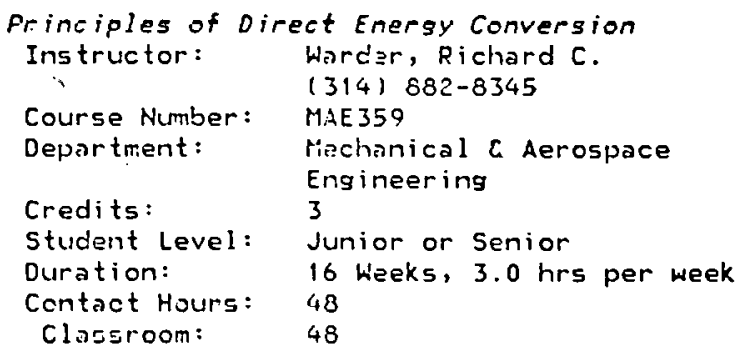

Topics Covered Extensively: Energy

Conversicn; Fhotovoltaics

Number of Times Taught: 2

Average Enrol Iment: 20

Solar Energy Utilization

Instructor: roore, Gordon L.

(314) 882-2785

Course Number: MAE339

Department: Mechanical \& aerospace Erizineering

Credits: 3

Stuelent Level: Junior or Senior

Duretioti

16 Heeks, 3.0 his per week

Contact Hours: 48

Classroom: 48

Tcpics Covered Extensively: Energy

Concorwation; Energy Storago; Hant and

Energy Transfer; Intro. to Solar

Energy; Passive Solar Technology; Solar

Hone Construction; Domestic Hot Water;

Space Heating; Space Cooling

Number of Times Taught: 1

Average Enrollment: 22

Teachers Energy Sympcsium

Inș truç tor :

Mlejer, W./ Bull, S.R.

(314) 852-3550

Course Number: NE301

Department: Energy Systems and Resources

Credits:

I

Student Level: Collese Graduate

Duration: 1 Heeks, 20.0 hrs per week

Contact Hours: 20

Tcpics Covered Extensively: Alternate

Energy Sources; Energy Conservation;

Intro. to Solar Energy
Number of Times Taught: 3

Average Enrollment:

70

HISSCURI- KANSAS CITY, U OF
KAISAS CITY, MiSSOUR' 64110
$(816) 276-1000$

$(2518)$

SOLAR RELATED COURSES

Solar Energy Utilization

Instructor: Stewart, Jr. W. E. $(816) 276-1672$

Course Number: 301

Department: Mechanical Engineering

Credits: 3

Stucleat Level: Junior or Senior

Duration: 16 Heeks, 3.0 hrs per week

Contact Hours: 48

Tcpics Covered Extansively: Appropriate

Technology; Energy Conversion; Energy

Storage; Heat and Energy Transfer;

Materials Fesearch; Passive Solar

Techrology; Solar Economics; Solar

Collector EvaluationiDesign; Solar Systems Design

Number of Times Taught: 1

Average Enrollment:

$\hat{6} 5$

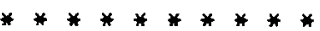

MISSOURI - ROLLA, U OF

ROLLA, Missouri 65401

$(2517)$

(314) 341-4114

PROGRAMS AHD CURRICULA

Solar Energy Conversion

Degree: MS, Electrical Engineering

Contact: Boone, Jack L.

(314) 341-4357

\section{SOLAR RELATED COURSES}

Solar. Energy Conversion

Instructor: Boone, Jack $\mathbf{L}$.

(314) 341-4357

Course Number: EE 335

Department: Elec. Engineering

Program or

Curriculum: Solar Energy Conversion

Credits:

3

Student Level: College Graduate

Duration: $\quad 15$ Weeks, 3.0 hrs per week

Contact Hours: 45

Topics Covered Extensively: Appropriate Technolosy; Energy Conversion; Energy

Storage; Heat and Energy Transfer;

Intro. to Solar Energy; Photovoltaics;

Solar Collector Evaluation/Design;

Solar Systems Design

Average Enrollment:

10

Solar Energy Conversion-Lab

Instructor: Boone, Jack L.

Course Number: EE 336 


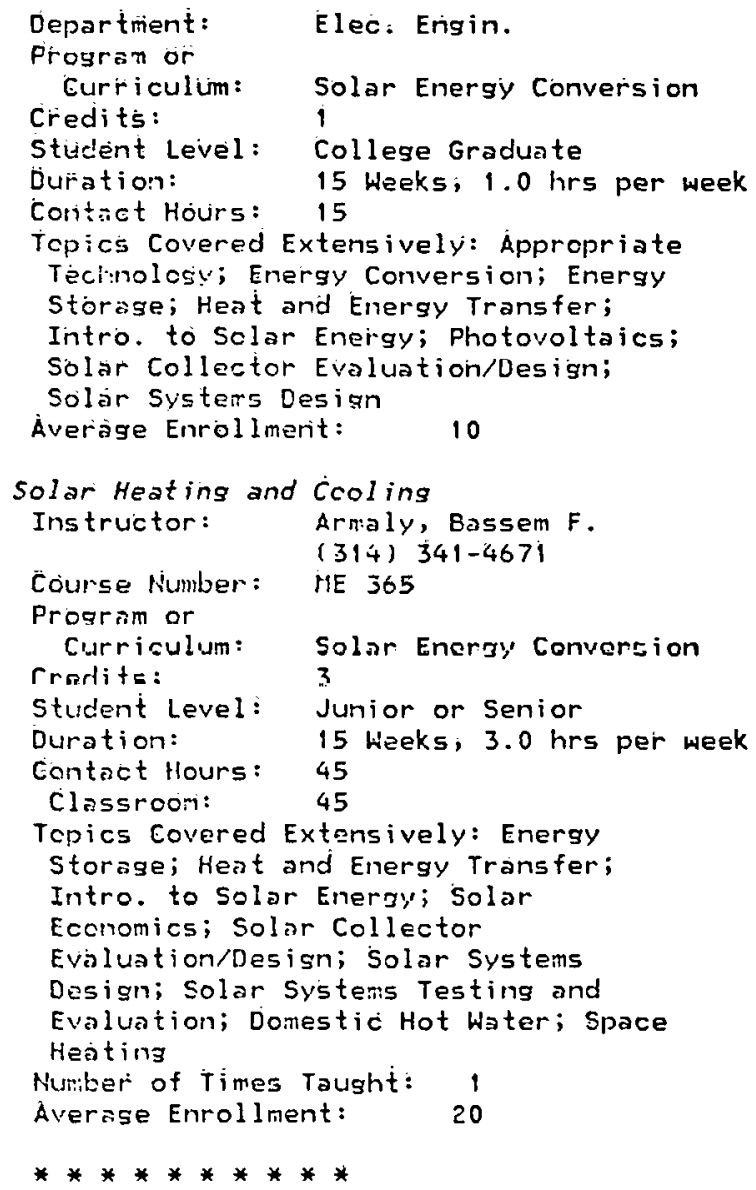

(2501)
Conservation; Energy Conversion; Energy Storage; Heat and Energy Transfer; Intio. to Solar Energy; Solar Collector Evaluation/Design; Domestic Hot Water; space Heating

\section{SOUTRLESTERH RO ST UHIVERSITY \\ SPRINGFIELD, Missouri 65802 \\ (417) $836-5000$}

PROGRAMS AND CURRICULA

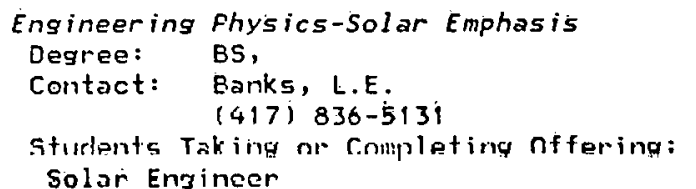

\section{SOLAR RELATED COURSES}

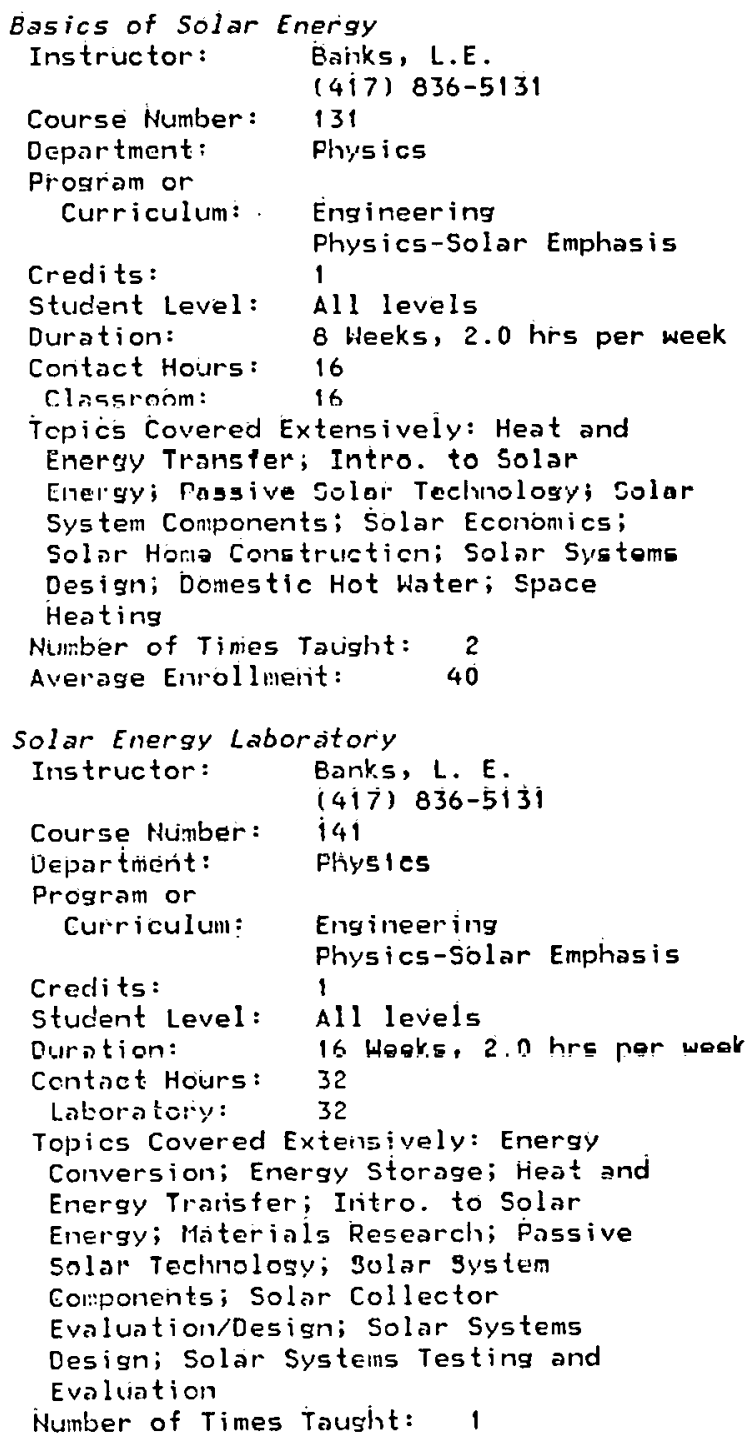


Solar system Analysis

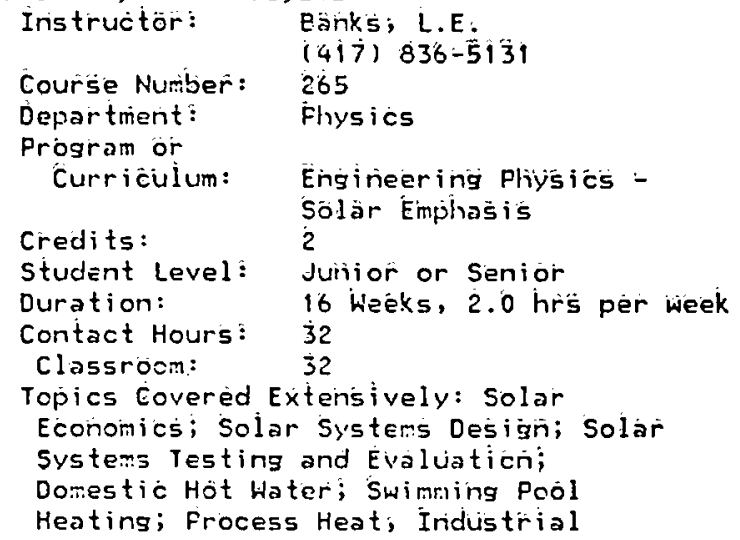

Solar system besisn

\begin{tabular}{|c|c|}
\hline tor: & $\begin{array}{l}\text { Bonks, L:É: } \\
(417) 836-\overline{5} i 3 i\end{array}$ \\
\hline $\begin{array}{l}\text { Course Hurber: } \\
\text { Department: }\end{array}$ & $\begin{array}{l}265 \\
\text { Fhysics }\end{array}$ \\
\hline $\begin{array}{l}\text { Program or } \\
\text { Curriculum: }\end{array}$ & $\begin{array}{l}\text { Engineer ing } \\
\text { Fhysics-Solar Emphàsis }\end{array}$ \\
\hline $\begin{array}{l}\text { Credits: } \\
\text { Student Level : } \\
\text { Duration: } \\
\text { Contact Hours: } \\
\text { Laboratory: }\end{array}$ & $\begin{array}{l}\text { Junior or Senior } \\
16 \text { he=ks, } 2.0 \mathrm{hrs} \text { per wee } \\
32 \\
32\end{array}$ \\
\hline $\begin{array}{l}\text { Topics Covered } \\
\text { Solar Technolo } \\
\text { Constructicn; } \\
\text { Evaluation/Des } \\
\text { Averase Enrollm }\end{array}$ & $\begin{array}{l}\text { Extensively: Passive } \\
\text { ogy; Solar Home } \\
\text { Solar Collector } \\
\text { sign; Solar Systems Design } \\
\text { gent: }\end{array}$ \\
\hline
\end{tabular}

Solar Thermal Analysis

Instructor: Banks, L.E.

(417) 836-5131

Course Number: 265

Department: Physics

Program or

Curriculum: Engineering

Credits :

Fhysics-Solar Emphasis

2

Student Level: Junior or Senior

Duration: 16 Heeks, 2.0 hrs per week

Centaet Heurgi 32

Clàssroon: $\quad \dot{3} 2$

Topics Covered Extensively: Energy

Storage; Heat and Energy Transfer;

Passive Solar Technolozy; Space Heating

Number of Tires Tausht: 1

Averase Enrollmenti io

\begin{tabular}{|c|c|}
\hline Instructor: & $\begin{array}{l}\text { Fortlirip, J.W. } \\
(417) 836-5405\end{array}$ \\
\hline Course Nurber: & 131 \\
\hline $\begin{array}{l}\text { Department: } \\
\text { Program ör }\end{array}$ & Flinges ios \\
\hline Curriculum: & $\begin{array}{l}\text { Engineering } \\
\text { Fhysics-Solar Emiphasis }\end{array}$ \\
\hline $\begin{array}{l}\text { Credits: } \\
\text { Student Level: }\end{array}$ & All levels \\
\hline $\begin{array}{l}\text { Duration: } \\
\text { Contact Hours: }\end{array}$ & 8 Heeks, 2.0 hirs per week \\
\hline Classroom: & 16 \\
\hline
\end{tabular}

Topics Covered Extensivelyy: Wind Power, Ceritral Systems; Wind Power; Sniall Systeinis

Number of Times Taught: 1

Avèráse Érióliment: 40

$* \ddot{*} \ddot{*} \ddot{*} * \ddot{*} \ddot{*} * \ddot{*}$

\section{WASHIRETON UHIVERSSTTY}

SAINT LOUIS, Miśsoüi 63130

i $252 \hat{0}$ )

(314) 889-5000

\section{SOLÄR REELATED COUR̂́SËS}

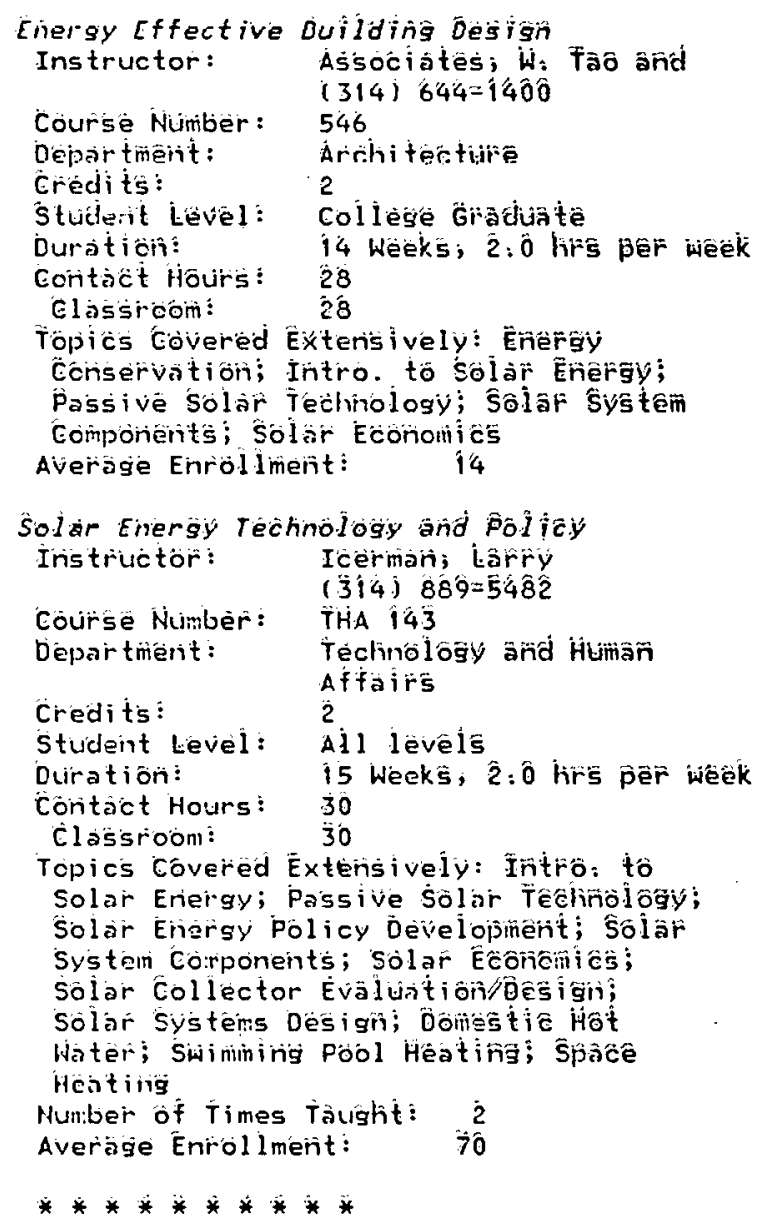

UEBSTER COLLEGE

SAIHT LOUIS, Missoúri 63119

$(2521)$

(314) $968-0500$

ŠÖLAR̂̉ RELATED́ GÖURTSESS

Energig Appopiate to the Task

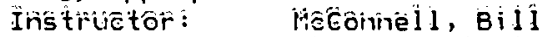

Dêpartnient: $\quad$ Sçience

Credits? 3

stiudent levề: All levels

Dúration

Cófitât Hoùrs:
16 Wék's; 3.0 hr's per wèk 
Topics Covered Extensively: Energy

Conservation; Passive Solar Technology

Nuriber of Tires Tausht: 2

Averase Enrollment:

10

'*********

\author{
Community/Junior colleses \\ CROWDER COLLEGE \\ (2459) \\ NEOSHO, MissCURi 64350 \\ $(417) 451-3223$ \\ SOLAR RELATED COURSES \\ Basie solar Degisn \\ Instruetor: Boyt, Art \\ (417) $451-5365$ \\ Department: Ssience \\ Credits: 3 \\ Student Level: All levels \\ Duration: 16 heeks, 3.0 hrs per week \\ Contact Hours: 48 \\ Classrcom: $\quad 45$ \\ Topics Covered Extensively: Alternate \\ Energy Sources; Appropriate Teclinology; \\ Energy Conservation; Energy Conversion; \\ Enersy Storase; Intro. to Solar Energy; \\ Passive Solar Technology; Solar System \\ Cormenents; Solar Economics; Solar Home \\ Construction; Solar Collector \\ Evaluation/Desisn; Solar Systems \\ Uesisn; Solar Sistens Maintenance; \\ Domestic Mot Hater; Elec'l Generation, \\ Snall Scale; Space Heating; Wind Power, \\ Small syatemis \\ Number of Times Taught: 4 \\ Averase Enrollment: \\ 23 \\ SAIHT LOUIS CC- MERATEC \\ (2472) \\ KIRKWOOD, Missouri 63122 \\ (314) $956-7500$ \\ SOLAR RELATED COURSES \\ Fund. of Solar Ener. and Ener. Cons. \\ Instructor: Strutman, Harren \\ 1314) $966-7747$ \\ Coures llumber: 12,706 \\ Department: Ener. and Tech.-Cont. \\ EO. \\ Student Level: High School Graduate \\ Duration: 8 Weeks, 2.0 hrs per week \\ Contact Hours: 16 \\ Tcpics Covered Extensivaly: Energy \\ Conservation; Energy Storage; Heat and \\ Energy Transfer; Intro. to Solar \\ Energy; Plumbing Techniques; Solar Home \\ Construction; Solar Collector \\ Evaluaticn/Design; Domestic Hot Water; \\ Space Heating; Space Cooling
}




\section{Colleges/Universities}

MOITAHA C MIKRL SCI- TECHK

(2531)

BUTTE, Montana 59701

(406) 792-8321

SOLAR RELATEO COURSES

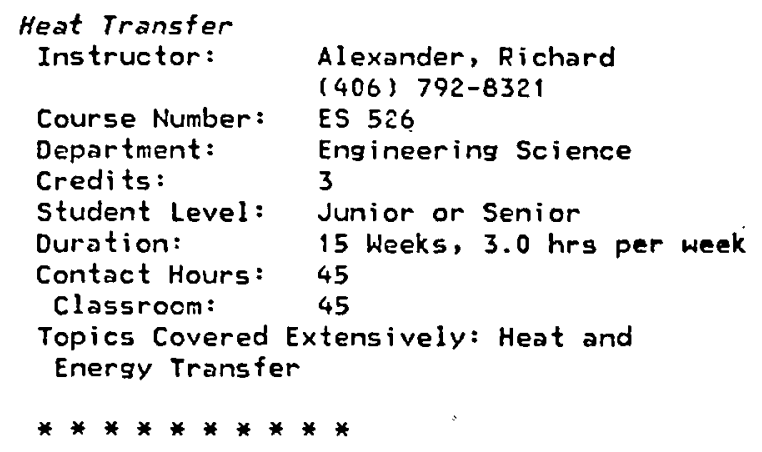

MONTANA STATE UNIVERSITY

(2532)

BOZEMAN, Montana 59715

(406) 994-4361

\section{SOLAR RELATED COURSES}

Ener. Train. Shop-Second. Sci. Instructs.

Instructor: Mussulman, R. L.

(406) 994-2203

Course Number: $\quad 570$

Department: Mechanical Engineering

Credits: 4

Student Level: College Graduate

Duration:

Classrocm: $\quad 30$

Laboratory: $\quad 10$

Topics Covered Extensively Alternate

Energy Sources; Energy Conservation;

Energy Storase; Heat and Energy

Transfer; Intro. to Solar Energy;

Passive Solar Technology; Solar System

Components; Solar s-ornomics; Domestic

Hot Water; Space Mating

Number of Times Taught:

Average Enrollment:

5

Enersy Course for Homebuilders

Instructor: Martindale, W. R.

(406) 994-2203

Course Number: $\mathbf{5 7 0}$

Department: Mechanical Engineering

Student Level: All levels

Duration: 10 Weeks, $2.0 \mathrm{hrs}$ per week

Contact Hours: 20

Classroom: 20

Topics Covered Extensively: Alternate Energy Sources; tnergy Conservation; Heat and Enersy Transfer; Intro. to Solar Energy; Passive Solar Technology; Plumbing Techniques; Solar System Components; Solar Econonics; Solar Home Construction; Solar Systems Design; Solar Systems Installation; Solar Systems Maintenance; Solar Systems Testing and Evaluation; Domestic Hot
Water; Space Heating

Number of Times Tought: 1

Average Enrollment: $\quad 65$

Energy Efficient BIdg. for Contractors

Instructor: Warrington, Robert

(406) 994-2203

Department: Continuing Education

Student Level: All levels

Number of Times Tausht: 2

Energy: Limits, Problems and Prospects

Instructor: Kirkpatrick, Larry

(406) 994-3614

Course Number: PHY 252

Department: Letters \&

Credits: 3

Student Level: Freshninn or Sophomore

Duration: 12 heeks, $3.0 \mathrm{hrs}$ per week

Contact Hours: 36

Classroom: $\quad 36$

Number of Times. Taught: 5

Average Enrollment: $\quad 35$

Solar Energy Design

Instructor: k'arrington, Robert 0 .

(406) 994-2203

Course Number: ME 480

Department: Mechanical Engineering

Credits: 3

Student Level: Junior or Senior

Duration: 16 Weeks, 3.0 hrs per week

Contact Hours: 48

Classroon: $\quad 48$

Tcpics Covered Extensively: Heat and

Energy Transfer; Passive Solar

Techuology; Solar System Components;

Solar Economics; Solar Collector

Evaluationidesign; Solar Systems

Design; Solar Systems Testing and

Evaluation; Space Heating

Number of Times Taught: 2

Average Enrollment: 10

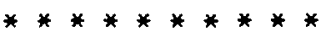

MOHTANA, UHIVERSITY OF

(2536)

MISSOULA, Montana 59801

(406) 243-0211

SOLAR RELATED COURSES

Alternative Energy \& the Ecosphere

Instructor: Sheridan, R.

Course Number: $178-9$

Uepartmeint: Botany-Liberaí Arts

Credits: 3

Student Level: All levels

Duration: 9 heeks, 3.0 hrs per week

Contact Hours: 27

Topics Covered Extensively: Alternate

Energy Sources; Appropriate Technology;

Bionass Conversion; Energy

Conservation; Energy Storage; Heat and

Energy Transfer; Intro. to Solar

Energy; Passive Solor Technology;

Thotovoltaics; Plumbing Techmiques; 
Sclar System Eomponents sol ar Home Eonstruction: Solar Col lector Evaluation/0esign; Solar Systems Oesign; Solar systens Installation: Solar Systens Maintenanoe; Solar Systens Testins and Evaluation; Qomestic Hot Water; Space Heating; Wind Power, Central systems; Wind Power, Strall Șystems

Number of Times Taught: 3

Aversge Enrol lmenti 200

\section{HESTERH MONTAHA COLLEGE}

$(2537)$

QILLOR! Monțana 59725

$1406 ! 68 ?=725 !$

\section{SOLAR RELATED COURSES}

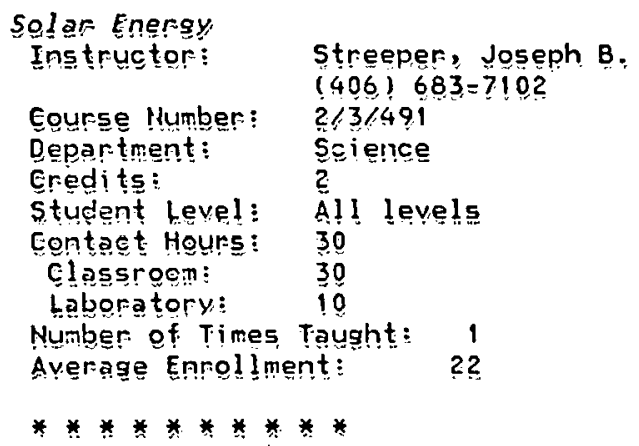

communi ty/suniop colleses

FLATYEAD YLY ETTY EOLLEEE

KALISPELL, Montana 5990 !

(67??)

$(406) 755=5222$

SELAR RELATED EOUPSES

Alternative Encroy \& Conscruation

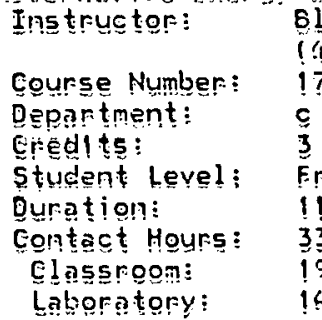

ropics Eovered Extens ively: Al ternate

Energy Scurces; Appropriate Technology;

Di entoss Convel:sioni Ene!:gy

Gonseryation; Enersy storage; Intro: to

Solar Energy; Passive Solar Technology;

Fhotovoltaics; Soląr Energy Policy

Devel ermenti Sol ap System Gonponents;

Sol ar Economies; Sola! Heme

construttion; Solar Lew/Legislationi
Soler Collector Evalunțion/Design;

Solar Systems Desisn; Domestic Hot

Water: Elec!l Generation, Small Scale;

Process Heat, Agricul tural; Space

Heating; Wind Power, Eentral Systems;

Wind Power, small systems

Number of Times Taught: I

Average Eniol lnient: 50

Energy for A Technological society

Instructor: Blood, Lex

Course Number: $\quad 173$

Department: Earth Sciences, Geology

Credits: 3

Student Level: Freshman or Sophomore

Duration: 11 Weeks, $3.0 \mathrm{hrs}$ per week

[entent Hollus: 33

Classroom: 19

Laboratory: 14

Topics Covered Extensively: Energy

Eonservation

Number of Times Taught: 2

Average Enrol lment: $\quad 25$

\section{other Educational Institutions}

ALTERNATYYE EHERGY RESOURCE ORG.

$(90270)$

435 stapleton Bldg.

Bill ings, Monta!n Eq10!

SOLAR REELATEO EOURSES

* Solar Eneray Horkshor

üuration: I Weeks

Topics Covered Extensively: Intro. to

Solar Enengy; Solar System Components;

Solar Collector Evaluation/Design:

Solar Systems Design; Space Heating

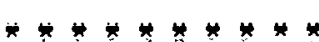




\author{
colleges/Universities \\ HASTIH:GS COLLEGE \\ HASTINGS, Nebraska 68901 \\ (2548) \\ (402) $463-2402$ \\ SOLAR RELATED COURSES \\ Environmental science 260 \\ Course Nurbar: 260 \\ Department: Physics \\ Credits: 4 \\ Student Level: Junior or Senior \\ Duration: $\quad 14$ Weeks, 5.0 hrs per week \\ Contąct Hours: 70 \\ Classroom: $\quad 56$ \\ Laboratory: 14 \\ Number of Times Taught: 6 \\ Average Enrollment: $\quad 24$ \\ Environmental Science 460 \\ Course Number: 460 \\ Department: Plyysics \\ Credits: 4 \\ Student level: Junior or Senior \\ Duration: $\quad 14$. Weeks, $5.0 \mathrm{hrs}$ per week \\ Contact Hours: 70 \\ Classroom: $\quad 56$ \\ Laboratory: $\quad 14$ \\ Number of Tines Taught: 2 \\ Average Enrollment: \\ 20 \\ $* * * * * * * * * *$
}

\section{MIDLAYID LUTTERAM COLLEGE \\ FREMONT, Ne!raska 68025 \\ (402) $721-5480$}

(2553)

SOLAR RELATEO COUURES

Solar Home Heat ing

Instructor: Kruse, James

(402) 721-5480

Department: Continuing Ed.

Student Level: All levels

Duration: 7 Heeks, 3.0 hrs per week

Contact Hours: 21

Classroom: 21

Topics Covered Extensively: Energy

Storage; Heat and Energy Transfer;

Intro: to Solar Energy; Solar Home

Constructicn; Solar Systens Designi

Space Heating

Number of Times Taught: 4

Average Enrollment:

30

**********

NEBRASTKA- LINCOLN, U OF

$(2565)$

LINCOLN, Nebraska 68588

$(402) 472-721$ !

\section{SOLAR RELATED COUURSTES}

Solar Energy Engineering

Instructor: Anderson, Edward $E$.

(402) $472-1678$

Course Number: $\quad 414 / 814$

\author{
Department: \\ Credits: \\ Mechanical Engịneering \\ Student Level: \\ Level: Junior or Senior \\ Contact Hours: 42 \\ Classroom: 42 \\ Topics Covered Extensively: Intro. to \\ Solar Energy; Solar System Components: \\ Solar Collector Evaluation/Design; \\ Solar: Systems Design; Domestic Hot \\ Water; Process Heat, Agricultural; \\ Process Heat, Industrial; Space \\ Heating; Space Cooling \\ Number of Times Taught: 3 \\ Average Enrollment: \\ 20 \\ Summer Instit. and Curr. Dev,-Ener. Edu, \\ Instructor: MeCurdy, Donald $W$. \\ Course Number: $\quad \begin{aligned} & (402) \\ & 9935\end{aligned}$ \\ Department: Teachers College-Sec. \\ Ecluc. \\ Credits: 3 \\ Student Level: College Graduate \\ Duration: $\quad 3$ Weeks, 30.0 hrs per week \\ Contact Hours: 90 \\ Classroom: $\quad 30$ \\ Laboratory: 60 \\ Topics Covered Extensively: Al ternate \\ Energy Sources; Energy Conservation; \\ Heat and Enersy Transfer; Intro. to \\ Solar Energy; Passive Solar Technology; \\ Photovoltaics; Solar Home Construction; \\ Elec'l Generation, Central; Space \\ Heating \\ Number of Times Taught: 2 \\ Average Enrollment: \\ 30
}


Number of Times Taught: 46

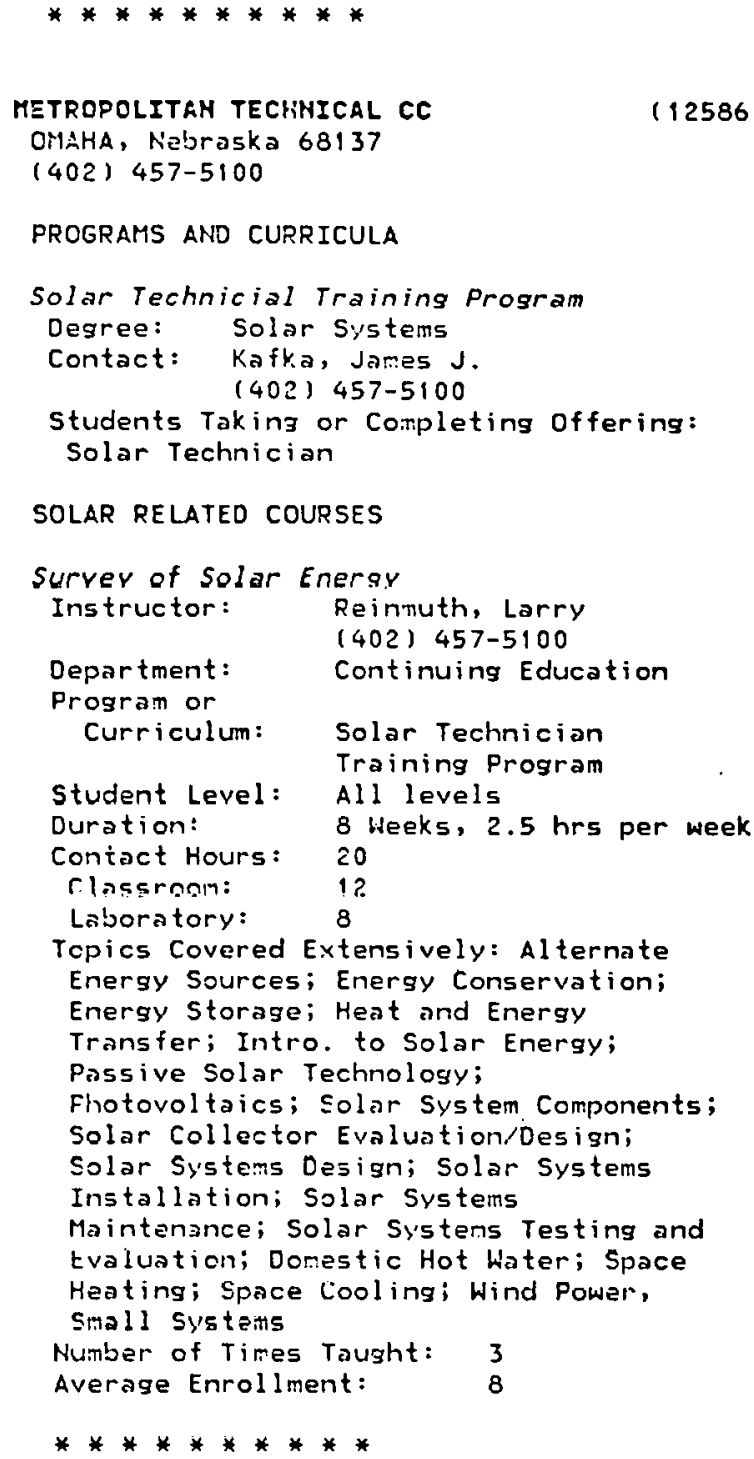

SOUTHEAST CC MILFORD CAM

MILFORD, Nebraska 68405

(402) 761-2131

\section{SOLAR RELATED COURSES}
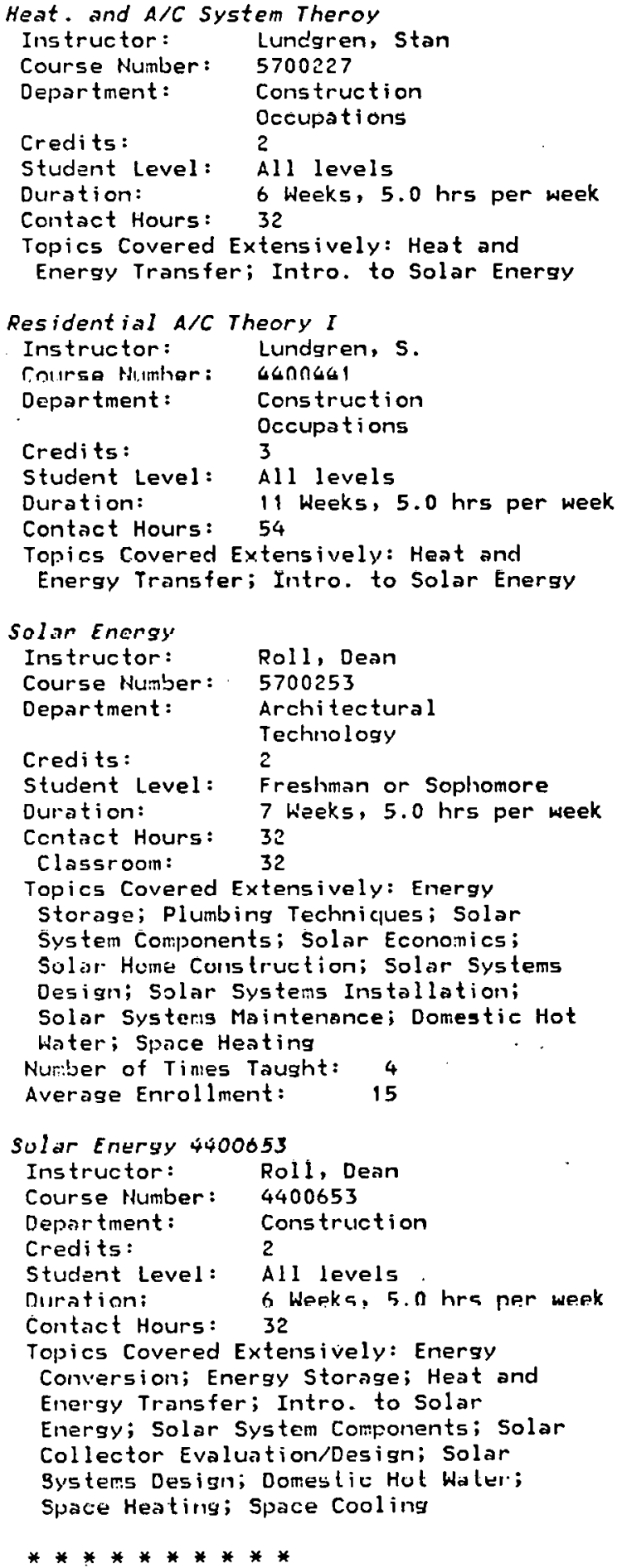


\section{Colleges/Universities}

HEVADA LAS VEGAS, U OF

LAS VEGAS, Nevada 89154

$(2569)$

$(702) 739-3011$

PROGRAMS AND CURRICULA

Solar Systems

Contact: Tryon, John G.

(702) 739-3701

students Taking or Completing offering:

Contractor, Installer-Residential

(Solar System), Installer-Commercial

(Solar System). Solar Technician,

Heating, Ventilation, and Air Cond.

Horker

\section{SOLAR RELATED COURSES}

\section{Solar Heating}

Instructor:

Tryon, John $G$.

(702) 739-370

Course Number: EGG475X

Department: Engineering

Credits: 3

Student Level: Junior or Senior

Duration: 16 Weeks, 3.0 hrs per week

Contact Hours: 48

Classrocm: $\quad 48$

Topics Covered Extensively: Intro. to

Solar Elrergy; Solar System Components;

Solar Economics; Solar Collector

Evaluation/Desisn; Solar Systems

Design; Solar Systens Installation;

Domestic Hot Hater; Swimming Fool

Heating; Space Heating; Space Cooling

Nur:-ber of Times Taught: 1

Averase Enrollment: $\quad 10$

Solar Systems-Domest ic Hot hater

Instructor: Tryon, John G.

(702) 739-3701

Department: Cont. Ed., Hev. Sol.

Ener. Assoc.

Program or

Curriculum:

Solar Systems

All levels

Ouration: 5 heeks, 3.0 hrs per. week

Contact Hours: 16

Classicom: 13

Tcpics Covered Extensively: Solar System

Corponents; Solar Economics; Solar

Collector Evaluation/Desisn; Solar

Sy'sters Design; Solar Systems

Installation; Domestic Hot Water

Number of Times Tausht: 1

Average Enrollment:

20

Solar Systers-Economics

Instructor: Tryon, John $G$.

(702) 739-3701

Department:

Cont. Ed., Nev. Sol.

Program or

Curriculum:

Student Level:

Duration:

Contact Hours:

Ener. Asso.

Solar Systems

All levels

2 Heeks, $10.0 \mathrm{hrs}$ per week

20

20
Topics Covered Extensively: Solar Economics; Domestic Hot Water; Swimming Pool Heating; Space Heating

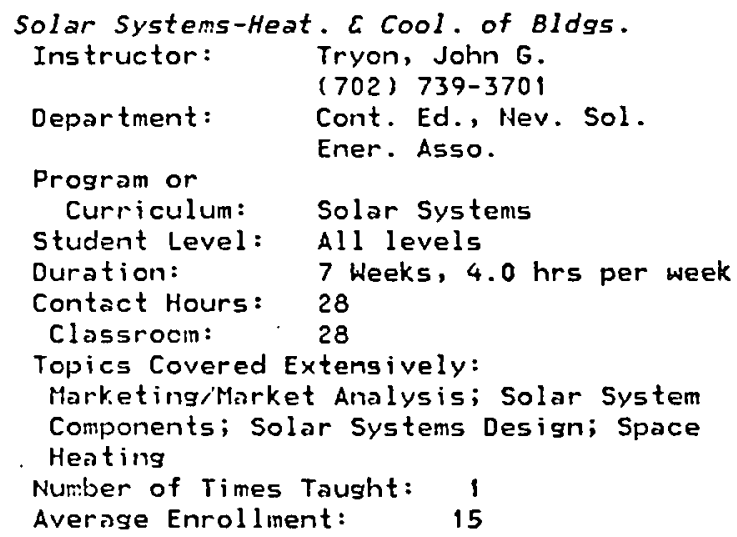

Course Number: 483-783

Department:

Crodits: 


\author{
Student Level: Junior or Senior \\ Duration: 15 heeks, 3.0 hrs per week \\ Contact Hours: 45 \\ Classroom: 45 \\ Topics Covered Extensively: Appropriate \\ Technolosy; Energy Conversion; Energy \\ Storase; Heat and Energy Transfer \\ Number of Times Tausht: 2 \\ Average Enrollment: $\quad 15$ \\ Solar Engineering \\ Instructor: Mckee, R. $B$. \\ Course Number: 374 \\ Department: Mechanical Engineering \\ Credits: 3 \\ Student Level: Junior or Senior \\ Dulaliull: 15 keeks, 5.0 hrs per week \\ Contact Hours: 75 \\ Classroom: $\quad 30$ \\ Laboratory: 45 \\ Topics Covered Extensively: Intro. to \\ Solar Enersy; Solar Collector \\ Evaluation/Uesisn; Solar Systems \\ Design; Domestic Hot Water; Space \\ Heating \\ Number of Times Taught: 1 \\ Averase Enrollment: $\quad 10$ \\ **********
}

Technology; Energy Conservation; Energy

Conversion; Energy Storage; Heat and

Energy Transfer; Materials Research;

Plumbing Techniques; Solar System

Components; Solar Econouics; Solar Home

Construction; Solar Law/Legislation;

Solar Collector Evaluation/Designi

Solar Systems Desisn; Solar Systems

Installation; Solar Systems

Maintenance; Solạr Systems Teșting and

Evaluaticn; Domestic Hut Water;

Swiming Pool Heating; Space Heating;

Space Cooling

$\begin{array}{ll}\text { Introduction to Solar Technology, } \\ \text { Instructor: } & \text { Comarow, David } \\ & (702) 643-6060 \\ \text { Course Number: } & \text { SOL } 119 \\ \text { Deportment: } & \text { Scicnce }\end{array}$

Prnaram no

Curriculum: Solạ Energv Terhhningy

Credits:

student Level: Freshman or Sophomore

Nirnatinn:

Contact Hours: 135

Classroom: $\quad 90$

Laboratory: 45

Topics Covered Extensively: Energy

Conservation; Energy Storage; Heat and

Energy Transfer; Intro. to Solar

Energy; Plumbing Techniques; Solar

Systen! Components; Solar Economics;

Solar Home Construction; Solar

Collector Evaluatien/Design; Solar

Systems Deșisn; Solar Systems

Installation; Solar Systems

Maintenance; Solar Systeris Testing and

Evaluation; Doricstic llot Mulei"s

Swiming Panl Hesting: Spare Heating;

Space Cooling

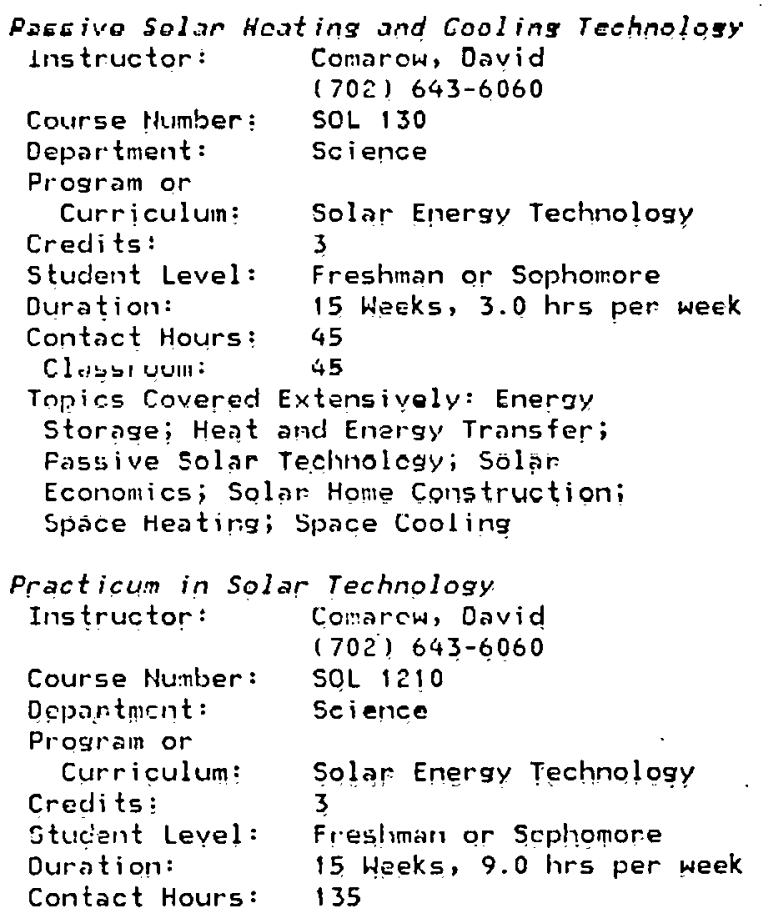

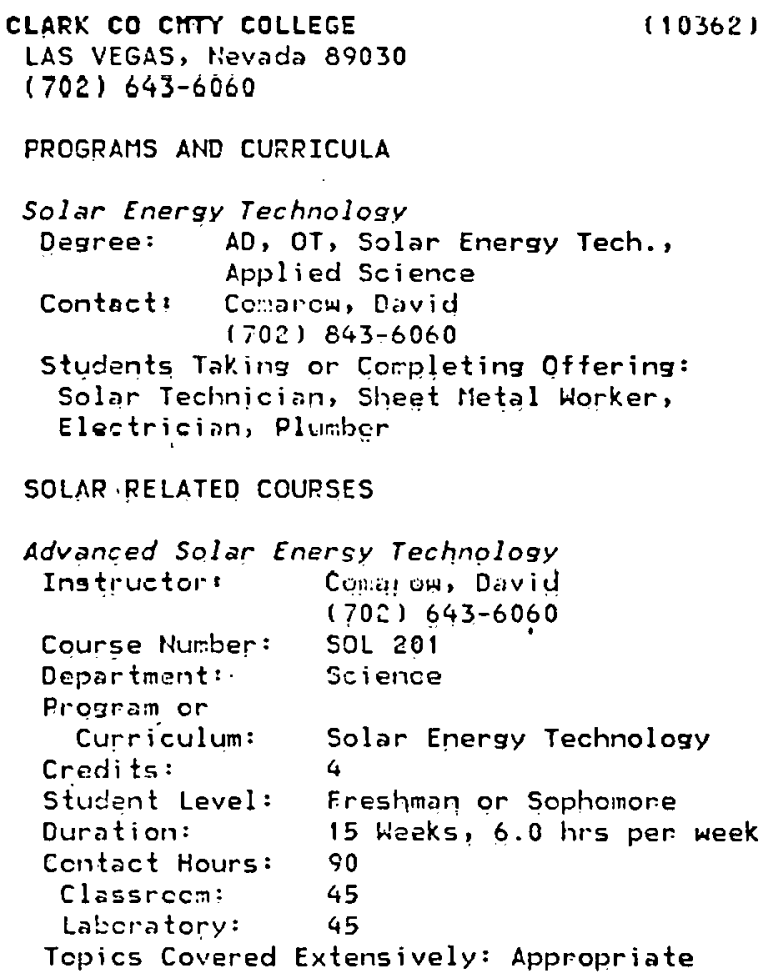


Topics Covered Extensively: Domestic Hot Water; Swimming Pool Heating; Process Heat, Industrial; Space Heating; Space Cooling

\author{
Solar Energy Technology--Home Cuner \\ Instructor: Comarow, David \\ (702) 643-6060 \\ Course Number: Elv 1183 \\ Department: Science \\ Prosram or \\ Curriculum: Solar Energy Technology \\ Student Level: Ali levels \\ Duration: \\ Conţą̧ Hours: \\ Classroom: \\ I Heeks, 15.0 hrs per week \\ Topics Covered Extensively: Alternate \\ Enersy Scurces; Apprcpriate Technology; \\ Enersy Conservation; Energy Converșion; \\ Enersy Storase; Heat and Energy \\ Transfer; Intro. to Solar Energy; \\ Passive Solar Technolcgy; Plumbing \\ Techniques; Solar System Components; \\ Solar Eccnomics; Solar Home \\ Construction; Solar Collector \\ Evaluationidesign; Solar Systems \\ Desisn; Solar Systems Installation; \\ Solar Systems Maintenance; Solar \\ Systems Testing and Evaluation; \\ Domestic Hot Hater: Swiming Pool \\ Heating; Space Heating; Space Cooling \\ Number of Times Taught: \\ Average Enrollment:
}




\section{colleges/Universities}

DARTMOUTH COLLEGE

HANOVER, Hew Hampshire

(603) 646-1110

FROGRAMS AND CURRICULA

* Solar studies-Blds. Heat. and Photor.

SOLAR RELATED COURSES

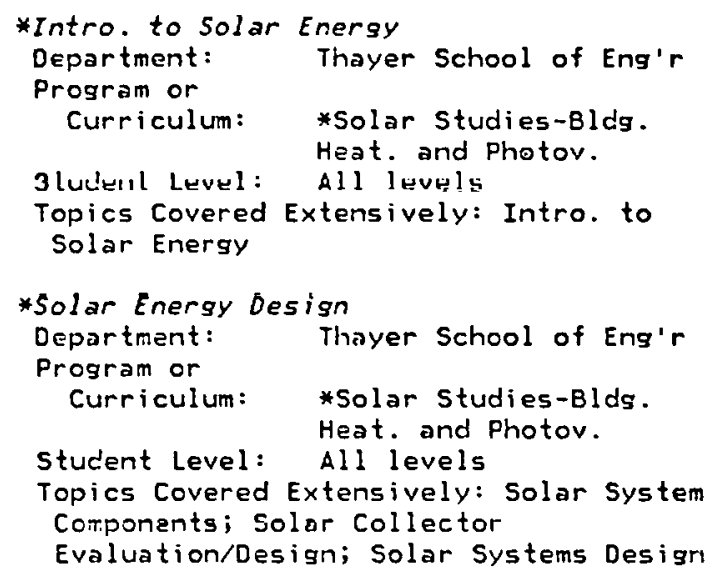

HII PLYROUTI ST COLLECE,U PLYMOUTH, New Hampshire

(603) 536-1550

\section{SOLAR RELATED COURSES}

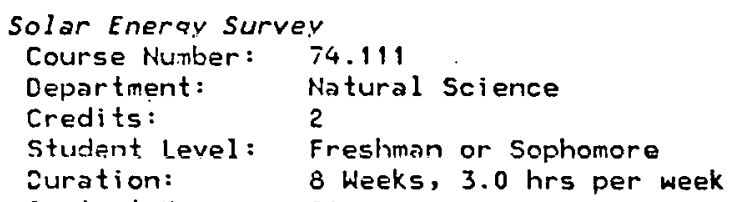

Classroom: 24

Topics Covered Extensively: Alternate Energy Sources; Energy Conversion:

Energy Storage; Intro. to Solar Energy Number of Times Taught: 3 Average Enrollment: 40

\section{Vocational/Technical colleges}

NH VO:- TELH C MANCHESTER

(2582) MAHCHESTER, Hew Hampshire (603) 663-6706

FROGRAMS AND CURRICULA

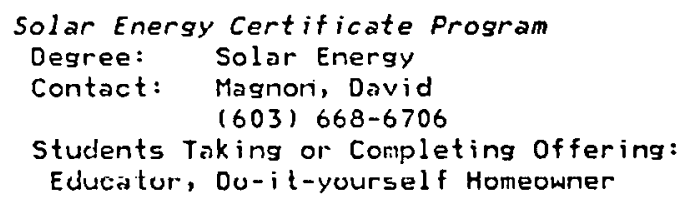

SOLAR RELATED COURSES

Energy Conservation - Principles Instructor: Magnon, David (603) 668-6706

Course Number: MSÁlEV

Department: Evening

Progrom or Curricultum: Solar Energy

Credils: 3

Stueiant Leval: All levels

Duration: 12 Weeks, 3.0 hrs per week

Contact Hours: 36

Tcpics Covered Extengively: Energy

Conservation

Number of Times Taught: 1

Average Enrollment: $\quad 20$

Energy Survey $\varepsilon$ Alternative Systems

Instructor: Magnon, David

(603) 668-6706

Course Number: M9ioEV

Department: Evening

Program or

Curriculum, Solar Energy

Crevils: 3

Student Level: All levels

Duration: 12 Weeks, 3.0 hrs per week

Contact Hours: 36

Topics Covered Extensively: Alternate

Energy Sources; Appropriate Technology;

Fnergy Conservatinn; Intro. to Solor

Energy; Passive Solar Technology

Number of $T i$ ries Tausht: 1

Average Enrollment: 20 


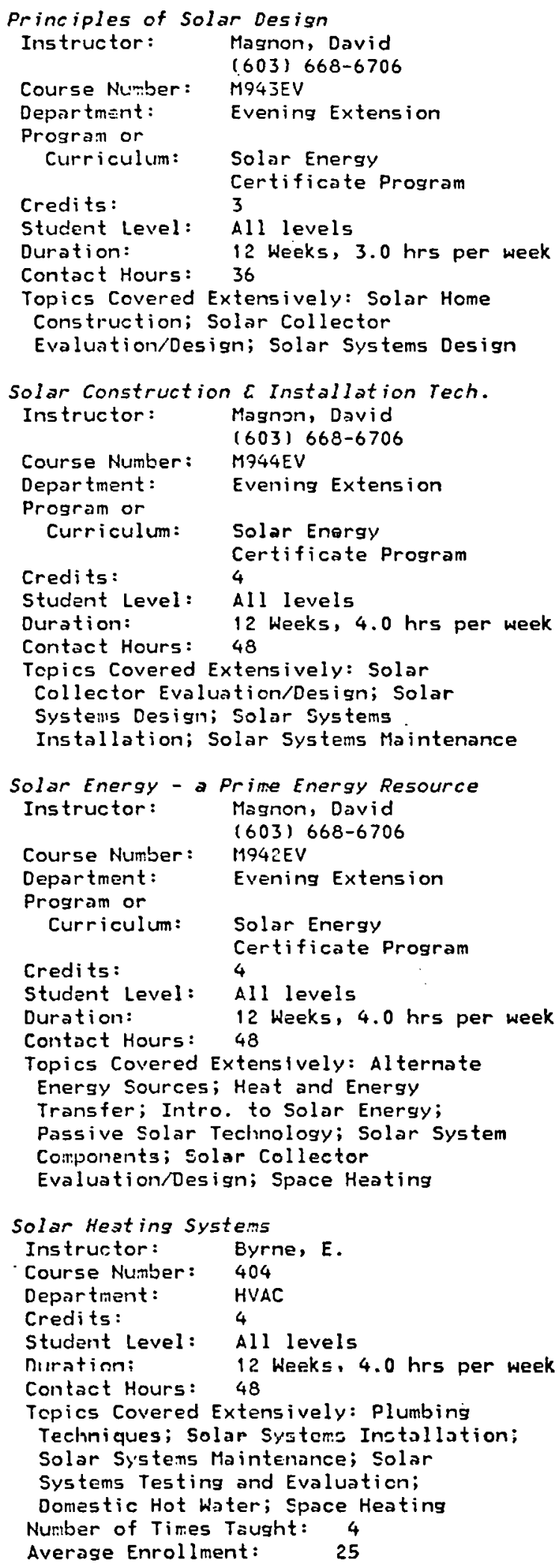

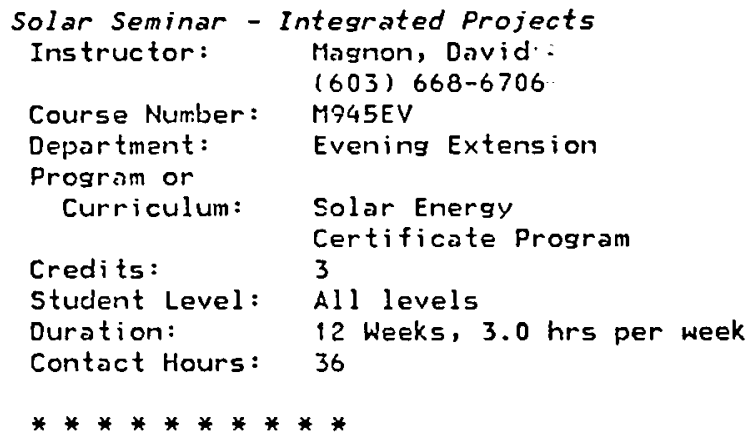




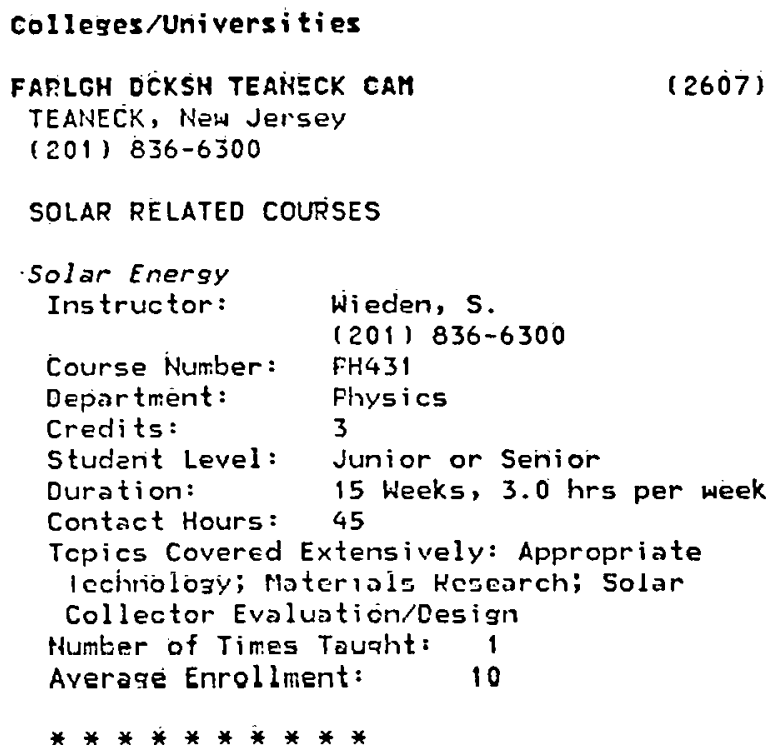

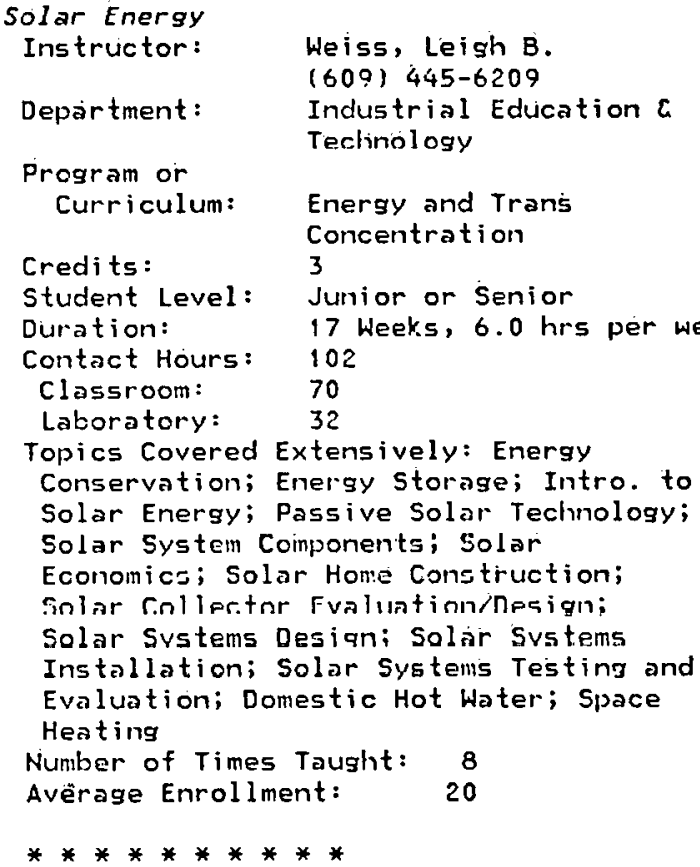

\section{SOLAR RELATED COURSES}

Alternate Energy Conversion Systems Instructor: Greeniald, Martin

(201) 893-4163

Course Number: 484

Uepartiment: Industriai teduc: Technology

Fi usi dill UI.

Curriculum: Industrial Powers

Ciedilst 3

Student Level: All levels

Duration: $\quad 16$ Weeks, $4.0 \mathrm{hrs}$ per week

Contret Hours: 64

Classroom: $\quad 32$

Laboratory: 32

Topics Covered Extensively: Alternate Energy Sources; Energy Conservation; Energy Coinversion; Energy Storage; Intro. to Solar Energy; Solar Economics; Solar Systems Design; Solar Systems Installatiun; Jular Systemin Maintenance; Solar Systel:s Testing and Evaluation; Elec'l Generation, Central; Elec'l Generation, Small Scale; Space Heating; Wind Power, Central Systenis; Wind Power, Small Systems

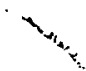




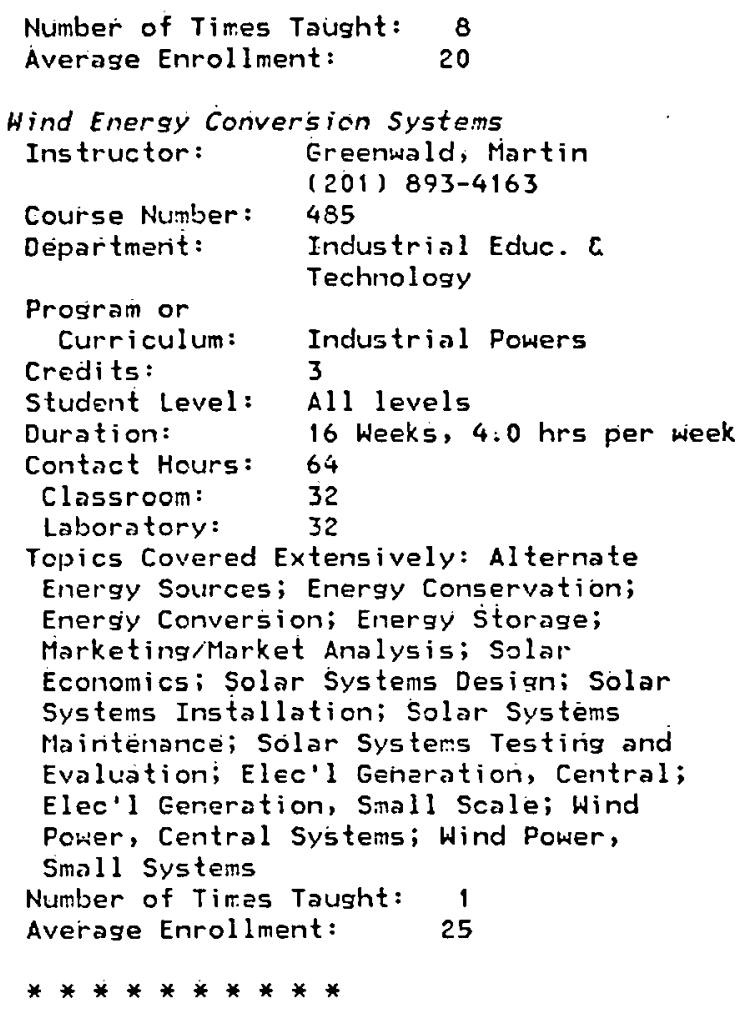

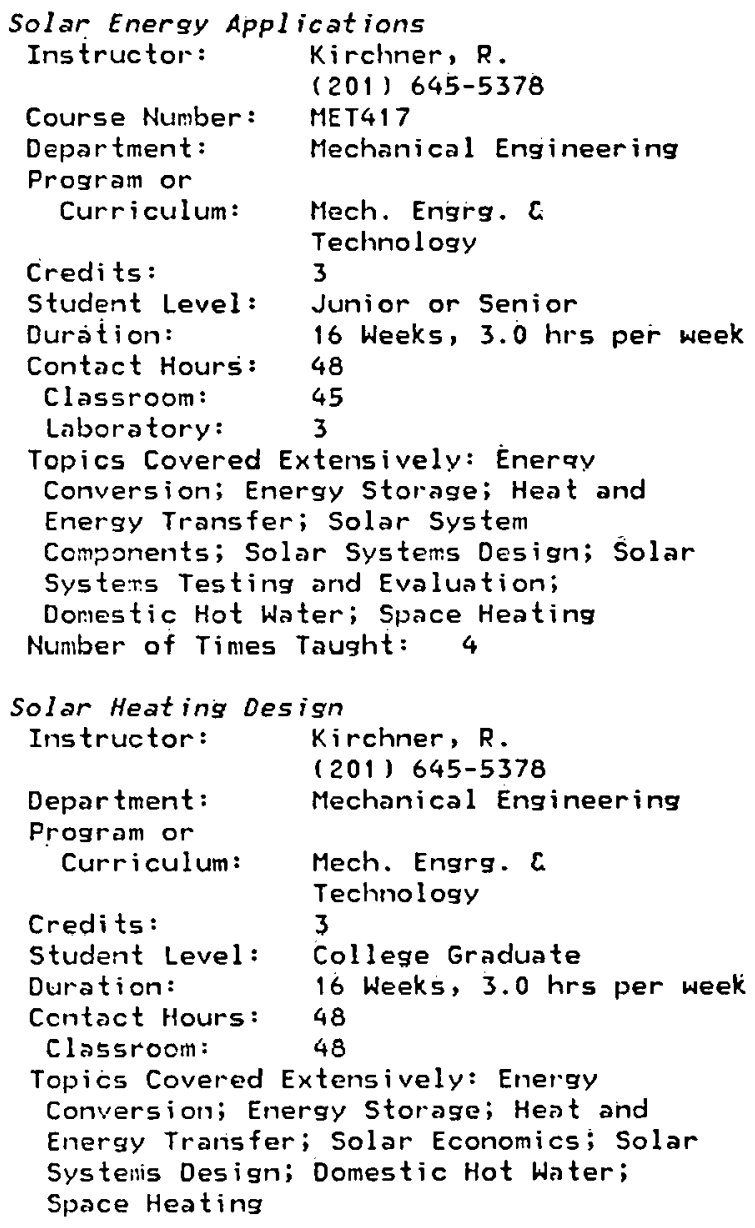


Credits: 3 Student Level: Freshman or Sophomore Duration: 13 Weeks, $3.0 \mathrm{hrs}$ per week Contact Hours: 39

Topics Covered Extensively: Materials Research; Photcvoltaics

Nurber of Tires Tausht: 5

Average Enrollrient: 13

Ener. and the Envir.: A Quant itative App.

Instructor: Sccolow, R.

(609) 452-5446

Course Number: EliGP 213

Department: Mechanical and

Program or

Curriculum: Energy Conversion and Pesources

Croveli is:

3

Student Level: Freshman or Sophomore

$\begin{array}{ll}\text { Duration: } & 13 \text { theeks, } 3.0 \text { hrs per week } \\ \text { Centact Hours: } & 39\end{array}$

Topics Covered Extensively: Alternate

Energy Sources; Appropriate Technolcay;

Energy Conservaticn; Energy Conversion;

Energy Storage; Intro. to Solar Energy;

Passive Solar Technology; Solar Energy

Policy Development; Solar System

Corponents; Solar Economics; Space

Heating

Nurber of rimes Taught: 7

Average Enrollment: 30

Intro. to Solar Thermal Engineering

Instructor:

Antal, i. J.

(60?) 452-5136

Course Nutber:

Erig? 101

Department :

Mechanical and

Aeroepace Engingering

Proaram or

Curriculum: Energy Conversion and

Resources

Credits:

3

Student Level: Freshman or Sophomore

Duration: 13 Hooks, $3.0 \mathrm{hre}$ per moek.

Contact Hours: 39

C.lassroom: 20

Laboratory: 19

Topics Covered Extensively: Alternate

Energy Sources; Appropriate Technology;

Enersy Conservation: Energy Conversion;

Energy Storaco; Hat and tnorgy

Transfer; Intro. to Solar Enersy;

Passive Solar Technology; Solar System

Corponents; Solar Hore Construction;

Solar Systems Desisn; Space Heating

thubiei of Tinies Tought, a

Average Enrollment: $\quad 50$

Fhysical frocesses of Energy Conversion

Instructor:

Course Number:

Miles, R. B.

$16091452-5131$

Department:

MAE 328

Mechanical and

Aerospace Engineering

Program or

Curriculum:

Energy Conversion and

Resources

Credits:

3

Student Level: Junior or Senior
Duration:

13 Weeks, 3.0 hrs per week

Contact Hours: 39

Topics Covered Extensively: Energy

Conversion; Energy Storage

Number of Times Taught: 5

Average Enrollment: $\quad 20$

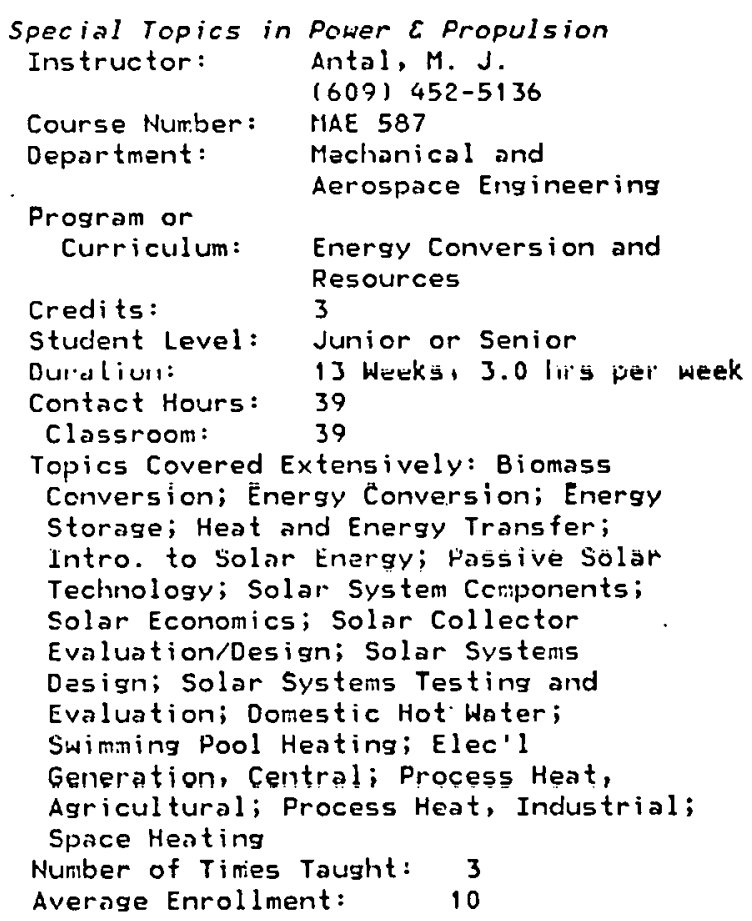

States of Matter

Instructar:

Pnyere, R. S. H.

$1609) 452-4681$

Course Number: MAE 324

Department: Mechanical and

Aerospace Engineering

Program or

Curriculum: Energy Conversion and

Credits: Resources

Student Level: Junior or Senior

Duration: 13 Weeks, 3.0 hrs per week

Contact Hours: 39

Classroom: $\quad 39$

lopics Loverod tutensivoly: risterials

Research; Fhotovoltaics

Number of Times Taught: 1

Average Enrollment: $\quad 10$

$* \times * * \times \times \times * *$

RAMAPO C OF HEH JERSEY

(9344)

MAHWAH, New Jersey

(201) 825-2800

PROGRAMS AND CURRICULA

Alternative Energy

Degree: BA, BS, Environmental

Contact: Harrison, Eugene

(201) 825-2800 
Students Taking or Completing Offering: Educator, Researcher, Do-it-yourself Homeowner, other

\section{SOLAR RELATED COURSES}

Alternative Energy Desisn

Instructor: Makofske, $W$. (201) 825-2800

Course Number: 400

Department: Environmental studies

Program or

Curriculum: Alternative Energy

Credits: 4

Student Level: Junion or Senior

Duration: 16 Weeks, $4.0 \mathrm{hrs}$ per week

Contact Hours: 64

Classroom: $\quad 48$

Laboratory: 16

Topics Covered Extensively: Alternate

Energy Sources; Appropriate Technology;

Biomass Conversion; Energy

Conservation; Energy Storage; Heat and

Energy Transfer; Passive Solar

Technolosy; Solar System Components;

Solar Home Construction; Solar

Collector Evaluation/Design; Solar

Systers Desisn; Solar Systems

Installation; Solar Systems

Maintenance; Solar Systems Testing and

Evaluation; Domestic Hot Water; Space

Heating; Wind Power, Small Systems

Alternative Energy Sources

$\begin{array}{ll}\text { Instructor: } & \text { Makofske, William } \\ & (201) 825-2800 \\ \text { Course Number: } & 300 \\ \text { Department: } & \text { Environmental Studies } \\ \text { Program or } & \\ \quad \text { Curriculum: } & \text { Alternative Energy } \\ \text { Credits: } & 4 \\ \text { Student Level: } & \text { Junior or Senior } \\ \text { Duration: } & 16 \text { Weeks, } 4.0 \text { hrs per week } \\ \text { Contact Hours: } & 64 \\ \text { Classroom: } & 64\end{array}$

Topics Covered Extensively: Al ternate

Energy Sources; Energy Conservation;

Energy Conversion; Heat and Energy

Tranisfer; Intro. to Solar Energy;

Photovoltaics; Solar System Components

Number of Times Tausht: 3

Average Enrollment: $\quad 40$

Alternative Energy fiorkshop

$\begin{array}{ll}\text { Instructor: } & \text { Greenwald, M./ Makoske, } \\ & \text { H. } \\ & (201) 825-2800 \\ \text { Course Number: } & 300 \\ \text { Department: } & \text { Environmental Studies } \\ \text { Program or } & \\ \text { Curriculum: } & \text { Alternative Energy } \\ \text { Credits: } & 4 \\ \text { Student Level: } & \text { All levels } \\ \text { Duration: } & 16 \text { Weeks, } 4.0 \text { hrs per week } \\ \text { Centact Hours: } & 64 \\ \text { Classroom: } & 24 \\ \text { Laboratory: } & 40 \\ \text { Topics Covered Extensively: Solar System } \\ \text { Components; Solar Collector } \\ \text { Evaluation/Design; Solar Systems }\end{array}$

Design; Solar Systems Installation;

Solar Systems Testing and Evaluation;

Domestic Hot Water; Space Heating; Wind

Power, Snall Systems

Number of Times Taught: 6

Average Enrollment: $\quad 25$

Energy Efficient Solar Desisn

Instructor: Makofske, $W$.

$\begin{array}{ll} & 1201) 825-2800 \\ & 300\end{array}$

Department: Environmental Studies

Prosram or

Curriculum: Alternative Energy

Credits:

Student Level: Junior or Senior

Duration: 16 Weeks, $4.0 \mathrm{hrs}$ per week

Contact Hours: 64

Classroom: $\quad 64$

Topics Covered Extensively: Energy

Consarvation; Energy Conversion; Energy.

Storase; Heat and Energy Transfer;

Intro. to Solar Energy; Passive Solar

Technology; Solar System Components;

Solar Economics; Solar Hone

Construction; Solar Collector

Evaluation/Design; Solar Systems

Design; Domestic Hot Water; Space

Heating; Space Cooling

Number of Times Taught: 3

Average Enrollment: $\quad 50$

Energy, Power and the Environment

Instructor: Mokoske, W.

(201) 825-2800

Course flumber: 200-300

Department: Environmental Studies

Program or

Curriculum: Alternative Energy

Credits:

Student Level: All levels

Duration: 16 Heeks, $4.0 \mathrm{hrs}$ per week

Contact Hours: 64

Classroom: $\quad 64$

Topics Covered Extensively: Energy

Conversion; Heat and Energy Transfer;

Intro. to Solar Energy

Nunber of Times Taught: 3

Average Enrollment: 40

**********

RUTGERS U HEH BRUNS'JICK

HEH BRUIISWICK, Hew Jersey

(6964)

(201) 932-1766

SOLAR RELATED COURSES

Solar Thermal Ener. Collect. and stor.

Instructor: Briggs, David G.

(201) $923-3656$

Course Number: $650: 474$

Department: Mech. Indus. and

Credits: Aerospace Engineering

Student Level: Junior or Senior

Duration: 16 Weeks, 3.0 hrs per week

Contact Hours:- 48

Topics Covered Extensively: Energy 
Storase; Heat and Energy Transfer; Intro. to Solar Energy; Solar Collector Evaluation/Design; Solar Systems Desisn; Domestic Hot Water; Space Heating; Space Cooling Number of Times Taught: Averase Enrollment: 1

**********

\section{STOCKTON STATE COLLEGE}

FO: HOH.A, H:W Jersey

(609) 652-1776

SOLAR RELATED COURSES

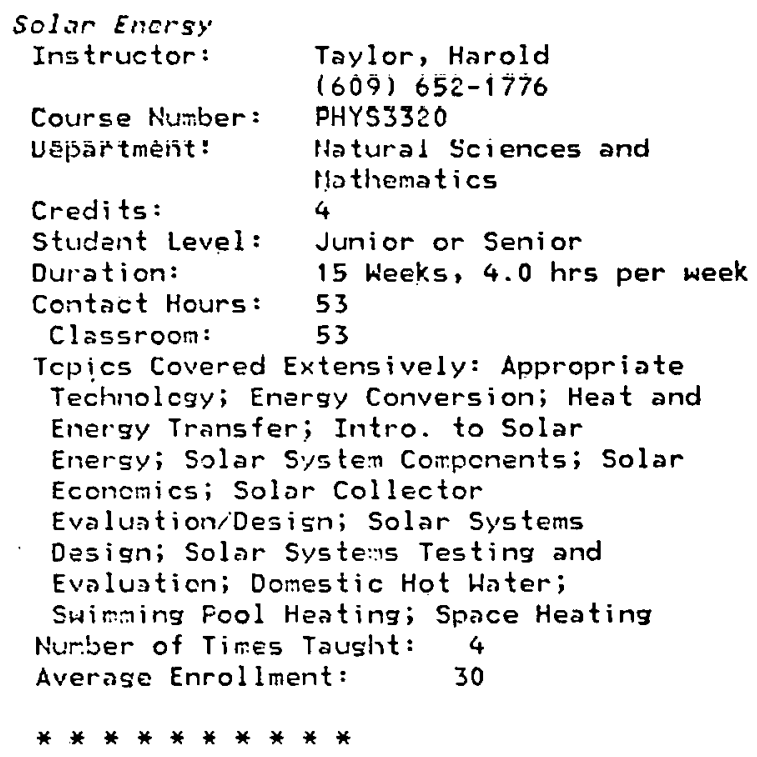

\section{Community/Junior Colleges}

\section{BROOKDALE CRTY COLLECE}

LIIICROFT, NiE Jersey

(201) $842-1900$

SOLAR RELATED COURSES
Collese Gradurte 15 Heeks, 2.0 hrs per week

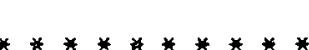

HIDOLESEX COUHTY COLLEGE

$(2615)$

EOISON, New Jersey

(201) 548-6000

SOLAR RELATED COURSES

*Horkshop Appr. to Teach. Train. in Energ. Department: Project Watte

**********

\section{Vocational,Technical colleses \\ MERCER CO AREA VOC. TECH. SCHOOLS \\ 1055 old Trenton Rd. \\ TRENTON, Hew Jersey 08690 \\ SOLAR RELATED COURSES \\ * Install Solar Heat. a Cool. \\ Department: Pluming. Heating, $c$ Refrig. \\ Topics Covered Extensively: Plumbing Techniques; Solar System Conponents; Solar Systems Installation; Domestic Hot Water; Space Heating; Space Cooling

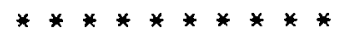

Jacksom, New Jersey 08527

FROGRAMS AIID CURRICULA

*Sol. Ener. Theory - Heat., Vent., A/C

Tech.

Degree: Evening School Certificate

SOLAR RELATED COURSES

*5ol. Ener. Theory - Heat., Vent., a/C Tech. Department: Evening School Program or Curriculum: *sol. Ener. Theory Heat., Vent.; A/ C Tech.

Studont Laval. All lovols Duraticn: 15 Weeks Topics Covered [xtensively. Solor System Corponents; Solar Collector Evaluation/Design; Solar Systems Design; Domastic Hot Water; Space Heating

* Solar Enersy Horkshop

Department: Evening School Student Level: All levels

Topics Covered Extensively: Solar System Components; Solar Fconomics; Solar Collector Evaluation'Design; Solar Systens Design; Solar Systems 
Installation; Domestic Hot Water

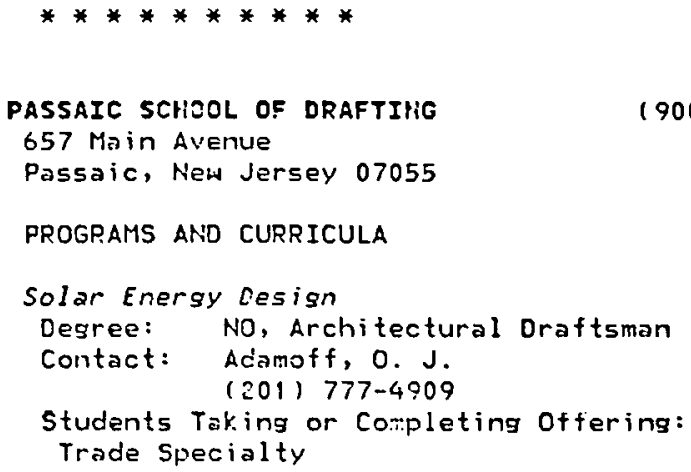

\section{SOLAR RELATED COURSES}

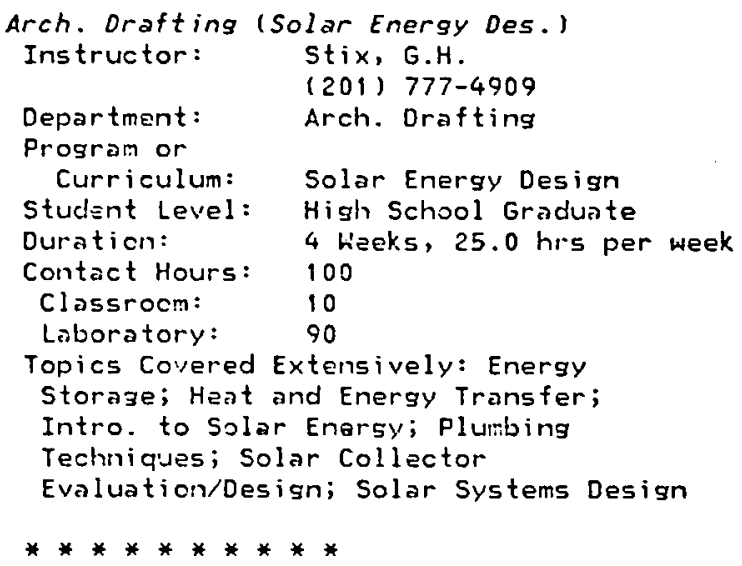

SALEM COUHTY VOCATIONAL TECHYICAL SCHOOLS

R.D. $\# 2$, Box 350

Hoodstown, Hew Jersey 08098

PROGRAMS AHD CURPICULA

* Plumbing and Heating Trades

SULAR RELATEU CUURSES

* Introduction to Solar Heating

Department: Continuing Education

Contact Hours: 36

Topics Covered Extensively: Space

Heatina; space Cooling

* Unit on Solar Energy

Program or

Curriculum: *Plumbing and Heating Trac!es

Student Level: All levels

Topics Covered Extensively: Solar System

Components; Solar Collector

Evaluation'Design; Solar Systems

Design: Comestic hot hater

UHYOY CO TECHHICAL IHST

SCOTCH PLAINS, New Jersey

(201) 889-2000

\section{SOLAR RELATED COURSES}

\section{Solar Heating I}

Instructor:

Course Number:

Department:

(201) $889-2000$

$\mathrm{HV}-201-71$

Heating Ventilating-Air

Conditioning

Student Level: All levels

Duration:

Contast Hours: 45

Classronm: $\quad 15$

Laboratory: $\quad 30$

Topics Covered Extensively: Alternate

Energy Sources; Appropriate Technology;

Energy Conservation; Energy Conversion;

Energy Storage; Heat and Energy

Transfer; Intro. to Solar Energy;

Plun:jing. Techniques; Sheet Metal

Techniques; Solar System Components;

Solar Economics; Solar Collector

Evaluation/Design; Solar Systems

Desisn; Solar Systens Installation;

Solar Systers Maintenance; Solar

Systerns Testing and Evaluation;

Domestic Hot Water; Swinming Pool

Henting; Space Henting; Space Cooling

Number of Times Tousht: 2

Averase Enrollment: $\quad 20$

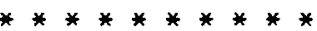

\section{other Educational Institutions}

ESSEX COUATY TECHIYICAL CAREERS CENTER (90390)

91 Weśt Market St.

Newark, New Jersey

FROGRAMS AFID CURRICULA

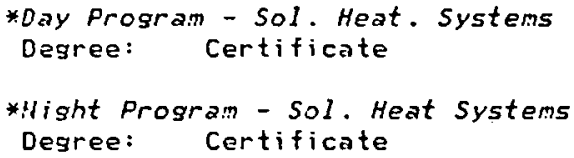

SOLAR RELATEO COURSES

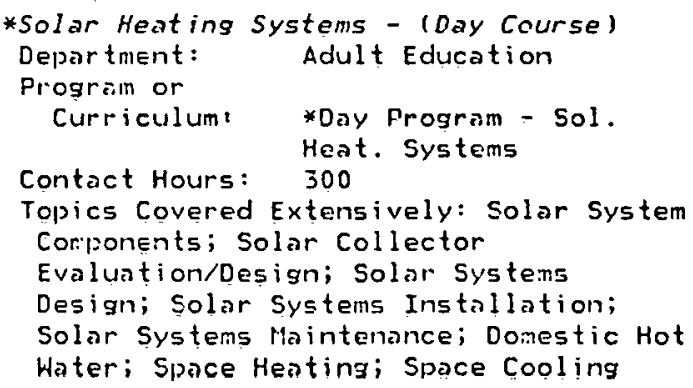




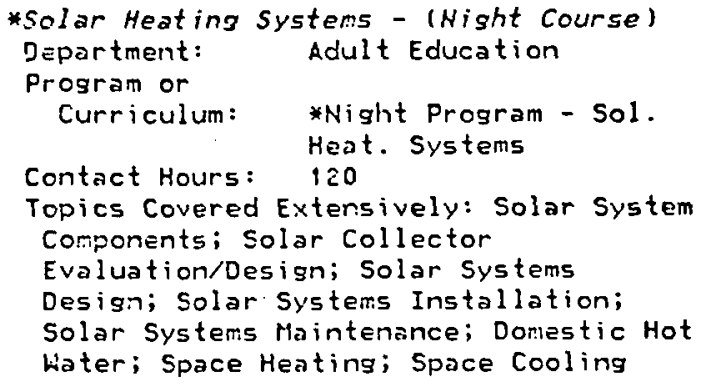




\section{Colleges/Universities}

NEH MEXICO HIGHLAHDS U

(2653)

LAS VEGAS, Hew Mexico 8770

(505) 425-751!

PROGRAMS AND CURRICULA

Solar Greenhouse Construction

Degree: No,

Contact: Martinez, E. Eloy

(505) 425-7511

\section{SOLAR RELATED COURSES}

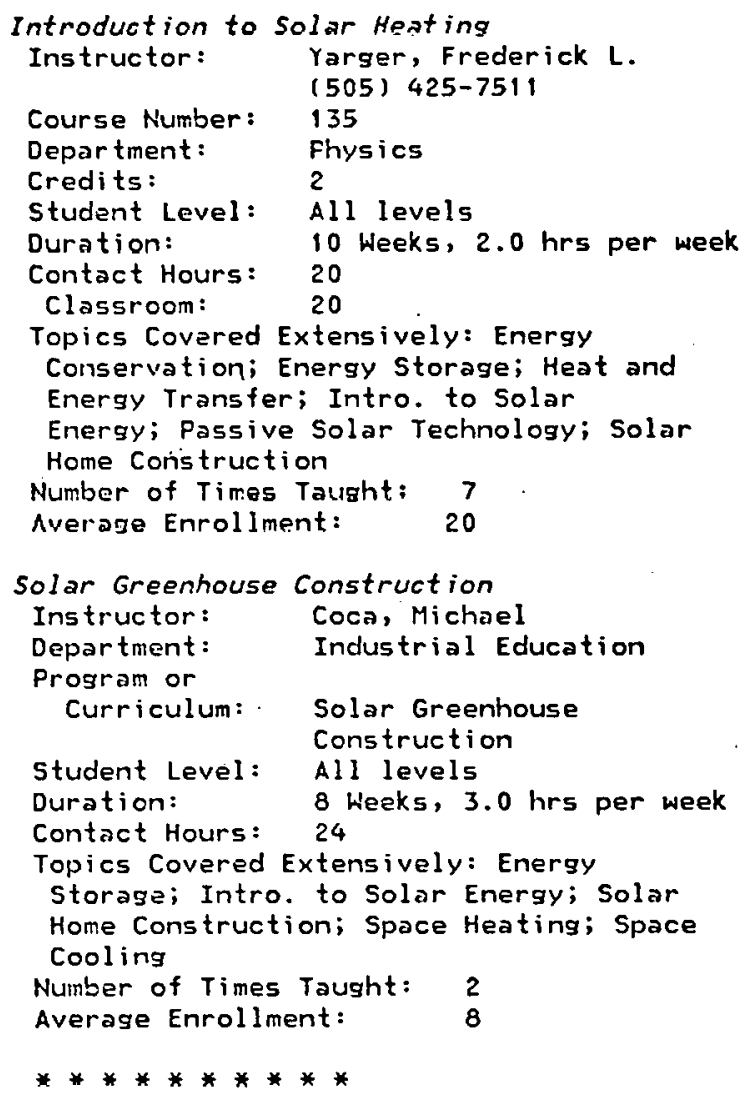

NH MAIN CAMPUS, U OF

$(10313)$

ALBUqUeRque, New Mexico 87131

(505) 277-0111

\section{SOLAR RELATED COURSES}

Applied Solar Energy-Engineering Systems

Instructor: Hessling, F. C.

(505) 277-4937

Course Number: ME 425

Department: Mechanical Engineering

Credits: 3

Student Level: Junior or Senior

Duration: 16 Weeks, $3.0 \mathrm{hrs}$ per week

Contact Hours: 48

Topics Covered Extensively: Energy

Conservation; Energy Storage; Heat and

Energy Transfer; Passive Solar
Technology; Solar Collector

Evaluation/Design; Solar Systems Design

Number of Times Taught: 3

Average Enrollment: $\quad 18$

Energy Utilization and Conversion

Instructor: Houghton, A. V.

Course Number: 382

Department: Mechanical Engineering

Credits: $\quad 3$

Student Level: Junior or Senior

Duration: 15 Weeks, $3.0 \mathrm{hrs}$ per week

Contact Hours: 45

Classroom: 45

Topics Covered Extensively: Alternate

Energy Sources; Biomass Conversion;

Energy Conservation; Energy Conversion;

Energy Storage; Intro. to Solar Energy;

Photovoltaics; Solor Energy Policy

Development; Solar System Components

Number of Times Taught: 7

Average Enrolliment: 22

\section{Power Generating Systems}

Instructor: Houghton, A. V.

Course Number: 483

Department: Mechanical Engineering

Credits: 3

Student Level: Junior or Senior

Duration: 15 Weeks, 3.0 hrs per week

Contict Hours: 45 .

Classroom: 45

Topics Covered Extensively: Energy

Conservation; Marketing/Market

Analys is; Materials Research; Plumbing

Techniques; Sheet lletal Techniques

Number of Times Taught: 3

Average Enrollment: $\quad 25$

Solar Energy System Design and Analysis

Instructor: Wessling, F. C.

(505) 277-4937

Course Number: M.E. 525

Department: Mechanical Engineering

Credits: 3

Student Level: College Gracluate

Duration: 16 Heeks, 3.0 hrs per week

Contact Hours: 48

Topics Covered Extensively: Energy

Conservation; Energy Storage; Heat and

Energy Transfer; Intro. to Solor

Energy; Passive Solar Technology; Solar

Collector Evaluation/Design; Solar

Systemis Design

Number of Times Taught: 2

Average Enrollment: 10

Solar Energy Use

Instructor:

Ebenezer, J.

Course Number: 385

Department: Engineering

Credits: 3

Student Level: Junior or Senior

Duration: 16 Weeks, 3.0 hrs per week

Contact Hours: 48

Classroom: $\quad 48$

Topics Covered Extensively: Passive 
Solar Technology; Solar System Components; Domestic Hot hater; Space Heating

Number of Times Taught: 3

Average Enrollment: 170

$* * * * * * * * * *$

NM STATE U MAIH CAMPUS

(2657)

LAS CRUCES, New Mexico 88003

(505) 646-2035

\section{PROGRAMS AND CURRICULA}

\section{Solar Engineering}

Degree: Pho, MS, Mechanical Engr.

Contact: Smith, P.R.

(505) 646-3501

Students Taking or Completing Offering:

Pesearrhar, Solsp Engineer

SOLAR RELATED COUPSES

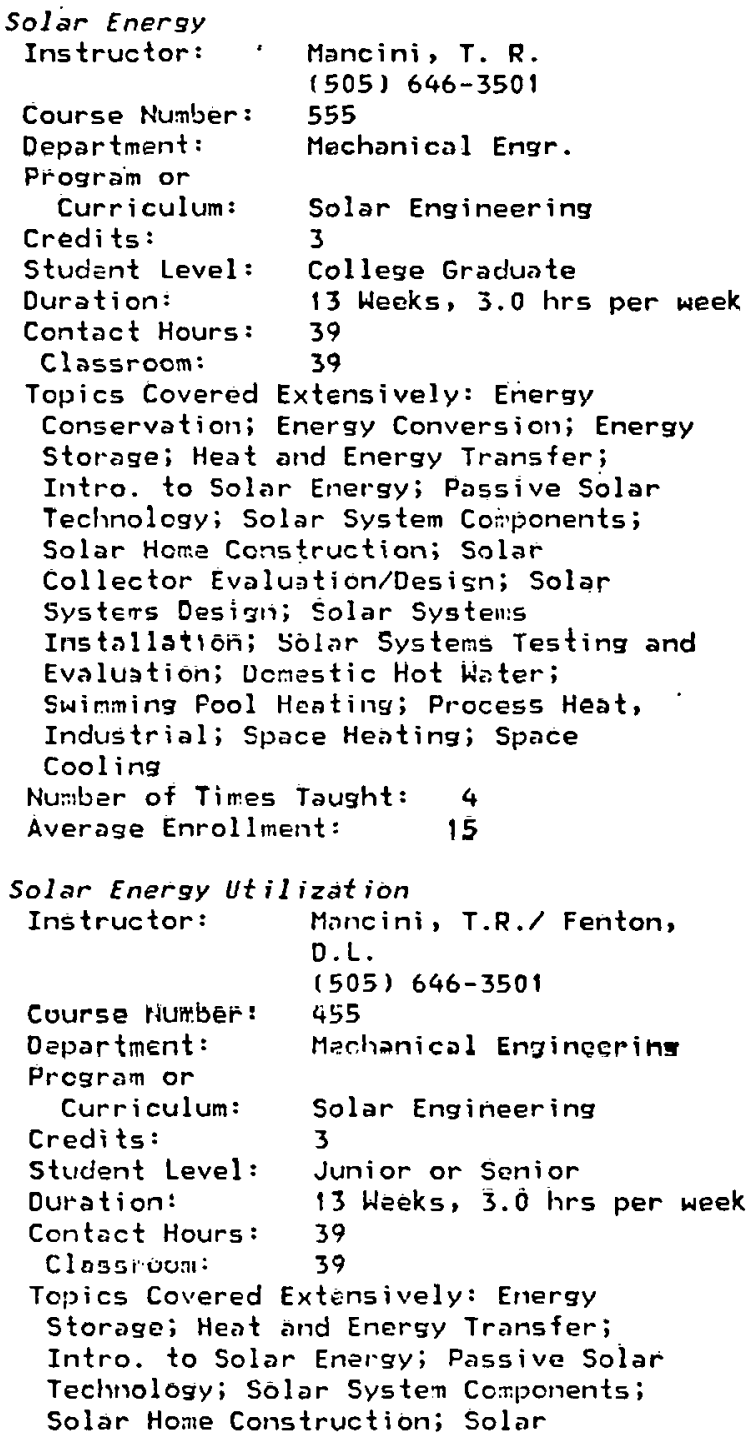

Collector Evaluation/Design; Solar Systers Design; Dorrestic Hot Hater; Swimming Pool Heating; Space Heating; Space Cooling

Number of Tines Taught: 10

Average Enrollment: $\quad 30$

Solar Heating and Cooling

Instructor: Lumsdaire, E./ Mancini,

$$
\text { T.R. }
$$
(505) 646-3501

Course Number: 565

Department: Mechanical Engr.

Prosiam or

Curriculum: Solar Engineering

Credits: 3

Student Level: College Graduate

Duration. 13 Heeks, 3.0 hrs per Hook

Contact Hours: 39

Classroom: $\quad 39$

lcpics Covered Extensively: Energy Lonservation; Energy Storage; Heat and Energy Transfer; Intro. to Solar Energy; Passive Solar Technology; Fhotovoltaics; Solar System Components; Solar Economics; Solar Home

Construction; Solar Law/Legislation; Solar Collector Evaluation/Design; Sprice Heating; Solar Systems Testing and Evaluation; Domestic Hot Water; Swiming Fool Heating; Space Heating; Space Cooling

Number of Times Taught: 1

Average Enrollment: 10

Solar Thernal Fower

Instructor: Mulholland, G. P.

(505) 646-3501

Course Number: $\mathbf{5 7 5}$

Dcpartment: Mecharical Engr.

Program or

Curriculum: Solar Engineering

Erodite.

Sturlent lavol: College Crocluste

Duration: $\quad 13$ Weeks, $3.0 \mathrm{hrs}$ per week

Contact Hours: 39

Classroom: $\quad 39$

Topics Covered Extensively: Energy Storase; Hoat and Energy Trarlsfer; Materials Research; Solor System Conponentsi Julal Law'l wyialstion: Solar Systems Design; Solar Systems Testing ond Evaluation; Elec'l Generation, Central

Number of Times Taught: 1 Average Enrollimant: 8

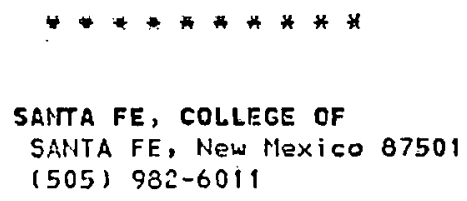




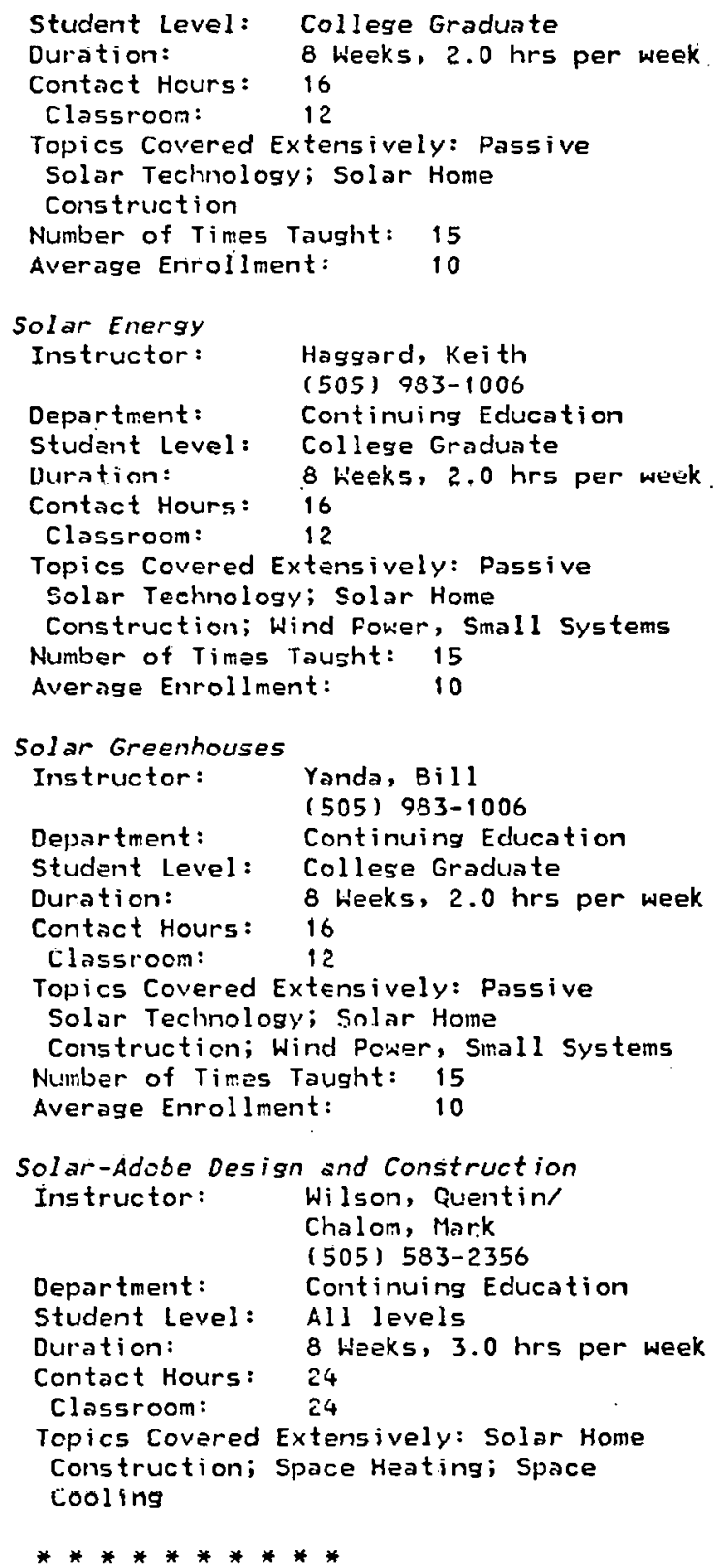

Community/Junior colleges
NORTHERH NH COMYUNITY COLLEGE

(29087)

$$
\text { EL RITO, New Mexico } 87530
$$

(505) 581-4501

SOLAR RELATED COURSES

Solar-Adobe Design and Construction

Instructor:

Wilson, Quent in $C$.

(505) 583-2356

Department: Continuing Education/Community

Student Level: All levels

Duration: 8 Weeks, $3.0 \mathrm{hrs}$ per week

Contact Hours: 24

Classroom: $\quad 24_{4}$

Topics Covered Extensively: Solar Home

Construction; Solar Systems Design

Number of Times Taught: 7

Average Enrollment:

12 


\section{Colleses/Universities}

ADELPHI UHIVERSITY

(2666)

GARDEH CITY, NEW YOrK 11530

(516) 294-8700

\section{PROGRAMS AND CURRICULA}

Energy Institute

Degree: MS, BS, Physics, Energy

Studies

Contact: Docher, Jehn

(516) 294-8700

Studerits Taking or Completing offering:

Educator, Researcher

SOLAR RELATED COURSES

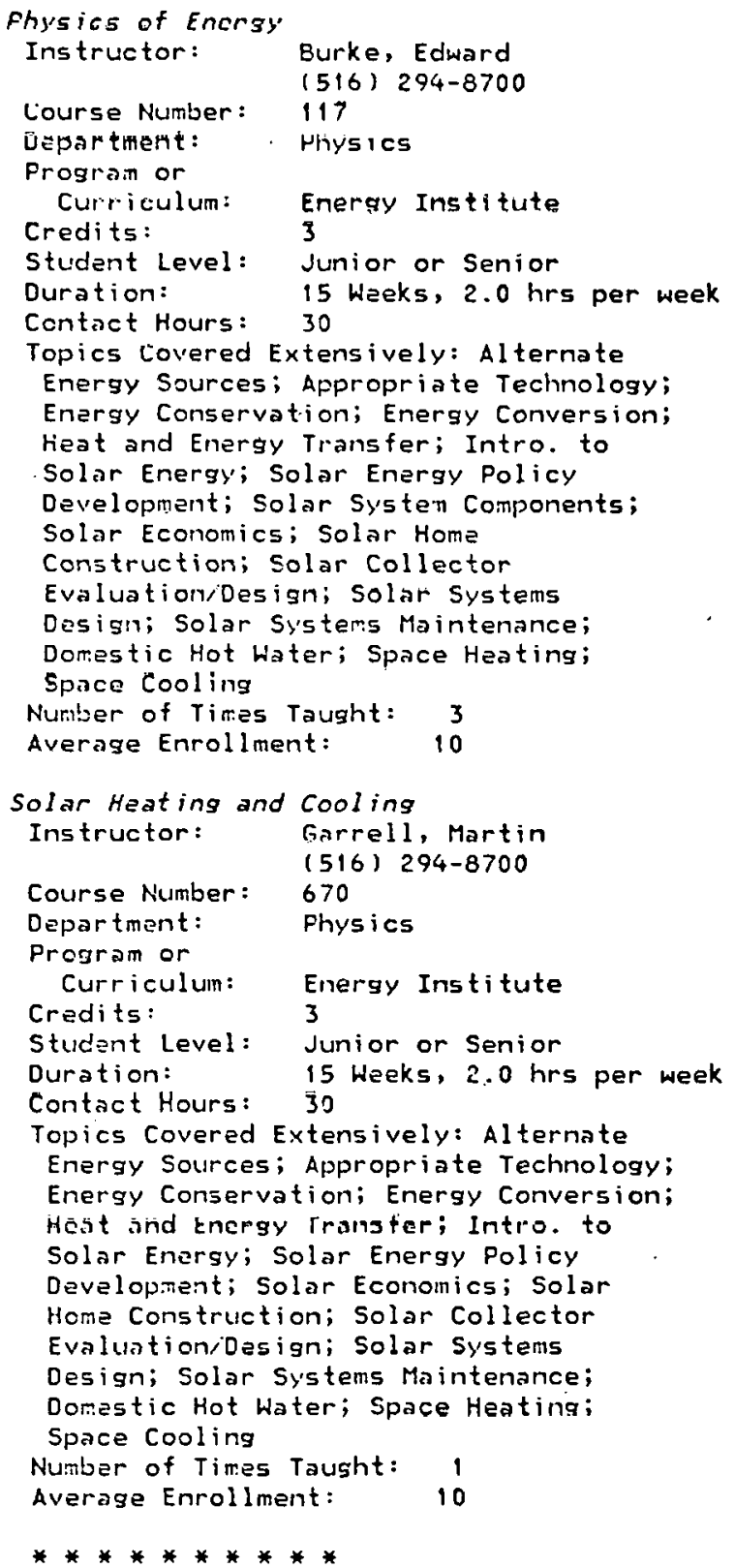

BARO COLLEGE

(2671)

ANHJANDALE-ON-HUDSON, New York 12504

(914) 758-6822

SOLAR RELATED COURSES

Alternative Energy Sources

Instructor: Brody, Burton

Department: $\quad(914) 758-6822$

Credits:

Student Level: All levels

Duration: $\quad 13$ Weeks, $4.0 \mathrm{hrs}$ per week

Contact Hours: 42

Classroom: $\quad 32$

Laboratory: 2

Topics Covered Extensively: Alternate

Energy Sources; Apṕropriate Technology;

Intro. to Solar Energy; Elec'l

Generation. Small Scale; Space Heating Number of Times Taught: 1

Average Enrollment: $\quad 30$

$* * * * * * * * * *$

CLARKSON COLLEGE OF TECHN

(2699)

POTSOAM, New York 13676

(315) 268-6400

SOLAR RELATED COURSES

Alternate Sources of Energy

Instructor: Clark, James

Course Number: ME 315

Department: Mechanical and

Credits: 3

Student Level: Junior or Senior

Duration: 15 Heeks, $3.0 \mathrm{hrs}$ per week

Contact Hours: 45

Topics Covered Extensively: Alternate Entriyy Sources; Energy Conservation; Energy Conversion; Energy Storage; Intro. to Solar Energy; Solar Systems Design; Wind Power, Central Systems; Wind Power, Small Systems Number of Times Taught: 3 Average Enrollinent: $\quad 35$

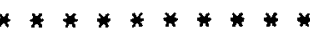

COLUMBIA U HAIK DIVISIOK

NEW YORK, Naw York 10027

(2707)

(212) 280-1754

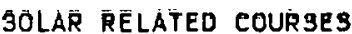

\section{Solar Energy Applications}

Instructor: Sanders, W.T.

Course Number: MEÉ4224X

Department: Mechanical Engineering

Credits:

Student Level:

Duration:

Contact Hours:

Classroom:
3

College Graduate

14 Weeks, $3.0 \mathrm{hrs}$ per week

42

36 
Topics Covered Extensively: Energy Storage; Heat and Energy Transfer; Intro. to Solar Energy; Solar System Components; Solar Collector

Evaluation/Design; Solar Systems Design; Domestic Hot Water; Space Heating; Space Cooling

***********

CORNELL U STATUTORY C

$(19693)$

ITHACA, New York 14853

(607) 256-1000

SOLAR RELATED COURSES

Energy and Man

Instructor:

Albright,. L. D.

(607) 256-4535

201

Course Number:

Agr. and Life Sci.,

Department:

Agr. Engineering

Credits:

Student Level: Junior or Senior

Duration: 14 Weeks, $3.0 \mathrm{hrs}$ per week

Contact Hours: 42

Classroom: $\quad 42$

Topics Covered Extensively: Alternate

Energy Scurces; Energy Conservation;

Heat and Energy Transfer; Intro. to

Solar Energy

Number of Times Taught: 4

Average Enrollment:

25

***********

CUNY BROOKLYN COLLEGE

(2687)

BROOKLYiN, New York 11210

(212) 780-5485

PROGRAMS ANIO CURRICULA

Energy Related Topics

Contact: Celenza, L. S.

(212) 780-5813

\section{SOLAR RELATED COURSES}

Energy in a Technological Society

Instructor: Celenza

(212) $780-5813$

Department:

Physics

Program or

Curriculum: Energy Reloted Topics

Credits:$$
3
$$

Student Level: College Graduate

Duration: 2 Heeks, 23.0 hrs per week

Contact Hours: 45

Topics Covered Extensively: Alternate

Encrgy' Sourcesi Bionses Conversinn:

Enersy Conservation; Enersy Comversion;

Energy Storage; Heat and Energy

Transfer; Intro. to Solar Energy;

Passive Solar Technolegy;

Photovoltaics; Solar Energy Policy

Develcpment; Solar Systen Components;

Solar Econonics; Solar Home

Construction; Elec'l Generation,
Central; Space Heating; Space Cooling;

Wind Power, Central Systems; Wind

Power, Small'Systems

Energy in a rechnological Society Phys 0.3

Instructor: Celenza

$\begin{array}{ll} & (212) 780-5813 \\ \text { Course Number: } & \text { PHYS } 0.3\end{array}$

Department: Physics

Program or

Curriculum: Energy Related Topics

Credits: 3

Student Level: All levels

Duration: 15 Weeks, 3.0 hrs per week

Contact Hours: 45

Classroom: 45

Topics Covered Extensively: Al ternate

Energy Sources; Biomass Conversion;

Energy Conservation; Energy Conversion;

Energy Storage; Heat and Energy

Transfer; Intro. to Solar Energy;

Photovoltaics; Solar Economics; Solar

Home Construction; Elec'l Generation,

Central; Elec'l Generation, Small Scale

Number of Times Taught: 3

Average Enrollment: $\quad 70$

Energy Technology

Ins tructor:

Skorinko

(212) 780-5817

Course Number: FHYS 38

Department: Physics

Credits: 3

Student Level: Junior or Senior

Duration: 15 Weeks, 3.0 hrs per week

Contact Hours: 45

Classroom: 45

Topics Covered Extensively: Alternate

Energy Sources; Biomass Conversion;

Energy Conversion; Energy Storage; Heat

and Energy Transfer; Intro, to Solar

Energy; Photovoltaics; Solar System

Components; Solar Economics; Elec'l

Generation, Central; Space Heating;

Space Cooling; Wind Power, Central

Systems; Wind Power, Small Systems

Seminar Series on Energy and Solar Energy

Instructor: Schwartz, Brian

Department: Science

Student Level: Junior or Senior

Topics Covered Extensively:

Photovoltaics

CUNY C OF STATEN ISLAND

$(29040)$

STATEN ISLAND, New York 10301

(212) 720-3000

SOLAR RELATED COURSES

Solar Energy

Instructor:

Nankivell, John

Course Number:

(212) 390-7524

Department:

MET 108

Credits:
Mechanical Technology 3 


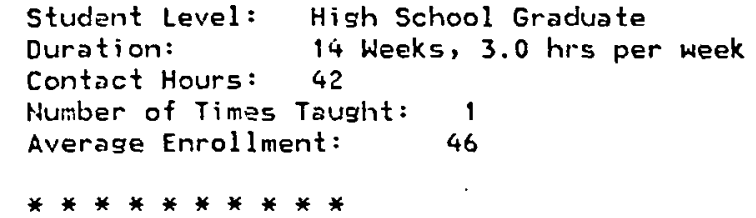

\section{HAHILTOH COLLEGE \\ CLINTON, New York 13323 \\ (315) 859-4011}

SOLAR RELATED COURSES

Fhysics of Energy

Instructor:

Department:

Student Level: Freshman or Sophomore

Duration:

Contact Hours: 72

classroom: $\quad 36$

Laboratory: 36

Number of Times Taught: 3

Average Enrollment:

20

$* * * * * * * * * *$

MAKIIATTAK COLLEGE

ERCNX, New York 10471

(212) 548-1400

SOLAR RELATED COURSES

$\begin{array}{ll}\text { Direct Energy Conversion } & \\ \text { Instructor: } & \text { Ley, James } \\ & (212) 548-1400 \\ \text { Course Number: } & 735 \\ \text { Department: } & \text { Engineering, Electrical } \\ & \text { Engin. } \\ \text { Credits: } & 3 \\ \text { Student Level: } & \text { College Graduate } \\ \text { Duration: } & 15 \text { Weeks, } 3.0 \text { hrs per week } \\ \text { Contact Hours: } 45 & \\ \text { Topics Covered Extensively: Energy } \\ \text { Conversion }\end{array}$


Energy Sources; Energy Conversion Number of Times Tausht: 5

Averase Enrollment:

15

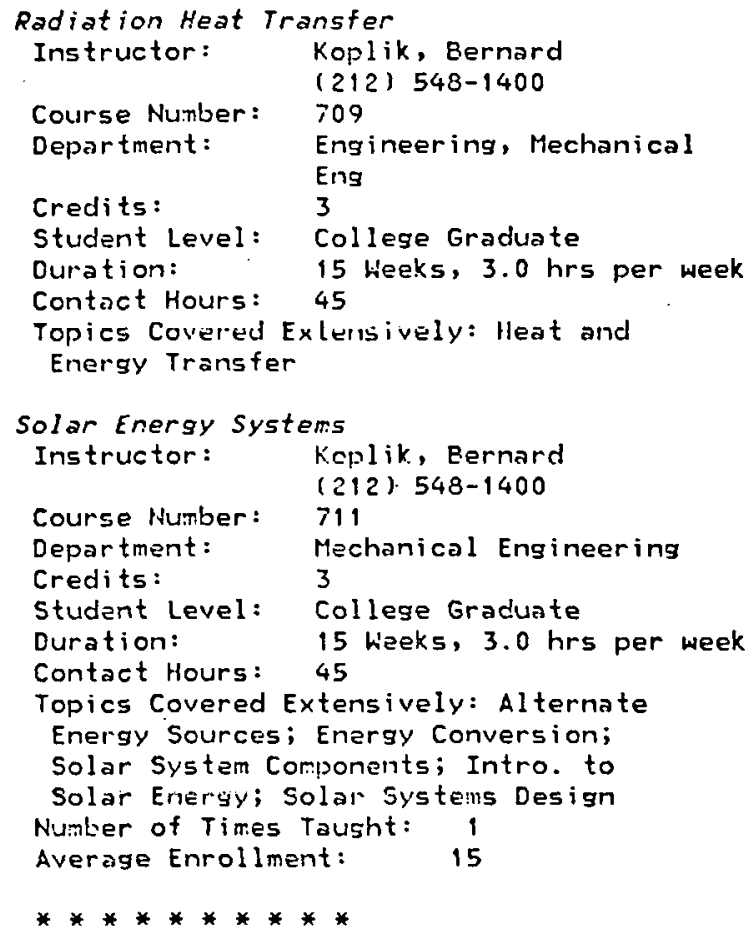

RCCHESTER, NEW YOrK 14610

(716) 5.36-2525

\section{SOLAR RELATED COUPSES}

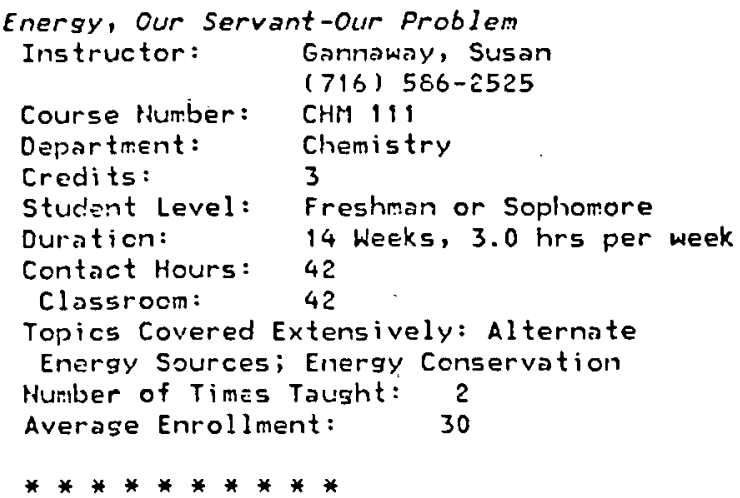

NEU YORK UYIYERSITY

137851

HEW YOPK, liew York 10012

( $乞 12) 593-1212$

\section{FROGRAMS AND CUPRICULA}

\footnotetext{
Solar Energy

Degree: Certificate of Participation

Contact: Philipps, Denis Sinclair $(212) 598-2.101$
}

\section{SOLAR RELATED COURSES}

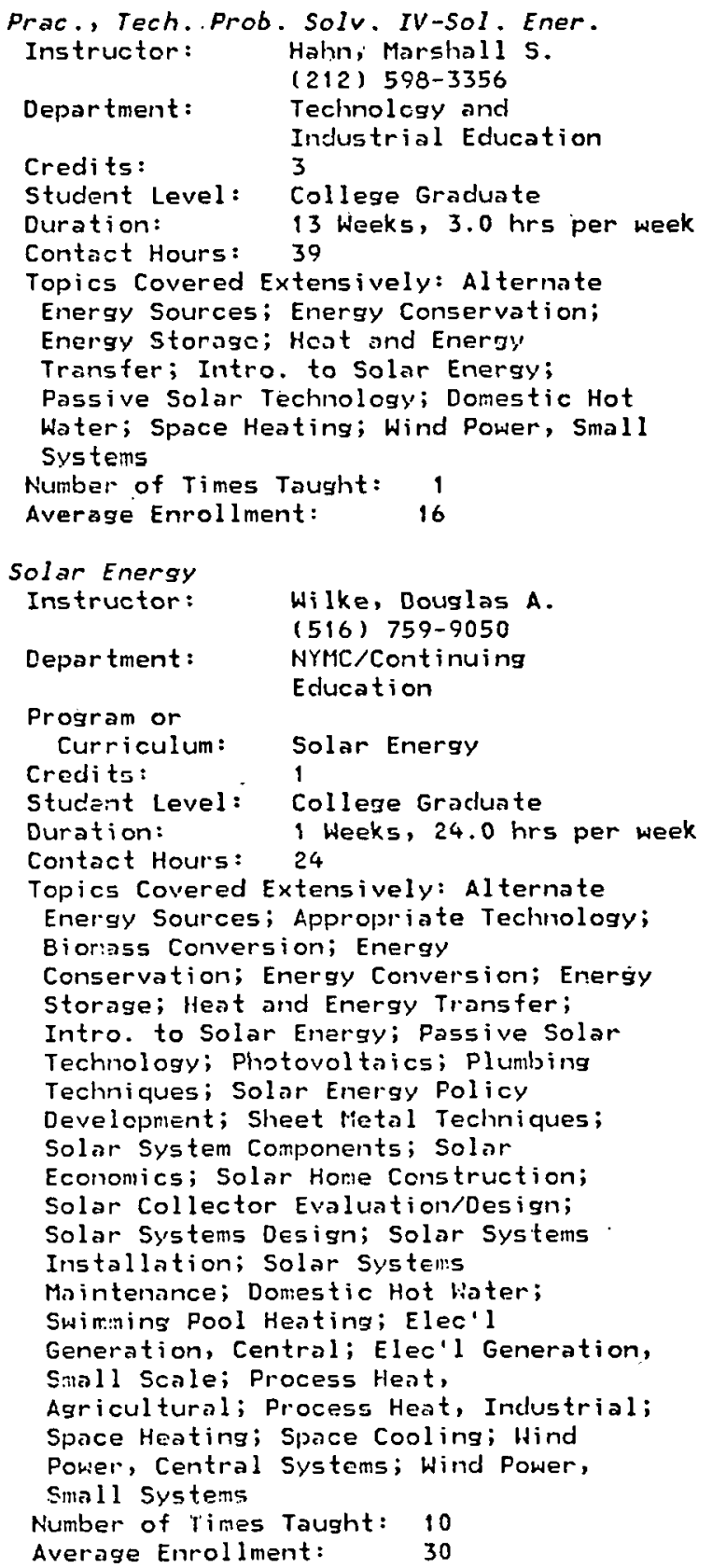

********** 
POLYTECHNIC INST KEH YORK

(2796) BROOKLYN, New York 11201 (212) 643-5000

\section{SOLAR RELATED COURSES}

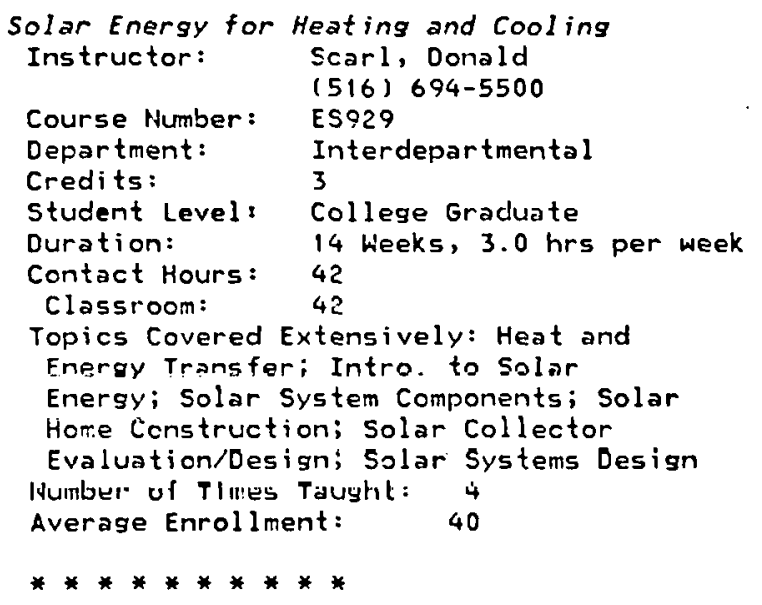

REHSSELAER POLY IKSTITUTE

(2803)

TROY, New York 12181

(518) $270-6000$

\section{SOLAR RELATED COURSES}

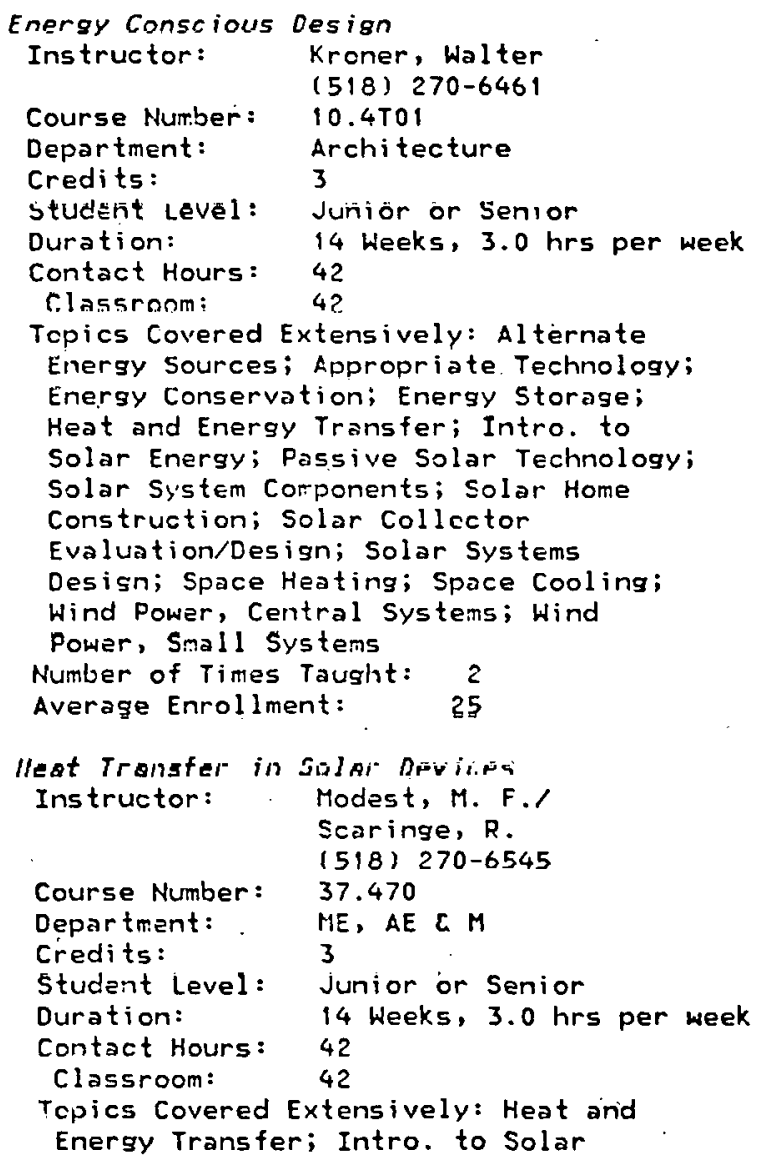

Energy; Solar Collector

Evaluation/Design; Domestic Hot Water;

Space Heating; Space Cooling

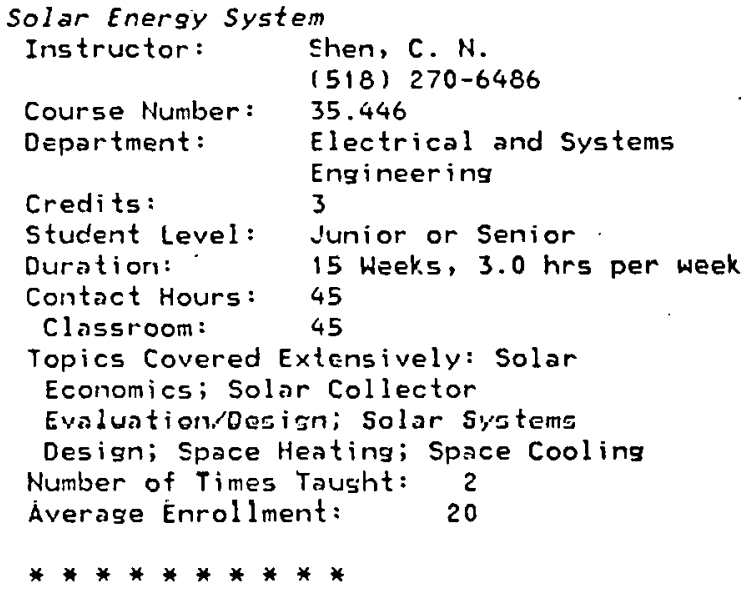

(2806)

$(2806)$


Class room:

42

Topics Covered Extensively: Energy Conversicn

Number of Times Taught: 5

Average Enrollment:

20

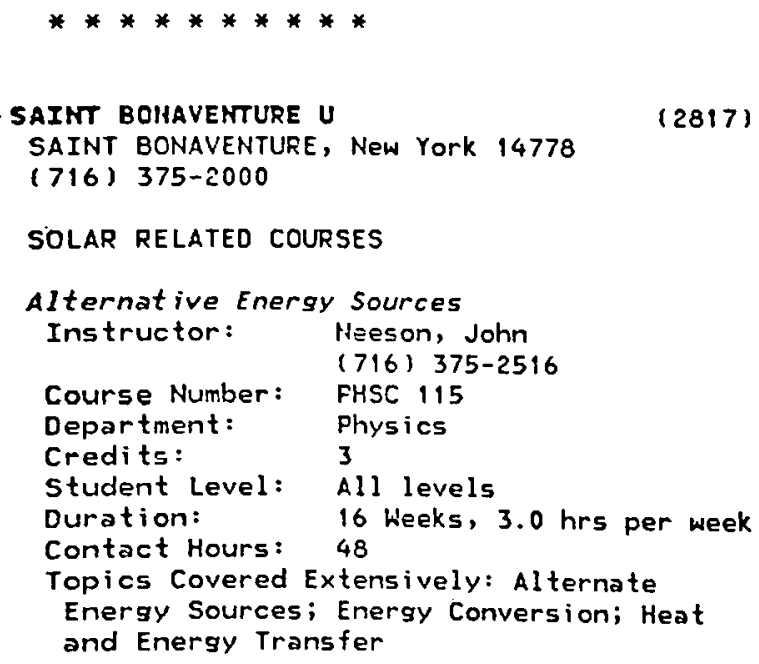

SAINT BOHAVENTURE U

SAINT BONAVENTURE, New York 14778

128171

(716) 375-2000

\section{SOLAR RELATED COURSES}

\section{SAIMT JOHH FISHER COLLEGE}

(2821)

ROCHESTER, New York 14618

( 716$) 586-4140$

SOLAR RELATED COURSES

Energy: Its Science and Technology

Instructor: Heininger, Clarence (716) 586-4140

Course Number: SCI 200

Department: Chemistry

Credits: 3

Student Level: All levols

Duration: 15 Weeks, $3.0 \mathrm{hrs}$ per week

Contact Hours: 45

Classroom: $\quad 45$

Topics Covered Extensively: Alternate Enpray Sources; Appropriate Technnlngy; Energy Conservation; Energy Conversion; Energy Storage; Intro. to Solar Energy; Passive Solar Technology:

Photovoltaics; Solar System Components; Solar Collector Evaluation/Design; Wind Power, Central Systens; Wind Power, Small Systems

Number of Times Taught: 4

Average Enrollment: 12

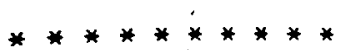

SUNY AT ALBAHY

Albany, New York 12222

$(2835)$

(518) 457-3300

PROGRAMS AND CURRICULA

Solar Energy Meteor. and Train. Site

Degree: PhD, MS, BS, Atmospheric

Sciences

Contact: Stewart, Ronald (518) 457-7584

Students Taking or Completing offering:

Educator, Researcher, Solar Technician

SOLAR RELATED COURSES

Solar Energy Horkshop

Instructor: Stewart, Ronald

(518) 457-7584

Department: Atmospheric Sci.

Program or

Curriculum: " Solar Energy Meteor.

Credits: and Train. Site

Student Level: College Graduate

Duration: 1 Heeks, $40.0 \mathrm{hrs}$ per week

Contact Hours: 40

Tópics Covered Extensively: Alternate

Energy Sources; Energy Conservation;

Intro. to Solar Energy; Wind Power,

Central Systems; Wind Power, Small

Systems

Number of Times Taught: 2

Average Enrollment: 25

Solar Radiation

Instructor: Czapski, Ulrich

(518) 457-3991

Course Number: 2639

Department: Atmospheric Science

Program or

Curriculum: Solar Energy Meteor.

Credits: 3

and Train. Site

Student Level: College Graduate

Duration: $\quad 20$ Weeks, $3.0 \mathrm{hrs}$ per week

Contact Hours: 60

Humber of Times Toight: 1

Average Enrollment: $\quad 10$

**********

SUYY AT BIHGHAMTON

BINGHAMTON, New York 13901

(2836)

(607) $798-2000$

PROGRAMS AND CURRICULA

Physics-Specialization in Solar Energy

Degree: $B A, B S$,

Phys ics-5pecialization in

Solar Energy

Contact: Stannard, C.R.

(607) 798-2217

Students Taking or Completing Offering:

Educator, Researcher, Solar Engineer 


\section{SOLAR RELATED COURSES}

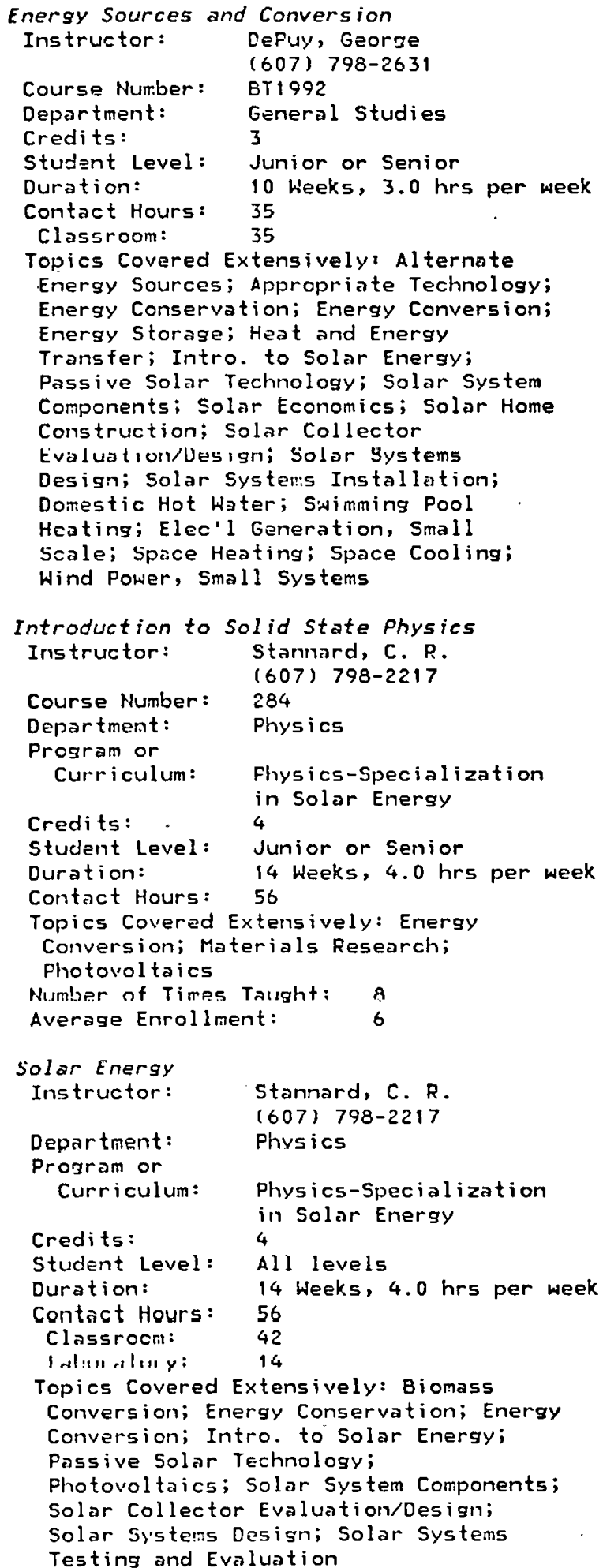

The Environment and Fhysical Principles

Instructor: Stannard, C. R.

(607) $798-2217$

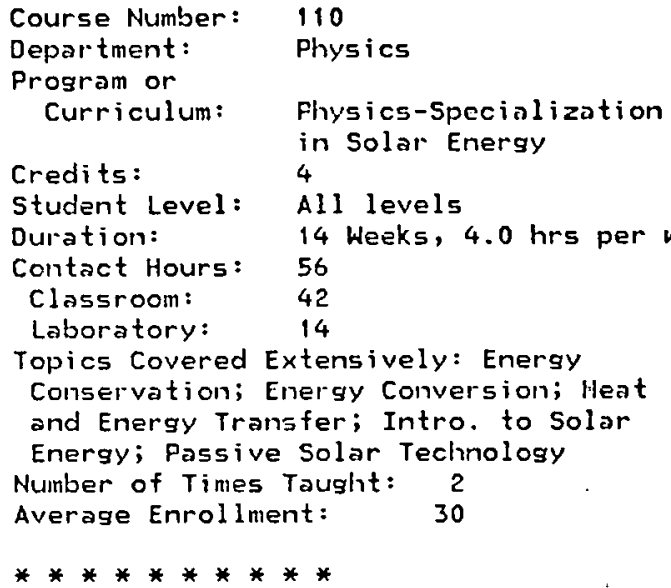

SU:T AT BUFFALO MAIH CAM

(2037)

BUFFALO, New York 14260

(716) $831-9000$

\section{SOLAR RELATED COURSES}

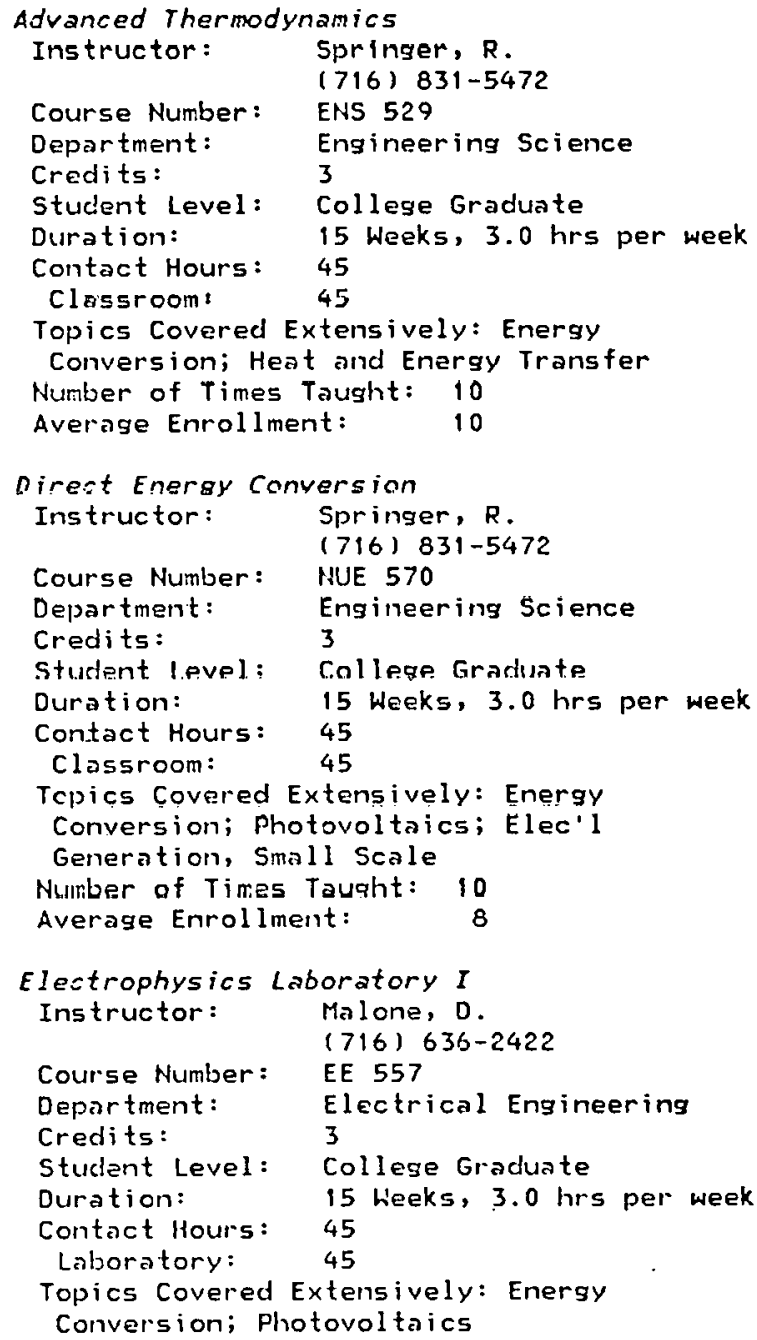




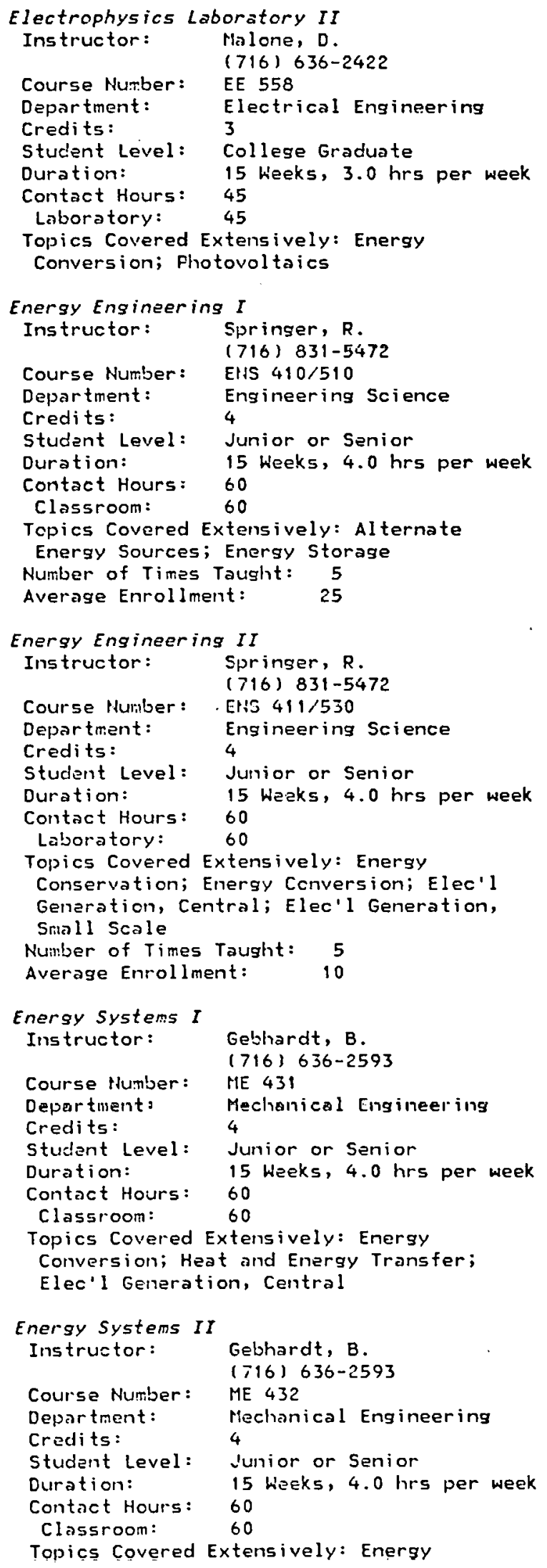

Conversion; Heat and Energy Transfer;

Elec'l Generation, Central

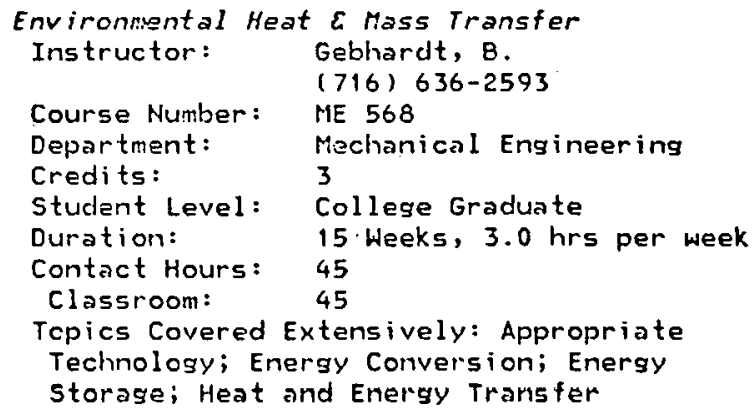

Physical Electronics

Instructor:

Course Number:

Department:

Credits:

Student Level: Junior or Senior 


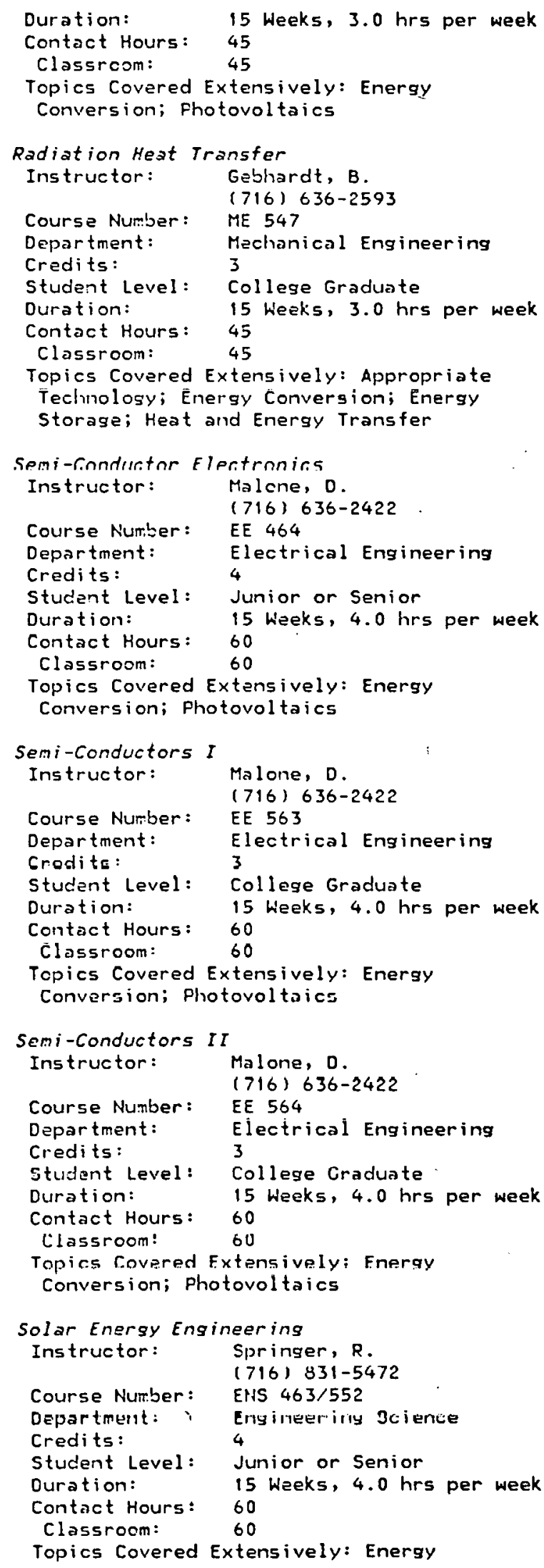

Storage; Intro. to Solar Energy;

Passive Solar Technology;

Fhotovoltaics; Solar System Components;

Solar Economics; Solar Collector

Evaluation/Design; Solar Systems

Design; Domestic Hot hater; Elec'l

Generation, Small Scale; Space Heating;

Space Cooling

Number of Times Taught: 5

Average Enrollment: 5

$\begin{array}{ll}\text { Solid-state Electrical Engineering I } \\ \text { Instructor: } & \text { Malone, D. } \\ & (716) 636-2422 \\ \text { Course Number: } & \text { EE } 554 \\ \text { Department: } & \text { Electrical Engineering } \\ \text { Credits: } & 3 \\ \text { Student Level: } & \text { College Graduste } \\ \text { Duration: } & 15 \text { Weeks, } 3.0 \text { hrs per week } \\ \text { Cnntart Hnurs: } 45 \\ \text { C.lassroom: } 45 \\ \text { Topics Covered Extensively: Energy } \\ \text { Conversion; Photovoltaics }\end{array}$

Sol id-state Electrical Engineering II

Instructor: Malone, $D$.

Course Number: $\quad$ EE 555

Uepartment: Electrical Engineering

Credits: 3

Student Level: College Graduate

Duration: 15 Heeks, 3.0 hrs per week

Contact Hours: 45

Classroom: $\quad 45$

Topics Covered Extensively: Energy

Conversion; Photovoltaics

Solid-state Optical Devices

Instructor: Malono, $\mathrm{n}$

(716) 636-2422

Course Number: EE 656

Department: Electrical Engineering

Credits: 3

Student Level: College Graduate

Duration: 15 Weeks, 3.0 hrs per week

Contact Hours: 45

Classroom: $\quad 45$

Topics Covered Extensively: Energy

Conversion; Photovoltaics

Transport Phenomena in Chem. Engin II

Instructor: Ulbrecht, $\mathrm{J}$.

(716) 636-2911

Course Number: CHE 510

Department: Chemical Engineering

Credits: 3

Student Level: College Graduate

Duration: 15 Weeks, 3.0 hrs per week

Contact Hours: 45

Classroom: $\quad 45$

Topics Covered Extensively: Energy

Conversion; Heat and Energy Transfer

Number of Times Taught: 10

Transport Phenomena in Chemical Engineering I

Instructor:

Ulbrecht, $J$.

Course Number:

Department:

Credits :

\section{CHE509}

Chemical Engineering 3 


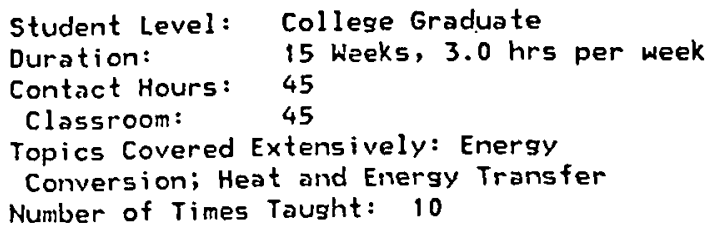

**********

SUHY AT STONY BROOK MAIH CAM

(2838)

STONY BROCK, NEW YOrK 11794

(516) 246-5000.

\section{SOLAR RELATED COURSES}

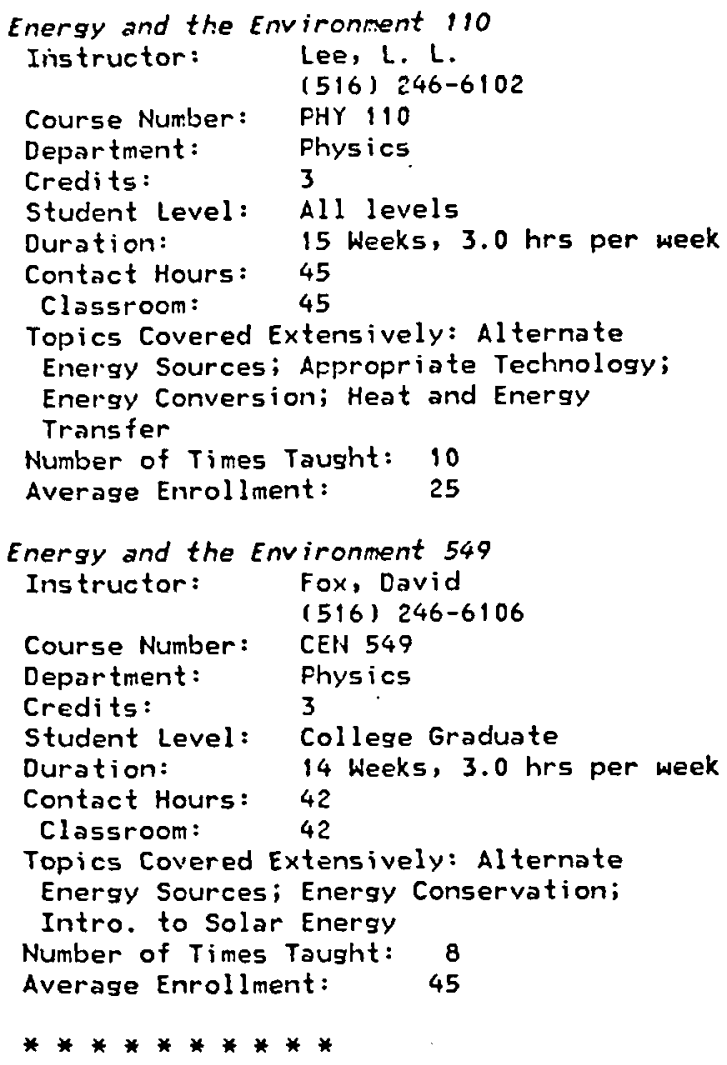

\section{SUMY C ENVRNITL SCI- FORESTRY}

SYRACUSE, New York 13210

(315) 473-6611

\section{SOLAR RELATED COURSES}

Energy: Froduction and Conservation

Instructor: Palmer, David $G$.

(315) 473-8796

Course Number:

ERE 11

Department:

Environmental and Resource Engineering

Credits: 3

Student Level: College Graduate

Duration: 13 Weeks, $3.0 \mathrm{hrs}$ per week

Contact Hours: 39

Topics Coverod Extensively: Alternste

(2851)
Energy Sources; Biomass Conversion;

Energy Conservation

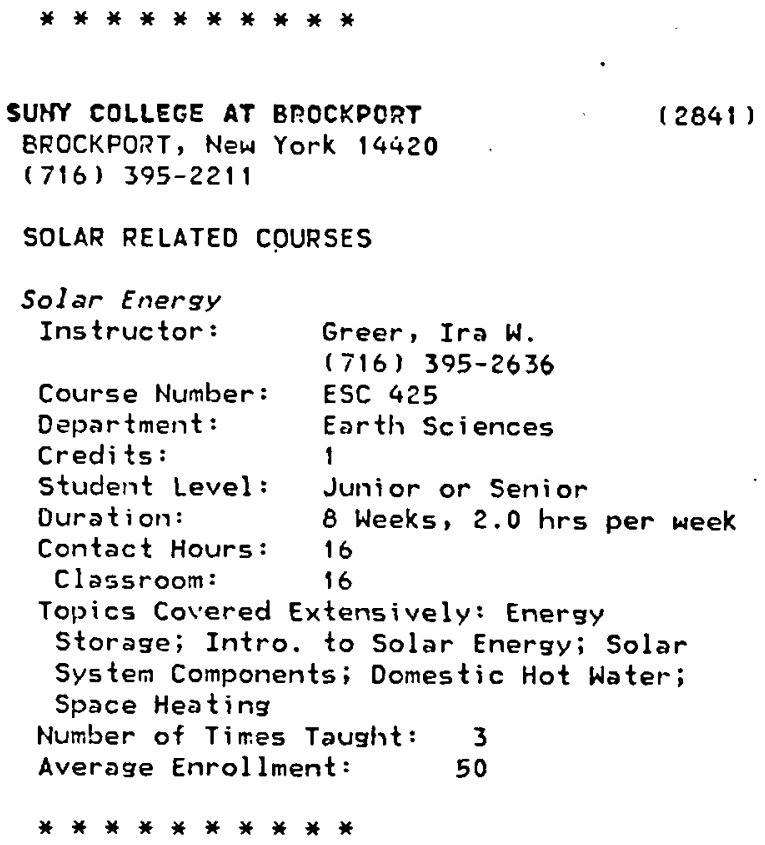

(2841)

\section{SUHY COLLECE AT GENESEO}

GENESEO, Hew York 14454

(716) 245-5211

\section{SOLAR RELATED COURSES}

Ener. Ed. Horkshop/Solar Energy

Instructor: Kinsey, K. F. (716) $245-5283$

Course Number: INT 979

Departnent: Physics

Credits: 1

Student Level: College Graduate

Duration: 1 Weeks, 15.0 hrs per week

Contoct llours: 15
(2844) 
Number of Times Taught: 2

Average Enrollment: $\quad 15$

***********

SUMY CELLECE AT OSHECO

(2848)

OSHEGS, N.W YORK 13126

$(315) 341-2500$

\section{SOLAR RELATED COURSES}

Ener. Tech., Eff. Res. Fuel util.

Instructor: Hinrichs, R./ Salvagin,

Department: Phys/Earth Sci/Indus.

Arts

Credits: 3

Stucient Level: All levels

Duration: 6 heeks, $6.7 \mathrm{hrs}$ per week

Contact Hours: 40

Topics Covered Extensively: Alternate

Energy Sources; Energy Conversion; Heat

and Energy Transfer; Intro. to Solar

Energy; Materials Research; Passive

Solar Tecinnology; Solar System

Components; Solar Econcmics; Solar Home

Construetion; Solar Collector

Evaluation'Desisn; Solar Systems Design

Energy From Nature to Man

Instructor: Hinrichs, R.

Course Number: 105

Department: Fhysics

Credits: 3

Student Level: Freshman or Sophomore

Duration: 15 Heeks, $3.0 \mathrm{hrs}$ per week

Contact Hours: 45

Classroom: $\quad 4,5$

Topics Covered Extensively: Alternate

Eniersy Sources; Appropriate Technolegy;

Enersy Conservation; Energy Conversion;

Heat and Energy Transfer; Intro. to

Solar Energy; Passive Solar Technology;

Solar Systemis Design; Domestic Hot

Water; Space Heating

Number of Times Taught: 8

Averase Enrollment: $\quad 40$

Energy Technologies and Efficient Res. Fuel Utilization Instructor: Schneider, Raymond

(315) $341-42.52$

Course Number: ES 315-515

Department: Earth Sciences and

Tnriustrial Arts

Credits: 3

Student Level: n1l lovele

Duration: $\quad 6$ Heeks, 6.5 hrs per week

Contact Hours: 39

Classroom: $\quad 39$

Topics Covered Extensively: Alternate

Energy Sources; Appropriate Technology;

Enersy Conservation; Heat and Energy

Transfer; Intro. to Solar Energy;

Passive Solar Technology; Solar

Economics; Solar Home Construction;

Solar Collector Evaluation/Design;

Solar Systems Design; Domestic Hot

Water; Space Heating; Space Cooling;
Wind Power, Small Systems

Number of Times Taught: 1

Average Enrollment: $\quad 48$

Thermodynamics and Applications

Instructor: Hinrichs, R.

Course Number: $\quad 340$ 341-2388

Department: Fhysics

Credits: 3

Student Level: Junior or Senior

Duration: 15 Heeks, 3.0 hrs per week

Contact Hours: 45

Classroom: $\quad 45$

Topics Covered Extensively: Energy

Conservation; Energy Conversion; Heat

and Energy Transfer; Space Heating

Number of Tinnes Tought: 3

Averase Enrollment: $\quad 30$

Using Solar Energy for Res. Heat ing

Instructor: Gerbracht, Carlton

(315) 341-3028

Course Number: CE 380

Uepartment: Lindustirial arts

Stuc!ent Level: Collese Graduate

Duration: 4 Weeks, 1.5 hrs per week

Contact Hours: 6

Clsssroom: 6

Tepics Covered Extensively: Intro. to

Solar Energy; Solar Systems

Installation

Number of Times Taught: 2

Averase Enroliment:

40

SUMY COLLEGE PLATTSBUREH

(2849)

PLAIISBUTGH, NeW Yörk. I¿Ỹủ

(518) 564-2000

PROGRAMS ANO CURRICULA

Environmental Sci. (Alternate tn̈ergy)

Degree: BA, Environmental Science

Contact: Dawson, James C. (518) 564-2178

students Taking or Completing Offering: Contractor, Do-it-yourself Homeowner,

Solar Technician

\section{SOLAR RELATED COURSES}

Energy Systems

Instructor:

Courna Humber:

Kiss!ner, Fritz

(518) 564-2178

Department:

E!IV 3 ?

Program or

Curriculun: Environmental Sci.

Credits:

Institute for Man and

Environment

Student Level: Jumior or Senior

Duration: 15 Weeks, $3.0 \mathrm{hrs}$ per week

Contact Hours: 45

Clissroom: 45

Topics Covered Extensively: Alternate

Enersy Sources; Appropriate Technology; 
Energy Conversion; Energy Storase; Heat and Energy Transfer; Intro. to Solar Energy; Solar Energy Folicy

Developisint; Solar System Components Number of Times Taught: 6 .

Average Enrollment: 60

Energy-Rescurces and Conservation Instructor: Barnett, S, G. (518) 564-3107 GEL 385

Course Number Earth Sciences

Program or

Curriculum: Environmental Sci.

Credits: 3 (Alternate Energy)

Stucient Level: Jumior or Senior

Duration: 15 hieeks, 3.0 hrs per week

Contract Hours: 45

Classroom: $\quad 45$

Topics Covered Extensively: Alternate

Energy Sources; Energy Conservation;

Energy Storase; Heat and Energy

Transfer

Number of Times Taught: 6

Average Enrollnient:

40

Residential fesearch Semester

Instructor: Dawson, James C.

(51s) $564-2178$

Course Number: ENV 313-318

Department: Institute for Man and

Environment

Program or

Curriculum: Environmental Sci.

Credits: (Alternate Energy) 15

Student Level: Junior or Senior

Duration: 15 heeks, $45.0 \mathrm{hrs}$ per week

Contact Hours: 675

Classroom: $\quad 75$

Laboratory: 600

Tcpics Covered Extensively: Alternate Energy Sources; Appropriate Technology;

Biomass Conversion; Energy

Conservation; Energy Conversion;

Plumbing Techniques; Sheet lietal

Techniques; Solar Home Construction;

Solar Collector Evaluation/Design;

Solar Systems Design; Domestic Hot

Water; Space Heating

Number of Times Tausht: 4

Average Enrollment: 35

\section{Solar Energy}

Instructor:

Course Number: PHY 301

Szydl ik, Paul

(518) 564-2048

Department: Physics

Program or

Curriculum:

Credits:

Environmental Sci. (Alternate Energy)

Student Level:

Duration:

Contact Hours:

Classroom: 3

Junior or Senior

15 Heeks, $3.0 \mathrm{hrs}$ per week

45

45

Topics Covered Extensively: Energy

Conservation; Energy Conversion; Energy

Storage; Heat and Energy Transfer;
Intro. to Solar Eneray; Passive Solar Technology; Photovoltaics; Solar System Components; Solar Collector

Evaluation/Design; Solar Systems

Design; Donestic Hot Water

Nu:mber of Tines Taught: 3

Average Enrollment: $\quad 15$

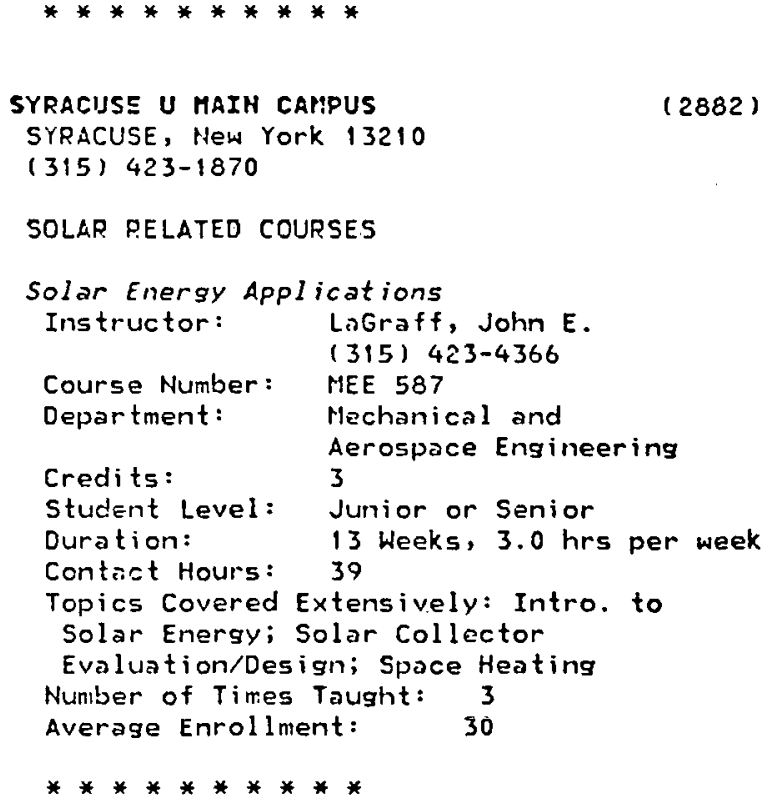


US MEPCHAST MARIHE ACAD

KIHISS POINT, NEW YCRK, New York 11024

(516) $48 \varepsilon-8200$

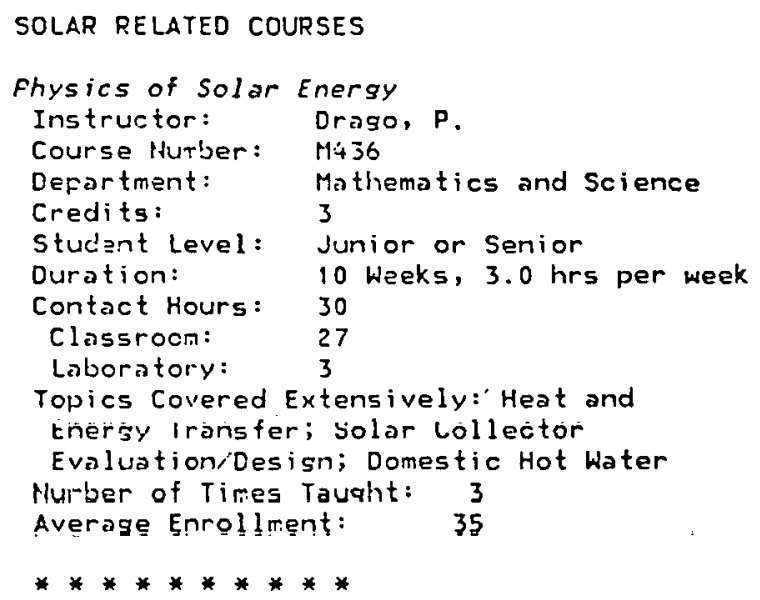

\section{Community/Junior colleges}

ADIROMBACK CTIY COLLEGE

(2860)

GLENS FALLS, New York 12801

(518) 793-4491

PROGRAMS ANO CUPRILULA

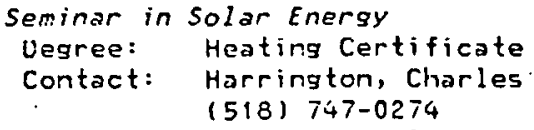

Students Taking or Completing Offering: Solar Technician

(2892)

(2895)

20

(2) 
COLUMBIA-GREENE CC

(6789)

HUDSON, New York 12534

(518) 828-4181

\section{SOLAR RELATED COURSES}

\section{Solar Energy}

Instructor: Drum, Donald A.

(518) 828-4181

Course Number: CE 005

Department: Centinuing Education

Student Level: All levels

Duration: 10 Weeks, $2.0 \mathrm{hrs}$ per week

Contact Hours: 20

Topics Covered Extensively: Alternate

Energy Sources; Energy Conservation;

Energy Conversion; Energy Storage; Heat

and Energy Transfer; Intro. to Solar

Energy; Passive Solar Technology; Solar

Home Construction; Solar Collector

Evaluation/Design; Solar Systems

Uesign; Domestic Hot Water; Space

Heating; Space Cooling

Number of Times Taught: 3

Average Enrollment:

71

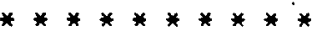

CUNY NEU YORK CITY CC

(2696)

BROOKLYN, Hew York 11201

(212) 643-4033

PROGRANIS AND CURRICULA

Environmental Control Technology

Degree: AD, Applied Science

Contact: Lorask, Samuel

(212) 962-0407

\section{SOLAR RELATED COURSES}

Environmental Design I

Instructor: Farkas, stanley

(212) 239-1662

Course Number: EC 110

Department: Environmental Control

Technology

Program or

Curriculum: Envirumenlal Cuntrol Technology

Credits: 3

Student Level: Freshman or Sophomore

Duration: 15 Heeks, 3.0 hrs per week

Contact Hours: 45

Classroom: $\quad 45$

Topics Covered Extensively: Energy

Conservation; Heat and Energy Transfer

Number of Tiries Taught: 70

Average Enrollment: $\quad 25$

Environmental Design Laboratory

Instructor: Farkas, Stanley

(212) 239-1662

Course Number: EC11!

Department: Environmental Control

Technology

Program or Curriculum: Environmental Control

Technolosy

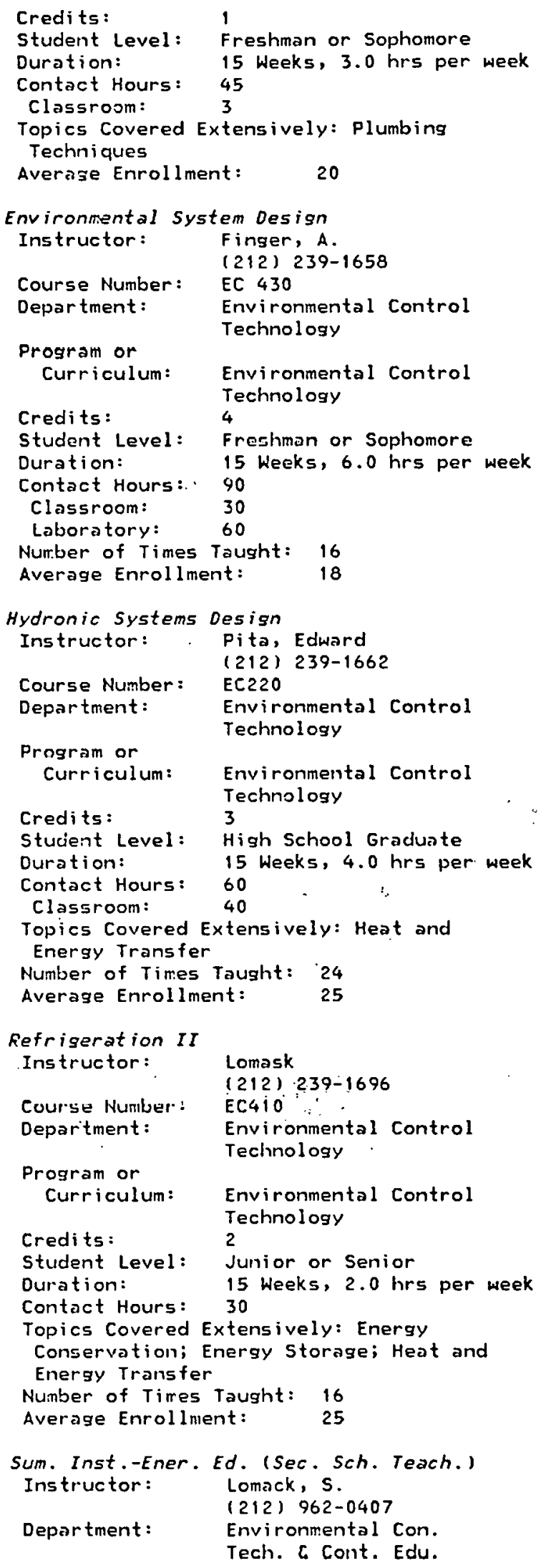




\author{
Student Level: College Graduate \\ Duration: $\quad 3$ Weeks, 30.0 hrs per week \\ Contact Hours: 90 \\ Classrocn: $\quad 80$ \\ Topics Covered Extensively: Al ternate \\ Energy Sources; Appropriate Technology; \\ Energy Conservation; Energy Conversion; \\ Energy Storase; Heat and Energy \\ Transfer; Intro. to Solar Energy; \\ Passive Solar Technology \\ Average Enrollment: 80 \\ **********
}

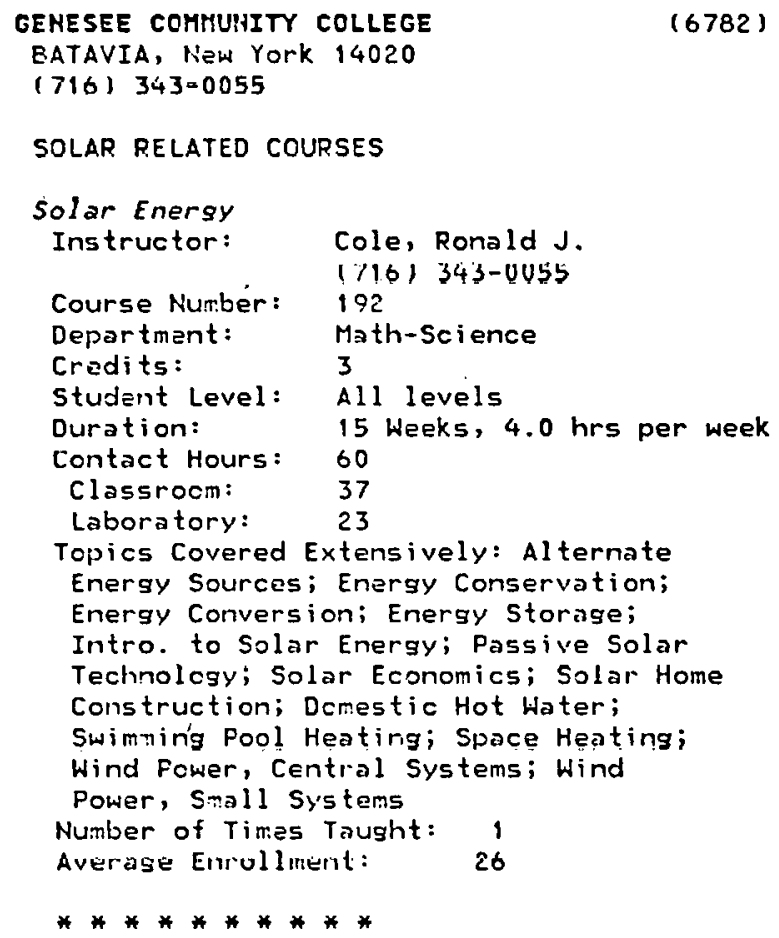

SOLAR RELATED COURSES

$\begin{array}{ll}\text { Solar Energy I-Ener. and Ener. Cons. } & \text { Dunning, Francis } \\ \text { Instructor: } & (315) 792-5514 \\ & \text { Cosing } \\ \text { Course Nilmer: } & \text { Fhysics and Engineering } \\ \text { Department: } & \text { Science } \\ & \\ \text { Prcgram or } & \\ \text { Curriculum: } & \text { Solor Encrgy Technology } \\ \text { Credits: } & 3 \\ \text { Student Level: } & \text { All levels }\end{array}$

\author{
Duration: \\ Contact Hours: 30 \\ Classroom: $\quad 30$
}

Topics Covered Extensively: Appropriate

Technolosy; Energy Conservation; Energy Storage; Heat and Energy Transfer;

Intro. to Solar Energy; Solar System

Components; Solar Economics; Solar

Systems Installation; Donestic Hot

Water; Space Heating

Number of Times Taurht: 5

Average Enrollment: $\quad 30$
Solar Energy II (Installation)
Instructor: Dunning, Francis
(315) 792-5514
Course Number: D2985
Depar. Lment: Plysics
Program or
Curriculum: Solar Energy Technology
Credits:
Student Level: Freshman or Sophomore
Duration: 10 Weeks, $5.0 \mathrm{hrs}$ per week
Contoct Hours: 50
Classroom: $\quad 30$
Laboratory: 20
Tcpics Covered Extensively: Appropriate
Techuology; Energy Storage; Heat and
Energy Transfer; Intro. to Solar
Energy; Plumbing Technicues; Solar
System Comucnents; Solar Economics;
Solar Howe Construction; Solar Systems
Design; Solar Systems Installation;
Solar Systenis Maintenance; Solar
Systems Testing and Evaluation;
Domestic Hot Hater; Space heating
Number of Times Tausht: 1
Averase Enrollment: $\quad 30$

Solar III (Sol. Ener. Sys. Des. and Ana.)

Instructor: Duinning, Francis

$\begin{array}{ll}\text { Department: } & (315) 792-5514 \\ \text { Program or } & \text { Pliysics } \\ \text { Curriculum: } & \text { Solar Energy Technology } \\ \text { Credits: } & 4 \\ \text { Student Level: } & \text { Freshman or Sophomore } \\ \text { Duration: } & 10 \text { Weeks, } 5.0 \text { hrs per week } \\ \text { Contact Hours: } & 50 \\ \text { Classroon: } & 30 \\ \text { Laboratory: } & 20\end{array}$

Topics Covered Extensively: Appropriate Technolosy; Energy Conversion; Energy Storase; Heat and Energy Transfer; Materials Research; Pluribing Teelmiciues; Sheet Metal Tecluniques; Solar System Components; Solnr Ecclionics; Solar Home Corstructiun; Solar Collector Evaluation/Design; Solar Systens Desisn; Solar Systems Installation; Solar Systems Testing and Evaluaticn; Domestic Hot Water; Swintming Fool Heating; Space Heating; Space Cooling

Solar IV (Alternate Energy Sources)

Instructor: Dunning, Franc is

(315) 792-5514

Departiment: Physics

program or 
Curriculum: Credits: Student Level: Duration:

Contact Hours: Classroom: Laboratory:

Topics Covered Extensively: Alternate

Energy Sources; Energy Conservation; Energy Conversion; Intro. to Solar

Energy; Fassive Solar Technology; Photovoltaics; Solar Eccnomics; Solar Law/Lesislation; Frocess Heat, Agricultural; Process Heat, Industrial; Wind Power, Central Systems; Wind Power, Small Systems

Solar system fabrication I

Instructor: Duming, Francis (315) 792-5514

Department: Physics

Program or $\begin{array}{ll}\text { Curriculum: } & \text { Solar Energy Technology } \\ & 2\end{array}$ Credits:

Student Level: Freshman or Sophomore

Duration: 10 heeks, 3.0 hrs per week

Contact Hours: $\quad 30$

Classroom: $\quad 10$

Laboratory: 20

Topics Covered Exterisively: Appropriate Technology; Plumbing Techniques; Sheet Metal Techniques; Solar Systems Installation; Solar Systems Maintenance; Solar Systens Testing and Evaluation; Doriestic Hot Water; Space Heating; Space Cooling

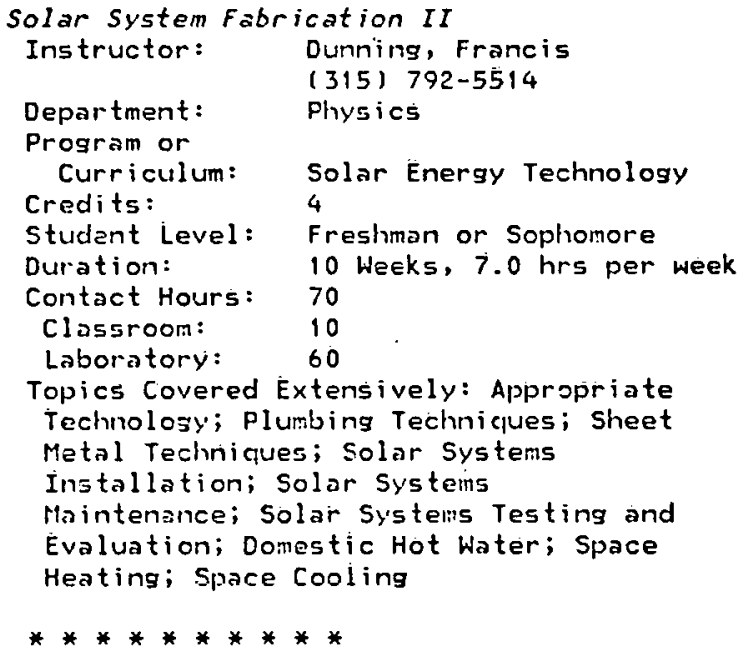

ROCHESTER, HeW York 14623

(716) 442-9950

\section{SOLLAR RELATED COURSES}

Alternative Sources of Energy

Instructor: Dowd, Janis

(716) 275-9318

Course Number:
CEU $036=181$
Department:

Community Services

Student Level: All levels

Duration:

Contact Hours: 8

Classroom: 8

Topics Covered Extensively: Alternate

Energy Sources; Appropriate Technology;

Energy Conservation; Energy Colversion;

Enersy Storase; Heat and Energy

Transfer; Intro. to Solar Enersy; Solor

System Components; Solar Home

Construction; Solar Collector

Evaluation/Design

Number of Times Taught: 3

Average Enrollment: $\quad 40$

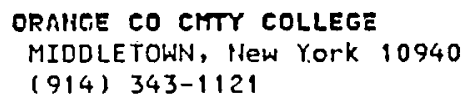

ROCKLAY'D CMTY COLLEGE

SUFFERI,, New York 10901

(914) 356-4650

\section{SOLAR RELATED COURSES}

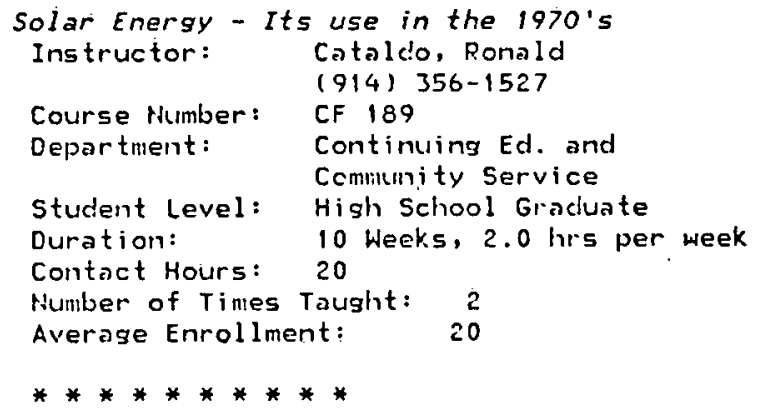


SUIY AGRL C TECH C CAMTOH

$(2855)$

CANIUH, NEW YORK 13617

(315) 386-7204

\section{SOLAR RELATED COURSES}

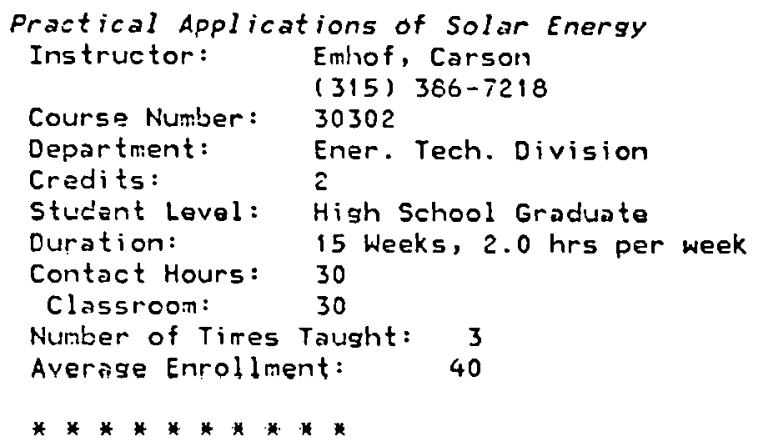

SUNY AGRL $C$ TECH C DELHI

(2857)

DELHI, New York 13753

$(607) 746-4111$

PROGRAMS ANO CURRICULA

Construction Tech./ Civil Tech.

Degree: AD, Applied Science

Contact: Duncan, Eeorge (607) 746-42:5

Students Taking or Cotipleting Offering:

Mechanical or Electrical Contractor,

Contractor, Other

\section{SOLAR RELATEO COUPSES}

General Chemistry

Instructor: Onasch, Frederick

(607) 746-4377

Course Number: 9512

Devartment: Phyșical Sciences

Program or

Curriculum: Construction Tech./

Credits : Civil Tech.

4

Student Level: Freshman or Sophomore

Duration: $\quad 15$ hieeks, 5.0 hrs per week

Contact hours: 75

Classroom: $\quad 30$

Latoritory: 45

Topics Covered Extensively: Alternate

Energy Sources; Appropriate Technology;

Biomass Conversion; Energy Conversion;

Energy Stcrase; Heat and Energy

Transfer; Materials Research;

Photovoltaies

General Chemistry 9513

Instructor: Cnasch, Frederick

(607) 746-4377

Course Number: 9513

Department:

Program or

Curriculum: Construction Tech./

Credits: Civil Tech.

Student Level: Freshman or Sophomore

Duration: 15 Heeks, 5.0 hrs per week $\begin{array}{ll}\text { Contact Hours: } & 75 \\ \text { Classroom: } & 30 \\ \text { Laboratory: } & 45\end{array}$

Topics Covered Extensively: Alternate Energy Sources; Appropriate Technology; Biomass Conversion; Energy Conversion; Energy Storase; Heat and Energy

Transfer; Materials Research; Photovoltaics

General Physics

Instructor:

Vetter, Willard

(607) 746-4374

Course Number: 9521

Department: Physical Sciences

Program or

Curriculum: Construction Tech./

Credits:

Civil Tech.

Student Level: Freshinan or Sophomore

Uurntion:

Contaot Hourc:

Freshinan or Sophomore

15 Heeks, 4.0 hre por lioek

60

Classroom: $\quad 30$

Laboratory: $\quad 30$

Topics Covered Extensively: . Al ternate Enersy Sources; Apprcpriate Technology;

Energy Conservation; Energy Conversion;

Energy Storage; Heat and Energy

Transfer; Intro. to Solar Energy

Average Enrollinent: $\quad 100$

General Physics 9522

$\begin{array}{ll}\text { Instructor: } & \text { Vetter, Willard } \\ & (607) 746-4374 \\ \text { Course Number: } & 9522 \\ \text { Department: } & \text { Physical Sciences }\end{array}$

Program or

Curriculum: Construction Tech/

Credits: 3

Student Level: Freshman or Sophomore

Duration: 15 Weeks, $4.0 \mathrm{hrs}$ per week

Contact Hours: 60

Classroom: $\quad 30$

Laböratory: $\quad 30$

Topics Covered Extensively: Alternate

Energy Sources; Appropriate Technolcgy;

Energy Conservation; Enersy Conversion;

Energy Storage; Heat and Energy

Transfer; Intro. to Solar Energy

Average Enroliment: $\quad 100$

Mechanical Equipment for Buildings

Instructor: Hampel, John

Course Number: 3741

Department: Construction Technölogy

Program or

Curriculum: Construction Tech./

Credits:

Civil Tech.

Stucient Level: Freshman or Sophomore

Duration: 15 Weeks, $4.0 \mathrm{hrs}$ per week

Contact Hours: 60

Classroom: $\quad 43$

Laboratory: $\quad 30$

Topics Covered Extensively: Energy

Conservation; Energy Conversion; Energy

Storage; Heat and Energy Transfer;

Plumbing Techniques 
Number of Times Taught: 20

Average Enrollment:

65

Thermodynamics and Heating

Instructor: Hampel, John

(607) 746-4386

Course Number:

3711

Department :

Construction Technology

Program or

Curriculum: Construction Tech/

Credits:

Civil Tech.

4

Student Level: Freshman or Sophomore

Duration: 15 Weeks, $4.0 \mathrm{hrs}$ per week

Contact Hours: 60

Classroom: $\quad 45$

Laboratory: 30

Topics Covered Extensively: Energy

Conservation; Energy Conversion; Energy

Storage; Heat and Energy Transfer;

Plumbing Techniques

Number of Times Taught: 20

Average Enrollment:

65

Hater Resources

Instructor:

Singer, Darrell

(607) 746-4391

Course Number:

3554

Department:

Program or

Curriculum:

Civil Technology

Credits: 4

Construction Tech./

Civil Tech

Student Level: Freshman or Sophomore

Duration: 15 Weeks, 6.0 hrs per week

Contact Hours: 90

Classroom: $\quad 30$

Laboratory: $\quad 60$

Topics Covered Extensively: Alternate

Energy Sources; Biomass Conversion;

Energy Conservation; Energy Conversion;

Energy Storage

Number of Times Taught: 20

Average Enrollment:

30

**********

TOMPKINS-CORTLAND CC

(6788)

DRYDEN. New York 13n53

(607) 844-821)

\section{SOLAR RELATED COURSES}

Home Use of the Sun's Energy

Instructor: Klein, Gary

(607) 844-8211

CEET 708

Course Nurber:

$\begin{array}{ll}\text { Department: } & \text { Lifelong L } \\ \text { Student Level: } & \text { All levels }\end{array}$

Duration: 8 bieeks, 3.0 hrs per week

Contact Hours: 24

Topics Covered Extensively: Alternate

Energy. Sources; Afprcpriate Technology;

Energy Conservation; Enersy Storage;

Intro. to Solar Energy; Solar System

Components; Solar Systems Installation

Number of Times Tausht: 3

Average Enrollment:
**********

HESTCHESTER CHTY COLLEGE

VALHALLA, New York 10595

(2881)

(914) 347-6800

\section{SOLAR RELATED COURSES}

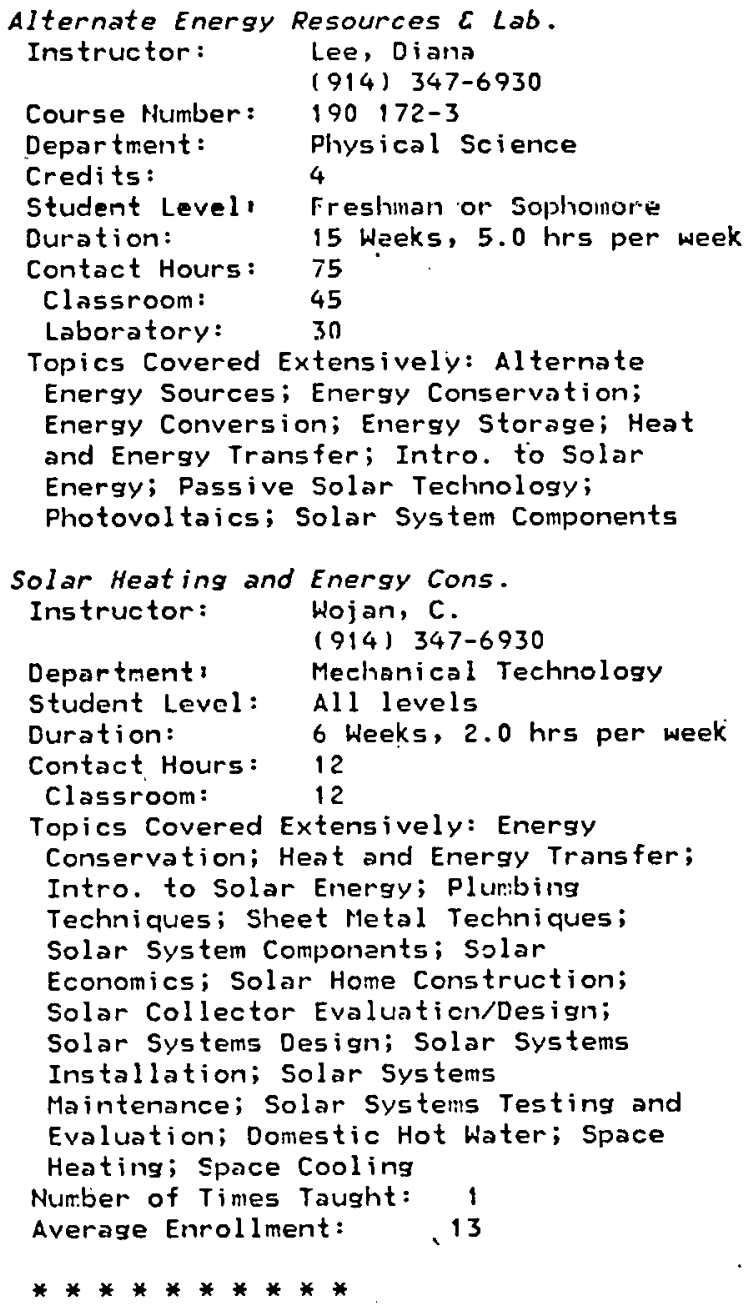

\section{other Educational Institutions}

ALAA PROFESSIONAL STUDY SERIES

(90500)

1290 Avenue of the Americas

NY, New York 10019

SOLAR RELATED COURSES

*H ind Engineering

Instructor: Sforza, Pasquale

Duration: 2.0 Days 


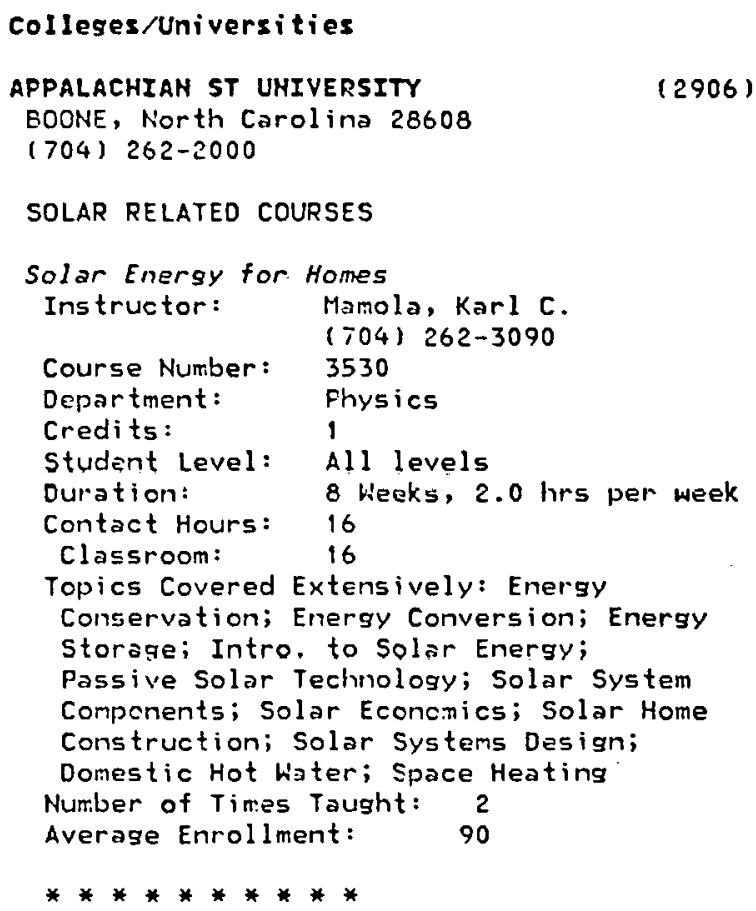

DURHAM, Horth Carolina 27706

(919) 684-6111

\section{PROGRAMS AHD CURRICULA}

Energy Conservation

$$
\text { Desree: MS, Civil, Electrical, }
$$$$
\text { Mechanical Engineering }
$$

Contact: Chaddock, Jack B.

(919) 684-2632

\section{SOLAR RELATED COURSES}

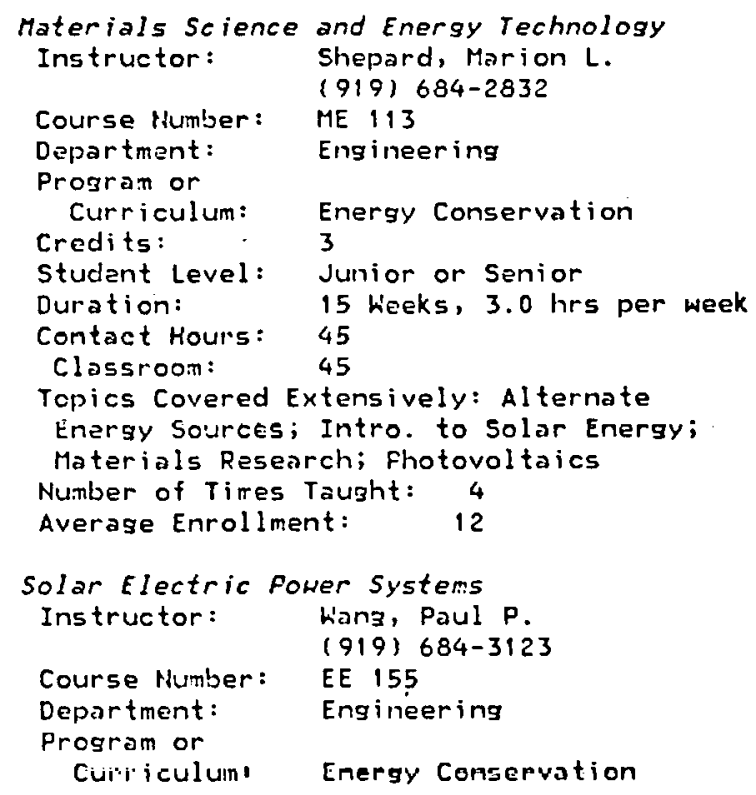

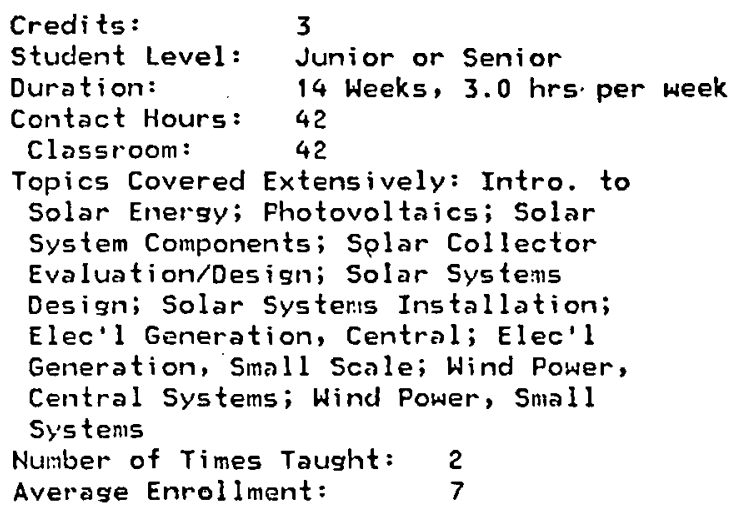

EAST CAROLIHA UHIVERSITY

GREENVILLE, North Carolina 27834

(2923) (919) 757-6212

\section{SOLAR RELATED COURSES}

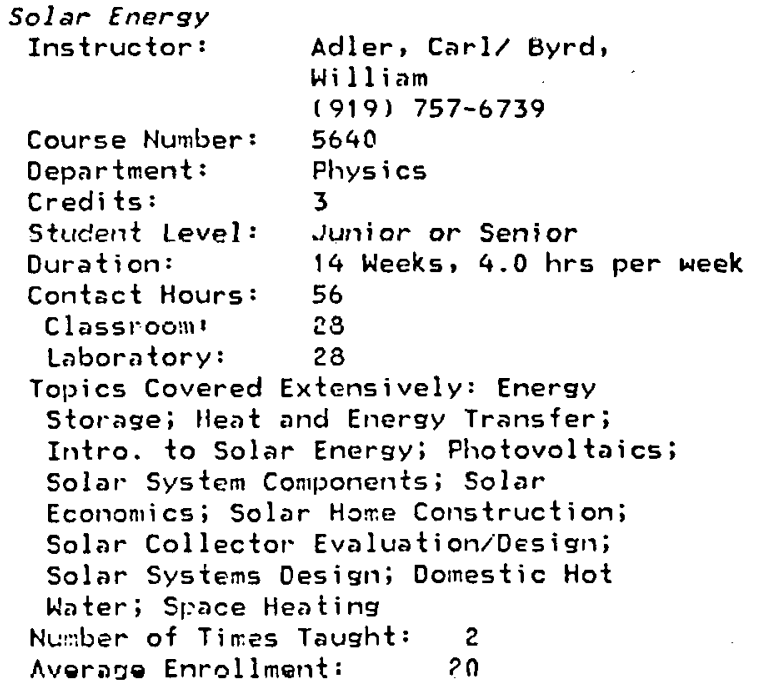




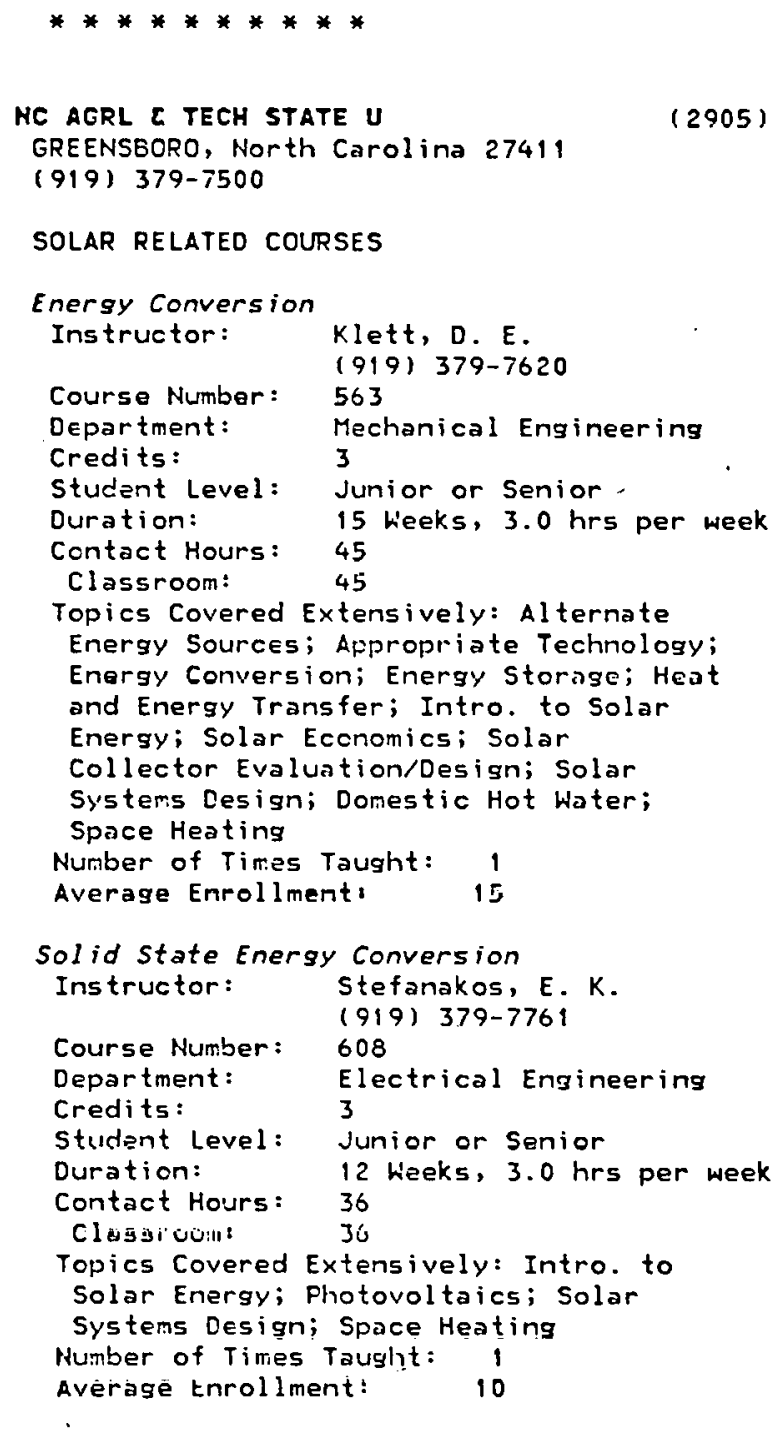

Solar Collector Evaluation/Design:

Solar Systems Design; Domestic Hot Water

Number of Times Taught: 4

Average Enrollment: $\quad 60$

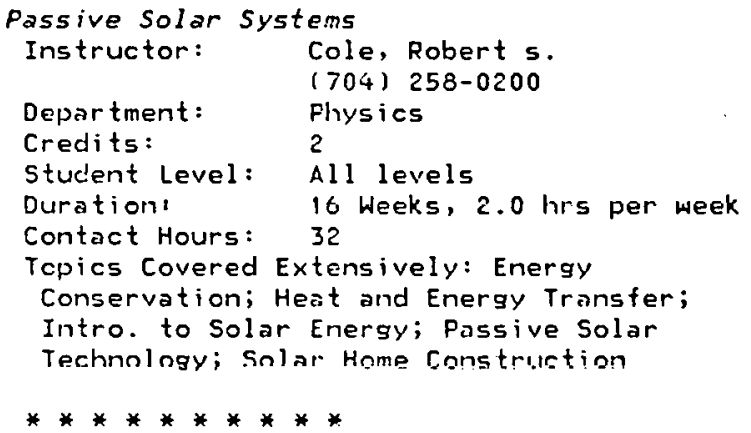

MC AT GREEHSBORO, U OF GREEHSEORU, North Carolina 27412 (919) 379-5000

SOLAR RELATED COURSES

Energy Options and the Environment

Instructor: Meisner, Gerald $W$. (919) 379-5330

Course Number: PHYS. 334

Department: Arts and

Sciences/Physics

Student Level: All levels

Duration: 15 Weeks, $3.0 \mathrm{hrs}$ per week

Contact Hours: 45

Tcpics Covered Extensively: Alternate Energy Sources; Energy Conservation;
(2975) 
Passive Solar Technology Nuniber of Times Taught: 10 Average Enrollment:

(2972)

\section{NC STATE U RALEIGH \\ RALEIGH, North Carolina 27607 \\ (919) 737-2011}

\section{SOLAR RELATED COURSES}

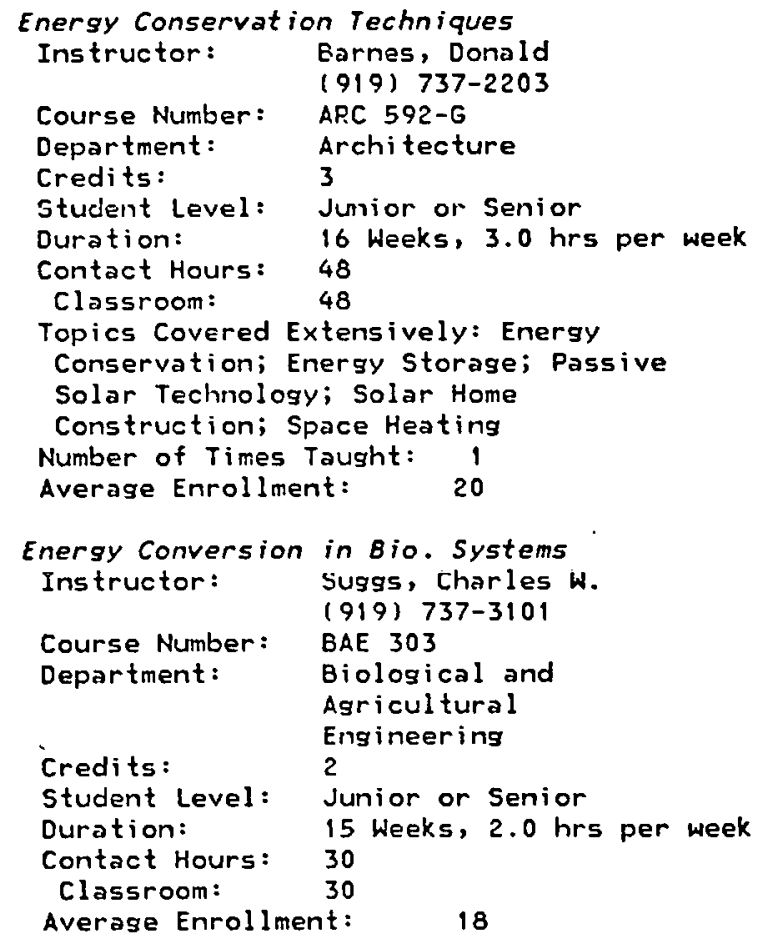

\section{WSTH CAROLIMA UNIVERSITY}

CULLOWHEE, North Carol ina 28723

(2981)

( 7 Uि4) 293-7211

\section{PROGRAMS AND CURRICULA}

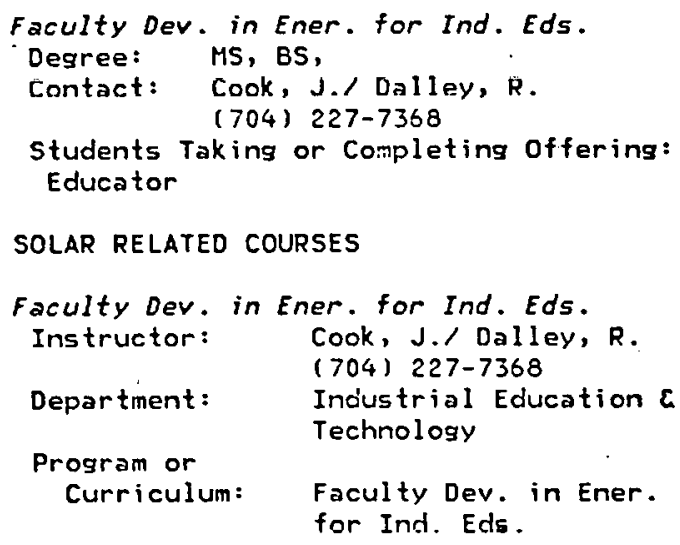

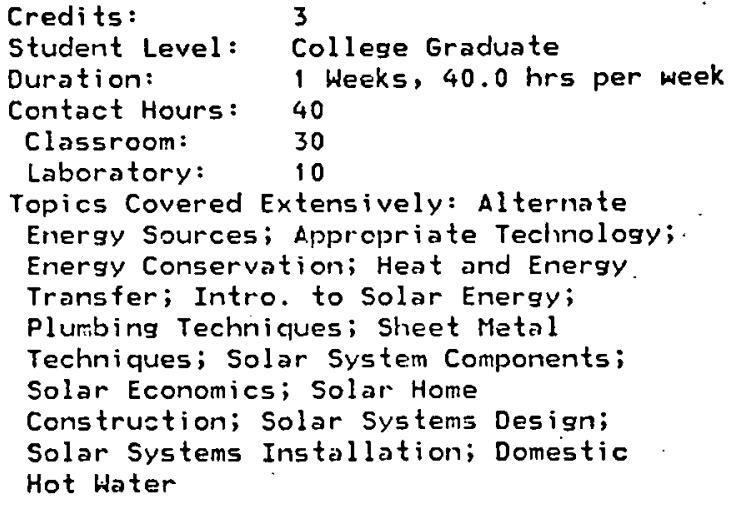

\section{Community/Junior Colleges}

CARTERET TECHNICAL IHST

(8081)

MOREHEAD CITY, North Carolina 28557

(919) 726-2811

PROGRAMS AND CURRICULA

Solar Energy: Fundamentals and

Construction

Degree: Institute Certificate

Contact: Nelson, J. Lenn (919) 726-1171

students Taking or Completing Offering: Do-it-yourself Homeowner

\section{SOLAR RELATED COURSES}

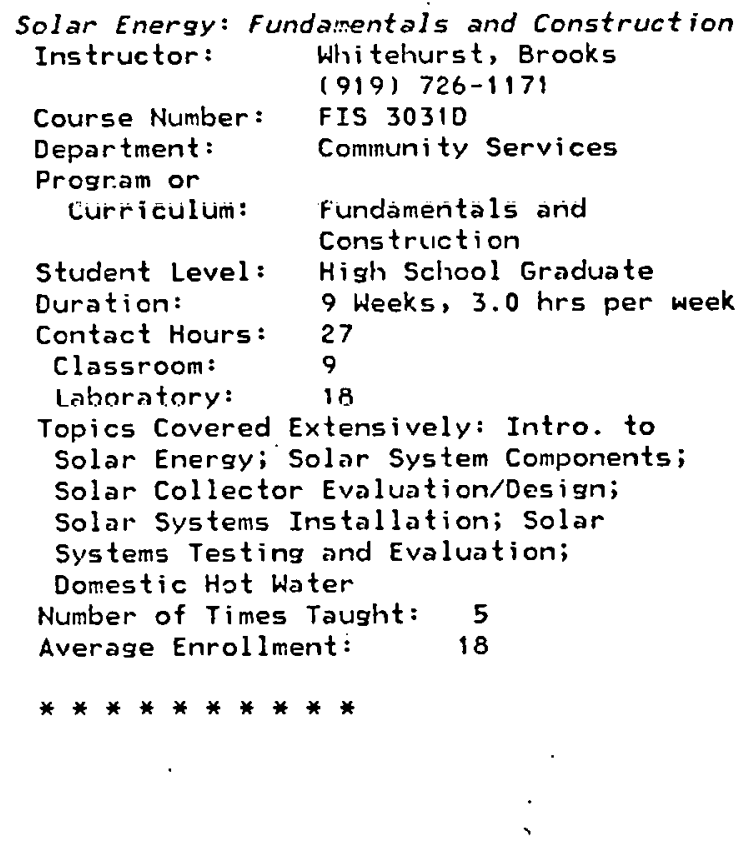


CEN PIEOMONT CMTY COLLEGE

CHARLOTTE, North Carolirsa 28204

(2915)

(704) 373-6566

SOLAR RELATED COURSES

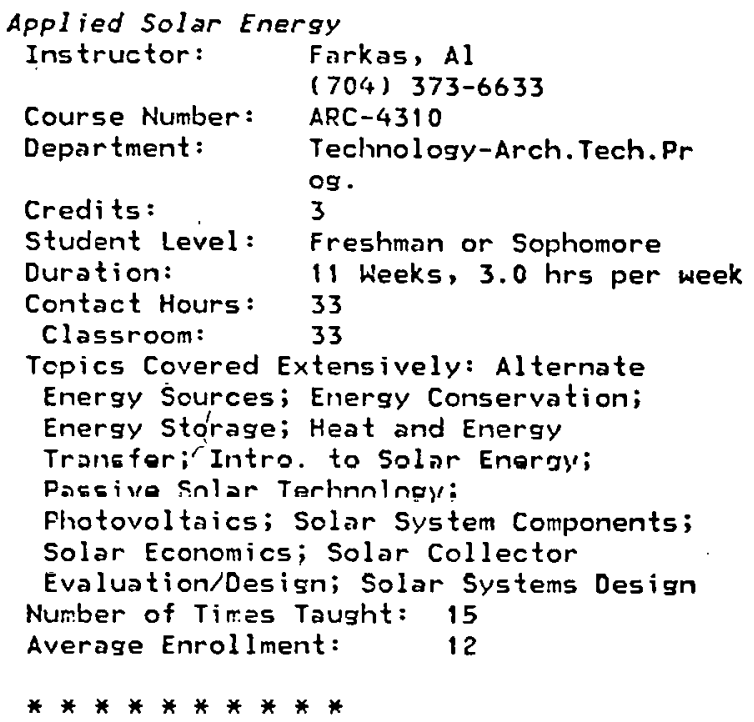

GASTON COLLEGE

DALLAS, North Carolina 28034

(704) $922-3136$

(2973)

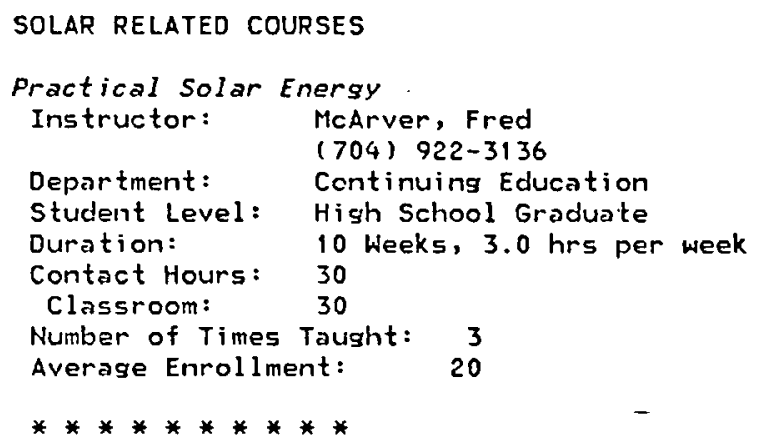

(7687) 
SAMPSON TECHMICAL IHST

CLINTON, North Carolina 28328

( 919 ) 592-8081

\section{PROGRAMS ANO CURRICULA}

Air Conditioning, Heating, and Refrig.

Degree: Diploma

Contact: Peacock, Sherwood

(919) 592-8081

Students Taking or Corpleting offering:

Installer-Commercial (Solar System),

Solar Technician

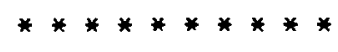

STANLY TECHYICAL INST

(11194)

ALBEMARLE, North Carolina 28001

(704) 982-0121

SOLAR RELATED COURSES

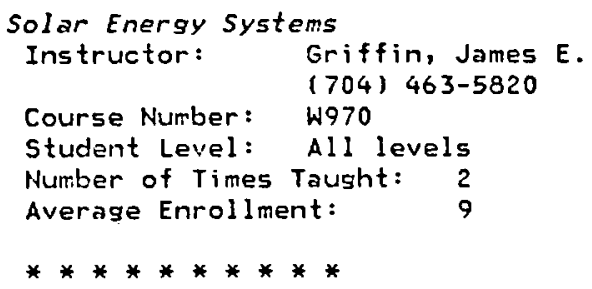

TRI-COUSTY TECHIHICAL IHST

(9430)

MURPHY, Nor th Carolina 28906

(704) 837-6810

\section{SOLAR RELATED COURSES}

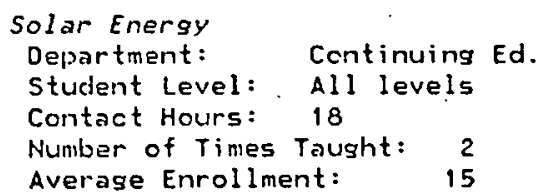

VAMCE-GRAHVL CMTY COLLEGE

HENDERSON, North Carolina 27536

(919) 492-2061

(9903)

\section{SOLAR RELATED COURSES}

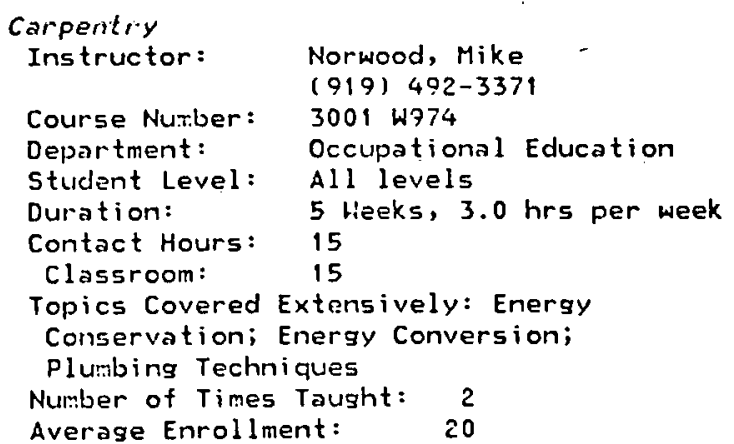

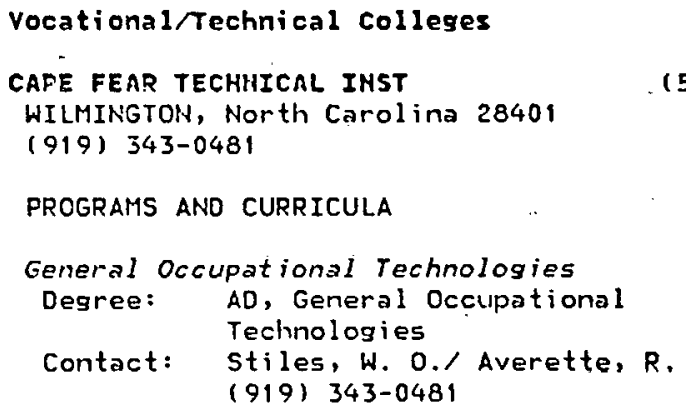

SOLAR RELATED COURSES

Introduction to Energy Resources

Instructor: Bordeaux, Ralph

(919) 343-0481

Course Number: T-EGY101

Department: Engineering Division

Program or

Curriculum: General Occupational

Technologies

Credits: 3

Student Level: All levels

Duration: $\quad 11$ Weeks, 3.0 hrs per week

Contact Hours: 33

Classroon: $\quad 33$

Topics Covered Extensively: Alternate Energy Sources; Energy Storage; Intro. to Solar Energy

Number of Times Taught: 2

Average, Enrollment:

Introduction to Solar Energy Systems (Elect.) Instructor: Bordaaux, Ralph

(919) 343-0481

$\begin{array}{ll}\text { Course Number: } & \text { T-EGY } 103 \\ \text { Department: } & \text { Engineering }\end{array}$

Curriculum

General Occupational

Credits:

Student Level: All levels

Duration: $\quad 11$ Weeks, $6.0 \mathrm{hrs}$ per week

Contact Hours: 66

Classroom: 44

Laboratory: 22

Topics Covered Extensively: Appropriate Technology; Photovoltaics; Solor Energy Policy Develoument; Elec'l Generation, Small Scale; Wind Power, Small Systems

Introduction to Solar Energy Systems (Thermal) Instructor: Stiles, Warren 0 .

(919) 256-3146

Course Number: T-EGY-102

Department: $\quad$ G.O.T./Evening

Program or

Curriculum: General Occupational

Technologies 


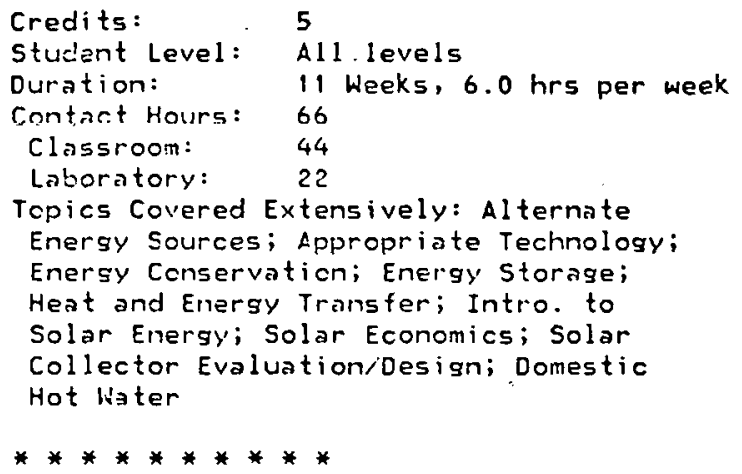

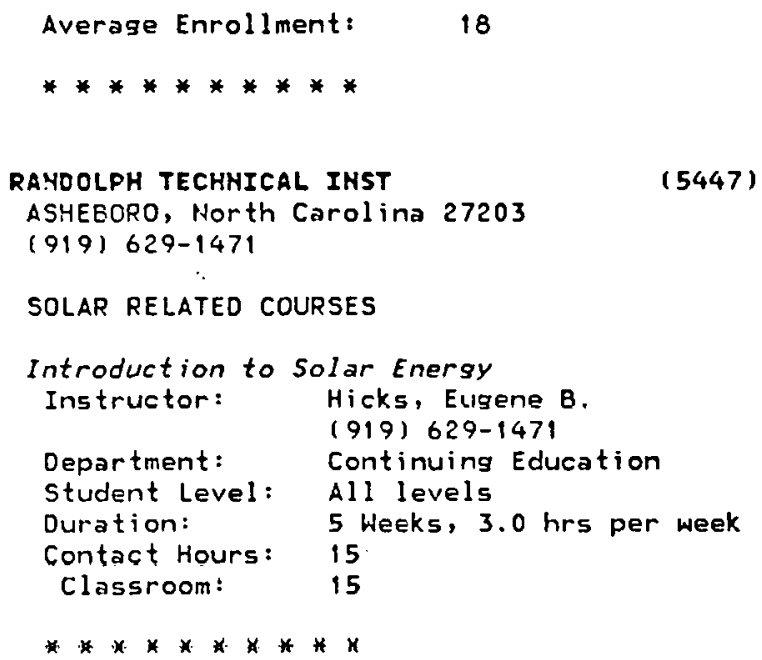

(5447) 


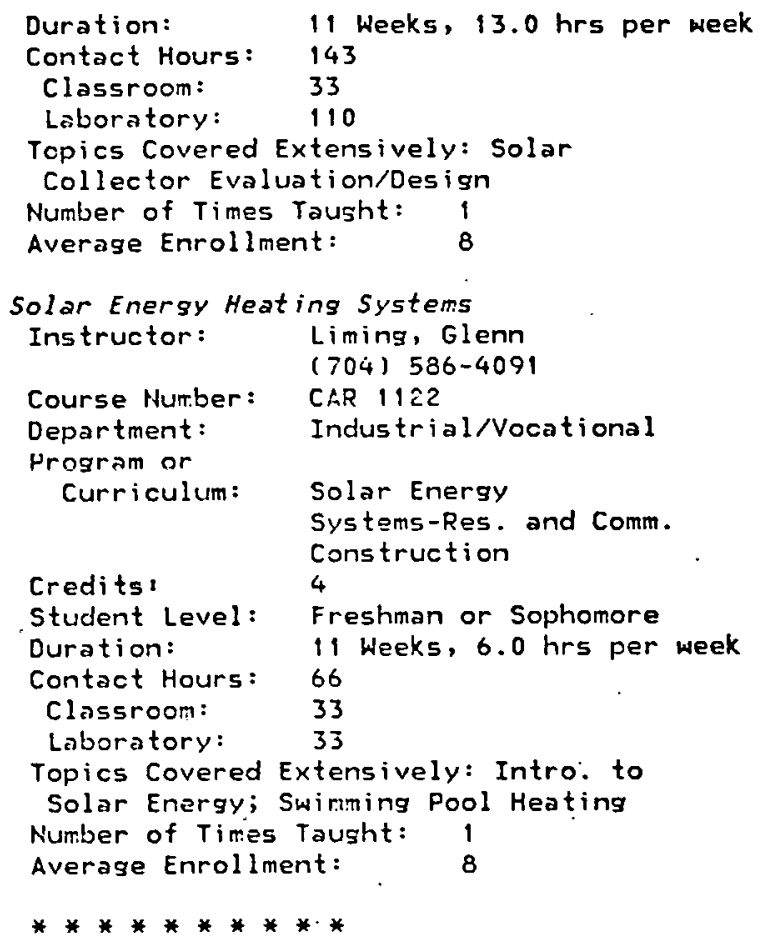

TECH INST OF ALARANCE

HAW RIVER, North Carolina 27258

(919) 578-2002

\section{SOLAR RELATED COURSES}

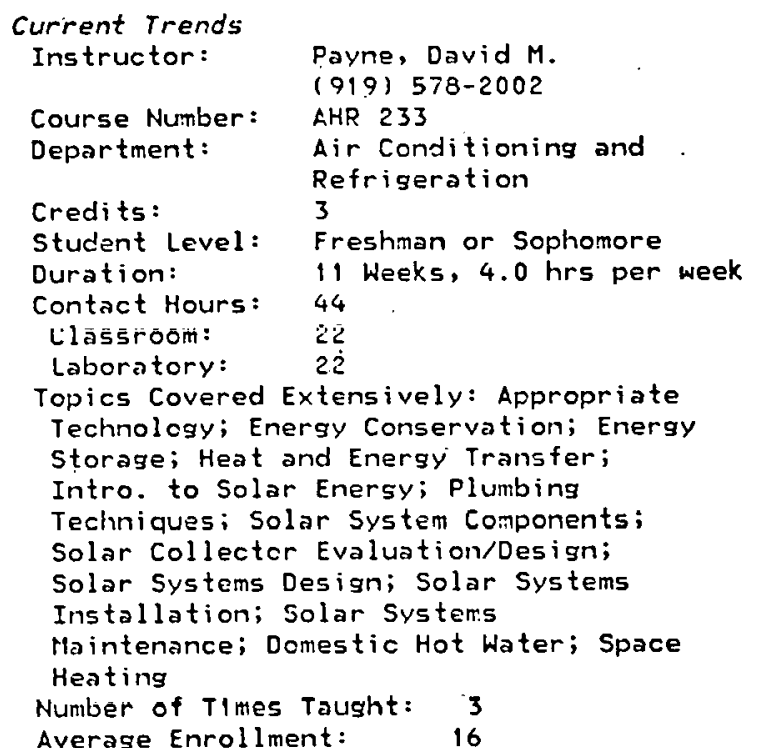




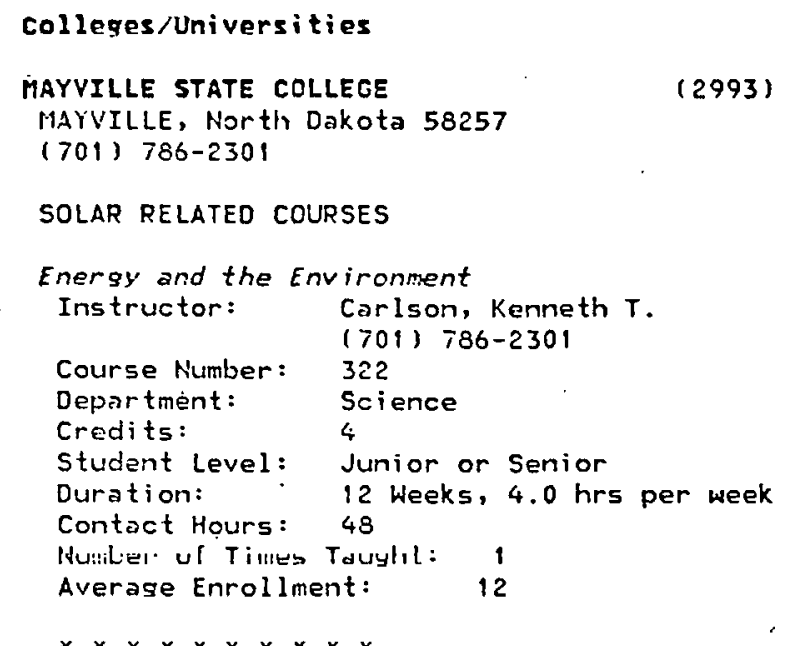

ND STATE U MAIN CAMPUS

192651

FAFGO, North Dakota 58102

(701) 237-8011

SOLAR RELATED COURSES

* Energy Conservation in Bldgs.

Department: Eng'r and Architecture

Student Level: Junior or Senior

$* * * * * * * * * *$
Laboratory: 193

Topics Covered Extensively: Plumbing

Techniques; Sheet Metal Techniques;

Solar Home Construction; Solar Systems

Design; Solar Systems Installation;

Solar Systems Maintenance; Solar

Systems Testing and Evaluation;

Domestic Hot Water; Space Heating;

space Cooling

**********
(2996)

HD STATE SCHOOL SCIENCE
WAHPETON, North Dakota 58075
$(701) 671-1130$

PROGRAMS AND CURRICULA

Environmental Systems Design

Degree: Certificate, Diploma,

Contact: Whitcomb, Larry $17013671-2529$

Students Taking or Completing offering: Installer-Residential (Solar System), Installer-Commercial (Solar System), Trade Specialty, Plumber, Sheet Metal Worker

\section{SOLAR RELATED COURSES}

Systems and Equipment

Instruetor: Whil tcomb, Larry

(701) 671-2529

Course Number: ESD 203

Departaint: Eivirurtmenlal gystems

Credits: 3

Student Level: All levels

Duration: 12 Weeks, $5.0 \mathrm{hrs}$ per week

Contact Hours: 60

Classroom: $\quad 60$

Tujics Coveled [xtensively. Alternete

Energy Sources; Heat and Energy

Transfer; Intro. to Solar Energy;

Plumbing Techniques; Sheet Metal

Tortuniques; Snlar Hnme rnnitritrtinn:

Solar Collector Evaluation/Design;

Solar Systens Installation; Solar

Syjtens Haintenanee

Number of Times Taught: ,

Average Enroliment:

25
Solar Energy

Instructor:

Department:

Program or Curriculum :

Credits:

Student Level:

Duration:

Contact Hours:

Classroom:
Mckinney, David

(701) 255-0566

Heating, Refrigeration, and Air Conditioning

Solar Heating

8

Freshman or Scphomore

8 Weeks, 32.0 hrs per week 256

63 


\section{Colleges/Universities \\ AIR FORCE INST TECHMOLOGY \\ (3009) \\ DAYTON, Ohio 45433 \\ (513) 255-2079 \\ SOLAR RELATED COURSES \\ Contemporary Energy Applications \\ Instructor: Stan, Robert \\ (513) 255-4552 \\ Course Number: 462 \\ Department: Engineering Technology \\ Credits: 3 \\ Student Level: College Graduate \\ Duration: I Heeks, 35.0 hrs per week \\ Contact Hours: 35 \\ Number of Times Taught: \\ Average Enrollment: $\quad 30$ \\ Radiation Heat Transfer \\ Instructor: Hitcheock, James E. \\ (513) 255-3069 \\ Course Number: ME 6.73 \\ Department: Aero and Astro. School \\ Credits: of Engineering \\ 4 \\ Student Level: College Graduate \\ Duration: 10 heeks, 4.0 hrs per week \\ Contact Hours: 40 \\ Classrocm: $\quad 40$ \\ Tcpics Covered Extensively: Heat and \\ Energy Transfer \\ Number of Times Taught: 15 \\ Average Enrollment: \\ 8}

AKRON MAIN CAMPUS, $U$ OF

(3123)

AKRON, Ohio 44325

(216) 375-7111

SOLAR RELATED COURSES

Energy Conversion

Instructor: Gross, Richard J.

(216) 375-7736

Course Number: $4600: 415$

Department: Engineering/ Mechanical

Engr.

Credits: 3

Student Level: Junior or Senior

Duration: 15 Weeks, $3.0 \mathrm{hrs}$ per week

Contact Hours: 45

Classroom: 45

Topics Covered Extensively: Energy

Conversion; Intro. to Solar Energy;

Solar System Components; Solar

Collector Evaluaticn/Design; Solar

Systems Desisn; Solar Systens

Installation; Wind Power, Central

Systers; hind Fower, Small Systems

Nuniber of Times Taught: 7

Averase Enrollment: $\quad 20$

Fhysics, Energy and Man

Instructor: Wilson, C.W.

(216) 375-7079

Cuirse Number: 36501141
Department: Physics/Arts and

Credits: 3

Student Level: Freshman or Sophomore

Duration: 15 Weeks, 3.0 tirs per week

Contact Hours: 45

Classroom: 45

Topics Covered Extensively: Al ternate

Energy Sources; Energy Conservation;

Intro. to Solar Energy

Nuriber of Times Taught: 7

Average Enrollment: $\quad 35$

$* * * * * * * * * *$

ANTIOCH COLLEGE

YELLOW SPRINGS, Ohio 45387

(8795)

(513) 767-1424

\section{SOLAR RELATED COURSES}

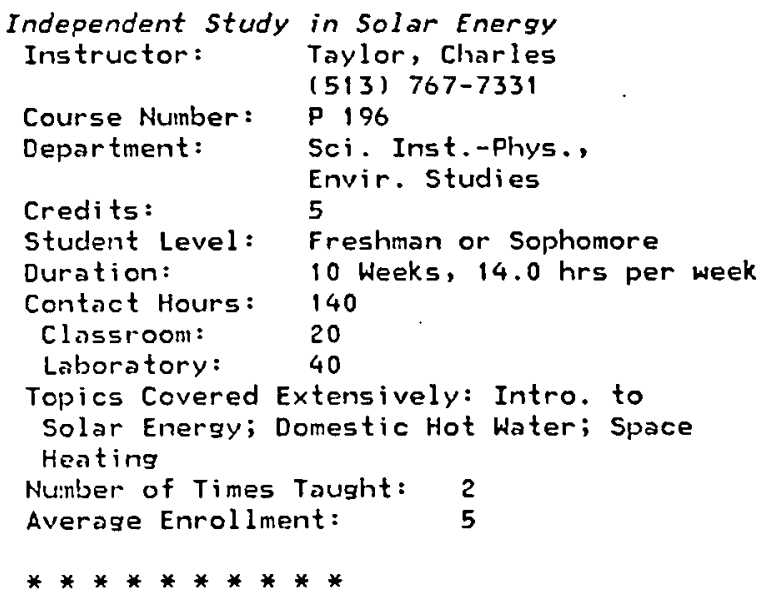

SOLAR PELATED COURSES

E.T. Lab on Alt. Energy Systems

Instructor: Smith, David Lee

(5)3) 475-6426

Course Number: 23-300-413

Cepartment: Architecture

Credits: 3

Student Level: Junior or Senior

Duration: 10 heeks, $4.0 \mathrm{hrs}$ per week

Contact Hours: 40

Laboratory: $\quad 10$ 
Topics Covered Extensively: Alternate Energy Sources; Passive Solar Teshnology; Solar System Corponents; Solar Collector Evaluation/Design; Solar Systems Design; Wind Power, Small Systems

Number of Times Taught: 5

Average Enrollment: 8

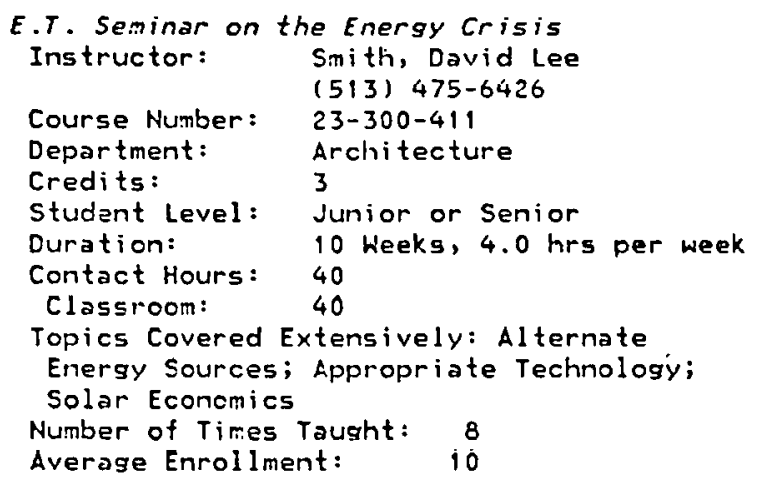

E.T. Studio on Ener. Conscious Ces. Instructor: $\quad 5 \mathrm{mith}$, David Lee

(513) 475-6426

Course Number: 23-300-510

Department: Architecture

Credits: 3

Student Level: Junior or Senior

Duration: $\quad 10$ heeks, $9.0 \mathrm{hrs}$ per week

Contact Hours: 90

Laboratory: 90

Topics Covered Extensively: Energy

Conservation; Intro. to Solar Energy;

Passive Solar Technology; Solar Home,

Construction; Solar Collecter

Evaluation/Design; Solar Systems

Design; Space Heating; Space Cooling

Numbor of Times Taught: 12

Aversue Finrollment: 12

Solar Heating and Cooling

Instructor:

Garrett, Ponald W.

(513) 475-6541

Course Nurber: 32-195-467

Departiment: Applied

Science/Mechanical

Ensr. Tech.

Program or

Curriculum: Mechanical Engineering

r.reditsi

Techinolosy

Student Level: Junior or Senior

Qurstion: il lijeke, l.i hre për weck

Contact Hours: 44

Classroom: $\quad 44$

Topics Covered Extensively: Energy

Storage; Heat and Energy Transfer;

Intro. to Solar Enersy; Solar Home

Construction; Solar Collector

Evaluation/Design; Solar Systems

Design; Domestic Hot Water; Space

Heating

Number of Times Taught: 1

Average Enrollment:

35
DAYTOH, UNIYERSITY OF

DAYTON, OhIO 45469

(513) 229-0123

PROGRAMS AHD CURRICULA

Energy Conversion

Degree: MS, BS, Mechanical

Engineering

Contact: Smith, Howard E. (513) 229-2835

Students Taking or Completing Offering:

Educator, Researcher, Other

SOLAR RELATED COURSES

Direct Energy Conversion

Instructor: Chuang, Henry $\mathrm{N}$.

(513) 229-2835

Course Humber: HEE 514

Department: Mechanical Engineering

Program or

Curriculum: Energy Conversion

Credits:

3

Student Level: Junior or Senior

Duration:

Contact Hours: 45

Classroom: 45

Tupics Covered Extensively: Energy

Conversion; Heat and Energy Transfer;

Intro. to Solar Energy; Materials

Research; Photovoltaics; Elec'l

Generotion, Central; Elec'l Generaiion,

Small Scale

Number of Times Taught: 5

Average Enroliment: 10

Enersy Conversion Systems

Instructor: Chuang, Henry $N$.

(513) 229-2835

Course Number: MEE 402

Department: Mechanical Cngineering

Program or

Curriculum: Energy Eaviversion

Credits: 3

Student Level: Junior or Senior

Duration: 15

Contact Hours: 45

Classroom: 45

Tcpics Covered Extensively: Alternate

Energy Sources; Appropriate Technolcgy;

Energy Conservation; Energy Conversion;

Intro. to Solar Energy

Number of Times Tausht: 4

Avarage Enrollmant; 25

Solar Heating Analyigis

Instructor: Chuang, Henry $\mathrm{N}$.

(513) 229-2835

Course Humber: MEE 567

Department: Mechanical Engineering

Program or

Curriculum: Energv Conversion

Credits:

Student Level: Junior or Senior

Duration: 12 Weeks, 4.0 hrs per week

Contact Hours: 48

Classroom: $\quad 45$

Laboratory: 3

Topics Covered Extensively: Energy 


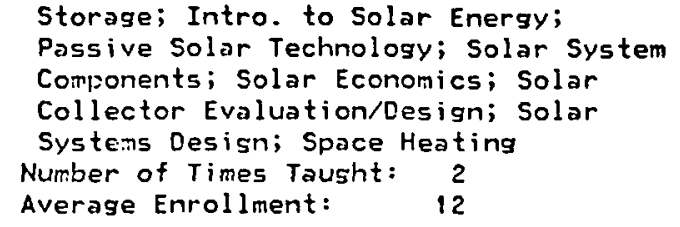

Storage; Intro. to Solar Energy; Passive Solar Technology; Solar System Components; Solar Economics; Solar Collector Evaluation/Design; Solar Systens Desisn; Space Heating Number of Times Tausht: 2 Average Enrollment: 12

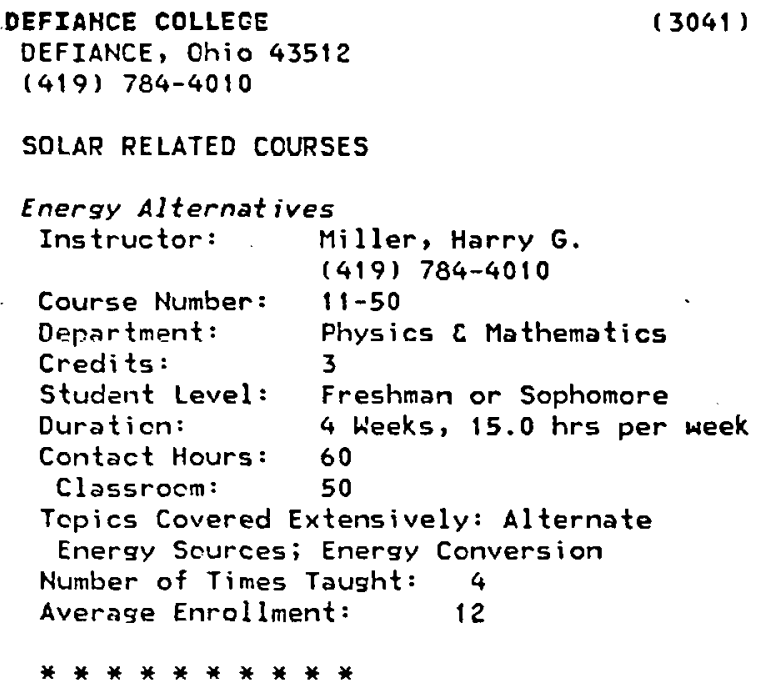

Students Taking or Completing Offering: Architect, Do-it-yourself Homeowner

\section{SOLAR RELATED COURSES}

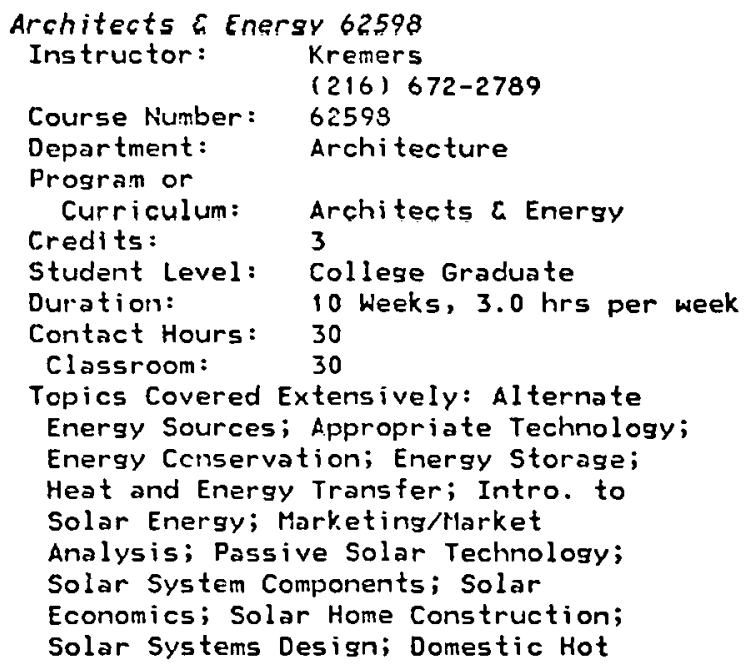

Water; Space Heating; Space Cooling;

Wind Power, Small Systems

Number of Times Taught: 1

Average Enroliment: $\quad 10$

\section{Energy Conversion Utilization}

Instructor: Loughridge, R./ Lees, J./ Fhillips, J. (216) 672-2892

Course Number: 20001

Department: Technology

Credits: 3

Student Level: All levels

Duration: 10 Weeks, $6.0 \mathrm{hrs}$ per week

Contact Hours: 60

Classroom: $\quad 30$

Laboratory: $\quad 30$

Topics Covered Extensively: Energy

Conversion; Heat and Energy Transfer;

Process Heat, Industrial; Space

Heating; Space Cooling

Power Technology

Instructor:

Lees, James

(216) 672-2892

Course Number: $\quad 31032$

Department: Technology

Credits: 5

Student Level: All levels

Duration: 10 Weeks, $10.0 \mathrm{hrs}$ per week

Contact Hours: 100

Classroom: $\quad 50$

Laboratory: $\quad 50$

Number of Times Taught: 21

Average Enrollment: 20

Solar Energy Today

Instructor: Kremers

(216) 672-2789

Course Number: CONT.ED.

Department: Architecture

Program or

Curriculum: Architects 2 Energy

Credits: 2

Student Level: All levels

Duration: 10 Weeks, $2.0 \mathrm{hrs}$ per week

Contact Hours: 20

Topics Covered Extensively: Alternate

Enersy Sources; Appropriate Technology;

Energy Conservation; Energy Storage;

Heat and Energy Transfer; Intro. to

Solar Energy; Passive Solar Technology;

Solar Economics; Solar Home

Construction; Solar Systems Design;

Domestic Hot Water; Space Heating; Wind

Power, Simall Systems

Number of Times Tausht: 2

Average Enrollment: $\quad 25$ 
OHIO HORTHERH UNIVERSITY

(3089)

ADA, Uhio 45810

(419) 634-9921

\section{SOLAR RELATED COURSES}

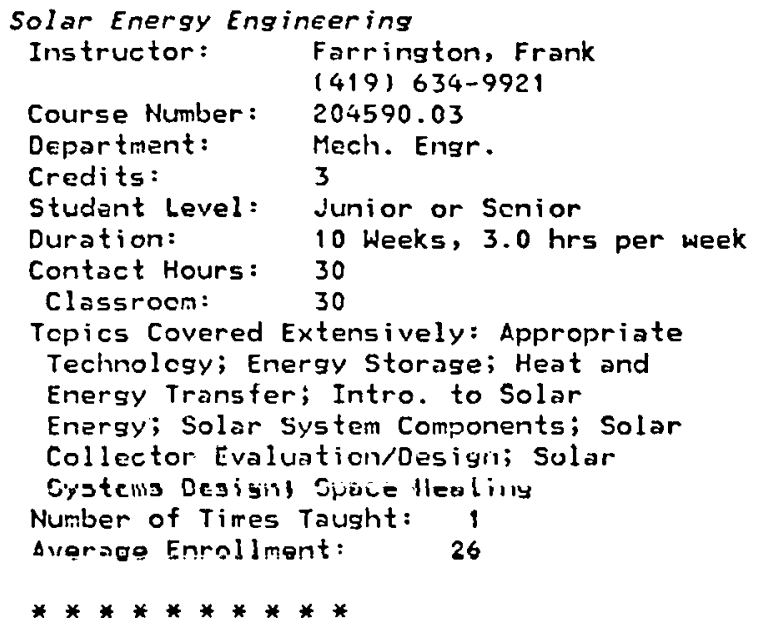

OHIO STATE U MaIH CaMPUS

(6883)

COLUHBUS, OhiO 43210

(614) 422-6446

\section{SOLAR RELATED COURSES}

Intro. To Agricultural Engin. Design

Instructor: Eondurant, Btron L. (611) $422-6131$

Course Number: AE 223

Department: Agricultural

Student Level: Freshman or Sophomore

Duration: 10 Weeks, $4.0 \mathrm{hrs}$ per week

Centact Hours: 40

Classroom: $\quad 10$

Laboratory: $\quad 30$

Number of Times T'aught: 9

Averase Enrollment: $\quad 15$

Solar Energy Thermal systems

Instructor: Sepsy, C.F.

Course Number: ME 614

Department: Mach. Engr.

Credits: 3

Student Level: Junior or Senior

Duration: 10 Weeks, 3.0 hrs per week

Contact Hours: 30

Topies Covered [xtenaively: rineluy

Conversion; lleat and Energy Transfer;

Solar System Components; Solar

Economics; Solar Systens Design;

Domestic Hot Water

Nuriber of Tires Taught: 2

Average Enrollment: $\quad 35$
OHIO STATE U HANSFIELD BR

(3093)

MANSFIELD, Ohio 44906

(419) 747-6561

\section{SOLAR RELATED COURSES}

Solar Energy

Instructor:

Clark, D. L.

(419) 755-4011

Department: Continuing Education

Student Level: All levels

Duration:, 4 Heeks, $2.5 \mathrm{hrs}$ per week

Contact Hoursi 10

Classroom: $\quad 10$

Topics Covered Extensively: Energy

Conservation; Intro. to Solar Energy;

Solar Collector Evaluation/Design;

Space Heating

Averase Enrollment:

65

Wind Energy

Ins lrut lur.

Clark, D. L.

(419) 755-4011

Department:

Centinuing Educotion

Student Level : Ali levels

Duration: $\quad 4$ Weeks, $2.5 \mathrm{hrs}$ per week

Contact Hours: 10

Classroom: $\quad 10$

Toplcs Covered Extensively: Energy

Conservation; Intro. to Solor Energy;

Solar Collector Evaluation/Design;

space Heating; Wind Power, Small

Systems

Average Enrollment:

45

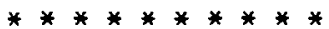

TOLEQO, UHIVERSITY OF

$(3131)$

IULEUU, Uhio 43606

(419) 537-2072

\section{SOI_AR RELATED COURSES}

Energy Conversion I

Instructor: Eltimsahy, Adel $H$.

(419) 537-2638

Course Number: $436: 361$

Department: Electrical Engineering

Credits: 3

Student Level: Junior or Senior

Duration: 10 Weeks, 3.0 hrs per week

Contact Hours: 30

Topics Covered Extensively: Energy

Conversion

Number of Tinies Taught: 2

Average Enrollment: $\quad 40$

Fower: Electronics I

Instructor: stuart, $T$.

(419) 537-2251

Course Number: $436: 468$

Department: Electrical Engineering

Credits : 3

Stuc!eist Level: Junior or Senior

Duration: 10 Weeks, 3.0 hrs per week

Centact Hours: 30

Classroom: 30

Tcpics Covered Extensively: Energy

Conversion 


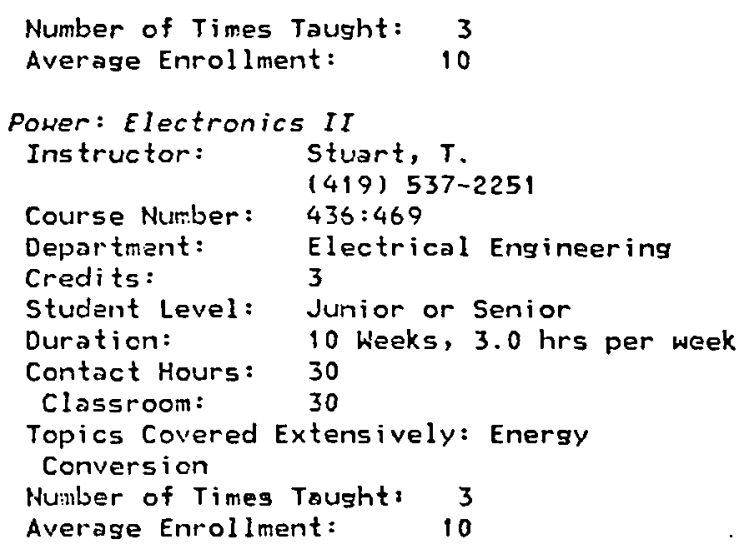

Solar Energy Utilization

Instructor: Eltimasahy, Adel $\mathrm{H}$. (419) 537-2638

Course Number: $436-4 / 563$

Department: Electrical Engineering Credits: $\quad 4$

Student Level: Junior or Senior

Duration: $\quad 10$ Weeks, $4.0 \mathrm{hrs}$ per week

Contact Hours: 40

Classroom: $\quad 30$

Topics Covered Extensively: Energy

Storage; Intro. to Solar Energy;

Fhotovoltaies; Solar System Components;

Solar Systems Desien; Elec'l

Generation, Central; Elec'l Generation,

Srall Scale; Space Heating; Space

Cooling

Number of Times Taught: 4

Average Enrollment: $\quad 15$

\author{
Solar Heating and Cooling \\ Course Number: 434:641 \\ Department: Mechanical Engineering \\ Credits: 3 \\ Student Level: College Graduate \\ Duration: $\quad 11$ Weeks, 3.0 hrs per week \\ Contact Hours: 33 \\ Classroom: $\quad 33$ \\ Topics Covered Extensively: Energy \\ Storage; Heat and Energy Transfer; \\ Intro. to Solar Energy; Passive Solar \\ Technology; Solar System Ccraponents; \\ Solar Economics; Solar Collector \\ Evaluation/Design; Solar Systems \\ Design; Dorestic Hot Water \\ Number of Times Taught: 1 \\ Average Enrollment: $\quad 10$
}

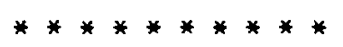

HALSH COLLEEE

(3135)

CANTON, ONIO 44720

(216) 499-7090

SOLAR RELATEO COURSES

Solar Heating

Instructor:

Course Hutiser:

Department:

Over, Calvin $\mathbf{S}$.

CEOTO9

Student Level:

Continuing Education

All levels

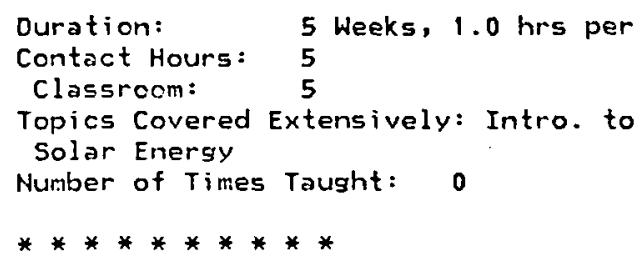

Introduction to Modern Technology

Instructor: Alexander, Charles

Course Number: EE 555

Department: Elec. Engin.

Credits:
$(3145)$ 


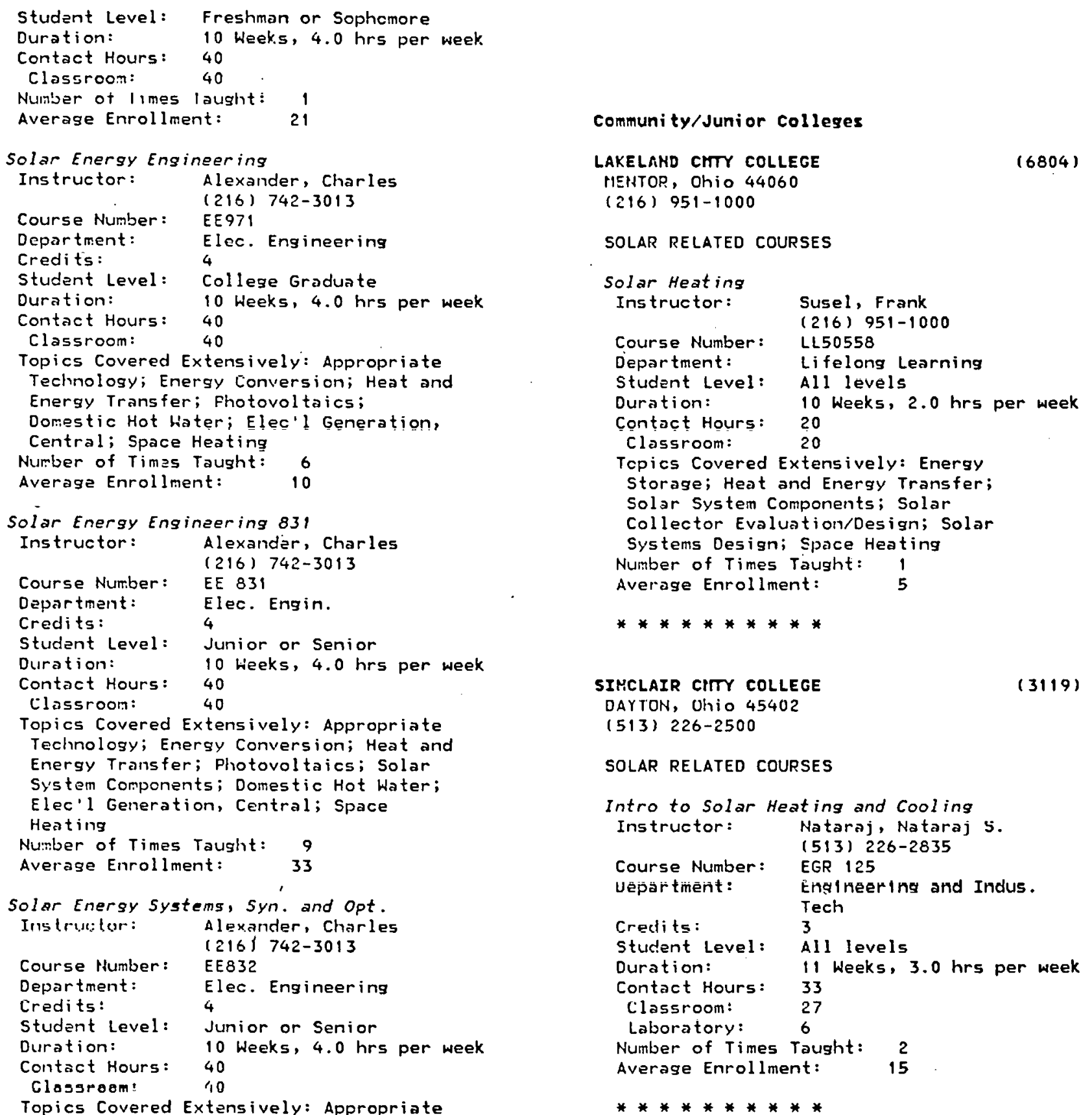

Technology; Energy Storage; Solar

System Components; Solar Economics;

Solar Heme Construction; Solar

Collector Evaluation/Design; Solar

Systens Design; Solar Systems

Installation; Solar Systems Testing and

Evaluation; Domestic Hot Water;

Swimming Pool Heating; Eled'l

Generation, Central; Process Heat,

Industrial; Space Heating; Space

Cooling

Number of Times Taught: 3

Averase Enrollment: $\quad 13$

Vocational/Technical colleges 


\section{Colleges/Universities}

OKLA STATE U MAIN CAMPUS

$(3170)$

STILLWATER, CK lahoma 74074

(405) 624-5000

\section{PROGRAMS AND CURRICULA}

Elec. Engin.-Emphas is on Energy

Degree: FHD, MS, BS, Electrical

Contact: Eacon, C.M.

(405) 624-5156

\section{SOLAR RELATED COURSES}

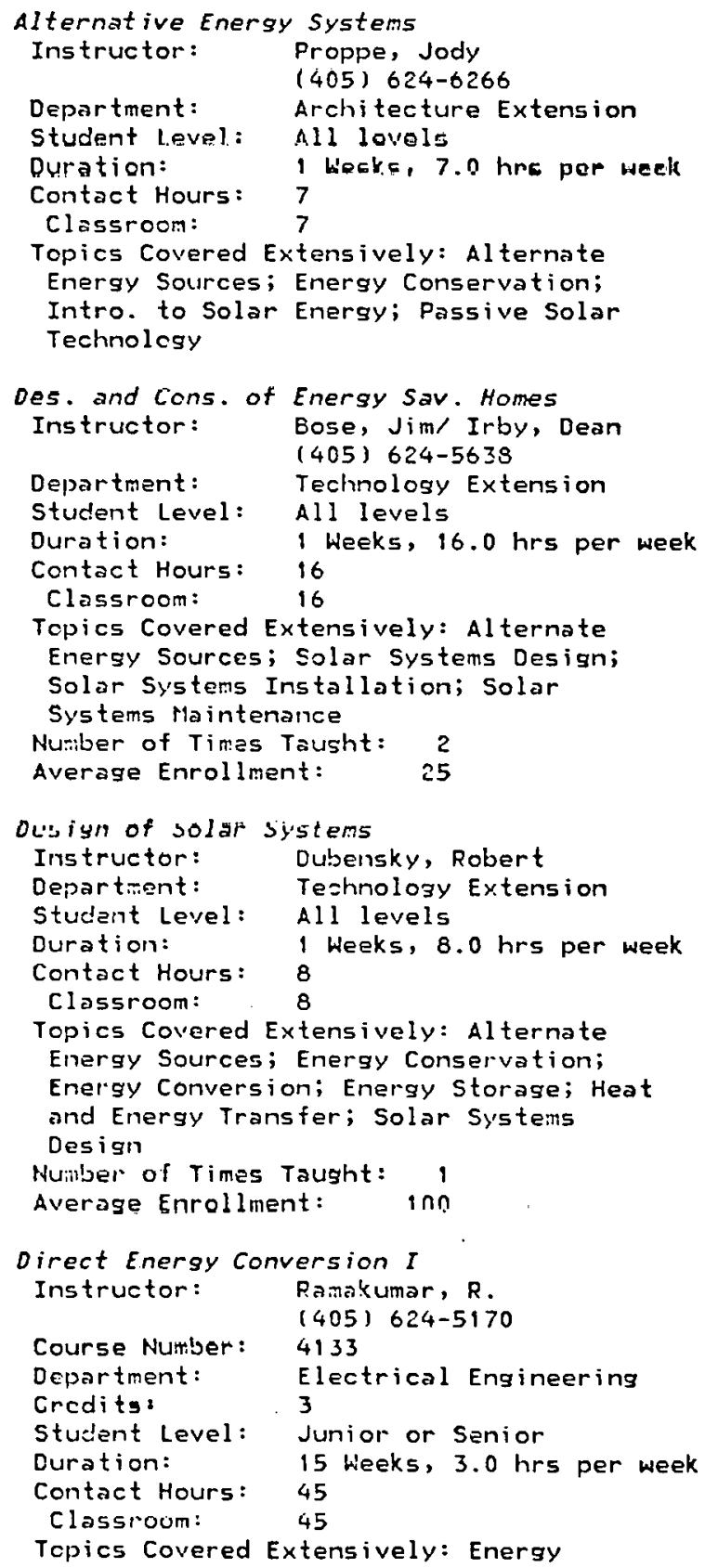

Conversion; Intro. to Solar Energy Number of Times Taught: 17

Average Enrollment: $\quad 25$

Direct Energy Conversion II

Instructor: Ramakumar, $R$.

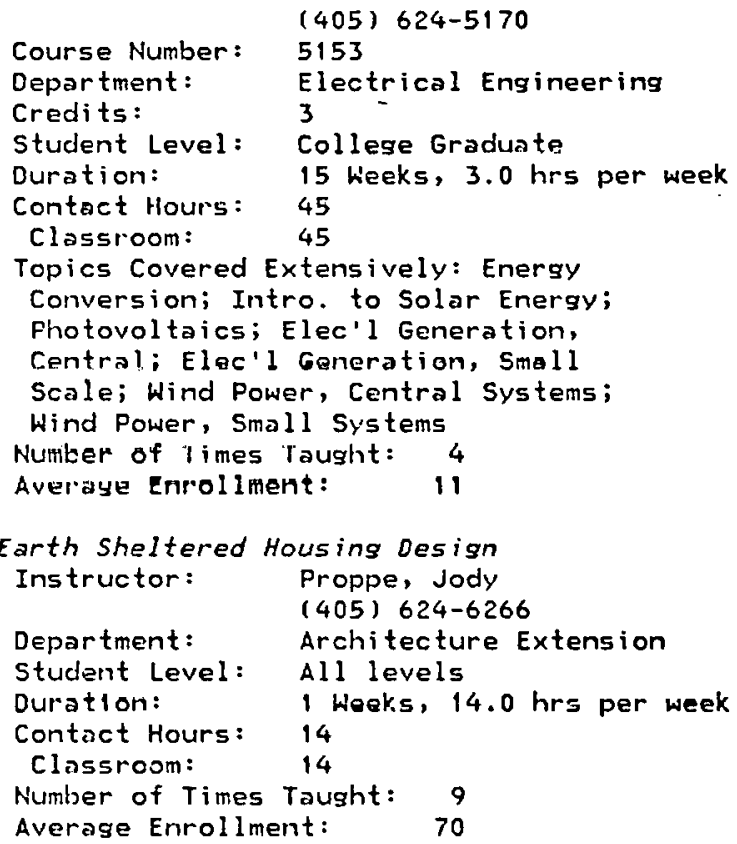

Elect. Engr. Aspects-Hind Ener. Systems

Instructor: Hughes, W.L./ Lingelbacti, $D$. (405) 624-5168

Department: Cont. Education

Student Level: All. levels

Duration: it Weeks, $7.0 \mathrm{hrs}$ per week

Contict Hours: 7

Tnpire Govarod Estensively's Enelgy

Conversion; Fler'l Fanoration; Contral;

Elec'l Generation, Small Scale; Wind

Power, Small Systems

Energy Conservation and Management

Instructor: Turnar, W.C.

- (405) 624-6055

Department: Industrial Engineering

Credits: and Manogement

Student Level: Junior or Senior

Duration: 16 Heeks, $3.0 \mathrm{hrs}$ per week

Contact Hours: 48

Clissroom: $\quad 30$

lehing tory: is

Topics Covered Extensively: Energy

Conservation

Energy Conservation and Management

Instructor: Turner, W.C.

(405) 624-6055

Course Number: IHDEH 4923

Department: Indusu. Engr. and

Credits: 3

Student Level: Junior or Senior

Duration: 16 Weeks, 3.0 hrs per week 


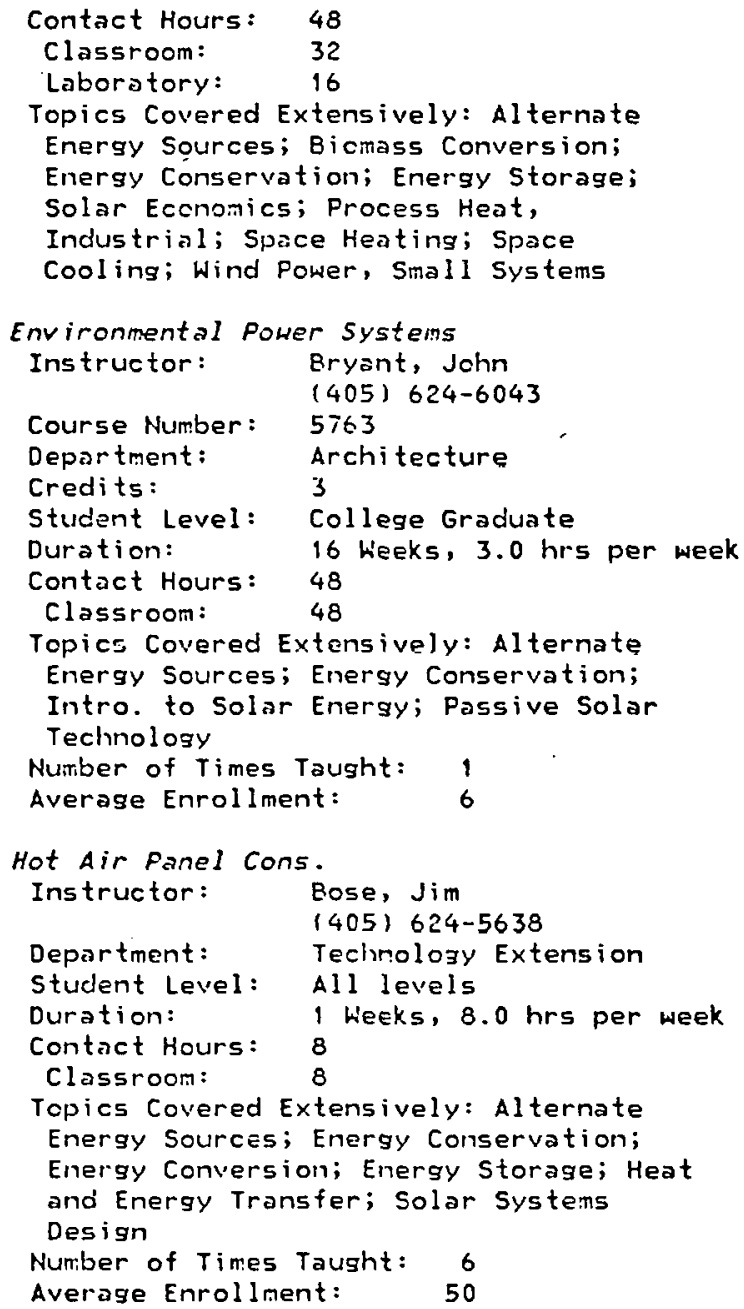

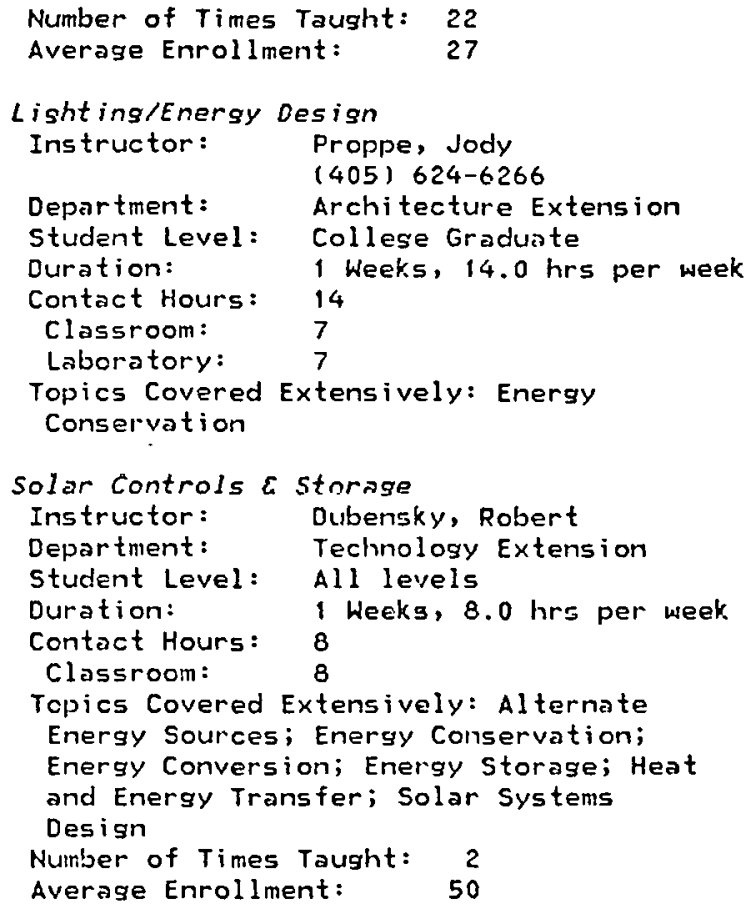


Solar Heat ing Appl.

$\begin{array}{ll}\text { Instructor: } & \text { Bose, Jim } \\ \text { (405) 624-5638 } & \text { Technolcsy Extension } \\ \text { Department: } & \text { All levels } \\ \text { Student Level: } & 1 \text { Weeks, } 8.0 \text { hrs per week } \\ \text { Duration: } & 8 \\ \text { Contact Hours: } & \\ \text { Classroom: } & 8 \\ \text { Topics Covered Extensively: Alternate } \\ \text { Energy Sources; Solar Economics; Solar } \\ \text { Hon:e Constructicn } \\ \text { Number of Times Taught: } 2 \\ \text { Average Enrollment: } \quad 20\end{array}$

***********

oklahoma norman caH, U OF

$(3984)$

NORMAN, OKI ahoma 73019

(405) 325-0311

SOLAR RELATED COURSES

Energy Conservation in Buildings - Seminar

Instruetör: calyert, Floyd 0 . (405) 325-2444

Course Number: 6023

Department: Architecture

Credits: 3

Student Level: Junior or Senior

Duration: 15 Weeks, $3.0 \mathrm{hrs}$ per week

Contact Hours: 45

Classroom: 45

Topics Covered Extensively: Energy

Conservation; Fassive Solar Technology

Number of Tires Taught: 4

Averase Enrollment: $\quad 20$

Energy Conservation Seminar

Instructor: Calvert, Flnurd $n$.

(405) 325-2444

Course Number:

ARCH 6023

Department: Architecture

Ciedils: 3

Sturiont lovol: donion or scnior

Duration: 15 Weeks, 3.0 hrs per week

Contact Hours: 45

Topics Covered Extensively: Energy

Conservation; Passive Solar Technology

Number of Times Taught: 4

Average Enroliment:

Solar Energy Thermal Processes

Instructor: Turkington, D.B.

(405) 325-5011

Course Number: AllE 6750

Department: Aerospace, Mech. and

Credits: 3

Student Level: College Graduate

Duration: 15 Weeks, 3.0 hrs per week

Contact Hours: 45

Classroom: 45

Topics Covered Extensively: Intro, to

Solar Fnergy; Solar Collegtor

Evaluation/Desisn; Solar Systems

Design; Domestic Hot hater; Space

Heating

Number of Times Tousht:

Average Enroliment: 13

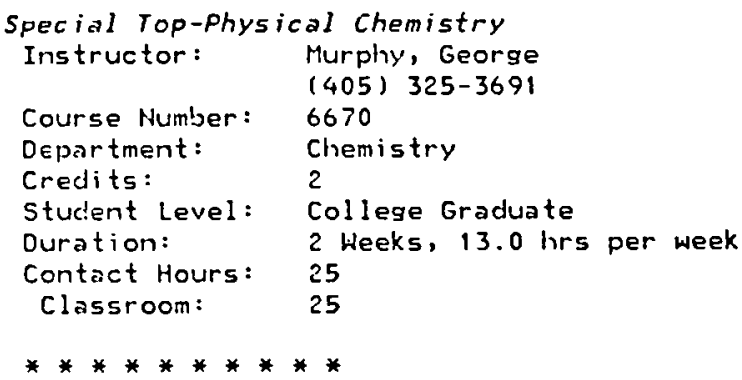

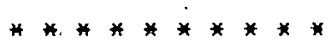




\section{Colleges/Universities}

OREGON INST OF TECHNOLOGY

(3211)

KLAMATH FALLS, Oregon 97601

(503) 882-6321

\section{SOLAR RELATED COURSES}

Seminar (Solar Heating)

Instructor: King, William $\mathrm{N}$.

(503) 882-6321

Course Number: MET 207

Department: Mechanical Engineering Technology

Credits: 1

Student Level: All levels

Duration: 5 Heeks, $2.0 \mathrm{hrs}$ per week

Contact Hours: 10

Classroom: $\quad 10$

Topics Covered Extensively: Passive

Solar Technology; Solar System

Components; Solar Economics; Solar

Collector Evaluation/Design; Solar

Systems Design; Domestic Hot Water;

Space Heating

Number of Times Taught: 1

Average Enrollment: $\quad 74$

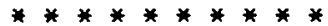

OREGON MAIH CAMPUS, $U$ OF

(3223)

EUGENE, Oregon 97403

(503) 686-3111

\section{PROGRAMS AND CURRICULA}

Solar Energy Center

Degree: no,

Contact: Reynolds, John S.

(503) 686-3631

Students Taking or Completing Offering: Architect, Educator, Researcher, Solar Technician

\section{SOLAR RELATED COURSES}

Environmental Control systems 321

Instructor: Reynolds, John s.

(503) 686-3631

Course Number: ARCH 321

Department: Architecture

Program or

Curriculum: Solar Energy Center

Crodits:

Student Level: Junior or Senior

Duration:

Contact Hours:

Classroom:

10 Weeks, 4.0 hrs per week

40

40

Topics Covered Extensively: Alternate

Energy Sources; Appropriate Technology;

Energy Conservation; Energy Storage;

Heat and Enersy Transfer; Intro. to

Solar Energy; Materials Research;

Passive Solar Technology; Plumbing

Techniques; Solar System Components;

Solar Economics; Solar Home

Construction; Solar Collector

[valuationidesign; Solar syctems

Design; Domestic Hot Water; Space
Heating; Space Cooling

Number of Times Taught: 5

Average Enrollment: $\quad 140$

Environmental Control systems 322

Instructor: Reynolds, Jolnn 5 .

(503) 686-3631

Course Number: ARCH 322

Department: Architecture

Program or

Curriculum: Solar Energy Center

Credits:

4

Student Level: Junior or Senior

Duration:

10 Heeks, 4.0 hrs per week

Contact Hours: 40

Classroom: $\quad 40$

Topics Covered Extensively: Alternate

Energy Sources; Appropriate Technology;

Enersy Conservation; Energy Storase;

Heat and Energy Transfer; Intro. to

Solar Energy; Materials Research;

Passive Solar Technology; Plumbing

Techniques; Solar System Components;

Solar Economics; Solar Horre

Construction; Solar Collector

Evaluation/Design; Solar Systems

Design; Domestic Hot Water; Space

Heating; Space Cooling

Number of Times Taught: 5

Average Enrollment: $\quad 140$

Environmental Control Systems 323

Instructor: Reynolds, John 5 .

(503) 686-3631

Course Number: APCH 323

Department: Architecture

Program or

Curriculum: Solar Energy Center

Credits:

4

Student Level: Junior or Senior

Duration: 10 Weeks, $4.0 \mathrm{hrs}$ per week

Contract Hours: 40

Classroom: $\quad 40$

Topics Covered Extensively: Alternate

Energy Sources; Appropriate Technology;

Energy Conservation; Energy Storage;

Heat and Energy Transfer; Intro. to

Solar Energy; Materials Research;

Passive Solar Technology; Plumbing

Techniques; Solar System Components;

Solar Economics; Solar Hone

Construction; Solar Collector

Evaluation/Design; Solar Systems

Design; Domestic Hot Water; Space

Heating; Space Cooling

Number of Times Taught: 5

Average Enrollment:

140

Sun 25 a future Energy Source

Instructor: McDaniels, D. K.

Course Number: $\quad$ PH. 116

Department: Physics

Program or

Curriculum: Solar Energy Center

Credits:

3

Student Level: All levels

Duration:

Contast Hours: 30

Classroom: $\quad 30$ 
Topics Covered Extensively: Energy Conservation; Energy Conversion; Energy Storase; Heat and Energy Transfer; Intro. to Solar Energy; Passive Solar Technolosy; Fhotovoltaics; Solar System Components; Solar Home Construction; Solar Collector Evaluation/Design; Solar Systems Design; Elec'1 Generation, Central; Space Heating Number of Times Tausht: 10 Average Enrollment: $\quad 200$

***********

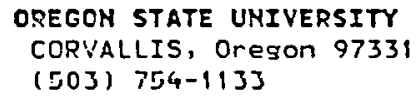

$\begin{array}{ll}\text { Contact Hours: } & 30 \\ \text { Classroom: } & 30\end{array}$

Topics Covered Extensively: Energy

Storage; Heat and Energy Transfer;

Intro. to Solar Energy; Solar System

Components; Solar Economics; Solar

Collector Evaluation/Design; Solar

Systems Design; Solar Systems

Installation; Solar Systems

Maintenance; Solar Sy'stems Testing and

Evaluation; Domestic Hot Water; Space Heating

Number of Times Taught: 1

Average Enrollment: 12

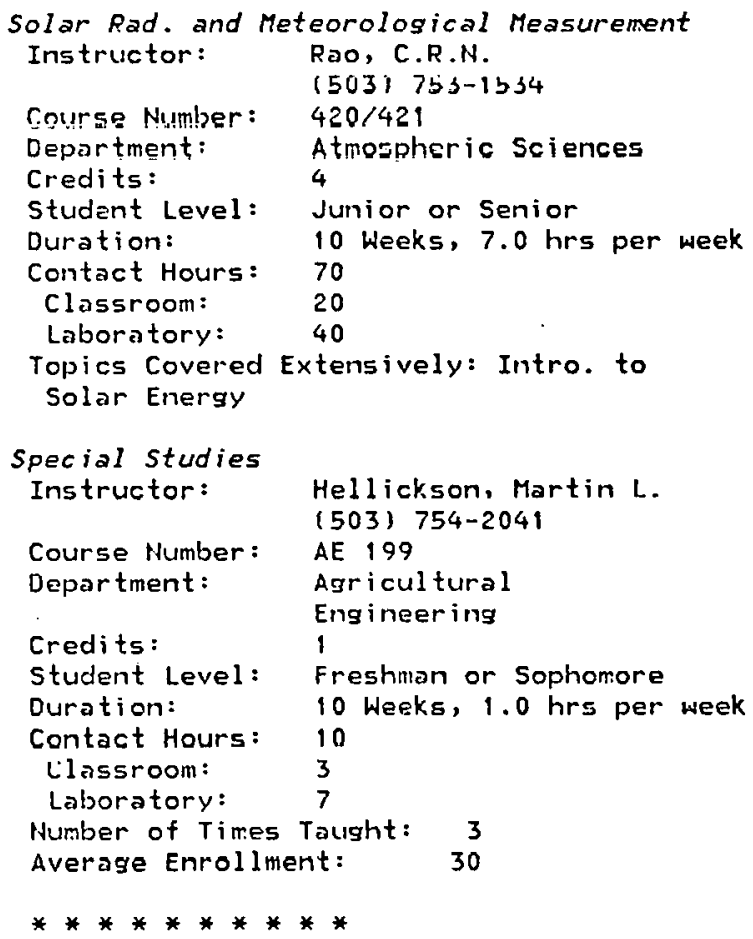

PACIFIC UMIVERSITY

(3212)

FOPEST EROVE, Oregon 97116

(503) 357-6151

SOLAR RELATED COURSES

Ener. Cons. in the fesidential Sector

Instructor: Griffith, Tom/ Story,

Jue
(503) 357-6151

Course Number: 555

Departrent: Science/Social Science

Credits: 3

Student Level: Collese Gracluate

Duration: 3 Heeks, 15.0 hrs per week

Contact Hours: 45

Classroem i 45

Topics Covered Extensively: Energy

Conservation; Energy Conversion; Heat and Energy Transfer; Intro. to Solar Eriergy; Passive Solar Techmology; Solar System Components; Solar Home Construction; Solar Collector 
Evaluation/Design; Solar Systems Design; Solar Systems Installation; Solar Systems Testing and Evaluation; Domestic Hot hater; Swirming Pool Heating; Elec'l Generation, Central; Elec'l Generation, Small Scale; Space Heating

Number of Times Taught: 1

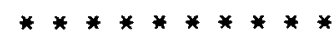

\section{Community/Junior colleges \\ ClackaHAS CITTY COLLEGE \\ OREGOH CITY, Oregon 97045 \\ (503) 656-2631 \\ SOLAR RELATED COURSES \\ Introduction to Appropriate Energy \\ Instructor: Aronson, Mike \\ (503) 656-2631 \\ Credits: \\ Student Level: All levels \\ Duration: 11 Weeks, $3.0 \mathrm{hrs}$ per week \\ Contact Hours: 33 \\ Classroom: 22 \\ Laboratory: $\quad 11$ \\ Topics Covered Extensively: Intro. to \\ Solar Energy; Passive Solar Technology; \\ Solar Systems Design; Space Heating}

\section{LIPIN-BEMTON CITY COLLEGE \\ ALBANY, Oregon 97321}

(6938)

(503) $928-2361$

\section{PROGRAMS AID CURRICULA}

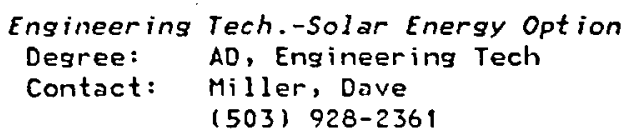

\section{SOLAR RELATED COURSES}

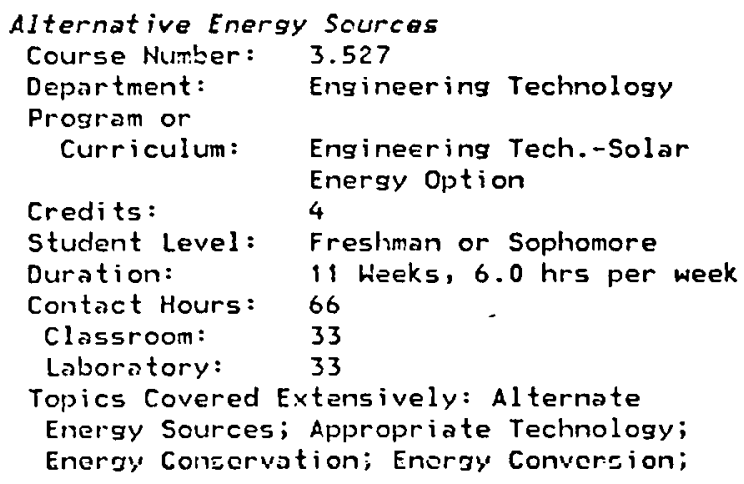

Energy Storage; Heat and Energy

Transfer; Intro. to Solar Energy; Solar

System Coniponents; Solar Collector

Evaluation/Design; Solar Systems

Design; Elec'l Eeneration, Small Scale;

Wind Power, Small Systems

Nurrber of Timas Taught: 4

Averase Enrollment: $\quad 15$

Energy Systems Management

Course Number: 6.220

Department: Engineering Technology

Program or

Curriculum: Engineering Tech.-Solar

Credits: Energy Option

Student Level: Freshman or Sophomore

Duration: 11 Weeks, 3.0 hrs per week

Contact Hours: 33

Classroom: $\quad 33$

Topics Covered Extensively: Energy Conservation; Energy Conversion; Heat and Energy Transfer; Intro. to Solar Energy; Solar System Components; Solar Economics; Solar Systems Maintenance; Solar Systems Testing and Evaluation; Elec'l Eeneration, Central; Elec'l Generation, Small Scale; Process Heat, Industrial; Space Heating; Space Cooling; Wind Power, Central Systems; Wind Power, Small Systems

Solar Energy

Course Number:

Department :

Program or Curriculum: Engineering Tech.-Solar Energy Options

Credits: 3

Student Level: Freshman or Sophomore

Duration:

Contact Hours: 33

Classroom: $\quad 33$

Topics Covered Extensively: Biomass Conversion; Energy Conservation; Energy Conversion; Enersy Storage; Heat and Energy Transfer; Intro. to Solar Energy; Materials Research; Passive Solar Technolozy; Solar System Components; Solar Economics; Solar Home Construction; Solar Collector Evaluation/Design; Solar Systems Design; Solar Systems Testing and Evaluation; Domestic Hot Water; Elec'l Generation, Small Scale; Space Heating; Wind Power, Small Systems Number of Times Taught: 2 Average Enrollment: $\quad 12$

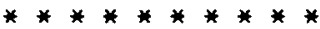




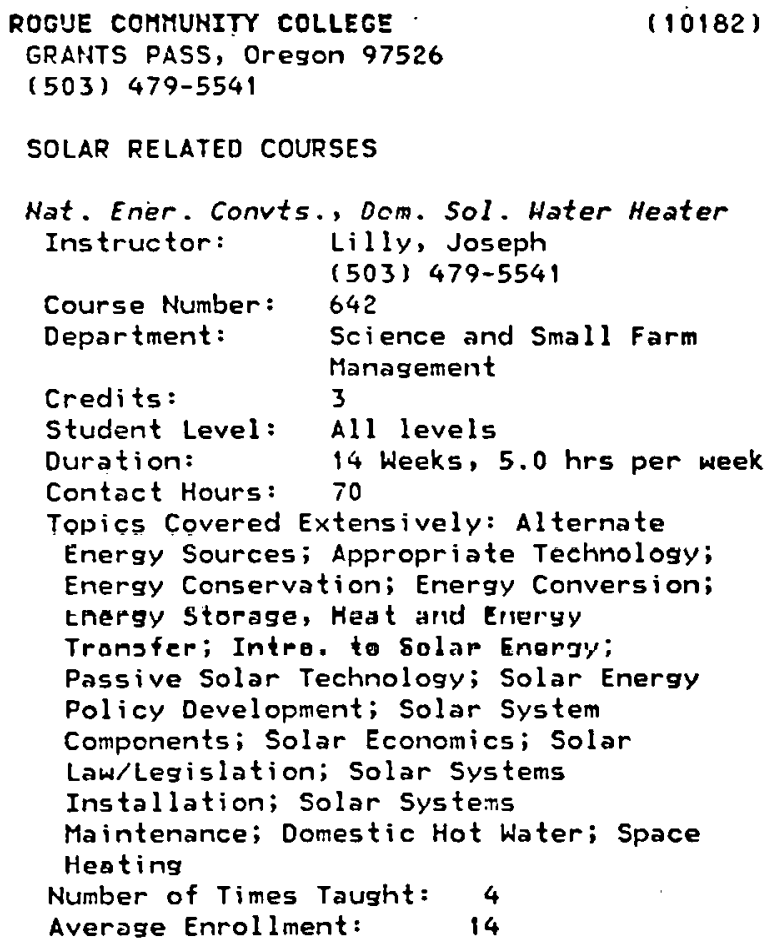




\section{Colleses/Universities \\ CALIFORNIA STATE COLLEGE \\ CALIFORHIA, Pennsylvania 15419 \\ (3316) \\ (412) $938-4000$ \\ SOLAR RELATED COURSES \\ Energy and Fower

$\begin{array}{ll}\text { Instructor: } & \text { Hallidy, William } \\ & (412) 938-4153 \\ \text { Department: } & \text { Physical Science } \\ \text { Credits: } & 3 \\ \text { Student Level: } & 411 \text { levels } \\ \text { Duration: } & 15 \text { Weeks, } 3.0 \text { hrs per week } \\ \text { Contact Hours: } & 45 \\ \text { Classroom: } & 45\end{array}$ \\ Topics Covered Extersively: Alternate \\ Energy Sources; Appropriate Technology; \\ Energy Conservation; Energy Comversion; \\ Energy Storase; Heat and Energy \\ Transfer; Intro. to Solar Energy; \\ Elec'l Generation, Central; Elec'l \\ Generation, Small Scale \\ Number of Times Taught: 2 \\ Averase Enrollment: 25 \\ **********}

\section{DICKINSOY COLLEGE}

CARLISLE, Pennsylvania 17013

(717) 243-5121

SOLAR RELATED COURSES

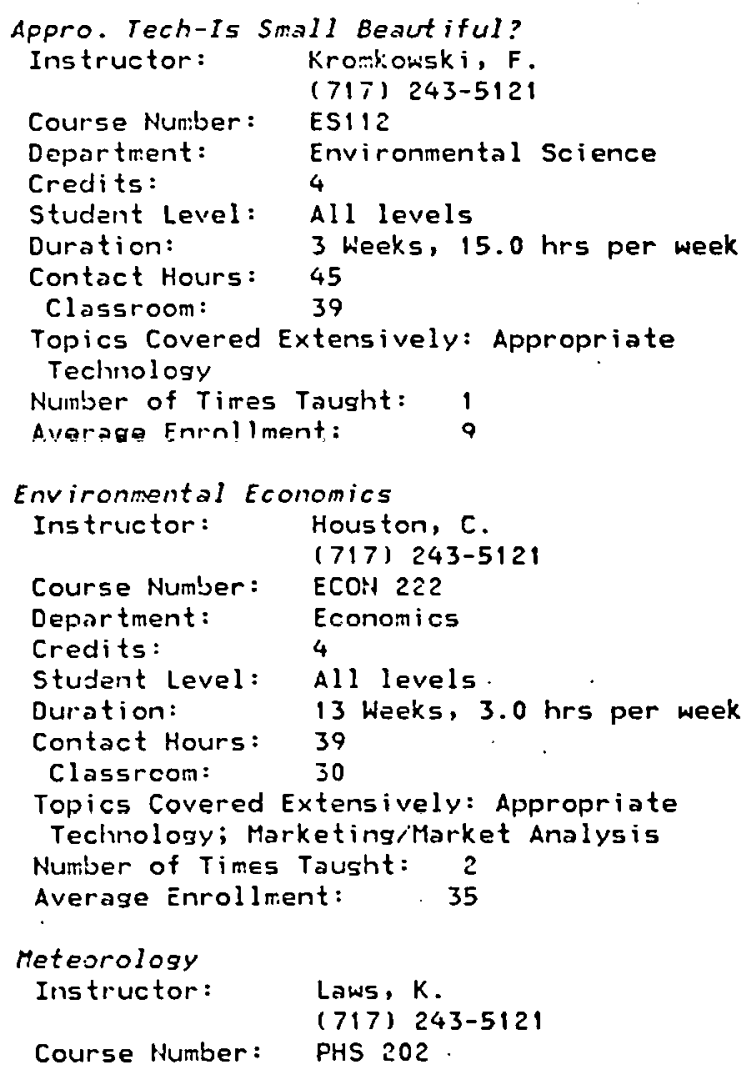

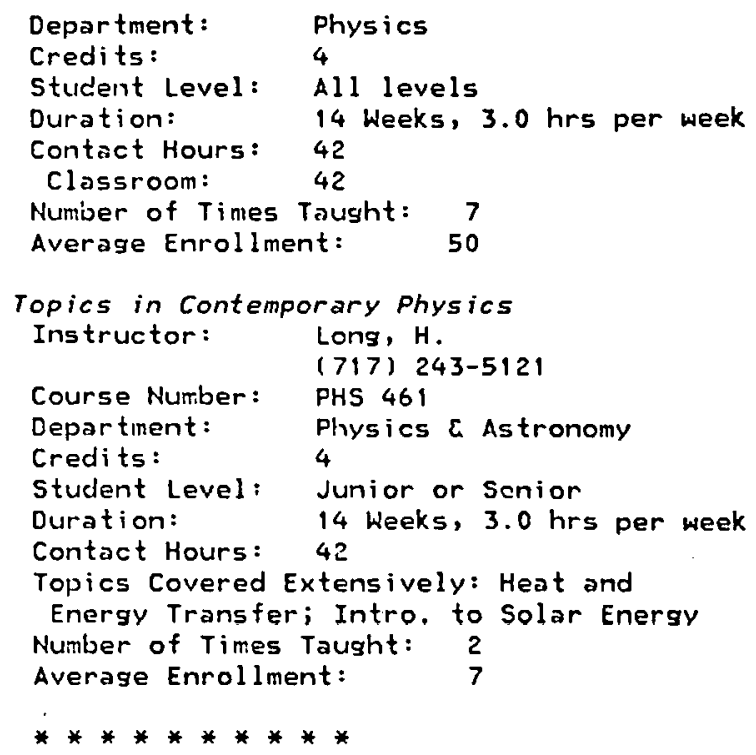


GANREN COLLECE

ERIE, Pennsylvania 16501

(814) $456-7523$

SOLAR RELATED COURSES

Desisn Project for Solar Heat

Instructor: Dowell, Milt

Course Number: $\quad$ E160

Department: Engineering

Credits: 3

Student Level: Junior or Senior

Duration: $\quad 14$ Weeks, $6.0 \mathrm{hrs}$ per week

Contact Hours: 84

Topics Covered Extensively: Space

Heating

Average Enrollment:

1

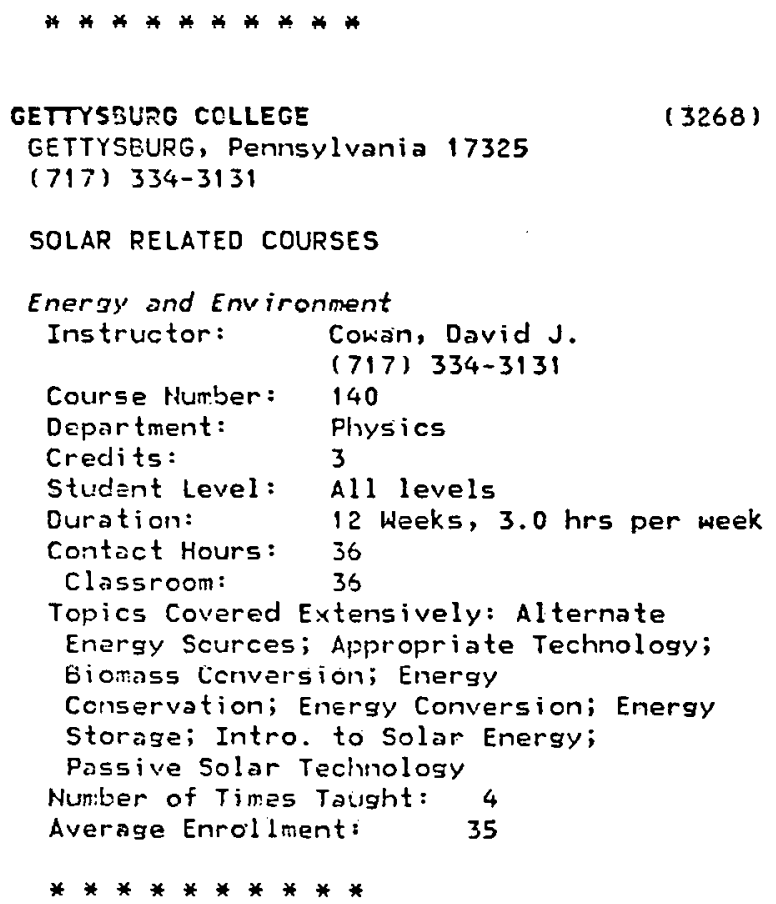

(3268)

(3266)

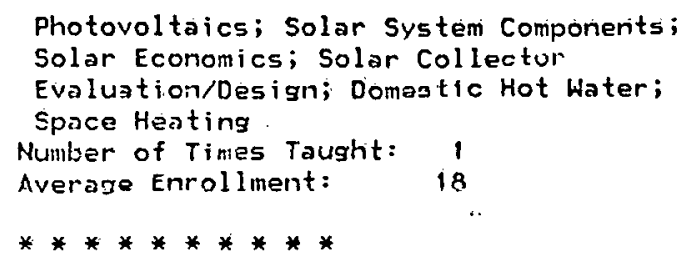

PA ST U SKEHAKGO VLY CAN

(3345)

SHARON, Pennsylvania 16146

(412) $981-1640$

PROGRAMS ATHU CURRICULA

Solar Heat ing and Cooling Technology Desree: Short Course Certificate Contact: Houl ihan, John F. (412) $981-1640$

\section{SOLAR RELATED COURSES}

Fundamentals of solar Eriergy Instructor: Houlitian, J.F.

$\begin{array}{ll} & (42 i) 981 \\ \text { Course Number: } & \text { PHYS. } 296\end{array}$ Department: Fhysics:

Prosram or Curriculum: Solar Heating and

Credits: Student Level: Freshman or Sophomore Duration: 10 heeks, 2.0 hrs per week Contact Hours: 20

Classroom: $\quad 15$ Laboratory: 5

Topics Covered Extensively: Energy Conversion; Energy Storage; Heat and Ellersy Tiansfer; Intro. to Solor Energy; Passive Solar Technology; Photovoltaics; Solar Sy'stem Components; Solar Home Construction; Solar Collector Evaluatión/Design; 5olar Systens Oesign; Space Heating; Space 
Cooling; Wind Power, Central Systems Number of Times Taught: 2

Average Enrollment: 12

Intro.

Topics Covered Extensively: Intro. to Solar Energy; Space Heating; Wind

Power, Central Systems

Intro. to Solar Energy

Instructor: Houl ihan, John F.

(412) $981-1640$

Course Number: FHYS. 297

Department: Physics

Program or

Curriculum: Solar Heating and

Credits: Cooling Technology

Student Level: Freshman or Sophomore

Duration: 10 Heeks, 3.0 hrs per week

Contact Hours: 30

Classroom: $\quad 28$

Laboratory: 2

Topics Covered Extensively: Intro. to

Solar Energy; Space Heating; Wind

Power, Central Systems

Number of Times Taught: $\overline{2}$

Averase Enrollment: $\quad 15$

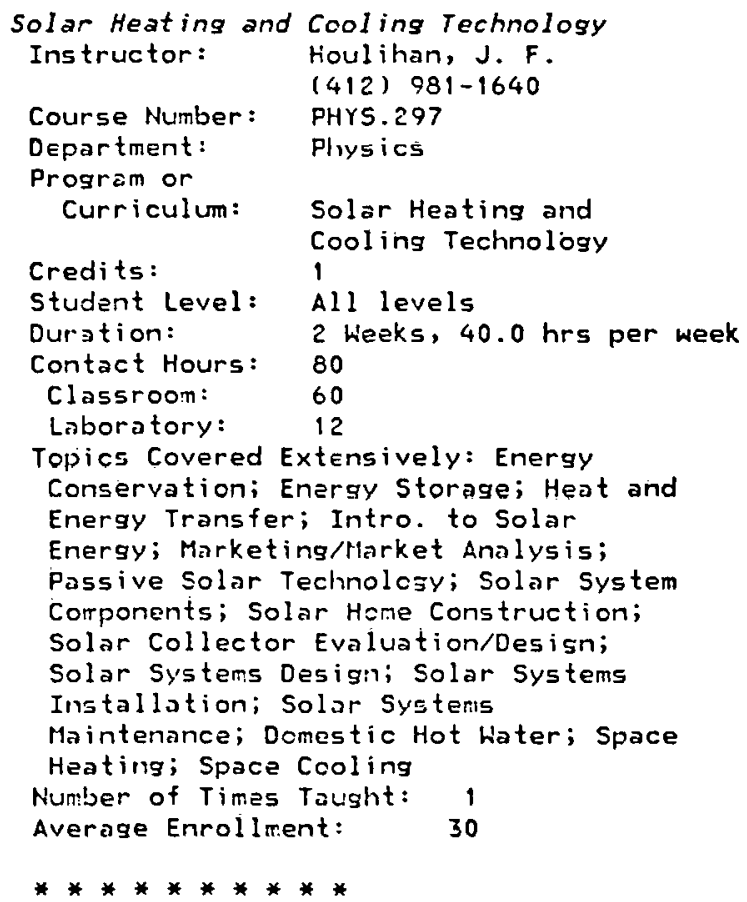

PA STATE U ALLEHTONH CAY

$(3330)$

FOGELSVILLE, Pennsylvania 18051

(2:5) 285-4811

SOLAR RELATED COURSES

* Solar horkshop-Cooling for Homes

Topics Covered Extensively: Space

Cooling

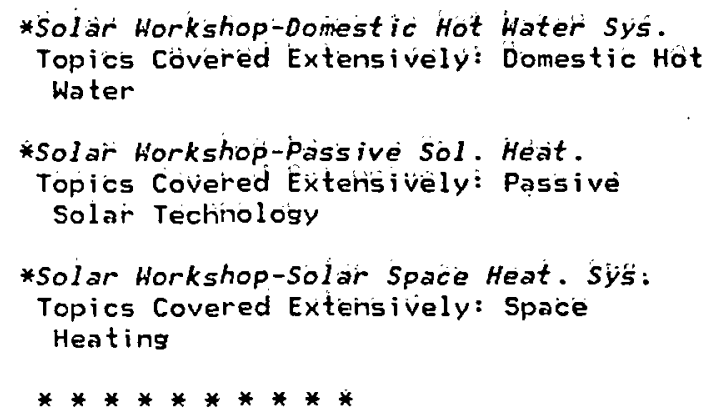

PA STATE U MAIH CAMPUS

UNIVERSITY PARK, Pentisylvanita 16802

$(6965)$

(814) 865-4700

SÖLAR RELATED COURSES

Solar Energy Building System Desigh

Instructor: Gilmain, stâniley $F$ :

(814) $865-6344$

Course Number: AE-497

Department: Architectúral

Engineering

Student Level: Junior or Seniôn

Duration: 10 Weeks; 7.00 hirs pèr week

Contact Hours: 70

Classroom: $\quad 40$

Topicg Covered Extensivelý Energý

Storage; Heat arid Energy Tranisfer;

Intro. to Solar Energy; Solar System

Conporients; Solar Economics; Sollar

Collector Evaluation/Désigni Solar

Systems Design; Domestic Hot hater;

Space Heating

Nuriber of Times Taught: 4

Average Enroliment: $2 i$

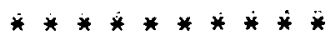

PERTSYLVAATIA,U OF

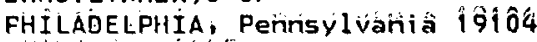

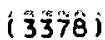

(215) 243-5000

PROGRAMS AND CURRICULÁ

Energy Engineer ing

Degree: MS, Science iñ Éngineeringig

Contact: Eisenberig; Larry

(215) $243=8507$

Students Taking oi Eonipleting offering:

Educator, Researcher; Solàr Éng i neer

Sólár related cóvíses

Eriergy Converisioh

Instructor

Couirse llivimber?

Depar tment:

Progirain ố

Euirriculún:

Credits:

Studeiat tével:

Durstion :

Fegleÿ; Ken

566

Systems Erigineering

Energiy Engineering

3

'collége Graduate

14 Hikaks. 3:0 hirs per week 
Contact Hours: 42

Classroom: $\quad 42$

Topics Covered Extensively: Appropriate

Technology; Energy Conversion; Energy

Storage; Heat and Energy Transfer

Number of Times Taught: 4

Average Enrollment: $\quad 15$

Materials for Energy Engineering

Instructor: Laird, CampbelI

Course Number: 555

Department: Material Science

Program or

Curriculum: Energy Engineering

Credits:

Student Level: College Graduate

Duration: 14 heeks, 3.0 hrs per week

Contacl Huw's: 42

Classroom: $\quad 42$

Topics Covered Extensively: Energy

Storage; Materials Research

Number of Times Taught: 4

Average Enrollment: $\quad 15$

Prin. of Solar Energy Utilization

Instructor: Lior, Noam

Course Number: 591

Department: Mechanical Engineering

Program or

Curriculum: Energy Enginieering

Credits: 3

Student Level: College Graduate

Duration: $\quad 14$ Weeks, $3.0 \mathrm{hrs}$ per week

Contact Hours: 42

Tcpics Covered Extensively: Alternate

Enersy Sources; Appropriate Technology;

Biomass Conversion; Energy

Conservation; Energy Storage; Intro. to

Solar Energy; Passive Solar Technology

Number of Times Taught: 4

Average Enrollment: $\quad 15$

Solid stale Energy Conversion

Instructor:

Course llumber: EES 524

Department: Electrical Eng. $\varepsilon$

Science

Prosram or

Curriculum: Energy Engineering

Credits: 3

Student Level: College Graduate

Duration: 14 heeks, 3.0 hrs per week

Contact Hours: 42

Topics Covered Extensively: Energy

Conservation; Energy Conversion; Energy

Storage; Heat and Energy Transfer;

Intro. to Solar Energy; Photovoltaics;

Elec'l Oerieraliun, Central; Elec'l

Generation, Small Scale

Number of Times Taught: 3

Average Enrollment:

15

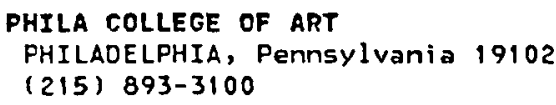

Credits:

Student Level: All levels

Duration: 16 Weeks, $3.0 \mathrm{hrs}$ per week

Contact Hours: 48

Tcpics Covered Extensively: Energy Conservation; Intro. to Solsr tnergy;

Passive Solar Technology; Solar Home Construction

Number of Times Taught: 3

Average Enrollment: 18

**********

SAINT VINCENT COLLEGE

LATROBE, Pennsylvania 15650

(412) 539-9761

SOLAR RELATED COURSES

Solar Energy

Instructor:

Heid, Roland $L$.

Department:

(412) 539-9761

Student Level: All levels

Duration: $\quad 6$ Weeks, $3.0 \mathrm{hrs}$ per week'

Contact Hours: 18

Classroom: $\quad 18$

***********

SHARTHMORE COLLEGE

$(3370)$

SIVARTHMORE, Pennsylvania 19081

(215) 544-7900

SOLAR RËLATED COURSES

Alternate Energy Technologies

Instructor: Bowler, D. L./ Barns,

Department: Engineering

Credits: 1

Student Level: Freshingin or sophomore

Duration:

14 Weeks, 3.0 hrs per week

Contact Hours: 70

Classroom: $\quad 52$

Laborotory: $\quad 18$

Number of Times Taught: 3

Average Enrollment: 6

Solar Heating-Design

Instructor: Bowler, D. L./ Barns,

C.f Orthlieb, $F$.

(215) 544-7900

Course Number: E 90

(3368) 


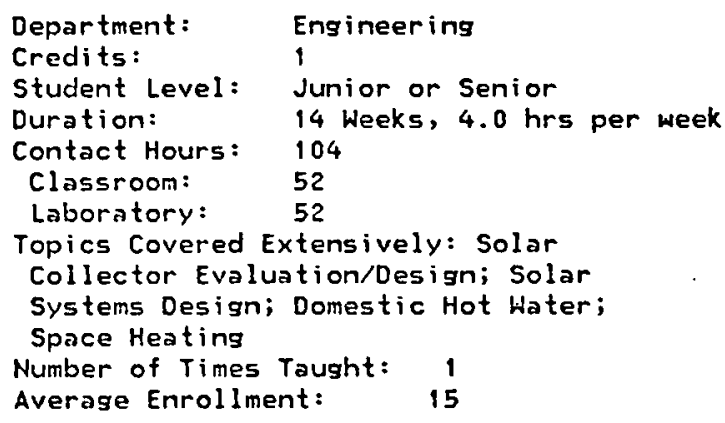

\section{HESTHINSTER COLLEGE}

NEW WILMIYGTON, Pennsylvania 16142

(3392)

(412) $946-8761$

\section{SOLAR RELATED COURSES}

Energy Use and Alternate Energy Sources

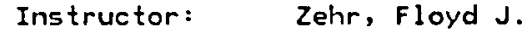

\section{HIDEKER COLLEGE}

CHESTER, Pennsylvania 19013

(3313)

(215) 876-5551

\section{SOLAR RELATED COURSES}

\section{Energy $I$}

Instructor: Madonna, L. A.

Course Number: 437

Department: Center of Engineering Credits:

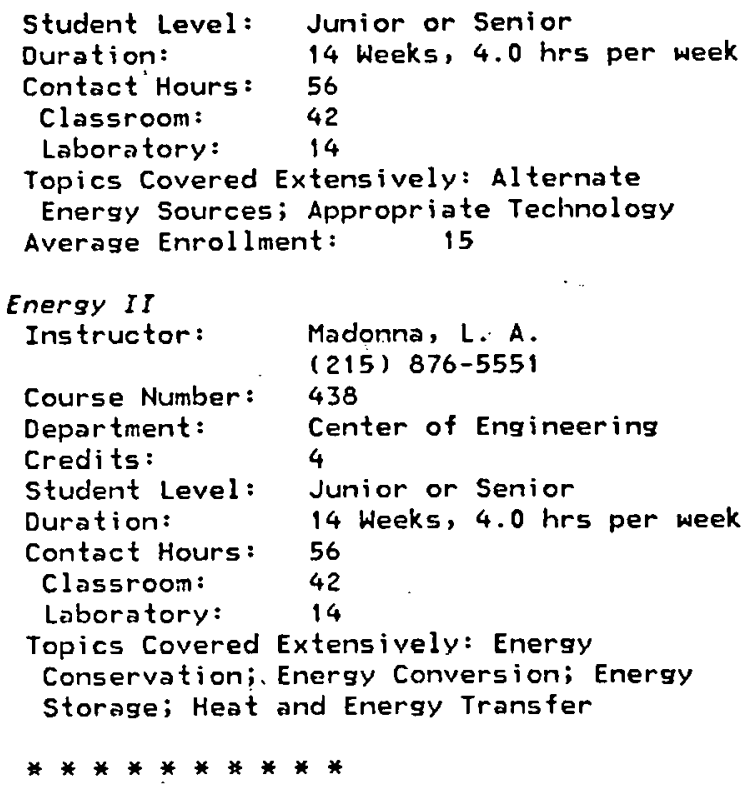

\section{HILKES COLLEGE}

WILKES-BAPRE, Pennsylvania 18703

(717) 824-4651

(3394)

\author{
SOLAR RELATED COURSES \\ Alternate Energy Sources \\ Instructor: Nejib, U.R. \\ (717) 824-4651 \\ EE 390 \\ Course Number: \\ Department: \\ Credits: \\ Student Level: Junior or Senior \\ Duration: \\ Contact Hours: 46 \\ Classroom: 28 \\ Topics Covered Extensively: Alternate \\ Energy Sources; Heat and Energy \\ Transfer; Passive Solar Technology; \\ Photovoltaics; Elec'l Generation, \\ Central; Wind Power, Small Systems \\ Number of Times Taught: 2 \\ Average Enrollment: $\quad 15$
Energy Cons.-Alts. and Methods
Instructor: Nejib, Umid R. (717) 824-4651
Course Number: E0594
Department: Engineering
Credits: 3
Student Level: College Graduate
Duration: $\quad 3$ Weeks, $38.0 \mathrm{hrs}$ per week
Contact Hours: 114
Classroom: $\quad 36$
Laboratory: 24
Topics Covered Extensively: Alternate
Energy Sources; Appropriate Technology;
Energy Conservation; Intro. to Solar
Energy; Solar Energy Policy
Development; Elec'l Generation, Central
Number of Times Taught: 1
Average Enrollment: $\quad 32$




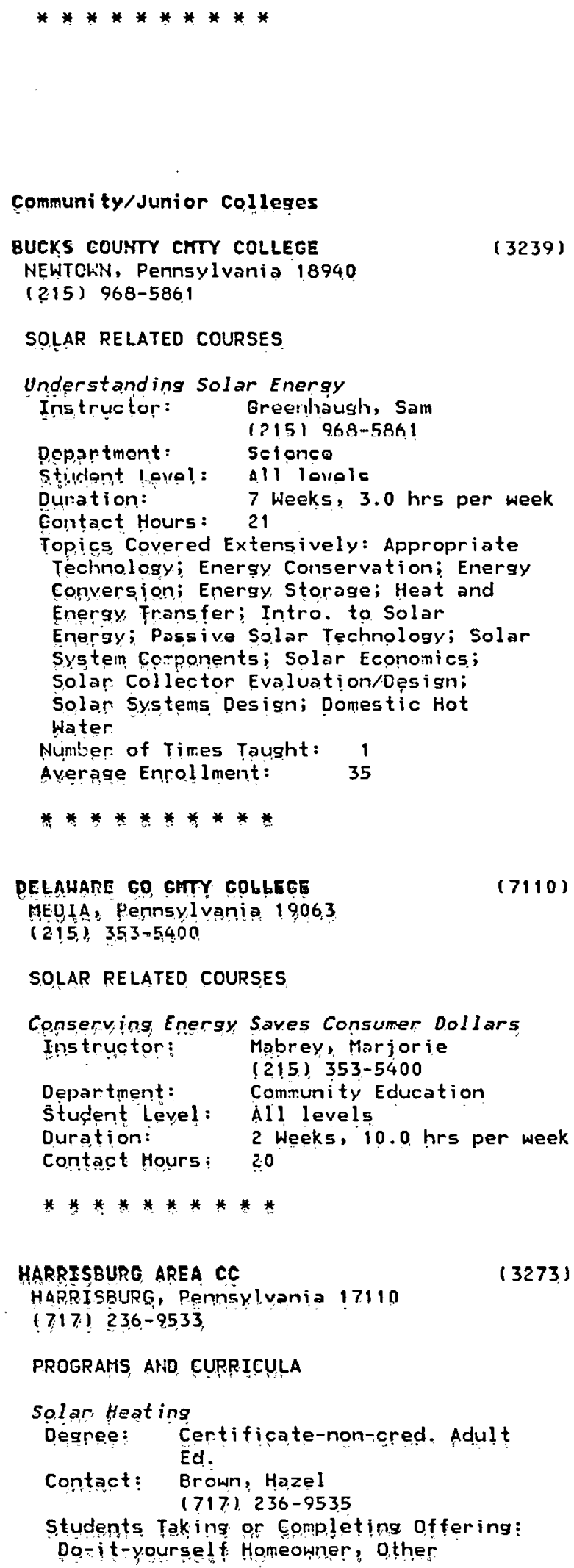

SOLAR RELATED COURSES

Sol. Heat.-Food, Heat Prod. Greenhouse

Department: Community Resources Institute

Program or

Curriculum: Solar Heat

Student Level: All levels

Duration: 3 Heeks, 3.0 hrs per week

Contact Hours: 9

Classroom: 9

Tcpics Covered Extensively: Alternate

Energy Sources; Appropriate Technology;

Energy Conservation; Energy Conversion;

Energy Storage; Heat and Energy

Transfer; Intro. to Solar Energy; Solar

System Components; Solar Economics;

Solar Home Construction; Solar

Collactor Evalubtigunesien; Snlar

systems Mesian: Snlar Sustems

Installation; Elec'l Generation, Small

Scale; Process Heat, Industrial; Space Heating

Number of Times Taught: 2

Average Enroliment: $\quad 20$

KEYSTOHE JUHIOR COLLEGE

(3280)

LA PLUHE, Pennsylvania 18440

(717) 945-5141

PROGRAMS AND CURRICULA

Solar. Engineering Technology

Degree: AD, Applied $30 i$. in 501.

Contact: Kutch, Dennis/ Cupilleri, Tom $171719.45-5141$

Sluderts Taking or Completing Offering! Solar Teclinician

\section{SOLAR RELATED COURSE'S}

Siz., Inst., and Oper.-Sol. Heat. (Res. BI.) Instructor: Kutch, Dennis

(717) 945-5141

Department: $\quad$ Solar Energy Study $a$

Program or Curriculum: Solsr Engineering

Res. Cill.

Technolozy

Student Level: All levels

Duration: 2 Weeks, 36.0 hrs per week

Contact Hours: 72

Classroom: $\quad 30$

Laboratory: 42

Topics Covered Extensively: Energy Conservation; Plumbing Techniques; Sheet Metal Techniques; Solar System Components; Solar Home Construction; Solar Bystems I!nstallation; Sular. Systems plaintenance; Solar Systems Testing and Evaluation; Domestic Hot Water; Swimming Pool Heating; Suace Heating 


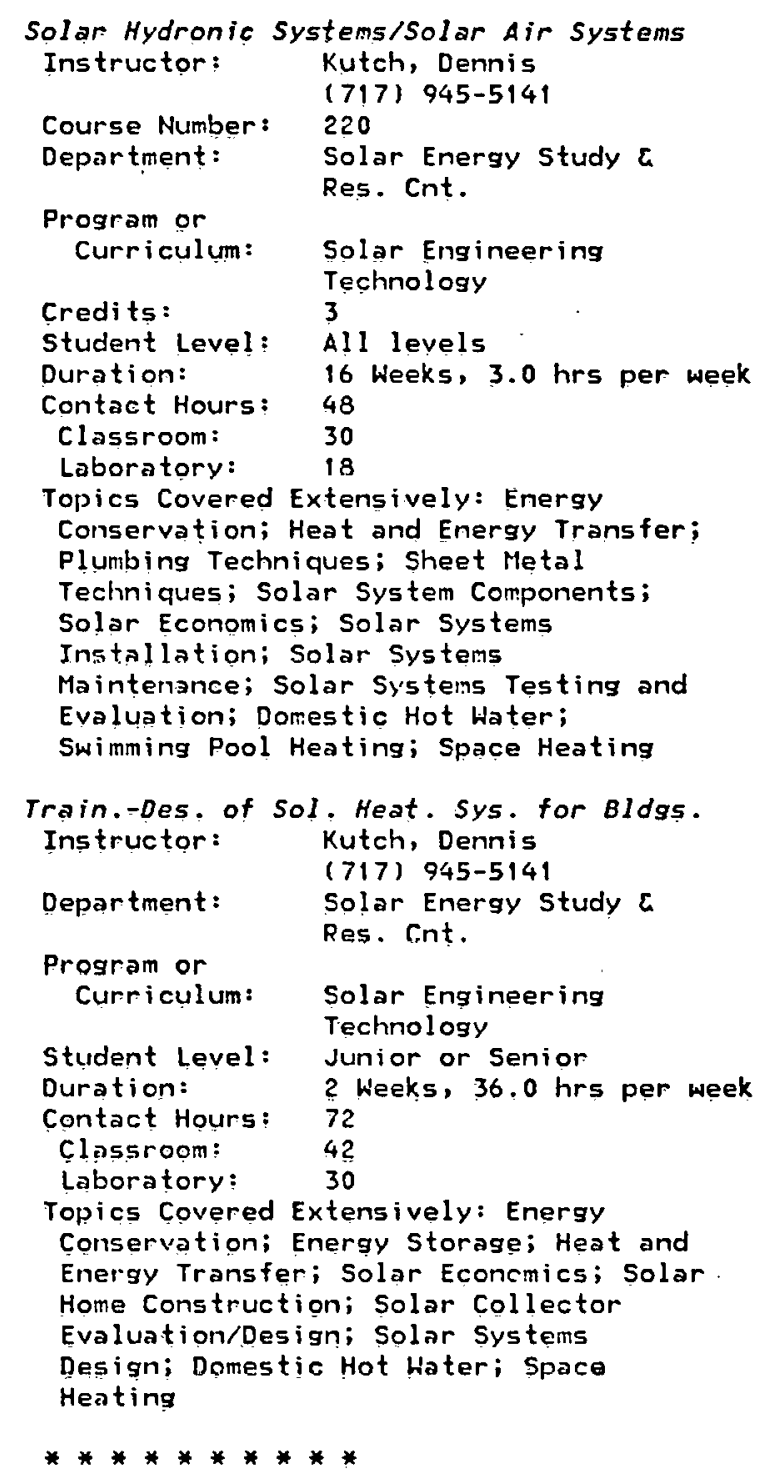

\section{LEHICTH CO CITY COLLEGE}

SCHNECKSVILLE, Pennsylvania 18078

(215) $799-2121$

(6810)

\section{Alternate Energy Teçholog̣les \\ Degree: $A D$, Applied Science \\ Contact: Walker, J. Robert \\ ( 215 ) $799=1515$}

\section{SOLAR RELLATED COURSES}

$\begin{array}{ll}\text { Alternate Energy } & \text { Technologies } \\ \text { Instructor: } & \text { Walker, J. Rebert } \\ & (215) 799-1515 \\ \text { Course Humber: } & \text { PHY } 102 \\ \text { Department: } & \text { Fhysics and } \\ \text { Pechnologies } & \\ \text { Program or } & \text { Alternate Energy }\end{array}$

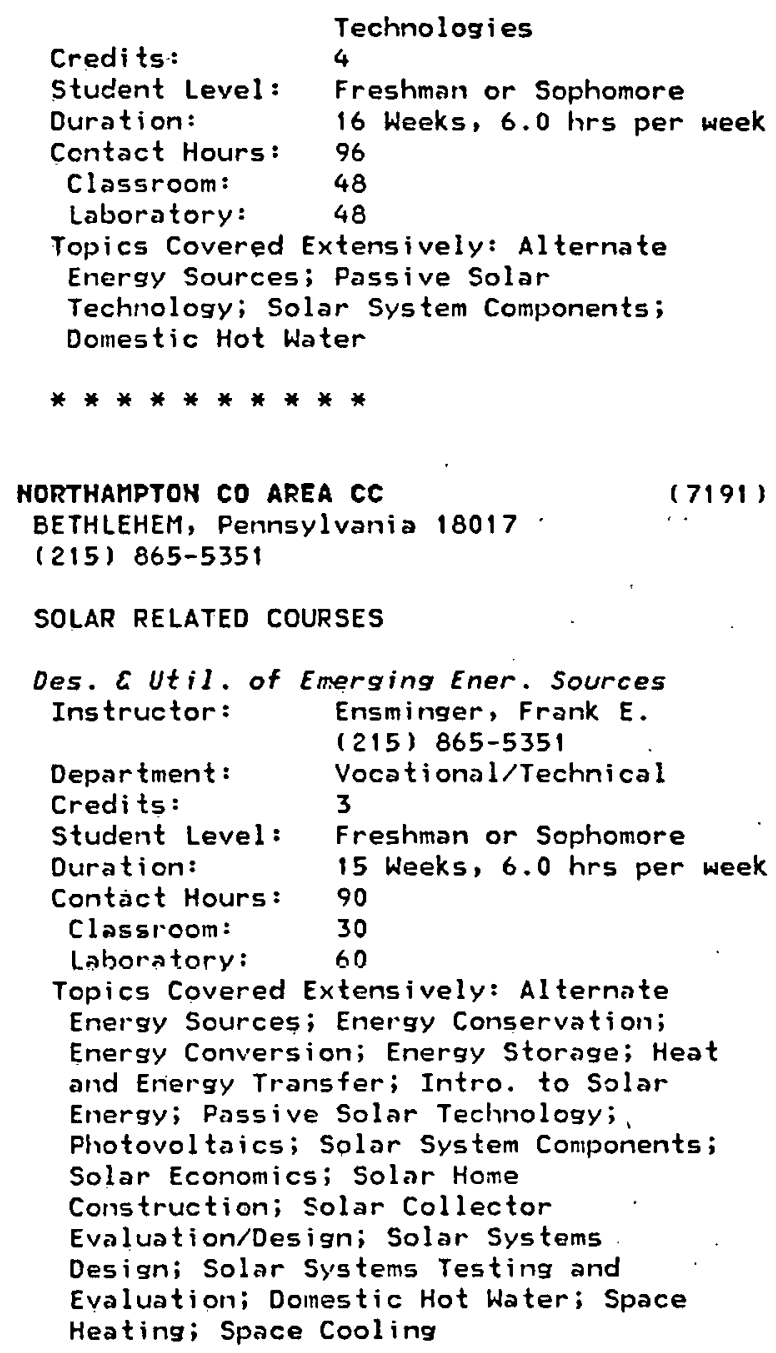

\section{PROGRAMS AND CURRICULA}

Plumbing and Heating

Oegree: Certificate of Applied Arts

Contact: Krause, George $C$. (717) 326-3761

Students Taking or Completing offering: Installer-Residential (Solar System), Plumber, Trade Specialty

\section{SOLAR RELATED COURSES}

Plumbing and Heating

Instructor: Beatty, Franklin P.

Course Number: 842

Department: Building Technology,

Program or

Curriculum: Plumbing and Heating 


$\begin{array}{ll}\text { Student Level: } & \text { Freshman or Sophomore } \\ \text { Duraticn: } & 8 \text { Weeks, } 25.0 \text { hrs per week } \\ \text { Contact Hours: } & 200 \\ \text { Classroom: } & 64 \\ \text { Laboratory: } & 136 \\ \text { Number of Times Taught: } & 4 \\ \text { Averase Enrollment: } & 18\end{array}$

**********
Program or

Curriculum: Energy Technology

Credits:

Stucient Level: High School Graduate

Duration: 12 Weeks, $5.0 \mathrm{hrs}$ per week

Contact Hours: 60

Topics Covered Extensively: Alternate

Energy Sources; Energy Conversion;

Intro. to Solar Energy; Wind Power,

Small Systems

Number of Times Taught: 1

Average Enrollment: $\quad 35$

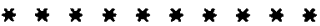

\section{Other Educational Institutions}

NEH ENGLAND FUEL IKSTITUTE

20 Sunmer St. Box 888

Watertown, Pennsylvania 02172

$(90230)$

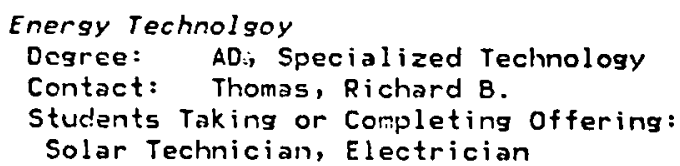

SOLAR RELATED COURSES

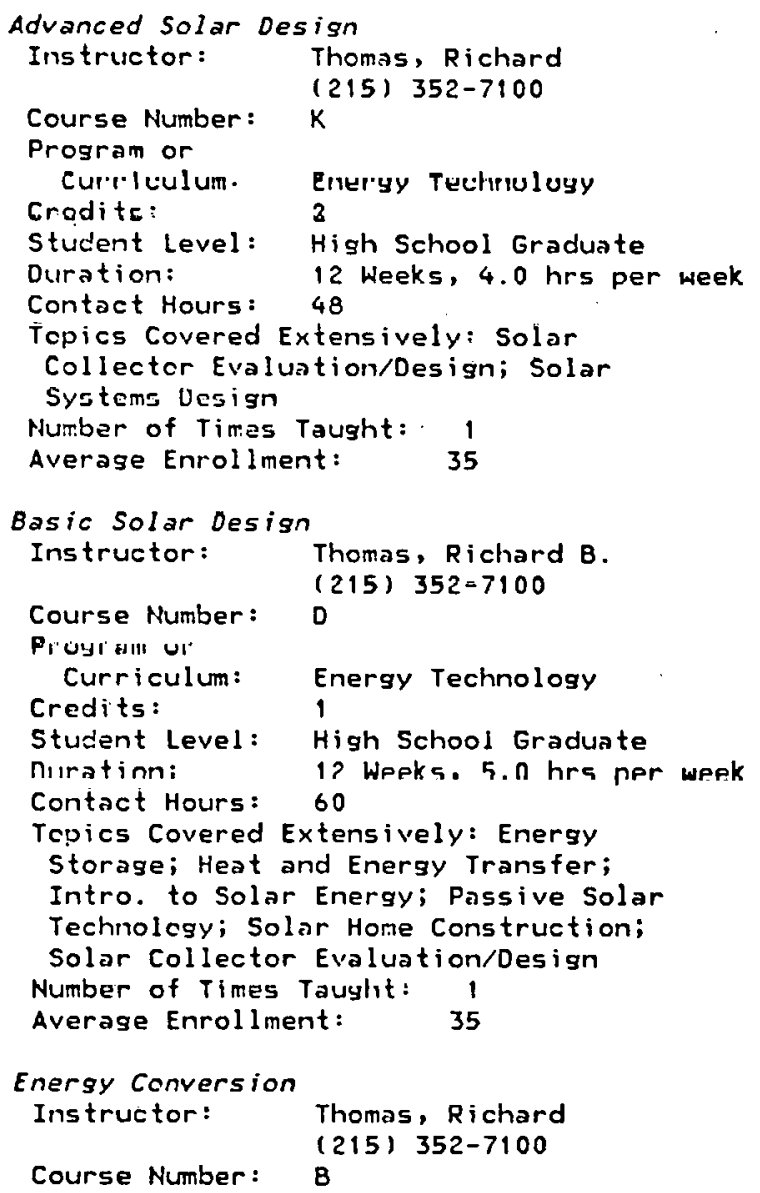

SOLAR RELATED COURSES

* Basic Solar Heating Tech.

Topics Covered Extensively: Space Heating

* Sol ar Installation and Maintenance

Instructor: Tavino, R.t Taylor, R.

(617) 924-1000

Student Level: All levels

Duralluil. 4 Wueks; 40.0 liss pei week

Contact Howra: 160

Classroom: 80

Laboratory: 80

Topics Covered Extensively: Solar System Components; Solar Systems Installation; Solar Systems Maintenance; Domestic Hot Water; Space Heating

***********

PA STATE U CAPITOL CAMPUS

MIŌólétöidid; Fenusylvania 17057

(6814)

(717) 787-7737

SOLAR RELATED COURSES

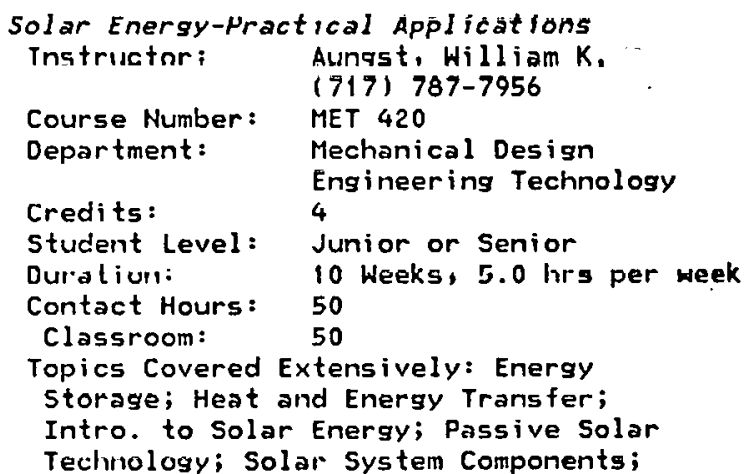


Solar Economics; Solar Collector Evaluation/Design; Solar Systems Design; Domestic Hot Water; Space Heating

Number of Times Taught: 2

Average Enrollment: 33

THE SCHOOL OF LIVIMG

PO BOX 3233

York, Pennsylvania 17402

SOLAR RELATED COURSES

*alternat ive Energy

Topics Covered Extensively: Alternate

Energy Sources

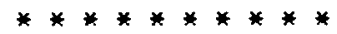

TRIANGLE INSTITUTE OF TECHNOLOGY, INC (90110)

$635 \mathrm{Smithfield} \mathrm{St.}$

Pittsburgh, Pennsylvania 15222

(412) 255-6170

PROGRAMS AND CURRICULA

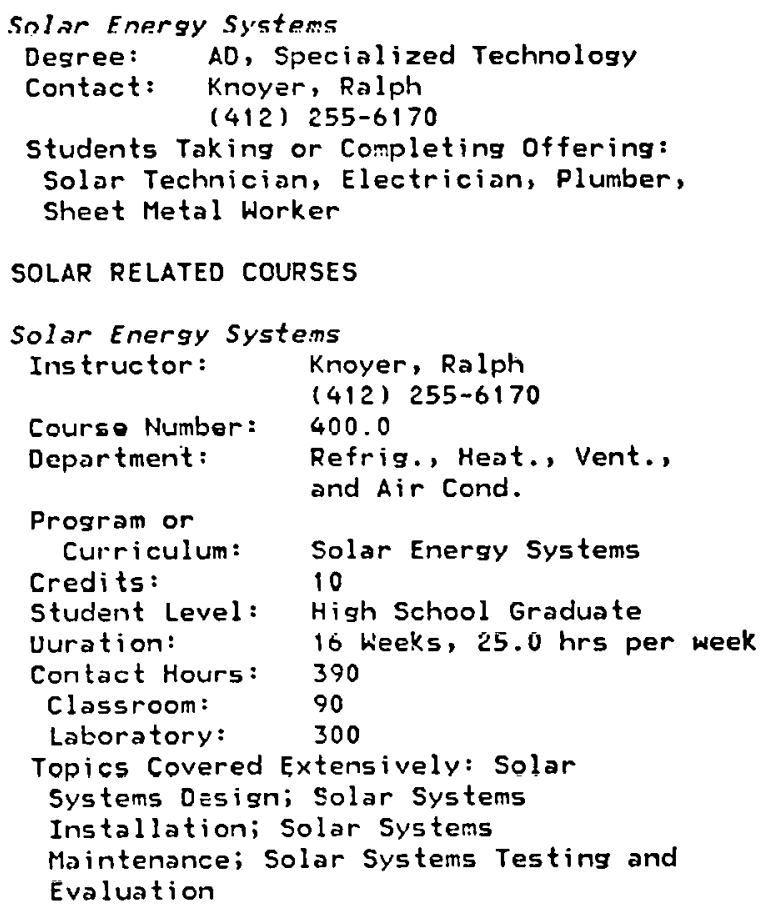




\section{Colleges/Univerșities}

PR MAYAGUEZ,U OF

MAYAGUEZ, Puerto Rico

$(809) 832-4040$

PROGRAMS AND CURRICULA

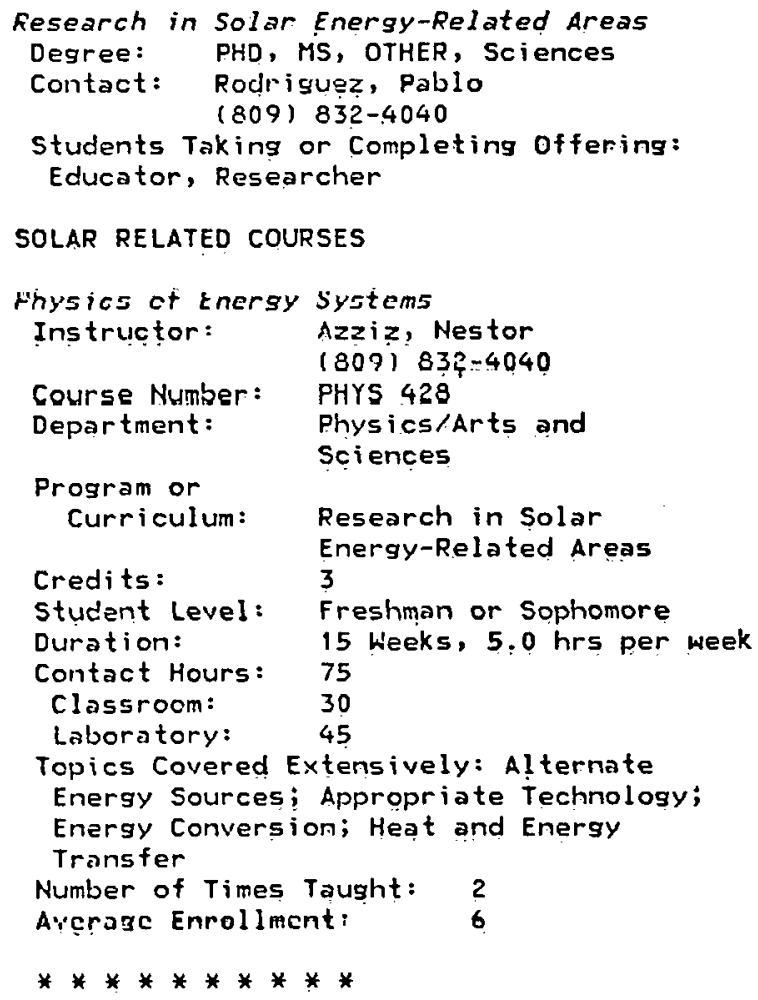




\section{Colleges/Univerșities}

BROHH UHIVERSITY

FROVIDENCE, Rhode Is land

(401) 863-1000

SOLAR RELATED COURSES

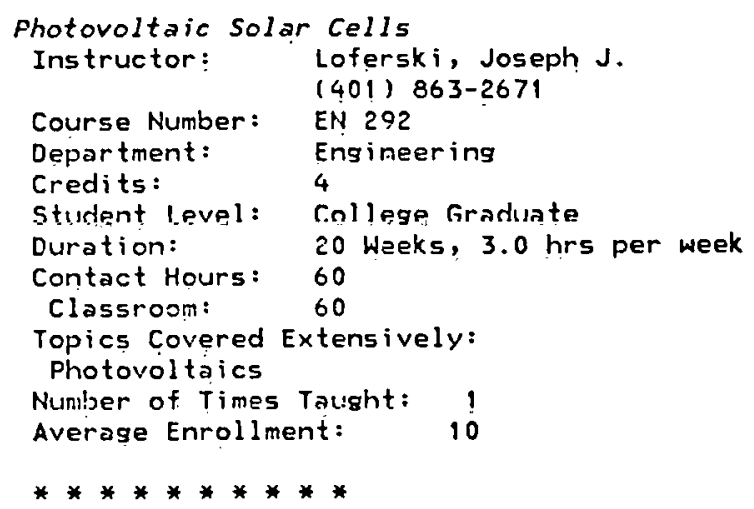

RHODE ISLAKO, U OF $(3414)$ KINGSTON, Rhode Is land (401) 792-1000

\section{SOLAR RELATED COURSES}

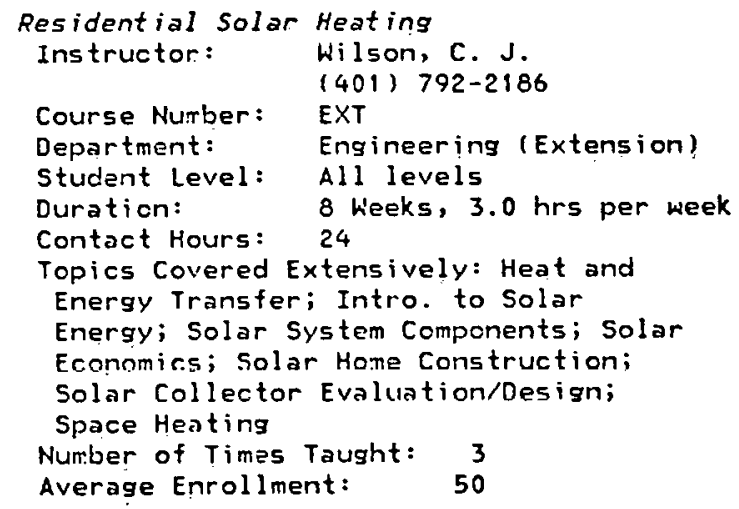

\section{vocational/Tcchnical colleges}

\section{HALL INSTITUTE}

330 Harborside Blvd.

Providence, Rhode Is land 02905

PROGRAMS AIṬ CUPRICULA

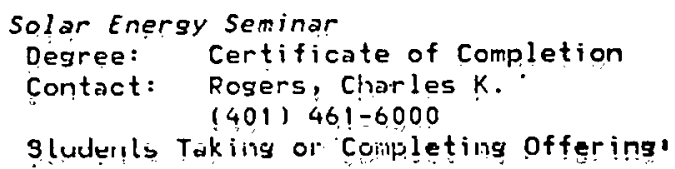

Architect, Educator, Do-it-yourself Homeowner

\section{SOLAR RELATED COURSES}

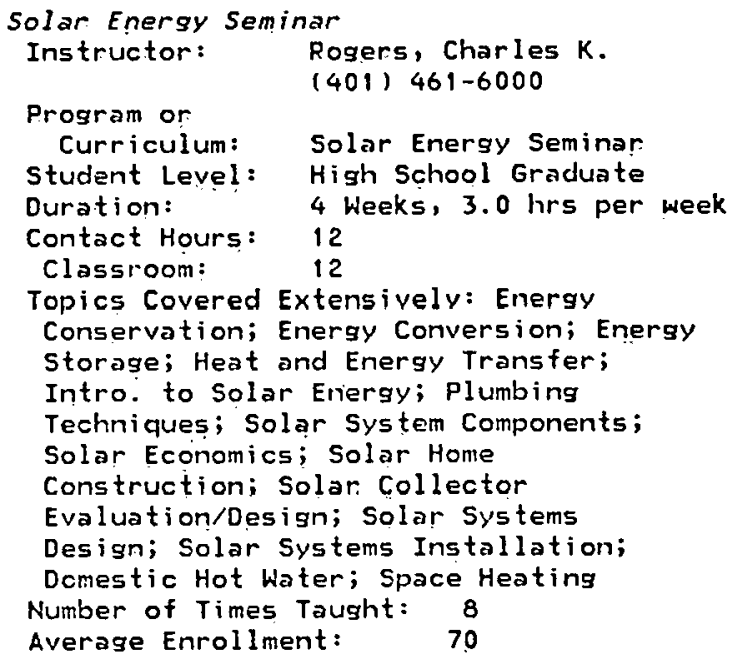




\author{
Colleges/Universities \\ CENTRAL hesleyan COLLEGE \\ (3422) \\ CENTRAL, South Carolina 29630 \\ (803) 639-2453

\section{SOLAR RELATEO COURSES}

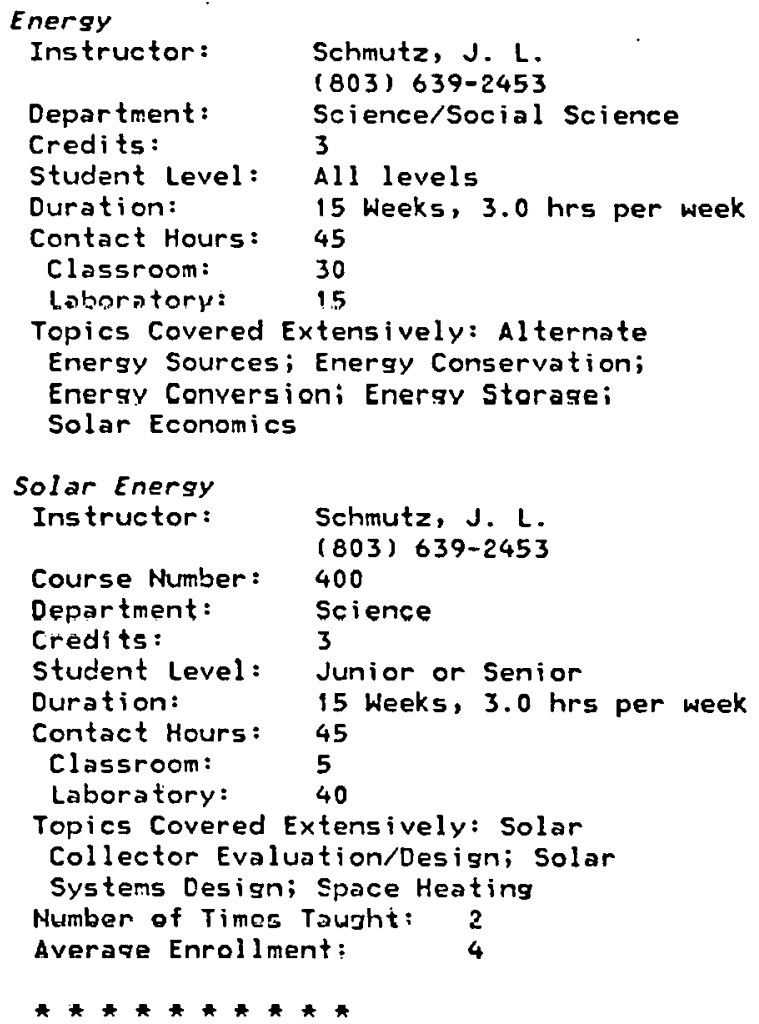

\section{SOLAR RELATED COURSES}

$\begin{array}{ll}\begin{array}{l}\text { Energy Conversion } \\ \text { Instructor: }\end{array} & \text { Lathrop, J. W. } \\ & (803) 656-3371 \\ \text { Course Number: } & 403 \\ \text { Department: } & \text { Electrical and Computer } \\ & \text { Engineering } \\ \text { Program or } & \\ \text { Curriculum: } & \text { Energy Systems } \\ \text { Eredits: } & 3 \\ \text { Student Level: } & \text { Junior or Senior }\end{array}$

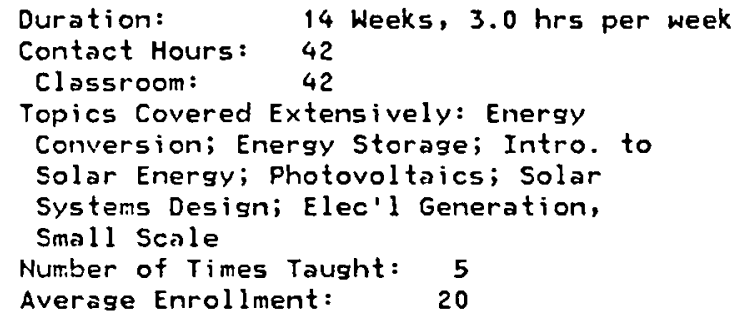

(3448) 
Laboratory: 6

Topics Covered Exterisively: Energy

Storage; Heat and Energy Transfer;

Intro. to Solar Energy; Solar System

Components; Solar Systems Design

Number of Times Tausht: 3 .

Average Enrollment: $\quad 40$

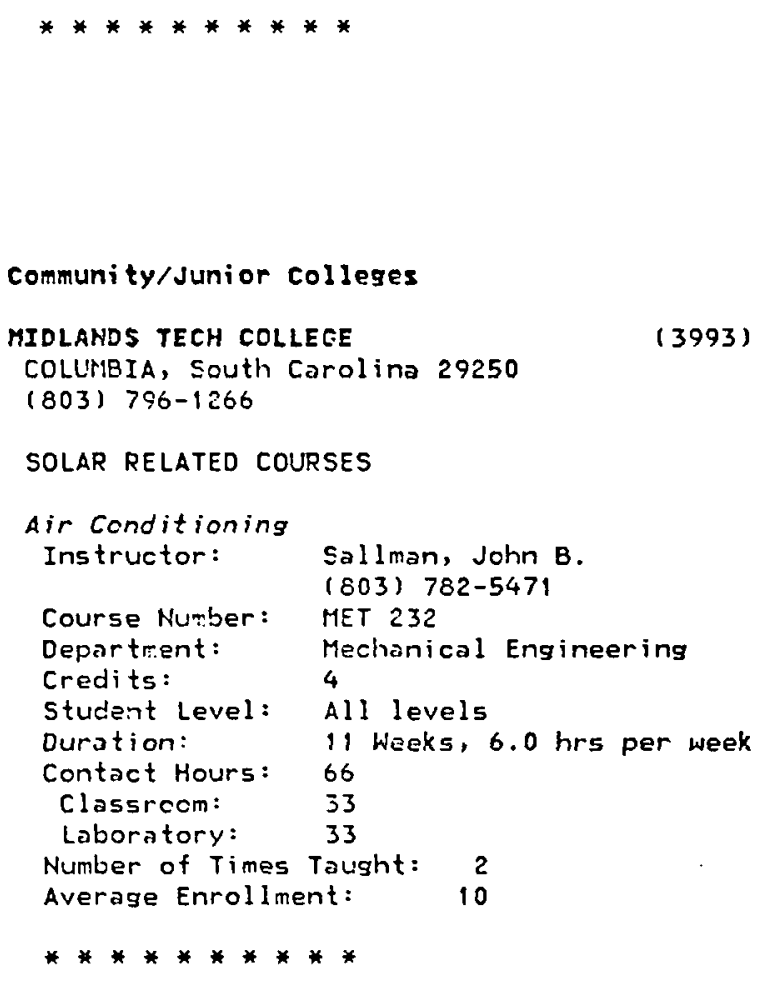

Community/Junior colleges

MIDLANDS TECH COLLEGE

COLLNBIA, South Carolina 29250

(3993)

(803) $796-1266$

SOLAR RELATED COURSES

\section{PIEDMONT TECH COLLEGE}

(3992)

GREENHSOOD, South Carolina 29646

(803) 223-8357

\section{SOLAR RELATED COURSES}

Solar Energy for Climate Control

Instructor: Ledford, John

(803) 223-8357

Course Number: ACR 204

Department: Heating and Air

Credits: Conditioning

Student Level: Freshman or Sophomore

Duration: $\quad 11$ Heeks, $4.0 \mathrm{hrs}$ per week

Contact Hours: 44

Classroom: 44

Topics Covered Extensively: Energy

Conservation; Energy Storage; Intro. to

Solar Energy; Solar System Components;

Solar Collector Evaluatinn/Mesign;

Solar Systems Installation; Solar

Systems Maintenance; Domestic Hot

Water; Space Heating

Number of Times Tausht: 3

Average Enrollment:
TRI-COU:TY TECH COLLEGE

(4926)

PENOLETON, South Carolina 29670

(803) 646-3227

\section{SOLAR RELATED COURSES}

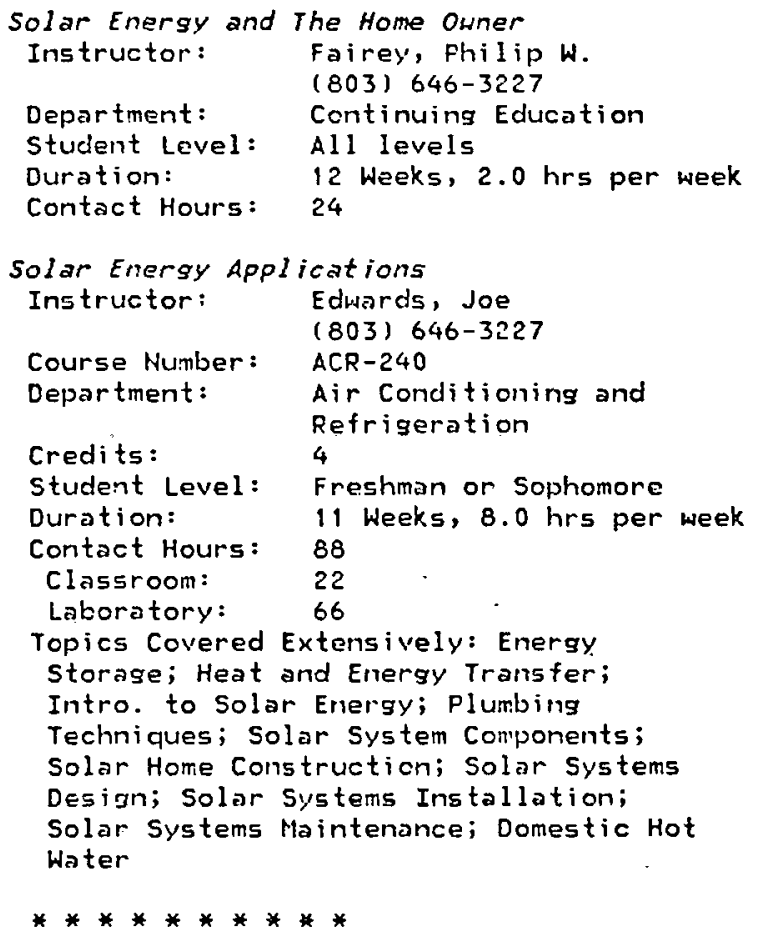

YORK TECHMICAL COLLECE

(3996)

ROCK HILL, South Carolina 29730

(803) 328-3843

PROGRAMS ANO CURRICULA

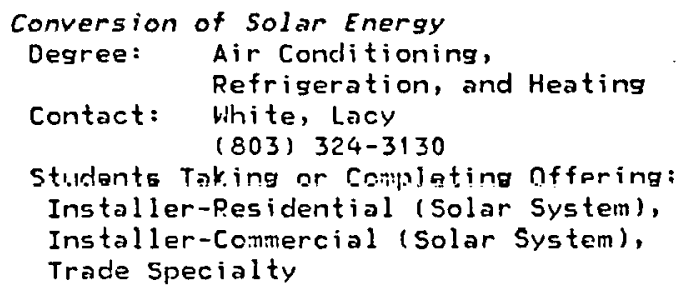

SOLAR RELATED COURSES

$\begin{array}{ll}\begin{array}{l}\text { Conversion of Solar Energy } \\ \text { Instructor: }\end{array} & \text { White, Lacy } \\ & (803) 324-3130 \\ \text { Course Number: } & A C R 204 \\ \text { Department: } & \text { Air Conditioning } \\ \text { Prngram or } & \\ \text { Curriculum: } & \text { Conversion of Solar } \\ & \text { Energy } \\ \text { Credits: } & 4 \\ \text { Student Level: } & \text { High School Graduate } \\ \text { Duration: } & 11 \text { Weeks, } 8.0 \text { hrs per week } \\ \text { Contact Hours: } & 88 \\ \text { Classroom: } & 22 \\ \text { Laboratory: } & 66\end{array}$


Topics Covered Extensively: Plumbing Techniques; Solar Systems Installation; Donestic Hot Water; Space Heating Number of Times Taught: 1 Average Enrollment:

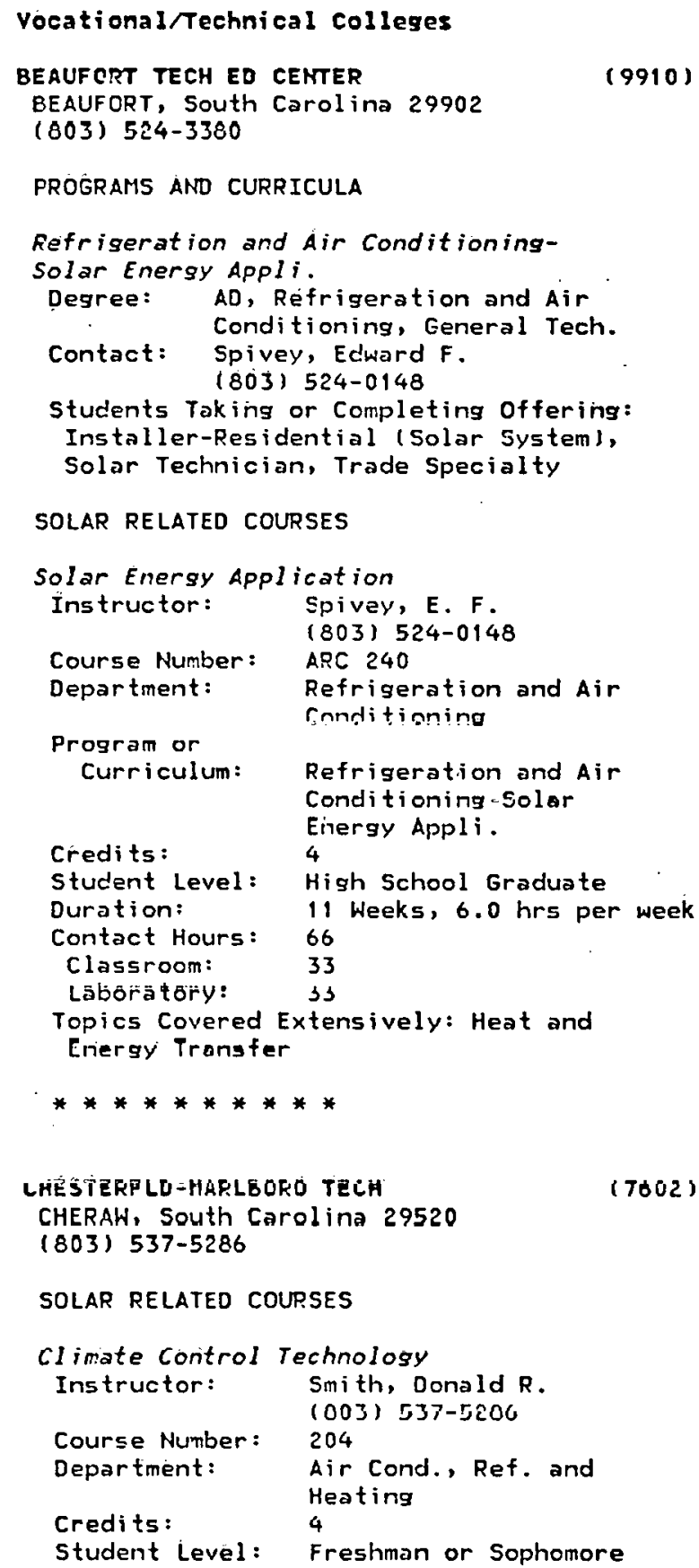

Duration:

Contact Hours:

11 Weeks, 4.0 hrs per week

Classroom:

***********

FLOREHCE DARLINGTON TECH

(3990)

FLORENCE, South Carolina 29502

(803) 662-8151

PROGRAMS AND CURRICULA

Conversion of Solar Energy

Degree: Climate Control

Contact: Jackson, Edward

(803) 662-8151

\section{SOLAR RELATED COURSES}

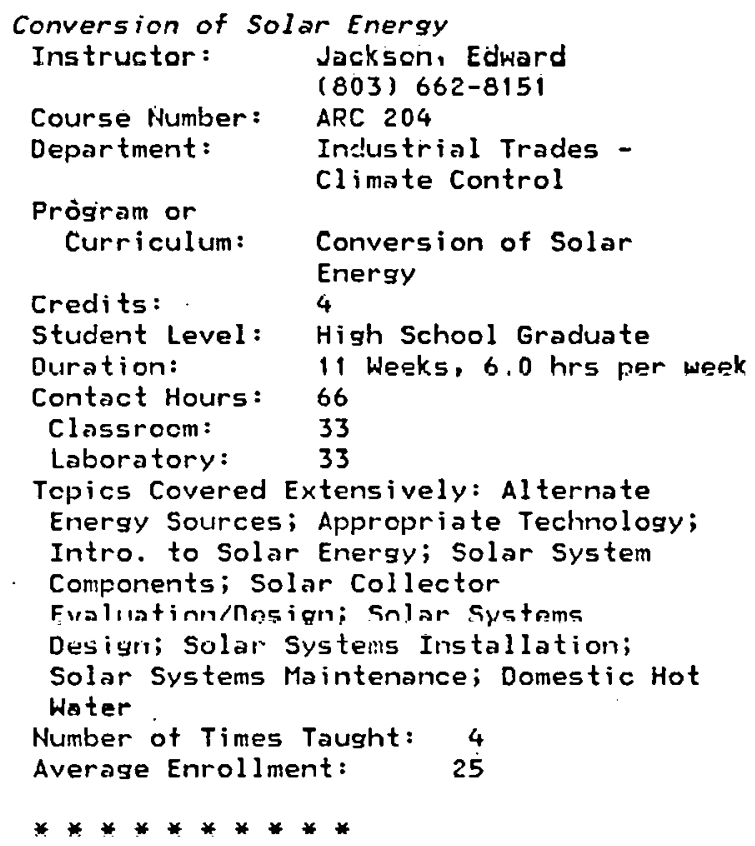


HORRY-GĖORGETOLN TECH C

CONWAY, South Carolina 29526

(803) 347-3186

SOLAR RELATÉ COURSES

* Air Conditión Tech. Coúrses

Department: Air Eond. Tecti:

$* * * * * * * * * *$

SPARTANBURG TECH COLLEGE

$(3994)$

SPARTANBURG, South Carolina 29303

(803) $576-5770$

\section{GOLAR RELATED COURSES}

Basic Solar Heating

Instructor: Watts, John R. (803) 576-5770

Department: Continuing Education

Student Levél: All levels

Duration: 2 Weeks, 4:0 hrs per week

Contact Hours: 8

Clasśrocri : 8

Topics Covered Extensively: Intro. to

Solar Energy; Sollar System Components;

Space Heatting

Number of Times Taughit: 2

Average Enrollment: 35 .

**********

\section{SUITER AREA TECH COLLEGE}

(3995)

SUMTER, South Carolina 29150

(803) 773-9371

SOLAR RELATËD COURSES

* Air Cond. Tech. Coursés

Department: Air Condtioning Tech:
$(4925)$

Number of Times Taught: 4

Averaǵe Enrollment:

17

\section{TRIDEKT TECHIIICAL COLLEGE \\ P O BOX 10367 \\ CHARLESTON, South Carolina 29411 \\ (803) 553-2375}

PROǴRAMS AND CURRICULÁ

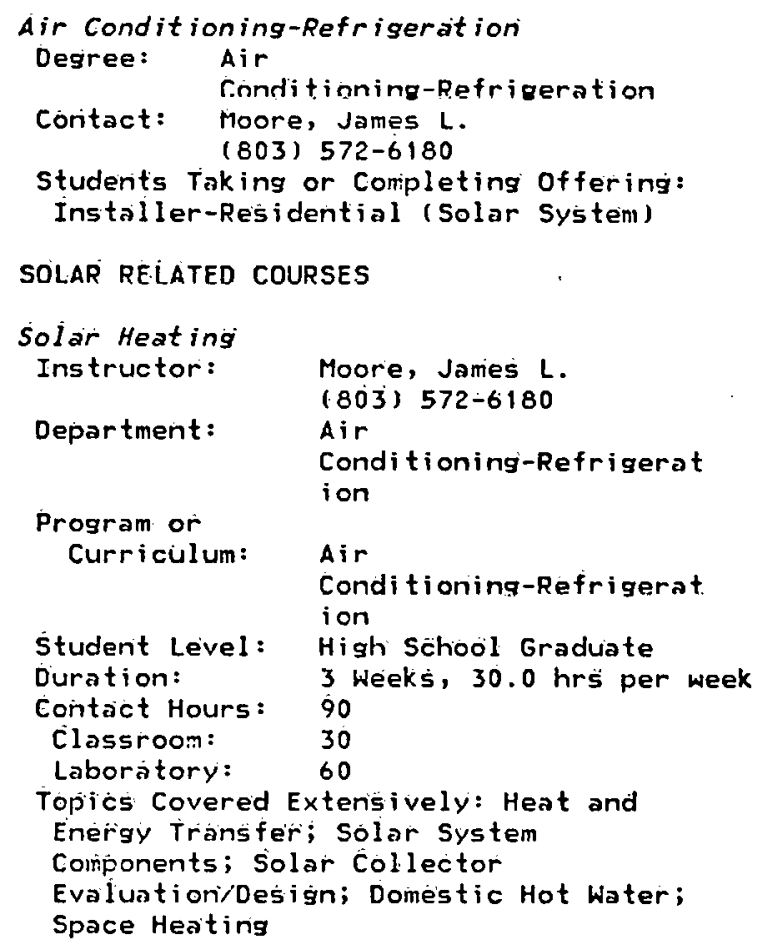

(8818)
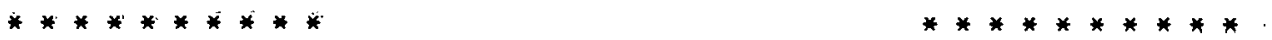


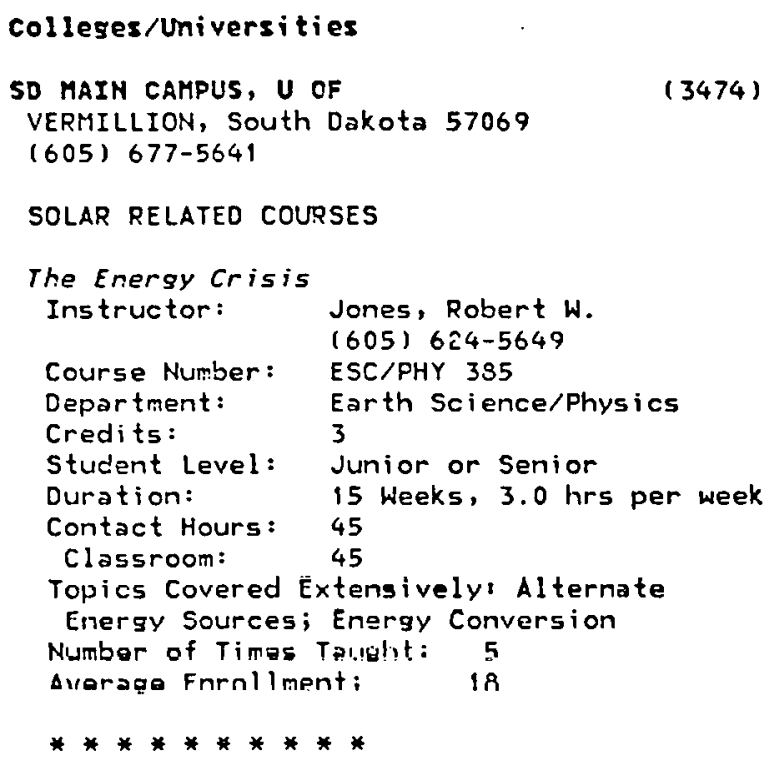

SO STATE UHIVERSITY

RROOKINGS, South Dakota 57007 .

(3471)

(605) 688-4111

\section{SOLAR RELATED COURSES}
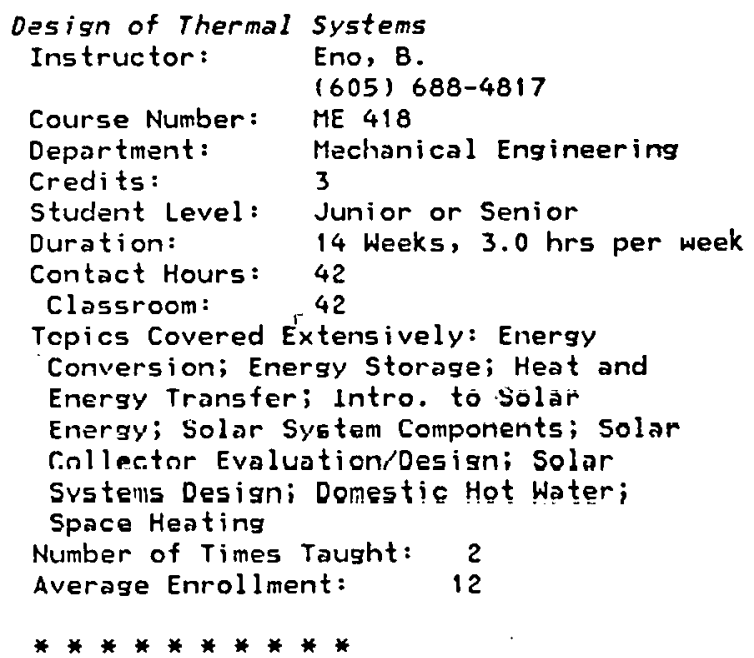


\author{
colleses Universities \\ MEHPHIS STATE UNIVERSITY \\ MEMPHIS, Tennessee 38152 \\ (901) $454-2000$ \\ SOLAR RELATED COURSES \\ Fundamentals of Solar Engineering \\ Instructor: Perry, Edward $H$. \\ (901) 454-2174 \\ Course Number: MECH 4317 \\ Department: Mechanical Engineering \\ Credits: 3 \\ Student Level: Jumior or Senior \\ Duration: 15 Meeks, 3.0 hrs per week \\ Contact Hours: 45 \\ Classroom: 45 \\ Topics Covered Extensively: Heat and \\ Energy Transfer; Intro. to Solar \\ Energy; Solar System Components; Solar \\ Collector Evaluation/Design; Solar \\ Systems Design \\ Number of Times Taught: 4 \\ Average Enrollment: \\ 20 \\ $* * * * * * * * * *$
}

MIODLE TEWH ST UHIVERSITY

MURFREESBORO, Tennessee 37130

(3510)

(615) $898-2300$

SOLAR RELATED COURSES

Solar Home Design

Instructor: Mathis, William H.

(615) $898-2778$

Course Number: $470 \mathrm{C}$

Department: Industrial studies

Credits: 3

Student Level: Junior or Senior

Duration: 16 Weeks, $3.0 \mathrm{hrs}$ per week

Contact Hours: 48

Classroom: $\quad 40$

Laboratory: 8

Topics Covered Extensively: Energy

Storage; Heat and Energy Transfer;

Intro. to Solar Energy; Passive Solar

Technology; Solar System Components;

Solar Home Construction; Space Heating

Number of Times Taught: 2

Average Enrollment: 17

$* * * * * * * * * *$

TEMH AT CHATTANOOGA,U OF

(3529)

CHATTANOOGA, Tennessee 37401

(615) 755-4011

SOLAR RELATED COURSES

Energy Systems

Instructor:

Russel1, Lym D.

(615) 755-4121

Course Number:

586

Department:

Credits?

School of Engineering

Student Level:
Duration:

Contact Hours:

15 Weeks, $4.0 \mathrm{hrs}$ per week 60

Topics Covered Extensively: Energy

Conversion

Number of Times Taught: 2

Average Enrollment:

10

***********

TENKESSEE KNOXVILLE, $U$ OF

KNOXVILLE, Tennessee 37916

(3530)

(615) 974-2591

SOLAR RELATED COURSES

* Solar Energy Utilization

Course Number: EN4740

Department: Mech. C Aero Eng'r

Credits: 3

Student Level: Junior or Senior

Topics Covered Extensivelys Energy

Conversion; Energy Storage; Heat and

Energy Transfer; Intro. to Solar

Energy; Solar Collector

Evaluation/Design; Solar Systems

Design; Solar Systems Testing and

Evaluation; Space Heating; Space

Cooling

**********

TENHESSEE NASHVILLE, $U$ OF

(3533)

NASHVILLE, Tennessee 37203

(615) $251-1111$

SOLAR RELATED COURSES

Energy Conversion systems

Instructor: Knight, Charles V.

(615) 251-1341

Course Number: ME4150

Department: Engineering

Credits: 3

Student Levels Junior or Senior

Duration: 10 Weeks, 3.0 hrs per week

Contact Hours: 30

Classroom: $\quad 30$

Topies Covared Extensively: Alternate Energy Sources; Energy Conservation; Energy Conversion; Energy Storage; Heat and Energy Transfer; Intro. to Solar Energy

Number of Times Taught? 5

Avorage Enrollment:

\section{2}

***********

TENKESSEE TECKNOLOGICAL $U$

( 3523 )

COOKEVITLE, Tennessege 3B5n

(615) $528-3241$

SOLAR RELATED - COURSES

Solar Energy Processes and Systems

Instructor: Hewitt, Jr. Hudy C.

Course Number: ME541 


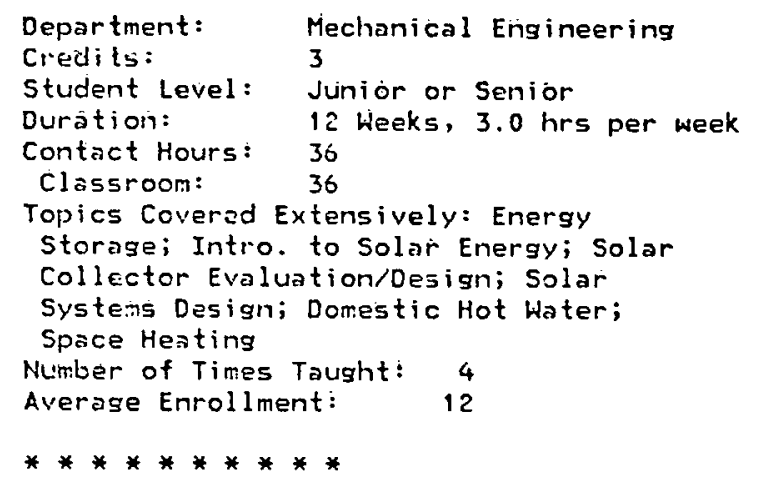

\section{Community/Junior Collezes}

CLEVELARD ST CMTY COLLEGE

(3999)

CLEVELAND. Tennessee 37391

(615) 472-7141

PROCRAMS AHID CURRICULA

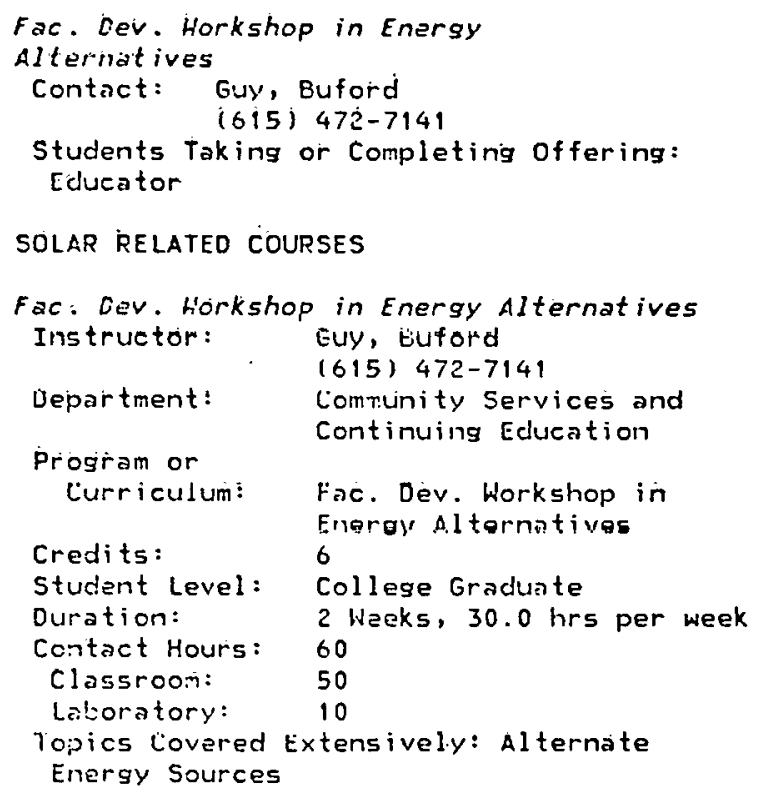

MOTLOW STATE CHTY COLLEGE

(6836)

TULLAHOMA, Tennessee 37388

(615) 455-8511

\section{PROGRAMS AND CURRICULA}

Energy Engineering Technology

Degree: $A D$, Engr. Tech.-Ener. Engr.

Contact: Thornton, otis $B$. (615) 455-8511

students Taking or Completing offering: Installer-Residential ISolar System), Researcher, Solar Technician

\section{SOLAR RELȦTED COURSES}

Solar Energy Applications Lowndes, Richard (615) 455-8511

Course Number: ERG 205

Deportmont: Coroor Educotion

Program or

Curriculum: Energy Engineering

Credits:

Student Level:

Duration: Technology

Contact Hours:

Classroom:

Laboratory: 3

All levels

10 Weeks, 5.0 hrs per week 50

20

30

Topics Covered [x'tensivelyi [nergy

Storage; Heat and Energy Transfer;

Intro. to Solar Energy; Solar System

Components; Solar Economics; Solar Home

Construction; Solar Collector

Evaluation/Design; Solar Systems

Design; Solar Systems Installation;

Solar Systems Testing and Evaluation;

Domestic Hot Hoter; Spoec Henting

Solar Energy Theory

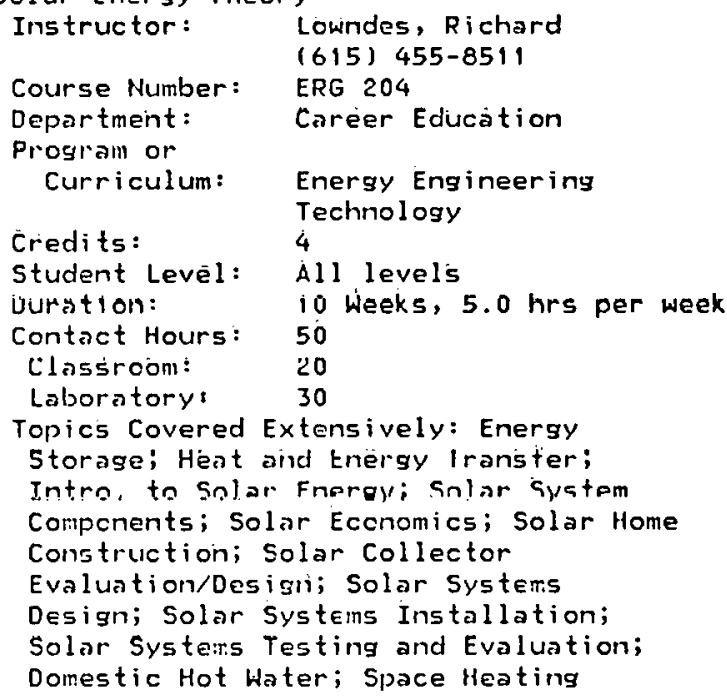

Domestic Hot Water; Space Heating 


\section{Colleses/Universities}

\section{AMERICAN TECHNOLOGICAL U \\ KILLEEN, Texas 76541 \\ (817) 526-1261}

\section{PROGRAMS AND CURRICULA
Energy Manasement Sciences
Desree: MS, Science
Contact: Smith, Rcbin/ Kincel, John (817) 526-1171
Students Taking or Ccmpleting offering: Educator, Researcher, Solar Engineer, Dther

\section{SOLAR RELATED COURSES}

\section{Alternative Energy Sources \\ Department: Mgmnt. \& Business \\ Program or \\ Curriculum: Energy Managrment Sciences \\ Student Level: College Graduate \\ Topics Covered Extensively: Alternate Energy Sources; Biomass Conversion; Energy Conversion; Wind Power, Small Systems}

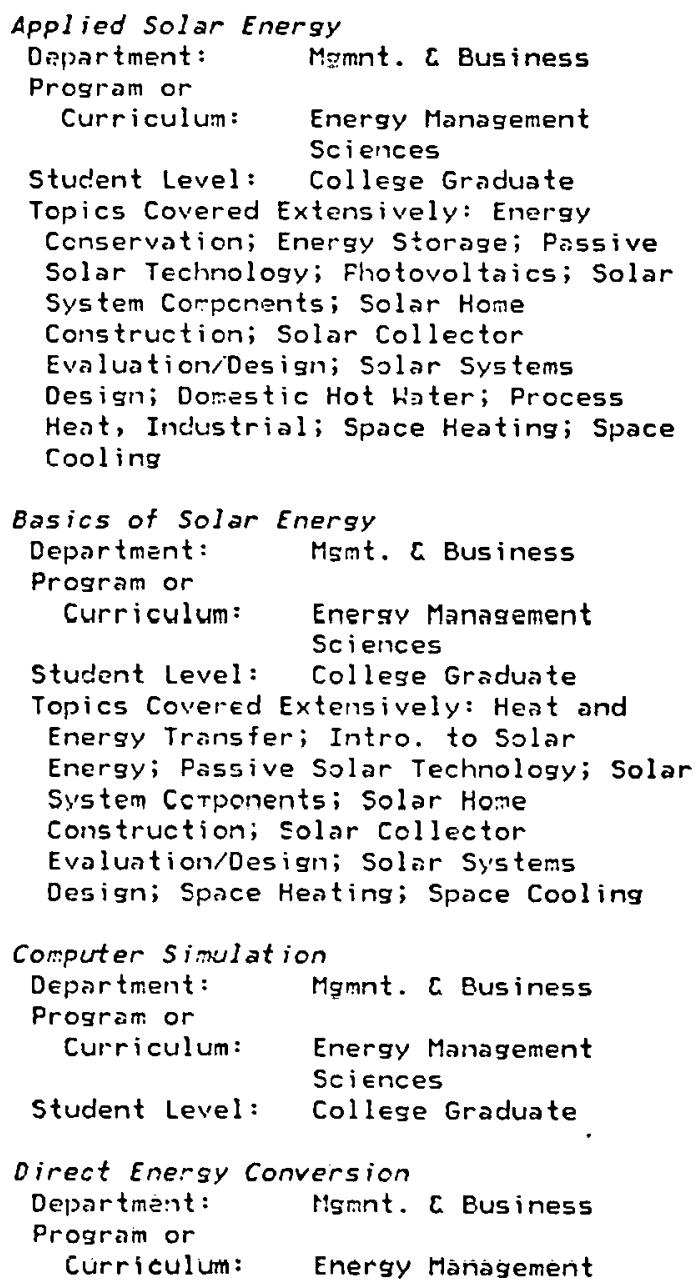

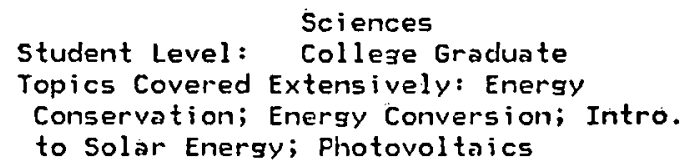




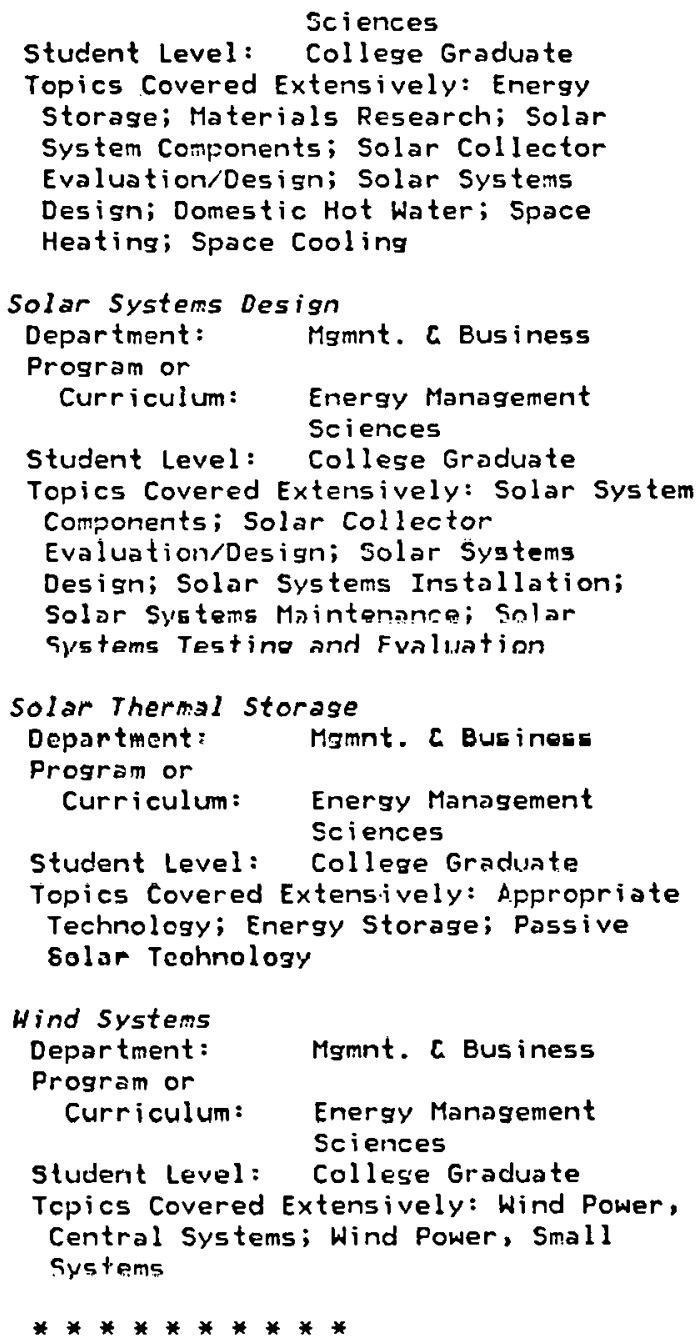

NORTH TEXAS ST UMIVERSITY

(3594)

DENTON, Texas 76203

(817) 788-2026

\section{SOLAR RELATED COURSES}

Energy and our Physical Environment

Instructor: Hehn, Jack

(817) 788-2626

Course Number: PHYS 308

Department: Arts $\varepsilon$ Sciences,

Credits: $\quad 3$

Student Level: Junior or Senior

Duration: $\quad 16$ Weeks, $3.0 \mathrm{hrs}$ per week

Contact Hours: 48

Topics Covered Extensively: Alternate

Energy Sources; Apprepriate Technology; Energy Conservation; Heat and Energy

Transfer; Intro. to Solar Eneray; Marketing/Mlerket Analysis; Passiye Solar Technology; Fhotovoltaics; Solar Energy Policy Development; Solar System Components; Solar Econonics; Salar Home Construction; Solar Collector

Evaluation/Design; Solar Systems Design; Solar Systems Testing and Evaluation; Domestic Hot W'ater; Elec'l Generation, Central; Elec'l Generation, Small Scale; Space Heating; Space

Cooling; Wind Power, rentral Systems; Wind Power, Small Systems Number of Times Taught: 5 Average Enrollment: 21

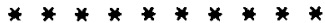

OUR LADY OF LAKE U

SAN ANTOHIO, Texas 78285

(3598)

(512) 434-6711

SOLAR RELATED COURSES

Orner Built Solar Alr Heaters

Instructor: Nawrocki, David

(512) 822-9935

Department: Continuing Education

Studeirt Level: All levels

Duration: 1 Weeks, 5.0 hrs per week

Contact liours! 5

Classroom: 2

Laboratory: 3

***********

RICE UHIVERSITY

HOUSTON, Texas 77001

$(3604)$

(713) 527-8101

PROGRAMS AND CURRICULA

Space Solar Power Researeh
Degree: PhD, MS,
Contact: Freeman, John W.
Students Taking or Completing Offering:
Researcher 


\section{SOLAR RELATED COURSES}

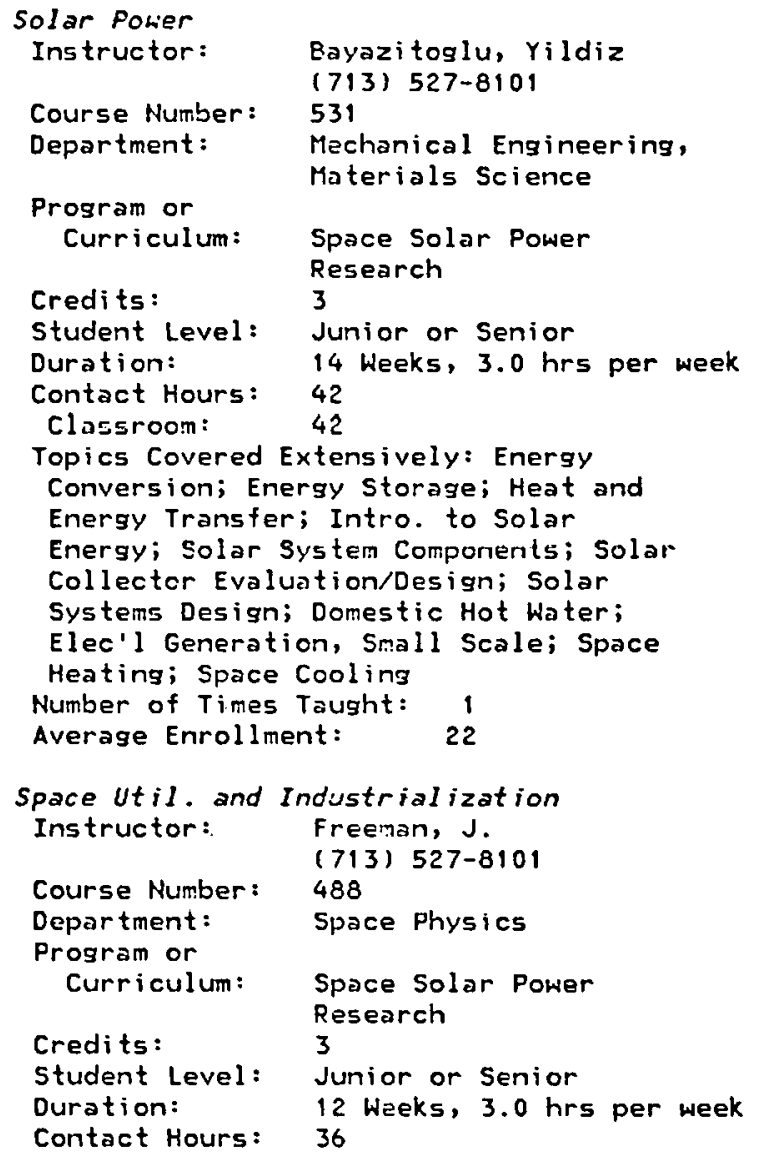

***********

\section{SOUTHERN METH UHIVERSITY \\ DALLAS, Texas 75275}

(214) 692-2000

\section{SOLAR RELATED COURSES}

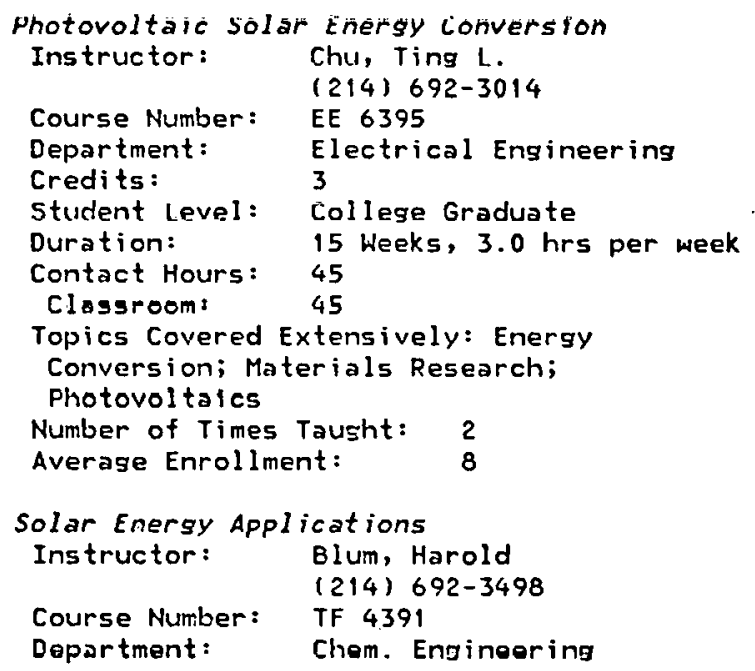

Credits: 3

Student Level: Junior or Senior

Duration: $\quad 5$ Weeks, 9.0 hrs per week

Contact Hours: 45

Classroom: $\quad 39$

Laboratory: 6

Topics Covered Extensively: Intro. to

Solar Energy; Solar System Corponents;

Solar Economics; Solar Systems Design;

Domestic Hot Water; Process Heat,

Agricultural; Process Heat, Industrial;

Space Heating

Number of Times Taught: 3

Averase Enrollment: $\quad 10$

***********

STHHST TEX ST UHIVERSITY

SAll MARCOS, Texas 78666

(512) 245-2111

SOLAR RELATED COURSES

Physical Sciences

Instructor: Michalk, Victor E.

(512) 245-2131

Course Number: PHY $\$ 314$

Department: Physics

Credits: 3

Student Level: Freshman or Sophomore

Duration: 16 Wetks, 4.0 hrs per week

Contact Hours: 64

Classronm: $\quad 48$

Laboratory: 16

Average Enrollment:

30

Physical Sciences

Instructor:

Michalk, Victor E.

(512) 245-2131

Course Number: PHY 1313

Department: Physics

Credits: 3

Student Level: Freshman or Sophomore

Duration: 16 Weeks, $4.0 \mathrm{hrs}$ per week

Contact Hours: 64

Classroom: $\quad 48$

Liboratory: $\quad 16$

Averase Enrollment:

30

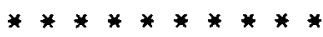

TEXAS ACI UHIVERSITY

(3639)

KINGSVILLE, Texas 78363

(512).595-2111

\section{SOLAR RELATED COURSES}

Environmental Chemistry

Instructor: Beram, J.A.

Cuurbe Numbei: 304

Department: Chemistry

Credits: 3

Duration: 14 Weeks, 3.0 hrs per week

Contact Hours: 42

Humber of Times Taught: 4

Average Enrollment: 15

********** 
TEXAS REM U MAIN CAMFUS

COLLEEE STATION, Texas 77843

$(10366)$

(713) 845-3211

PROGRAMS ATID CURRICULA

\author{
Graduate Program- Undersraduate \\ Frosram \\ Degree: Pho, MS, Mechanical \\ Engineering \\ Contact: Jenkins, Peter $\varepsilon$. \\ (713) 845-1251 \\ Students Taking or Completing Offering: \\ Educator, Researcher, Solar Engineer
}

SOLAR RELATED COURSES

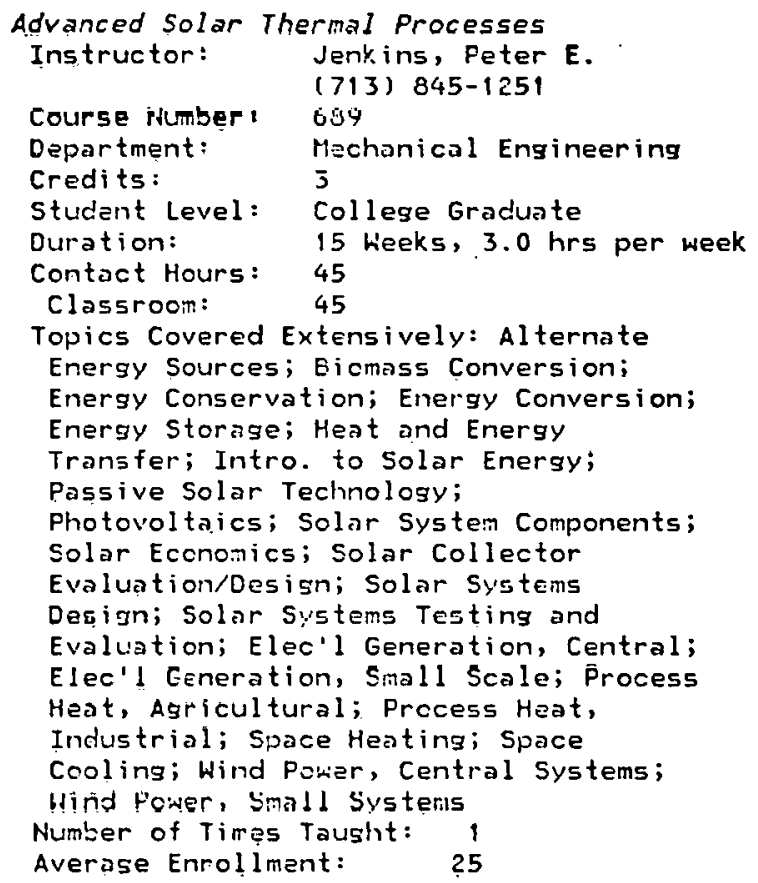

Alternative Architecture

$\begin{array}{ll}\text { Instructor: } & \begin{array}{l}\text { Zweig, Peter } \\ (713) 845-1015\end{array} \\ \text { Course Number: } & 645 \\ \text { Department: } & \text { Architecture } \\ \text { Credits: } & 3 \\ \text { Studant Level: } & \text { College Graduate } \\ \text { Duration: } & 3 \text { Weeks, } 16.0 \mathrm{hrs} \text { per week } \\ \text { Contact Hours: } 48 & \\ \text { Number of Times Taught: } \quad 2 \\ \text { Averase Enrollment: }\end{array}$

Energy; Solar System Components; Solar Economics; Solar Collector

Evaluation/Design; Solar Systems

Design; Solar Systems Testing and

Evaluation; Domestic Hot Water; Elec'l

Generation, Simall Scale; Space Heating;

Space Cooling

Number of Times Taught: 3

Average Enrollment: $\quad 45$

Direct Energy Conversion

Instructor: Jenkins, Peter E.

(713) 845-1251

Course Number: ME 473

Department: Mechanical Engineering

Credits: 3

Student Level: Junior or Senior

nuration: 15 Heeks, $3.0 \mathrm{hre}$ por week

Contact Hours: 45

Classroom: $\quad 45$

Topics Covered Extensively: Alternate

Energy Sources; Biomass Conversion;

Energy Conversion; Energy Storage; Heat

and Energy Transfer; Intro. to Solar

Energy; Fhotovoltaics; Solar System

Corponents; Solar Economics; Solar

Collector Evaluation/Design; Solar

Systems Desisn; Dornestic Hot Water;

Elec'l Generation, Central; Elec'l

Generation, Small Scale; Process Heat,

Industrial; Space Heating; Space

Cooling; Wind Pcwer, Central Systems;

Wind Power, Small Systems

Nun:ber of Times Taught: 3

Average Enrollment: 32

Enersy Optimization Techniques

Instructor: Degelman, Larry 0 .

(713) 845-1015

Course Number: ARCH 689

Department: Architecture

Credits: 3

sturdent Level: Colloge Graduato

Duration: $\quad 15$ Weeks, $3.0 \mathrm{hrs}$ per week

Contact Hours: 45

Classroom: $\quad 40$

Laboratory: 5

Topics Covered Extensively: Appropriate

Technolngy; Fnergy rnngervation; Intro. to Solar Energy; Solar Economics; Solar Colleotor Evaluation'Design; Space. Hesting

Number of Times Taught: 2

Average Enroilment: 8

Environmental rontrol syatama

Instructor: Trost, F.J.

(713) 845-1017

Course Number: APCH 633

Department: Architecture

Credits: 3

Student Level: College Graduate

Duration: 15 Weeks, 3.0 hrs per week

Contact Hours: 45

Clossroom: $\quad 40$

Laboratory: 5

Topics Covered Extensively: Energy

Ccnservation; Heat and Enersy Transfer;

Intro. to Solar Energy; Solar

Economics; Space Heating 
Average Enrolliment:

15

\section{TEXAS AT ARLINGTON, $U$ OF \\ ARLINGTON, Texas 76019}

(3656)

(817) 273-2011

\section{SOLAR RELATED COURSES}

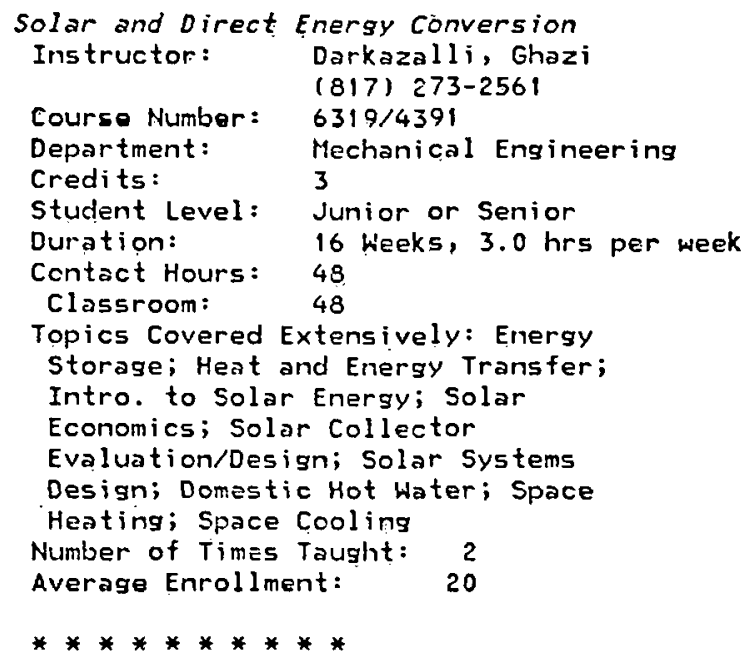

AUSTIN, Texas 78712

(512) $471-3434$

\section{PROGRAMS AND CURRICULA}

Solar-related Courses

Degree: $\mathrm{Ph}, \mathrm{D}, \mathrm{MA}, \mathrm{BA}$, Architecture related

Contact: Arumi-Noe, Francisco (512) 471-4911

Students Taking or Completing offering: Architect

\section{SOLAR RELATED COURSES}

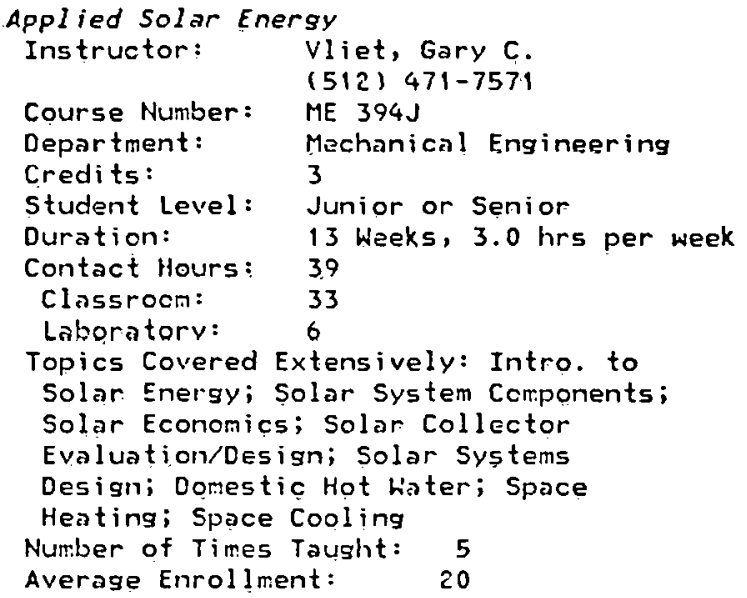

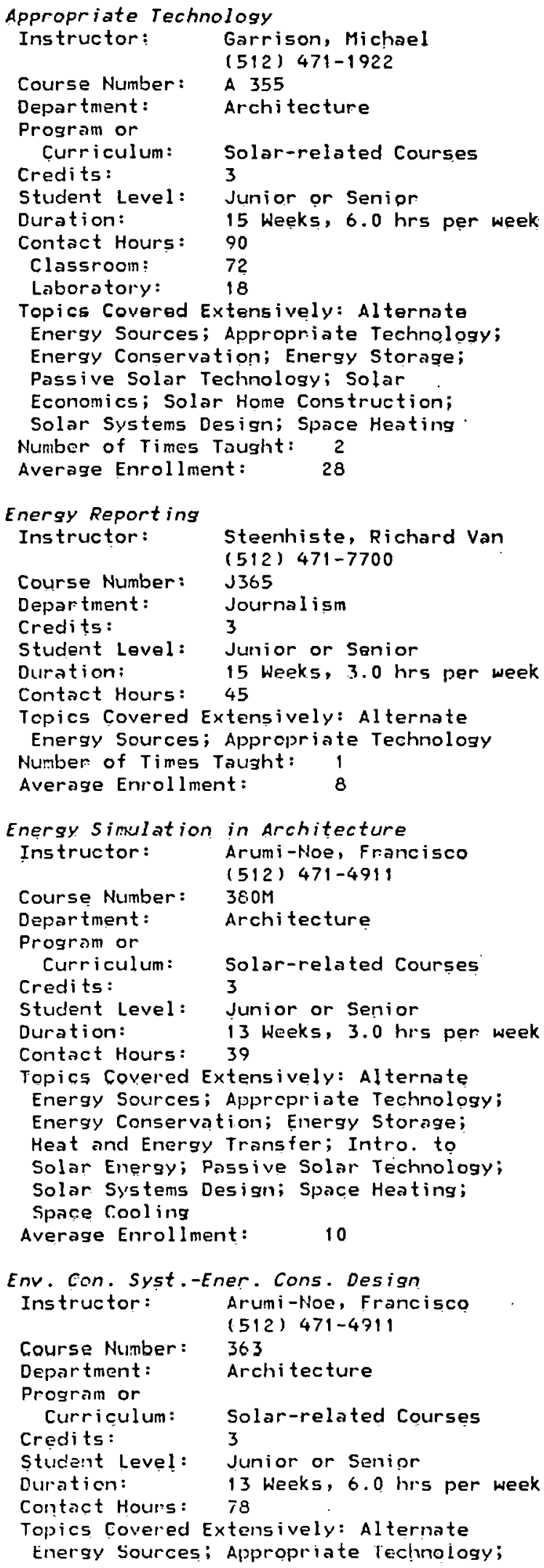


Energy Conservation: Energy Conversion; Energy Storage; Heat and Energy Transfer; Intro. to Solar Energy; Materials Research; Passive Solar Technology; Fhotovoltaics; Solar System Components; Solar Economics; Solar Home Construction; Solar Collector Evaluation/Design; Solar Systems Design; Solar Systems Installation; Domestic Hot Hater; Swimning Pool Heating; Elec'l Generation, Central; Space Heating; Space Cooling; Wind Power, Central Systems; Wind Power, Small Systems

Average Enrollment:

Sol. Heat., Cool., Ener. Cons.-Bldgs.

Instructor: Vliet, G.t Jones, $J$. (512) $471-7571$

Department: Continuing, Fnginepring Education

Student Level: All levels

Duration: I Weeks, $26.0 \mathrm{hrs}$ per week

Contact Hours: 26

Classrocm: 26

Topics Covered Extensively: Intro. to Solar Energy; Solar System Corponents; Solar Economics; Solar Collector Evaluation/Design; Solar Systems Desisn; Domestic Hot Water; Space Heating; Space Cooling Nurtber of Times Taught: Average Enrollment: 4 15

\section{Solar Energy}

Instructor: Amstead, R. H.

(512) 471-1331

Counse Plumber: ME $378 \mathrm{M}$

Department: MF

Credits: 3

Studẹnt Level: Junior or Senior

Duration: 17 Weeks, $3.0 \mathrm{hrs}$ per week Contact Hours: 51

Topics Covered Extensively: Alternate

Energy Sources; Energy Conservation;

Intro. to Solar Energy

Number of Times Taught: 3

Average Enrollment: $\quad 25$

\begin{tabular}{|c|c|}
\hline $\begin{array}{l}\text { Solar Technology } \\
\text { Instructor: }\end{array}$ & $\begin{array}{l}\text { Assessment } \\
\text { Blissett, Marlan } \\
(512) 471-4962 \\
\text { PA } 552\end{array}$ \\
\hline $\begin{array}{l}\text { Course Number: } \\
\text { Department: }\end{array}$ & $\begin{array}{l}\text { PA } 852 \text { A } \\
\text { LBJ School of Public } \\
\text { Affairs }\end{array}$ \\
\hline $\begin{array}{l}\text { Credits: } \\
\text { Student Level: } \\
\text { Duration: } \\
\text { Contact Hours: } \\
\text { Classroom: } \\
\text { Laboratory: }\end{array}$ & $\begin{array}{l}8 \\
\text { Collese Graduate. } \\
32 \text { Weeks, } 4.0 \text { hrs per week } \\
123 \\
100 \\
18\end{array}$ \\
\hline $\begin{array}{l}\text { Tcpics Covered } \\
\text { Technology; Ene } \\
\text { to Solar Energs } \\
\text { Analy'sis; Pass } \\
\text { Solar Eccnomics } \\
\text { Construction; } \\
\text { Dcrestic hot Ha } \\
\text { Asricultural; } \\
\text { Space Heating }\end{array}$ & $\begin{array}{l}\text { Extensively: Appropriate } \\
\text { ergy Conservation; Intro. } \\
\text { y; Marketing/Market } \\
\text { ive Solar Technology; } \\
\text { s; Solar Home } \\
\text { Solar Law/Legislation; } \\
\text { ater; Process Heat, } \\
\text { Process Heat, Industrial; }\end{array}$ \\
\hline
\end{tabular}

Average Enrollment: $\quad 20$

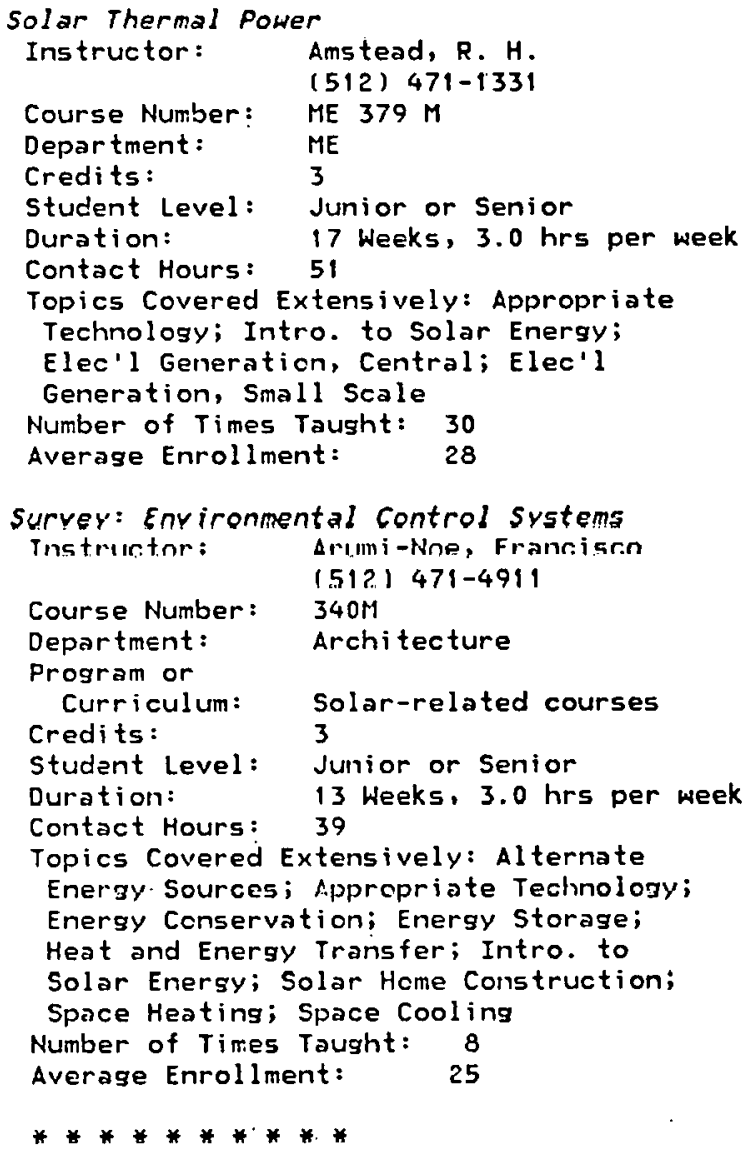


Topics Covered Extensively: Energy Conversion; Enersy Storage; Heat and Energy Transfer; Solar Economics; Solar

- Systems Desisn; Elec'l Generation, Central; Elec'l Generation, Small Scale; Space Heating; Space Cooling Number of Tires Tausht: 3

Average Enrollment:

8

Energy

Instructor: Rapp, D./ Fenyves, E. (214) 690-2970

Course Number: ES5354

Department: NSM'ES

Program or Curriculum: Sol. Ener. in Environ.

Credits: Studs.

3 3

Student Level: College Graduate

Duration: 15 heeks, 3.0 hrs per week

Contact Hours: 45

Classroom: 45

Topics Covered Extensively: Alternate Energy Sources; Appropriate Technology; Energy Conservation; Energy Conversion Number of Times Taught: 1

Average Enrollment: 30

Energy Consumption-Resources \& Impact Instructor: Fenyves, $E$.

Course Nurber: ENSC5320

Department: HSM/ES

Program or

Curriculum: Sol. Ener. in Environ.

Credits: 3 Studs.

Student Level: College Graduate

Duration: 15 Weaks, 3.0 hrs per week

Contact Hours: 45

Classrcom: $\quad 45$

Topics Covered Extensively: Alternate Energy Sources; Appropriate Technology;

Energy Conservation; Energy Conversion;

Intro. to Solar Energy

Number of Times Taught: 5

Average Enrollment:

20

Solar Energy

Instructor:

Course Number:

Rapp, 0 .

(214) 690-2474

Department:

ENHEG6347

Program or

Curriculum:

ISECM/ES

Sol. Ener. in Environ. Studs.

Credits: 3

Student Level: College Graduate

Duration: 15 heeks, $3.0 \mathrm{hrs}$ per week

Contact Hours: 45

Classroom:

45

Topics Covered Extensively: Intro. to

Solar Energy; Solar Systelts Design;

Domestic Hot Water; Space Heating

Number of Times Tausht: 4

Averase Enroliment: $\quad 15$

Solar Enersy Laboratory

Instructor: Rapp, D.

(214) 690-2970

Course Number: ENEG6356

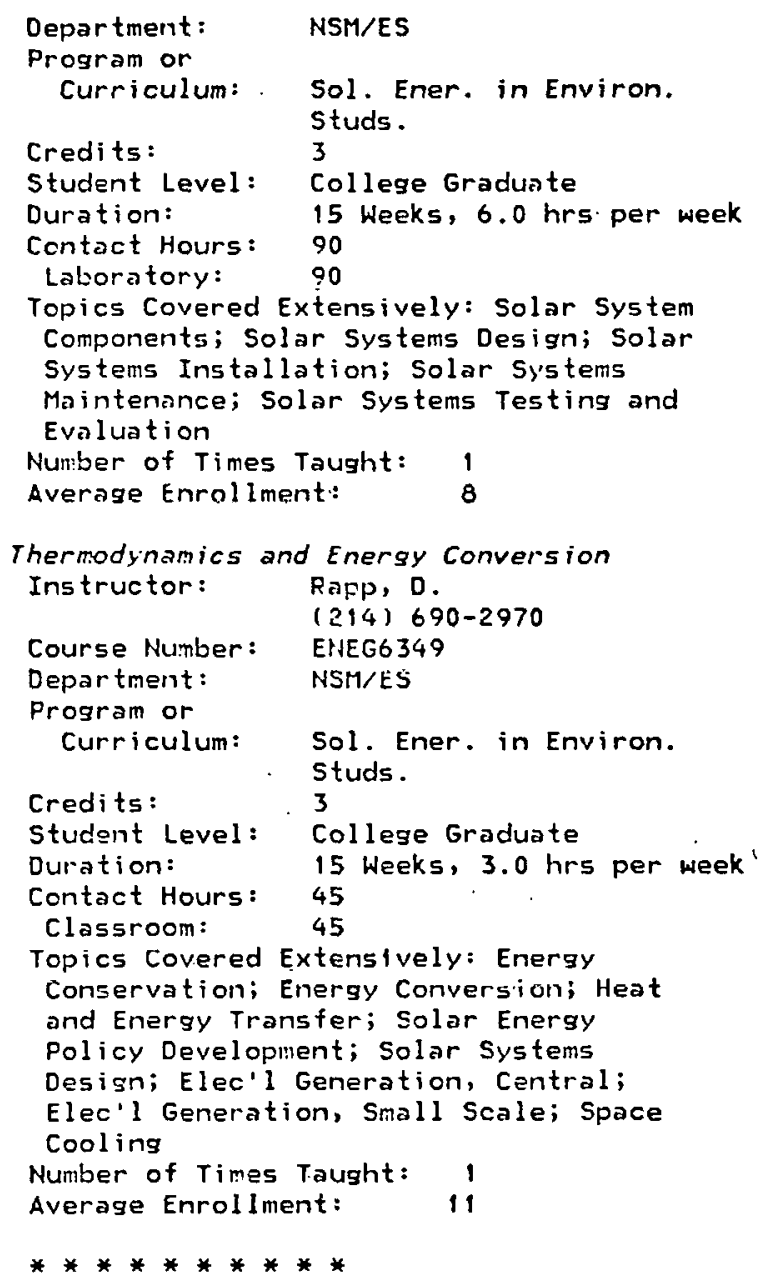

TEXAS AT EL PASO, U OF

EL PASO, Texas 79968

(915) 747-5000

PROGRAMS AND CURRICULA

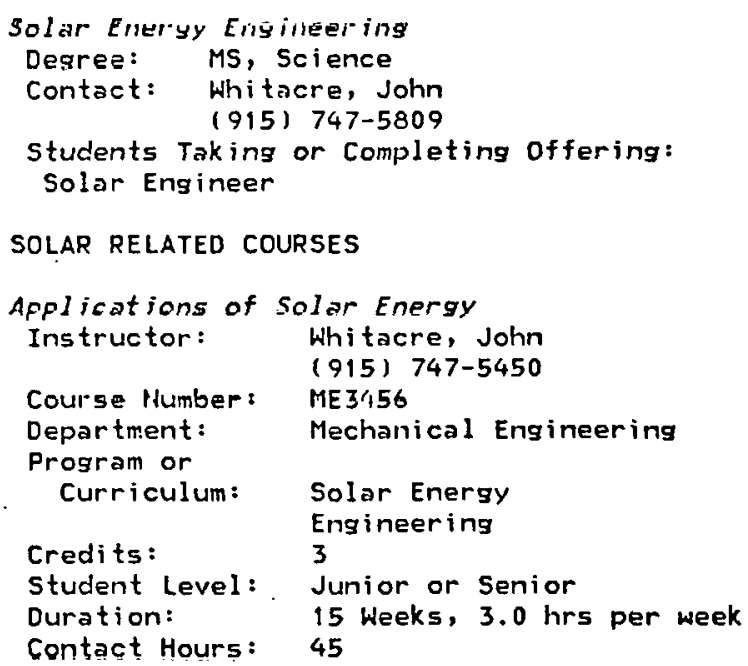


Classroom:

45

Topics Covered Extensively: Heat and Energy Transfer; Solar System

Corponents; Solar Systems Design; Space

Heating; Space Cooling

$* * * * * * * * * *$

TEXAS CHRISTIAAN U

FORT HCRTH, TEXas 76129

(3636)

(817) 926-2461

\section{SOLAR RELATED COURSES}

Energy and the future

Instructor:

Course Number:

Quarles, C. A.

(817) $921-7375$

Department:

6413

Physics

Credits I

3

Studant terel, All levels

Duration:
Contact Hours: 45 heeks, 3.0 hrs per week

Topics Covered Extensively: Alternate

Energy Sources; Energy Conservation;

Solar Econcmics; Solar Collector

Evaluationidesion

Number of Times Tought: 2

Average Enrollment:

40

× $\times$ ×******

TEXAS TECH UNIVERSITY

LUBBOCK, Texas 79409

(806) $742-2011$

PROGRAHS ARIO LURKILULA

Interdisc ipl inary Engineer ing

Degree: PhD, Philosophy-Inter. Engin.

Contact: Gully, A.J.

(8.06) $742-3456$

students Takin̄s or Completing Offering:

Researcher, Solar Engineer

\section{SOLAR RELATED COURSES}

Energy and Housing

Instructor:

Kiesling, E. H. (896) 742-3472

Department: Cat., Ener. Res. and

Program or

Curriculum: Interdisciplinary

Engineering

Student Lovol: Collese Graduote

Duration: i heeks, 32.0 hrs per week

Contact Hours: 32

Classroom: $\quad 32$

Topics Covered Extensively: Energy

Conservation; Intro. to Solar Energy;

Solar Sy'stem Components; Solar

ECsiomics; Solar Hon:a Construction;

Solar Systems Desisn; Domestic Hot

Water; Space Heating
Engin. Aspects-Res., Frocess., Util.

Instructor: Parker, Harry $W$.

(806) 742-3553

Course Number: CH.E. 4333

Department: Chemical Engineering

Prosram or

Curriculum: Interdisciplinary

Credits: 3

Student Level: Junior or Senior

Duration: 15 Heeks, 3.0 hrs per week

Contact Hours: 45

Classroom: $\quad 45$

Topics Covered Extensively: Alternate

Energy Sources; Biomass Conversion

Number of Times Taught: 4

Average Enroliment: is

Housing and Energy as Consumer Issues

Instructor: Mckown, Cora

Course Number: 518

Department: Home Eco., Fam Mgmnt.,

Program or Hous. Cons. Sci.

Curriculum: Interdisciplinary

Credits: 3

Student Level: College Graduate

Duration: 1 Weeks, 45.0 hrs per week

Contact Hours: 45

Topics Eovered Extensively: Energy

Conservation; Marketing/Market analysis

Number of Times Taught: 1

Average Enrollment:

25

Interdis. Appr.-Res, Energy Ed.

Instructor: Mekown, Cora

$(806) 742-3153$

Course Number: 1518

Department: Home Eco., Fam. Mamnt.,

Program or

Curriculum: Interdisciplinary

Credits Engineering

Student Level: Junior or Senior

Duratinn: 3 Heoks, 15.0 hrs per week Contact Hours: 45

Tepice Covercd Eulursively Alternate Energy Sources; Apprepriate Teehnolosy;

Energy Conservation: Tutrn. to solar Energy; Marketing/Market Analysis;

Solar System Components; Solar

Feonnmics; Solor Herio Confotruction;

Solar Low/Legislation; Solar Systems

Design; Solsr systems Installation

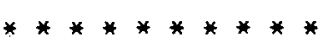


TRINIT UNIVERSITY

(3647)

SAN ANTONIO, Texas 78284

(512) 736-7011

\section{PROGRAMS AND CURRICULA}

Solar Energy Graduate frog.

Degree: MS, Sci.-Appld. Sol. Ener. or Sol. Ener. Stud.

Contact: Clark, Eusene

(512) 736-7504

Students Taking or Completing Offering:

Researcher, Solar Engineer, Solar

Technician

\section{SOLAR RELATED COURSES}

Economic Analysis of Energy and Capital Projects

Instructor:

Dorner, Fred $\mathrm{H}$.

(512) 736-7238

BSH 390

Course Humber

Business Administration

Program or

Curriculum: Solar Energy Graduate Prog.

Credits: 3

Studant Level: College Graduate

Duration: 15 Heeks, 3.0 hrs per week 45

Classroom: 45

Topics Covered Extensively: Alternate

Energy Sources; Solar Economics; Elec'l

Generation, Central; Space Cooling

Number of Times Tausht: 1

Average Enrollment:

18

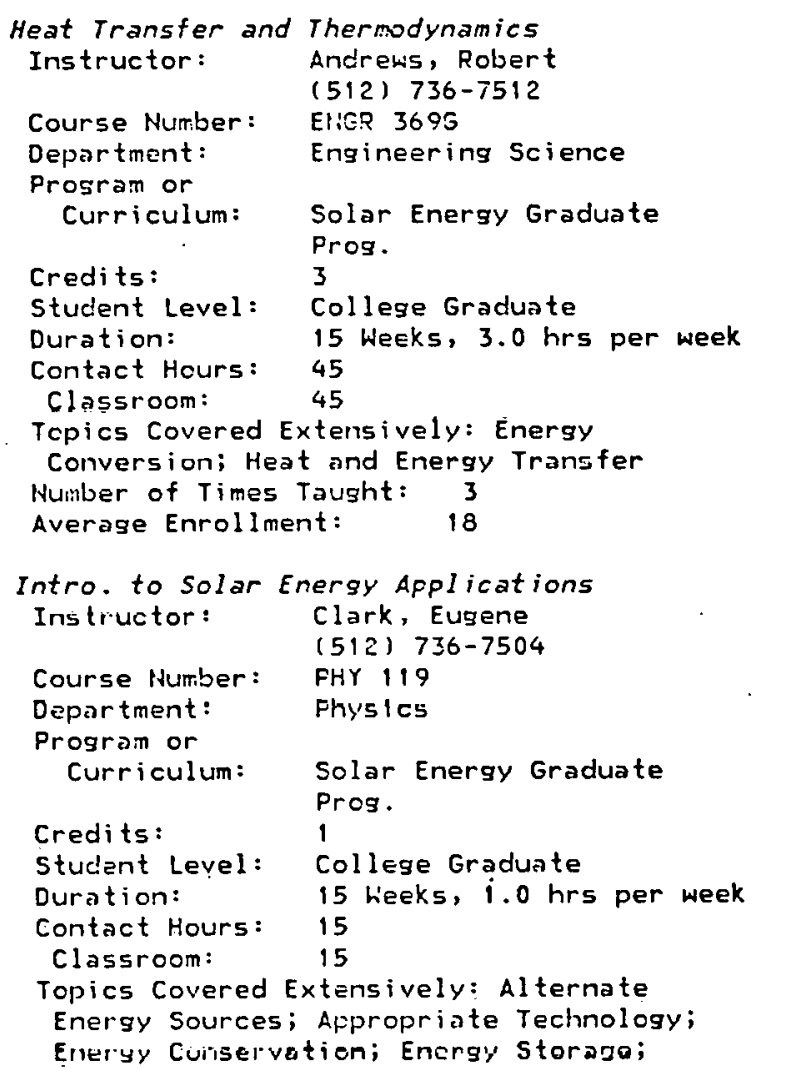

Intro. to Solar Energy; Passive Solar: Technology; Solar System Components;

Solar Economics; Solar Collector

Evaluation/Design; Domestic Hot Water;

Space Heating

Number of Times Taught: 3

Average Enrollment: $\quad 15$

Modeling and Computer Analysis

Instructor: Treat, C. H.

(512) 736-7512

Course Number: EHGR 3835

Department: Engineering Science

Program or

Curriculum: Solar Energy Graduate

Pros.

Credits: 3

Student Level: College Graduate

Duration: 15 Weeks, 3.0 hrs per week

Curitact llours: 45

Classroom: $\quad 45$

Topics Covered Extensively: Elec'I

Generation, Central; Elec'l Generation,

Small Scale; Process Heat, Inclustrial;

Space Cooling

Number of Times Taught: 2

Average Enrollment: $\quad 15$

Fhotovoltaics

Instructor: Loxsom, Fred

(512) 736-7421

Course Number: PHYS 3506

Department: Physics

Program or

Curriculum: Solar Energy Graduate

Credits: 3

Student Level: College Graduate

Duration: 15 Weeks, 3.0 hrs per week

Contact Hours: 45

Classroom: $\quad 45$

Topics Covered Extensively: Energy

Conversion; Photovoltaics; Solar

Systems Design; Elec'l Eeneration,

Small Scale

Number of Times Taught:

Average Enrollment: $\quad 10$

Physics of Solar Collectors

Instructor: Claik, Eugene

(512) 736-7504

Course Number: PHYS 356

Department: Physics

Program or

Curriculum: Solar Energy Graduate

Pros.

Credits: 3

Student Level: College Graduate

Duration: is Heaks, $3.0 \mathrm{hrs}$ per week

Contact Hours: 45

Classroom: 45

Topics Covered Extensively: Alternate Energy Scurces; Appropriate Technology; Heat and Energy Transfer; Intro. to Solar Energy; Passive Solar Technology; Solar Systen Components; Solar Collecter Evaluation/Design; Solar Systens Desisn; Dcriestic Hot Water; Swiming Pool Heating; Elec'l

Generation, Central; Elec'l Generation, 
Small Scale; Process Heat, Agricultural; Process Heat, Industrial; Space Heating; Space Cooling Number of Times Taught: 2 Average Enrollment: 2

Solar and Atmospheric Radiation Instructor: Clark, Eugene (512) 736-7504

Course Number: PHYS 387

Department: Physics

Program or

Curriculum: Solar Energy Graduate Prog.

Credits: Student Level: College Graduate Duration: 15 Weeks, 3.0 hrs per week

$\begin{array}{ll}\text { Contact Hours: } & 45 \\ \text { ilassroom: } & 45\end{array}$

ilas5rooin: 45

Topics Covered Extensively: Alternate Energy Sources; Intro. to Solar Energy; Solar Elieryy Fulicy Develupment; Solar Collector Evaluation/Design; Solar Systers Design; Domestic Hot Hater; Swiming Pool Heating; Elec'l Generation. Central; Elec'l Generation, Small Scale; Process Heat, Agricultural; Process Heat, Industrial; Space Heating; Space Cooling Number of Times Taught: 3 Average Enrollment: $\quad 15$

Solar Energy system Components

Instructor: Treat, C. H. (5i2) $736-7512$

Course Number: EH:GR 3626

Department: Engineering

Program or

Curriculum: Solar Energy Graduate

Credits: Prog.

Student Level, College Graduale vursţoñ :

Contact Hours:

15 Weeks, 3.0 hrs per neak. 45

Classroom: 45

Topics Covered Extensively: Heat and Energy Transfer; Marketing/Market Analysis; Materials Research; Solar System Components; Solar Collector Evaluation/Design; Solar Systems nesign: Solar Systems Testing and Evaluation; Domestic Hot Water;

Swimming Pool Heating; Elec'l

Generation, Central; Elec'l Generation,

Small Scale; Process Heat,

Agricultural; Process Heat, Industrial;

Space Heating; Space Cooling

Number of Times Taualut \&

Average Enrollment:

10

Solar Energy System Design

Instructor: Treat, C. $H$.

(512) 736-7512

Course Number: EFIGR 3955

Department: Engineering Science

Program or

Curriculum:

Credits:

Solar Energy Graduate Prog.

Student Level: College Graduate
Duration:

15 Weeks, 3.0 hrs per week

Contact Hours:

45

Classroom: 45

Topics Covered Extensively: Solar System

Components; Solar Economics; Solar

Collector Evaluation/Design; Solar

Systems Desisn; Solar Systens

Installation; Solor Systems Testing and

Evaluation; Domestic Hot Hater;

Swimmina Pool Heating; Elec'l

Generation, Central; Elec'l Generation,

Small Scale; Process Heat,

Agricultural; Process Heat, Industrial;

Space Heating; Space Cooling

Number of Times Taught: 2

Average Enrollment: $\quad 13$

*********x

U OF HOUSTON CEN CAMPUS

(3652)

HUUSTUN, lexas 77004

(713) 749-2214

\section{SOLAR RELATED COURSES}

\author{
Man, Architecture and Energy \\ Instructor: \\ Kay, George E. \\ (713) 749-1188 \\ Cuurse Number: $430 \mathrm{~T}$ \\ Department: Architecture \\ Credits: 3 \\ Student Level: Junior or Senior \\ Duration: $\quad 14$ keeks, 3.0 hrs per week \\ Contact Hours: 42 \\ Classrcom: 42 \\ Topics Covered Extensively: Alternate \\ Energy Sources; Appropriate Terhmologv; \\ Energy Conservation; Intro. to Solar \\ Energy; Passive solar Techmology; Solar \\ System Components; Solar Economics; \\ Solar Home Constructioni Space Heating: \\ spooe Cooling \\ Number of Times Taught: 3 \\ Average Enrollment: $\quad 15$
}

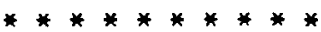

WEST TEXAS ST UNIVERSITY

(3665)

CAHYYOI! TERHS 79016

(806) 656-0111

\section{SOLAR RELATED COURSES}

Silar fnergy: fes, and Rural syetems

Instructor: Helson, Vaughn

Course Number: $39901-1$

Department: Phisics

Credits: 3

Student Level: All lavels

Duration: $\quad 17$ Weeks, $3.0 \mathrm{hrs}$ per week

Contact Hours: 51

Classroom: $\quad 51$

Topics Covered Extensively: Alternate Energy Sources; Appropriate Technology; Energy Conservation; Energy Conversion; Energy Storage; Intro. to Solar Energy; Domestic Hot Water; Space Heating; Wind 
Power, Small Systems

\begin{tabular}{|c|c|}
\hline $\begin{array}{l}\text { Wind Energy and } \\
\text { Ins tructor: }\end{array}$ & $\begin{array}{l}\text { Hind rurbines } \\
\text { Nelson, Vaughn } \\
\text { (806) 656-3904 }\end{array}$ \\
\hline Course Number: & $39902-1$ \\
\hline Department: & Physics \\
\hline $\begin{array}{l}\text { Credits: } \\
\text { student level: }\end{array}$ & $\begin{array}{l}3 \\
\text { All levels }\end{array}$ \\
\hline Duration: & 17 Weeks, 3.0 hrs per week \\
\hline $\begin{array}{l}\text { Contact Hours: } \\
\text { Classroom: }\end{array}$ & $\begin{array}{l}51 \\
51\end{array}$ \\
\hline $\begin{array}{l}\text { Topics Covered } \\
\text { Conservation; } \\
\text { Storagc; Heat } \\
\text { Solar Systems } \\
\text { Systems }\end{array}$ & $\begin{array}{l}\text { Extensively: Energy } \\
\text { Enersy Conversion; Energy } \\
\text { and Energy Transfer; } \\
\text { Design; Wind Power, Small }\end{array}$ \\
\hline
\end{tabular}

**********

\section{Community/Juni or Colleges}

CENTRAL TEXAS COLLEGE

(4003)

KILLEEN, Texas 76541

(817) 526-1211

\section{PROGRAMS AND CURRICULA}

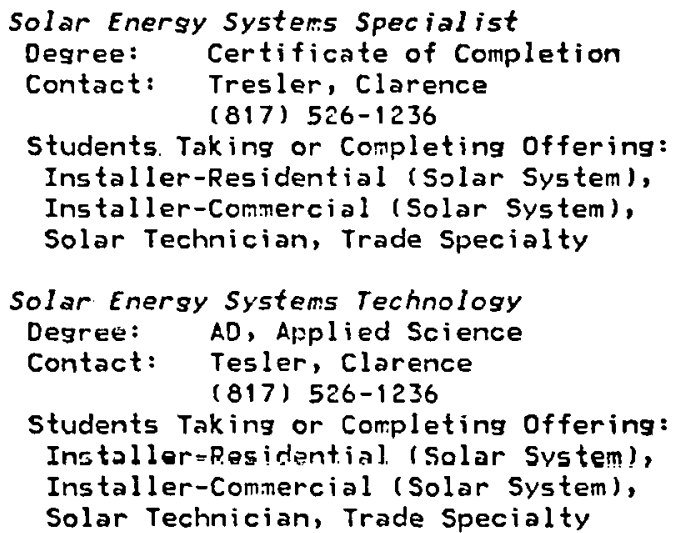

SOLAR RELATED COURSES

$\begin{array}{ll}\text { Principles of Solar Energy } \\ \text { Instructor: } & \text { Tresler, Clarence } \\ & (817) 526-1236 \\ \text { Course Number: } & \text { SESY 1314 } \\ \text { Department: } & \text { Industrial Technology } \\ \text { Program or } & \\ \text { Curriculum: } & \text { Solar Energy Systems } \\ & \text { Technology/ Solar } \\ & \text { Energy Systems } \\ & \text { Specialist } \\ \text { Credits: } & 3 \\ \text { Student Level: } & \text { Freshman or Sophomore } \\ \text { Duration: } & 16 \text { Weeks, } 3.0 \text { hrs per week } \\ \text { Contact Hours: } & 48 \\ \text { Classroom: } & 43 \\ \text { Topics Covered Extensively: Intro. to }\end{array}$

Solar Energy

Number of Times Taught: 2

Average Enrollment: $\quad 25$

Solar Cooling Systems

Instructor: Tresler, Clarence

(817) 526-1236

Course Number: SESY 241

Department: Industrial Technology

Prosram or

Curriculum: Solar Energy Systems

Technology

Credits: 4

Student Level: Freshman or Sophomore

Duration: 16 heeks, $6.0 \mathrm{hrs}$ per week

Contact Hours: $\quad 96$

Classroom: $\quad 48$

Laboratory: 48

Topics Covered Extensively: Energy

Storage; Heat and Energy Tranefer;

Plumbing Techniques; Solar System

Components; Solar Home Construction;

Solar Collector Evaluation/Design;

Solar Systems Design; Solar Systems

Installation; Solar Systems

Maintenance; Solar Systems Testing and

Evaluation

\author{
Solar Energy Special Projects \\ Instructor: Tresler, Clarence \\ (817) 523-1236 \\ Course Number: SESY 231 \\ Department: Industrial Technology \\ Program or \\ Curriculum: Solar Energy Systems \\ Credits: \\ Technology \\ Credits: 3 \\ Student Level: Freshman or Sophomore \\ Duration: 16 keeks, $6.0 \mathrm{hrs}$ per week \\ Contact Hours: 96 \\ Classroom: $\quad 16$ \\ Laboratory: 80 \\ Topics Covered Extensively: Energy \\ Conversion; Energy 5torage; Heat and \\ Energy Transfer; Plumbing Techniques; \\ Solar System Components; Solar Home \\ Construction; Solar Collector \\ Evaluation/Design; Solar Systems \\ Design; Solar Systems Installation; \\ Solar Systems Maintenance; 3ular. \\ Systems Testing and Evaluation; \\ Domestic Hot Water; Swimming Pool \\ Heating; Space Heating; Space Cooling
}

Solar Heating 5ystems

Instructor: Tresler, Clarence

(817) 526-1236

Course Number: SESY 141

Departinent: Industrial Technology

Program or

Curriculum: Solar.Energy Systems

Technolusy/ Solar

Energy Systems

Credits: 4 Specialist

Student Level: Freshman or Sophomore

Duration:

Contact Hours: 96

Classroom: $\quad 48$

Laboratory: $\quad 48$ 
Techniques; Solar System Components; Solar Collector Evaluation/Design; Solar Systems Design; Solar Systems Installation; Solar Systems Maintenance; Solar Systenis Testing arid Evaluation; Domestic Hot Water; Space Heating; Space Cooling

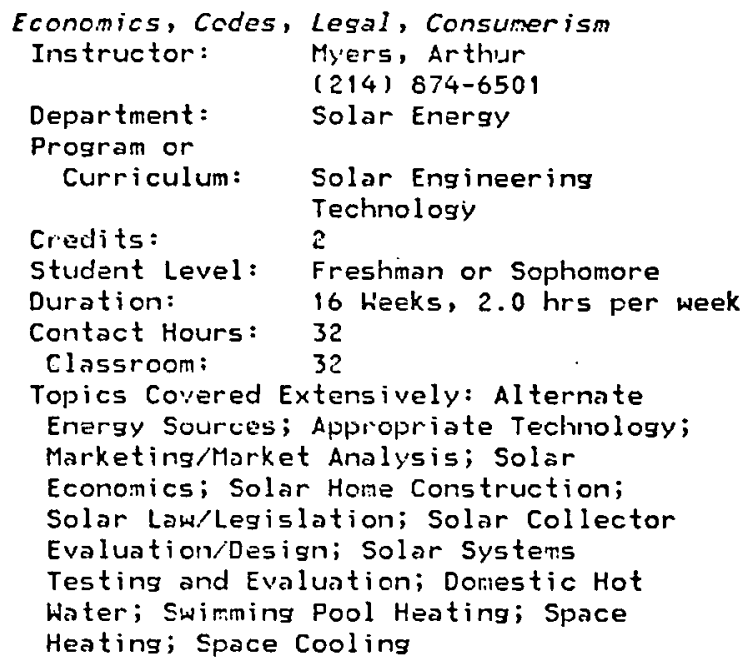

Energy Science I

Ins tructor:

Myers, Arthur

(214) 874-6501

Department:

Solar Energy

Program or

Curriculum: Solar Engineering

Credits: Techrology

Student Level: Freshman or Scphomore

Duration:

16 Heeks, 6.0 hrs per week

Contact Hours: 96

Classrocm: $\quad 48$

Laboratory: 48

Tepics Covered Extensively: Alternate

Energy Sources; Appropriate Technolosy;

Energy Conservation; Energy Conversion;

Energy Storage; Heat and Energy

Transfer; Intro. to Solar Energy;

Plumbing Techniques; Sheet Metal

Techicues; Solar Systom Componente;

Solar Systems Design; Solar Systems

Installation; Solar Systems

Maintenance; Domestic Hot hater; Space

Heating; Space Cooling

Enersy Science II

Instructor: Myers, Arthur

$\begin{array}{ll} & (214) 874-6501 \\ \text { Department: } & \text { Solar Energy }\end{array}$

Program or

Curriculum: Solar Engineering

Credits: Technology

Student Level: Freshman or Sophomore

Duration: 16 Weeks, $6.0 \mathrm{hrs}$ per week

Contact Hours: 96

Classroon: $\quad 49$

Laboratory: 48

Topics Covered Extensively: Alternate

Enersy Sources; Appropriate Technology;
Energy Conservation; Energy Conversion; Energy Storage; Heat and Energy

Transfer; Intro. to Solar Energy;

Plumbing Techniques; Sheet Metal

Techniques; Solar System Components;

Solar Systems Design; Solar Systems

Installation; Solar Systems

Maintenance; Domestic Hot Water; Space

Heating; Space Cooling

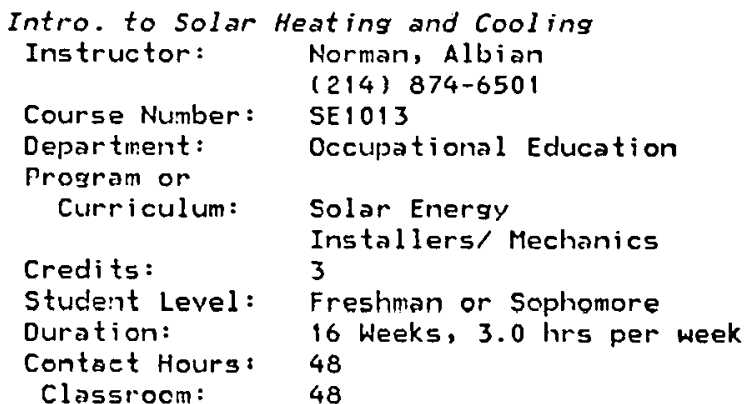

Topics Covered Extensively: Alternate

Energy Sources; Appropriate Technolosy;

Energy Conservation; Heat and Energy

Transfer; Intro. to Solar Energy;

Passive Solar Technology; Plumbing

Techniques; Solar System Components;

Solar Home Construction; Solar Systems

Design; Domcstic Hot hater; Elec'l

Generation, Small Scale; Space Heating;

Space Ccoling

Number of Timas Taught: 1

Average Enrollment: 23

Materials and Fabrication

Instructor: Vaughn, Ralph

Course Number: $\quad$ SE1034 874-6501

Department: Occupational Education

Program or

Curriculum: Solar Energy

Credits:

Student Level: Freshman or Sophomore

Duration: $\quad 16$ Weeks, $6.0 \mathrm{hrs}$ per week

Contact Hours: 96

Classroom: 32

Laboratory: $\quad 64$

Topics Covered Extensively: Energy

Storage; Heat and Energy Transfer;

Materials Research; Plumbing

Techniques; Sheet Metal Techniques;

Solar System Components; Solar Home

Construction; Solar Systems Design;

Solar Systems Installation; Solar

Systeris Maintenance; Solar Systems

Testing and Evaluaticn; Donestic Hot

Water; Space Heating; Space Cooling

Materials and Material Handling

Instructor: Myers, Arthur

Prosrain or

(214) 874-6501

Curriculum:

Solar Engineering

Credits: Technology

Student Level: $\quad$ Freshman or Sophomore

Duration: 


$\begin{array}{ll}\text { Contact Hours: } & 80 \\ \text { Classroom: } & 16\end{array}$

Laboratory: 64

Topics Covered Extensively: Materials

Research; Plumbing Techniques; Sheet

Metal Techniques; Domestic Hot Water;

Space Heating; Space Cooling

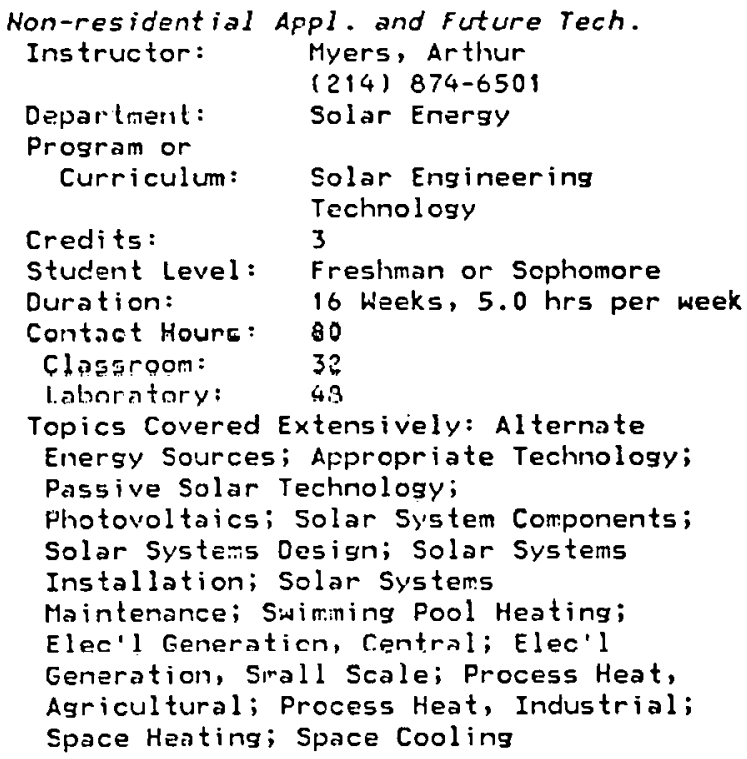

Operational Diagnosis

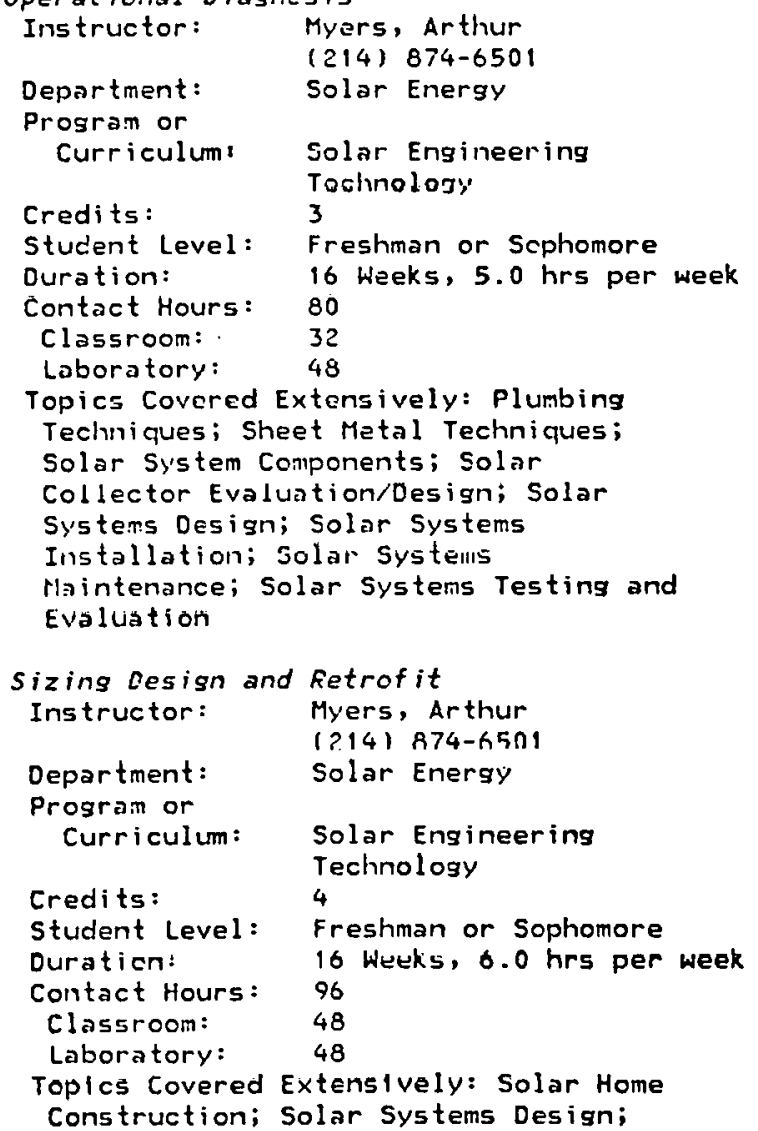

Solar Systems Installation; Solar Systems Testing and Evaluation; Domestic Hot Water; Space Heating; Space Cooling

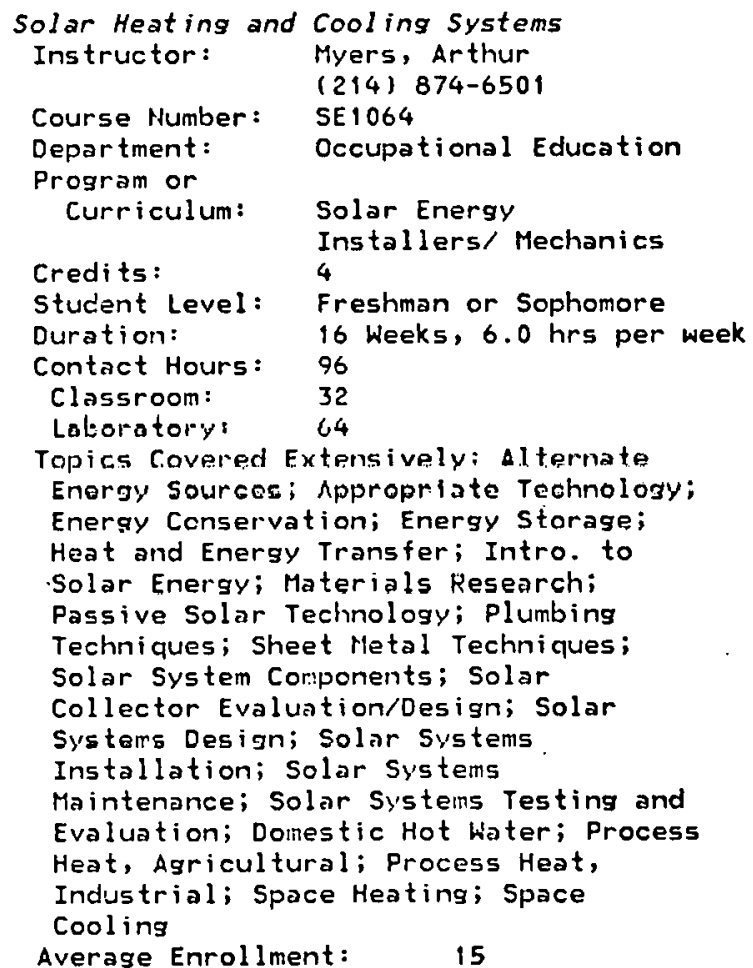

Solar Practicum

Instructor:

Myers, Arthur

(214) 874650

Program or Solar Energy

Curriculum

Solar Engineering Technology

Credits: Student Level: Freshman or Sophomore

Duration:

Contact Hours: 48

Laboratory: 48

Topics Covered Extensively: Alternate Energy Sources; Appropriate Technology; Intro. to Solar Energy; Materials Research; Plumbing Techniques; Sheet Hetal Techniques; Solar Systems Design; Solar Systems Installation; Solar Systems Maintenance; Solar Systems Testing and Evaluation; Domestic Hot Watar; Space Heatina; Space Cooling

$\begin{array}{ll}\begin{array}{l}\text { Technical Surveys of Energy Sources } \\ \text { Instructor: }\end{array} & \begin{array}{l}\text { Myers, Arthur } \\ (214) \text { 874-6501 }\end{array} \\ \text { Department: } & \text { Solar Energy } \\ \text { Program or } & \\ \text { Currlculum: } & \text { Solsr Englneering } \\ & \text { Technology } \\ \text { Credits: } & 3 \\ \text { Student Level: } & \text { Freshman or Sophomore } \\ \text { Duration: } & 16 \text { Weeks; } 3.0 \text { hrs per week } \\ \text { Contact Hours: } 48\end{array}$


Classroom: 48

Topics Covered Extensively: Alternate Energy Scurces; Appropriate Technology; Biomass Conversion; Energy

Conservation; Energy Conversion; Energy Storase; Heat and Enerzy Transfer; Intro. to Solar Energy; Passive Solar Technology; Fhotovoltaics; Domestic Hot Water; Swimming Fool Heating; Elec'l Generation, Central; Elec'l Generation, Small Scale; Process Heat, Agricultural; Prccess Heat, Industrial; Space Heating; Space Cooling; Hind Power, Central Systems; Wind Power, Small Systems

\section{MORTH LAKE COLLEGE}

IRVING, Texas 75062

(214) 255-5229

\section{PROGRAMS ARTD CURRICULA}

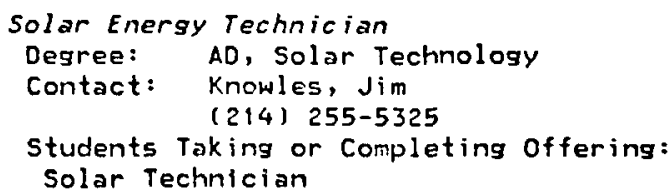

\section{SOLAR RELATEO COURSES}

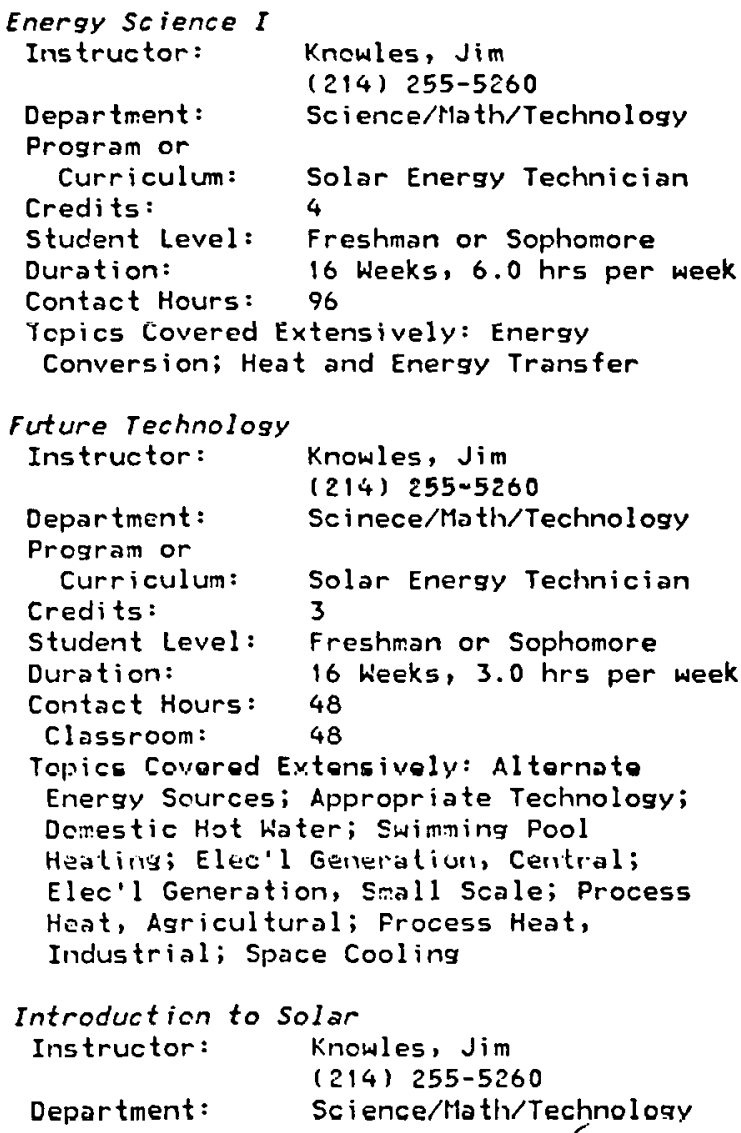

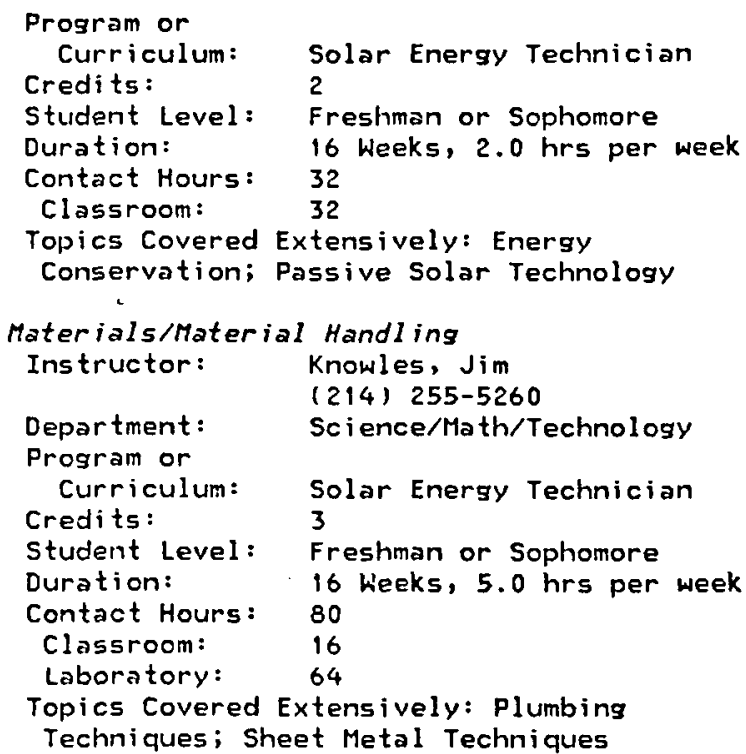




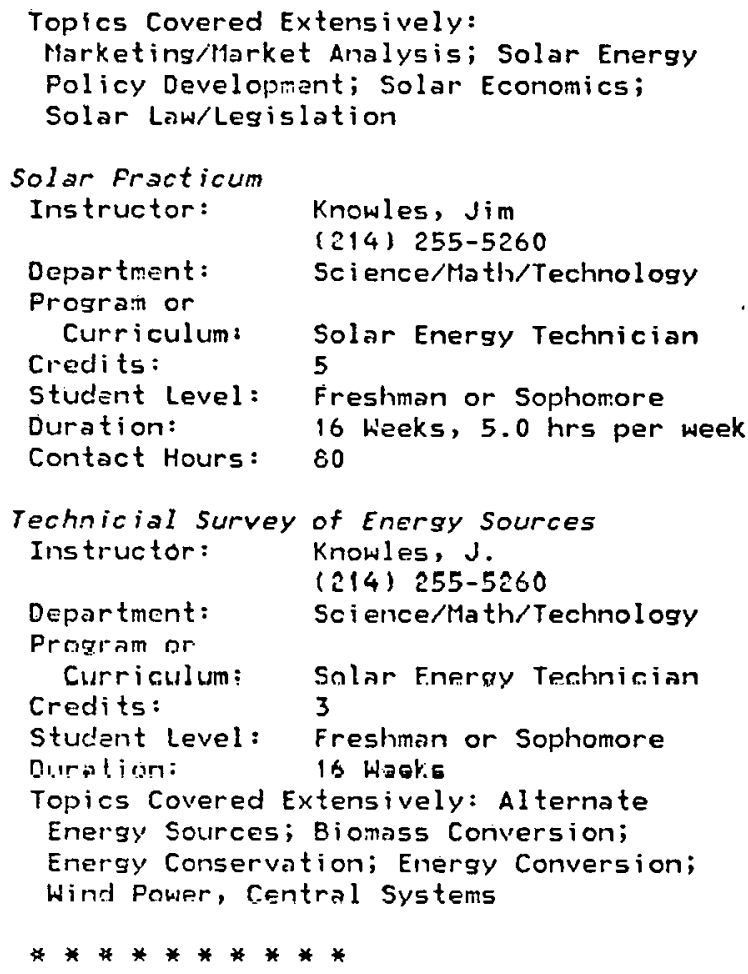

RAIIGER JUHIOR COLLEGE
RANGER, TEXas 76470
(817) $647-3234$

(3603)
PROGRAMS AND CURRICULA

$A / C$ and Refrig. - Sol. Ener. Option

Degree: AD, Applied Science

Contact: Stiles, Alton

(817) 647-3234

Students Taking or Completing Offering: Solar Technician

\section{SOLAR RELATED COURSES}

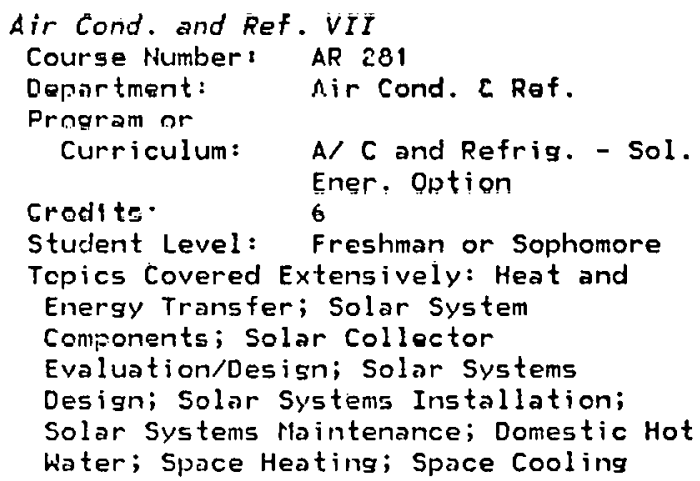

Fundanentals of Solar Heat. and Cool.

Course Number: AR 263

Department: Air Cond. C Refrig

Program or Curriculum: Á $C$ and Refrig. - 301.

Credits: Ener. Option

Student Level: Freshman or Sophomore

Topics Covered Extensively: Energy Conversion; Energy Storage; Heat and Energy Transfer; Intro. to Solar Energy; Solar System Components; Solar Collector Evaluation/Design; Solar Systems Design; Space Heating; Space Cooling

Solar Thermal Energy Systems

Instructor: Stiles, Alton

(817) 647-3234

Cuurse Nurnibr: AR 204

Department: Air

Conditioning/Refrigerat

Prngram nr

Curriculum: $\quad A / C$ and Refrig. - Sol.

Credits:

Student Level: Freshman or Sophomore

Duration:

Contact Hours:

Classroom:

Laboratory:

Techniques; Solar System Components;

Solar Collector Evaluation/Design;

Solar Systems Design; Solar Systems 
Installation; Solar Systems Maintenance; Solar Systems Testing and Evaluation; Domestic Hot Water; Elec'l Generation, Small Scale; Space Heating; Space Cooling

**********

TYLER JUHIOR COLLEGE

TYLER, TEXas 75701

(214) 597-4281

\section{PROGRAMS AND CURRICULA}

Air Conditioning \& Refrig. Tech.

Degree: AD, Applied Science

Contact: Minter, Richard $T$.

(214) 593-4401

students Taking or Cumpleting offering:

Trade Specialty

\section{SOLAR RELATED COURSES}

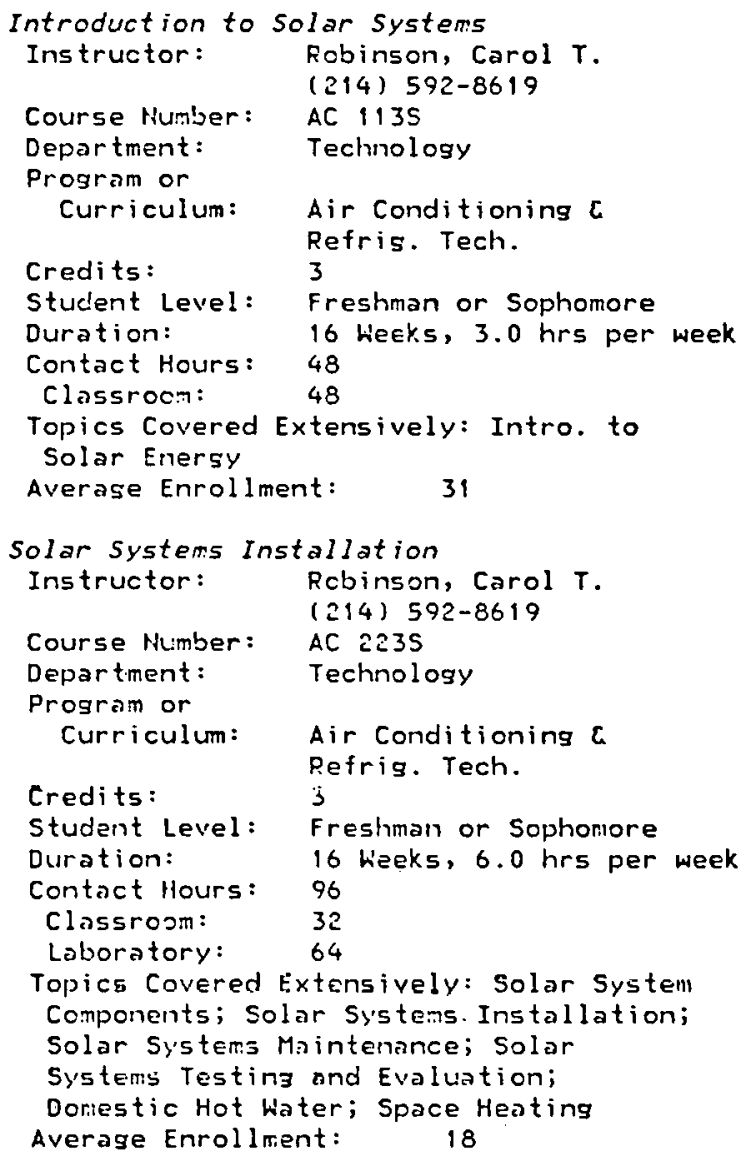

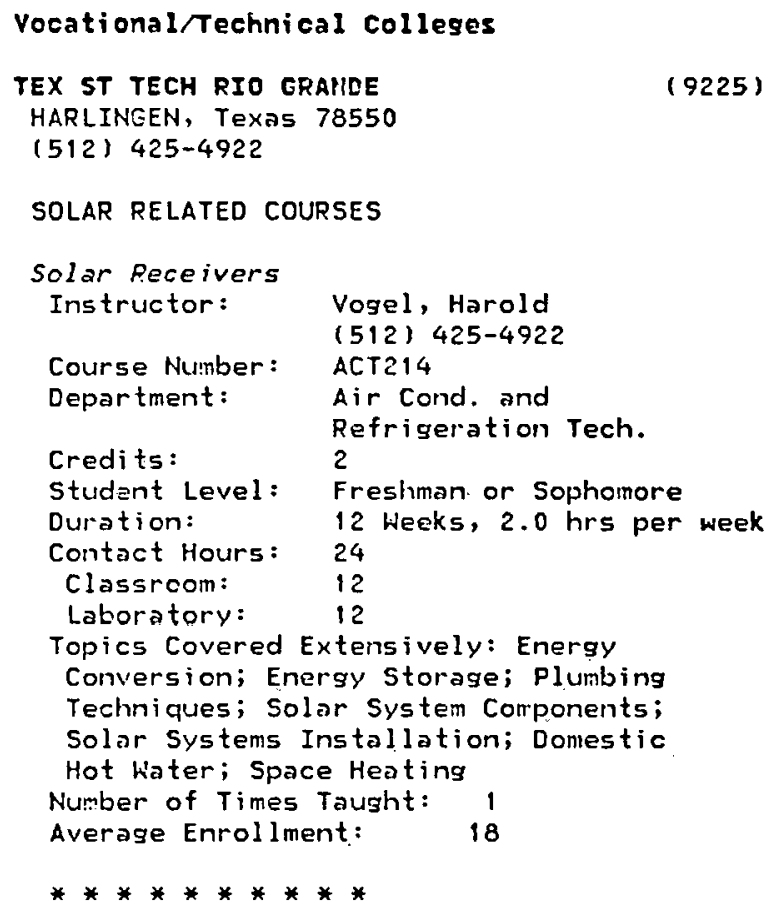

\section{SOLAR RELATED COURSES}

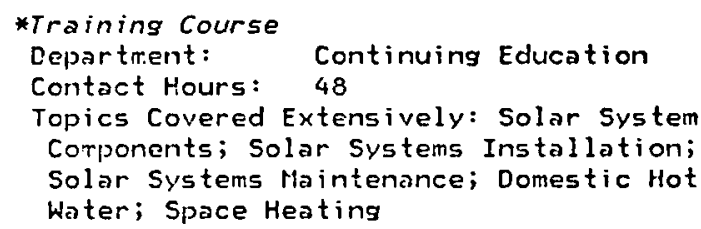

\section{other Educational Institutions}

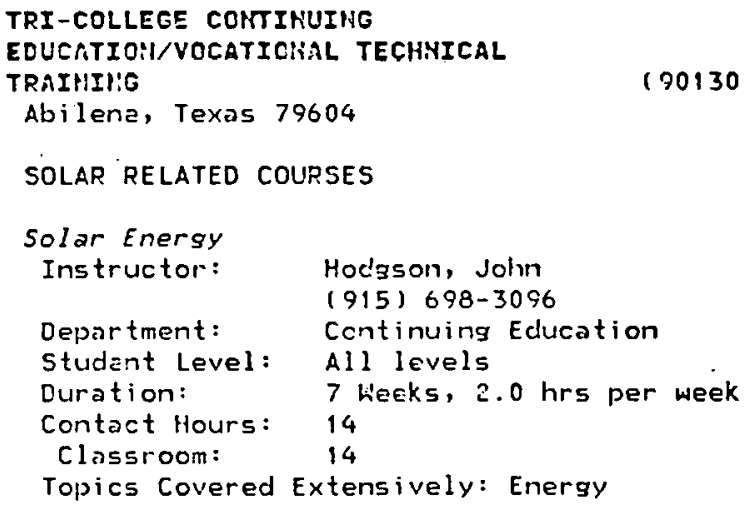


Conservation; Intro. to Solar Energy;

Passive Solar Technology; Solar System

Components; Solar Systems Design; Solar

Systems Installation; Solar Systems

Maintenance; Space Heating

Number of Times Taught:

Average Enrollment:

1

*********** 


\section{Colleges/Universities}

UTAH STATE UNIVERSITY

(3677)

LOGAN, Utah 84322

( 801 ) $752-4100$

SOLAR RELATED COURSES

Biometeorolsy Instruments

Instructor: Dirmhirn

(801) 752-4100

Course Number: 652

Department: Azri/Soil Sci. and

Biometeorology

Credits: 3

Student Level: College Graduate

Duration: 12 heeks, 3.0 hrs per week

Contact Hours: 36

Classroom: 9

Laboratory: 27

Topics Covered Extensively: Appropriate

Technology; Heat and Energy Transfer;

Intro. to Solar Energy; Photovoltaics

Number of Times Taught: 5

Average Enrollment:

Biometeorology

Instructor:

Dirmhirn/ Hanks/

Hooldrige

(801) 752-4100

Course Number:

709 .

Department:

Argi./Soil Sci, and Biometeorology

Credits: 3

Student Level: College Graduate

Duration: 12 Weeks, 3.0 hrs per week

Contact Hours: 36

Classroom: $\quad 36$

Topics Covered Extensively: Appropriate Technology; Heat and Energy Transfer;

Intro. to Solar Energy; Fhotovoltaics

Nuniber of Times Tausht: 5

Average Enrollment:

10

Envirnomental Remate Sensing

Instructor: Dirmhirn, I./ Baker, K.

(801) 725-7100

Course Number: 707

Department: Soil Sci C Biomet.

Argiculture

Credits:

3

Student Level: College Graduate

Duration: 12 Weeks, $3.0 \mathrm{hrs}$ per week

Contact Hours: 36

Classroom: $\quad 36$

Topics Covered Extensivoly: Appropriate

Technology; Heat and Energy Transfer;

Intro. to Solar Energy; Fhotovoltaics

Number of Times Tausht: 4

Average Enrollment:

Environmental field Experiments

Instructor: Dirmhirn

(801) $752-4100$

Course llumber: 693

Department: Argi/Soil Sci. and

Credits: 3

Biometeo

Student Level: College Graduate

Duration:

12 Weeks, 3.0 hrs per week $\begin{array}{cl}\text { Contact Hours: } & 36 \\ \text { Classrocm: } & 9\end{array}$

Laboratory: 27

Topics Covered Extensively: Appropriate

Technology; Heat and Energy Transfer;

Intro. to Solar Energy; Photovoltaics

$\begin{array}{ll}\text { Number of Times Taught: } & 4 \\ \text { Average Enrollment: } & 9\end{array}$

Instrumentation Lab

Course Number: 752

Department: Arti/Soil Sci. and

Bicmeteo.

Credits:

Studant Level: College Graduate

Duration: 12 Weeks, 3.0 hrs per week

Contact Hours: 36

Classrocm: 9

Laboratory: 27

Topics Covered Extensively: Appropriate

Technology; Heat and Energy Transfer;

Intro. to Solar Energy; Photovoltaics

Number of Tin:es Taught: 4

Average Enrollment:

Solar Enersy Systems

Instructor: Fhillips, W.F.

(801) 752-4100

Course Number: ME546

Department: Mech. Engrg.

Credits: 3

Student Level: Junior or Senior

Duration: 10 Weeks, 3.0 hrs per week

Contict Hours: 30

Clossroom: $\quad 30$

Topics Covered Extensively: Energy

Storage; Heat and Energy Transfer;

Intro. to Solar Enersy; Solar System

Corrfonents; Solar Collector

Evaluation/Design; Solar Systems Design

Number of Times Taught: 1

Averoge Enrollment:

12

$* * * * * * * * * *$

UTAH, UHIVERSITY OF

(3675)

SAL.T I,AKE CITY, Utah 84112

(801) 581-7291

SOLAR RELATED COURSES

Energy Conversion

Instructor:

Boehm, R. F.

Course Number:

$1801) 581-6441$

Department:

HiE $56:$

Credits:

Engin., Mech. and

4

Student Level: Junior or Senior

Duration: $\quad 10$ Weeks, 4.0 hrs per week

Contact Hours: 40

Classroom: $\quad 40$

Topics Covered Extensively: Alternate

Energy Sources; Energy Conversion

Number of Times Taught: 10

Average Enroliment: $\quad 35$ 


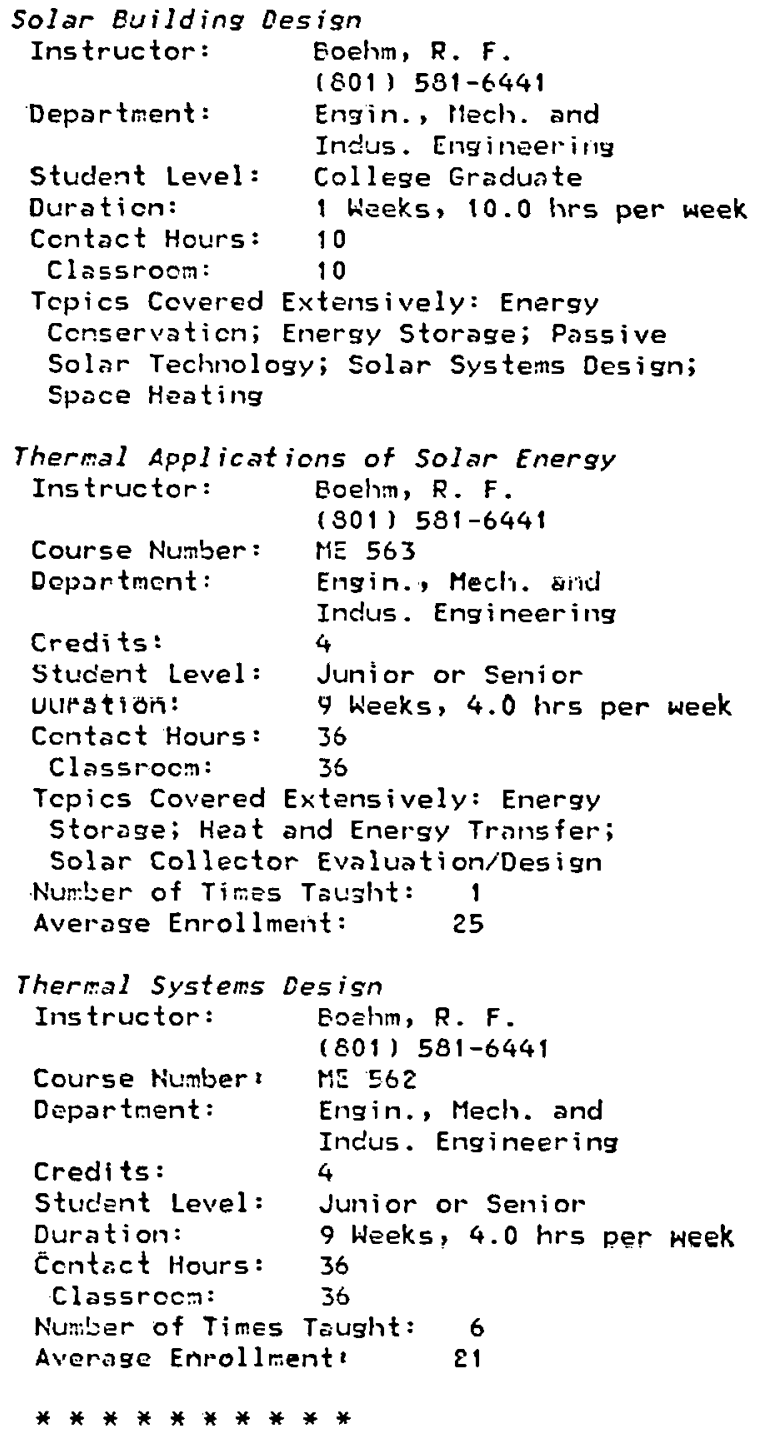

Community/Junior colleges
DIXIE COLLEGE

SAINT GECRGE, Utah 84770

(3671)

(801) 673-4811

PROGRAMS AHD CURRICULA

$\begin{array}{ll}\text { Solar Technolosy } \\ \text { Degree: Certificate of Completion } \\ \text { Contact: } & \text { Hacking, Jchn } \\ & (801) 673-4811\end{array}$

SOLAR RELATED COURSES

Intro. to Applied

Tait, Don (801) 673-4811

Course Number: ST 150

Department: Engineering Tech.

Prosram or

Curriculum: Solar Technolozy

Crediț:

4

Student Level: Freshman or sophomore

Duration: 11 heeks, 5.0 hrs per week

Contact Hours: 55

Classroom: $\quad 55$

Topics Covered Extensively: Energy

Conversion; Energy Storage; Heat and

Energy Transfer; Intro. to Sclar

Enersy; Marketins/Market Analysis;

Materials Research; Passive Solar

Technology; Plunibing Techniques; Solar

System Corponents; Solar Economics;

Solar Home Construction; Solar

Collector Evaluation/Desisn; Solar

Systems Design; Solar Systems

Installation; Solar Systens

Maintenance; Solor Systems Testing and

Evaluation; Domestic Hot Water; Space

Heating; Space Cooling

Number of Times Taught: 12

Averase Enrollment:

20

Solar Energy-Home Use Appls.

Instructor: Tait, Don

(801) 67j-4811

Course Number: ST 123

Deportment: Engineering Tech.

Program or

Curriculum: Solar Technology

Credits: 3

Student Level: Freshman or Sephomore

Duration: if lieeks, 3.0 hr's per week

Contact Hours: 33

Claceroom: $\quad 16$

Laboratory: 15

Topics Covered Extensively: Alternate Erielyy guurces, Energy ionservation; Energy Conversion; Energy Storace; Intro. to Solar Enersy; Passive Solar Techolosy; Plumbing Techmiques; Solar System Components; Solar Ecciomics; Solar Collector Evaluntion'Design;

Solar Systens Design; Solar Systems Installation; Solar Systens

Maintcinnce; Solar Sisteris Testing and Evaluation; Domestic Hot Hater; Space

Heating; Space Cooling

Number of Times Taught: 9

Average Emollment: $\quad 18$ 


\section{Vocational/Technical colleges}

UTAH TECH COLLEGE SALT LK

SALT LAKE CITY, Utah 84107

(801) 299-3411

SOLAR RELATED COURSES

Alternate Energy

Instructor:

Course Number:

Department:

Student Level:

Duration:

Contact Hours:

Classroom:

Laboratory:

Ingram, William W.

(801) 969-3411

ADT 057E

Architect

All levels

10 Weeks, 5.0 hrs per week

Topics Covered Extensively: Energy

Conservaticn; Intro. to Solar Energy;

Passive Solar Technology; Process Heat,

Agricultural; Prccess Heat, Industrial

Number of Tires Tausht: ?

Average Enrollment:

22 


\section{Colleges/Universities}

GOLOARD COLLEGE

(3686)

FLAINF IELO, Vermont

(802) 454-8311

PROGRAMS AND CURRICULA

*Social Ecology

Contact: Brookchin, Murray

SOLAR RELATED COURSES

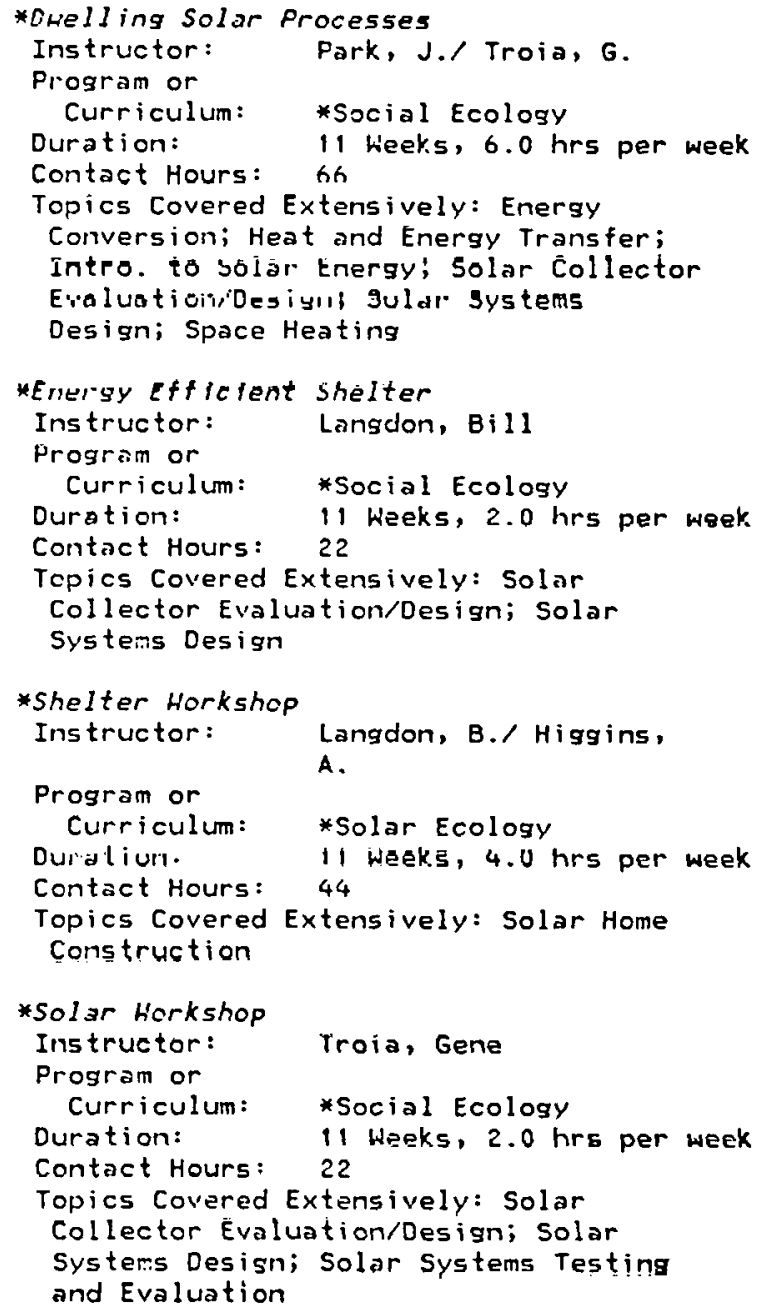

\section{SOLAR RELATED COURSES}

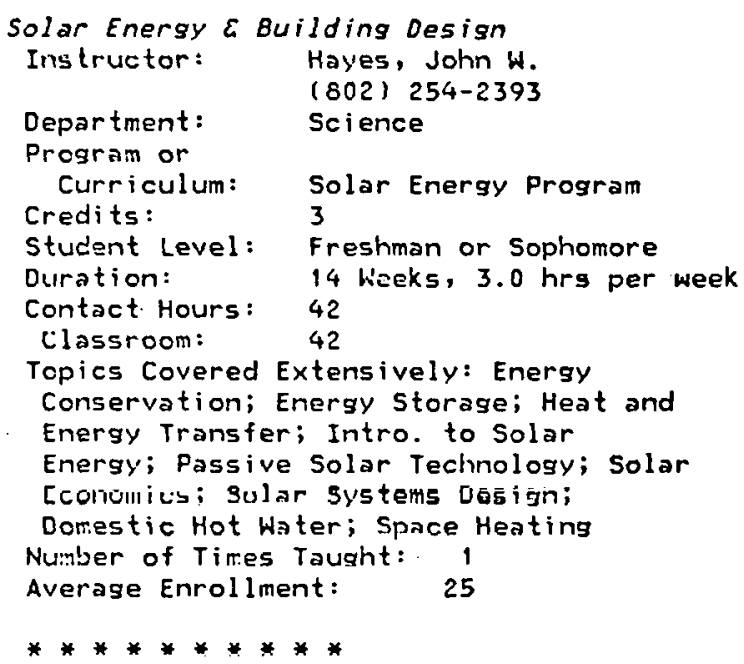

Community/Junior Colleges 
VERHONT, CHTY COLLEGE OF

(11167)

MONTPELIER, Vermont

(802) 828-2401

SOLAR RELATED COUPSES

Alternative Energy

Student Level: All levels

Duration: $\quad 3$ Heeks, $12.0 \mathrm{hrs}$ per week

Contact Hours: 36

Topics Covered Extensively: Alternate

Energy Sources; Intro. to Solar Energy;

Passive Solar Technology

Number of Times Taught: 3

Averase Enrollment:

10

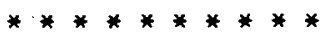


Colleges/Universities

FERRUN COLLEGE

FERRUM, Virsinia 24088

(703) 365-2121

PROGRAMS AND CURRICULA

Energy Technology

Degree: BS,

Contact: Bier, James/ Talbert, Roy

(703) 365-2121

students Taking or Completing Offering:

Solar Technician

\section{SOLAR RELATED COURSES}

Energy

Instructor: Bier,

(703) 365-2121

Department; Fnvirnnmantal sturipgs

Program or

Curriculum: Energy Technology

Credits:

Student Level: Junior or Senior

Duration: 15 Weeks, 5.0 hrs per week

Contact Hours: 75 .

Classroom: $\quad 45$

Laboratory: $\quad 30$

Topics Covered Extensively: Energy

Conservation; Space Heating

Number of Times Taught: 1

Average Enrollment: 20

**********

LYKCHBURG COLLECE

$(3720)$

I YNCHA!!FG, Virginia 24509

(804) 845-9071

SOLAR RELATED COURSES

Solar Energy

Instructor: Sigler, J.A.

Course Number: 538

Department: Fhysics

Credits: 3

Student Level: College Graduate

Duration: $\quad 15$ heeks, $3.0 \mathrm{hrs}$ per week

Contact Hours: 45

Clisil Unill: 45

Topics Covered Extensively: Appropriate

Technology; Energy Storage; Heat and

Energy Transfer; Intro. to Solar

Energy; Materials Research:

Fhotovoltaics; Solar System Components;

Solar Collector Evaluation/Design;

Elec'l Generation, Central; Elec'l

Generation, Small Scale; Space Heating

Number of Times Taught: 1

Average Enrollment: 10

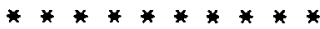

OLD DOMIHIOH UHIVERSITY

(3728)

NORFOLK, Virginia 23508

(604) 489-6000

PROGRAMS AND CURRICULA

Power/Energy Conversion Option

Desree: PhD, MS, BS, Mechanical

Engineering

Contact: Goglia, G. L. (804) 489-6485

Students Taking or Completing Offering:

Pesearcher, Solar Engineer, Other

Solar Energy Systems

Degree: BS, OT,

Contact: Crossman, Gary R.

(804) 489-6574

SOLAR RELATEO COURSES

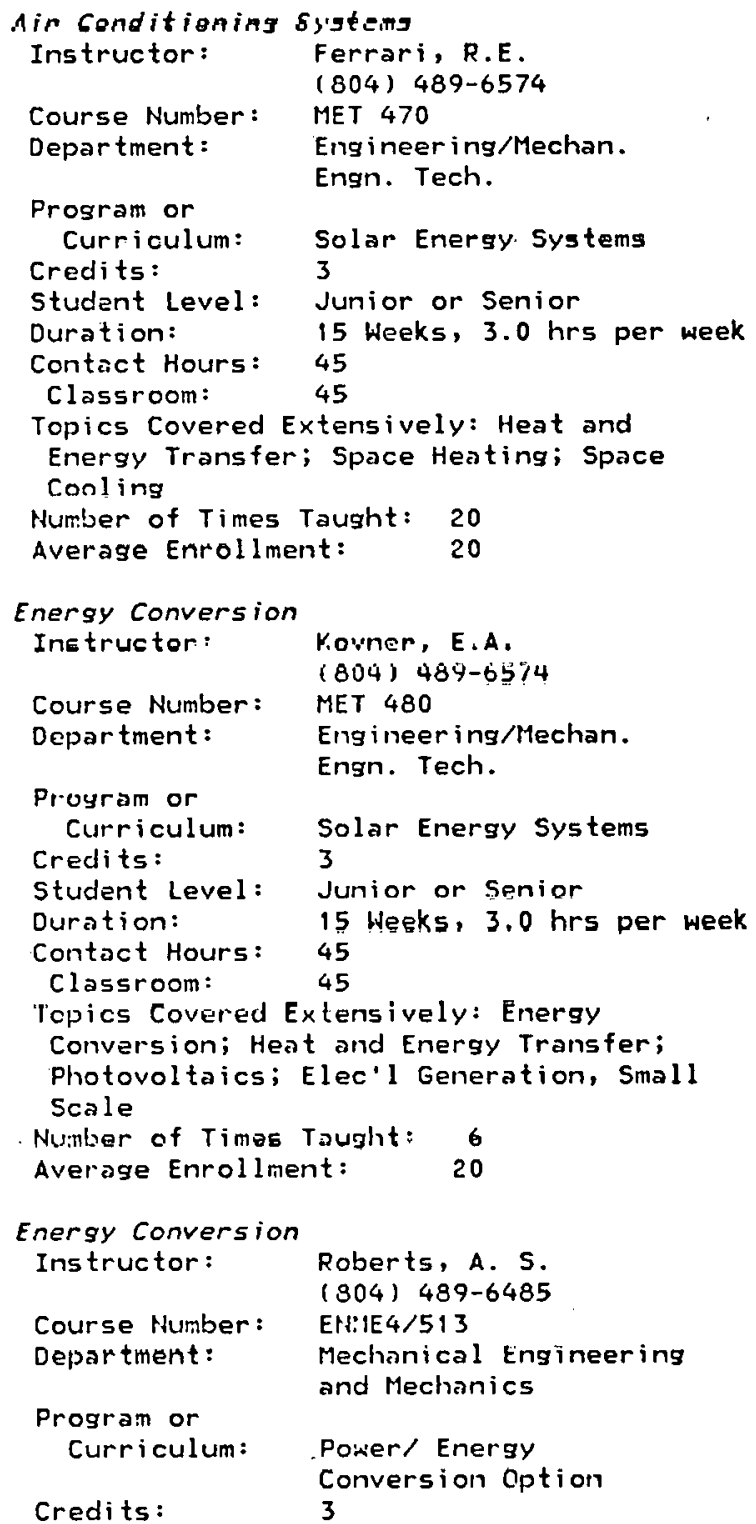




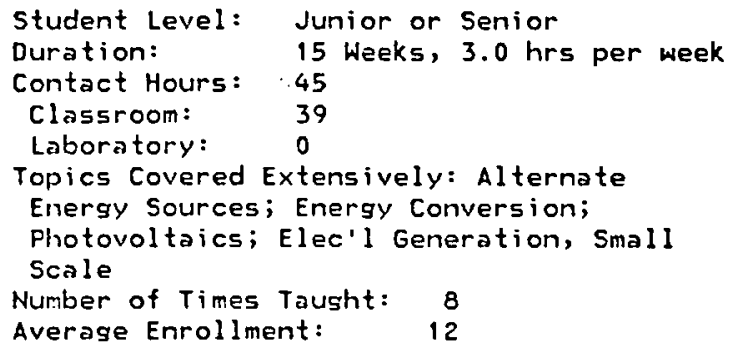

\section{Energy Systems}

Instructor:

Deal, Walter $F$. (804) 489-6461

Course Number: 243

Department: Vocational and Inclustrial Arts Education

Program or Curriculum: Power/ Energy

Credits: Conversion Option

Student Level:

Duration:

Contact Hours:

Classroom:

Laboratory:

Topics Covered Extensively: Energy

Conversicn; Intro. to Solar Energy;

Solar Collectur Evaluation/Design;

Solar Systems Testing and Evaluation;

Domestic Hot hater

Number of Times Tausht: 4

Average Enrollment: $\quad 15$

Envircnmental Control

Instructor: Roberts, A.5.

(804) 489-6485

Course Number: Eriile4/512

Department: Mechanical Engineering

and Mechanics

Curriculum: Power/ Energy

Credits:

Conversion Option

Student Level: Junior or Senior

Duration: 15 Weeks, $3.0 \mathrm{hrs}$ per week

Contact Hours: 38

Classiroom: 28

Topics Covered Extensively: Energy Conservation; Energy Conversion; Heat and Energy Transfer; Intro. to Solar Energy

Number of Times Taught: 10

Average Enrollment: 10

$\begin{array}{ll}\begin{array}{l}\text { Power and Erergy } \\ \text { Instructor: }\end{array} & \begin{array}{l}\text { Crosatory } \\ (804) 489-6574\end{array} \\ \text { Course Number: } & \text { MET } 465 / 485 \\ \text { Depaptment. } & \begin{array}{l}\text { Enginaaring/Merhan. } \\ \text { Engn. Tech. }\end{array} \\ \text { Program or } & \\ \text { Curriculum: } & \text { Solar Energy Systems } \\ \text { Credits: } & 2 \\ \text { Student Level: } & \text { All levels } \\ \text { Duration: } & 30 \text { Weeks, 3.0 hrs per week } \\ \text { Contact Hours: } & 90 \\ \text { Laboratory: } & 90\end{array}$

Topics Covered Extensively: Energy

Conversion; Heat and Energy Transfer Number of Times Taught: 20

Average Enrollment: $\quad 15$

Refrigeration systems

Instructor: Kovner, E.A.

$\begin{array}{ll}\text { Course Number: } & (804) 489-6574 \\ \text { MET } 460\end{array}$

Department: Engineering/Mechan.

Program or

Curriculum: Solar Energy Systems

Credits:

Student Level: Junior or Senior

Duration: 15 Weeks, 3.0 hrs per week

Contact Hours: 45

Classroom: 45

Topics Covered Extensively: Heat and

Energy Transfer; Space Cooling

Number of Times Taught: 20

Averige Enrollment: $\quad 20$

Solar Energy Systems 490

Instructor: Crossinan, G.R.

Course Number: $\quad(804) 498-6574$

MET 490

Department: Engineering/Mechan.

Program or

Curriculum: Solar Energy Systems

Credits:

Studeint Level: Junior or Senior

Duration: 15 Weeks, $3.0 \mathrm{hrs}$ per week

Contact Hours: 45

Classroom: 45

Topics Covered Extensively: Energy

Conversion; Heat and Energy Transfer;

Intro. to Solar Energy; Solar System

Components; Solar Collector

Evaluation/Design; Solar Systems

Design; Solar Systens Installation;

Domestic Hot Water

Solar Power Engineering

Instructor: Roberts, A, Sidney

(804) 489-6485

Course Number: ENME 4/516

Department: Mechanical Engineering

and Mechanics

Program or

Curriculum: Power/Energy

Credits:

Conversion Option

Student Level: Junior or Senior

Duration:

Contact Hours:

Classroom:

15 Weeks, 3.0 hrs per week

45

45

Topics Covered Extensively: Energy

Conversion; Heat and Energy Transfer;

Intro. to Solar Energy; Solar System

Coriponents; Solar Collector

Evaluation/Design; Solar Systems

Desisn; Domestic Hot Hater; Space

Heating

Number of Times Taught: 3

Average Enrollment: $\quad 15$ 


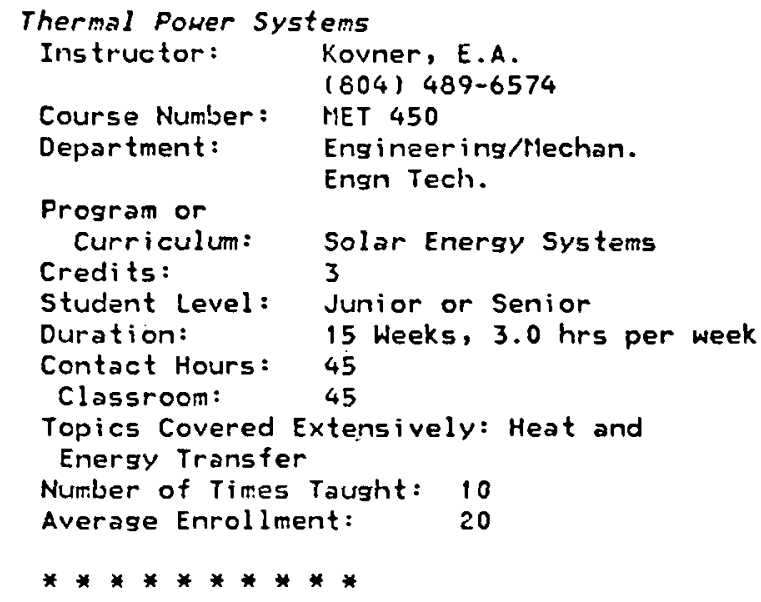

\section{VA POLY TNST ANM STATE U \\ BLACKSBURG, Virginia 24061 \\ $(703) 951-6000$}

$(3754)$

\section{SOLAR RELATED COURSES}

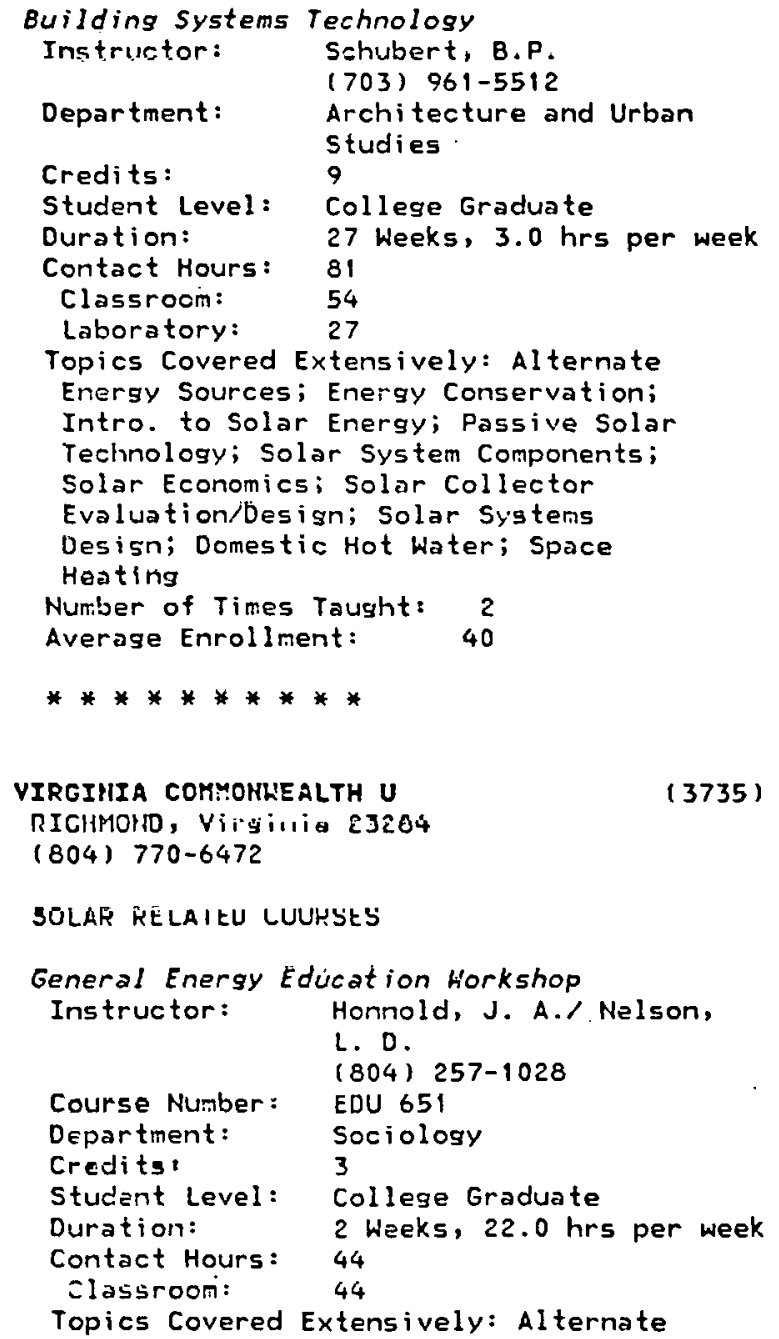

Energy Sources

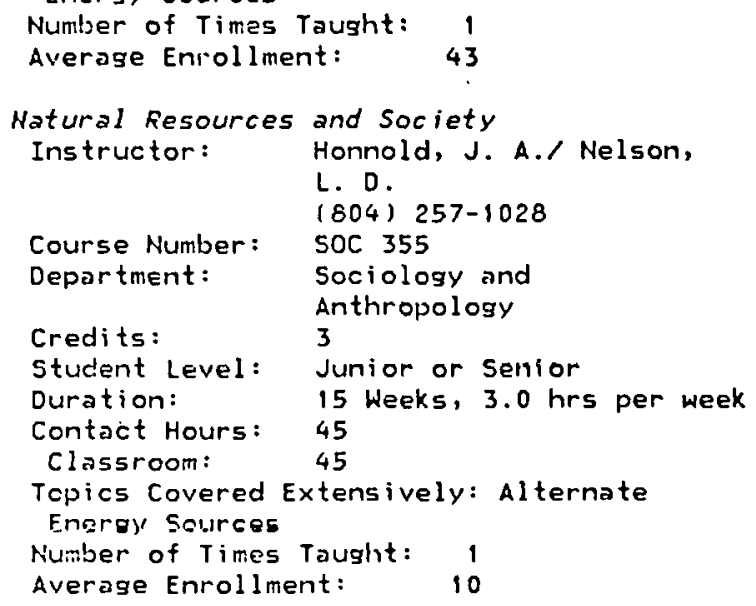


VIRGINIA HILITARY INST

(3753)

LEXINGTON, Virginia 24450

(703) 463-6311

\section{SOLAR RELATEO COURSES}

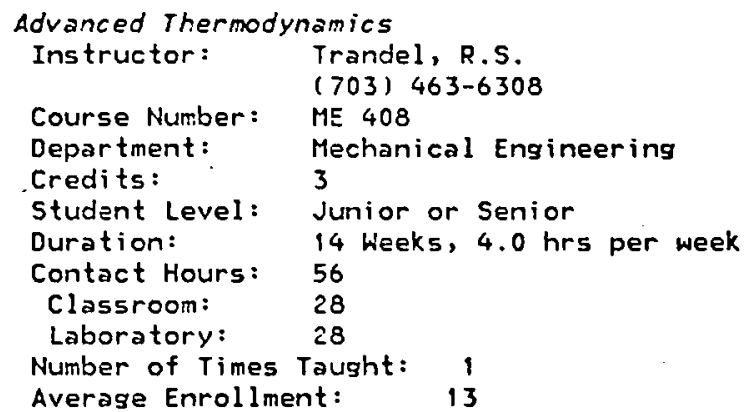

\section{Community/Junnior Colleges}

\section{BLUE RIDCE CTTY COLLEGE}

WEYERS CAVE, Virginia 24486

(703) 234-246)

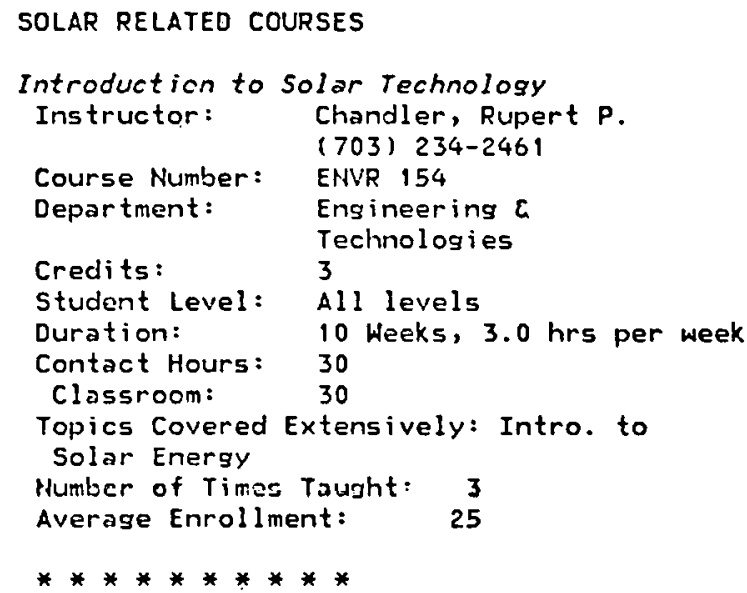

\section{DANVILLE CITY COLLEGE}

(3758)

DANVILLE, Virginia 2454

(804) 797-3553

\section{SOLAR RELATED COURSES}

A Survey of Alternate Energy

Instructor: Solomon, Michael

(703) 797-3553

Course Number: PH103

Department: Fhysics

Credits: 3

Student Level: Freshman or Sophomore

Duration: 12 Weeks, 3.0 hrs per week

Contact Hours: 36

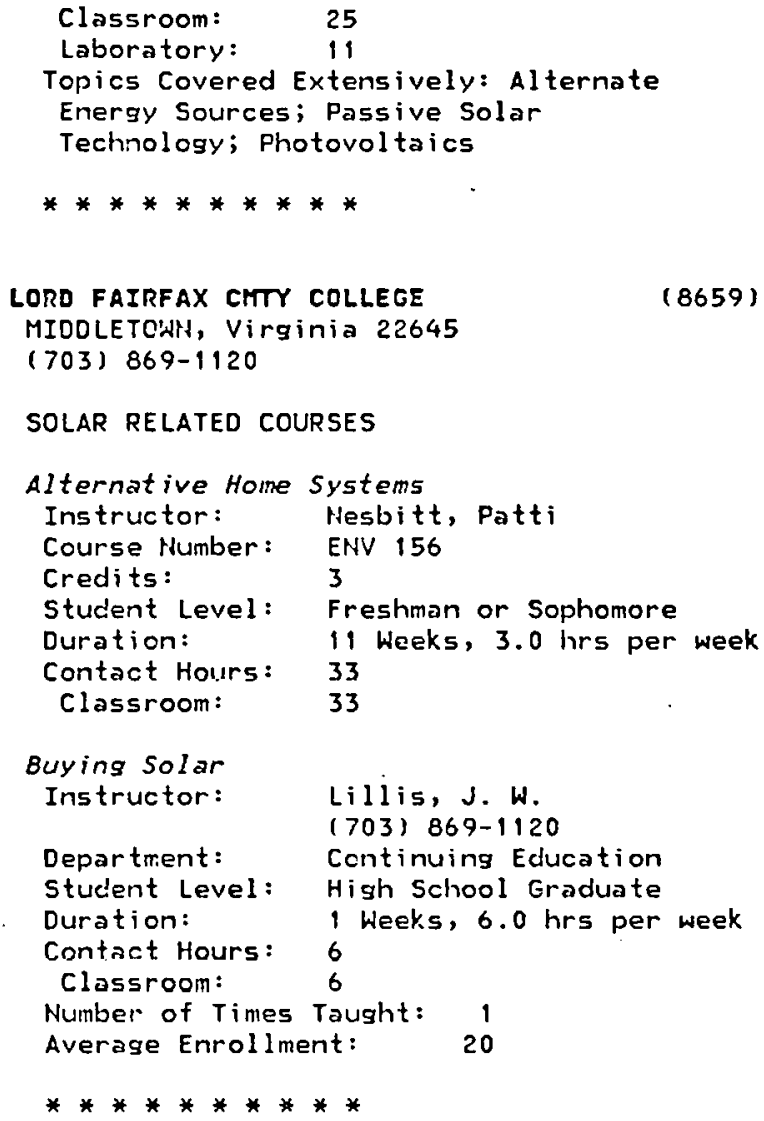


SOUTHSIOE VA CMTY COLLEGE

$(8661)$

ALBERTA, Virginia 23821

$(804)$ 949-7111

PROGRAMS AND CURRICULA

Air Cond. Heat. E Refrig.

Degree: A, C Heat. E Refrig.

Contact: Varyhan, C.G. (804) 949-7111

students Taking or Completing offering:

Contractor, Installer-Residential

(Solar System), Installer-Commercial

(Solar System)

THCHAS KELSH CMTY CULLEGE
HAMPTON, VIrginia 23670

(6871)

(804) $826-4800$

SOLAR RELATED COURSES

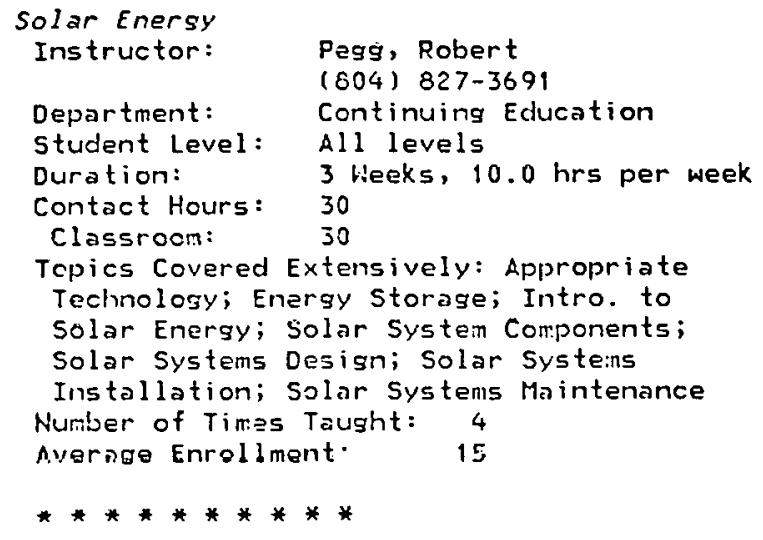

Tcpics Covered Extensively: Appropriate

Technology; Energy Storage; Intro. to Solar Energy; Solar System Components;

Solar Systems Design; Solar Systens

Installation; Solar Systens Maintenance

Number of Times Taught: 4

Ayernee Enreliment. 15

VA NESTERH CITT COULLELE

ROANOKE, Virginia 24015

(703) 344-2031

SOLAR RELATED COURSES

Solar Energy Systems Design

Instructor: Arminio, Robert

(703) 982.7305

Course Number: APCG167

Department: Arch. Technology

Credits: 3

Student Level: All levels

Duraliur. 10 heeks, 3.4 hrs për week

Contact Hours: 30

Topičs Covered Extensively: Solar

Systems Design 


\section{Colleges/Universities \\ HASHIHGTCN ST UNIVERSITY \\ $(3800)$ \\ FULLMAH, Washington 99163 \\ (509) 335-3564 \\ SOLAR RELATED COUPSES

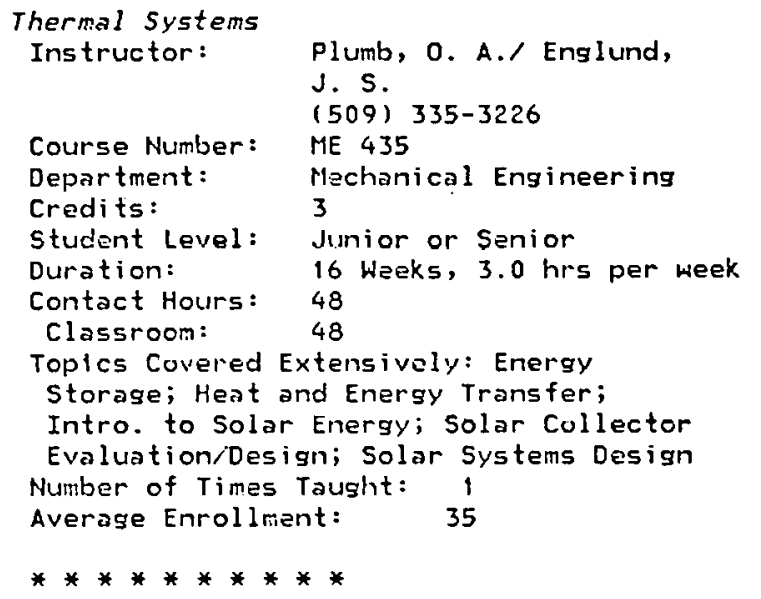

WASHIHSTON, UIIIVERSITY OF

(3798)

SEATTLE, hashington 98195

(206) 543-2100

\section{PROGRAMS AND CURRICULA}

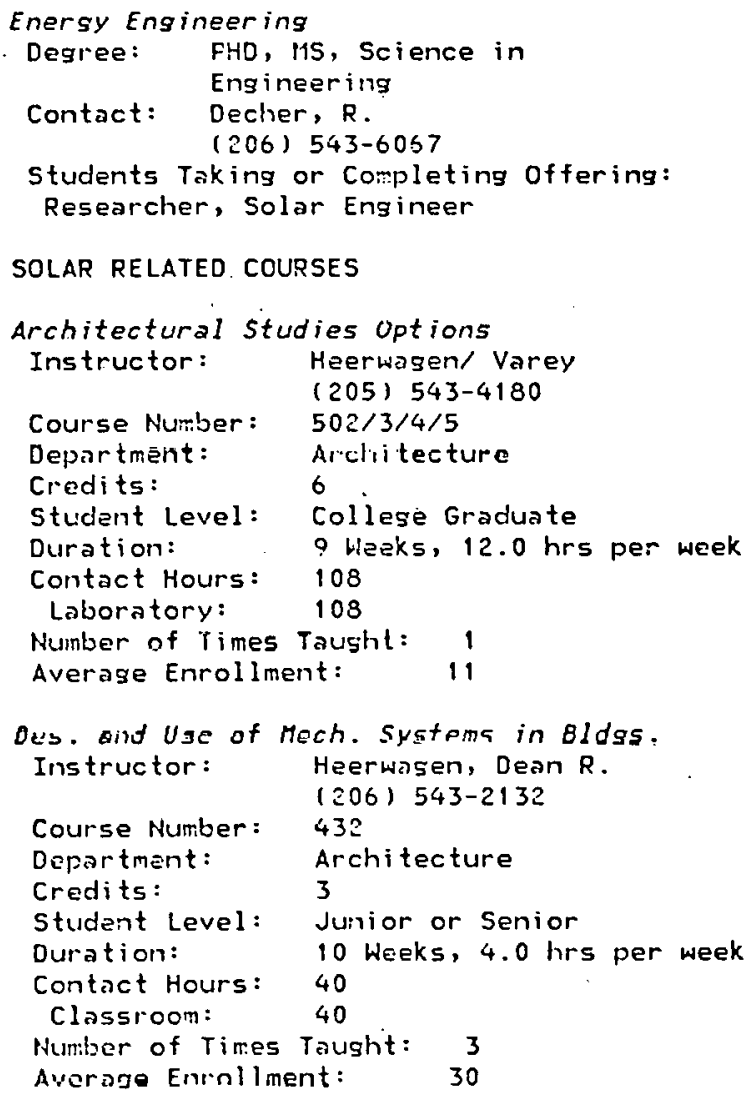

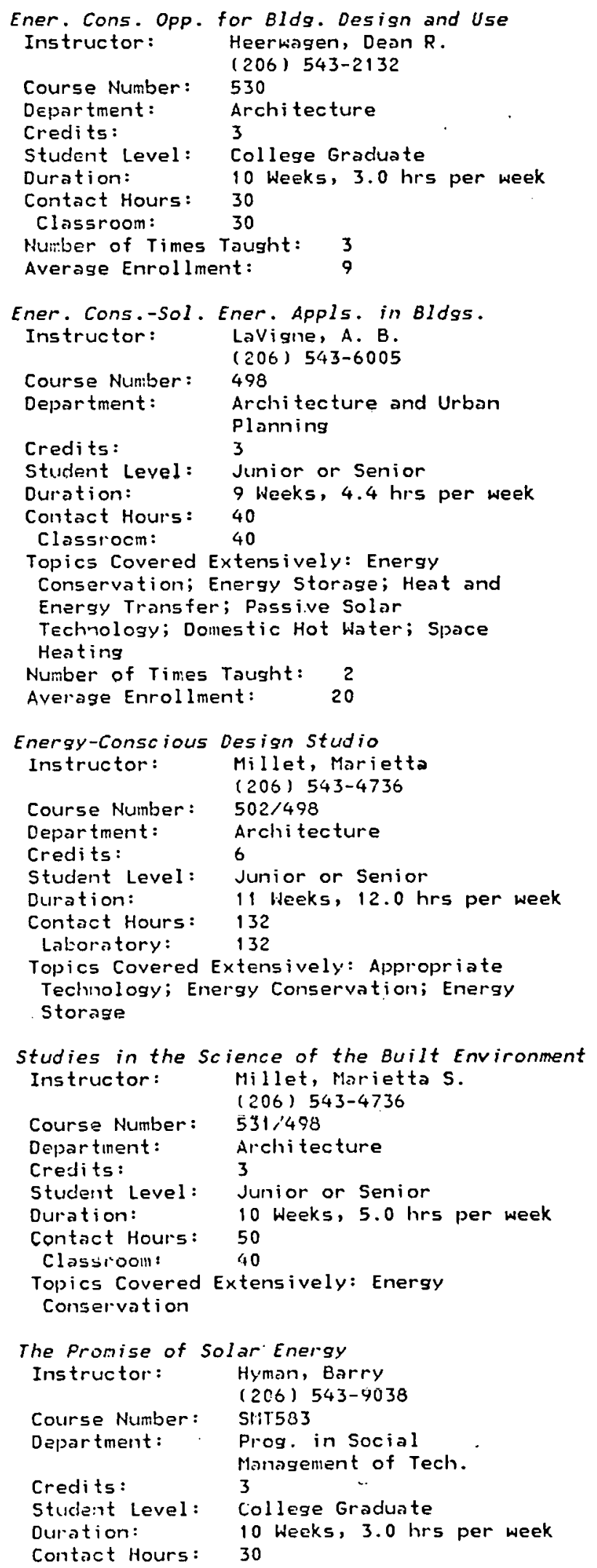


Topics Covered Extensively: Energy

Storage; Intro. to Solor Energy;

Marketing/Plarket Analysis; Passive

Solar Technology; Solar Energy Policy

Develcfrent; Solar Economics; Solar

Law/Legislation

Numiber of Times Taught: 5

Average Enrollment: $\quad 20$

Thermal Performance of Buildings

Instructor: Heerwagen, Dean R.

Course Number: 431

Department: Architecture

Credits: 3

Student Level: Junior or Senior

Duration: 10 Weeks, $4.0 \mathrm{hrs}$ per week

Contact Hours: 40

Clinesroom: $\quad 40$

Nurtber of Times Taught: 1

Averase Enrollment: $\quad 45$

\author{
Community/Junior Colleges \\ FORT STEILACOOH CC \\ $(5000)$ \\ TACOHA, Washington 98498 \\ (206) 552-3948 \\ SOLAR RELATED COURSES \\ Envircnmental Science \\ Instructor: Harding, Karen \\ Course Number: 120 \\ Department: Fhysical Science \\ Crodite: 5 \\ Student Level: Freshman or Sephomore \\ Duration: 10 Weeks, 5.0 hrs per week \\ Contact.Hours: 50 \\ Classroem: $\quad 50$ \\ Number of Times Taught: , \\ Average Enrollment: $\quad 25$ \\ **********
}

\section{NORTH SEATTLE CC}

(9704)

SEATTLE, Hashington 98103

(2.06) $634=4444$

PRÚGRARIS ATIU CURRICULA

Heat ing-Solar Energy

Contoct: Swenson, Don (206) 634-4419

Students Taking or Completing Offering: Sheet Metal Workpr

\section{SOLAR RELATED COURSES}

$\begin{array}{cc}\text { Heating -Solar Energy } & - \\ \text { Instructor: } & \text { Swenson, Don } \\ & (206) 634-4419 \\ \text { Department: } & \text { Engineering Technology }\end{array}$

Program or

Curriculum: Heating-Solar Energy

Student Level: High School Graduate

Duration: 6 Weeks, $15.0 \mathrm{hrs}$ per week

Contact Hours: 90

Classroom: $\quad 45$

Laboratory: 45

Topics Covered Extensively: Alternate Energy Sources; Appropriate Technology;

Intro. to Solar Energy; Solar System

Components; Solar Collestor

Evaluation/Design; Solar Systems

Design; Donestic Hut Water; Space

Heoting

Solar Energy

Instructor:

Stepnich, Ivan

(206) 634-4423

Course Number: ECT 207

Department: Engineering Related

Technologies

Program or

Curriculum: Heating-Solar Energy

Credits:

3

Student Level: Freshman or Sophomore

Duration: $\quad 11$ Heeks, $3.0 \mathrm{hrs}$ per week

Contact Hours: 33

Classroom: $\quad 33$

Topics Covered Extensively: Alternate Energy Sources; Appropriate Technoloyy; Energy Conservation; Energy Conversion; Energy Storage; Heat and Energy

Treinglel; Intro. to Solar Energy:

Passive Solar Technology; Plumbing

Techniques; Solar System Components;

Solar Home Construction; Snlor

Collector Evaluation/Design; Solar

Systers Design; Solar Systems

Installation; Solar Systens Testing and

Evaluation; Domestic Hot Hater; Elec'l

Generation, Central; Process Heat,

Industrial; Space Heating

Number of Times Taught: 1

Average Enrollment: . 25

$* * * * * * * * * *$

FEIIISSULA COLLEGE

FORT ANGELES, Washington 98362

(206) $152 .-2277$

\section{SOLAR RELATED COURSES}

Residential Solar Heating Systems

Instructor: Vandeusen, George

(206) 452-9277

Course Number: T.E.275

Department: Engineering Technology

Credits:

Student Level: All levels

Duration:

Contact Hours: 30
0 Weeks, $3.0 \mathrm{hrs}$ per week 
Classroom:

30

Topics Covered Extensively: Energy Conservation; Intro. to Solor Energy; Passive Solar Technolozy; Solar System Components; Solar Home Construction; Solar Systems Design; Space Heating Number of Times Taught: i Average Enrollment: $\quad 40$

**********

SPOKAKE FLS CITY COLLEGE

(9544)

SPOKAHE, Hashington 99204

(509) $456-2810$

SOLAR RELATED COURSES

How To Bld. Your OWn Sol. H/W Heater

Instructor: Roscher, Ted

(509) 456-2840

Department:

Eveningl Adult

Continuing Edu.1

Student Level: All levels

Duration: 10 Weeks, $3.0 \mathrm{hrs}$ per week

Contact Hours: 30

Classroom: $\quad 10$

Laboratcry: 20

Topics Covered Extensively: Appropriate

Technolcgy; Energy Conservation; Energy

Conversion; Energy Storase; Heat and

Eneray Transfer; Intro. to Solar

Enercy; Materials Research; Plumbing

Techniques; Sheet Metal Techniques;

Solar System Components; Solar

Collector Evaluation/Desisn; Solar

Systems Design; Solar Systems

Installation; Solar Systems

Maintenance; Solar Systems Testing and

Evaluation; Domestic Hot Water

Number of Times Taught: 1

Average Enrollment:

13

$* * * * * * * * * *$

TACCHA COMMUHIT COLLEGE

TACOMA, Washington 98465

(206) 756-5100

\section{SOLAR RELATEO COURSES}

Energy: Fast-Present-future

Course Nurber: ENER. 120

Department: Engineering

Credits:

2

Student Level: Freshman or Sophomore

Ouration: $\quad 10$ haeks, 2.0 hrs per week

Contact Hours: 20

Classroom: 20

Topics Covered Extensively: Alternate

Energy Sources; Biomass Conversion;

Energy Conservation; Energy Conversion;

Energy Storage; Heat and Energy

Transfer; Intro. to Solar Energy;

Passive Solar Technology

Number of Times Tausht: 2

\section{Vocational/Technical colleges}

OLYMPIA TECH CrTY COLLEGE

(5372)

OLYMPIA, Washington 98502

(206) $753-3000$

SOLAR RELATED COURSES

Solar Energy for Space Heating and Hot Hater Instructor: Oatman, Martin

(206) 753-3005

Course Number: C.ONS 198

Department: Continuing Education

Student Level: College Graduate

Duration: $\quad 11$ Weeks, $3.0 \mathrm{hrs}$ per week

Contact Hours: 33

Classroom: 33

Topics Covered Extensively: Energy

Storage; Heat and Energy Transfer;

Intro. to Solar Energy; Solar System

Components; Solar Economics; Solar

Collector Evaluation/Design; Solar

Systems Design; Solar Systems

Installation; Solar Systems Testing and

Evaluation; Domestic Hot Water

Number of Times Taught: 1

Average Enrollment:

20 


\section{colleges/Universities}

HEST VIRGIHIA UNIVERSITY

MORGAHTOdiv, West Virginia 26506

(304) 293-01:1

\section{SOLAR RELATED COURSES}

\section{Aercspace Froblems}

Instructor: Toth, John.

(30.4). 2.93-5339

Course Number: AE280:

Department: Engineering Aerospace

Credits: 3

Student Level: All levels

Duration: $\quad 16$ Weeks, 4.0 hrs per week

Contact Hours: 64

Classroom: $\quad 48$

Laboratory: 16

Tepics Covered Extensively: Energy

Conservation; Energy Conversion; Energy

Storsae; Heat and Frergyi Tranefer;

Intro. to Soliar Fneroy: Solar Syetem

Components; Solar Home Construction;

Solar Collector Evaluation/Design:

Solar Systems resting and Evaluation

Nuriber of Times Taught: 4

Average Enrollment: $\quad 15$

Appropriate Technology Seminar

Instructor: Devore, Paul W.

(304) 293-3803

Course Nurber: 390

Department: Technology Education

Credits: 3

Student Level: Junior or Senior

Duration: 15 heeks, $3.0 \mathrm{hrs}$ per week

Contact Hours: 45

Tcpics Covered Extensively: Alternate

Enersy Sources; Appropriate Technology;

Intro. to Solar Energy; Passive Solar

Technology

Number of Times Taught: 2

Averaze Enmollment? 15

Energy and Society

Iristructor: Depue, David

(304) 293-3803

Course Number: 280

Department: Technology Education

Credits: 3

Student Level: College Graduate

Duration: 15 keeks, 3.0 hrs per week

Contact Hours: 45

Clessi urill: 43

Tcpics Covered Extensively: Alternate

Energy Sources; Bicnass Conversion;

Enrergy Conservation; Enëray Conversion:

Passive Solar Technology

Number of Times Taught: 1

Averase Enrollment: $\quad 12$

Production Systems - Technical

Instructor: Fytlik, Eclward

(304) 293-3.503

Course Number: 321

Departrent: Technology Education

Credits: 3

Studant Level: College Graduate

Duration: 15 Wieks, 4.0 hrs per week
Contact Hours: 60

Classroom: $\quad 60$

Topics Covered Extensively: Energy

Conversion; Energy Storage;

Marketing/Market Analysis; Materials

Research; Passive Solar Technology

Number of Times Taught: 10

Average Enrollment: $\quad 10$

Technology - History and Development

Instructor: Pytlick, Edward

Course Number: 400

Department: Technology Education

Credits: 3

Student Level: College Graduate

Duration: 15 Weeks, $3.0 \mathrm{hrs}$ per week

Contact Hours: 45

Classroom: $\quad 45$

Number of Times Taught: 12

Average Enrollment: $\quad 12$

$* * x \times x$ * $* x$

\section{Community/Junior colleges}

PARKERSEURE CMTY COLLEGE

PARKERSBURG, West Virginia 26101

(3828)

(304) 424-8000

\section{SOLAR RELATED COURSES}

Spec. Top. in Air Cond/Refrig: Solar Heat

Instructor: Schmidt, Larry

$1304) 424-8256$

Course Number: ACR 299

Department: Air Cond./Ref.

Credits

Technolnay

4

Student Level: Freshman or Sophomore

Duration: 15 Weeks, 6.0 hrs per week

Contact Hours: 90

Topics Covered Extensively: Appropriate

Technology; Energy Conservationi Enerqy

Storage; Heat and Energy Transfer;

Intro. to Solar Energy; Passive Solar

Technology; Plumbing Techniques; Sheet

Metal Techniques; Solar System

Colmponents; Solar Collector

Evaluation/Design; Solar Systems

Design; Solar Systers Installation:

Solar Systems Maintenance; Solar

Systems Testing and Evaluation;

Doliestic Hot Water; Space Heating

Nuniber of Tines Taught: 1

Average Enrollment: 13

********** 
POTOMAC STATE COLLEGE

(3829)

KEYSER, hest Virginia 26726

(304) 788-3011

SOLAR RELATED COURSES

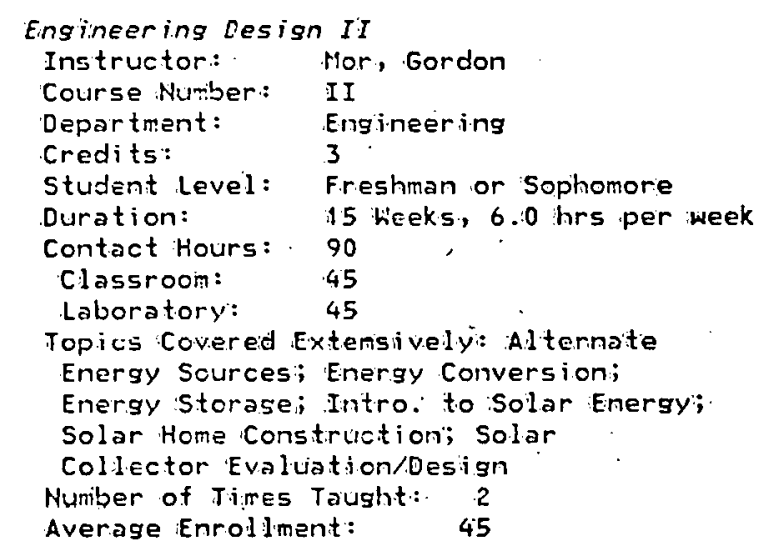




\section{Colleges/Universities}

LAWREKEE UNIVERSITY

APPLETON, Wiscons in 54911

(3856)

(414) 739-3681

\section{SOLAR RELATED COURSES}

Energy and the Environiment

Instructor: Joel, Peteranne

(414) 739-3681

Course Number: CHEM 9

Department: Chemistry

Credits: 3

Student Level: All levels

Duration: $\quad 10$ Weeks, $3.5 \mathrm{hrs}$ per week

Contact Hours: 35

Classrocm: $\quad 35$

Number of Times Taught: 6

Average Errollment: 22

$* * * * * * * * *$

\section{MARQUETTE UNIVERSITY}

(3863)

1515 H. Wisoong in Ave.

MILHAL'KEE, Hisconsin 53233

(414) 224-7700

PRDGRAMS AND CURRICULA

Energy Engineering

Degree: $\quad A D$, Engineering

Contact: Jaskovski, S.V.

(414) 224-6820

Students Taking or Completing offering:

Solar Engineer

\section{SOLAR RELATED COURSES}

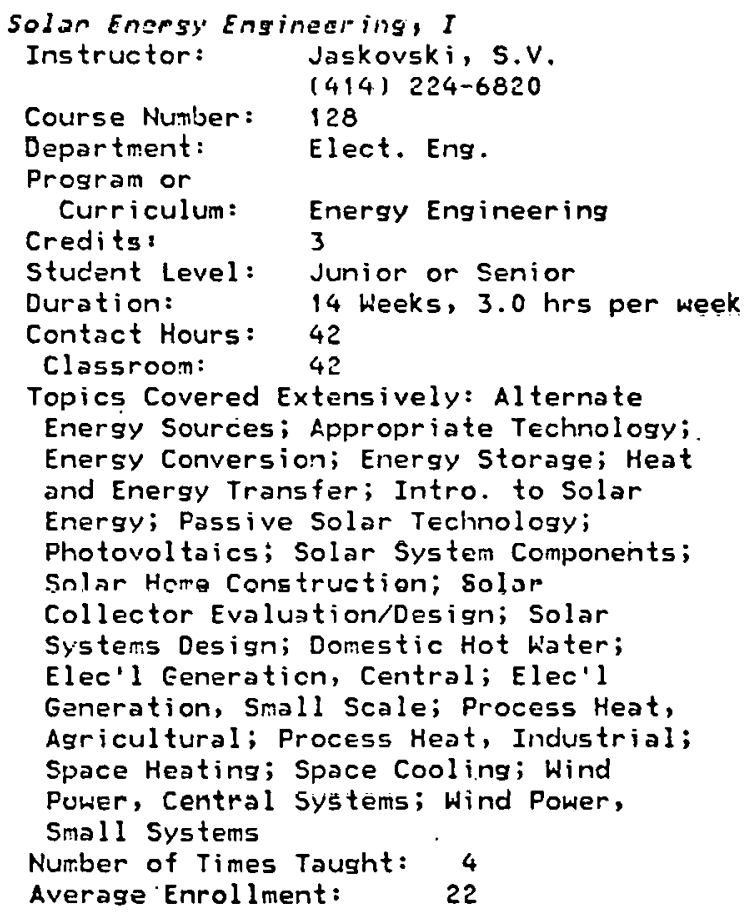

Solar Energy Engineering, II

Instructor:

Jaskovski, S.V.

(414) 224-6820

Course Niumber: 129

Department: Elect. Eng.

Program or

Curriculum: Energy Engineering

Credits: 3

Studient Level: Junior or Senior.

Duration: 14 Weeks, 3.0 hrs per week

Contact Hours: 42

Classroom: $\quad 42$

Topics Covered Extensively: Alternate

Energy Sources; Arpropriate Technology;

Enersy Conversion; Energy Storage; Heat and Energy Transfer; Intro. to Solar Energy; Passive Solar Technology;

Fhatovoltaics; Solar System Components; Solar Hore Construction; Solar

Collector Evaluation/Design; Solar

Systeris Uesign; Domestic Hot Water;

EleE'l Generation, Central; Elec'l

Generation, Small Scale; Process Heat,

Agrieultural; Frocess Heal, Industrlal;

Space Heating; Space Cooling; Wind

Power, Central Systems; Wind Power,

Small Systems

Nuriber of Times Taught: 4

Average Enrollment: 22

***********

MILL!AUYEE SCH EHGIV!EERING

$(3868)$

MILWAUKEE, Wiscons in 53201

(414) 272-8720

\section{SOLAR RELATED COUTSES}

Sojar Energy"

Ins tructor:

Courga Humber:

Mallmonn, A. James

Department:

Credits:

Student Level: Junior or Senior

Duration: il Weeks, $3.0 \mathrm{hrs}$ per week

Contact Hours: 33

Clisssrom: $\quad 33$

Topics Covered Extensively: Energy Conservation; Energy Conversion; Energy Storage; Heat and Energy Transfer; Intro. to Solar Energy; Solar Collector Evaluation/Design; Solar Systems Design; Space Heating; Wind Power, Sniall Systems

Number of Times Taught: 4 Average Enrollment:

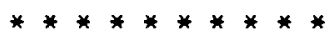


HICOLET COLLEGE- TECH IHST

(8919)

RHINELANDER, Wiscons in 54509

(715) 369-4410

\section{SOLAR RELATED COURSES}

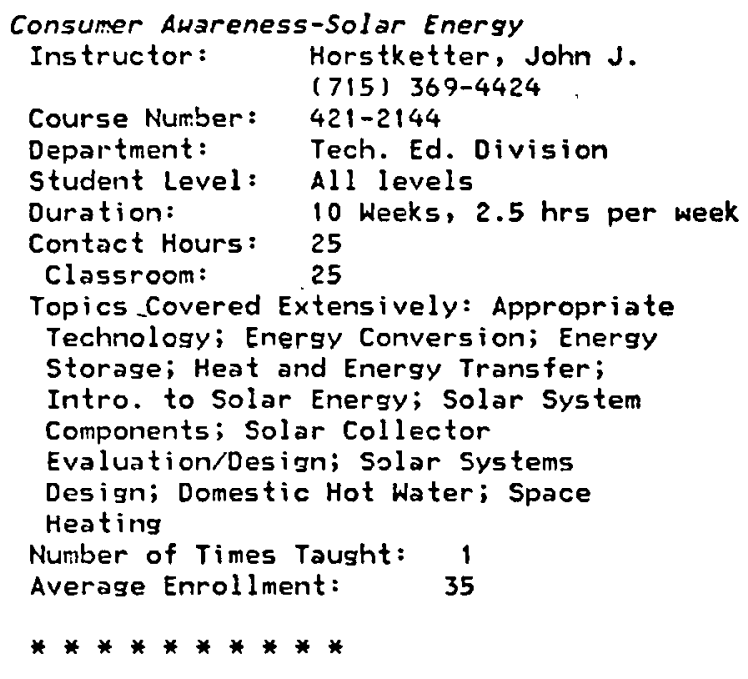

\section{RIFON COLLEGE}

(3884)

RIPON, Wiscons in 54971

(414) 748-8118

\section{SOLAR RELATED COURSES}

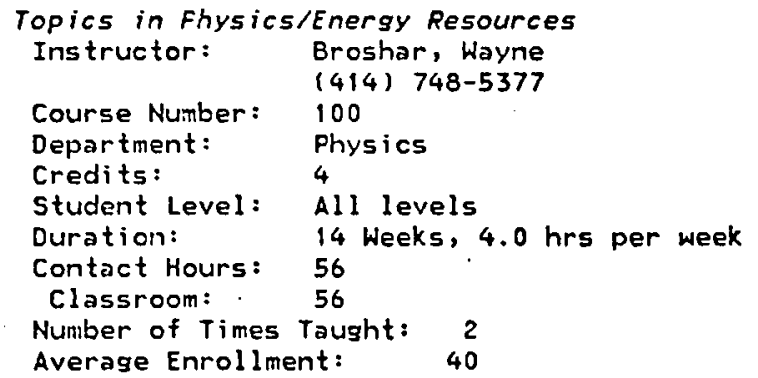

***********

HISCONSIH EAU CLAIRE, U OF

EAU CLAIRE, Wiscons in 54701

(3997)

(715) 836-0123

\section{SOLAR RELATED COURSES}

Solar Enersy

Instructor:

Course Number:

Janke, Robert

(715) $836-3718$

Department:

Credits:

336

Geography

Student Level: Junior or Senior

Duration: 16 Weeks, $3.0 \mathrm{hrs}$ per week

Contact Hours: 48

Topics Covered Extensively: Energy

Conservation; Energy Conversion; Energy

Storage; Heat and Energy Transfer;

Intro. to Solar Energy; Solar System
Components; Solar Economics; Solar

Collector Evaluation/Design; Domestic

Hot Water; Space Heating

Number of Times Taught: 3

Averase Enrollment: $\quad 70$

***********

UISCONSIN GREEN BAY, $U$ OF

(3899)

GREEN BAY, Wiscons in 54302

(414) 465-2121

\section{SOLAR RELATED COURSES}

Introduction to Solar Energy

Instructor: "Norman, Jack

(414) 465-2276

Course Number: $862 / 483 X$

Department: Science Environmental

Credits: 3

Student Level: Freshman or Sophomore

Duration: 8 Weeks, 5.2 hrs per week

Contact Hours: 42

Topics Covered Extensively: Al ternate

Energy Sources; Appropriate Technology;

Biomass Conversion; Energy Conversion;

Energy Storage; Heat and Energy

Transfer; Intro. to Solar Energy;

Passive Solar Technology;

Photovoltaics; Solar Energy Policy

Development; Solar System Components;

Solar Systems Design; Domestic Hot

Water; Space Heating; Wind Power, Small

Systems

Number of Times Taught: 1

Average Enrollment: 5

Solar and Alternate Energy Systems

Instructor: Mehra, Anjani/ Lanz,

Robert

1414) 465-2371

Course Number: $862 / 415$

Department: Interdis. - Sci. and

Credits: $\quad 3$

Student Level: Junior or Senior

Duration: 14 Weeks, 3.0 hrs per week

Contact Hours: 42

Topics Covered Extensively: Alternate

Energy Sources; Photovoltaics; Solar

Collector Evaluation/Design; Solar

Systems Design; Domestic Hot Water;

Space Heating

**********

HISCONSIH LA CROSSE, U OF

LA CROSSE, Wiscons in $\mathbf{5 4 6 0 1}$

(3919)

(608) 785-8000

SOLAR RELATED COURSES

Energy Problems-Solar Options

Instructor; Egbert, G./ Gystrom, $D$.

(608) 785-8431

Department! University Outreach and Physics 


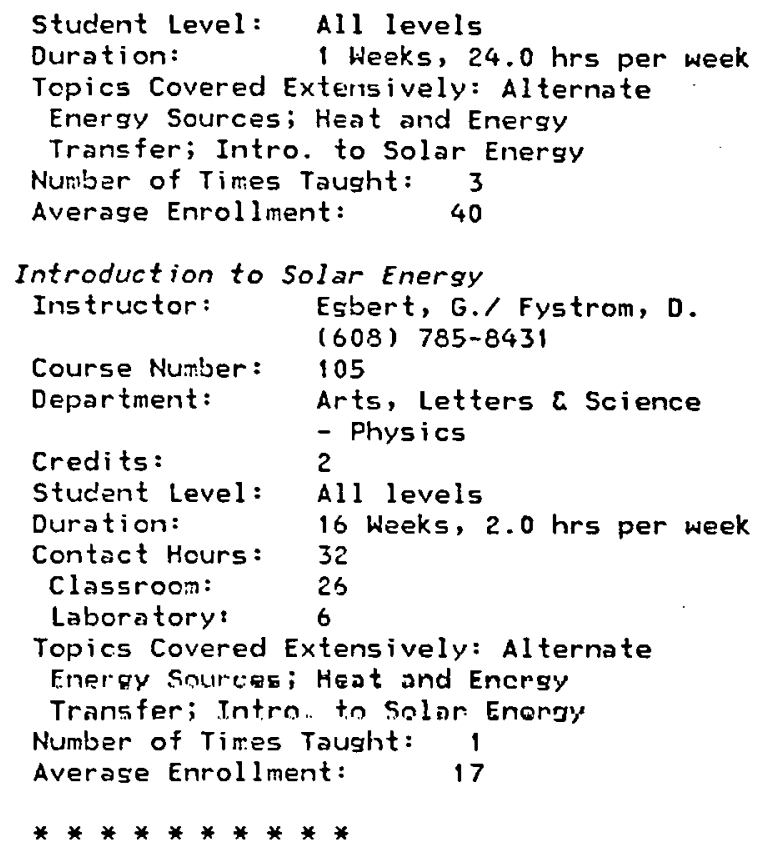

Student Level: Junior or Senior

Duration: 16 Weeks, $3.0 \mathrm{hrs}$ per week

Contact Hours: 48

Classroom: 48

Topics Covered Extensively: Alternate

Energy Sources; Heat and Energy

Transfer; Intro. to Solar Energy; Wind

Power, Snall Systems

Number of Times Taught: 2

Average Enrollment: 21

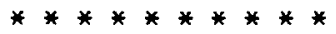

HISCONSIN MILHAUKEE, $U$ OF

(3896)

MILKAUKEE, Wiscons in 53201

(414) $963-4444$

PROGRAMS AND CURRICULA

Solar Architecture

Degree: Architecture

Cuntacl: Catanese, Anthoiny James

(414) 983-4016

Students Taking or Completing Offering:

Architect

\section{SOLAR RELATED COURSES}

Energy Conscious Design

Instructor: Dent, $5 . /$ ollwang, J./

Schade, J.

(414) $963-5650$

Course Number: $470 / 770$

nepartment: Architcoture and Urban Planning

Progran or Curriculum: Solar Architecture

Credits:

Student Level: Junior or Senior

Duration: 16 Weeks, 16.0 hrs per week

Contact Hours: 256

Classroom: $\quad 52$

lahoratory: 204

Topics Covered Extensively: Energy Conservation; Intro. to Solar Energy; Passive Solar Technology; Solar System Components; Solar Systems Design; Space Heating; Space Cooling

Number of Times Taught: 6

Average Enrollmenti 10

Energy Design Fundamentals

Instructor: Shade, John

(414) 963-4014

Course Nurber: ARCH 497

Devartment: Arrhitortion

Program or Currlculum: Solsr Architecture

Credits:

Student Level: Junior or Senior

Duration: 15 Wceks, 3.0 hrs per week

Contact Hours: 45

Topics Covered Extensively: Alternate Energy Sources; Appropriate Technology; Energy Conservation; Energy Storase; Intro. to Solar Energy; Passive Solar Technolcgy; Solar Economics; Solar Home Construction; Solar Systems nesign; Domestic Hot Water; Space Heating 
Number of Times Taught: 3

Average Enrollment: 30

Energy Design Fundimentals II

Instructor: Dent, Stephen D.

(414) $963-5650$

Course Number: 497

Department: Architecture and Urban Planning

Program or

Curriculum: Solar Architecture

Credits: 3

Student Level: Junior or Senior

Duration: 16 heeks, 3.0 hrs per week

Contact Hours: 48

Topics Covered Extensively: Alternate

Energy Sources; Energy Conservation;

Energy Storage; Intro. to Solar Energy;

Passive Solar Technology; Solar System

Components; Solar Collector

Evaluation/Design; Solar Systems

Design; Space Heating

Number of Times Taught: 2

Average Enrollment: $\quad 35$

opt. in Energy Conscious Design

Instructor: Schade, John

(414) 963-4014

Course Number: ARC $470 / 770$

Department: Architecture

Program or

Curriculum: Solar Architecture

Credits:

Student Level: 9

Duration:

Junior or Senior

15 Weeks, 9.0 hrs per week 135

Contact Hours:

Topics Covered Extensively: Alternate

Energy Sources; A.ppropriate Technology;

Energy Conservation; Energy Storage;

Intro. to Solar Energy; Passive Solar

Technolosy; Solar Economics; Solar Home

Construction; Solar Systems Design;

Domastic Hot Water; Space Heating

Number of Times Taught: 3

Average Enrollment:

30

Solar Owelling Design

Instructor: Dent, Stephen D.

(414) 963-5650

Course Number: 497

Department: Architecture and Urban

Plaming

Program or

Curriculum:

Credits:

Solar Architecture

Student Level: Junior or Senior

Duration:

Contact Hours: 40

Topics Covered Extensively: Energy

Conservation; Energy Storage; Heat and

Energy Transfer; Intro. to Solar

Energy; Passive Solar Technology; Solar

System Components; Space Heating

Number of Tin:es Taught: 1

Average Enrollment: 25

Solar Engineering

Instructor: Neusen, K.F. (414) 963-5001

Course Number:

$330-436$

\author{
Department: \\ Engr. C Appl. \\ Credits: \\ Sci./Energetics \\ Credits: 3 \\ Student Level: Junior or Senior \\ Duration: 15 Weeks, $3.0 \mathrm{hrs}$ per week \\ Contact Hours: 45 \\ Clissroom: $\quad 40$ \\ Laboratory: 5 \\ Topics Covered Extensively: Heat and \\ Energy Transfer; Intro. to Solar \\ Energy; Solar System Components; Solar \\ Collector Evaluation/Design; Space \\ Heating \\ Number of Times Taught: 2 \\ Average Enrollment: $\quad 18$

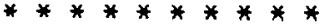

HISCONSIM OSHKOSH, U OF

(9630)

OSHKOSH, Hisconsin 54901

(414) 424-\$234

SOLAR RELATED COURSES

Solar Energy

Instructor:

Passow, M. W.

(414) 424-4430

Course Number: $82-755$

Department: Physics

Credits: 3

Student Level: College Graduate

Duration:

Contact Hours: 60

Classroom: $\quad 30$

Laboratory: $\quad 30$

Topics Covered Extensively: Heat and

Energy Transfer; Intro. to Solar

Energy; Solar System Components; Solar

Economics; Solar Hone Construction;

Solar Collector Evaluation/Design;

Solar Systens Design; Domestic Hot

Hater; Space Heating

Number of Times Taught: 2

Average Enrollment:

16

Solar Heat ing

Instructor:

(414) 424-4430

Course Number: $82-355$

Department: Fhyslcs

Credits: 3

Student Level: Freshman or Sophomore

Duration: 14 Weeks, $3.0 \mathrm{hrs}$ per week

Contact Hours: 42

Classroom: $\quad 42$

Topics Covered Extensively: Energy

Storage; Heat and Energy Transfer;

Intro. to Solar Energy; Passive Solar

Technolosy; Solar System Components;

Solar Economics; Solar Home

Construction; Solar Collector

Evaluation/Design; Solar Systems

Design; Solar Systems Installation;

Solar Systens Testing and Evaluation;

Domestic Hot Water; Swinming Pool

Heating; Space Heating

Nuniber of Tinies Taught: 1

Average Enrollment:

18 


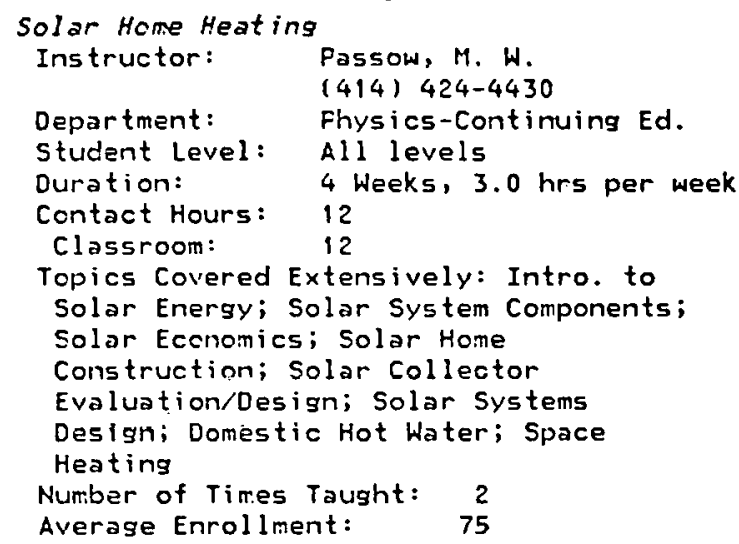

(3921)
HISCOHSIN STEVENS PNT, $U$ OF

(3924)

STEVENS POINT, Wiscons in 54481

(715) 346-0123

\section{SOLAR RELATED COURSES}

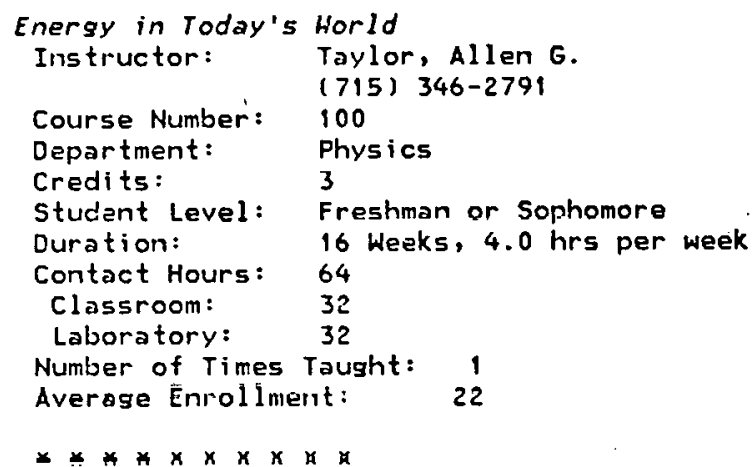

Energy in Industry Instructor: Rhoads, Charles (715) $232-1156$

Course Number: 110-101

Department: Industry and Technology Credits:

Student Level: Freshman or Sophomore

Duration: 9 Weeks, $6.0 \mathrm{hrs}$ per week Contact Houra: $\quad 54$

Classroom: 27

Laboratory: 27

Number of Times Taught: 20

Average Enrollment: $\quad 50$

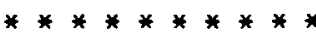


HISCONSIH HHITEHATER, $U$ OF

(3926)

WHITEWATER, Wiscons in 53190

(414) 472-1234

\section{SOLAR RELATED COURSES}

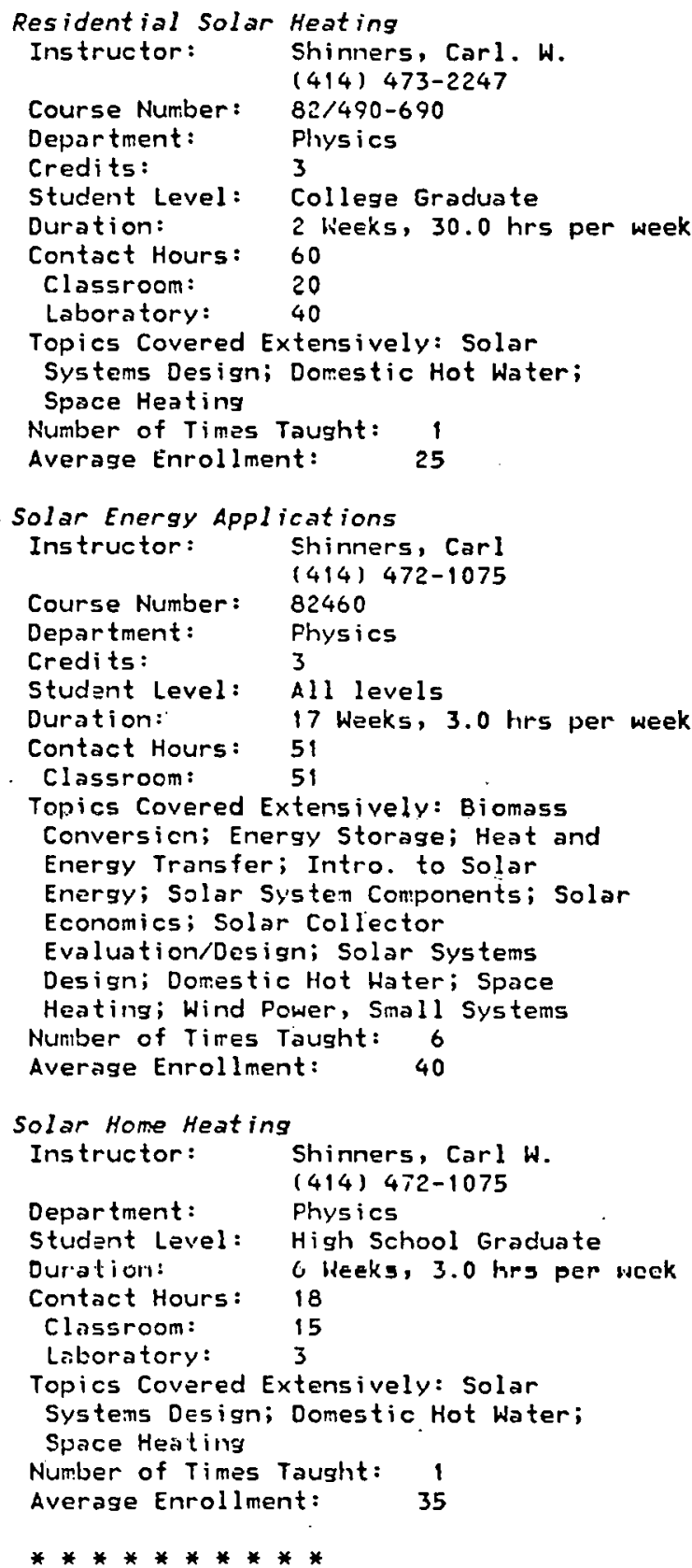

DISTRICT OKE TECH IHST

EAU CLAIRE, Wiscons in 54701

(715) 836-3975

\section{SOLAR RELATED COURSES}

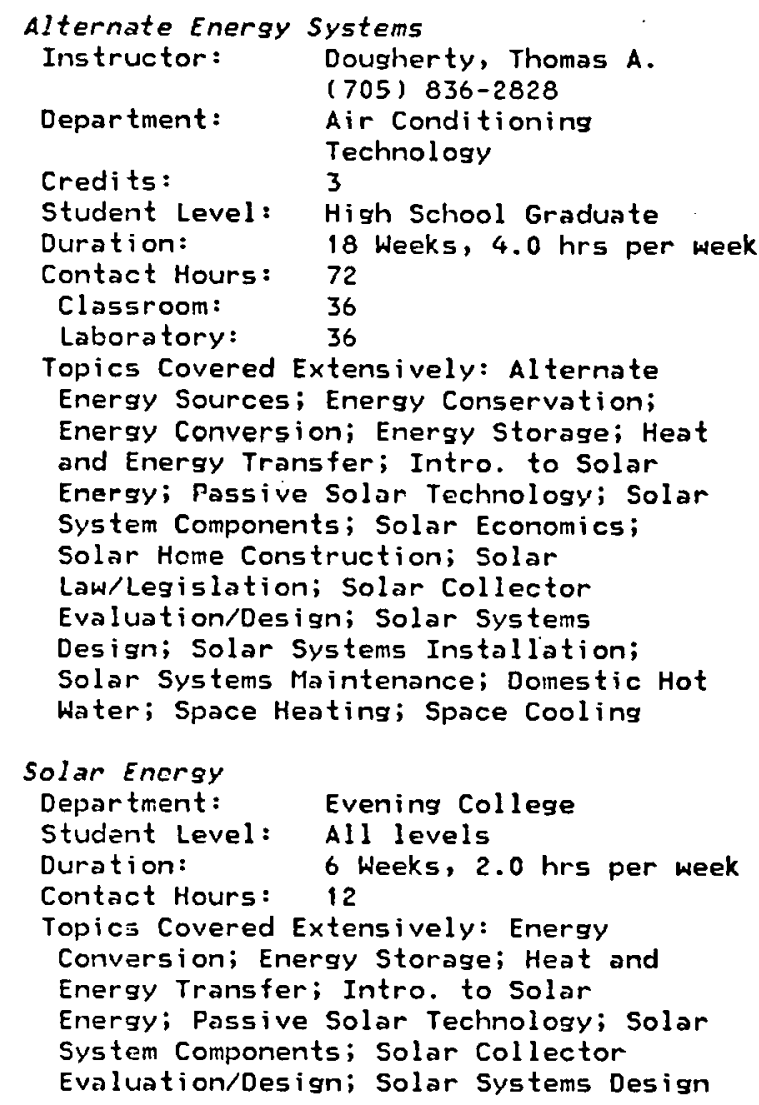

**********

MILHAUKEE AREA TECH C

MILWAUKEE, Wiscons in 53203

(3866)

(414) 278-6600

SOLAR RELATEN TOLIRSES

*Ener. Cons. E Alt. Ener. Sources

$* * * * * * * * * *$

MORAIIE PARK TECH INST

FOND DU LAC, Wiscons in 54935

(414) 922-8611

PROGRAMS AND CURRICULA

Solar Energy

Contact: Pasch, Rodney (414) 922-8611

Students Taking or Completing offering: Contractor, Do-it-yourself Homeowner, Electrician, Plumber, Sheet Metal Worker 


\section{SOLAR RELATED COURSES}

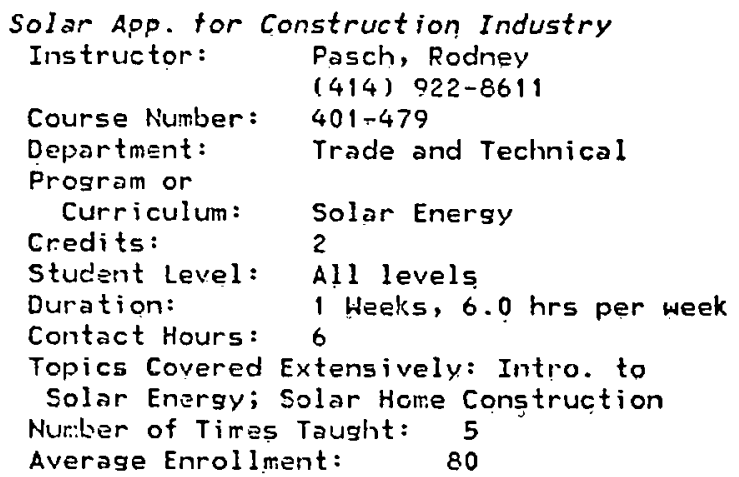
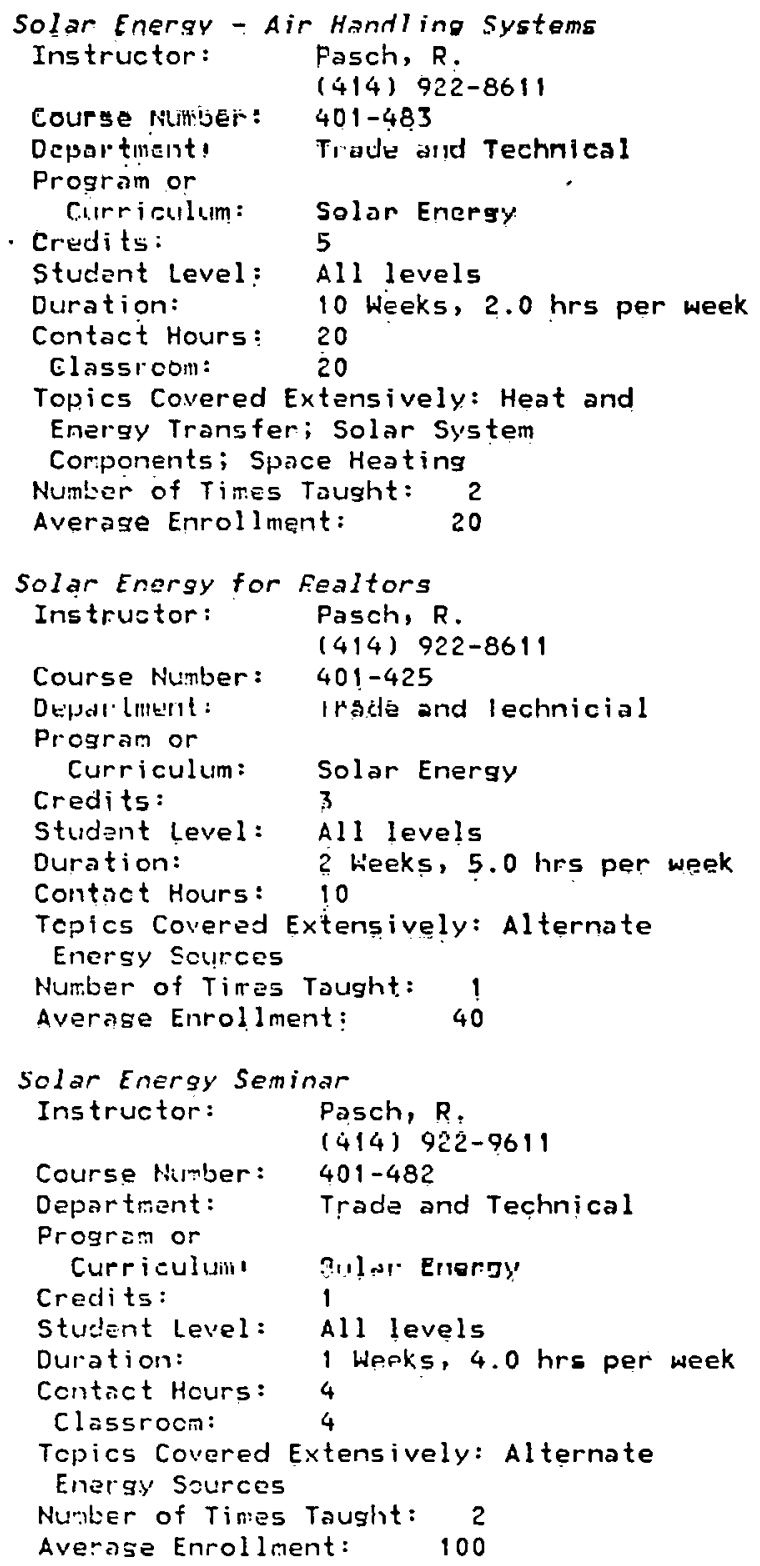

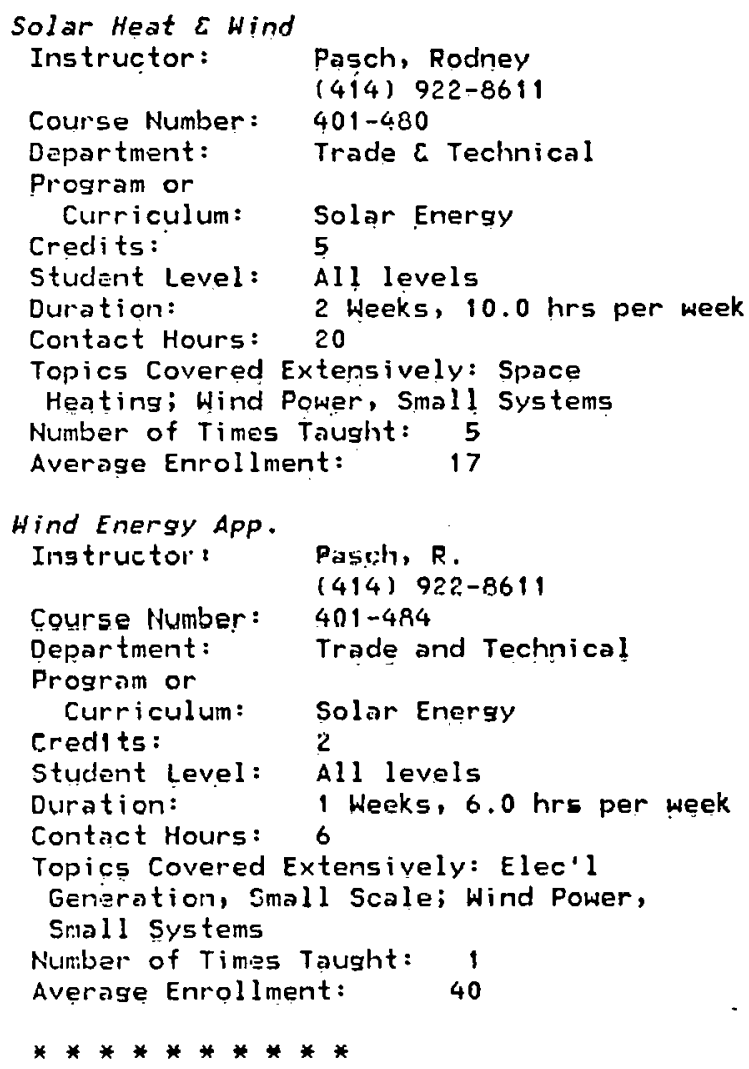




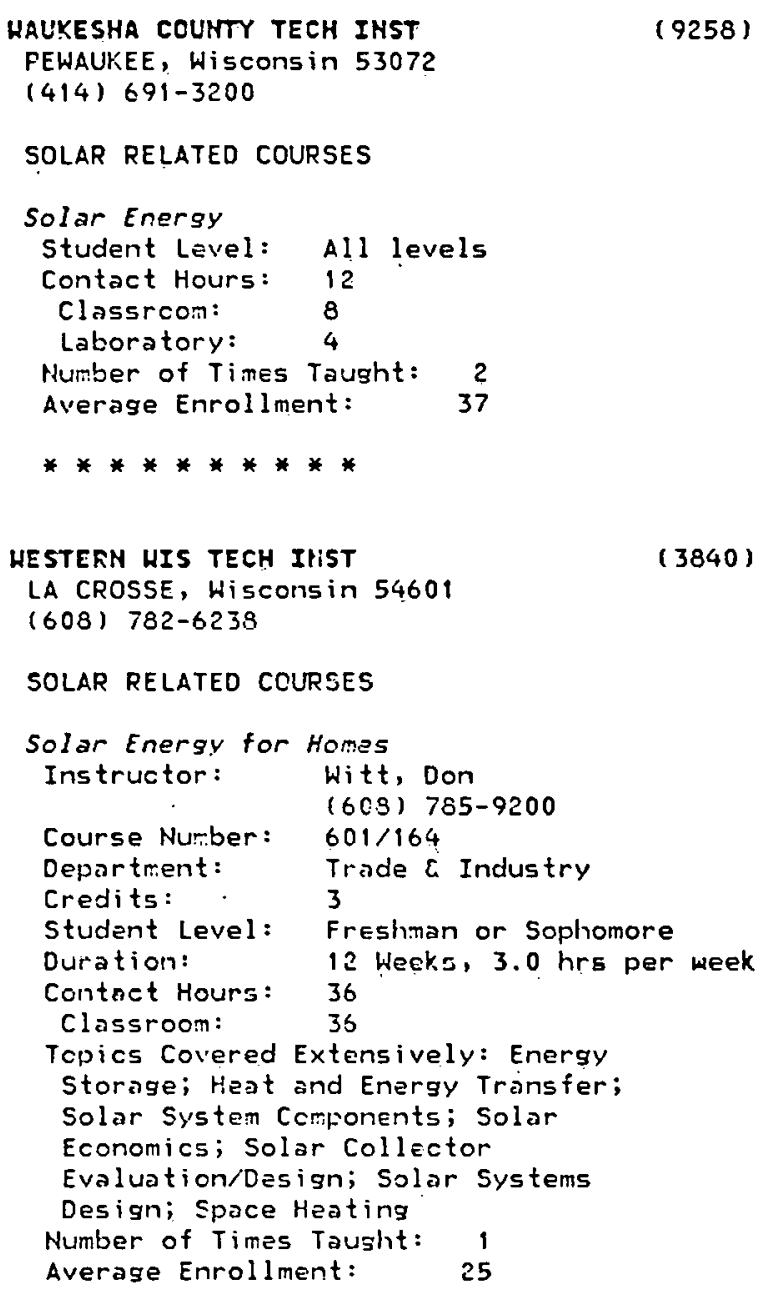

(9258)

(3840)

Duration:

Contact Hours:

Classroom:

Laboratory:

Energy Sources; Appropriate Technolosy;

Biomass Conversion; Energy.

Conservation; Energy Conversion; Energy

Storage; Intro. to Solar Energy;

Passive Solar Technology;

Photovoltaics; Solọr System Components;

Solar Economics; Solar Systems

Installation; Solar Systems.

Maintenance; Solar Systems Testing and

Evaluation; Wind Power, Small Systems 


\section{Colleges/Universities}

KYOHIHG, UNIVERSITY OF

LARAMIE, Hyoming 82071

(3932)

(307) $766-4121$

\section{SOLAR RELATEO COURSES}

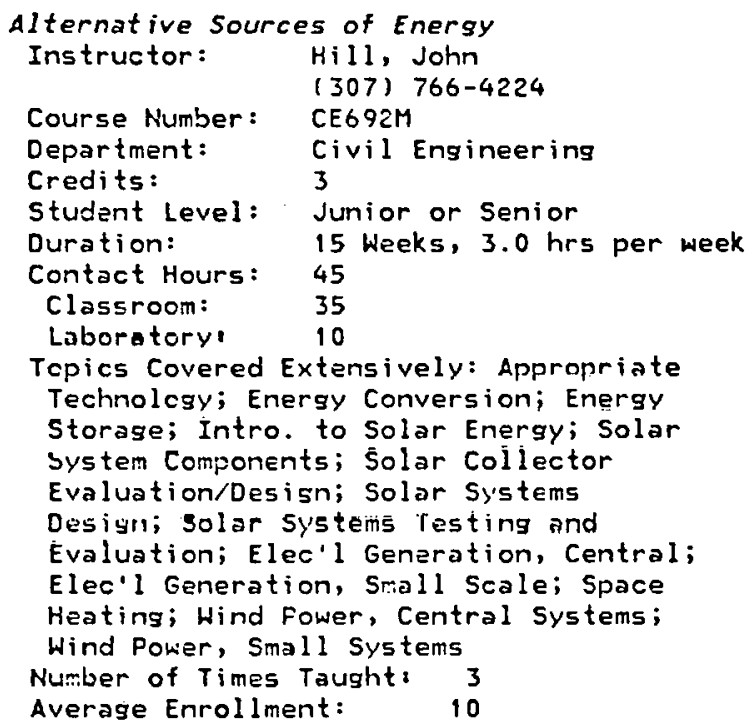

Atmos. Sci. Prob.: Atmospheric Radiation

Instructor: Veal, Donald L.

(307) $766-3245$

Course Number: $890 \mathrm{M}$

Department: Engineering/Atmospheric Science

Credits: 3

Student Level: College Graduate

Duration: 13 heeks, 3.0 hrs per week

Contact Hours: 39

Classroom: $\quad 39$

Number of Times Touglit: 2

Average Enrollment: 3

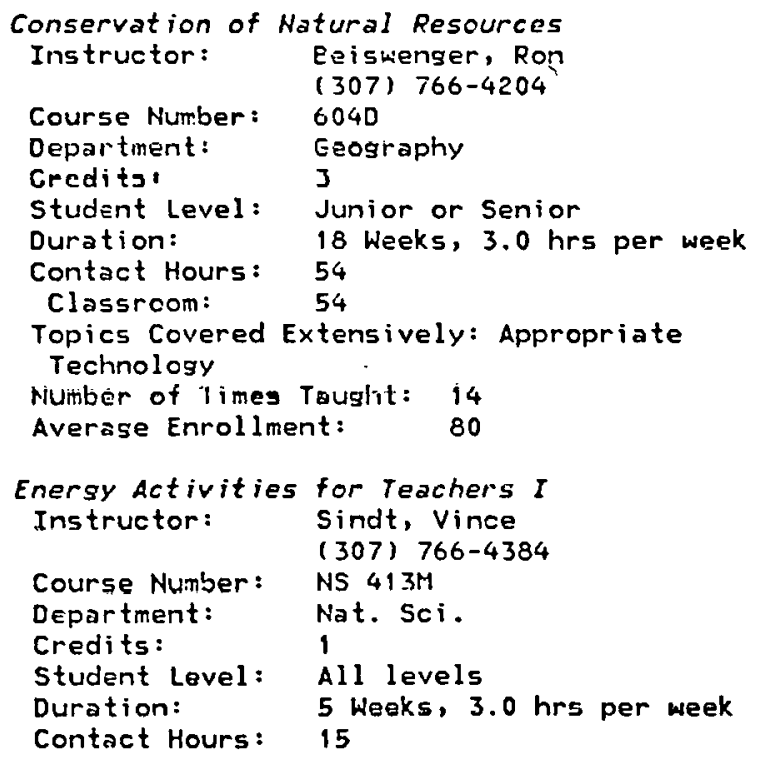

Topics Covered Extensively: Alternate Energy Sources; Appropriate Technology; Energy Conservation; Intro. to Solar Energy; Materials Research; Passive Solar Technology; Wind Power, Small Systems

Number of Times Taught: 5 Average Enrollment: $\quad 20$

Energy Activities for Teachers II

Instructor: Sindt, Vince

$\begin{array}{ll} & (307) 766-4384 \\ \text { Course Number: } & \text { N5679M }\end{array}$

Department: Nat Sci

Credits: 2

Student Level: Junior or Senior

Ouration: 2 Weeks, 25.0 hrs per week

Contact Hours: 50

Topics Covered Extensively: Alternate

Energy Sources; Appropriate Technology;

Fnergy Consorvation; Intro, to Solar

Energy: Materiale Pesenrch: Pagaive

Solar Technology; Wind Power, Small Systems

Nuriber of Times Taught: 5

Average Enrollment: $\quad 20$

Energy Conversion

Instructor: Amr, Abdel-Fattah

(307) 766-6139

Course Number: 602

Department: Mechanical Engineering

Credits: 3

Student Level: Junior or Senior

Duration: 15 Heeks, $3.0 \mathrm{hrs}$ per week

Contact Hours: 45

Classroom: $\quad 45$

Topics Covered Extensively: Alternate

Energy Sources; Energy Conversion;

Intro. to Solar Energ'; Sola! Syslem Cormponents

Number of Times Taught: 4

Avcrage Enrollment: $\quad 20$

Environmental Education for Teachers

Instructor: Beiswenger, Ron

(307) $766-4204$

Course Number: 6710

Departient: $\quad$ Ed. Curriculum and

Student Level: Junior or Senior

Duration: 18 Weeks, 4.0 hrs per week

Contact Hours: 72

Classroom: $\quad 36$

Laboratory: $\quad 36$

Number of Times Taught: 14

Average Enrollment: $\quad 15$

\section{Community/Junior Colleges}


CASPER COLLEGS

CASPER, Wyoming 82601

(307) 268-2110

\section{SOLAR RELATED COURSES}

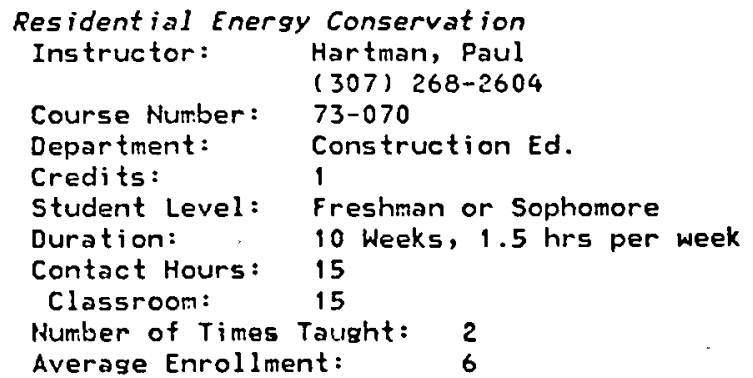

***********

CENTRAL HYOMING COLLEGE

RIVERTON, WYOTIII 82501

(307) 856-9291

\section{SOLAR RELATED COURSES}

Solar Energy

Instructor:

Course Humber:

Hansen, M. R.

(307) 856-9291

Department:

EPlGR 240

Life C Physical

Credits:

Sciences

Student Level: Freshman or Sophomore

Duration: 16 hieks, $3.0 \mathrm{hrs}$ per week

Contact Hours: 48

Classrocm: 0

Laboratory: 48

Topics Covered Extensively: Intro. to

Solar Energy; Solar Collector

Evaluation/Design; Solar Systems

Testing and Evaluation; Domestic Hot

Water

Number of Times Taught: 1

Average Enrollment:

6

**********

LARAMIE CO CHTY COLLEGE

CHEYENNE, Hyoming 82001

(9259)

(307) 634-5853

\section{SOLAR RELATEO COURSES}

\section{Energy and Man}

Instructor:

Edwards, William C.

Course Number: SCI103

Department:

Science

Credits: 3

Student Level:

Duration:

Freshman or Sophomore

18 Weeks, 3.0 hrs per week

Contact Hours:

Classroom:

54

40

Topics Covered Extensively: Alternate

Energy Sources; Energy Conservation

Number of Times Taught:

Average Enrollment:

$(7289)$ 


\section{SOLAR RELATED COURSES}

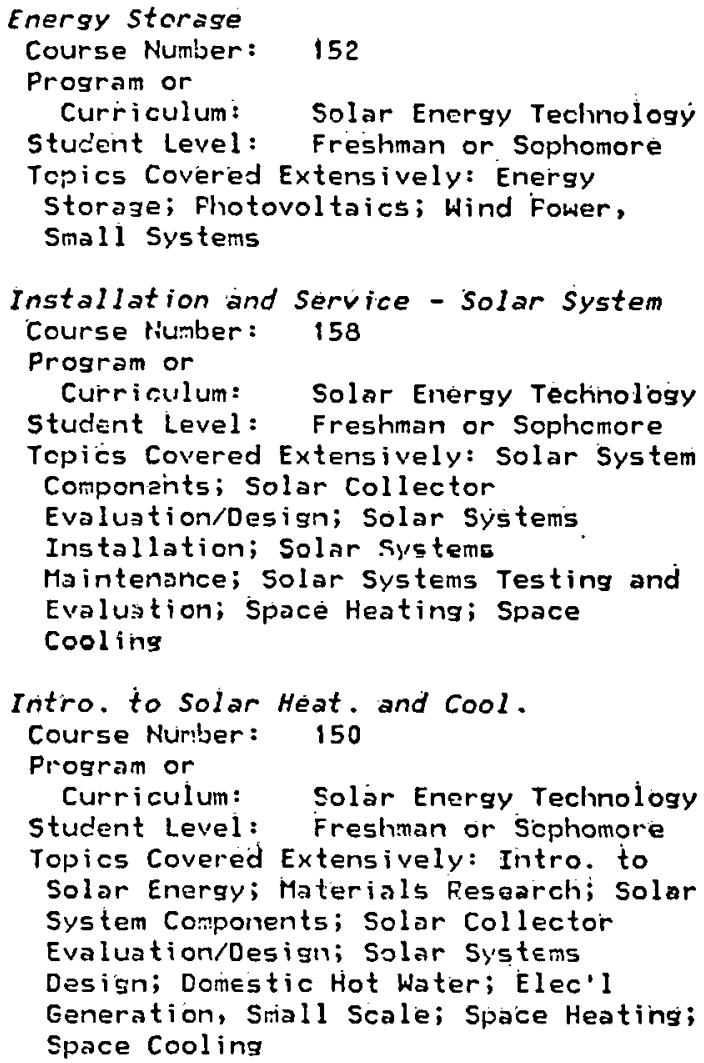

Intro. to Solar Heat. and Cool.

Course Nurber: 150

Program or

Curriculum: Solar Energy Technology

Student Level : Freshman or Sicphomore

Topics Covered Extensively: Intro. to Solar Energy; Materials Research; Solar System Components; Solor Collector Evaluation/Desisn; Solar Systems Design; Domestic Hot Water; Elec'l Generation, Sriall Scale; Space Heating; Space Cooling

Solar Collectors

Course licitber: 151

Program or

Curriculum: Solar Energy Technology

student Level: Freshman or Sophomore

Topics Covered Extensively: Solar System Components; Solar Collector

Evaluation/Desian; Snlar Sy, tems

Design; Solar Systems Testing and

Evaluation

Solar Energy fundamentals

Instructor: Ohm; Kenneth $\mathbf{R}$. $(307) 674-6446$

Course Number: $19-190$

Department:

r.aregr.Teoh

Program or

Curriculum:

Credits: Solar Energy Techniringij 3

Student Level: Freshman or Sophomore

Duration:

Contact Hours:

Closeroom: 16 Weeks, 4. 0 hrí per wèk 60

Laboratory:

40

12

Topics Covered Extensively: Al ternate Energy Sources; Appropirite Technology; Energy Storage; Heat and Energy Tranisfer; Intro. to Solar Energy; Solar Home Construction; Solar Collector Evaluation/Design; Solar Systems Design; Solar Systems Installation; Solar Systems Maintenance; Domestic Hot Water; Process Heat, Iridustrial; Space
Heating; Space Cooling; Wind Power, Central Systems; Wind Power, Small Sys tems

Number of Times Taught: 2

Average Enrollment: $\quad 35$

Solar Heating and Cooling systems

Course Number: 155

Program or

Curriculum: Solar Energy Technology

Student Level: Freshman or Sophomore

Topics Covered Extensively: Energy

Storage; Solar System Components; Solar

Collector Evaluation/Oesign; Solar

Systens Design; Domestic Hot Hater;

Process Heat, Agricultural; Frocess

Heat, Industrial; Space Heating; Space

Cooling

Hind systems

Course Number:

Prouram or

rurrirulum. 6olal Cirergy Technology

student Level: Freshman or Sophomore

Topics Covered Extensively: Wind Power,

Central Systems; Wind Power, Small

Systenis

**********

UESTERH HYO CHTY COLLEGE

(3933)

ROCK SPRIHGS, Wyoming 8290

(307) $382-2121$

\section{SOLAR RELATED COURSES}

Solar Home Planning

Instructor:

Bowles, Marvin

(307) 382-2121

Course liumbei: $52-299$

Department: Building Trades

Credits:

Student Level: High Srhnnl Graduate

Duratio

15 Weeks, 3.0 hrs per week

Contact Hours: 45

Classroom: $\quad 30$

Laborntory:

15

Tcpics Covered Extensively: Al ternate

Energy Sources; Apprcpriate Technology;

Bionisss Conversion; Energy

Conservation; Energy ronvarsion; Energy Storage; Heat and Energy Transfer;

Intro. to Solar Energy; Photovol taics;

Plumilung ieclinicques; Shest lietal

Techniques; Solar System Coniponents;

Solar Home Constructioni Snlar Sjatame

Design; Solar Systens Installation;

nomestic Hot Woter I Sined Hasting; Wind

Power, Small Systenis

Nunber of Times Taught: 4

Average Enrollment: $\quad 35$

**** $* \dot{*} * * \dot{*} * \dot{*}$ 
THIS PAGE

\section{WAS INTENTIONALLY LEFT BLANK}


Index to Institutions

$-\cdots--A$

A $-\cdots-$

ADAMS STATE COLLEEG, 45

ADELPHI UNIVERSITY, 165

ADIRONDACK CMTY COLLEGE, 177

AIR FOPCE IHIST TECHNOLCGY, 192

AKRON MAIN CAMPUS, $U$ OF, 192

ALAA PROFESSIOHAL STUDY SERIES, 182

ALABAMA A C M UNIVERSITY, 1

ALADAMA IRS BIRMINGHAM, $U, 1$

ALABAMA IN HUNTSVILLE, $U, i$

ALASKA AR:CHOPOAGE CC, $U$ OF, 3

ALASKA METH UNIIVERSITY, 3

ALLEGANY CMTY COLLEEE, 111

ALTERNATIVE ENEPGY PESOURCE ORG.; 147

AMERICAN RIVER COLLEGE, 30

AMERICAN SOCIETY OF MECHANICAL ENGINEERING, 183

AMERICAN TECHNOLOGICÁL U, 224

AHHERST COLLEGE, 1 II 3

ANTELOPE VALLEY COLLEGE; 30

ANIIOCH COLLEGE, 192

ANTIOCH COLLEGE/WEST, 43

APPALACHIAN ST UNIVERSITY, 184

ARIZOIIA STATE UIJIVERSITY, 5

ARIZONA; UNIVERSITY OF̈, 6

ARKANSAS MAIN CAMPUS, U OF; 11

ATLANTIC, COLLEGE OF THE, I08

AUEURN U AT MOHTGOMERY, 2

AUSURI, $U$ MAIM CAMFUS, 2

AUGSEURG COLLEGE, 135

BAKERSFIELO COLLEGE, 30

BALL STATE UNIVERSITY, 88

BARD COLLEGE, 165

BARRY COLLEGE, 64

EARTOH CO CIITY JR COLLEGE, 102

EEAUFORT TECH EO CEITTER; 219

BEMIDJI STATE $U, 135$

BENAEOICTINE COLLEGE, 93

BISHARCK JLWIOR COLLEGE, 191

BLUE HILLS PEG TECH ITIST; 121

BLUE RIOEE CIITY COLLEGE, 250

BOSTOI ARCHITECTURAL CENTER; 121

BOSTON COLLEGE, 113

EOSTCN LIVIVERSITY, 1 i3

BRADLEY, UNIV'ERSITY, 78

GREMAU COLLLESE, 70

BREVAFD CITTY COLLEGE, 66

BRIOGEWATER STATE COLLEGE, 113

BRISTOL CCHRTUIITY COLLEGE, II

BROOKÓALE CMTY COLLEGE; 159

BROONIE-DELAWARE-TIOSA BOLES; 183
BROWN UNIVERSITY, 216

GRUNSHICK JUHIOR COLLEGE, 71

BUCKS COUNTY CMTY COLLEGE, 211

BUINER HILL CMTY COLLEGE, I19

BUTTE COLLEGE, 31

CABR ILLO COLLEGE, 31

CAL IHIST OF TECHNOLOGY, 14

CAL POLY ST UNI. - SAN LUIS OB, 14

CAL STATE C- DOMINGUEZ HLS, 15

CAL STATE C- SAN BERIHAROIHO, 15

CAL STATÉ COLLEGE - SONOMA, I5

CAL STATE POLY U- FOMONA, 16

CAL STATE U- CHICO, 16

CAL STATE U- FRESHO, 16

CAL STATE U- FULLERTON, 16

CAL STATE U- HAYWARD; 16

CAL STATE U- LOHG BEACH, 17

CAL STATE U- LOS AHEELES, 18

CAL STATE U- NORTHRIDGE; 18

CAL- BERKELEY, U⿺ OF, 18

CAL- DAVIS, U OF, 21

CAL - LOS ANGELES; U OF, 21

CAL- RIVERSIDE, U OF; 23

CAL- SAN DIEGO, U OF, 23

CAL- SAHTA BAPBARA, U OF, 24

CAL- SAIITA CRUZ, U OF, 24

CALIFOPHIA STATE COLLEGE, 206

CAPE COD CMTY COLLEGE, 119

CAPE FEAR TECHIIICAL INST, 183

CARL SAIIDBURG COLLEGE, 84 CARTERET TECHINICAL IHST, 186

CASFER COLLLEE, 266

CATHOLIC U OF AMERICA, 62

CAYUSA CO CHTY COLLEGE, 177

CENN PIEDTONT CMTY COLLEGE, 187

CEITTER FOP EMPLOYMEHT TRAINIHG, 43

CEHTRRAL ARKAHSAS; $U$ OF, 11

CEIITRAL CONAI ST COLLEGE, 58

CENTRAL METHODISTT COLLEGE, 141

CEMTRAL MICH UHIVERSITY, 123

CEHTRAL MO ST UIIIVERSITY, 141

CEHITRAL OHIO TECHHICAL C, 198

CENTRAL TECH CHITY COLLEGE, 148

CENTRAL TEXAS COLLEGE, 234

CEHTRAL HESLEYAN COLLEGE, 217

CENTRAL WYOHIIHG COLLEGE, 266

CERRO COSO CHTY COLLEEE, 32

CHABOT COLLEGE-VALLEY CAMFUS, 32

CHAFFEY COLLEGE; 32

CHAS S MOTT CHTY COLLEGE, 131

CHESTEPFLD-MARLEORO TECH, 219

CHICAGO STATE UIJIVERSITY, 78

CHICAGO, UHIVEPSITY OF, 78

CIRCIHHATI MIAIN CAM, U OF, 192

CITRUS COLLEGE, 33

CITY C CHICAGO LOOP C, 84

CITY COLL OF CHICAGO- CITY WIDE C., 78 
CLACKAMAS CMTY COLLEGE, 204

CLARK CO CMTY COLLEGE, 151

CLARK UHIVERSITY, 113

CLARKSOIN COLLEGE OF TECHN, 165

CLEMSON UNIVERSITY, 217

CLEVELAND CO TECH INST, 189

CLEVELANO ST CIITY COLLEGE, 223

COASTAL CAROLIHA CC, 187

COASTLINE CMTY COLLEGE, 33

COCHISE COLLEGE, 7

COSSINELL COLLEGE, 24

COLBY COLLEGE, 108

COLLEGE OF SOLAR ENIERGY, 57

COLO MTN COLLEGE H!EST CAM, 52

COLO TECHINICAL COLLEGE, 45

COLO- COLO SFRINGS, U OF, 46

COLORADO AT GDULDEP, U OF, 47

COLORADO OFFICE OF ENEPGY CONSERVATION, 57

COLORADO SCHOOL OF MIIHES, 49

COLORADO STATE UAIVERSITY, 50

COLUIBIA JUIIIOR COLLEGE, IS

COLUMBIA U MAIH DIVISION, 165

COLUHSIA-CREEHE CG, 178

COLUHEUS TECHIIICAL IHST, 198

LUIN MAIN CAIFIUS, $U$ OF, 58

COPNELL U STATUTORY $C, 160$

CORNERSTUHLS, HIN'S SCHOHI OF SHELTER

TECHNOLO, 109

COSUINIES RIVER COLLEGE, 33

CRONOER COLLEGE, 145

CUESTA COLLEGE, 34

CUNY BROCKLYN COLLEEE, 166

CUNY C OF STAIEN ISLAIID, 166

CUNY CITY COLLEGE, 167

CUNY GRAD SCH \& U CENTER, 167

CUNY NEW YOPK CITY CC, 178

- - - D D - - - -

DANVILLE CMTY COLLEGE, 250

DARTHOUTH COLLEGE, 153

DAYTOH, UNIYERSITY OF, 193

DE ANZA COLLEGE, 34

DEFIANICE COLLEGE, 194

DEKALB COII:UNITY COLLEGE, 71

DELAWARE CO CMTY COLLEGE, 211

DELAWARE, UHIVERSITY OF, 61

DELGADO COLLEGE, 106

DELTA COLLEEE, 131

DELTA STATE LHIVERSITY, 139

DEHIVER NCRTH CAIFIS, CC OF, 53

DENVER RED ROCKS CAM, CC OF, 53

DENVER, UNIVERSITY OF, 50

DEPAUL UNIVERSITY, 78

DES MOINIES AT.CA CC, 34

DESERT, COLLEGE OF THE, 34

ULTROIT, LWIVERSITY UR, 123

DIABLO VALLEY COLLEGE, 34

DICKINSOH COLLEGE, 206

DISTRICT ONE TECH INST, 262

DIVIHE HCRD COLLEGE, 92

DIXIE COLLEGE, 243

DOMESTTR TECH:NOLOSY INSTITUTE, 5T

DREAEL UNIVERSITY, 206

DUKE UNIVERSITY, 184

DUNDALK CMTY COLLEGE, 111
DUPAGE, COLLEGE OF, 84

EARLHAM COLLEGE, 88

EAST CAPOLINA UNIVERSITY, 184

EASTERN ILL UNIVERSITY, 79

EASTERN MICH UHIVERSITY, 123

EOISONV COMPUNITY COLLEGE, 66

EMFORIA STATE UNIVERSITY, 98

ENERGY ED. SERVICES OF CONHNCTICUT, 60

ESSEX COUINTY TECHNICAL CAREERS CENTER, 160

EVERGREEN VALLEY COLLEGE, 35

-...- F - - - -

FARLGH DCKSN TEANECK CAM, 155

FEATHER RIYLR COLLEOE, : 35

FERRIS SIATE COLLEGE, 123

FERRUH COLLEGE, 247

FLATHEAD VLY CHTY COLLEGE, 147

FLORENCE DARLINGTON TECH, 219

FLORIDA IHST TECHNOLOGY, 64

FLORIDA INTERNATIOHAL U, 64

FLORIDA SOLAP. ENERGY CENTER, 69

FLORIDA TECHNOLOGICAL U, 65

FLORIDA, UHIVERSITY OF, 65

FOOTHILL COLLEGE, 35

FOPT LEHIS COLLEGE, 50

FORT STEILACOOM CC, 253

FRANIKLIN INST OF BOSTON, 120

FRESNO CITY COLLEGE, 35

FULLERTON COLLEGE, 35

- - $G$ - - - -

GA INST OF TECHN MAIN CAM, 70

GA INST TECHN- SOUTHERN TECH, 70

GANNOH COLLEGE, 207

GARDEN CITY COMMUNITY JC, 102

GASTON COLLEGE, 187

GAVILAN COLLEGE, 36

GEMERAL MOTORS INSTITUTE, 124

GEHESEE COHHiUNITY COLLEGE, 179

GECRGE WASH UHIVERSITY, 62

GEORGE WILLIAMS ROIIFGF, 7\%

GEORGIA, UNIVERSITY OF, 70

GETTYBRUPE COLLEGE, LU

GLASSBORO STATE COLLEGE, 155

GLEN OAKS CMTY COLLEGE, 131

GLEYIDALE CMTY COLLEGE, 7,36

GODDARD COLLEGE, 245

GOVERHORS ST UNIVERSITY, 79

GRADUATE CNT. OF RP : $\varepsilon$ CNT. ENVTRONMENT $\varepsilon$ $M A, 58$

GRAND RAPIOS JR COLLEGE, 131

GRAND VALLEY ST COLLEGES, 124

GRAYSON CO JUNIOR COLLEGE, 235 
GREENVILLE COLLEGE, 79 GREENVILLE TECH COLLEGE, 219

GUAM, UNIVEPSITY OF, 73 GUILFORD TECHHIICAL IHST, 187 GULF COAST CMTY COLLEGE, 66 GUSTAVUS ADOLPHUS COLLEGE, 135

HALL INSTITUTE, 216 HAMILTO:N COLLEGE, 167 HARFCRD CO: $:$ : UUAITY COLLEGE, 111 HARRISEUTG AREA CC, 211 HARTFORD GRADUATE CENTER, 58 HARVARO UHIVERSITY, 114 HARVEY MUDD COLLEGE, 24 HASTIHSS COLLEGE, 148 HAHAII AT MANOA, $U$ OF, 74 HAWAII HONOLULU CC, U OF, 74 HALAII KAUAI CC, $U$ OF, 74 HEARTHOOD, I21 HINDS JUNIOR COLLEGE, 139 HOOSUCK IHISTITUTE, 121 HORRY-GEORGETOWHI TECH C, 220 HOUSTO!I BAPT UIIIVERSITY, 225 HOHARD UNIVERSITY, 62 HUIEOLDT STATE U, 25 HUNTINSTON COLLEGE, 88

IDAHO STATE UNIVERSITY, 75 IDAHO, UNIVEPSITY OF, 75 ILL CHICAGO CIPCLE, $U$ OF, 80 ILL ESTN CC OLNEY CEN C, 84 ILL ESTN LIHCOLN TPAIL C, 85 ILL UREAHA CAYYFUS, $U$ OF, 80 ILLIR:OIS CERITPAL COLLEGE, 85 ILLIROIS IPIST TECHYOLOGY, 81 ILLIRUIS STATE UNIVERSITY, 82 IHID NORTHEFH GRAD SCH MGIT, 88 IHO VOC TECH C- EVARISVILLE, 90 IND VOC TECH - SELLERSSURG, 90 IND- PURDUL U TORT HAYHE, O9 IHO- FLPOUE U INOIANAFOLIS, 89 IHDIANA U OF FENHSYLVAHIA, 207 IPIDIANA VOCATICHAL TECHIICAL COLLEGE-NW, 91 IFIDIANAFOLIS CENTER FOR ADVANCED RESEARCH, 91 IOHA STATE U SCI \& TECHN, 92 IO'AA, UNIVERSITY OF, 93

JACKSON COMMUHITY COLLEGE, 132 JAMES SFRUNT INSTITUTE, 187 JOHN A LOEAN COLLEGE, 86 JORDAN COLLEGE, 125

KATIKAKEE CMTY COLLEGE, 86 KAISAS MAIH CAMFUS, $U$ OF, 98 KANSAS ST U AGR \& APP SCI, 98 KAISAS TECHIIICAL IHIST, 102 KENT STATE U MAIN CAMPUS, 194 KENTUCKY, UHIVERSITY OF, 104 KEYSTOHE JUIIIOR COLLEGE, 211 KIRKL'OOD CMTY COLLEGE, 94 KUTZTOLIN STATE COLLEGE, 207

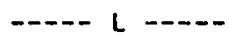

LA STATE U AND A C, 106

LA VERHE COLLEGE, 25

LAKELAIID CMTY COLLEGE, 197

LAKEWOOD CMTY COLLEGE, 138

LANSING COMIUNITY COLLEGE, 132

LAPAMIE CO CMTY COLLEGE, 266

LAHRENCE IHST TECHNOLOGY, 126

LAL'RENCE UNIVERSITY, 257

LEE COLLEGE, 235

LEHIGH CO CMTY COLLEGE, 212

LEWIS UIVIVEPSITY, 82

LINN-BEHTON CMTY COLLEGE, 204

LOMA LITIDA UNIVERSITY, 25

LONG BEACH CITY COLLEGE, 36

LORAS COLLEGE, 93

LORD FAIRFAX CMTY COLLEGE, 250

LOS AYIGELES PIERCE COLLEGE, 36

LOS AYIGELES TRADE TECH COLLEGE, 36

LOUISIAIIA TECH UNIVERSITY, 106

LO'NELL, UNIVERSITY OF, 114

LOYOLA COLLEGE, 110

LOYOLA MARYIIOUNT U, 25

LUTHER COLLEGE, 93

LYNCHBURG COLLEGE, 247

- - M M - - -

MACOMB CO CC- SOUTH CAMPUS, 133

MAINE AT FORT KENT, $U$ OF, 108

MAIIIE AT ORONO, U OF, 108

MAIHHATTAN COLLEGE, 167

MAHKATO STATE UNIVERSITY, 136

MARIN, COLLEGE OF, 37

MARLBORO COLLEEE, 245

MAPGUETTE UNIVERSITY, 257

MASS ATHERST CAMFUS, $U$ OF, 115

MASS INST OF TECH:IOLOGY, 115

MAYVILLE STATE COLLEGE, 191

MCHENRY COUNTY COLLEGE, 86

MO COLLEGE PARK CAM, U OF, 110

MEMPHIS STATE UNIVERSITY, 222

MERCED COLLEGE, 37

MERCER CO AREA VOC. TECH. SCHOOLS, 159 
MESA COLLEGE, 51

METROPOLITAN ST COLLEGE, 51 METROPOLITAN TECHNICAL CC, 149 MIAMI- DADE CMTY COLLEGE, 67 MIAMI, UNIVERSITY OF, 65 MICHIGAN STATE LNIVERSITY, 126 MICHIGAH TECH:OLLOGICAL U, 127 MICHIGAN- ARIN AREOP, U, 127 MICHIGAN- DEAREORH, U OF, 128 MICHIGAN- FLIHT, U OF, 128 MID MICHIGAN CMTY COLLEGE, 133 MIO PLAIHS CC-N PLATTE, 149 MIDDLE TENH ST UNIVERSITY, 222 MIDDLEBURY COLLEGE, 245 MIDDLESEX COUNTY COLLEGE, 159 MIDLAND LUTHERAN COLLEGE, 148 MIDLANDS TECH COLLEGE, 218 MILWAUKEE AREA TECH C, 262 MILWNUKEE SCH ENIGIHIEERII:G, 257 MINN MNIFLS SIIT PAUL, $U$ OF, 136 MINIJESOTA DULUTH, $U$ OF, 136 MISS CO CMTY COLLEGE, 11 MISSISSIPPI ST UIIIVERSITY, 139 MISSUUR 5 THN ST CULLEGE, 141 MISSOURI- COLUNSIA, U OF, 141 MIIJOOURI- KAHJA3 CITY; U OF, 142 MISSOUKI- ROLLA, U OF, $14 \hat{2}$ MISSOLRI- SAINT LCUIS, U OF, 143 MODESTO JUNIOP COLLEEE, 37 MOHAVE COMIUNITY COLLEGE, 7 MUHASEK VLY CMTY COLLEGE, 179 MOIROE COIMIUNITY COLLEGE, 180 MONTANA C MINPL SCI- TECHN, 146 MONTANA STATE UNIVERSITY, 146 MONTANA, UNIVERSITY OF, 146 MONITCLAIR STATE COLLEGE, 155 MONTEVALLO, UHIVERSITY OF, 2 MOORHEAD STATE UNIVEPSITY, 137 MOORPARK COLLEGE, 37 MIORAINE PARK TECH IHIST, 262 MORAINE VLY CMTY COLLEGE, 86 MOTLOW STATE CMTY COLLEGE, 223 MOUNT SAH ANTOHIO COLLEGE, 38 MT SAN JACINTO COLLEGE, 33 MUSCATIPIE CMTY COLLEGE, IS NUSKINGUM AREA TECH C, 198

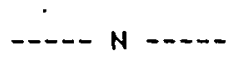

NAPA COLLEGE, 38 NASSAU COUITY BOLES, 183 NATIONAL TPAINIYG FUND, 62 NAVARPO COLLEGE, 235

NAZARETH O DF ROCHFSTFR, 168 NC AGRL \& TECH STATE U, 185 NC AT ASHEVILLE, U OF, 185 NC AT CHARLOTTE, $U$ OF, 185 NC AT GREEIISBCRO, U OF, 185 IIC STATE U RALEIGH, 186 ND STATE SCHOOL SCIEICE, 191 ND STATE U MAIH CAIIPUS, 191 MEBRASKA - LIHICOLN, U OF, 148 NEVADA LAS VEGAS, U OF, 150 NEVADA REIIO, U OF, 150 NEW COLLEGE OF CALIFCRNIA, 25 NEW EHGLAHD CENTER FOR APPROPRIATE TECH., 154
NEW ENGLAND COLLEGE, 153

NEH ENGLAND FUEL INSTITUTE, 213

NEH HAVEN, UNIVERSITY OF, 58

NEH MEXICO HIGHLAYIDS U, 162

NEW ORLEANS, UHIVERSITY OF, 106

NEW YORK UNIVERSITY, 168

NEHCASTLE COUNTY VOCATIOHAL TECHNICAL SCHOOL, 61

NH PLYMOUTH ST COLLEGE,U, 153

HH VOC- TECH C MANCHESTER, 153

NH VOC- TECH C NASHUA, 154

NHAW - HOHE STUDY IHSTITUTE, 198

NICOLET COLLEGE- TECH IIST, 258

NJ INSTITUTE TECHHOLOGY, 156

NM MAIN CALIFUS, $U$ OF, 162

NIM STATE U MAIN CAMPUS, 163

NORTH ADAFIS STATE COLLEGE, 116

NORTH CENTRAL TECH INST', 263

NORTH FLORIDA, U OF, 66

NORTH LAKE COLLEGE, 230

MORTH SEATTLE CC, 253

NORTH SHORE CMTY COLLECE, 120

NORTH TEXAS ST UNIVERSITY, 225

NOP THAMPTON CO AREA CC, 212

NORTHEAST INSTITUTE OF INDUSTRIAL TECHNOLOGY, 122

NOR THEASTERN UHIVERSITYY, 117

NORTHERN ARIZ UNIVERSITY, 6

NORTHEFIS COLORADO, U OF, 51

HORTHERIN ILL UNIVERSITY, 82

NORTHERN IOHA, $U$ OF, 93

NOPTHERH KY UNIVERSITY, 104

NORTHEPN MICH UNIVERSITY, 128

NORTHERN NM CORMUHITY COLLEGE, 164

NORTHERN VÁ CHTY COLLEGE, 250

NORTHLAYID PIOHCER COLLEGE, $\theta$

NORTHPOP UHIIVEPSITY, 26

NORTHHEST CHTY COLLEGE, 266

NORTHIHEST TECH COLLEGE, 198

HORTHHESTERH U:JIVERSITY, 83

NOTRE DAME, UHIVERSITY OF, 89

HY INST TECHP MAIN CAMPUS, 103

$---0$

OAKLAHD UNIVERSITY, 129

OCEAH COUHTY VOGATIONAL TECHMICAL SCHOOLS, 159

ODLSSA COLLEGE, 239

OFFICE OF APPFOFRIATE TECHNOLOGY, 43

OHIO NQRTHEPH UNIVERSITY, 195

OHIO STATE U MAIN CAIIPUS, 195

OHIO STATE U MAHSFIELD BR, 195

OHLDIE COLLEGE; 3 \%

OKLA STATE U MAIN CAPIPUS, 199

UKLAHUMA HOPHIAP CAM, U OF, 201

OLD DOHINIOH UHIVERSITY, 247

OLYMPIA TECH CMTY COLLEGE, 254

oraneE CO CHITY COLLEGE, 180

OPAHGE COAST COLLEGE, 39

OREGCH IHST OF TECHIOLOGY, 202

OREGON MAIH CAIIPUS, U OF, 202

OREGOH STATE UHIVERSITY, 203

OTERO JUIVIOR COLLEGE, 56

OUR LADY OF LAKE U, 225 
PA ST U SHENANGO VLY CAM, 207

PA STATE U ALLENTO'NH CAM, 208

PA STATE U CAPITOL CAMFUS, 213

PA STATE U MAIH CAMFUS, 208

PACIFIC UUIVERSITY, 203

PAMLICO TECHIICAL INST, 189

PARKEPSBURG CMIY COLLEGE, 255

PASADEHA CITY COLLEEE, 39

PASSAIC SCHOOL OF DRAFTING, 160

PENIHSULA COLLEGE, 253

PEIHSYLVAHIA IR!STITUTE OF TECHNOLOGY, 213

FEHINSYLVANIA,U OF, 208

PENSACOLA JUNIOR COLLEGE, 67

PHILA COLLEGE OF ART, 209

PIEC:ONT TECH COLLEGE, 218

PINELLAS VO-TECH IISSTITUTE, 68

PITTSBLIFG ST UHIVERSITY, 101

POLYTECH:IIC INST NEH YORK, 169

Portland Vocational Center, 109

POTOMAC STATE COLLEGE, 256

PR MAYAGUEZ,U OF, 215

FRINCE TON UHIVERSITY, 156

PRIRICIPIA COLLEGE, 83

PURDUE U MAIN CAHFUS, 89

RATAFO C OF NEW JERSEY, 157

RAHDOLPH TECHHICAL IHST, 189

RAHSER JUIICR COLLEGE, 239

REDLAFDS, U:IIVERSITY OF, 28

RED:HOODS, COLLEGE OF THE, 39

RENSSELAER FOLY IHSTITUTE, 169

RETS TECH CEIITER, 112

RHCDE ISLAND, U OF, 216

RICE LHIVEF.SITY, $2 \hat{5} 5$

RIO SALACO COMHULIITY COLLEGE, B

RIFON COLLEEE; 258

RIVERSIDE CIYY COLLEGE, 40

ROCHESTER CMTY COLLEGE, 138

ROCHESTER IHST TECHI:LLOGY, 169

ROCHESTEP, LUIIVEFSITY OF, 169

ROCKLARD CMTY COLLEEE, 180

RCGUE CCHMUITY COLLEGE, 205

ROSE-HULMAYH IHST OF TECHI, 90

RUTGERS U NEW BRUNSHICK, 158

SACRAMEITO CITY COLLEGE, 40 SAGINAW VLY STATE COLLEGE; 129 SAIHT BOIIAVEHTURE U; 170 SAIHT CLOUD ST UHIVERSITY, 137 SAINT JOH: FISHER COLLEGE, 170 SAINT JOSEEFH COLLEGE, 59

SAINT LOUIS CC-MEFAMEC, 145

SAINT MICHAEL'S COLLEGE, 245

SAIHT OLAF COLLEGE, 137
SAIHT TERESA, COLLEGE OF, 137

SAINT VIMCENT COLLEGE, 209

SALEM COUNTY VOCATIONAL TECHNICAL SCHOOLS, 160

SAMFSON TECHNICAL INST, 188

SAN BERNARDINO VLY COLLEGE, 40

SAN DIEGO CC- CITY COLLEGE, 40

SAH DIEGO CC- EVENING COLLEGE, 40

SAN DIEGO CC- MESA COLLEGE, 41

SAN DIEGO STATE U, 28

SAN FRANCISCO STATTE U, 28

SAN FRAHCISCO, U OF, 28

SAN JOAQUIN DELTA COLLEGE, 41

SAIJ JOSE CITY COLLEGE, 42

SAN JOSE STATE U, 29

SAHGAHON STATE UNIVERSITY, 83

SANTA ANA COLLEGE, 42

SANTA CLARA, UNIVERSITY OF, 29

SAITTA FE CIITY COLLEGE, 68

SANTA FE, COLLEGE OF, 163

SARATOGA-WHRREN BOCES-MIYERS OCC. CNT., 183

SC AT SUHTER, U OF, 220

SC MAIN CAMFUS, U OF, 217

SCHOOLCPAFT COLLEGE, 133

SCOTT COMHUNITY COLLEGE, 95

SD MAIN CAMPUS, $U$ OF, 221

SD SCH MINHES \& TECHNOLOGY, 221

SD STATE UNIVERSITY, 221

SEQLOIAS, COLLEGE OF THE, 42

SHELTER INSTITUTE, 109

SHERIDAN COLLEGE, 266

SIERRA COLLEGE, 42

SIHCLAIR CHTY COLLEGE, 197

SISKIYOUS, COLLEGE OF THE, 42

SHT CLAIR CO CMTY COLLEGE, 133

SOLAR STORE INC, 87

SOLAR TECHIICIAN TRAINING PROGRAM - OFFICE OF , 43

SOLARCON, 43

SOUTH FLOPIDA TECHNICAL INSTITUTE, 69

SCUTHEAST CC MILFORD CAM, 149

SOUTHEAST MO ST UHIVEPSITY, 143

SOUTHERN CALIFORHIA, U OF, 29

SOUTHERN ILLIHOIS U CAPBOHDL, 84

SOUTHERN ILLIHOIS U EOWARDSVL, 84

SOUTHERN METH UNIVERSITY, 226

SOUTHEPH MISSISSIPPI, $U$ OF, 139

SOUTHERN HEW JERSEY OIC, 161

SOUTHSIDE VA CMTY COLLEGE, 251

SOUTHWIESTERN COLLEGE, 43

SOUTHHESTEPN MICH COLLEEE, 134

SOUTHLEESTERH HO ST UHIVERSITY, 143

SOUTHWESTERH TECH II!ST, 189

SFARTAIISURG TECH COLLEGE, 220

SFOKAIJE FLS CIITY COLLEGE, 254

SFR IHIGFIELD COLLEEE, 117

SFRINGFIELD TECHIICAL CC; 121

STAHFORD UIIIVERSITY, 30

STAHLY TECHNICAL IHST, 188

STHESTN MASS UNIVERSITY, 118

STHLST TEX ST UHIVEPSITY, 226

STOCKTON STATE COLLEGE, 159

SUMITER AREA TECH.COLLEGE, 220

SUR.j STRLICTURES, Ij'4

SUIIY AERL \& TECH C CANTOH, 181

SUIYY AGPL \& TECH C DELHI, 181

SUHAY AT ALBAHY, 170

SUIYY AT BIH:GHAMTOH, 170

SUHYY AT BUFFALO MAIN CAM, 171

SUIYY AT STONY BROOK MAIN CAM, 174 
SUNY C ENVRNMTL SCI- FORESTRY, 174

SUNY COLLEGE AT EROCKPCRT, 174

SUNY COLLEGE AT FFEDOHIA, 174

SUNY COLLEGE AT GENESEO, 174

SUNY COLLEGE AT OS'AEGO, 175

SUNY COLLEGE PLATTSEURGH, 175

SWARTHMORE COLLEGE, 209

SYRACUSE U MAIN CAMPUS, 176

- - $-\cdots+-$.

TACOMA COMMUNITY COLLEGE, 254

TECH INST OF ALAMAPICE, 190

TENPLE UNIVERSITY, 210

TENH AT CHATTANCOGA, $U$ OF, 222

TENIJESSEE KNOXVILLE, $U$ OF, $2 ? .2$

TEHINESSEE NASHVILLE, U CF, 222

TENNAESSEE TECHNOLOSICAL $U, \hat{\imath} \imath 2$

TEX ST TECH RIO GRAHDE, 240

TEYAS A UIIVEREITY, CCG

TEXAS A U MAIH CAMPUS, 227

IEXAS AT ARLINGTAH, II DF, 228

TEXAS AT AUSTIM, $U$ OP, 228

TEXAS AT DALLAS, U OF, 229

TEXAS AT EL PASO, U OF, 230

TEXAS CHRISTIAN U, 231

TEXAS STATE TECHHICAL INSTITUTE, 240

TEXAS TECH UNIVEPSITY, 231

THAMES VLY STATE TECH C, 60

THE ALTERNATE EHERGY INSTITUTE, 57

THE CAMBRIDGE SCHOOL - HESTON CNT. FOR OPEN

$E, 122$

THE QUINCY TECHNICAL SCHOOL, 87

THE SCHOOL OF LIVING, 214

THOMAS NELSN CMTY COLLEGE, 251

TOLEDO, UPIVERSITY OF, 195

TOHIPKINS-CORTLAND CC, 182

TONSON STATE UHIVEPSITY, 110

TRI-COLLEGE CONTINUIHG EDUCATION/NOCATIONAL

$T, 240$

TRI-COUNTY TECH COLLEGE, 218

TRI-COUHTY TECHHICAL IHST, 188

TRIAHGLE INSTITUTE OF TECHNOLOGY, INC, 214

TRIDENT TECHNICAL COLLEGE, 220

TRIHIDAD STATE JR COLLEGE; 56

TRINITY UNIVERSITY, 232

TRITOH COLLEGE, 87

TULANE U OF LOUISIANA, 106

TULSA, UNIVERSITY OF, 201

TYLER JUNIOR CULLEGE, 240

U

$U$ OF ALASKA KENAI $\mathrm{CC}, 3$

$U$ OF ALASKA NORTHWEST CC, 3

$U$ OF ALASKA TAHAHA VLY CC, 3

$U$ OF HOUSTON CEN CAMFUS, 233

UNION CO TECHIICAL INST, 160

UNION COLLEGE, $1 \%$

UHIVERSITY FOR MAN, 103

US AIR FORCE ACADEMY, 52

US MERCHANT MARINE ACAD, 177
US NAVAL ACADEMY, 110

UTAH STATE UNIVERSITY, 242

UTAH TECH COLLEGE SALT LK, 244

UTAH, UHIVERSITY OF, 242

$----V----$

VA POLY IPIST AND STATE U, 249

VA WESTEPN CMTY COLLEGE, 251

VALENCIA CMTY COLLEGE, 68

VAH:CE-GRAN'VL CHTY COLLEGE, 188

VANOEFBILT UNIVERSITY, 223

VASSAR COLLEGE, 177

VEFIICHST, CMTY COLLEGE OF, 246

VIRGIHIA COMHOHHEALTH U, 249

VIRGIHIA IIAIN CAHFUS, $U$ Ut, 249

VIRGINIA MILITARY INST, 250

$----H---$

WALSH COLLEGE, 196

WASHIIGTOH ST UHIVERSITY, 252

WASHIHIGTOH UHIVEPSITY, 144

WASHIPIGTCN, UHIVERSITY OF, 252

WAUKESHA COUINTY TECH INST, 264

WAYHE STATE UHIVERSITY, 129

WEBER STATE COLLEGE, 243

WEDSTER COLLEGE, 144

HEITTHCRTH IIIST OF TECH, 118

HESLEYAH U:JIVEPSITY, 59

WEST FLORIDA, U OF, 66

WEST TEXAS ST UNIVERSITY, 233

WEST VALLEY COLLEGE, 43

WEST VIPGIHIA UNIVERSITY, 255

WESTCHESTER CHTY COLLEGE, 182

WESTERN CONN ST COLLEGE, 59

HESTEFH IOHA TECH, 95

HESTERN FYY UNIVERSITY, 104

HESTERN MICH UHIVERSITY, 130

WESTERH MOITTAHA COLLEGE, 147

HESTEPN ST COLLEGE COLO, 52

WESTERN WIS TECH INST, 264

WESTERH WYO CHTY COLLEQE, 267

WESTHIISTER COLLEGE, 210

WICHITA STATE IIHIVEFSITY, 101

WIDEHER COLLEGE, 210

WTI.KES COLLEGE, 210

WILLIAMSPORT AREA CC, 212

WISCONSIN EAU CLAIPE, U OF, 258

WISTCHSIN GAEEN BAY, U OT, ESO

WISCOISIN IHDIAN VOCATIOHAL, TECH., AND

AUULT, 264

HISCONSIN LA CROSSE, $U$ OF, 258

WISCOPISIH MADISON, $U$ OF, 259

WISCOHSIN MILHAUKEE, $U$ OF, 259

WISCOHSIH OSHKIOSH, $U$ OF, 260

HISCCISIN PARKSIDE, $U$ OF, 261

WISCONSIN PIATTEVL, U OF, 261

WISCOISIN STEVEHS PHT, $U$ OF, 261

WISCONSIIN STOUT, $U$ OF, 261

WISCONSIN WHITEWATER, U OF, 262

WH1 MITCHELL COLLEGE LAW, 137 
WM RAINEY HARPER COLLEGE, 87 WORCESTER FOLY INSTITUTE, 118 WORCESTER STATE COLLEGE, 119

WSTN CAROLINA UNIVERSITY, 186

WYOMING, UHIVERSITY OF, 265

XAVIER UNIVERSITY, 196

$---Y---$

YALE UNIVERSITY, 59

YAVAPAI COLLEGE, 8

YORK TECHNICAL COLLEGE, 218

YOUNGSTOWN ST UNIVERSITY, 196 
THIS PAGE

\section{WAS INTENTIONALLY LEFT BLANK}


Index to Programs and Curricula

*Adult Continuing Edu., 84

*Air Cond. E Refrig., 36

*Day Prosram - Sol. Heat. Systems, 160

*Electrical Eng'r., 223

*Energy Program, 119

*Energy Resources \& Environ., 62

EEvivirur. Scl. and lech., 58

* Home Study Program, 198

* Mechanical Eng'r, 65

*Night Program - Sol. Heat Systems. Ifn

*Plumbing and Heating Tracles, 160

* Professional Development, 183

* Research in Heating and Photovoltaics, 14

* Sheet Metal - Apprentice, Journeyman, 62

* Social Ecology, 245

* Sol. Ener. Ed. for Installers, 87

*Sol. Ener. Theory - Heat., Vent., A/C Tech., 159

*Sol. Heat. Tech. Skills Training, 15

* Solar Ener. Main. and Tech, 40

* Solar Enersetics Technology, 95

* Solar Energy \& Desisn, 43

* Solar Energy Opticn, 121

* Solar Engineering Technology, 32

* Solar Heating of Buildings, 61

* Solar Studies-Blda. Heat. and Photov., 153

* Solar Technician, 33, 35, 42

* Solar Technician Training Fragram, 13

* Training Program for Installers, 43

*Vocational - Technical, 71

$----A-\cdots$

A/C and Refrig. - 501. Ener. Option, 239

Air Cond. Heat. E Refrig., 251

Air Cond., Heat., and Vent., 38

Air Cond., Heat., Refrig., and Sol. Tech., 40

Air Conditioning E Refrig. Tech., 240

Air Conditioning Engineering Technology, 67

Air Conditioning-Pefrigeration, 220

Air Conditioning, Heatiny, and Refrig., 188

Alt. Ener. Emph. - Urban, Envirnn. Sturls., 124

Alternate Energy Technologies, 212

Alternative Energy, 157

Appl. Fhysics - Solar Ener. Option, 114

Appropriate Technology, 103

Arch. Draft., 131

Arch. Tech. - Solar Heating Option, 56

Arch.-Energy Conscious Design, 92

Architects \& Energy, 194

Architecture, 98

Architecture/Urban Design, 21
Construction Energy Program, 84

Construction Tech./ Civil Tech., 181

Conversion of Solar Energy, 218, 219

D

Distributed Studies in Energy Science, 46

---- E -----

Econ.,Ener.-Formation of Horld Conscious, 25

Elec. Engin.-Emphasis on Energy, 199

Ener. Conserving Des. Elective Sequence, 21

Ener. Convs., Fower Systs. Engineering, 17

Energy and Environmental Studies, 125

Energy and Trans. Concentration, 155

Energy Conscious Desisn, 81

Energy Conservation, 184

Energy Conversion, 193

Elitryy Conversion and Power Systems, 5

Energy Conversion and Resources, 156

Energy Cunversiun Systeins, by

Eneray Enqinepring, 208, 252, 257

Energy Engireering Technology, 138, 223

Energy Institute, 165

Energy Management Sciences, 224

Energy Program, 115

Energy Prograin for High School Teachers, 139

Energy Related Topics, 166

Energy Systems, 217

Energy Systems Engineering, 6, 26

Energy Systems Technology, 119

Energy Technolgoy, 213

Energy Technology, 131, 247

Engineering Fhysics-Solar Emphasis, 143

Engineering Tech.-Solar Energy Option, 204

Envir. Des. -Intro. Sol. Ener. Systems, 33

Environiminal rontrol Technology, 178

Envirolunental Design, 108

Environmental Planning \& Management, 28

Envirommental Sci. (Alternate Energy), 175

Envirommental Systems Design, 191

External - Project Sunrise, 37 
Fac. Dev. Workshop in Energy Alternatives, 223

Facility Engineering Technician, 264

Faculty Dev. in Ener. for Ind. Ecis., 186

General Occupational Technologies, 188 Graduate Prosram- Undergraduate Program, 227 Graduate Solar Energy, 21 H - - .

Heat Pumps ạnd Electives, 187

Heat., Vent., A/C, 131

Heating-Solar Energy, 253

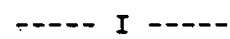

Industrial Powers, 155

Installing Solar Water Heaters, 122

Interdisciplinary Engineering, 231

Joint Inst. for Lab. Astrophysics, 47

$----\mathbf{L}--\cdot-$

Lab for Atmospheric and Space Phys, 47

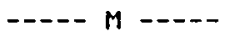

Major Qualifying Project, 118

Master of Fublic Administration, 66

Masters of Professional Managenent, 88

Mech. Engineering Tech. -Solar Option, 102

Mech. Engrg. \& Techuology, 156

Mechanical Engineering, 139.

Mechanical Engineering Technology, 6, 192

Mechanical Engr./Solar Energy, 110

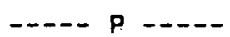

Physics-Specialization in Solar Energy, 170

Plumbing and Heating, 212

Power Technology, 75

Power/Energy Conversion Option, 247

Refrig., Climate Control and Clean Air, 112

Refrig., Heating and Air Conditioning

Technol, 123

Refrigeration and Air Conditioning-5olar

Ener, 219

Research in Solar Energy-Related Areas, 215

$----5----$

Self Sufficient Prog.-Solar, 116

Seminar in Solor Energy, 177

Senior Work in Solar Energy, 245

Sol. Ener. in Environ. Studs., 229

Sol. Envirn. Systs./Envir. Engnr., 14

Solar Architecture, 259

Solar Carpentry, 95

Solar Design/Engineering, 29

Solar Energy, 50, 75, 114, 124, 168, 189, 262

Solar Energy and Energy Sciences, 46

Solar Energy Applications, 50

Solar Energy Center, 202

Solar Energy Certificate Program, 153

Solar Energy Conversion, 142

Solar Energy Design, 160

Solar Energy Dynamics, 137

Solar Energy Engineering, 230

Solar Energy Graduate Prog., 232

Solar Energy I and II, 94

Solar Energy Installers/llachanics, 235

Solar Energy Meteor. and Train. Site, 170

Solar Energy Principles and Applications, 83

Solar Energy Program, 245

Solar Energy Research Program, 259

Solar Energy Seminar, 216

Solar Energy Solar Systems, 66

Solar Energy Ss'stems, 214, 247

Solar Energy Systems Specialist, 234

Solar Energy Systems Technology, 234

Solar Energy Systems-Res. and Comm.

Construct, 189

Solar Energy. Technician, 238

Solar Energy Technology, 8, 11, 64, 67, 102,

$151,177,179,266$

Solar Energy Unit Installer Program, 161

Solar Energy Workshop, 11, 75

Solar Energy-Instl. and Main., 53

Solar Energy: Fundamentals and Construction, 186 
Solar Eng'r Technology, 66

Solar Engineering, 18, 163

Solar Engineering Technology, 45, 211, 235

Solar Greenhouse Construction, 162

Solar Heating, 71, 191, 211

Solar Heating, and Cooling Technology, 207

Solar Power, 51, 239

Solar Systems, 150

Solar Systers Technology, 95

Solar Technicial Training Program, 149

Solar Technology, 3i, 243

Solar-related Courses, 228

Solar/Appropriate Technology, 47

Space Solar Power Research, 225

Special Probs. in Solar Energy, 29

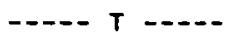

Technology for Industry-Energy, 82

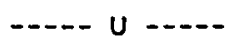

Use of Sol. Ener.-Homeowners, Builders, 95 